\title{
Developing a scalable synthesis of Peloruside A
}

by

Amira Brackovic

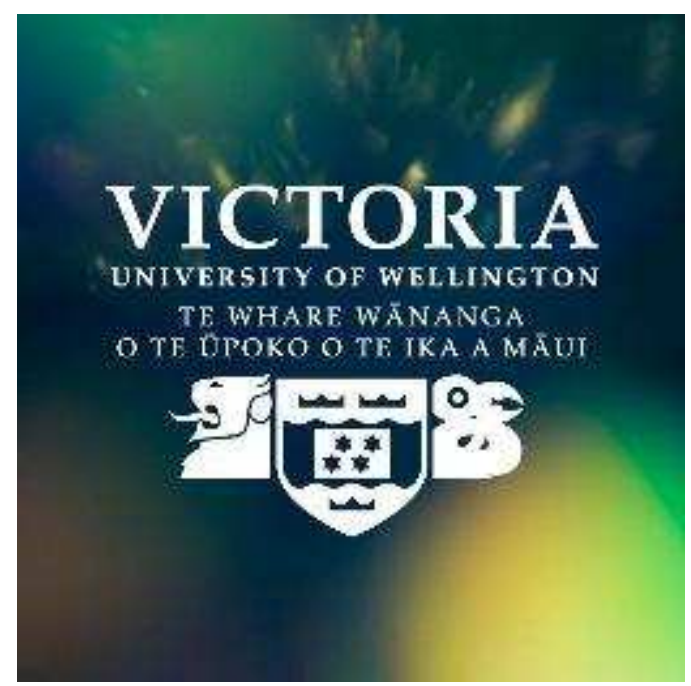

A thesis submitted to Victoria University of Wellington in fufillment of the requirements for degree of Doctor of Philosophy in Chemistry 



\section{Abstract}

Peloruside $A(P e I A, 1)$ is a marine natural product isolated from a sponge Mycale hentscheli found in Pelorus Sound, New Zealand. It is a microtubule-stabilising agent, active against various cancerous cell lines at nanomolar concentrations and offers several advantages over the current drugs on the market due to its unique mode of microtubule stabilisation, its potency and its activity in multidrug resistant cells. Since large-scale isolation of the compound from the sponge is unsustainable and an attempt to grow the sponge failed due to a sea-slug infestation, devising an efficient synthesis of peloruside $A$ that will be able to deliver larger quantities of this compound is essential in order to conduct further studies and enable the eventual manufacture of the drug.

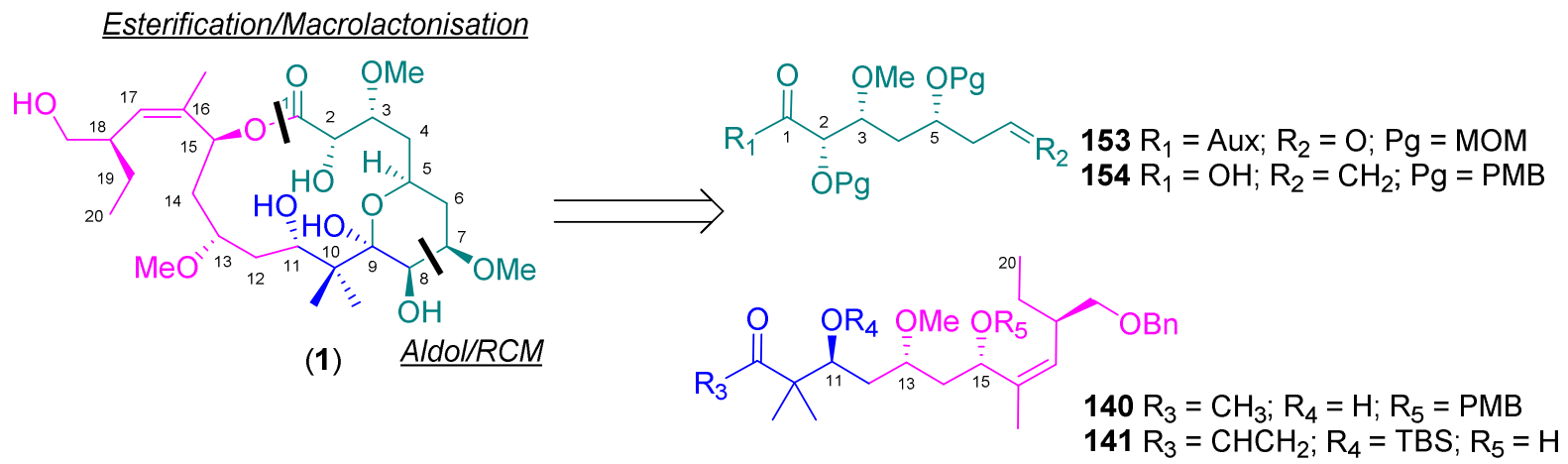

Peloruside $A$ is also a very interesting synthetic target as a macrolide with ten stereogenic centres, an internal pyran ring and a trisubstituted Z-double bond. Our synthetic strategy combines elements from previous total and partial syntheses with novel elements with an aim to make the synthesis more efficient. The synthesis of the side-chain fragment (C12C20) was based on Evans' methodology ${ }^{1}$ which was also utilised to couple this fragment with the $\mathrm{C} 8-\mathrm{C} 11$ fragments. It was envisioned to evaluate two different end-game strategies, and to this end it was necessary to synthesise two different versions of the C8C11 fragment. However, the synthesis of the C1-C7 fragments proved to be quite challenging and required a lot of alterations to the synthetic plan and the protecting group strategy. Various routes based on previous syntheses by Ghosh, Jacobsen and Taylor were explored. ${ }^{2-4}$ Eventually, the key intermediate was synthesised using a modified Taylor methodology. Our future work will focus on optimising and establishing fragment coupling 
methodologies and evaluating the two end-game approaches: macrolactonisation and a ring-closing metathesis. 


\section{Acknowledgements}

There have been many individuals that have contributed to my experience at Victoria University of Wellington. I would like to start by extending my sincere gratitude to my supervisors Dr Joanne Harvey, Professor Peter Tyler, and Associate Professor Paul TeesdaleSpittle. Joanne, you are the best. Your knowledge and passion for chemistry together with your encouraging and supportive nature have gotten me through this process. Peter and Paul thank you for always being there to answer my questions, your insights have been greatly appreciated.

I could not have completed this PhD without proper funding and thus, I would like to thank John Stewart and the Kathleen Stewart Postgraduate Scholarship fund who have provided me with financial support during my time here.

Next, to lan Vorster who has provided countless hours of support on the NMR and mass spectrometer. Additionally, I would like to thank Jingjing, Sophie, and Ethan for running my mass spec for me.

To Claire, our wonderful postdoc, thanks for all of your help in the lab but most of all for your friendship.

To the past and present members of the Organic Synthesis group, my time here would not have been the same without you. In the order of appearance thank you to Hemi, Kalpani, Jingjing, Thomas, Sarah, Loic, Sophie, Helena, Jasmin, José Luis, Dan, Hedley, Matt F., Chris, Ash, Will, Tao, Ethan, Jamie, Jordan, Paris, Phoebe, Mike, Olly and Matt H. It has been a real pleasure working in the lab with you.

A special thank you to the AM 204 office members. You have provided many laughs and good times over my time here. And a special special thank you to all of you who have spoilt me and helped me in the final days of the thesis-writing.

Last but not least, a huge thank you to my parents and my sister, for supporting me and believing in me. 


\section{Table of contents}

Abstract

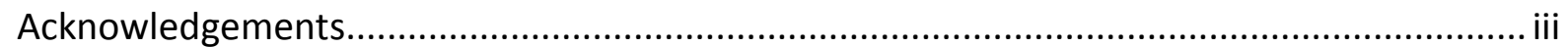

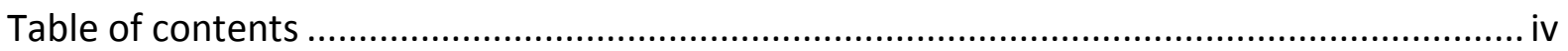

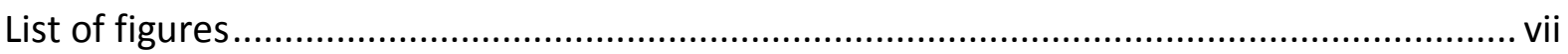

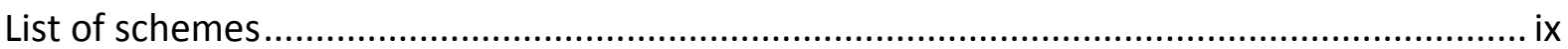

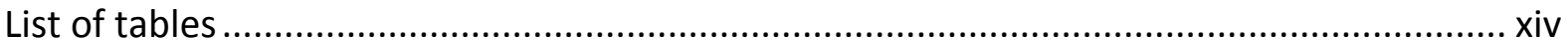

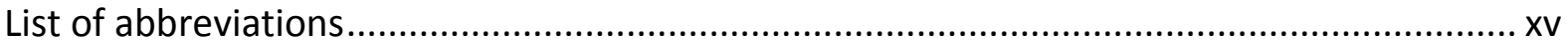

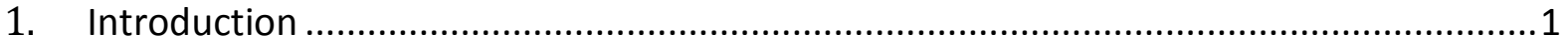

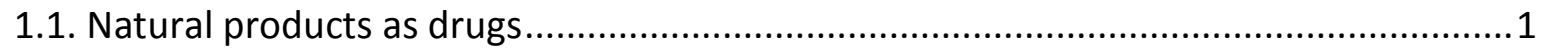

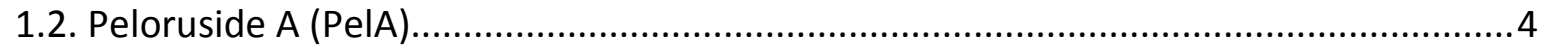

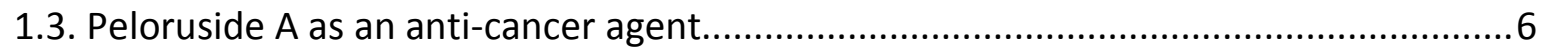

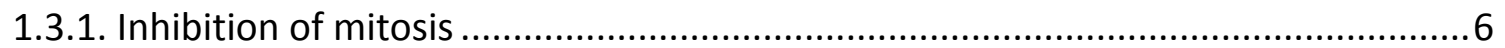

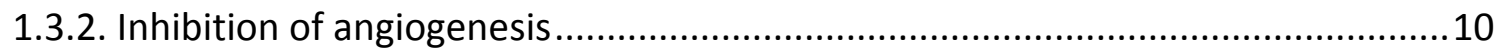

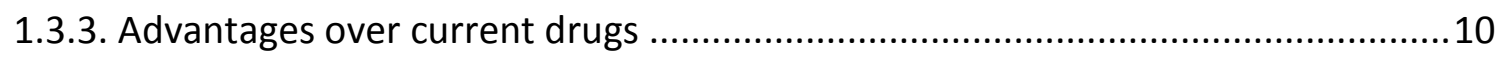

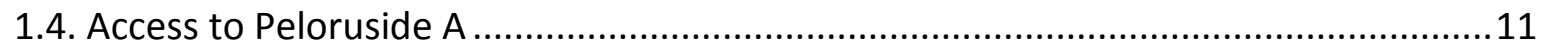

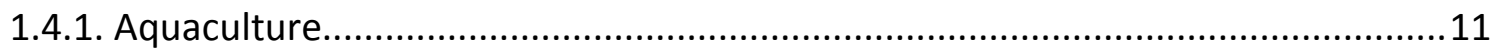

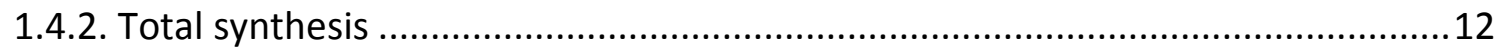

1.5. Thesis objective 1: devising a scalable synthesis of PelA …................................17

1.5.1. Overall approach to scalable synthesis of PelA ...................................................

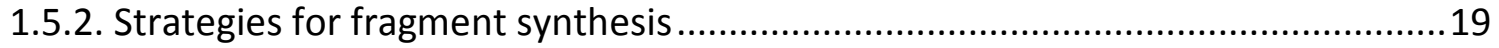

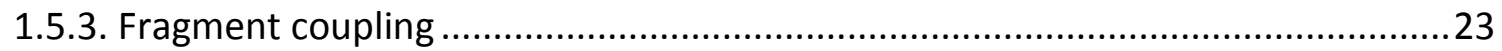

1.5.4. End-game strategy 1: Macrolactonisation approach ..........................................25

1.5.5. End-game strategy 2: Ring-closing metathesis approach..................................26

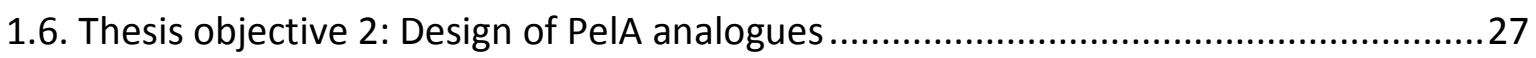




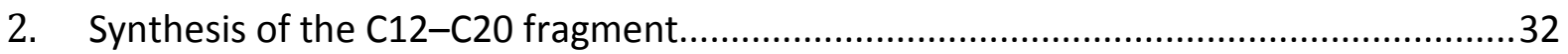

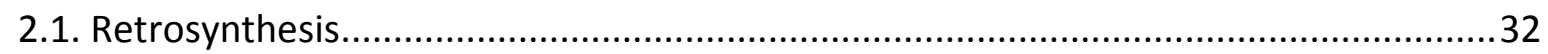

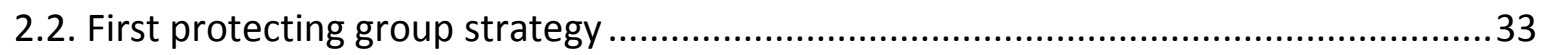

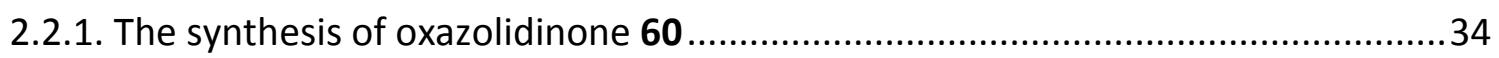

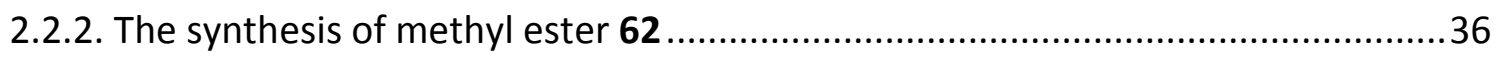

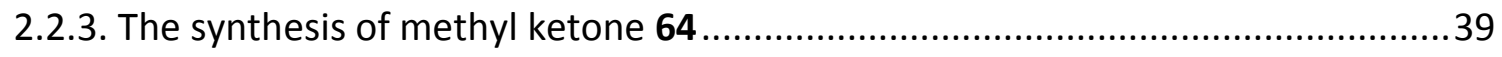

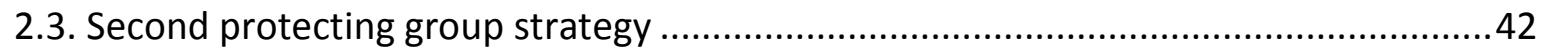

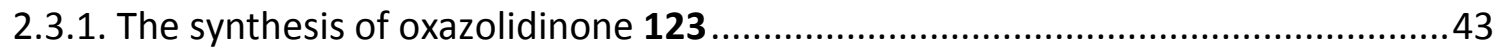

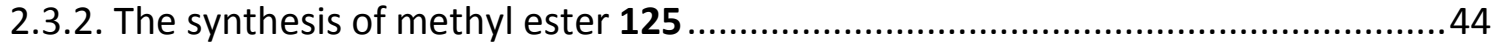

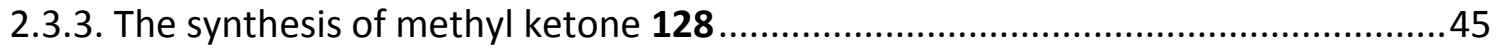

2.3.4. Determination of stereochemistry and the PMB protection ..............................51

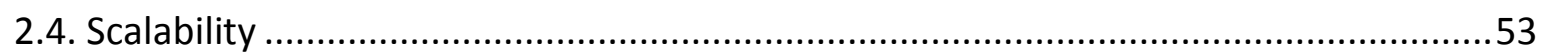

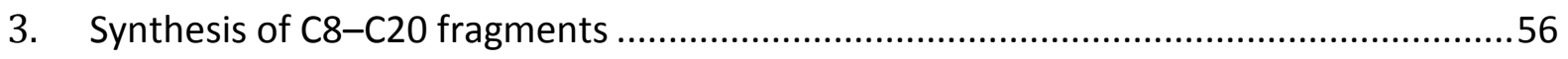

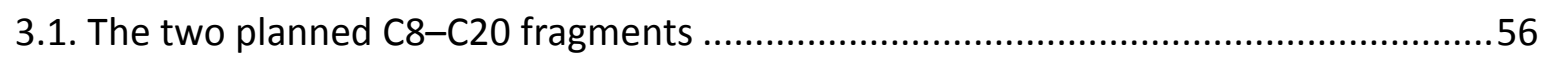

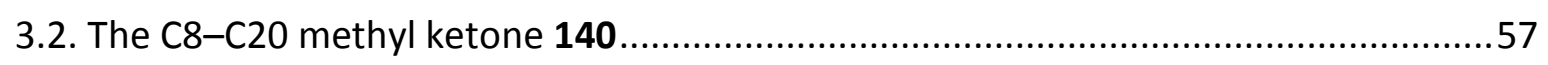

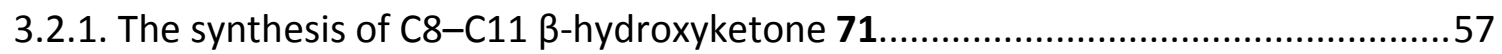

3.2.2. The aldol coupling and determination of configuration at $\mathrm{C} 11$...........................58

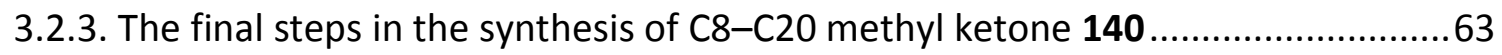

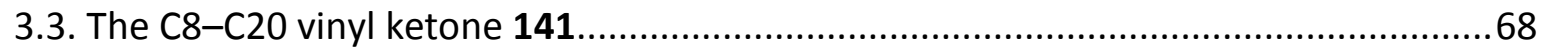

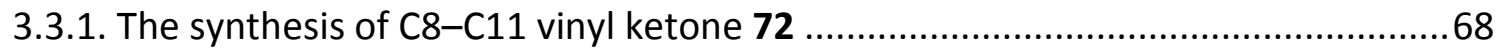

3.3.2. The attempted synthesis of C8-C20 vinyl ketone intermediate 143 ...................72

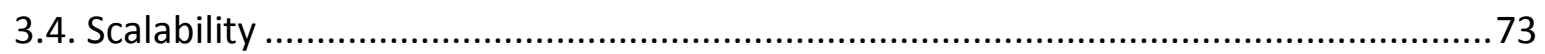

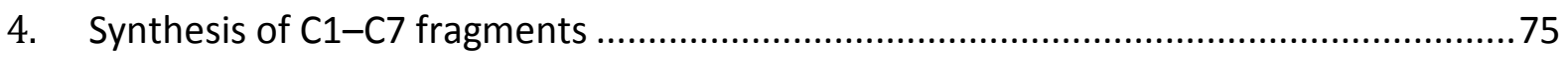

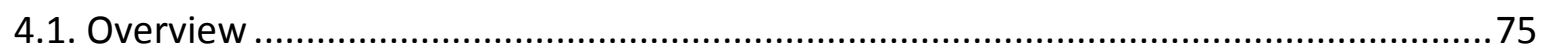

4.2. Ghosh-inspired approach: from (-)-2,3-O-isopropylidene-D-threitol ........................76

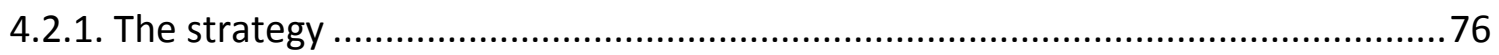




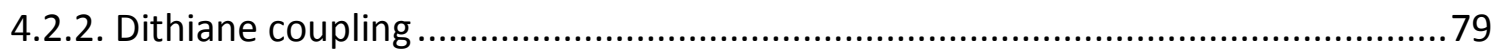

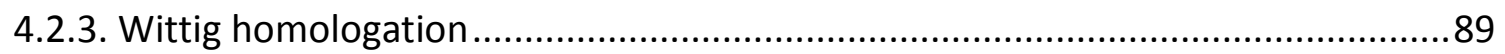

4.2.4. Ohira-Bestmann modification of the Seyferth-Gilbert homologation ..................94

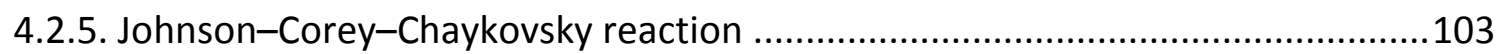

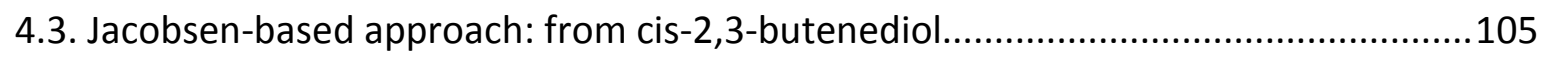

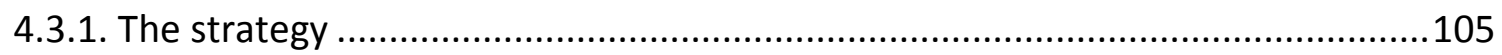

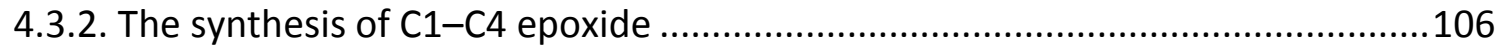

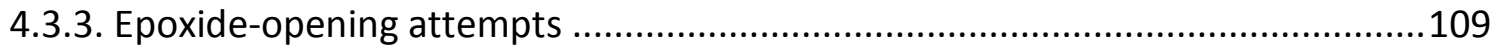

4.4. Taylor approach: from (S)-glycidyl tosylate ........................................................111

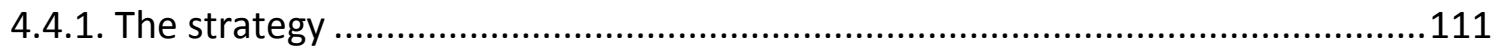

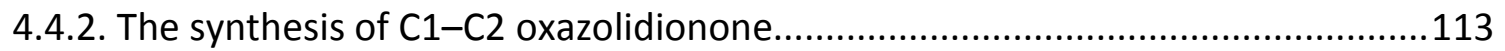

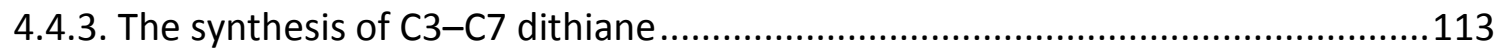

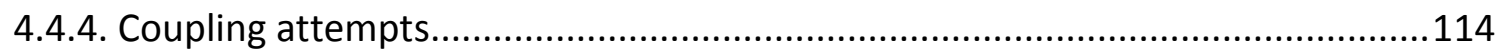

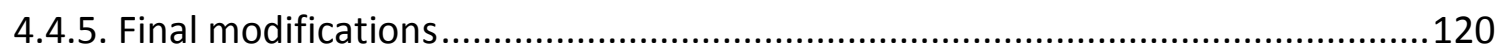

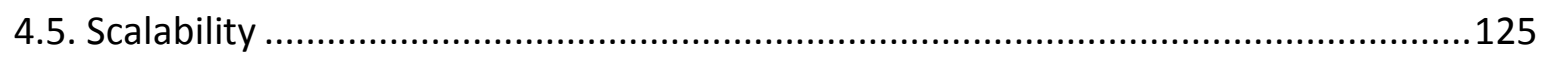

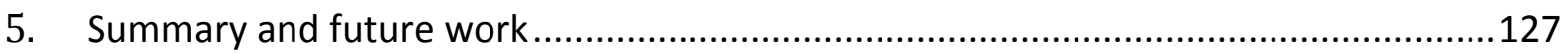

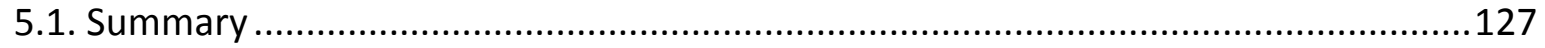

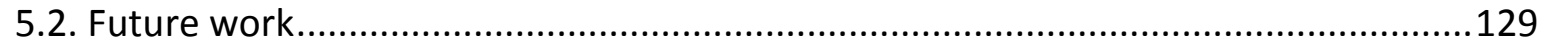

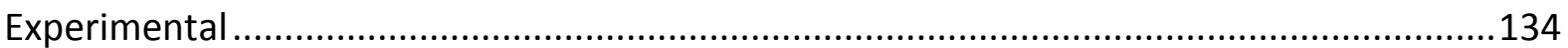

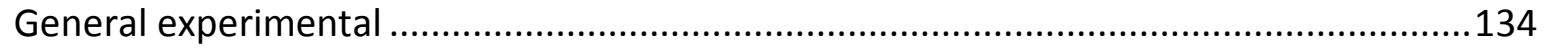

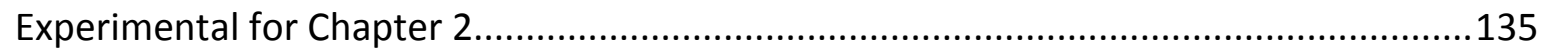

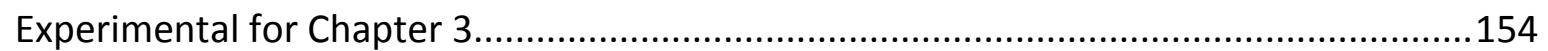

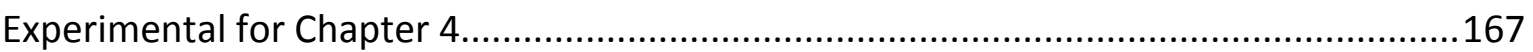

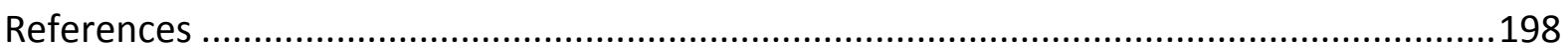

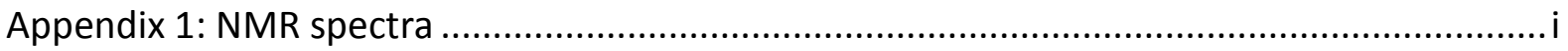




\section{List of figures}

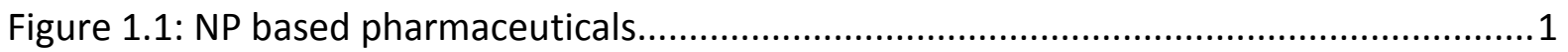

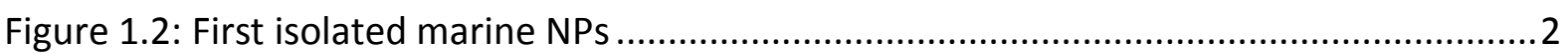

Figure 1.3: Marine NP derived anti-cancer drugs ................................................................

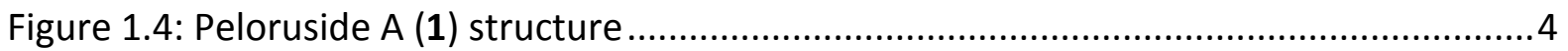

Figure 1.5: Selected examples of microtubule-targeting NPs ................................................

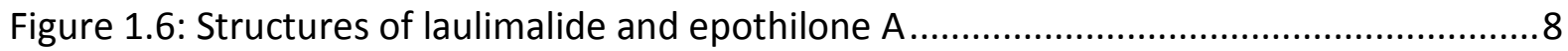

Figure 1.7: PelA and laulimalide binding to microtubules demontrated in a system comprised of $\alpha, \beta$-tubulin dimers, stathimin and TTL. ${ }^{29}$ Used with permission (2018) Copyright (C 2014 John Wiley \& Sons, Inc. All rights reserved.

Figure 1.8: PelA and laulimalide stabilisation of microtubules from $x$-crystal structure and modelling. ${ }^{29}$ Used with permission (2018).Copyright (C) 2014 John Wiley \& Sons, Inc. All rights reserved.

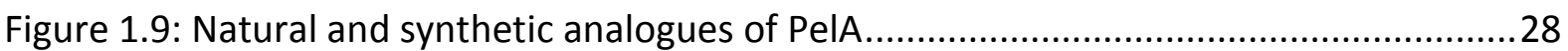

Figure 2.1: PelA structure with C12-C20 highlighted ...................................................... 32

Figure 2.2: ${ }^{1} \mathrm{H}$ NMR spectrum of the crude reaction mixture from the Ando olefination under Masamune-Roush conditions containing E/Z-62 (1:1.7 ratio).............................................38

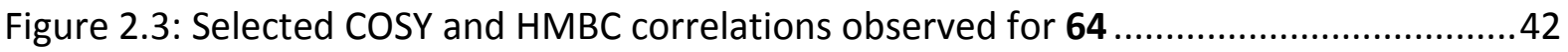

Figure 2.4: ${ }^{1} \mathrm{H}$ NMR of the crude mixture containing the major (123) and the minor (130) diastereomer

Figure 2.5: ${ }^{1} \mathrm{H}$ NMR spectrum of the recovered aldehyde 127 from the D-prolinol reaction.48

Figure 2.6: 2D NOESY spectrum of the isomerised aldehyde 127. .49

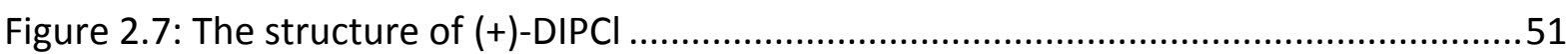

Figure 2.8: Mosher's ester analysis and the $\Delta \delta$ values obtained for compound $128 \ldots \ldots \ldots . . . .52$

Figure 3.1: PelA with C8-C20 fragment highlighted .........................................................56

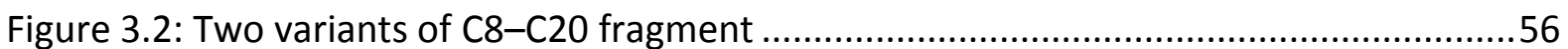

Figure 3.3: Goodman TS explaining the observed 1,5-anti induction ${ }^{139}$...............................60

Figure 3.4: Selected COSY and HMBC correlations observed for 142 ..............................61

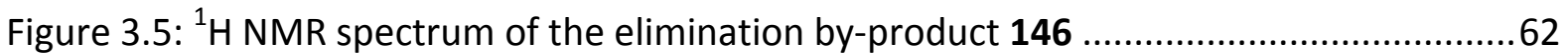

Figure 3.6: $\Delta \delta$ values obtained from ${ }^{1} \mathrm{H}$ NMR spectra of the two Mosher's esters 144 and 145 $\left(\Delta \delta=\delta_{S}-\delta_{R}\right)$ 
Figure 3.7: Diagrammatic representation of coupling constants and inferred relationships .65

Figure 3.8: Full structure after energy minimisation (truncated) .........................................66

Figure 3.9: Simplified structure after energy minimisation...............................................66

Figure 3.10: Newman projections based on the calculated angles ...................................67

Figure 3.11: Comparison of the proton spectra of the methyl ether 140 and diol 149 ........68

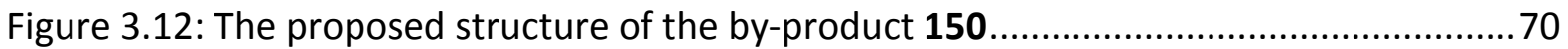

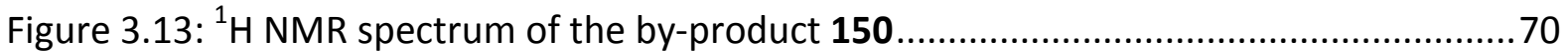

Figure 3.14: ${ }^{1} \mathrm{H}$ NMR spectrum of the crude reaction mixture after the attempted aldol

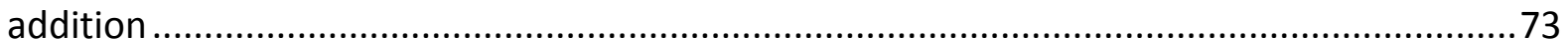

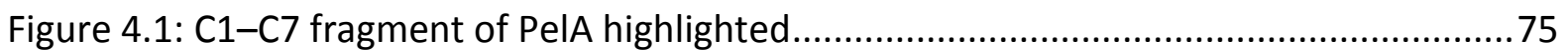

Figure 4.2: Target C1-C7 fragments using three different approaches ............................... 75

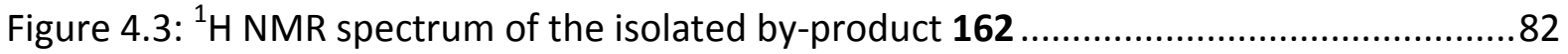

Figure 4.4: ${ }^{1} \mathrm{H}$ NMR spectra of a) sample containing by-product 163 and iodide $\mathbf{7 4} \mathrm{b}$ ) iodide

74 .83

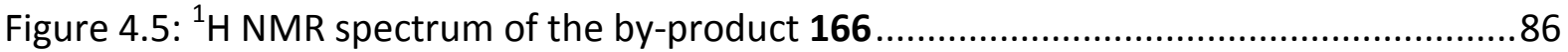

Figure 4.6: Part of the ${ }^{1} \mathrm{H}$ NMR spectrum of the methyl enol ether 173 ............................92

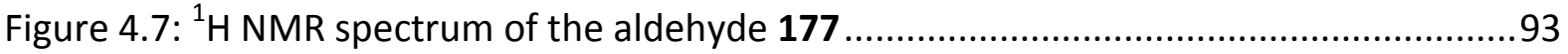

Figure 4.8: The lowest energy conformer of the diol 182 (conformer 1).............................98

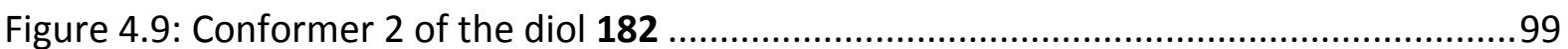

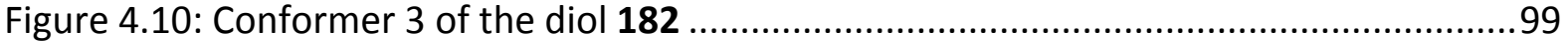

Figure 4.11: ${ }^{1} \mathrm{H}$ NMR spectrum containing alkyne starting material $\mathbf{1 8 0}$ and the protonolysis by-product 192

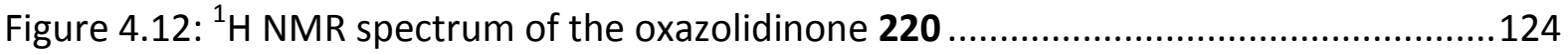




\section{List of schemes}

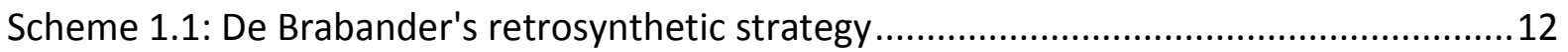

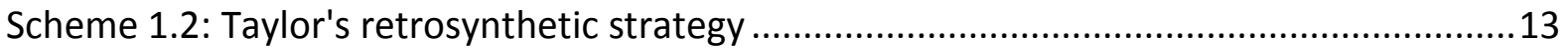

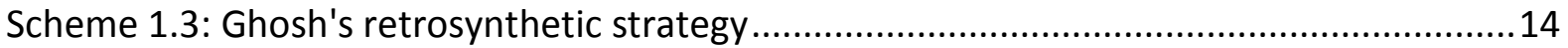

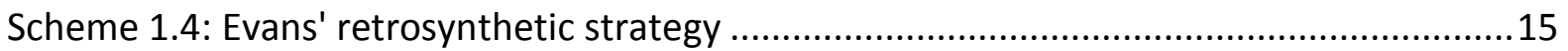

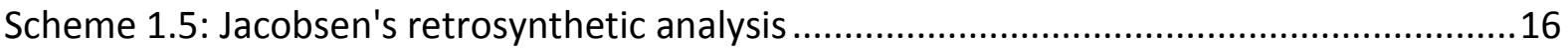

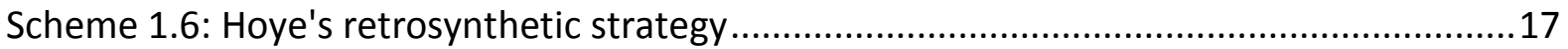

Scheme 1.7: Retrosynthetic approaches for the scalable synthesis of PelA .........................18

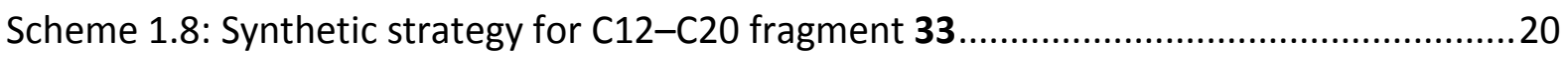

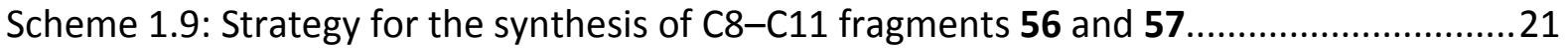

Scheme 1.10: Plan for the synthesis of C1-C7 fragments 52 and 53 ...............................22

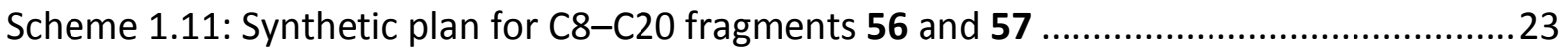

Scheme 1.12: C1-C20 complete carbon skeleton (91) of PelA through C7-C8 aldol coupling

Scheme 1.13: C1-C20 complete carbon skeleton (93) of PelA through C1 to C15

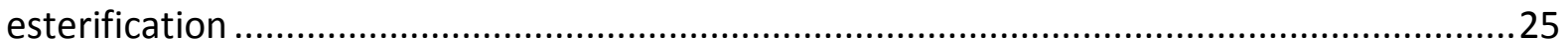

Scheme 1.14: PelA synthesis through Yamaguchi macrocyclisation ......................................25

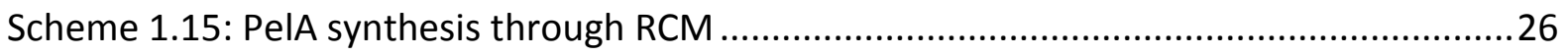

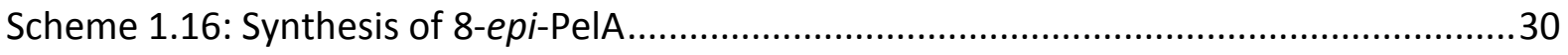

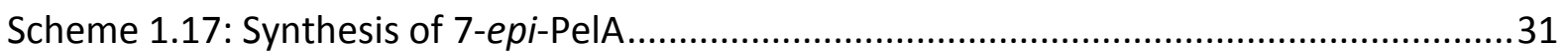

Scheme 2.1: Major disconnections for the C12-C20 fragment...........................................32

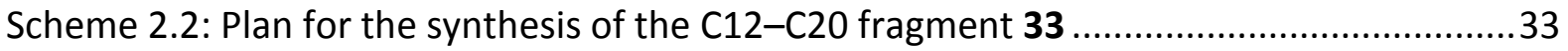

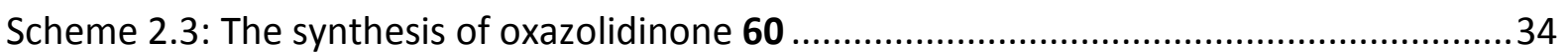

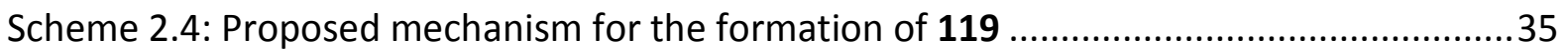

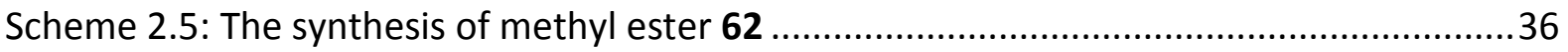

Scheme 2.6: a) General HWE olefination mechanism; b) Rationale for initial E/Z selectivity 37

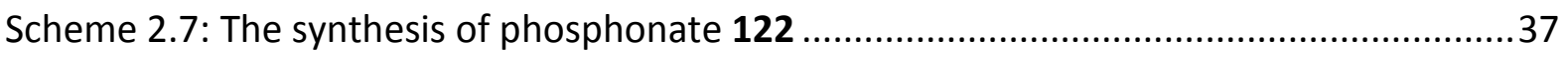

Scheme 2.8: Chelation effect under Masamune-Roush conditions ....................................38

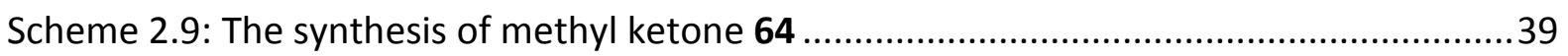

Scheme 2.10: The proposed mechanism of the L-proline catalysed aldol reaction ${ }^{110,113} \ldots . . . .40$

Scheme 2.11: D-Proline catalysed acetone aldol reaction ..............................................41 
Scheme 2.12: Second synthetic strategy for the synthesis of the side-chain fragment $129 . .43$

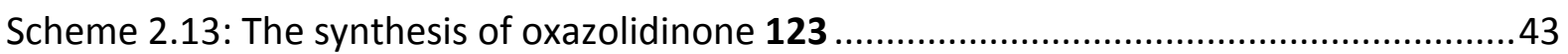

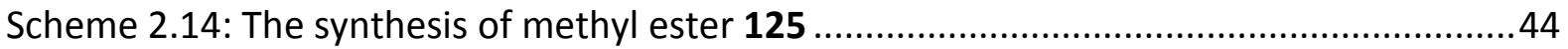

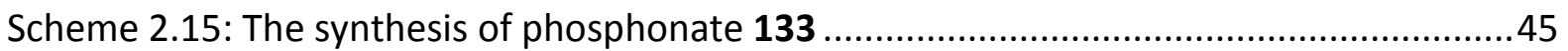

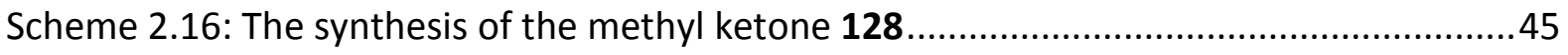

Scheme 2.17: Mechanism for the formation of the condensation product in the proline catalysed aldol reaction

Scheme 2.18: a) D-Prolinol; b) (R)-(+)- $\alpha, \alpha$-diphenyl-2-pyrrolidinemethanol catalysed reactions

Scheme 2.19: Proposed isomerisation mechanisms ............................................................50

Scheme 2.20: (+)-DIPCl transition state conformation ........................................................50

Scheme 2.21: The synthesis of Mosher's esters 136 and 137 and the complete side-chain

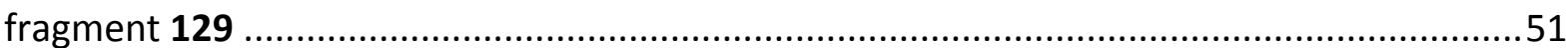

Scheme 2.22: Acetimidate to acetamide: the proposed rearrangement mechanism ............53

Scheme 2.23: Yields for each step in the synthesis of the side-chain fragment C12-C20 129

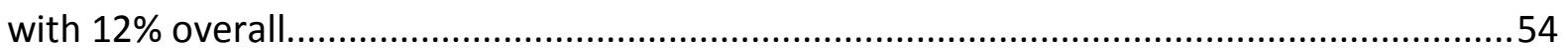

Scheme 3.1: Strategy for the synthesis of the C8-C20 intermediates 142 and $143 \ldots \ldots \ldots \ldots . . . .57$

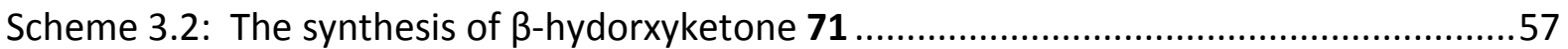

Scheme 3.3: The synthesis of the C8-C20 intermediate 142 through an aldol reaction........58

Scheme 3.4: Examples from the literature highlighting the importance of $\beta$-oxygenation in

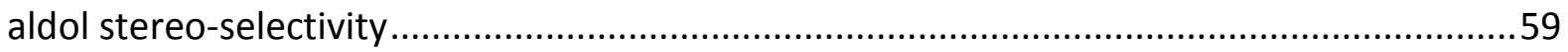

Scheme 3.5: The synthesis of Mosher's esters 144 and 145 .............................................61

Scheme 3.6: The synthesis of the methyl ketone C8-C20 fragment 140...........................63

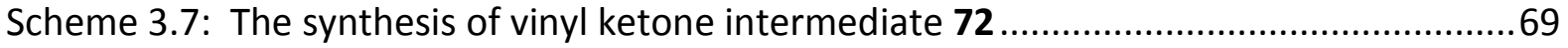

Scheme 3.8: a) benzyl deprotection; b) the proposed mechanism for the formation of $\mathbf{1 5 0} 71$

Scheme 3.9: Attempted aldol reaction for the formation of C8-C20 vinyl ketone 143 .........72

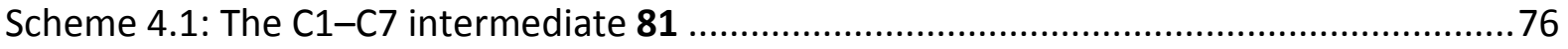

Scheme 4.2: The synthesis of the two C1-C7 fragments 52 and 53 ...................................77

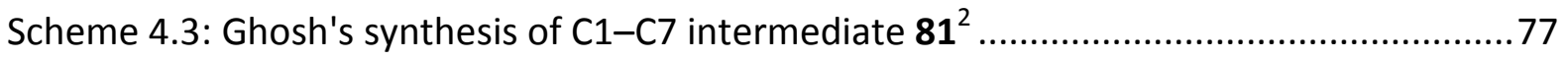

Scheme 4.4: Strategy for the synthesis of the C1-C7 intermediate 81..............................78

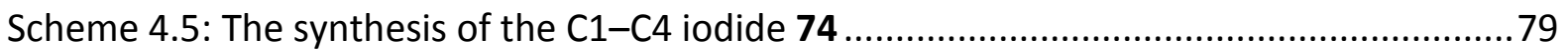

Scheme 4.6: Mechanism of the Appel reaction................................................................. 80 
Scheme 4.7: a) Normal carbonyl reactivity and b) reverse reactivity achieved by using dithiane.

Scheme 4.8: The synthesis of 2-allyl-1,3-dithiane 161

Scheme 4.9: Attempted coupling of the iodide 74 and the allyl dithiane 161 81

Scheme 4.10: Proposed mechanism for the formation of by-product 162 .82

Scheme 4.11: Mechanism for the formation of by-product 163 .84

Scheme 4.12: The synthesis of the bromide 164 .85

Scheme 4.13: The synthesis of the dithiane 165 .85

Scheme 4.14: Proposed mechanism for the synthesis of by-product 166

Scheme 4.15: The synthesis of the C1-C5 dithiane $\mathbf{1 6 9}$

Scheme 4.16: Regioselectivity of the methylation to give the $\mathrm{O} 2$ methylated product $171 . .88$

Scheme 4.17: Attempted synthesis of the dithiane $\mathbf{7 8}$

Scheme 4.18: Synthesis of the C1-C7 intermediate 176 through the Wittig homologation..90 Scheme 4.19: Mechanism of the Wittig reaction using the (methoxymethyl)triphenylphosphonium bromide.............................................................90

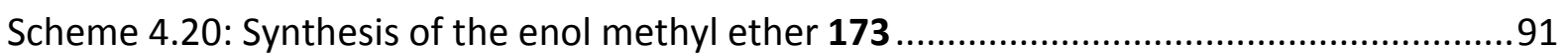

Scheme 4.21: Methyl enol ether conversion into aldehyde 177......................................92

Scheme 4.22: The proposed mechanism for the formation of 177 ....................................94

Scheme 4.23: Synthesis of the C1-C7 intermediate through Seyferth-Gilbert homologation

Scheme 4.24: a) Seyferth-Gilbert homologation mechanism; b) the Ohira-Bestmann reagent .96

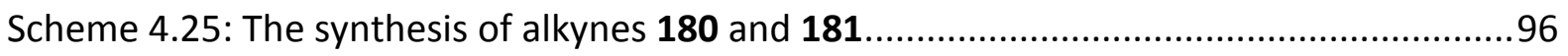

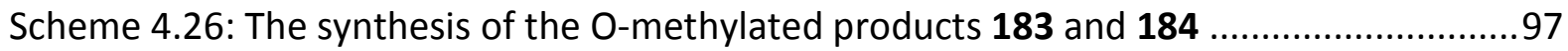

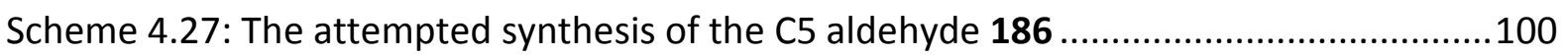

Scheme 4.28: Alkyne hydroboration/oxidation mechanism ..........................................100

Scheme 4.29: Results from hydroboration/oxidation attempts using different boranes.....101

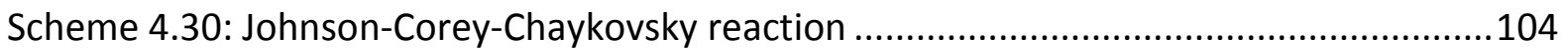

Scheme 4.31: Meinwald rearrangement mechanism ....................................................... 104

Scheme 4.32: Synthesis of the epoxide 193 and the attempted rearrangement to the aldehyde 194 105 
Scheme 4.33: Retrosynthetic analysis of the Jacobsen based plan for the synthesis of the C1-

C7 intermediate 198

Scheme 4.34: Strategy for the synthesis of the C1-C7 intermediate 198 from Jacobsen's epoxide 195 106

Scheme 4.35: The synthesis of the epoxide 195 107

Scheme 4.36: $m$-CPBA epoxidation mechanism 107

Scheme 4.37: a) the classic and b) the enantioselective versions of the Payne rearrangement 108

Scheme 4.38: Attempted synthesis of dithiane 196. 109

Scheme 4.39: Dithiane linchpin reaction mechanism 110

Scheme 4.40: Taylor's methodology for introducing the stereochemistry at C2 and C3 in a single step using an Evans aldol reaction 111

Scheme 4.41: Taylor's synthesis of the C1-C7 intermediate 208 .112

Scheme 4.42: Plan for the completion of synthesis of the two C1-C7 fragments.

Scheme 4.43: The synthesis of the oxazolidinone 28

Scheme 4.44: The synthesis of the dithiane 205 114

Scheme 4.45: Attempted synthesis of 206 114

Scheme 4.46: Methylative removal of the dithiane 115

Scheme 4.47: The synthesis of the a) aldehyde $\mathbf{2 1 3}$ and b) the oxazolidionone $\mathbf{2 1 4}$ 116

Scheme 4.48: Attempted coupling of the aldehyde $\mathbf{2 1 3}$ and the oxazolidinone $\mathbf{2 1 4}$ 116

Scheme 4.49: The synthesis of the C1-C7 oxazolidinone 216.

Scheme 4.50: Z-enolate formation

Scheme 4.51: Chirality transfer in reactions of metal enolates with a) alkyl electrophile b) aldehyde 118

Scheme 4.52: Enantioselectivity and diasteroselectivity in Evans aldol 119

Scheme 4.53: Result from the first attempt at the 03 methylation reaction 120

Scheme 4.54: Base-induced retro-aldol reaction of $\mathbf{2 1 6}$ 120

Scheme 4.55: The formation of the 2,3,5-tri-O-methylated product 218 with excess $\mathrm{Me}_{3} \mathrm{OBF}_{4}$ 121 Scheme 4.56: Proposed mechanism for the deprotection of PMB ethers in the formation of 218 121 
Scheme 4.57: The synthesis of the 03-methylated oxazolidinone $\mathbf{2 1 7}$ and the plan for the completion of synthesis of the two $\mathrm{C} 1-\mathrm{C} 7$ fragments

Scheme 4.58: The formation of the acetal 221 under MOM protection conditions

Scheme 4.59: Proposed mechanism for the formation of the acetal 221

Scheme 4.60: Efforts towards the synthesis of the C1-C7 fragments 153 and 154 .126

Scheme 5.1: Summary of the efforts towards the synthesis of two C8-C20 fragments

Scheme 5.2: Ghosh-inspired attempts at the synthesis of the C1-C7 fragments. 128

Scheme 5.3: Jacobsen-based attempt at the C1-C7 fragments synthesis 128

Scheme 5.4: The efforts towards the synthesis of the C1-C7 fragments 153 and 154 using Taylor's methodology 128

Scheme 5.5: The planned elaboration to the C8-C20 vinyl ketone fragment 141 130

Scheme 5.6: Macrocycle formation through RCM and final modifications to PelA 131

Scheme 5.7: Plan for the completion of the synthesis of PelA through macrolactonisation132 


\section{List of tables}

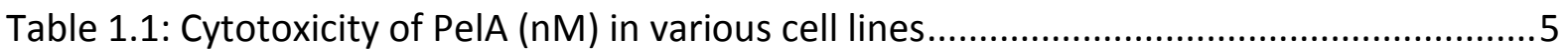

Table 1.2: Cellular growth inhibition $\mathrm{IC}_{50}$ values $(\mathrm{nM})$ in specified cell lines by PelA and its natural and synthetic analogues ${ }^{93}$ .29

Table 3.1: Chemichal shifts and coupling values observed for the silyl acetal 148. .65

Table 3.2: Calculated dihedral angle values for the silyl acetal 148 .....................................67

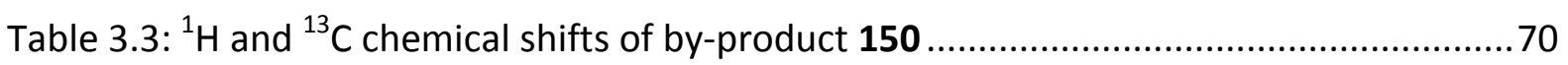

Table 4.1:Boltzmann percentage of different conformers of the diol 182 .........................98 


\section{List of abbreviations}

9-BBN

AD

$A E$

ALL

AML

Aux

aq.

B

BAIB

$\mathrm{Bn}$

$\mathrm{BOMCl}$

BRSM

CBS

conc.

COSY

CSA

d

DBU

DCC 9-borabicyclo[3.3.1]nonane

asymmetric dihydroxylation

asymmetric epoxidation

acute lymphocytic leukemia

acute myeloid leukemia

auxiliary

aqueous

base

(diacetoxyiodo)benzene

benzyl

benzyl chloromethyl ether

based on recovered starting material

Corey-Bakshi-Shibata

concentration

correlation spectroscopy

10-camphorsulfonic acid

doublet

1,8-diazabicyclo[5.4.0]undec-7-ene

$N, N^{\prime}$-dicyclohexylcarbodiimide 


\section{DCE}

DCM

DDQ

DIBAL-H

DIPCl

DIPEA

DMAP

DMF

DMP

DMPU

DMSO

DNA

E

$\mathrm{E} 1 \mathrm{cB}$

EA

eq./ equiv.

EWG

FDA

HBPin

HBTU

HMBC 1,2-dichloroethane

dichloromethane

2,3-dichloro-5,6-dicyano-1,4-benzoquinone

diisobutylaluminium hydride

chlorodiisopinocampheylborane

$\mathrm{N}, \mathrm{N}$-diisopropylethylamine (Hünig's base)

4-(dimethylamino)pyridine

$\mathrm{N}, \mathrm{N}$-dimethylformamide

Dess-Martin periodinane

$N, N^{\prime}$-dimethylpropyleneurea

dimethyl sulfoxide

2-deoxyribonucleic acid

electrophile

elimination unimolecular conjugate base

ethyl acetate (EtOAc)

equivalents

electron-withdrawing group

Food and Drug Administration (USA)

pinacolborane

$N, N, N^{\prime}, N^{\prime}$-tetramethyl-O-(1H-benzotriazol-1-yl)uronium

hexafluorophosphate

heteronuclear multi-bond correlation 


\begin{tabular}{|c|c|}
\hline HMPA & hexamethylphosphoramide \\
\hline HRMS & high resolution mass spectrometry \\
\hline HRFABMS & high resolution fast-atom bombardment mass spectrometry \\
\hline HSQC & heteronuclear single quantum correlation \\
\hline HWE & Horner-Wadsworth-Emmons \\
\hline $\mathrm{IC}_{50}$ & half maximal inhibitory concentration \\
\hline IR & infrared spectroscopy \\
\hline KHMDS & potassium bis(trimethylsilyl)amide \\
\hline LA & Lewis acid \\
\hline LiHMDS & lithium bis(trimethylsilyl)amide \\
\hline Ils & longest linear sequence \\
\hline $\mathrm{m}$ & multiplet \\
\hline M & metal \\
\hline MDS & myelodisplastic syndrome \\
\hline mCPBA & meta-chloroperbenzoic acid \\
\hline MOM & methoxy methyl acetal \\
\hline MT & microtubule \\
\hline MTPA & $\alpha$-methoxy- $\alpha$-trifluoromethylphenylacetic acid (Mosher's acid) \\
\hline NaHMDS & sodium bis(trimethylsilyl)amide \\
\hline NBS & $N$-bromosuccinimide \\
\hline NIS & $N$-iodosuccinimide \\
\hline
\end{tabular}




\begin{tabular}{|c|c|}
\hline NMO & 4-methylmorpholine 4-oxide \\
\hline NMR & nuclear magnetic resonance \\
\hline NOESY & nuclear Overhauser effect spectroscopy \\
\hline $\mathrm{Nu}$ & nucleophile \\
\hline PE & petroleum ether \\
\hline PelA & Peloruside A \\
\hline $\mathrm{Pg}$ & protecting group \\
\hline Pgp & P-glycoprotein efflux pump \\
\hline PMB & para-methoxybenzyl \\
\hline PMBTCA & para-methoxybenzyl-2,2,2-trichloroacetimidate \\
\hline PPTS & pyridinium $p$-toluenesulfonate \\
\hline PTSA & $p$-toluene sulfonic acid \\
\hline PTX & Paclitaxel \\
\hline $\mathrm{R}$ & alkyl group \\
\hline $\mathrm{RCM}$ & ring-closing metathesis \\
\hline $\mathrm{R}_{f}$ & retention factor \\
\hline RRCM & relay ring-closing metathesis \\
\hline S & singlet \\
\hline SAR & structure-activity relationship \\
\hline sat. & saturated \\
\hline SCUBA & self-contained underwater breathing apparatus \\
\hline
\end{tabular}


single electron transfer

$\mathrm{S}_{\mathrm{N}} 2$

substitution nucleophilic bimolecular

$\mathrm{t}$

triplet

TBAF

tetra-n-butylammonium fluoride

TBAI

tetra-n-butylammonium iodide

TBDPS

tert-butlydiphenylsilyl

TBS (TBDMS)

tert-butyldimethylsilyl

TEA

triethylamine

TEMPO

2,2,6,6-tetramethyl-1-piperidinyloxy

TES

triethylsilyl

THF

tetrahydrofuran

TLC

thin layer chromatography

TMEDA

tetramethylethylenediamine

TMS

trimethylsilyl

TMSI

trimethylsulfonium iodide

TPAP

tetra- $N$-propylammonium perruthenate

VUW

Victoria University of Wellington 


\section{Introduction}

\subsection{Natural products as drugs}

The term natural product (NP) is commonly used to designate secondary metabolites produced by living organisms. A secondary metabolite is a substance made by an organism that is not deemed essential for survival of the organism in a biological sense, although secondary metabolites often ensure survival of an organism in a particular environment or offer an evolutionary advantage. NPs have been, throughout history, exploited for the purpose of treating various diseases. The pharmaceutical industry still relies on NPs and their analogues as drug leads, and many of them have become the active substances in pharmaceutical products, several of which are shown in Figure 1.1.

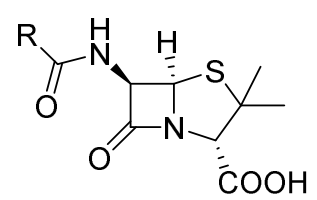

Penicillin family (2) fungus; antibiotic

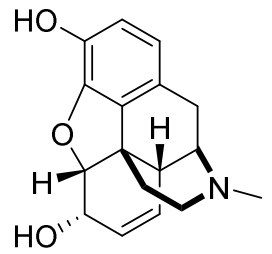

Morphine (3) plant; analgesic<smiles>CC(=O)CC(c1ccccc1)c1c(O)c2ccccc2oc1=O</smiles>

Warfarin (4) plant; anticoagulant

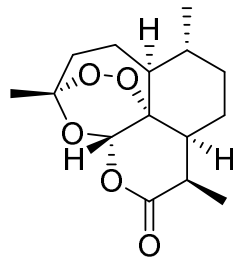

Artemisinin (5) plant; antimalarial

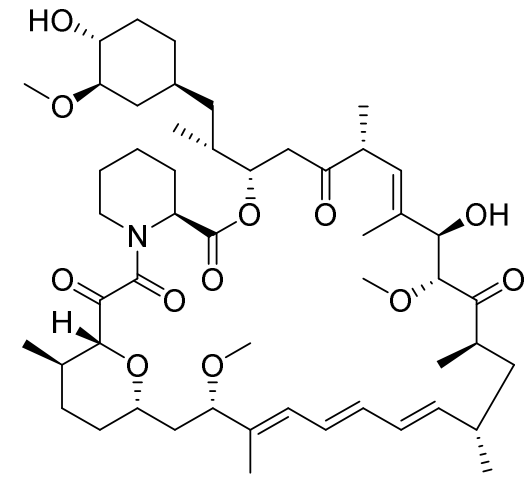

Sirolimus (6)

bacteria; prevention of transplant rejection<smiles>CC(C)c1c(C(=O)Nc2ccccc2)c(-c2ccccc2)c(-c2ccc(F)cc2)n1CC[C@H](O)C[C@@H](O)CC(=O)O</smiles>

Atorvastatin (7) plant; dyslipidemia

Figure 1.1: NP based pharmaceuticals

NPs represent an invaluable pool of potential anticancer agents. Based on the most recent review available ${ }^{6}, 83 \%$ of all small molecule new chemical entities (NCEs) approved for the treatment of cancer from 1981 to 2014 were either NPs and their derivatives or NP 
mimicking compounds. ${ }^{6}$ Natural products come from both terrestrial and marine organisms. The marine environment has much higher biodiversity representing $95 \%$ of earth's biosphere. ${ }^{7,8}$ The majority of bioactive compounds come from sessile organisms where they are produced to serve as a means of chemical defence. Bioactive compounds isolated from marine organisms are usually especially potent as they have to overcome dilution into sea water to exert their effect. ${ }^{9}$

The exploration of marine NPs was rapidly enabled by the development of submersible technology and improvement in diving techniques such as SCUBA that together made it possible to overcome the difficulties associated with marine sample collection. ${ }^{8}$ Early advances in the 1950s allowed isolation of two arabino-nucleotides (Figure 1.2), spongouridine (8, Ara-U) and spongothymidine (9, Ara-T), from the sponge Tectitethya crypta by Bergman and Feeney. ${ }^{10}$ Today, marine NPs are a fast growing collection of compounds and there are several reviews highlighting their structural diversity and therapeutic potential, especially as potential anticancer agents. ${ }^{11-14}$
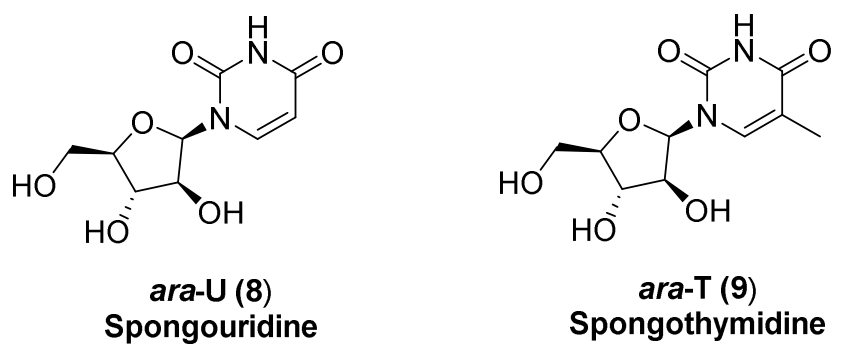

Figure 1.2: First isolated marine NPS

To date, four marine NP-derived drugs have been approved for the treatment of cancer (Figure 1.3). ${ }^{8,15}$ In 1969 cytrabine (10, Ara-C, Cytrosar-U ${ }^{\circledR}$ ), a fully synthetic analogue of Ara$U(8)$ and Ara-T (9), gained FDA approval and is still in use for the treatment of acute lymphocytic ( $A L L)$ and acute myeloid $(A M L)$ leukemias as well as for the treatment of nonHodgkin lymphoma and myelodysplastic syndrome (MDS). ${ }^{8,15}$ Cytrabine acts by inserting into the DNA chain where it inhibits the action of DNA polymerase. This inhibition arrests the cells in the $S$ phase of the cycle which leads to cell death. ${ }^{8,15}$

In 1990, the Rinehart and Wright groups, working on the same tunicate Ecteinascidia turbinata, simultaneously isolated the same tetrahydroisoquinoline alkaloid, trabectedin (ET-743) 11. ${ }^{16,17}$ Trabectedin has been shown to covalently bind to one of the strands in the 
minor groove of DNA where it interferes with cellular division, transcription and repair mechanisms resulting in cell death. ${ }^{18} \mathrm{~A}$ semi-synthetic method has been developed to allow access to larger quantities of the compound and subsequent commerciallisation. It was approved in Europe in 2007 under the name Yondelis ${ }^{\circledR}$ for the treatment of metastatic soft tissue sarcoma and ovarian cancer. Trabectedin also became FDA approved in $2015.8,15$<smiles>Nc1ccn([C@@H]2O[C@H](CO)[C@@H](O)[C@H]2O)c(=O)n1</smiles>

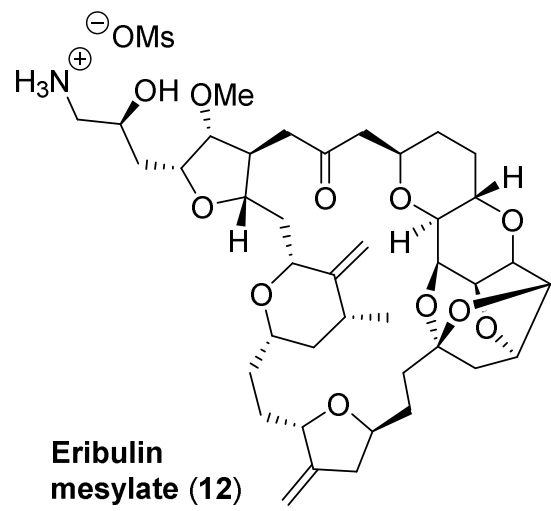

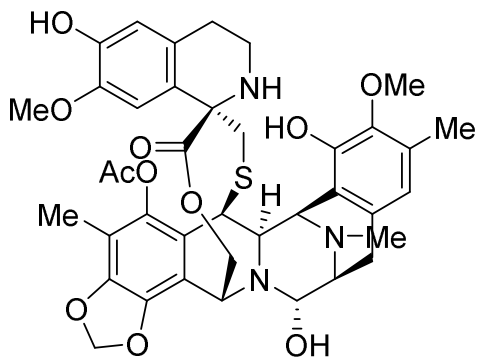

ET-743 (11) Trabectedin

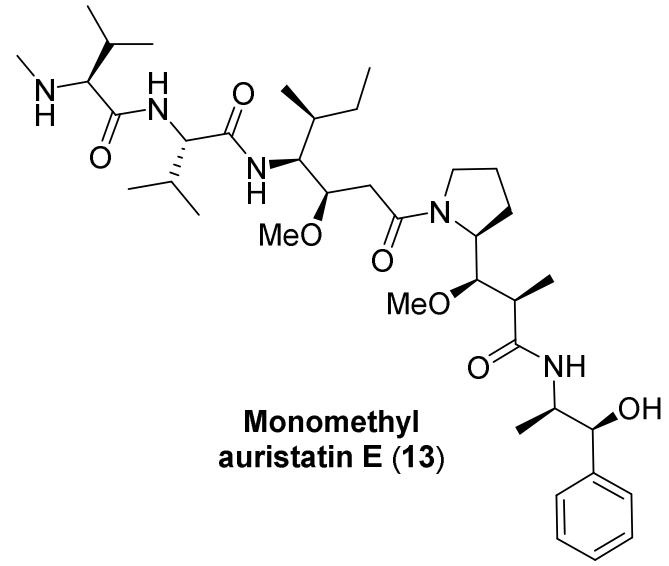

Figure 1.3: Marine NP derived anti-cancer drugs

Eribulin mesylate 12 (Halaven ${ }^{\circledR}$ ) gained FDA approval in 2010 for the treatment of metastatic breast cancer and some solid tumours. Eribulin is a fully synthetic (produced in 62 steps) truncated analogue of the macrocyclic polyether marine NP halichondrin B, isolated in 1986 from the sponge Halichondria okadai by Hirata and Uemura. ${ }^{19,20}$ Eribulin is a microtubule-depolymerising (destabilising) agent (vide infra), which binds to the $\beta$-tubulin subunit causing an irreversible mitotic blockade. ${ }^{21}$ Eribulin has also been shown to increase tumour blood delivery (perfusion) through blood vessel remodelling. ${ }^{20}$ This increase in perfusion is especially useful when treating solid tumours with hypoxic regions as it enhances the intratumoural drug delivery. ${ }^{20}$ Eribulin also decreases the expression of genes associated with angiogenesis, inhibiting the formation of new blood vessels. ${ }^{20}$ 
Lastly, monomethyl auristatin E (13), a synthetic derivative of a polypeptide marine NP dolastatin 10, was approved for cancer treatment as the drug component of an antibodydrug conjugate brentuximab-vendotin. ${ }^{8,15}$ Dolastatin 10 was isolated from the sea hare Dolabella auricularia by Pettit in $1987 .{ }^{22}$ The group subsequently produced several synthetic analogues of dolastatin 10 in order to gain more insight into structure-activity relationship (SAR). Of the analogues synthesised, monomethyl auristatin E was found to be the most promising drug candidate. ${ }^{23,24}$ Brentuximab-vendotin conjugate (Adcetris ${ }^{\circledR}$ ) gained FDA approval in 2011 for the treatment of Hodgkin lymphoma. ${ }^{8,15}$ The conjugate was also approved in Europe in 2015. Monomethyl auristatin E prevents mictotubule polymerisation and it has been shown to bind to the same domain as vinca alkaloids (vide infra). ${ }^{25}$

Several marine NP derived drug candidates have been approved for treatment of other diseases with many more in various stages of clinical trials. ${ }^{8,15}$

\subsection{Peloruside A (PelA)}

Peloruside A (1, Figure 1.4), is another marine NP with exciting biological activity against cancerous cells. It was discovered, isolated and characterised by researchers at Victoria University of Wellington (VUW) within the Northcote group. ${ }^{26}$ It was isolated in 1999 using a unique fractionation procedure, from the marine sponge Mycale hentscheli, which was found in the Pelorus Sound. Although the sponge species grows in other parts of the world, only members of the Mycale species that grow in Pelorus Sound have been found to produce significant quantities of peloruside $A$.

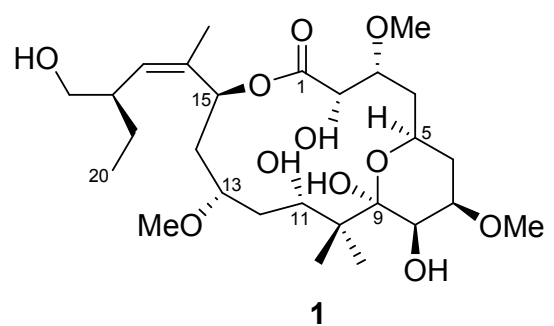

Figure 1.4: Peloruside A (1) structure

The molecular formula of the compound was established from high resolution fast atom bombardment mass spectrometry (HRFABMS) as $\mathrm{C}_{27} \mathrm{H}_{48} \mathrm{O}_{11}$. The structural elucidation of this highly oxygenated macrolide was performed using various $1 \mathrm{D}$ and $2 \mathrm{D}$ nuclear magnetic 
resonance (NMR) spectroscopy experiments to assign the peaks observed in ${ }^{13} \mathrm{C}$ and ${ }^{1} \mathrm{H} N M R$ spectra. This revealed the presence of ten stereogenic centres, an internal pyran ring and a trisubstituted $Z$-double bond. ${ }^{26}$

The bioactivity of peloruside A was first studied by researchers from VUW (led by Prof. John Miller) and it has since become a compound of interest to other institutions and teams around the world. ${ }^{27-33}$ In the first cellular assay, peloruside A exhibited cytotoxicity towards P388 murine leukaemia cells at a concentration of approximately $18 \mathrm{nM} .^{26}$ Peloruside A was subsequently identified as a microtubule stabilising agent that was cytotoxic to various cancerous cell lines at nanomolar concentrations (Table 1.1). ${ }^{27,33,34}$

Table 1.1: Cytotoxicity of PelA ( $\mathrm{nM})$ in various cell lines

\begin{tabular}{|c|c|}
\hline Cell line & ${ }^{\prime} C_{50}$ values (nM) \\
\hline P388 & 18 \\
\hline HL-60 & $7-35$ \\
\hline H441 & 6 \\
\hline SH-SY5Y & 15 \\
\hline 32D & 9 \\
\hline A2780 & 66 \\
\hline A2780AD & 455 \\
\hline $1 \mathrm{~A} 9$ & 16 \\
\hline MCF-7 & 4.9 \\
\hline MDA-MB-231/Luc & 50 \\
\hline PC-3 & 10 \\
\hline
\end{tabular}

P388 = mouse leukaemia cells, HL-60 = human leukaemia cells, H441 = human lung adenocarcinoma, SH-SY5Y = human neuroblastoma, $32 \mathrm{D}=$ Mouse myeloid precursor, $\mathrm{A} 2780=$ human ovarian carcinoma cells, $\mathrm{A} 2780 \mathrm{AD}=$ human ovarian carcinoma cells overexpressing P-glycoprotein, 1A9 = clone of A2780, MCF-7 = human breast carcinoma cells, MDA-MB$231 /$ Luc = human metastatic breast cancer, $\mathrm{PC}-3=$ human prostate cancer. 
The therapeutic potential of peloruside $A$ has mostly been attributed to its anti-cancer activity which will be the main focus of this introductory chapter, however it is also worth mentioning its activity against neurodegeneration ${ }^{35-38}$ (with implications in the treatment of Alzheimer's disease) and auto-immune disorders. ${ }^{38-42}$

\subsection{Peloruside $A$ as an anti-cancer agent}

\subsubsection{Inhibition of mitosis}

Cancerous cells exhibit increased cellular proliferation in comparison to healthy cells. Limiting cellular proliferation is an attractive mode of action for cancer therapy because of the inherent preference of antiproliferative agents for cancerous cells. Numerous cell components are involved in proliferation, however, not all of these components are essential for cell division. Microtubules are components of the cytoskeleton that are essential for proliferation, as they are responsible for division of cellular genetic information into the daughter cells. During mitosis, microtubules form a mitotic spindle that plays a major role in finding, attaching and separating chromosomes. ${ }^{43}$

Structurally, microtubules are polymers of globular proteins from the tubulin familyspecifically $\alpha$ and $\beta$-tubulins. These proteins form $\alpha, \beta$-heterodimers that polymerise into microtubules in a reversible process. Microtubules are dynamic structures with their ends switching between phases of polymerisation and depolymerisation. ${ }^{44}$ If this process is interrupted, cells cannot complete the division cycle which ultimately leads to apoptosis (programmed cell death). Interruption of microtubule dynamics involves either inhibition of polymerisation of tubulin units (microtubule-destabilising agents) or inhibition of depolymerisation of microtubules (microtubule-stabilising agents). ${ }^{44}$ Inhibition of microtubule dynamics is a well established mode of cancer therapy and two classes of NPs are already used as therapeutics that act on microtubules.

Vinca alkaloids (vinblastine, vincristine, vinorelbine, vindesine, and vinflunine) are a family of natural and semi-synthetic alkaloids isolated from Madagascar periwinkle plant Catharanthus roseus. ${ }^{45}$ They have been used for cancer treatment since 1963 when vincristine (14, Figure 1.5) gained approval from the United States Food and Drug Administration agency (FDA), and they still remain one of the most used classes of cancer 
drugs. Vinca alkaloids are microtubule-destabilising agents. They bind to $\beta$-tubulin subunit of the soluble dimer (vinca site) which induces a conformational change that prevents polymerisation. $^{44}$

Taxanes (paclitaxel, docetaxel) are a class of terpenoid NPs and semi-synthetic variants. Paclitaxel (15, Figure 1.5) was isolated from the bark of the Pacific yew tree Taxus brevifolia in 1966 and docetaxel (16, Figure 1.5) is a semi-synthetic analogue produced commercially from 10-deacetylbaccatin (natural-product precursor with hydroxyl functionality at C13). Paclitaxel was first approved by FDA in 1992 for the treatment of ovarian cancer and docetaxel gained approval in 1996 for use in treatment of breast cancer. ${ }^{44}$ Taxanes exert their anticancer activity through stabilisation of microtubules. They also bind to the $\beta$ tubulin subunit but, unlike vinca alkaloids which bind to the soluble tubulin dimer, taxanes bind to tubulin in the polymerised microtubules (taxoid site). This binding induces conformational changes that make polymerised microtubules more stable, thus inhibiting microtubule depolymerisation. ${ }^{44}$

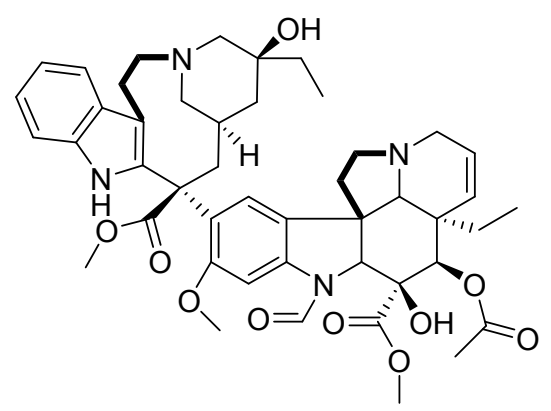

Vincristine (14)

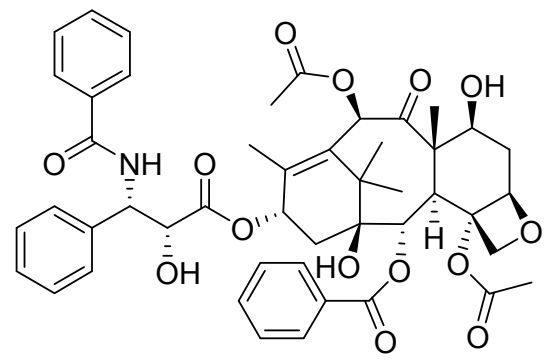

Paclitaxel (15)

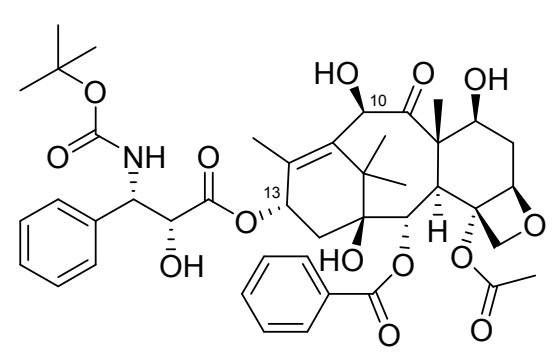

Docetaxel (16) 
Several marine NPs, including peloruside A, also act on microtubules. ${ }^{46}$ Like taxanes, peloruside $A$ binds to the $\beta$-tubulin subunit of the dimer, stabilising the polymerised microtubules, which arrests cells in the $G_{2}-M$ phase of the cell cycle. ${ }^{27}$ Microtubule stabilisation is a proven mode of action for cancer therapy and most of the identified microtubule-stabilising agents, like epothilone A (17, Figure 1.6) exert their action by binding to the taxoid site on polymerised microtubules. A study on the activity of PelA in paclitaxel-resistant cells in which a mutation of the $\beta$-tubulin gene caused modification of the taxoid binding site showed that these cell lines were not resistant to PelA. ${ }^{28}$ This indicated a different binding site through which peloruside stabilises microtubules.

In 2014, crystal structures of PelA, laulimalide (18, Figure 1.6) and epothilone A (17, Figure 1.6) binding to tubulin were published, providing information about the position, conformation and environment of these compounds when bound to microtubules. ${ }^{29}$

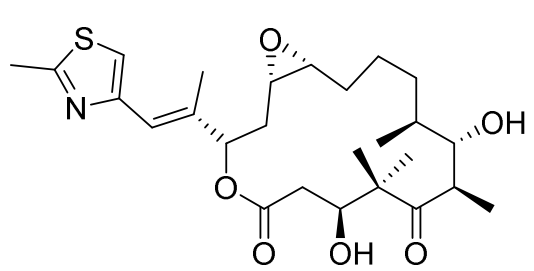

Epothilone A (17)

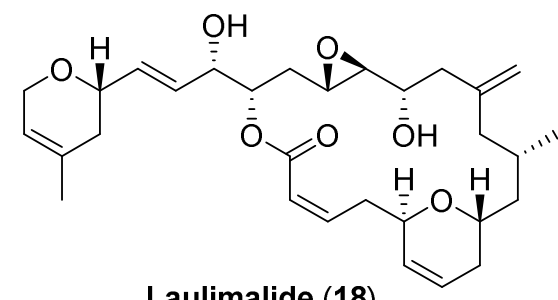

Laulimalide (18)

Figure 1.6: Structures of laulimalide and epothilone $A$

Crystal structures confirmed that whereas bacterial secondary metabolite, epothilone $A$, binds to the taxoid site on the exterior of the $\beta$-tubulin, peloruside $A$ and laulimalide, another marine NP and potent antimitotic agent, do not. Additionally, the study revealed that these two marine NPs share the same, unique binding site on the interior of the $\beta$ tubulin. PelA interacts with the binding site through five hydrogen bonds between oxygens O1, O2, O3, 011 and $\mathrm{O} 24$ on peloruside A and the side-chains of amino acids Ser298, Asp297/Arg308, Arg308, Gln293 and Tyr312, respectively, in the $\beta$-tubulin subunit (Figure 1.7c). ${ }^{29}$ This study also elucidated the ways in which PelA and laulimalide exert their microtubule stabilising action: through allosteric reorientation of the M-loop of $\beta$-tubulin, and by bridging two adjacent $\beta$-tubulin subunits (Figure 1.8b). ${ }^{29}$ The bridging of the two $\beta$ tubulin subunits on the neighbouring protofilaments is thought to occur through 
peloruside's 07 and 08 which are in reasonable hydrogen bonding distance to the Asp120', Arg123' and Lys124' (Figure 1.8c)..$^{29}$

a)

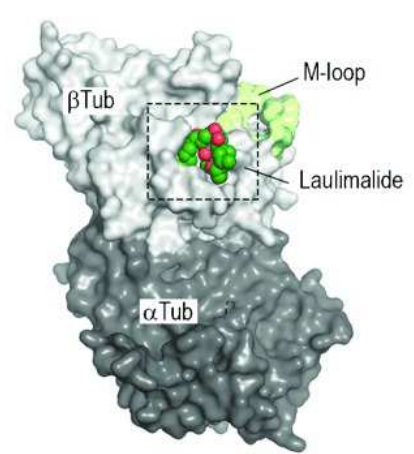

c)

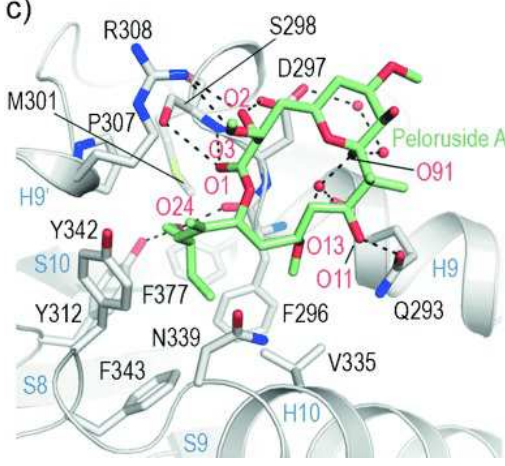

b)

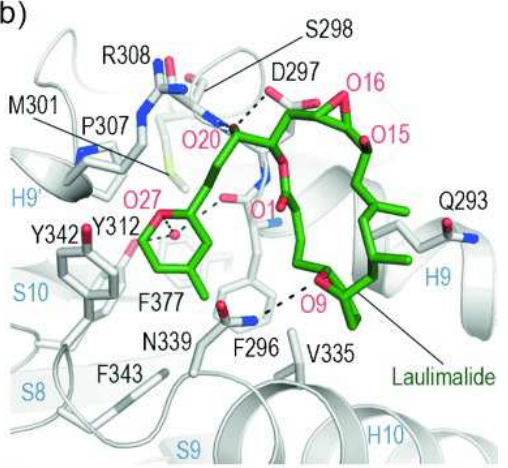

d)

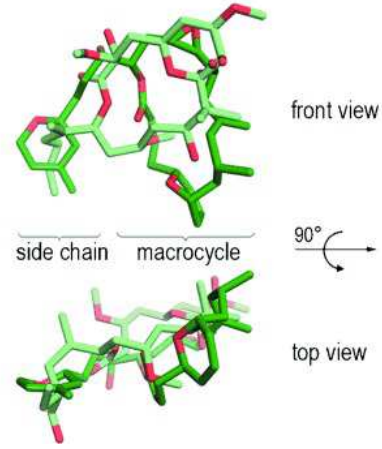

Figure 1.7: PelA and laulimalide binding to microtubules demontrated in a system comprised of $\alpha, \beta$-tubulin dimers, stathimin and TTL. ${ }^{29}$ Used with permission (2018) Copyright (C) 2014 John Wiley \& Sons, Inc. All rights reserved.

a)

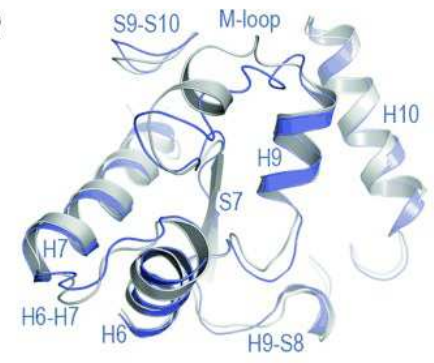

c)

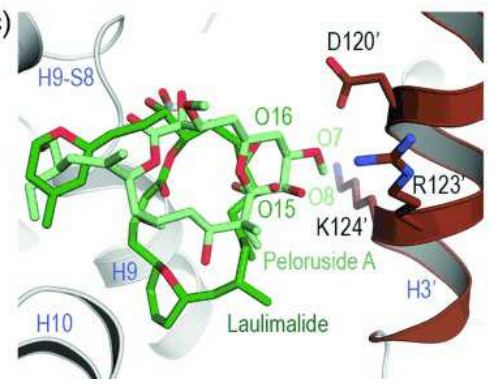

b)
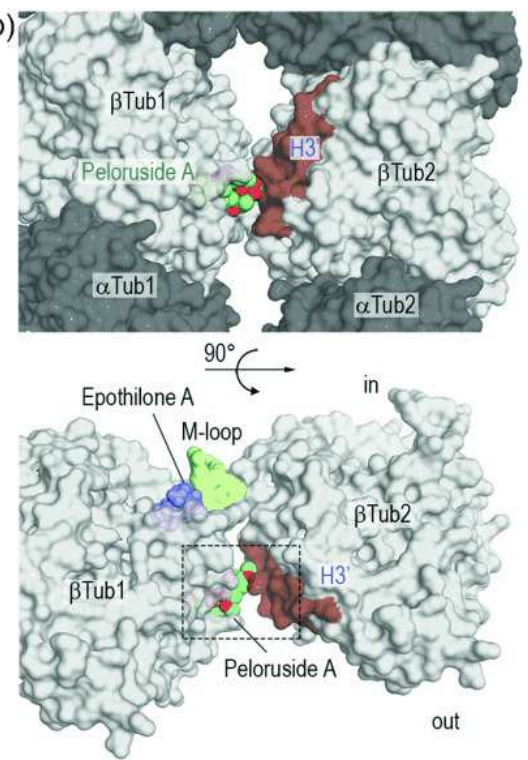

Figure 1.8: PelA and laulimalide stabilisation of microtubules from $x$-crystal structure and modelling. ${ }^{29}$ Used with permission (2018).Copyright @ 2014 John Wiley \& Sons, Inc. All rights reserved. 
Both of these actions stabilise the microtubules, causing the disruption of microtubular dynamics. An interesting finding that came out of this study is that concurrent binding of PelA or laulimalide and taxanes is possible, suggesting a synergistic effect and the potential to use these two types of microtubule-stabilising agents in combination. ${ }^{29}$ This synergistic effect of peloruside $A$ and laulimalide with some microtubule-stabilising agents, acting on the taxoid site, has been documented on a tubulin assembly system and in human ovarian carcinoma cells (1A9 cells). ${ }^{47,48}$ However, they did not synergise with each other as expected, nor with other microtubule-destabilising agents. ${ }^{47,48}$

A molecular dynamics study published in 2018 noted the new stabilising lateral contacts between two adjacent $\beta$-tubulin units (between the M-loop of $\beta 1$-tubulin and the H1-B2 loop of the $\beta 2$-tubulin) when PelA was bound. ${ }^{49}$ A similar stabilising effect was observed when epothilone is bound to the taxoid site on the interior of the microtubule and when either PelA or laulimalide are bound to their site, on the exterior. ${ }^{49}$

\subsubsection{Inhibition of angiogenesis}

Preventing the formation of new blood vessels is important as tumours need vasculature to deliver nutrients for their growth and to enable metastasis. Vasculature disrupting agents have been used as anticancer agents since $2004 .{ }^{32}$ Low, non-cytotoxic, concentrations of peloruside $A$ inhibit proliferation ( $\left(C_{50} 1.4 \mathrm{nM}^{32}\right)$, migration ( $\left(C_{50} 0.1 \mathrm{nM}\right.$ i.e. $1 / 200$ th of the antimitotic concentration ${ }^{31}$ ) and angiogenesis in human umbilical vein endothelial cells (IC 50 $\left.0.1 \mathrm{nM}^{31}\right)$.

\subsubsection{Advantages over current drugs}

The therapeutic selectivity of PelA and other microtubule-targeting agents is inherent in their mode of action, due to the increased proliferation rate of cancer cells compared to normal cells. However, cancer cells develop mechanisms to resist taxanes and some other microtubule-targeting drugs. Most common of these mechanisms is through overexpression of the P-glycoprotein (Pgp) drug efflux pump which normally removes toxins from the cell. The studies using multi-drug resistant cell lines indicate that PelA is a poor substrate for Pgp and retains its activity in these cell lines. ${ }^{28,50}$ The significance of PelA therefore also lies in its potential to be used in these taxane-resistant cancers. Furthermore, a recent preclinical 
study, conducted in vivo using nude immunocompromised mice using human xenografts, showed peloruside $A$ to be more effective than paclitaxel, docetaxel and doxorubicin in inhibiting tumour growth in two non-small cell lung carcinoma cell lines H460 and A549. This was also seen in one ovarian cancer cell line with an overexpressed P-glycoprotein pump ( $\mathrm{NCl} / \mathrm{ADR}-\mathrm{RES}$ ) while showing no overt toxicity to mice. ${ }^{9,33}$ The efficacy of PelA can also be increased through the synergistic effect that was observed with some of the other microtubule-stabilising agents (epothilone and paclitaxel). ${ }^{47,48}$ Another advantage of PelA is its increased polarity compared to PTX. ${ }^{9,38}$ Current formulations of PTX involve the use of solubilising vehicles for delivery (like Cremophore $E^{\circledR}{ }^{\circledR}$ or polysorbate 80 in $13 \% \mathrm{w} / \mathrm{w}$ ethanol) that are responsible for some of the toxic side-effects. ${ }^{9,38,51,52}$ The increased polarity of PelA circumvents the need for the use of these toxic delivery vehicles.

\subsection{Access to Peloruside A}

The nanomolar-range cytotoxicity of PelA, together with its unique binding site, and the many advantages over drugs that are currently on the market, make PelA an excellent candidate for Phase I clinical trials. However, the ease of supply often determines the future of a drug, and access to larger quantities of the compound is needed to further evaluate the compound's potential for the treatment of cancer, conduct pharmacokinetic, pharmacodynamic and toxicology studies, and progress the compound into clinical trials.

\subsubsection{Aquaculture}

In nature, PelA is present in tiny amounts within the sponge. An attempt to produce quantities suitable for clinical trials by growing the sponge on a marine farm in the Marlborough Sounds was unsuccessful because of the sudden growth in the population of a natural predator, dorid nudibranchs Hoplodoris nodulosa. ${ }^{53}$ Since large-scale isolation of the compound from the sponge is unsustainable and the attempt to grow the sponge failed, chemical synthesis represents an alternative method to gain access to this important compound. 


\subsubsection{Total synthesis}

Devising an efficient synthesis of peloruside $A$ that will be able to deliver larger quantities is essential to conduct further studies and enable the eventual manufacture of the drug. To date, there have been six total syntheses published. ${ }^{1-4,54,55}$

De Brabander, in 2003, was the first to synthesise peloruside A. ${ }^{54}$ His strategy (Scheme 1.1) envisaged the aldol coupling of the C14-C20 ketone fragment 19 to the $\mathrm{C} 1-\mathrm{C} 13$ aldehyde fragment 20 which gave the full carbon skeleton of PelA. Mitsunobu lactonisation ${ }^{56}$ was then employed to form a macrocycle. The side-chain fragment C14-C20 (19) was synthesised in five steps from 2,5-dihydrofuran (21), using ring-closing metathesis to form a Z-trisubstituted enone. The C1-C13 aldehyde functionality was installed through an allyl transfer with allyldiethylborane that gave a terminal double bond which after diol formation and cleavage, produced the C13 aldehyde $\mathbf{2 0}$. The tetrahydropyran ring in $\mathbf{2 0}$ was obtained by reduction and functionalisation of the dihydropyranone $\mathbf{2 2}$. This intermediate (22) was in turn synthesised through oxidation of the $\beta$-hydroxyketone resulting from the aldol addition between $\mathrm{C} 1-\mathrm{C} 7$ aldehyde (23) and the C8-C11 ketone (24).

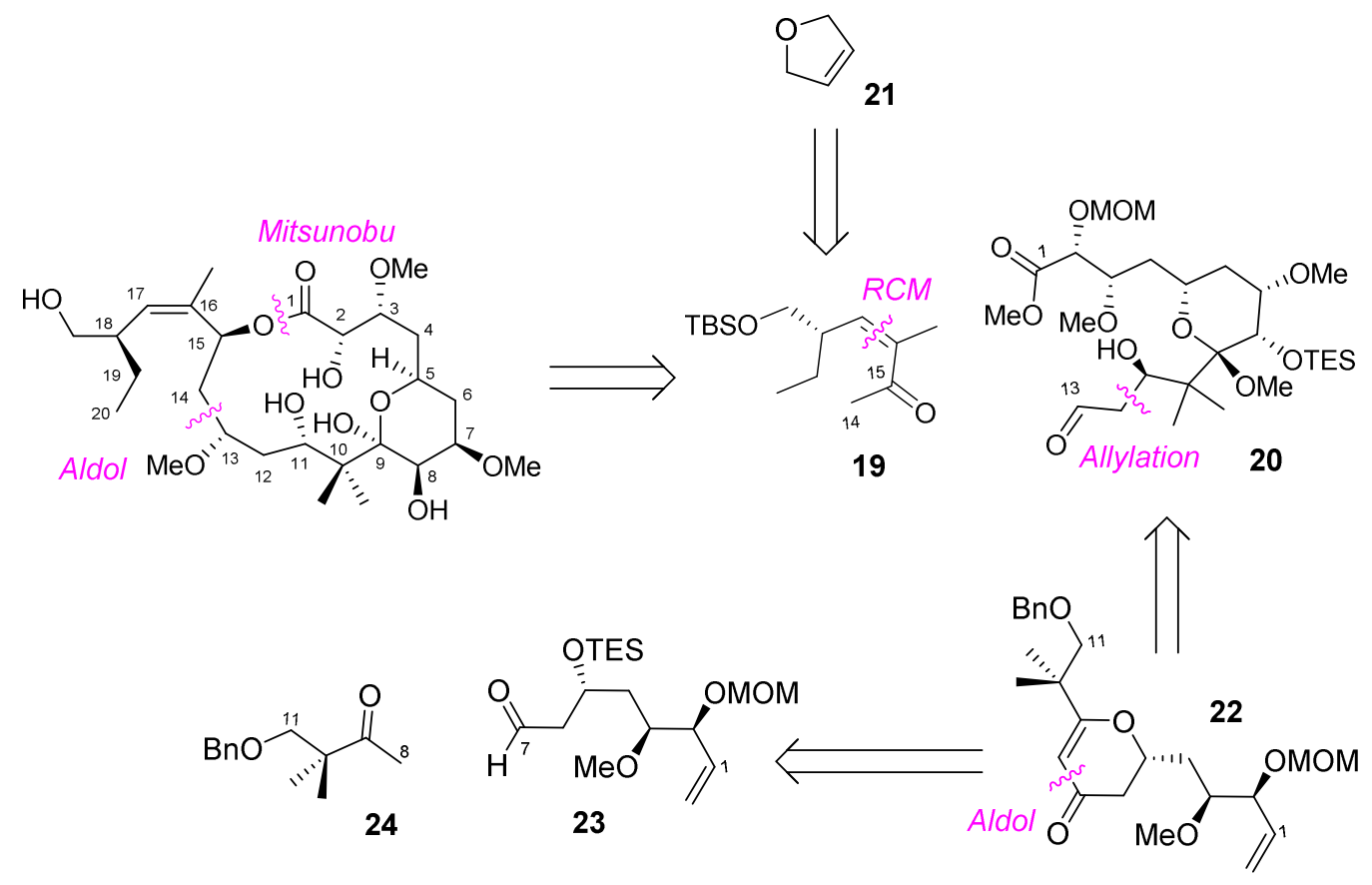

Scheme 1.1: De Brabander's retrosynthetic strategy

In 2005, Taylor reported another total synthesis of PelA. ${ }^{4}$ Here, the macrocyclisation was achieved using the Yamaguchi macrolactonisation protocol ${ }^{57,58}$ and the complete carbon 
skeleton of PelA was obtained through an aldol reaction between the C8-C20 ketone $\mathbf{2 5}$ and the C1-C7 aldehyde 26 fragments (Scheme 1.2). After the fragments were coupled, the pyran ring was formed in a similar manner to De Brabander's. ${ }^{54}$ The synthesis of the ketone fragment 25, starting from (R)-4-benzyl-3-butyryloxazolidin-2-one (27), involved the StillGennari $^{59}$ modification of the Horner-Wadsworth-Emmons (HWE) olefination which produced the trisubstituted Z-alkene. After further modifications, an anti-stereoselective Mukaiyama aldol ${ }^{60-63}$ reaction that gave the desired methyl ketone fragment. ${ }^{64}$ The key step in the synthesis of the $\mathrm{C} 1-\mathrm{C} 7$ aldehyde fragment (26) was a diastereoselective Evans aldol reaction between the C1-C2 oxazolidinone $\mathbf{2 8}$ and the C3-C7 aldehyde $\mathbf{2 9}$ that was used to establish the desired 2,3-syn 3,5-syn stereochemical relationship. ${ }^{65,66}$

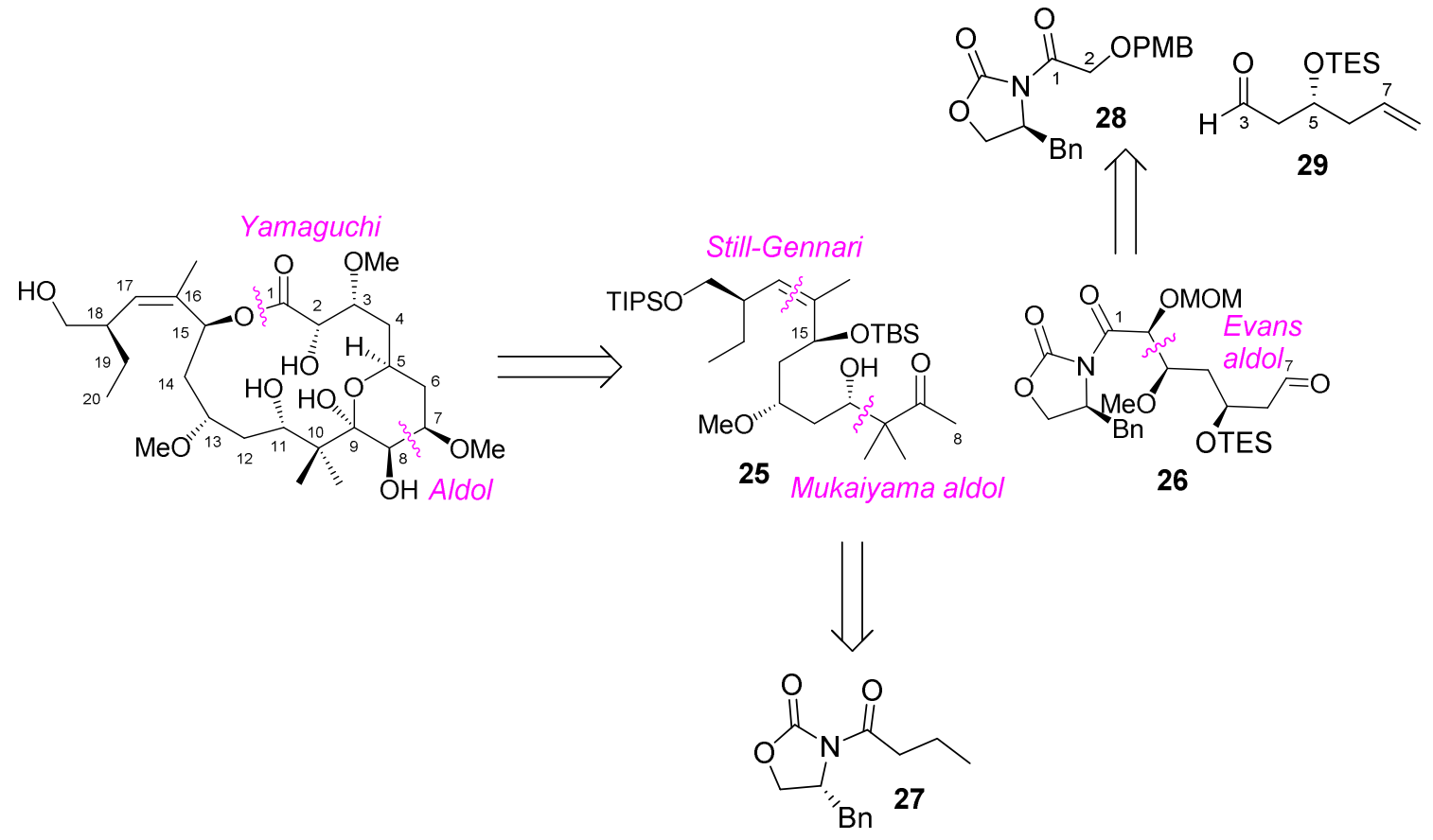

Scheme 1.2: Taylor's retrosynthetic strategy

The third total synthesis of peloruside A was reported in 2008 by Ghosh (Scheme 1.3) and it employed a reductive enolisation-aldol addition process to join the C11-C20 aldehyde 30 and the C1-C10 enone $\mathbf{3 1}$ fragments. ${ }^{2}$ Here, L-Selectride was employed to form an enolate from enone fragment $\mathbf{3 1}$ which was then added to the aldehyde fragment $\mathbf{3 0}$ in a reductive aldol reaction. After the selective removal of the protecting group at $\mathrm{C} 1$, two consecutive oxidations (TPAP/NMO followed by Pinnick ${ }^{67}$ ) gave the C1 carboxylic acid. Ghosh also employed Yamaguchi macrocyclisation ${ }^{57,58}$ and the pyran was formed in the subsequent step during the acid-mediated deprotection of the 05 protecting group. The synthesis of the 
enone-containing fragment 31 started from (-)-2,3-O-ispropylidene-D-threitol (32) that already posses two of the PelA stereocentres, C2 and C3. The C5 stereochemistry was introduced through a Brown allylation. ${ }^{68,69}$ The Ando modification ${ }^{70}$ of the HWE olefination followed by asymmetric Sharpless dihydroxylation ${ }^{71}$ of the Z-alkene produced the desired 7,8-diol. The enone functionality was installed by the addition of the Grignard reagent followed by oxidation. The side-chain aldehyde fragment $\mathbf{3 0}$ was synthesised from $(R)-4-$ benzyl-3-butyryloxazolidin-2-one $\mathbf{2 7}$, this time using the Ando olefination to form the Ztrisubstituted olefin. Brown asymmetric allylborations ${ }^{68,69}$ were employed to produce the required $\mathrm{C} 15$ and $\mathrm{C} 13$ stereochemistry and the oxidative cleavage of the terminal double bond gave the $\mathrm{C} 11$ aldehyde (30).

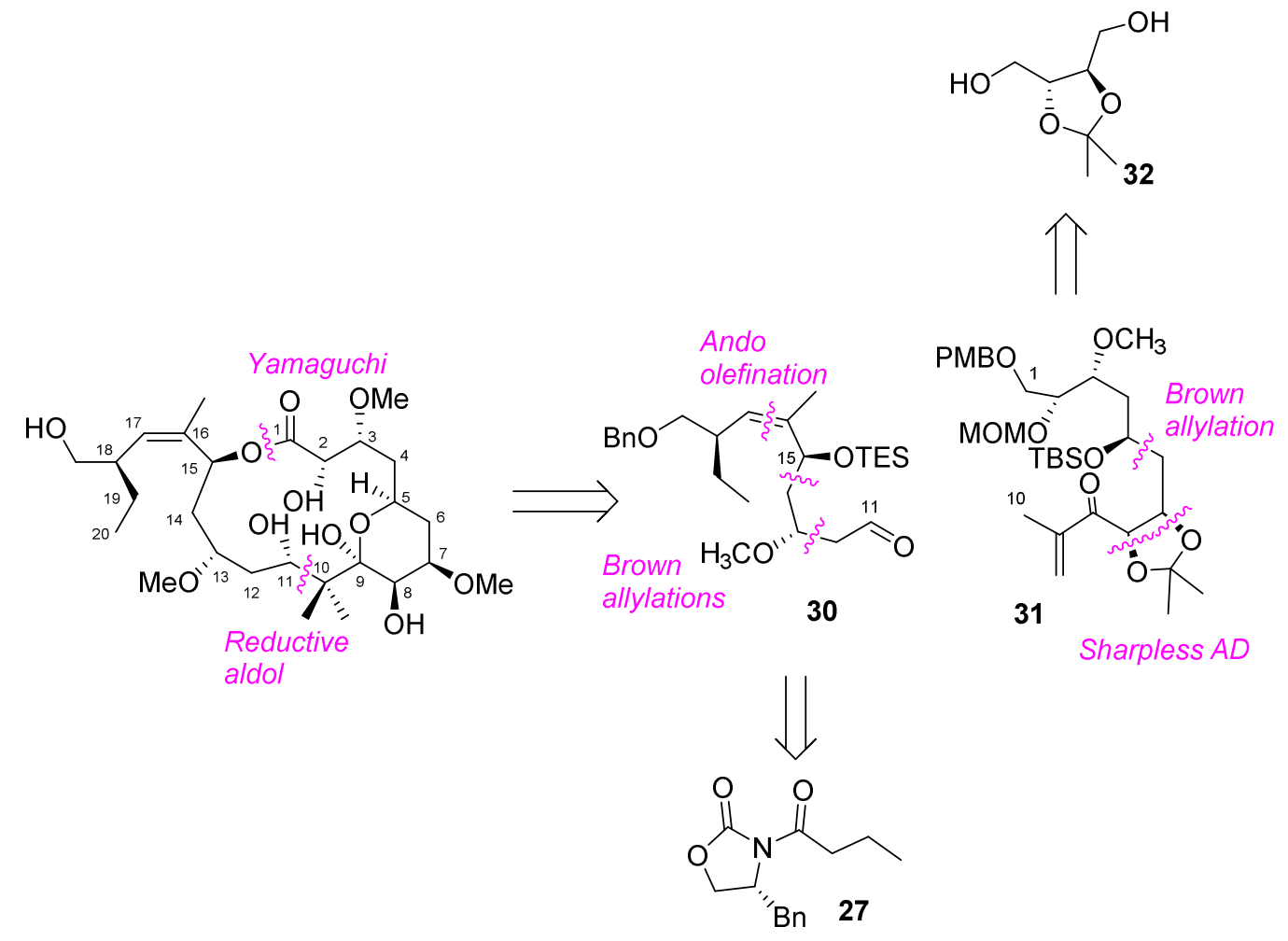

Scheme 1.3: Ghosh's retrosynthetic strategy

Evans published his total synthesis of PelA in 2009 (Scheme 1.4), where the carbon skeleton of PelA was obtained through an aldol reaction between the side-chain ketone fragment C12-C20 (33) and the C1-C11 aldehyde fragment (34) involving a chiral boron species. ${ }^{1}$ The macrocyclisation was achieved using the Yamaguchi protocol ${ }^{57,58}$ and the pyran ring was spontaneously formed in the very last step, after global deprotection. Boron-mediated aldol addition was also employed to obtain the C1-C11 fragment, through coupling of the C1-C6 
ketone fragment $\mathbf{3 5}$ with the C7-C11 aldehyde $\mathbf{3 6}$. The synthesis of the ketone fragment $\mathbf{3 5}$ started from (S)-4-benzyl-2-oxazolidinone (37) and the 2,3-syn configuration was established in an Evans aldol reaction. ${ }^{65,66}$ The synthesis of aldehyde fragment C7-C11 36 was achieved in seven high yielding steps from (S)-pantolactone (38). The side-chain 33 synthesis resembles that of Taylor but the Z-trisubstituted olefin was produced utilising the Ando olefination, ${ }^{70}$ employed previously by Ghosh. ${ }^{72}$ Evans' side-chain synthesis will be discussed in more detail later in the chapter (1.5.2.).

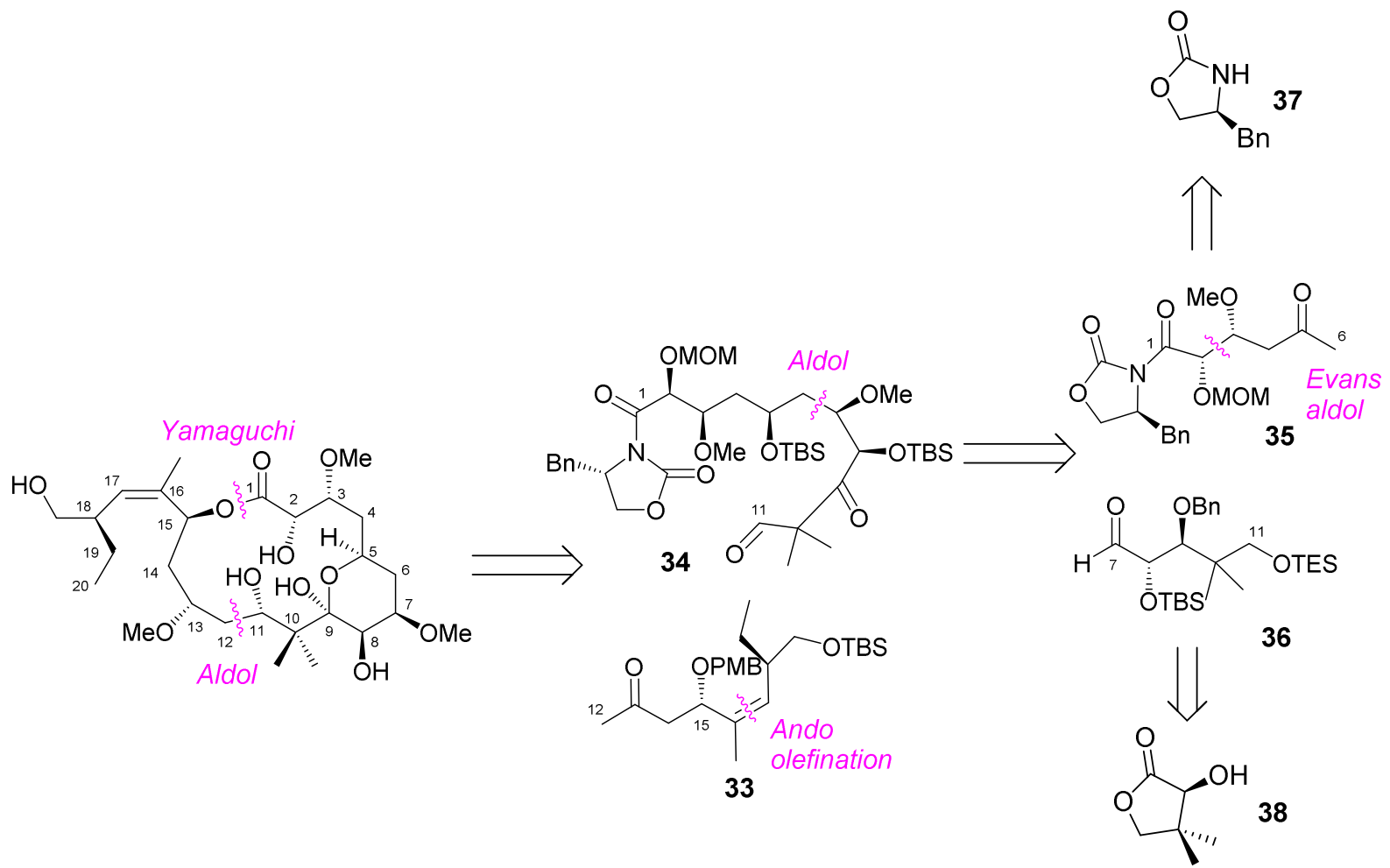

Scheme 1.4: Evans' retrosynthetic strategy

In 2010 Jacobsen also reported a synthesis of peloruside A. ${ }^{3}$ The two main fragments (Scheme 1.5), the $\mathrm{C} 1-\mathrm{C} 10$ enone 39 and the $\mathrm{C} 11-\mathrm{C} 20$ aldehyde $\mathbf{4 0}$, were joined together in a manner similar to that employed by Ghosh, ${ }^{2}$ using L-selectride-mediated reductive aldol reaction. After selective removal of the primary TBS protecting group and oxidation to secoacid, the macrocycle was formed by Yamaguchi macrocyclisation ${ }^{57,58}$ and the pyran ring closed upon global deprotection. The enone fragment $\mathbf{3 9}$ was obtained through elaboration of the intermediate $\mathbf{4 1}$ that was formed in a hetero-Diels-Alder reaction of the C1-C5 aldehyde $\mathbf{4 2}$ with a trioxy-substituted diene $\mathbf{4 3}$ using a chiral chromium-Schiffs base complex to control diastereoselectivity. In the C11-C20 aldehyde fragment 40 , the Z-trisubstituted 
olefin was obtained by bromine-for-lithium exchange of the intermediate $\mathbf{4 4}$ that then reacted with the aldehyde $\mathbf{4 5}$. The synthesis of the aldehyde fragments, $\mathbf{4 2}$ and $\mathbf{4 5}$ both started from enantioenriched epoxides.

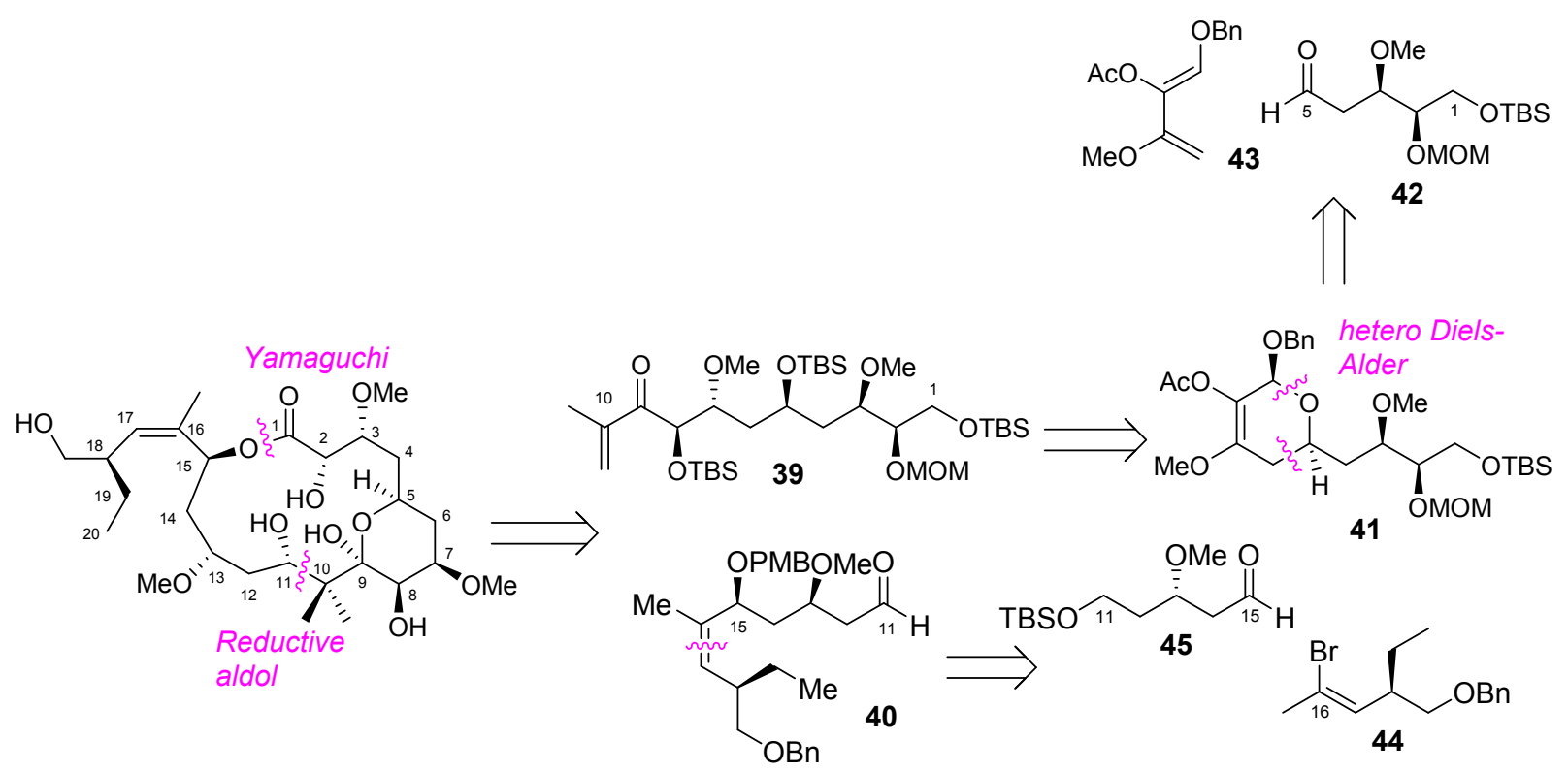

Scheme 1.5: Jacobsen's retrosynthetic analysis

The same year, Hoye published his total synthesis of PelA. ${ }^{55}$ As with the previous syntheses, the two main fragments, the $\mathrm{C} 1-\mathrm{C} 11$ aldehyde 46 and the $\mathrm{C} 12-\mathrm{C} 20$ methyl ketone 47 were coupled using aldol methodology and macrocycle formation achieved by the Yamaguchi protocol $^{57,58}$ (Scheme 1.6). The key feature in the synthesis of the C1-C11 aldehyde fragment 46 was the highly diastereoselective desymmetrisation through the tetramethylguanidine-promoted (TMG) lactonisation ${ }^{73}$ of $\mathbf{4 9}$ to produce a $\delta$-valerolactone as is 48. After the reduction of the lactone, the prenylation was employed to introduce the C10 gem-dimethyl moiety. The subsequent ozonolysis of the terminal olefin gave the C11 aldehyde (46). The ketone fragment $\mathbf{4 7}$ was synthesised from an ester intermediate $\mathbf{5 1}$ utilising relay ring-closing metathesis ${ }^{74}$ to first give a lactone $\mathbf{5 0}$. This was reduced to give the Z-trisubsituted olefin of the side-chain and the elaboration of the C13 nitrile involved a modified Blaise reaction followed by hydrolysis $(\mathrm{pH} 3)$ of the resulting $\beta$-aminoenoate to give a $\beta$-ketoester that then underwent decarboxylation to give the desired methyl ketone functionality (47). 


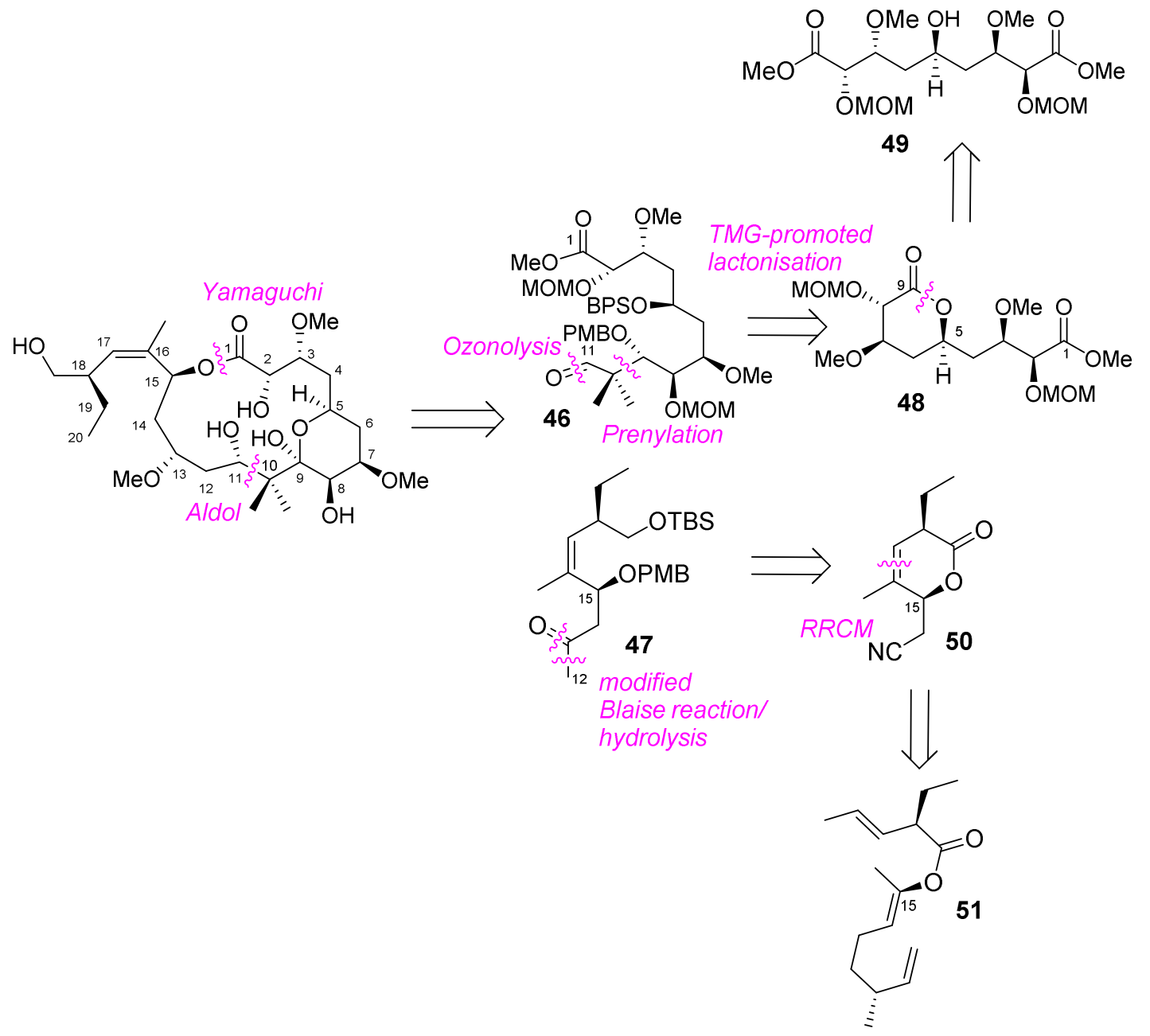

Scheme 1.6: Hoye's retrosynthetic strategy

\subsection{Thesis objective 1: devising a scalable synthesis of PelA}

\subsubsection{Overall approach to scalable synthesis of PelA}

The primary aim of this thesis project is to develop a synthetic strategy that will not only deliver enough material for preclinical and clinical studies but also enable manufacture of the drug for its eventual use as a cancer treatment. The scalable synthesis should be step efficient, convergent and suitable for large scale synthesis. It should also be cost efficient and, as much as possible, avoid the use of harsh reagents and conditions. With all that in mind, two plans for the scalable synthesis of peloruside $A$ have been proposed (Scheme 1.7), drawing from the previously published syntheses together with knowledge obtained from research in our labs and those of our collaborators. Additionally, these routes will give 
opportunities to access epimeric and diastereomeric analogues of peloruside $A$ from stereoisomeric intermediates produced in the chiral centre-forming reactions.

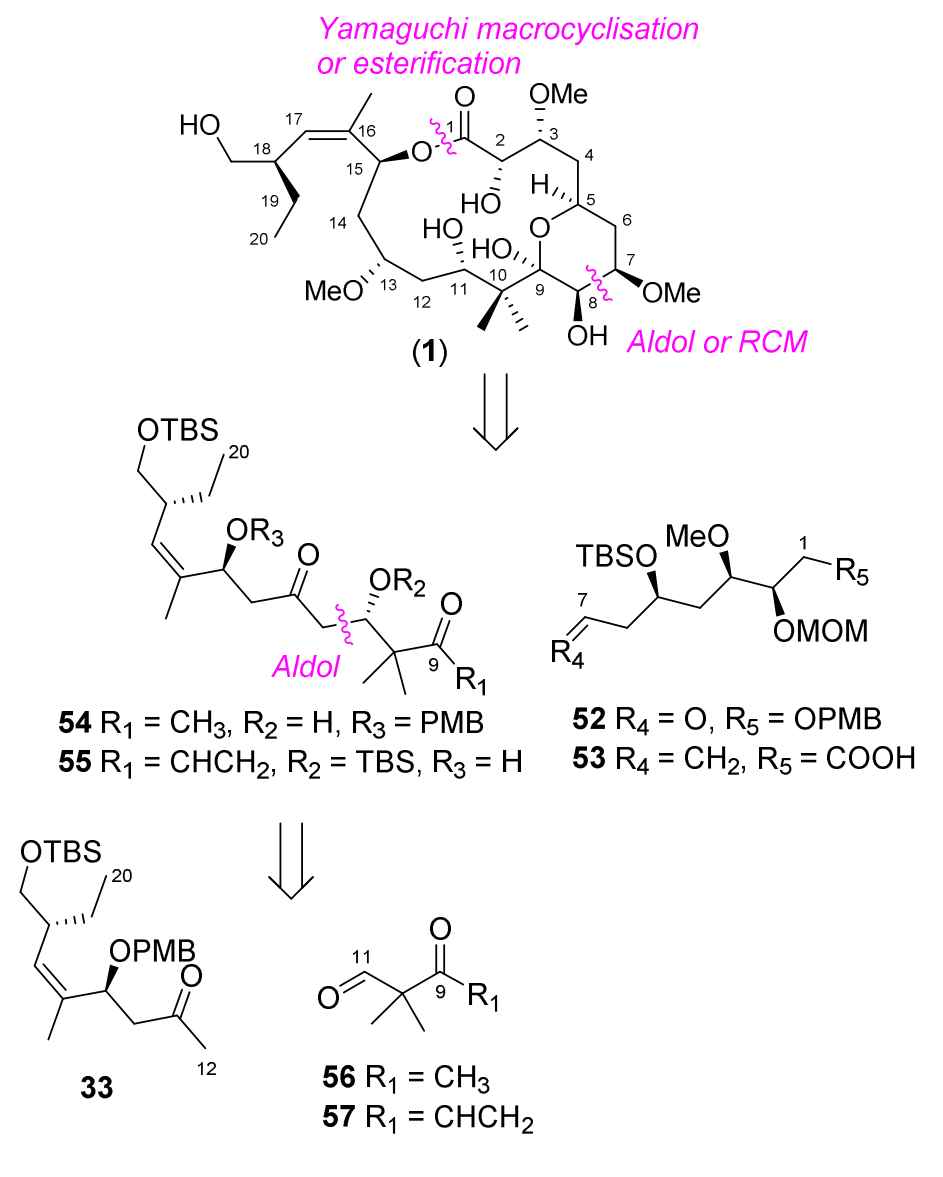

Scheme 1.7: Retrosynthetic approaches for the scalable synthesis of PelA

The goal of the two planned approaches to synthesis of PelA is to explore two different end-game strategies. The first end-game approach involves an aldol reaction to form the bond between $\mathrm{C} 7$ (of the $\mathrm{C} 1-\mathrm{C} 7$ aldehyde fragment 52) and $\mathrm{C} 8$ (of the C8-C20 methyl ketone fragment 54), which would complete the carbon skeleton of PelA, and a Yamaguchi macrolactonisation ${ }^{57}$ to close the macrocycle. In the second approach, an esterification reaction (Yamaguchi ${ }^{57,58}$ or Steglich ${ }^{75}$ ) would be used to synthesise the carbon skeleton of PelA through the establishment of the ester linkage between $\mathrm{C} 1$ (of the $\mathrm{C} 1-\mathrm{C} 7$ acid fragment 53) to $\mathrm{C} 15$ (of the $\mathrm{C} 8-\mathrm{C} 20$ vinyl ketone fragment 55), and the macrocycle would be closed subsequently by a C7 to C8 ring-closing methathesis (RCM). In both approaches, the pyran ring would be allowed to close spontaneously after the macrocyclisation as this was observed to happen in previous syntheses by Ghosh, Evans, Jacobsen and Hoye. ${ }^{1-3,55}$ 
The strategies for the fragment syntheses relies on the previously reported total and partial syntheses. Given the plan to explore two different end-game approaches we opted for fragments that will give us easiest access to both sets of functionalities we need. Additionally, with our second objective being the synthesis of analogues of PelA, a secondary criterion is fragment synthesis that enables ready access to different versions of the fragments necessary for the synthesis of the desired analogues.

The two synthetic plans, RCM and macrolactonisation, would give peloruside A with 22 and 23 steps in the longest linear sequence (IIs), respectively. In comparison, of the previous total syntheses of PelA, Jacobsen's was the shortest (20 steps IIs) but it relies on the use of multiple expensive and complex catalysts. ${ }^{3}$ Evans' total synthesis ${ }^{1}$ was 22 steps (Ils) but it involves the use of a highly pyrophoric trimethylaluminium (in the synthesis of the C7-C11 fragment). Other total syntheses were slightly longer: De Brabander's 28 steps $^{54}$, Taylor's 30 steps $^{4,64}$ (counted from the commercially available $(R)$-4-benzyl-1,3-oxazolidin-2-one), Ghosh's 29 steps $^{2}$ and Hoye's 36 steps $^{55}$ Ils. We envisaged that the use of readily available and generally inexpensive starting materials and reagents will compensate for the number of steps. Additionally, due to increased safety considerations associated with the scale-up process, the use of highly toxic and highly flammable reagents will be avoided where possible.

\subsubsection{Strategies for fragment synthesis}

The strategy for the C12-C20 fragment (33) synthesis was based on Evans' methodology with the added benefit that Taylor and Ghosh had similar aldol based approaches such that a "mix and match" approach to the reactions and structures might allow optimisation of the overall fragment synthesis. ${ }^{1,2,4}$ The synthesis starts from commercially available oxazolidone (58) that would be lithiated, then acylated using butyryl chloride (Scheme 1.8). The next step would be a trioxane aldol reaction that was adopted from Zhou's synthesis of the C11C20 fragment of peloruside $A .^{76}$ This change from Evans' methodology makes the overall fragment synthesis one step shorter. The resulting alcohol (59) would then be protected as a tert-butyldimethylsilyl ether (TBS). The auxiliary would then be reductively removed to give the alcohol 61. After oxidation, the aldehyde would undergo an olefination reaction to selectively give the Z-trisubstituted olefin (62). To achieve this both Evans and Ghosh used 
Ando's modification of the HWE olefination. ${ }^{1,72}$ Another option here was the use of the StillGennari modification of HWE olefination that was employed successfully by Taylor. ${ }^{4,64}$ After olefination the ester would be reduced to alcohol 63. From this alcohol, Evans methodology for the synthesis of the methyl ketone fragment $\mathbf{6 4}$ involves three more steps: (a) Oxidation to aldehyde, (b) Brown allylation ${ }^{68,69}$ to give the terminal alkene and (c) the Wacker oxidation. ${ }^{77}$ Here we decided to introduce novelty as well as shorten the sequence by using a proline-catalysed acetone aldol reaction which is expected to introduce the desired stereochemistry at $\mathrm{C} 15$, as well as the $\mathrm{C} 13$ ketone in a single step from the aldehyde. Then the para-methoxybenzyl (PMB) protection of the C15 hydroxyl group would conclude the synthesis of 33.

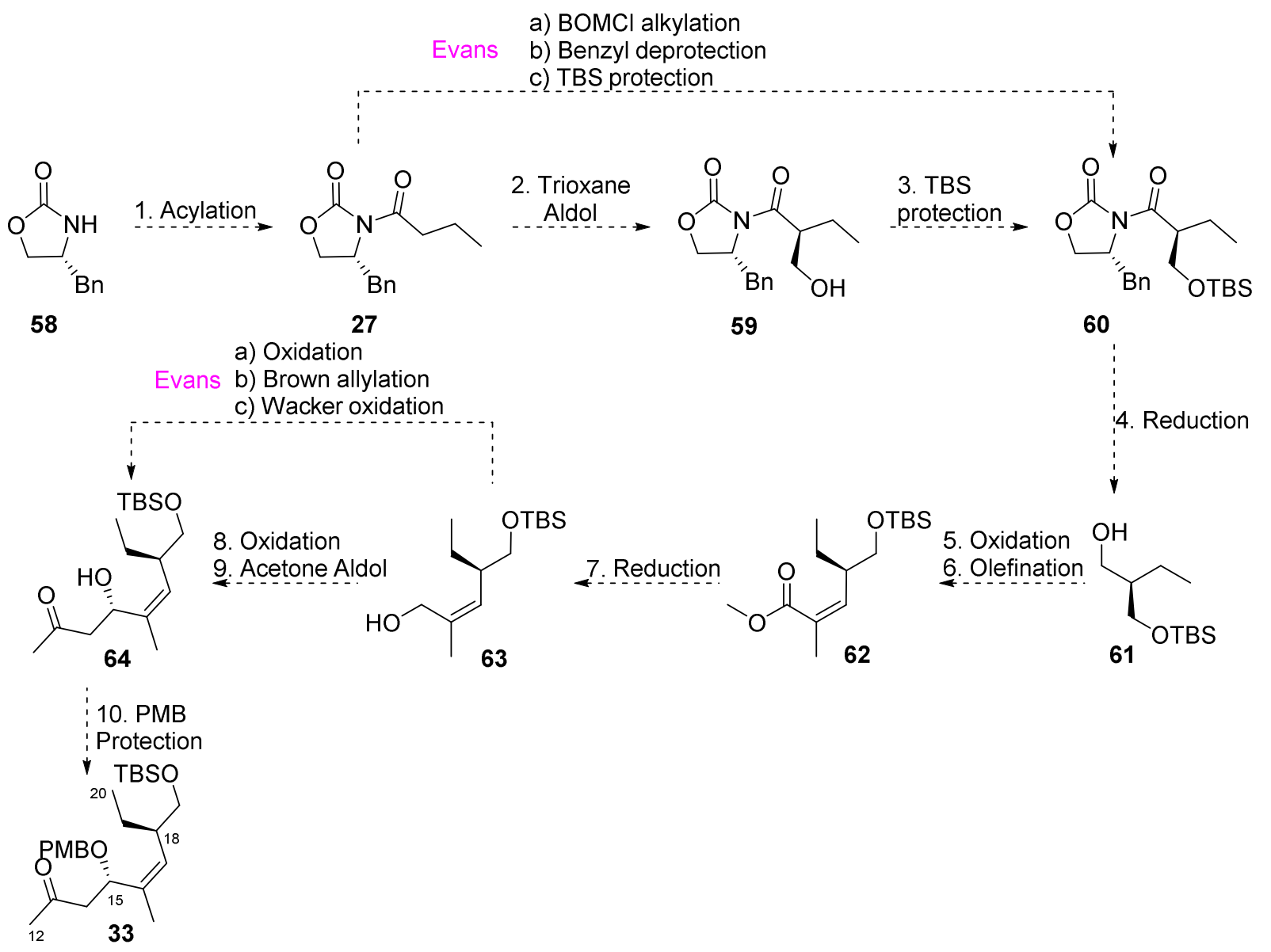

Scheme 1.8: Synthetic strategy for C12-C20 fragment 33

The synthesis of the C8-C11 fragments is based on De Brabander's route. ${ }^{54}$ This synthesis uses well established methodology and allows for easy introduction of different substituents, as required for our two strategies, at C8 by means of altering the Grignard 
reagent used (Scheme 1.9). The synthesis would start from commercially available 2,2-dimethylpropane-1,3-diol (65) that would be mono-benzyl protected and the resulting alcohol (66) oxidised to the corresponding aldehyde (67). This aldehyde would then be either methylated (MeMgl) or vinylated (vinylMgBr) to give the corresponding secondary alcohols 68 and 69. The oxidation of these alcohols would give C8-C11 methyl ketone (24) or vinyl ketone (70) intermediates, respectively. After the removal of the benzyl ( $\mathrm{Bn})$ protecting group, the oxidation of the primary alcohols 71 and $\mathbf{7 2}$ would the desired aldehyde fragments 56 and 57.
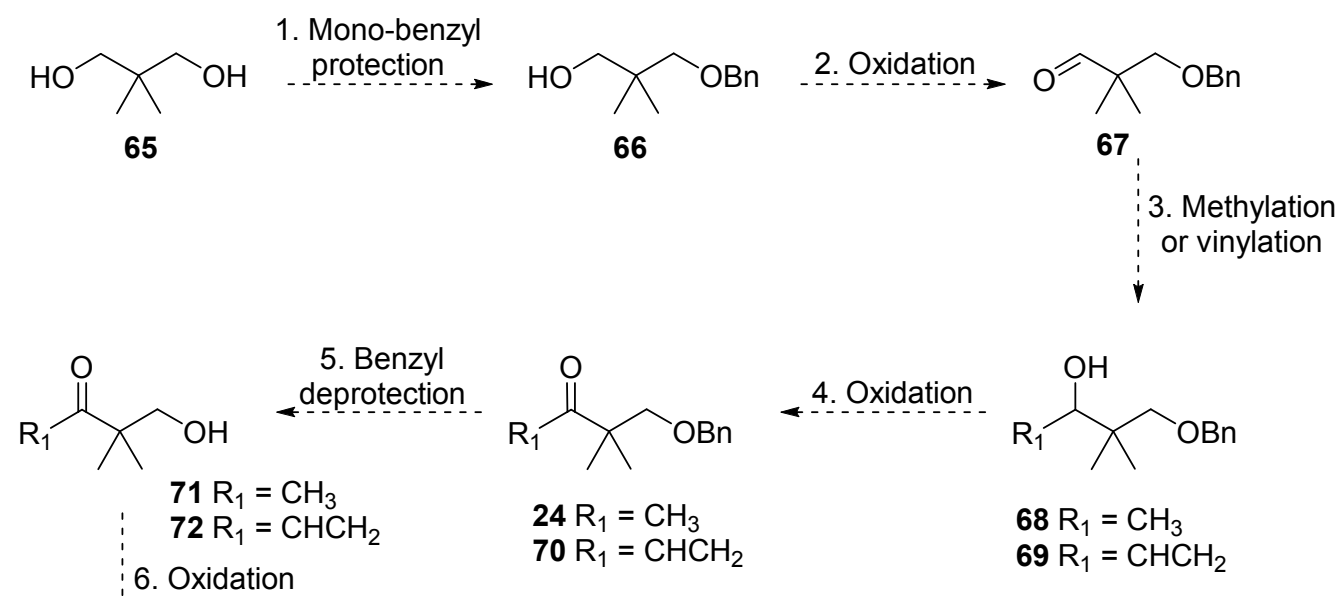

i

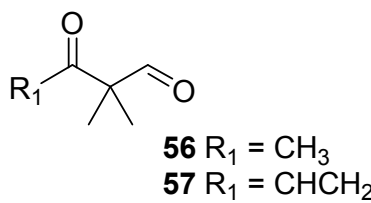

Scheme 1.9: Strategy for the synthesis of C8-C11 fragments 56 and $\mathbf{5 7}$

The synthetic strategy for the C1-C7 fragment is original (Scheme 1.10) but it borrows elements from Ghosh's synthesis of the C1-C10 fragment, mainly use of the same starting material, (-)-2,3-O-ispropylidene-D-threitol (32), and the protecting group strategy. ${ }^{2}$ In the same manner as Ghosh, the diol would be mono-protected ${ }^{2}$ with a PMB group and the resulting alcohol would be converted to an iodide (74) in an Appel reaction. ${ }^{78}$ This iodide would then be reacted with 2-allyl-1,3-dithiane to give the full seven-carbon skeleton of the fragment (75). Next, the acetonide protecting group would be removed under acidic conditions and the diol selectively $\mathrm{O}$-methylated at $\mathrm{O} 3$, based on the assumed steric differentiation of the two hydroxyls. The $\mathrm{C} 2$ hydroxyl would be protected as a MOM acetal. 


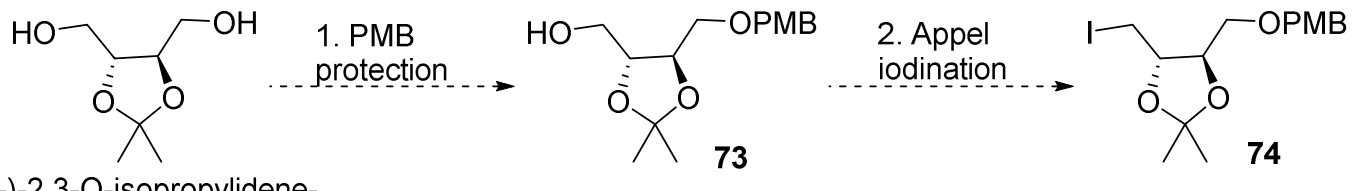

(-)-2,3-O-isopropylidene-

D-threitol 32

3. Allyldithiane coupling

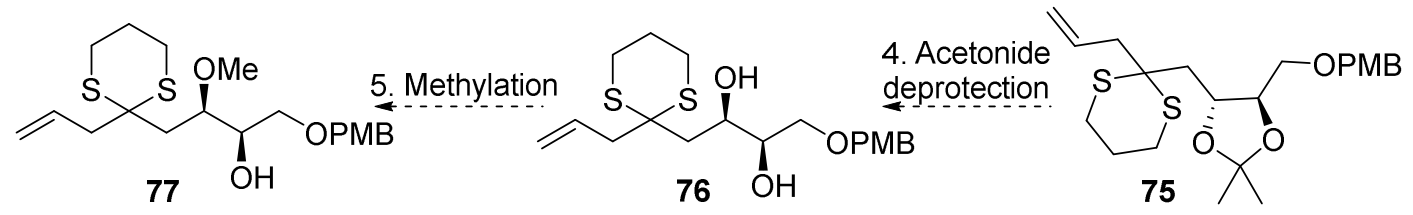

6. MOM protection

ì

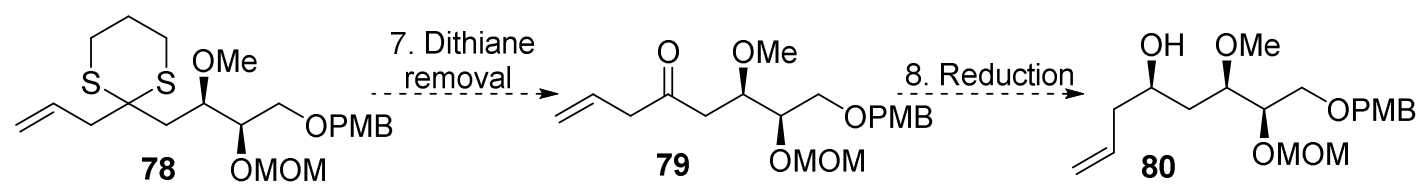

78 OMOM

79

10. Ozonolysis

52
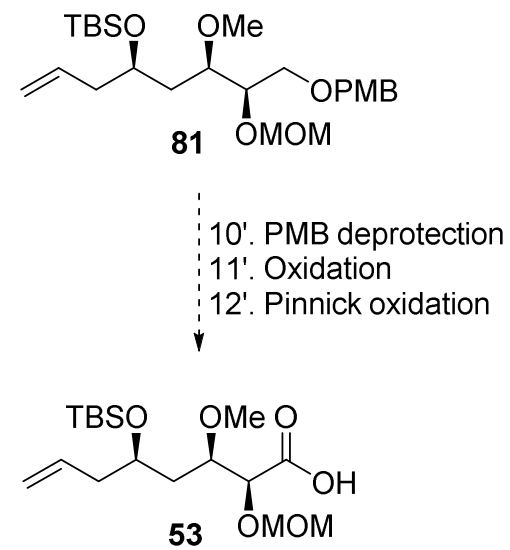

Scheme 1.10: Plan for the synthesis of C1-C7 fragments 52 and 53

The dithiane cleavage should reveal the C5 ketone (79), which will be stereoselectively reduced and the resulting alcohol $\mathbf{8 0}$ protected as a silyl ether (TBS). This would give the C1C7 intermediate 81, which is the last common precursor for the two C1-C7 fragments. Ozonolysis of the terminal double bond in $\mathbf{8 1}$ would give the C1-C7 aldehyde fragment $\mathbf{5 2}$ necessary for the macrocyclisation route. For the synthesis of the $\mathrm{C} 1-\mathrm{C} 7$ acid fragment (53), intermediate $\mathbf{8 1}$ would undergo PMB-deprotection at $\mathrm{O} 1$ and the resulting alcohol would be 
oxidised first to the aldehyde using TEMPO/BAIB oxidation, ${ }^{79}$ then to the carboxylic acid by the means of Pinnick oxidation. ${ }^{67}$

\subsubsection{Fragment coupling}

The C12-C20 fragment (33) would be first coupled to two different to the C8-C11 fragments (56 and 57) in a stereoselective and enantioselective boron-mediated 1,5-anti-aldol reaction previously employed by Evans. ${ }^{1}$ The resulting $\beta$-hydroxy diketones $\mathbf{8 2}$ and $\mathbf{8 3}$ subjected to the series of modifications (Scheme 1.11) used by Evans in order to achieve selective 1,3anti-reduction of the $\mathrm{C} 13$ ketone and the subsequent 013 methylation. ${ }^{1}$ This would complete the synthesis of $\mathrm{C} 8-\mathrm{C} 20$ methyl ketone fragment 54. The vinyl ketone $\mathrm{C} 8-\mathrm{C} 20$ intermediate $\mathbf{8 8}$ would undergo 011 TBS protection followed by 015 PMB deprotection (Scheme 1.11) to give the complete C8-C20 vinyl ketone fragment 55. Because of the steric effect of the $\mathrm{C} 10 \mathrm{gem}$-dimethyl it might not be necessary to protect the $\mathrm{C} 11$ hydroxyl group prior to the esterification and this will also be explored.
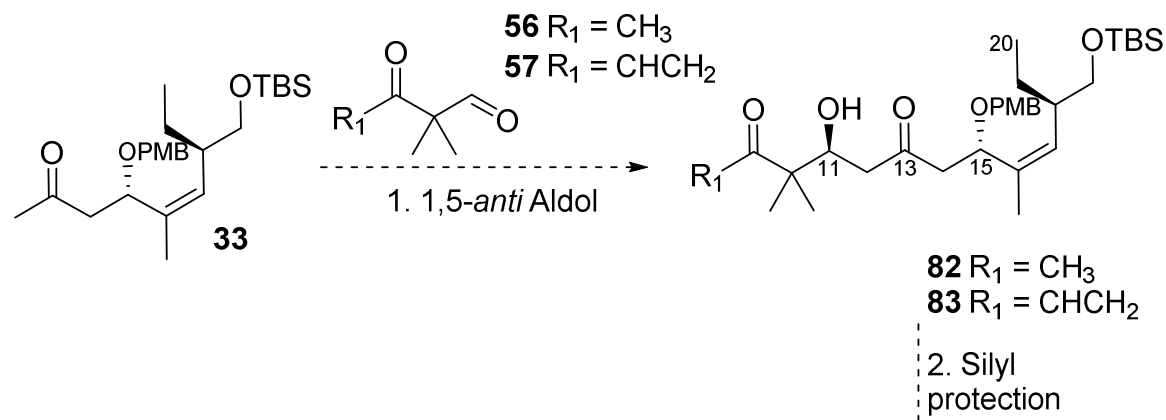

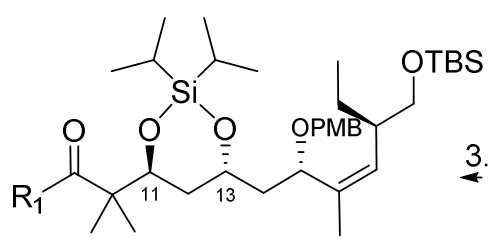

$86 \mathrm{R}_{1}=\mathrm{CH}_{3}$ 4. Silyl deprotection 5. Methylation

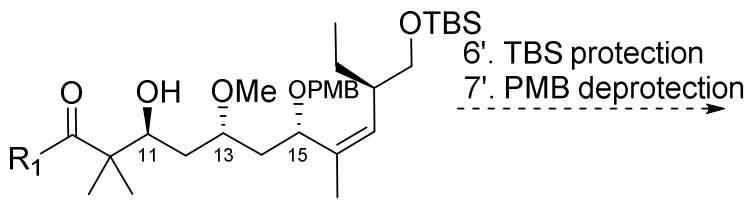
$54 \mathrm{R}_{1}=\mathrm{CH}_{2}$
3. 1,3-anti-reduction

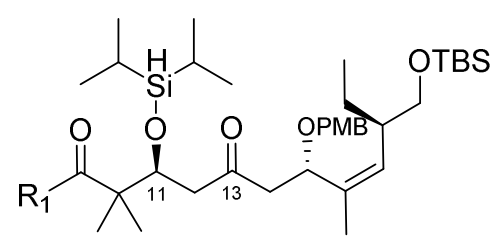

$84 \mathrm{R}_{1}=\mathrm{CH}_{3}$ $85 \mathrm{R}_{1}=\mathrm{CHCH}_{2}$

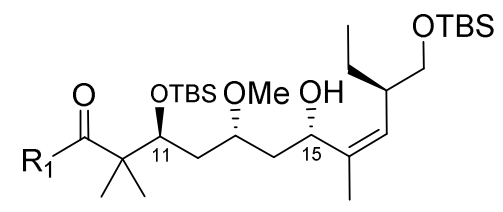

$55 \mathrm{R}_{1}=\mathrm{CHCH}_{2}$ 
The methyl ketone C8-C20 fragment 54 would then be reacted with C1-C7 aldehyde 52 in a Paterson aldol reaction using (+)-DIPCl to obtain the desired stereochemistry at C7 (Scheme 1.12). ${ }^{80,81}$ The $\mathrm{C} 7$ hydroxyl group would then be O-methylated selectively in the presence of the $\mathrm{C} 11$ hydroxyl due to steric effect imposed on the 011 by the $\mathrm{C} 10 \mathrm{gem}$-dimethyl group. In the next step, the resulting compound 90 would be hydroxylated at C8 (91). This hydroxylation, can be achieved using Davis' oxaziridine (92) as this methodology is commonly used to selectively $\alpha$-hydroxylate a ketone and was never employed on PelA system before. ${ }^{82,83}$ Here it would be possible to use chiral variants of the oxaziridine to obtain enantioselectivity ${ }^{84,85}$ but the non-chiral oxaziridine (92) would be tried first to obtain both epimers of $\mathbf{9 1}$, as the 8-epi-91 can be used for the analogue synthesis (vide infra).

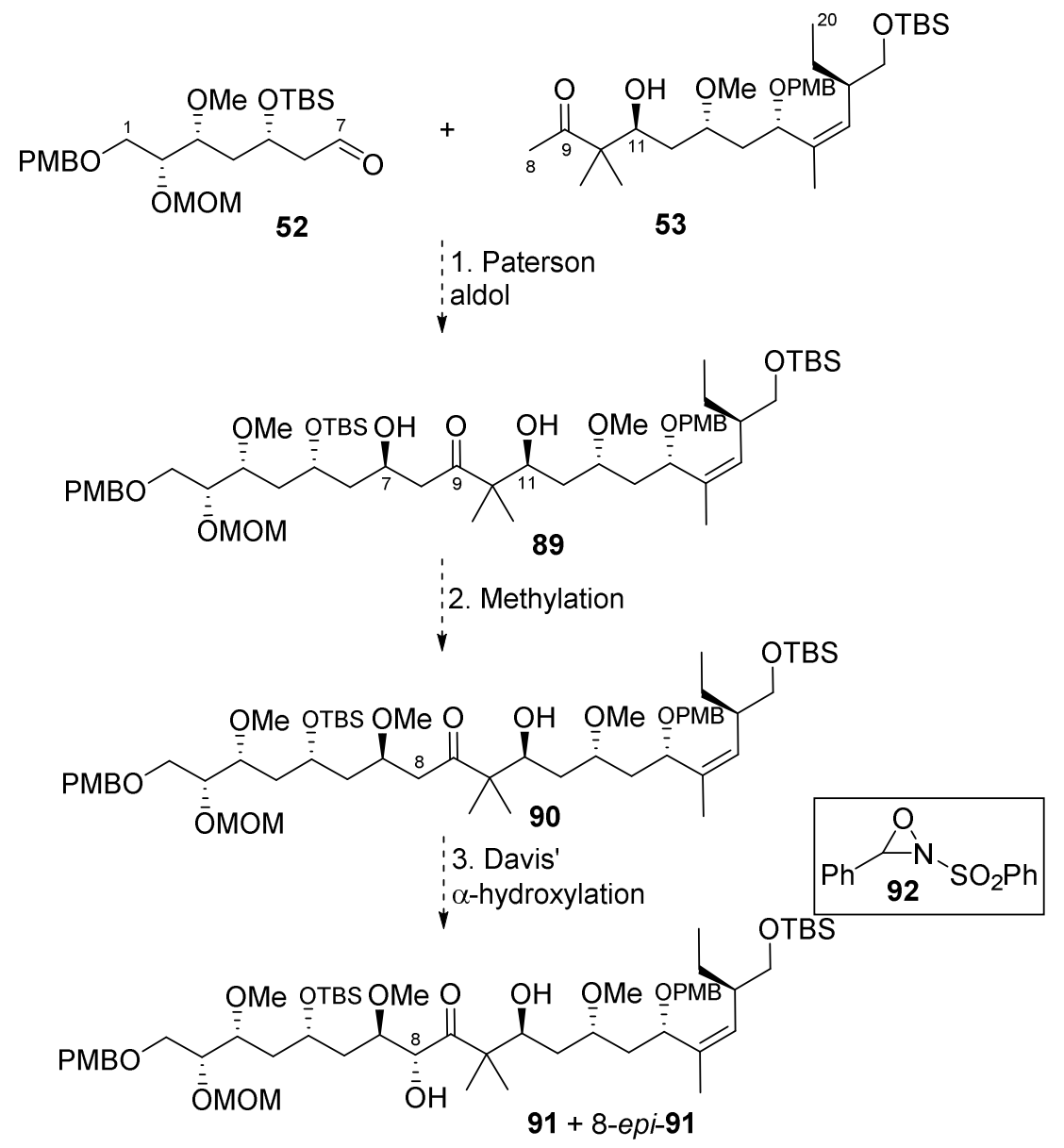

Scheme 1.12: $\mathrm{C} 1-\mathrm{C} 20$ complete carbon skeleton (91) of PelA through C7-C8 aldol coupling

The C8-C20 vinyl ketone 55 and the $\mathrm{C} 1-\mathrm{C} 7$ acid $\mathbf{5 3}$ fragment would be joined together through an esterification (Yamaguchi or Steglich) ${ }^{57,58,75}$ which will give the full carbon skeleton (93, Scheme 1.13) of PelA. 


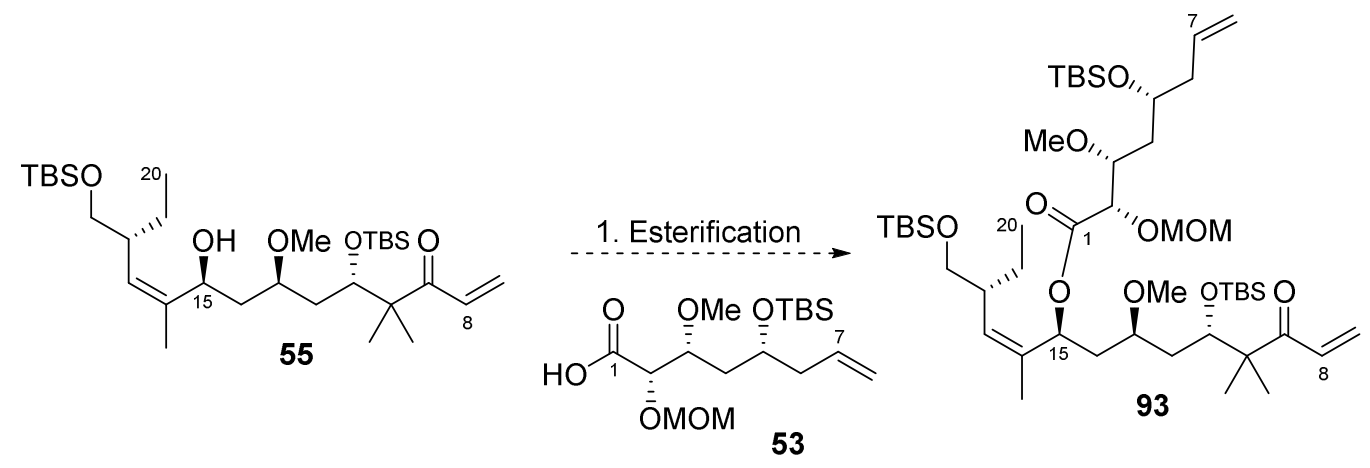

Scheme 1.13: C1-C20 complete carbon skeleton (93) of PelA through C1 to C15 esterification

\subsubsection{End-game strategy 1: Macrolactonisation approach}

On the complete carbon skeleton of PelA obtained through the aldol reaction ${ }^{80,81}$ (91), both PMB ethers (at 01 and 015 ) would be removed next (Scheme 1.14). This would be followed by a selective oxidation of the primary $\mathrm{OH}$ in the presence of a secondary which was already successfully achieved by Ghosh and Jacobsen. ${ }^{2,3}$ Then the aldehyde at C1 would be oxidised to give the seco-acid 94.

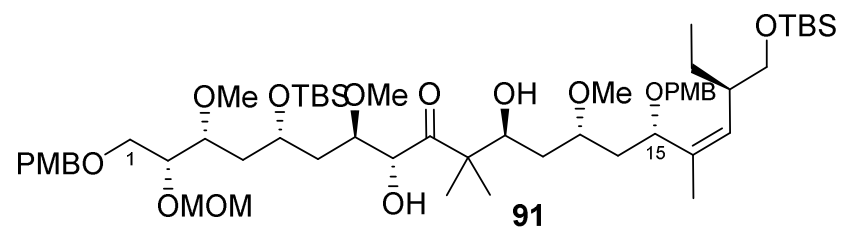

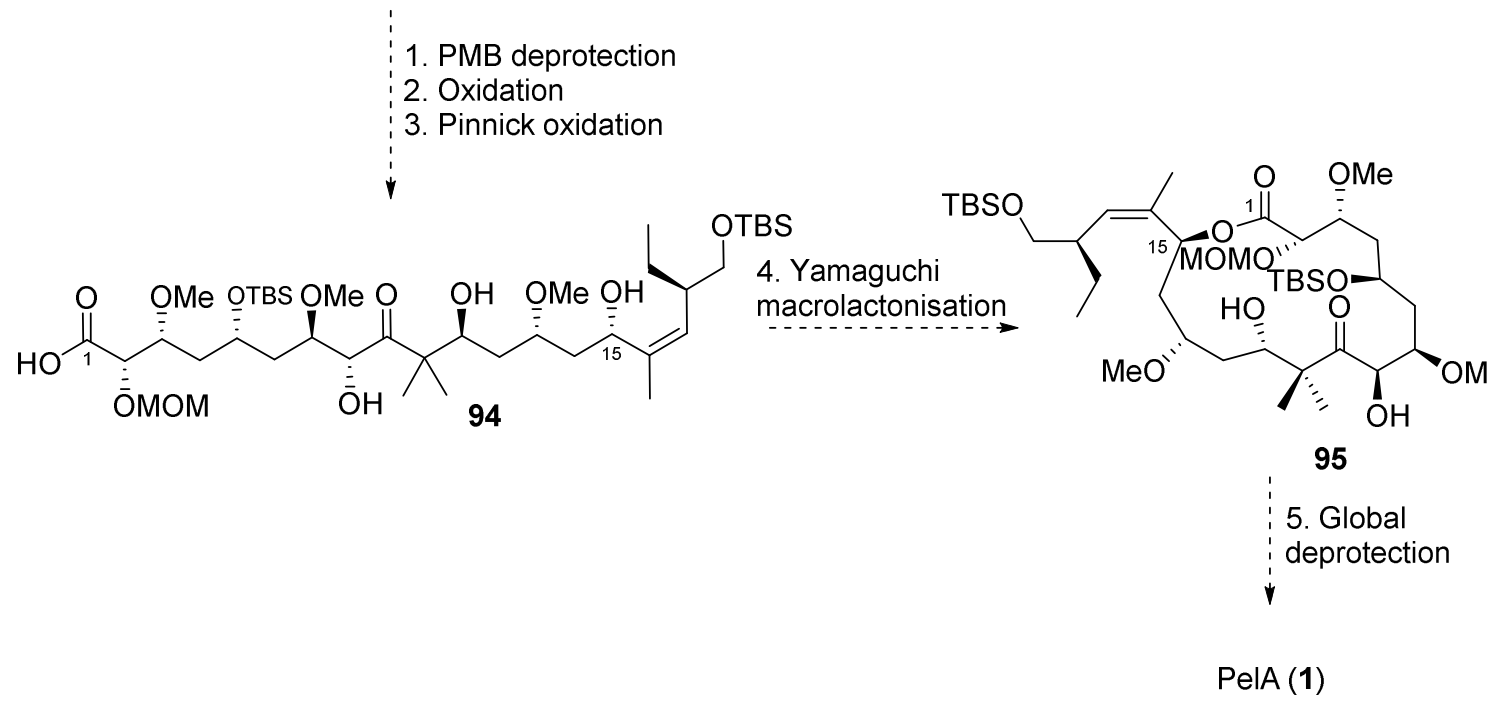

Scheme 1.14: PelA synthesis through Yamaguchi macrocyclisation

The Yamaguchi macrocyclisation will be used to form the macrocycle since it has already been used in all but one total synthesis of PelA. ${ }^{1-4,55}$ Finally global deprotection, as already 
observed in some previous syntheses, ${ }^{1,3,55}$ should facilitate spontaneous hemiketal formation and therefore result in formation of PelA.

\subsubsection{End-game strategy 2: Ring-closing metathesis approach}

The complete carbon skeleton of PelA obtained through esterification (93) would undergo a ring-closing metathesis using Grubbs' catalyst to give the C7-C8 olefin 96 (Scheme 1.15).

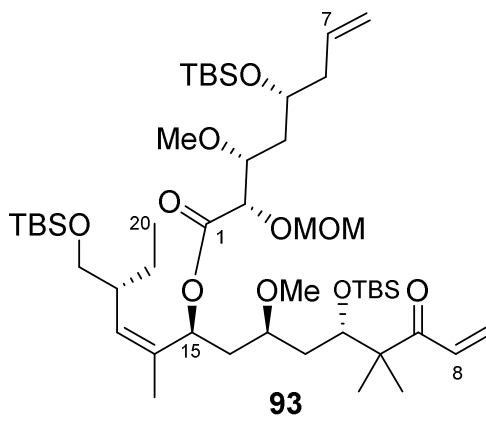

93

PelA (1)

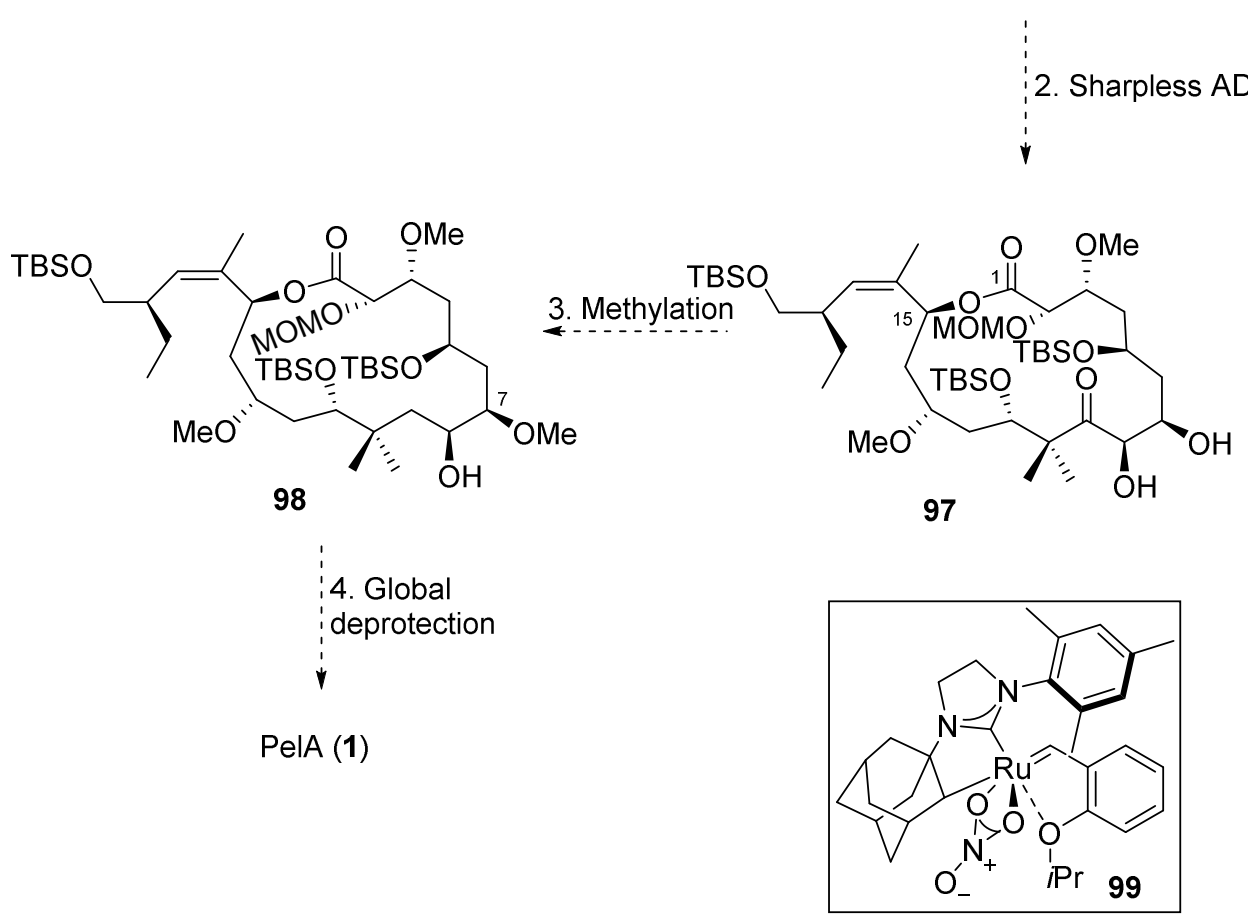

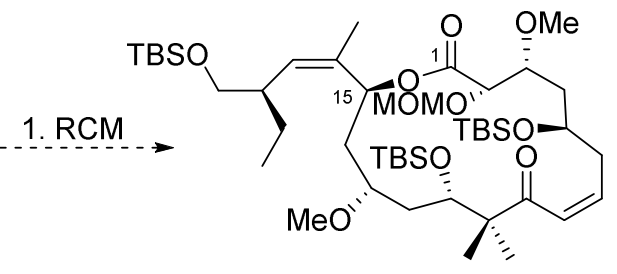

96

Scheme 1.15: PelA synthesis through RCM

Macrocyclisation by RCM has never been employed for PelA synthesis, but has been used for PelA analogue syntheses. Altmann's analogues ${ }^{86,87}$ of PelA were all synthesised through RCM macrocyclisation where the metathesis reaction constructed the C4-C5 olefin, whereas Lebreton and Mathé-Allainmat ${ }^{88}$ used RCM to construct a C2-C3 olefin. Taylor also employed RCM to obtain a $\mathrm{C} 12-\mathrm{C} 13$ double bond in the synthesis of his conformational 
analogues of PelA. ${ }^{89}$ In first two cases an E-olefin was obtained exclusively and Taylor obtained a 1:3.5 Z/E ratio in his analogues.

To selectively obtain the Z-7,8-alkene 96 we would use a Z-selective ruthenium Grubbs catalyst $^{90}$ (99), which is not much more expensive than the Grubbs II catalyst (Sigma Aldrich prices for $100 \mathrm{mg} Z$-selective Grubbs, NZ\$ 164.50; Grubbs II, NZ\$ 128.50). Then, the Sharpless asymmetric dihydroxilation ${ }^{71,91}$ would be used for functionalisation of the double bond to give the Z-7,8-diol 97. Even though Sharpless AD doesn't always have good enantioselectivity on Z-olefins, ${ }^{92}$ dihydroxylation of the Z-7,8-olefin was previously done on a fragment by Ghosh ${ }^{2}$ using AD-mix $\alpha$ and gave the diastereomeric ratio of 6.3:1 in favour of the desired diastereomer. The dihydroxylation would be followed by 07 methylation (98) where the regioselectivity would result from the steric imposition of the $\mathrm{C} 10 \mathrm{gem}$-dimethyl group on the hydroxyl at $\mathrm{C} 8$. The global deprotection should then lead to a spontaneous formation of the pyran ring (or an equilibrium mixture of hemiketal and macrocycle). ${ }^{1,2}$ With this, the synthesis of PelA would be completed.

\subsection{Thesis objective 2: Design of PelA analogues}

Several natural congeners of PelA have been isolated and other analogues synthesised, and their anticancer activity examined. ${ }^{93}$ So far, peloruside $A$ seems to be more active in inhibiting cellular growth than all the synthetic analogues and natural congeners (Table 1.2, Figure 1.9)..$^{93}$ Comparing the activity of PelA and analogues and relating it to the structural differences, it appears that the presence of decorations on the pyran ring and the pyran ring itself (although not essential for anticancer activity) is highly beneficial as these features increase the anticancer activity several-fold.

The lack of an oxygen substituent at C8 position drastically reduced the activity of Peloruside C (101) in human leukemia HL-60 cell lines (221 nM compared to PelA 7-35 nM, Table 1.2). ${ }^{94}$ Additionally, synthetic analogue 104 lacking both $C 7$ and C8 substituents was significantly less cytotoxic against breast cancer cell line MCF-7 (247 nM) than PelA (4.9 $\mathrm{nM}){ }^{88}$ These results are not surprising given that 07 and 08 hydrogen bonds are thought to be responsible for PelA bridging the two adjacent $\beta$-tubulin subunits, one of the ways in which PelA stabilises the microtubule. 


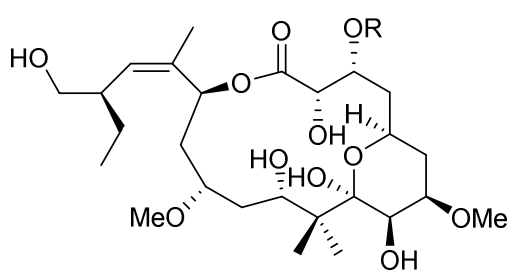

Peloruside A (PelA, 1, R = Me)

Peloruside $B(\mathbf{1 0 0}, R=H)$

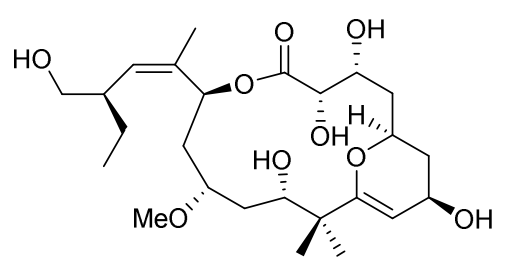

Peloruside C (101)

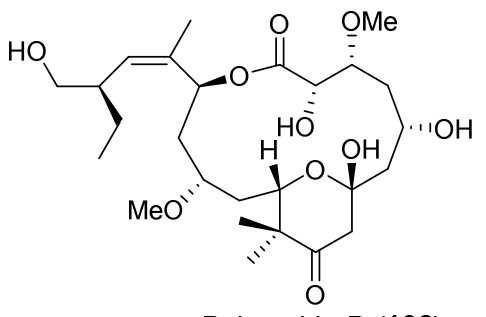

Peloruside D (102)

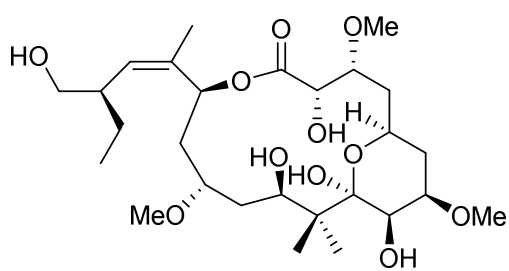

C11-epi-PelA (103)

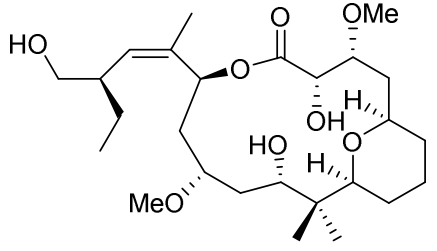

7-Desmethoxy-8,9-dideoxy-PelA

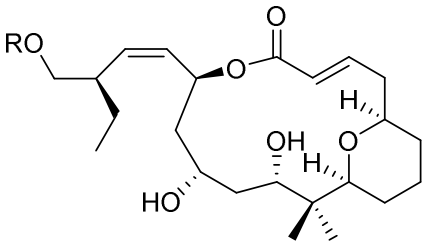

(104) 7-Desmethoxy-13-O-desmethyl16-desmethyl-2,3,8,9-tetradeoxydidehydroPelA $(105, \mathrm{R}=\mathrm{H})$

Silyl-protected $106(\mathrm{R}=\mathrm{SiMe} 2 \mathrm{tBu})$

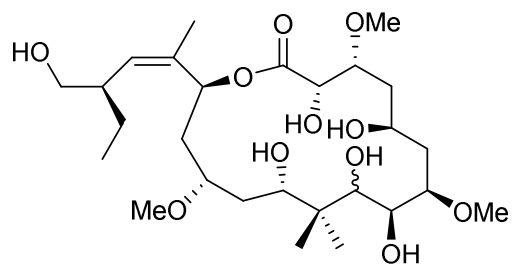

PelA reduction product 107

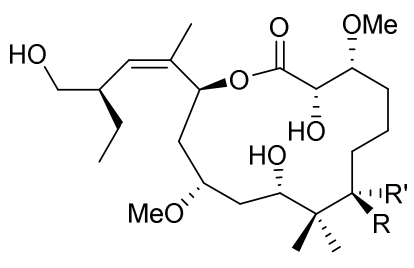

7R-des-THP-PelA (108, $\left.\mathrm{R}=\mathrm{OH}, \mathrm{R}^{\prime}=\mathrm{H}\right)$ 7S-des-THP-PelA $\left(109, \mathrm{R}=\mathrm{H}, \mathrm{R}^{\prime}=\mathrm{OH}\right)$

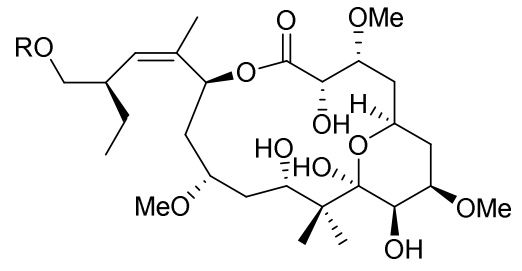

24-O-AcetylPelA $(\mathbf{1 1 0}, \mathrm{R}=\mathrm{Ac})$

24-O-ChloroacetylPelA

(111, $\mathrm{R}=\mathrm{ClCH} 2 \mathrm{C}(\mathrm{O})-$

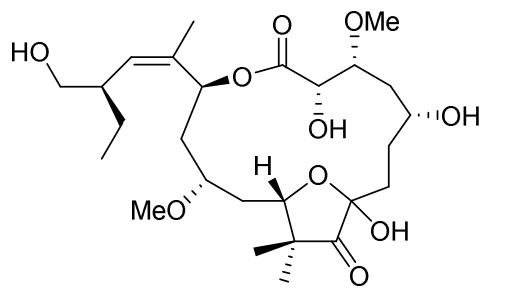

TFA-rearrangement product 112

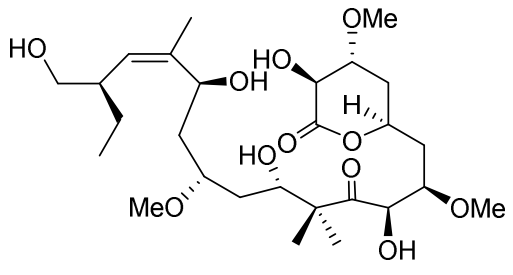

TfOH-rearrangement product 113

Figure 1.9: Natural and synthetic analogues of PelA 
Table 1.2: Cellular growth inhibition $I C_{50}$ values $(\mathrm{nM})$ in specified cell lines by PelA and its natural and synthetic analogues ${ }^{93}$

\begin{tabular}{|c|c|c|c|c|c|c|c|c|c|c|c|}
\hline & HL-60 & P388 & A2780 & A2780AD & $1 \mathrm{~A} 9$ & $\mathrm{CaCo} 2$ & HCT116 & PC3 & MCF-7 & A549 & $\begin{array}{l}\text { SK-MEL- } \\
\text { 5/HeLa }\end{array}$ \\
\hline $\begin{array}{ll}\text { Pel } & \text { A } \\
\text { (1) } & \end{array}$ & 7-35 & 18 & 19.2 & 880 & & & & & 4.9 & & \\
\hline ent-1 & & & & & & & & & & & $>10,000$ \\
\hline 100 & 33 & & & & $\begin{array}{l}48- \\
71\end{array}$ & & & & & & \\
\hline 101 & 221 & & & & & & & & & & \\
\hline 102 & 2,000 & & & & & & & & & & \\
\hline 103 & & 10 & & & & & & & & & \\
\hline 105 & & & & & & 12,000 & 15,000 & 15,000 & & & \\
\hline 106 & & & & & & $>25,000$ & $>25,000$ & $>25,000$ & & & \\
\hline 104 & & & & & & & 163 & & 247 & 124 & \\
\hline 107 & 221 & & & & & & & & & & \\
\hline 109 & & & & & & & $>20,000$ & & $>20,000$ & 16,400 & \\
\hline 108 & & & & & & & 1,170 & & 2,050 & 1,390 & \\
\hline 110 & & & 256.6 & 3,100 & & & & & & & \\
\hline 111 & & & 26.6 & 2,950 & & & & & & & \\
\hline 112 & $\begin{array}{l}15,00 \\
0\end{array}$ & & & & & & & & & & \\
\hline 113 & 7,000 & & & & & & & & & & \\
\hline
\end{tabular}

HL-60 = human leukaemia cells, P388 = murine leukaemia cells, A2780 = ovarian carcinoma cells, A2780AD = ovarian carcinoma cells overexpressing P-glycoprotein, $1 \mathrm{~A} 9=$ human ovarian carcinoma cells, $\mathrm{CaCo} 2$ = colon carcinoma cells, HCT116 $=$ colon carcinoma cells, $\mathrm{PC} 3=$ prostate carcinoma cells, MCF-7 = breast carcinoma cells, A549 = lung cancer cells.

The second project objective is to synthesise new analogues that explore the effects of stereochemistry at C7 and C8 on the bioactivity. The 8-epi-PelA analogue (Scheme 1.6) could be accessed in the macrolactonisation route from the 8-epi-91 intermediate produced in the non-enantioselective Davis $\alpha$-hydroxylation. ${ }^{82}$ 


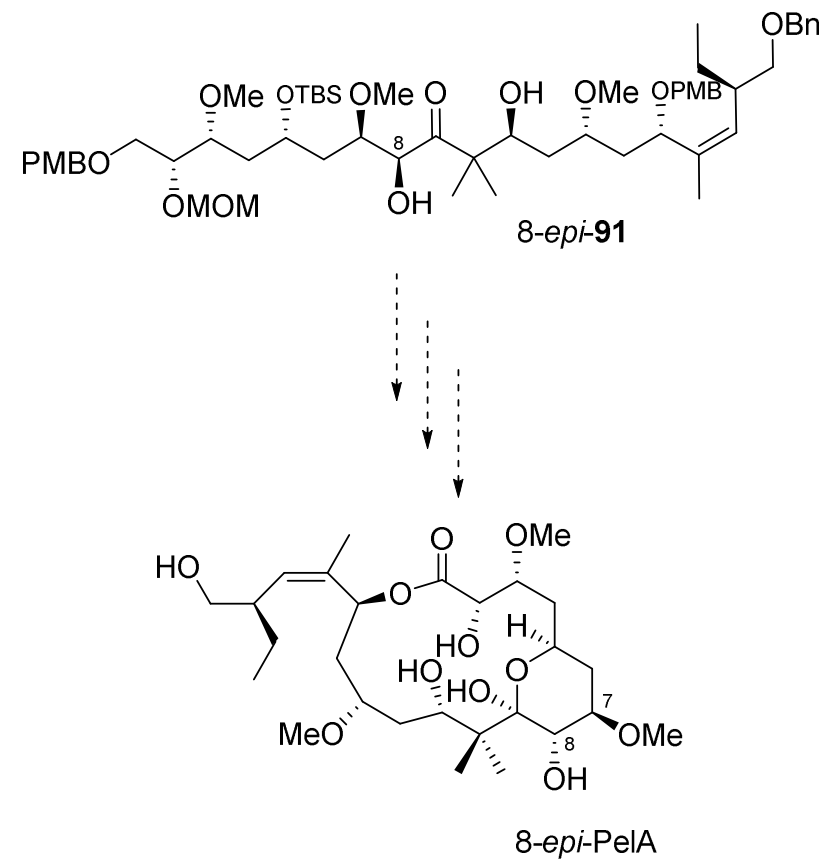

Scheme 1.16: Synthesis of 8-epi-PelA

The 7-epi-PelA analogue (Scheme 1.17) could be accessed from the RCM product 96 . Asymmetric epoxidation of the double bond would give epoxide 114, which can be opened in an $\mathrm{S}_{\mathrm{N}} 2$ reaction with a methoxy nucleophile. The regioselectivity here should give the $\mathrm{C} 7$ methoxy preferentially due to electronics and the significant steric bulk around C8 created by the $\mathrm{C} 10 \mathrm{gem}$-dimethyl substituents. There are several ways to achieve enantioselective epoxidation. Sharpless asymmetric epoxidation ${ }^{95}(A E)$ is one of them but it would require reduction of the C9 ketone before the epoxidation, in order to have the necessary allylic alcohol substrate, and the oxidation of the C9 hydroxyl after the epoxidation. This would introduce more steps into the overall synthesis. Alternative enantioselective epoxidations include the organocatalysed Shi epoxidation ${ }^{96,97}$ and the Jacobsen-Katsuki epoxidation ${ }^{98-100}$ that uses a chiral organomanganese catalyst species. It is also planned to try nonenantioselective epoxidation conditions $\left(\mathrm{NaOH}, \mathrm{H}_{2} \mathrm{O}_{2} / t-\mathrm{BuOOH} / m \mathrm{CPBA}\right)$ to ascertain whether there is any substrate control with these general epoxidation reagents which would bring down the cost and complexity of this reaction. 


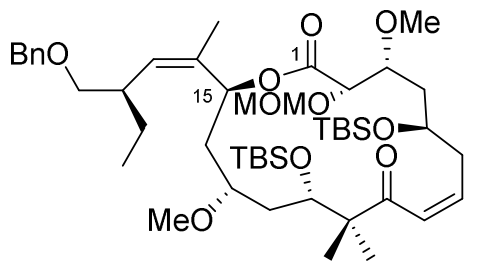

96

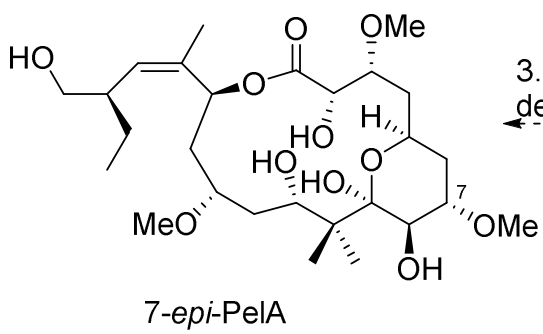

1. Asymmetric epoxidation

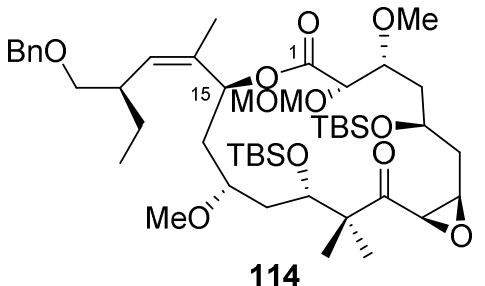

1. Epoxide opening -OMe

$\checkmark$

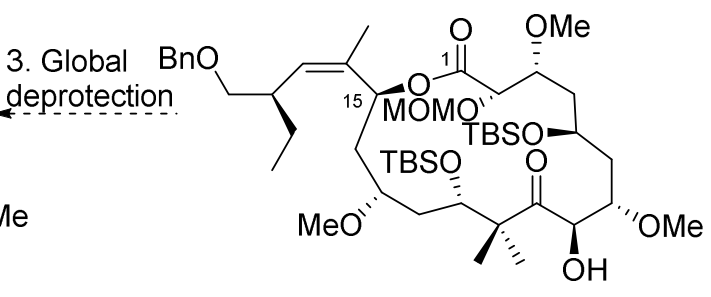

115

Scheme 1.17: Synthesis of 7-epi-PelA

With the plan developed, the synthesis of PelA and analogues could start. We commenced with the synthesis of the three fragments and their variations ( $\mathrm{C} 1-\mathrm{C} 7$ fragments $\mathbf{5 2}$ and $\mathbf{5 3}$, C8-C11 fragments 56 and 57 and the C12-C20 fragment 33). 


\section{Synthesis of the C12-C20 fragment}

\subsection{Retrosynthesis}

The plan for the synthesis of the side-chain fragment, C12-C20 (highlighted, Figure 2.1), was based mostly on Evans' synthesis of this fragment, although some transformations were adopted from other total and partial syntheses of PelA. ${ }^{1}$ This methyl ketone fragment will be coupled to the C8-C11 fragments using the aldol reaction also described by Evans ${ }^{1}$ (Chapter 1).

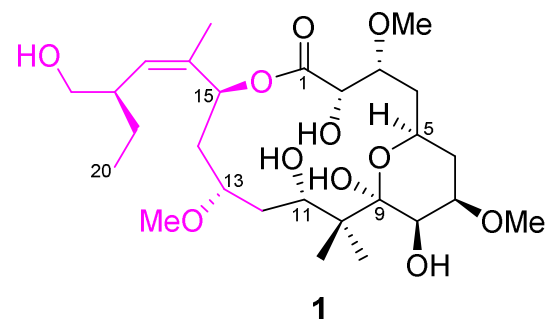

Figure 2.1: PelA structure with C12-C20 highlighted

Originally it was envisioned to use the same protecting groups (Pgs) as Evans, where Pg (Scheme 2.1) would be tert-butyldimethylsilyl (TBS) and Pg' would be a p-methoxybenzyl (PMB). This route was explored first. Later, due to the issues with some of the transformations and the changes in protecting group strategy for the synthesis of the $\mathrm{C} 1-\mathrm{C} 7$ fragment (Chapter 4), it was decided to change the Pg from TBS to benzyl (Bn) which also reduced the total number of steps in the synthesis of this fragment. A benzyl protecting group at this position is well precedented: it was used by both Ghosh and Jacobsen and in both cases the hydrogenolysis of the benzyl ether did not affect the side-chain double bond. ${ }^{2,3}$ Having the $\mathrm{C} 15$ hydroxyl protected as a PMB ether is of great importance for achieving the desired relative stereochemistry at C11 through 1,5-anti induction (Chapter 3).

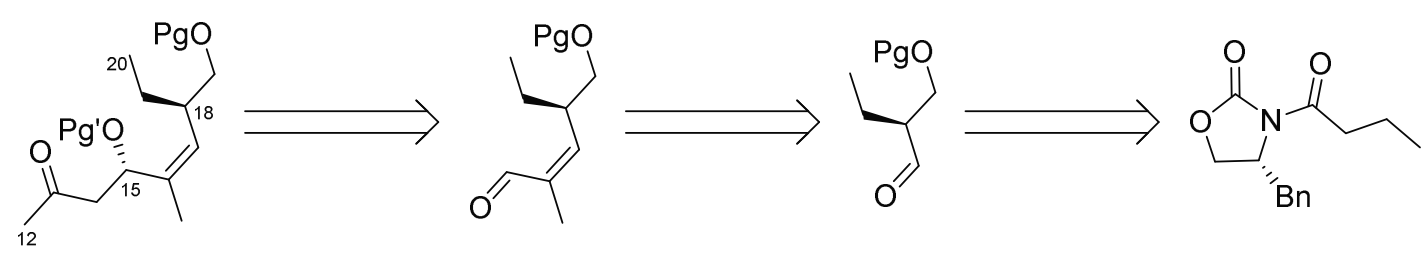




\subsection{First protecting group strategy}

Fragment 33 (Scheme 2.2) can be synthesised in ten steps from commercially available chiral oxazolidinone (58). The first step, $\mathrm{N}$-acylation, is followed by a trioxane aldol reaction and the resulting hydroxyl group (59) protected as a TBS ether. The chiral auxiliary is then reductively cleaved to give the primary alcohol (61). This alcohol can be oxidised to the corresponding aldehyde which undergoes olefination to install the trisubstituted Z-double bond (62). After the reduction of the methyl ester and the oxidation of the allylic alcohol (63), the resuting $\alpha, \beta$-unsaturated aldehyde (118, not shown in the scheme) is added to acetone in another aldol reaction, to give the methyl ketone 64 . The last step is the PMB protection of the hydroxyl at C15.

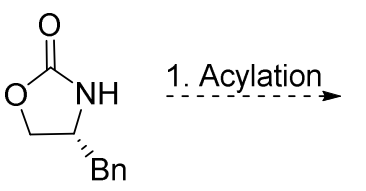

58<smiles>CCCC(=O)N1C(=O)OC[C@H]1Br</smiles>

27

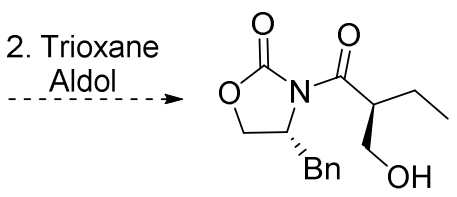

59

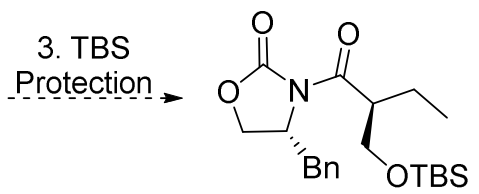

60<smiles>CCCCC(C=C(C)[C@@H](O)CC(C)=O)CO[AsH3]</smiles>

\section{Oxidation} 9. Acetone Aldol<smiles>CC[C@H](/C=C(/C)CO)C[O+]</smiles>

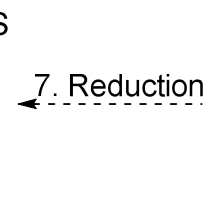<smiles>CC[C@H](/C=C(/C)C(=O)OC)CO</smiles>

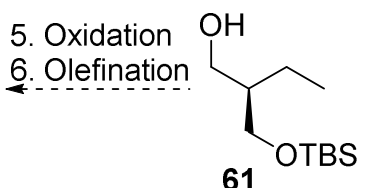

10. PMB

Protection

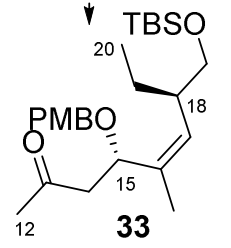

Scheme 2.2: Plan for the synthesis of the C12-C20 fragment 33

As discussed earlier, the plan for the synthesis of $\mathbf{3 3}$ was based on Evans' methodology. ${ }^{1}$ The trioxane aldol reaction (step 2, Scheme 2.2) was adopted from Zhou's partial synthesis and was used to reduce the number of steps in the synthesis of $60 .^{76}$ The commonly used sequence of steps, involving alkylation with benzyl chloromethyl ether (BOMCl), benzyl deprotection and TBS protection, was replaced with a trioxane aldol followed by TBS protection. The organocatalysed acetone aldol reaction (step 9, Scheme 2.2), to our 
knowledge, was never employed in the synthesis of this fragment and was included into the synthetic plan in order to reduce the number of steps as well as for novelty value.

\subsubsection{The synthesis of oxazolidinone 60}

The synthesis of the oxazolidinone 60 commenced (Scheme 2.3) via a well established $\mathrm{N}$ acylation of the commercially available $(R)$-4-benzyl-1,3-oxazolidin-2-one (58). ${ }^{86} n$ Butyllithium was used to deprotonate the oxazolidinone and butyryl chloride was then added as the acylating agent. This reaction was high yielding (94\%) and the product 27 generally did not require chromatographic purification.

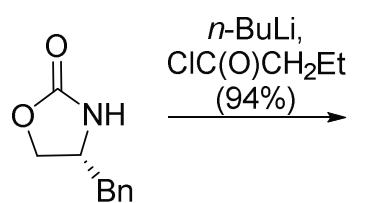

58

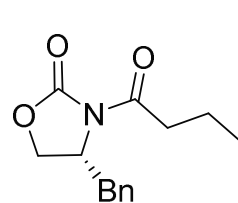

27

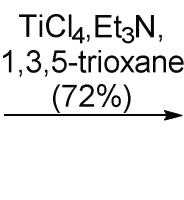

5 -trioxan

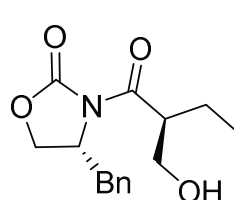

59

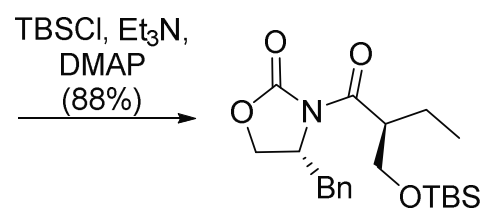

60

Scheme 2.3: The synthesis of oxazolidinone 60

The chiral oxazolidinone auxiliary was needed to direct the stereochemical outcome of the aldol reaction. The reaction gave the desired product 59 in $72 \%$ yield with a high stereoselectivity and the other diastereomer was never observed in the ${ }^{1} \mathrm{H}$ NMR spectrum of the crude reaction mixture. Experimental data for $\mathbf{5 9}$ matched those previously reported with the optical rotation value of -119.1 ( $c$ 1.15, $\mathrm{CHCl}_{3}$ ) being of the same sign but a different magnitude than the literature value $\left(-52.1, \mathrm{c} 1.0, \mathrm{CHCl}_{3}\right) .{ }^{101}$ Titanium tetrachloride serves a dual function in this reaction. The first equivalent is added at the start of the reaction and coordinates with the two carbonyl oxygen atoms, facilitating the $\alpha$ deprotonoation by triethylamine and the formation of the enolate. The second equivalent, added after trioxane, activates the trioxane making it more prone to a nucleophilic attack by the pre-formed enolate. This leads to in situ generation of formaldehyde (Scheme 2.4). ${ }^{76}$

Sometimes however, the dimer $\mathbf{1 1 9}$ was obtained as an inseparable by-product (Scheme 2.4). This dimer was first isolated after a surprisingly low yielding (50\% yield) TBS protection reaction, when it was at first mistaken for unreacted starting material. After the second attempt to TBS protect this "unreacted starting material" was completely unsuccessful, the material was fully characterised and identified as the dimer 119. The only new peak in the 
${ }^{1} \mathrm{H}$ NMR spectrum of the by-product (compared to the spectrum of the product) was of a singlet ( $\delta 4.69 \mathrm{ppm}$ ) corresponding to the $\mathrm{ROCH}_{2} \mathrm{OR}$ protons, and a corresponding peak in the ${ }^{13} \mathrm{C}$ NMR spectrum with a chemical shift of $95.5 \mathrm{ppm}$ for $\mathrm{ROCH}_{2} \mathrm{OR}$. These chemical shifts are quite typical for this type of acetal and are similar to proton and carbon chemical shifts observed with methoxymethyl acetal (MOM) protecting group for hydroxyls (R-OMOM, R$\left.\mathrm{OCH}_{2} \mathrm{OCH}_{3}\right)$. The IR spectrum showed no $\mathrm{OH}$ peak and the mass spectrum had its molecular ion peak at $\mathrm{m} / \mathrm{z} 567.2719$ for $\mathrm{C}_{31} \mathrm{H}_{39} \mathrm{~N}_{2} \mathrm{O}_{8}{ }^{+}[\mathrm{M}+\mathrm{H}]^{+}$in agreement with calculated $567.2714(\Delta$ $=2.3 \mathrm{ppm}$ ). The formation of $\mathbf{1 1 9}$ was attributed to the incomplete breakdown of trioxane (Scheme 2.4) after the initial aldol reaction. It was postulated that the amount of $\mathrm{TiCl}_{4}$ could be the key to efficient breakdown of trioxane. However, several attempts by fellow PhD colleague Ethan Woolly to suppress dimer formation by varying the quantity of $\mathrm{TiCl}_{4}$ were unsuccessful. Two attempts to hydrolytically break apart the dimer with $10 \%$ aq. $\mathrm{HCl}$ or PTSA were unsuccessful.

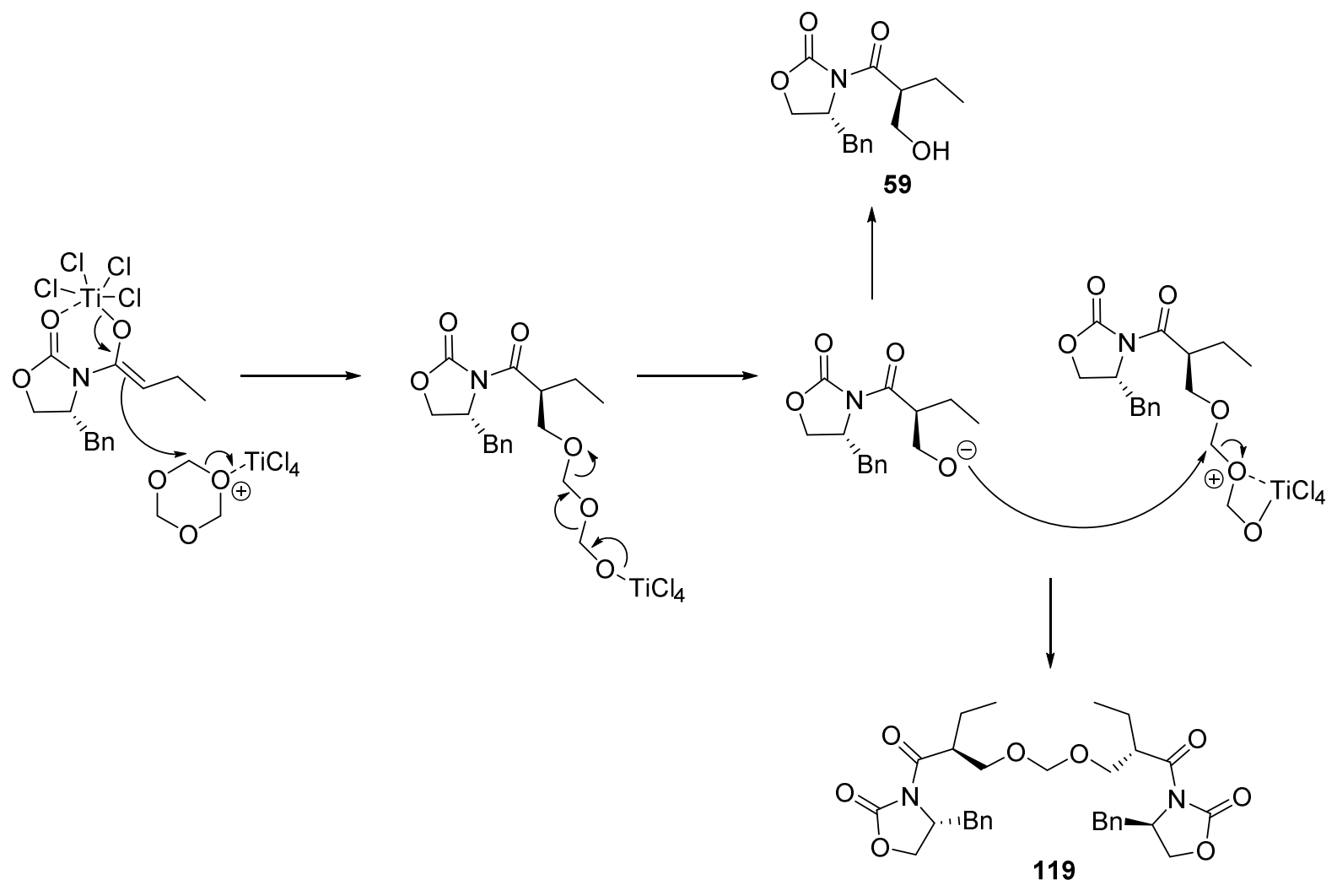

Scheme 2.4: Proposed mechanism for the formation of 119 
The last step in this sequence was the silyl protection of the new hydroxyl group to obtain the oxazolidinone $60 .^{101}$ The reaction gave the silyl ether in $88 \%$ yield. Experimentally derived spectroscopic data matched those previously reported for this compound. ${ }^{101}$

\subsubsection{The synthesis of methyl ester 62}

Reductive removal of the chiral auxiliary in 60 using lithium borohydride gave primary alcohol 61 (Scheme 2.5). Even though this reaction is well precedented, there was some concern about the possibility of TBS migration under the reaction conditions employed. However, the optical rotation of $[\alpha]_{\mathrm{D}}{ }^{24}=+14.8^{\circ}\left(\mathrm{c} 0.23, \mathrm{CHCl}_{3}\right)$ for the product obtained matched those previously reported. ${ }^{1,102}$ Oxidation of this primary alcohol was achieved using TEMPO/BAIB oxidation under anhydrous conditions and the resulting aldehyde was used directly in the next step, the olefination, without purification. ${ }^{79}$

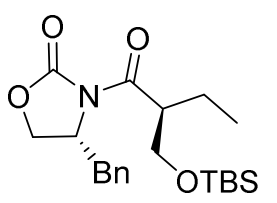

60
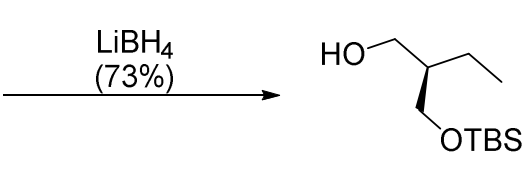

61

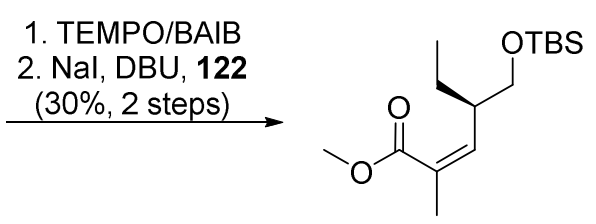

62

Scheme 2.5: The synthesis of methyl ester 62

To achieve the $Z$ selectivity, Still-Gennari and Ando modifications of the Horner-WadswordEmmons (HWE) reactions were considered. ${ }^{59,70}$ These modifications involve phosphonates with electron-withdrawing groups (EWG), phenyl and o-cresol (Ando) or trifluoroethyl (StillGennari), and strong bases which are together responsible for the Z-selectivity observed. In the classical HWE reaction (Scheme 2.6a), it is believed that the steps leading up to the final elimination are reversible and that this reversibility is responsible for predominant $E$ selectivity since the formation of the E-olefin is energetically favourable. In the Z-selective modifications of this reaction (Scheme 2.6b), the presence of the EWG on the phosphorus accelerates the elimination, making it faster than equilibration. ${ }^{59,70}$ With faster adduct elimination, the process becomes irreversible and the geometry set as in the initial addition step. Here, minimising steric interactions leads to the Z-olefin (kinetic product) selectively. ${ }^{70}$ 


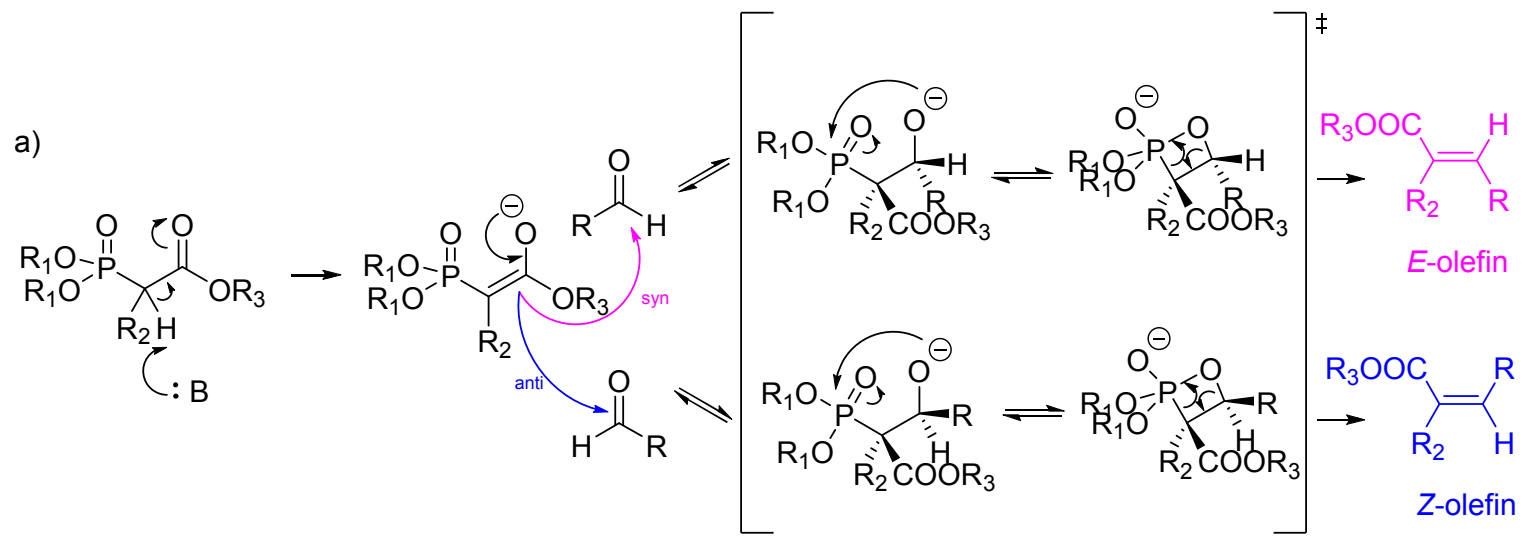

b)
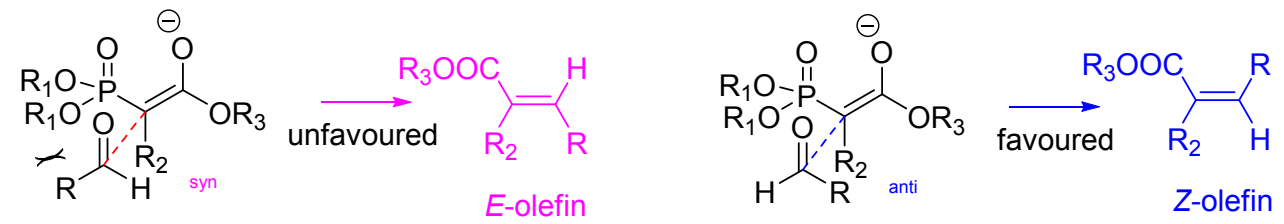

Scheme 2.6: a) General HWE olefination mechanism; b) Rationale for initial E/Z selectivity

Following Evans' methodology it was decided to use the Ando modification of the HWE reaction for which it was necessary to first synthesise the appropriate phosphonate (122, Scheme 2.7). The phosphonate 122, was synthesised in two steps (Scheme 2.7) in 41\% overall yield. $^{70,103}$ The first step, a nucleophilic substitution reaction between diphenylphosphite (120) and methyl bromoacetate in the presence of base, is followed by a-mono-methylation of the resulting methyl (diphenoxyphosphoryl)acetate (121). The modest yield over these two steps was attributed to the methylation reaction, which always resulted in formation of both mono and di-methylated products in various ratios. Fortunately, they were separable by silica gel chromatography.

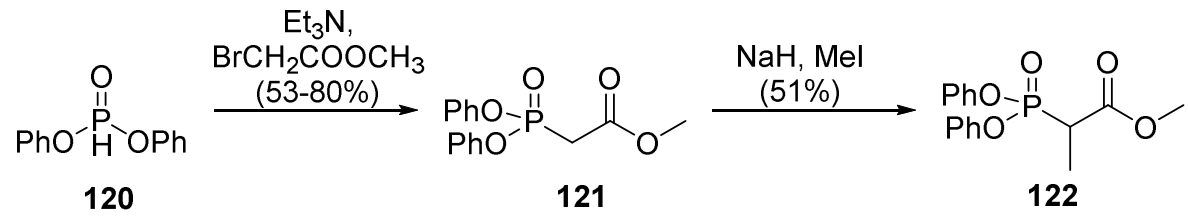

Scheme 2.7: The synthesis of phosphonate 122

With the phosphonate $\mathbf{1 2 2}$ in hand, the reaction was first performed under the same conditions as those employed by Evans, using sodium hydride (NaH) to deprotonate the phosphonate. However, under these conditions the reaction was very low yielding with the best obtained yield of $22 \%$ over two steps. In an effort to optimise the reaction, the 
Masamune-Roush conditions were used and involve a milder, non-nucleophilic base like DBU [1,8-diazabicyclo(5.4.0)undec-7-ene] (Scheme 2.8). ${ }^{104}$ Typically, under these conditions, $\mathrm{Na}$ or Li salts are used, as they may coordinate with the doubly-bonded oxygens and increase the acidity of the $\alpha$-proton (Scheme $\mathbf{2 . 8}$ ).

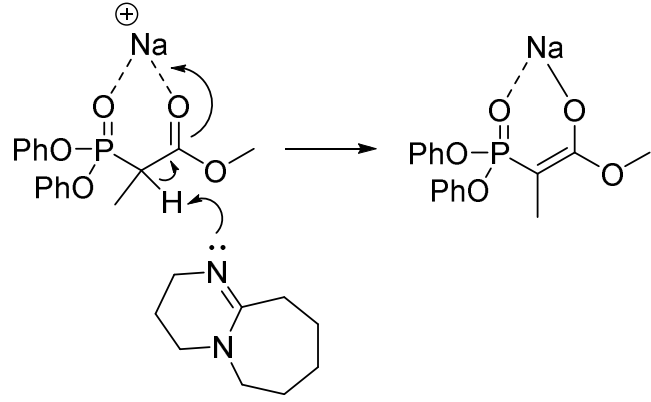

Scheme 2.8: Chelation effect under Masamune-Roush conditions

Under these conditions ( $\mathrm{Nal}, \mathrm{DBU}$ ), the yield improved slightly (30\% over 2 steps of the Z-62) but the $E$-isomer was also formed. The ${ }^{1} \mathrm{H}$ NMR spectrum of the crude product mixture (Figure 2.2) showed a ratio of $Z / E$ isomers of $1.7: 1 .^{105}$

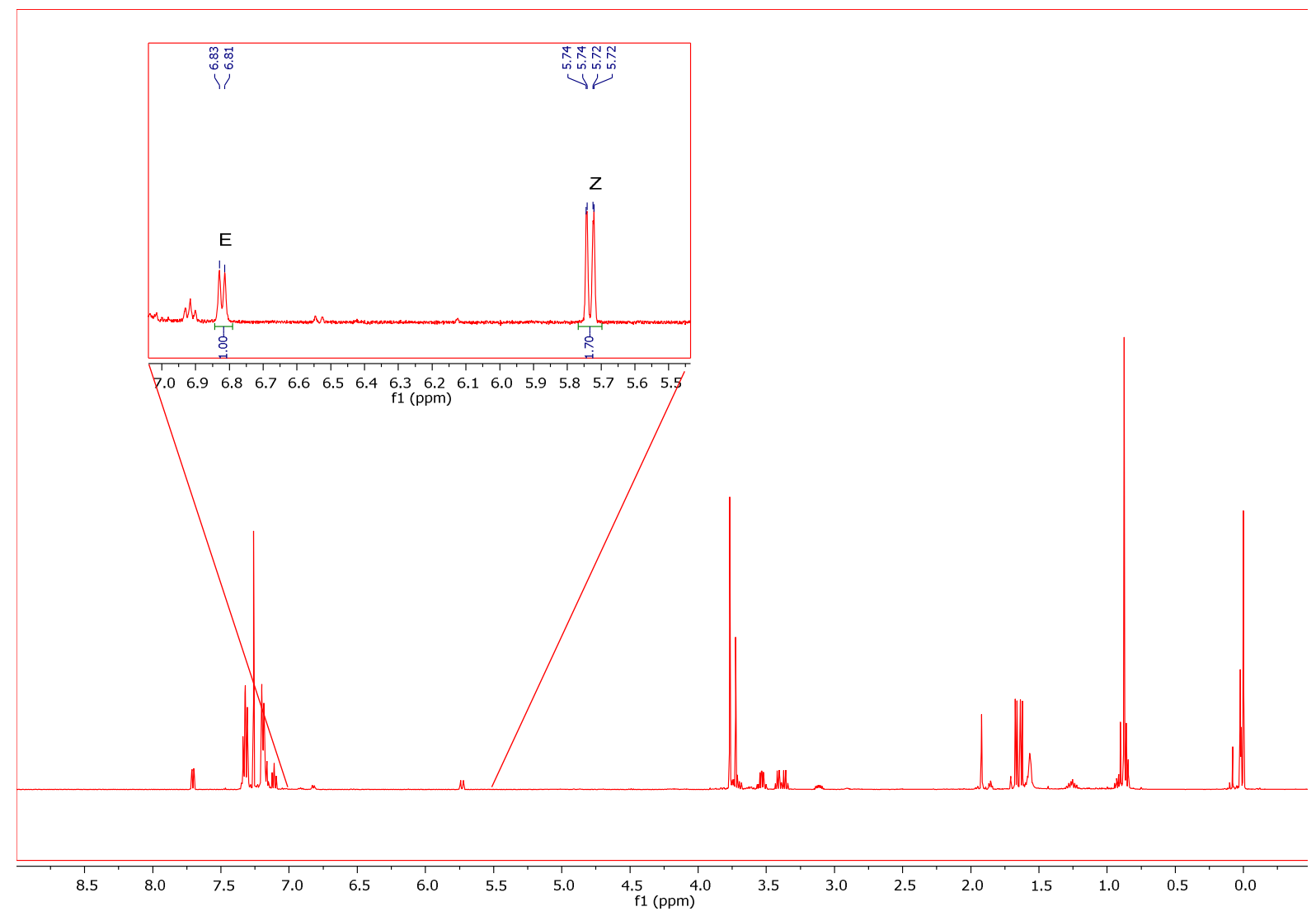

Figure 2.2: ${ }^{1} \mathrm{H}$ NMR spectrum of the crude reaction mixture from the Ando olefination under Masamune-Roush conditions containing E/Z-62 (1:1.7 ratio) 
The loss of selectivity was expected from the stabilising effect of chelation, which slows down rate of the elimination to some extent and allows equilibration and the formation of the thermodynamically favoured E-olefin. ${ }^{106}$ Fortunately, the two isomers were readily separable by chromatography.

The plan to further optimise this reaction involved the use of the Still-Gennari phosphonate but due to change in the protecting group strategy (vide infra) this was never explored.

\subsubsection{The synthesis of methyl ketone 64}

The reduction of the unsaturated methyl ester in Z-62 with DIBAI-H in anhydrous dichloromethane (DCM) gave the allylic alcohol 63 (Scheme 2.9). ${ }^{1}$ This alcohol was then oxidised using Dess-Martin periodinane in a reaction buffered with pyridine to avoid the acetic acid-mediated deprotection of the silyl ether. ${ }^{64,107}$ The aldehyde (118 not shown) was used in the next step, the organocatalysed acetone aldol addition, without purification.

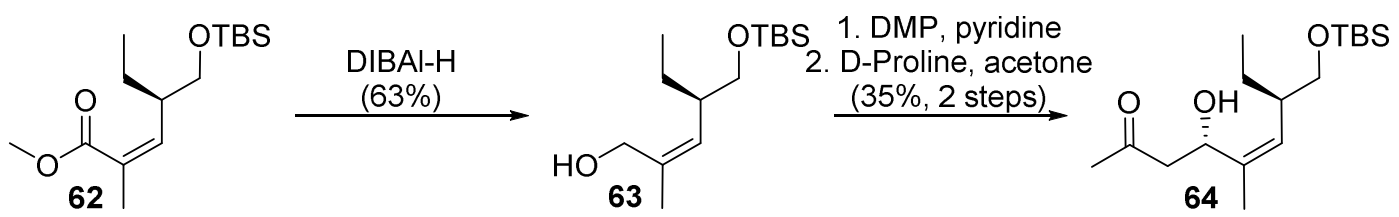

Scheme 2.9: The synthesis of methyl ketone 64

Asymmetric organocatalysis is a rapidly developing area of synthetic chemistry. The idea is to use readily available, non-toxic (and usually inexpensive) chiral pool reagents such as amino acids and alkaloids in sub-stoichiometric amounts. ${ }^{108}$

Proline (Scheme 2.10) is the prototypical organocatalyst. It is a natural amino acid that has already demonstrated its utility to catalyse a number of reactions such as aldol reactions, Mannich reactions, Michael additions, oxidations and reductions. ${ }^{18}$ As an amino acid it incorporates both base and acid functionality which means that it can act as an bifunctional catalyst. $^{110}$ It is also more nucleophilic than other amino acids because it is a secondary amine. ${ }^{109}$ All of these characteristics, in addition to its innate chirality, obtainability, price and low toxicity, makes proline an excellent asymmetric organocatalyst. ${ }^{108,110}$

The first L-proline catalysed asymmetric aldol reaction was an intramolecular reaction of a triketone, discovered in the 1970s and now known as the Hajos-Parrish-Eder-Sauer- 
Wiechert reaction. ${ }^{111,112}$ More recently, proline-catalysed asymmetric intermolecular aldol reactions were developed and investigated by List and Barbas. ${ }^{110,113}$ They explored intermolecular aldol reactions between ketones and aldehydes and obtained yields between $62-97 \%$ and e.e. values of $60-96 \%$, with $20-30$ mol\% catalytic loading. ${ }^{110,113}$ They also proposed and investigated the following reaction mechanism. ${ }^{110,113}$ The reaction starts with the nucleophilic attack of the amino group on the ketone to give a hemiaminal, which, after acid-assisted dehydration, gives the iminium ion (Scheme 2.10). This then tautomerises to an enamine which reacts with the aldehyde. This is the key step producing enantioselectivity. The iminium product of this addition hydrolyses to give the aldol product. Both amine (base) and carboxylic acid (acid) groups are deemed necessary for catalysis. The carboxylic acid is also responsible for the enantioselectivity of the reaction, which can be explained through a metal-free Zimmerman-Traxler-like transition state. In the proposed transition state, the acidic proton forms a tricyclic framework and the aldehyde is oriented so that the R substituent assumes a pseudo-equatorial position as this orientation minimises the 1,3-diaxial strain. ${ }^{110,113}$

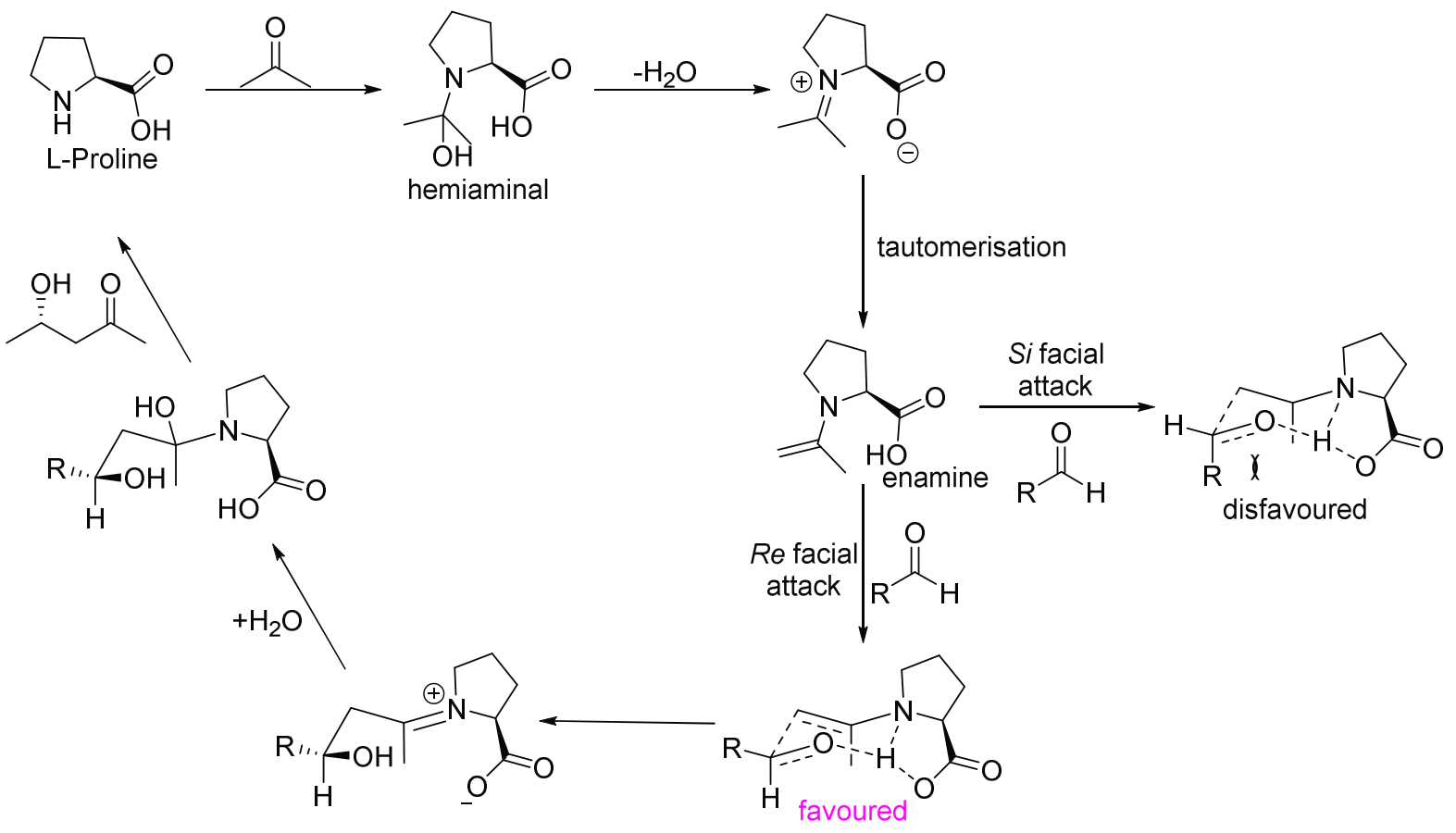

Scheme 2.10: The proposed mechanism of the L-proline catalysed aldol reaction ${ }^{110,113}$

In order to obtain the required C15 stereochemistry in 64, it was necessary to use D-proline as the catalyst (Scheme 2.11). The reaction was performed in a 4:1 mixture of 
DMSO:acetone, wherein acetone served dual function of reagent and solvent. DMSO is commonly used as a co-solvent for this reaction, mainly because the polarity of proline makes it insoluble (or partially soluble) in many organic solvents. ${ }^{110}$ Because of the small scale of the reaction (0.06 mmol starting material), a high catalyst loading was used, 95 mol\% instead of the usual $20-30$ mol\%. The first attempt at this reaction was successful in delivering 64 , but low yielding (35\%, 48\% BRSM).

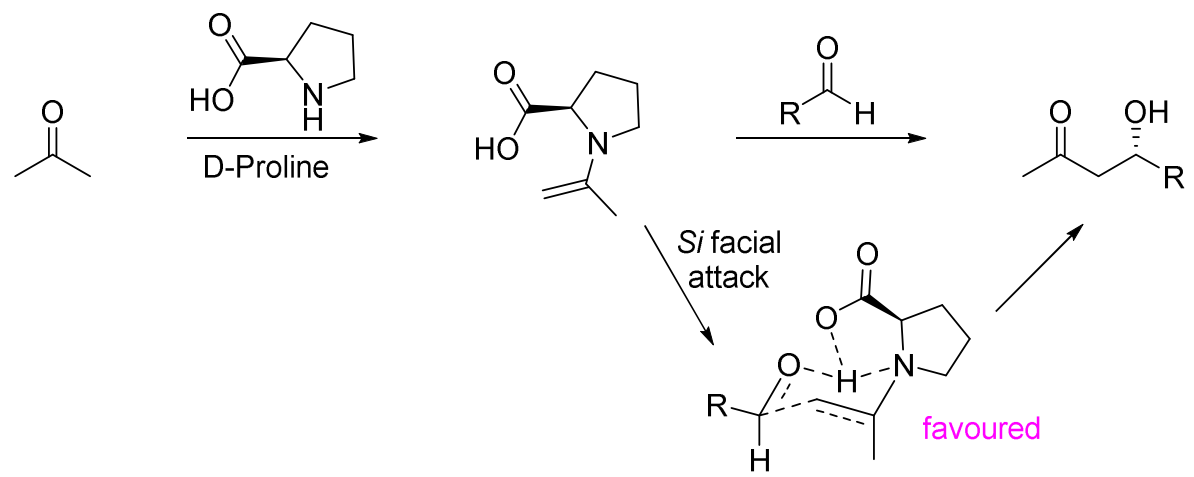

Scheme 2.11: D-Proline catalysed acetone aldol reaction

The structure of product 64 was determined using NMR spectroscopy, with full assignment of ${ }^{1} \mathrm{H}$ and ${ }^{13} \mathrm{C}$ NMR peaks and through-bond connections established using 2D NMR experiments (COSY, HSQC and $\mathrm{HMBC}$ ). The first noticeable ${ }^{1} \mathrm{H}$ spectral feature of this compound was the multiplet at $\delta 4.96$ integrating for two protons. Based on the magnitude of the chemical shift and what was previously observed for the allylic alcohol 63 , these were assumed to be the olefinic proton $\mathrm{H} 17$, and the new oxymethine proton $\mathrm{H} 15$. HSQC analysis confirmed this, and showed two correlations for the multiplet, one to the $\mathrm{sp}^{2}$ carbon at $131.3 \mathrm{ppm}$ (C17) and the other to a carbon at $65.5 \mathrm{ppm}$, typical for an oxymethine carbon (C15). The multiplet at $4.96 \mathrm{ppm}$ (due to H17) had a COSY correlation (Figure 2.3) to a multiplet at $\delta 2.55$ ( $\mathrm{H} 18$ methine). It also showed a correlation to the two methylene protons, the pair of doublets of doublets at $2.83(J=16.5,9.1 \mathrm{~Hz})$ and $2.49 \mathrm{ppm}(J=16.4,4.1$ $\mathrm{Hz}$ ), assigned as $\mathrm{H} 14 \mathrm{a}$ and $\mathrm{H} 14 \mathrm{~b}$, respectively, that are correlating to $\mathrm{H} 15$. These two protons had no additional COSY correlations but had an HMBC correlation (Figure 2.3) to a carbon at $208.6 \mathrm{ppm}$. This was assumed to be a two-bond correlation to the carbonyl carbon of the methyl ketone (C13). The singlet at $2.21 \mathrm{ppm}$, integrating for 3 protons, also had a two-bond $\mathrm{HMBC}$ correlation to this carbon and was assigned, therefore, as the methyl group of the methyl ketone (H12). Additionally, a three-bond correlation between this 
singlet and the C14 methylene carbon at $47.9 \mathrm{ppm}$ was present in the HMBC spectrum. HRMS analysis also confirmed the presence of the product (Experimental).

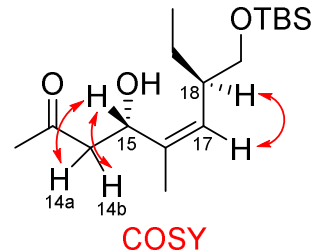

COSY

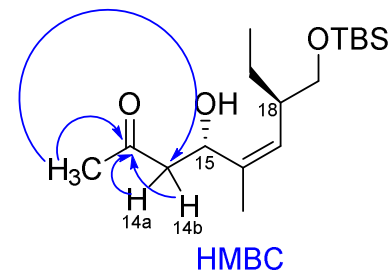

HMBC

Figure 2.3: Selected COSY and HMBC correlations observed for 64

The small scale of the reaction could have been partly responsible for the low isolated yield. Aldehyde intermediate $\mathbf{1 1 8}$ was also isolated during the purification (Experimental). This meant that the reaction did not go to completion, even after 24 hours of stirring at room temperature, which additionally contributed to the low yield. The isolation of the aldehyde implied that this $\alpha, \beta$-unsaturated aldehyde is stable on silica gel. Purifying it before the reaction, in future, might be beneficial for the outcome of the reaction.

However, at this point in time, it was decided to change the protecting group at 024 from TBS to $\mathrm{Bn}$, so the reaction was only attempted once and was not optimised. The plan was to optimise the reaction and confirm the stereochemistry at $\mathrm{C} 15$ for the benzyl protected material.

\subsection{Second protecting group strategy}

Changing the protecting group strategy decreased the number of steps required for the synthesis of this fragment (Scheme 2.12). This change, in combination with some additional modifications to the methodology, improved the yield to $12 \%$ overall ( $18 \%$ with respect to the methyl ketone 128), compared to the first synthetic plan ( $3 \%$ yield to the methyl ketone 64). 


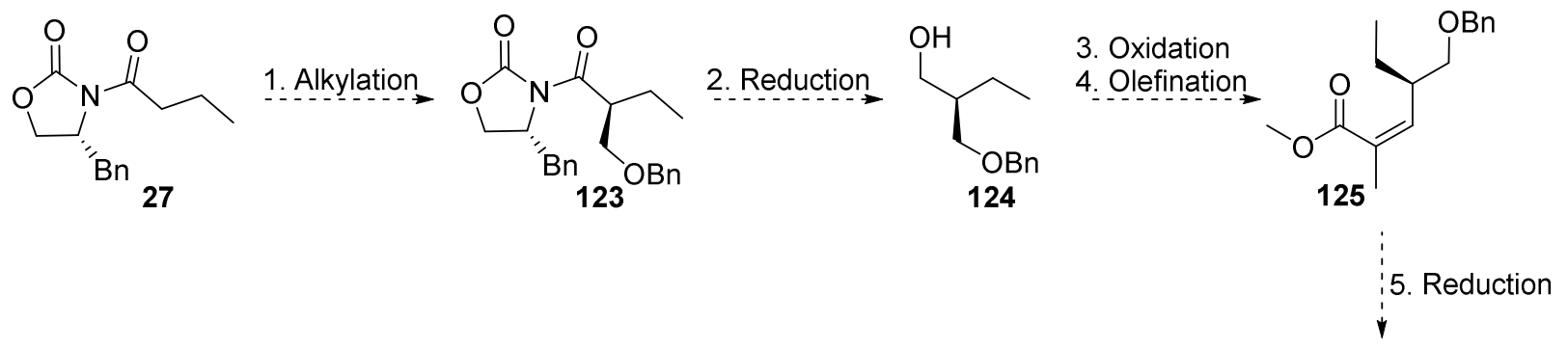

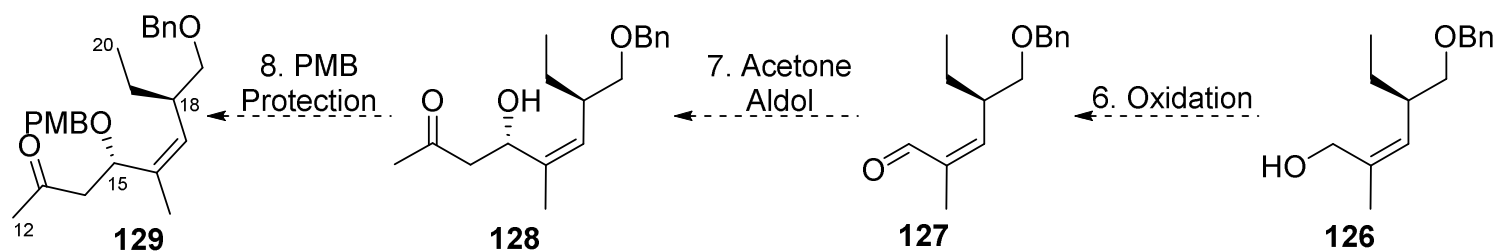

\subsubsection{The synthesis of oxazolidinone 123}

Previously synthesised oxazolidinone $\mathbf{2 7}$ was alkylated with benzyl chloromethyl ether (Scheme 2.13) using a procedure described by Altmann. ${ }^{86,101}$ The starting material was premixed with $\mathrm{TiCl}_{4}$ and triethylamine to form enolate and then the alkylating agent was added. The reaction was highly stereoselective in favour of the desired diastereomer $\mathbf{1 2 3}$ $\left([\alpha]_{D}{ }^{22}=-37.3\left(c 1.05, \mathrm{CHCl}_{3}\right.\right.$, lit. -39.1$),{ }^{86}$ giving the two products 123 and 130 in a 10:1 diastereomeric ratio as determined from integration of the peaks in the ${ }^{1} \mathrm{H} N M R$ of the crude reaction mixture (Figure 2.4). The reaction was generally high yielding, with the desired product yields varying from $73-83 \%$ and the total yield of both diastereomers from $82-88 \%$.

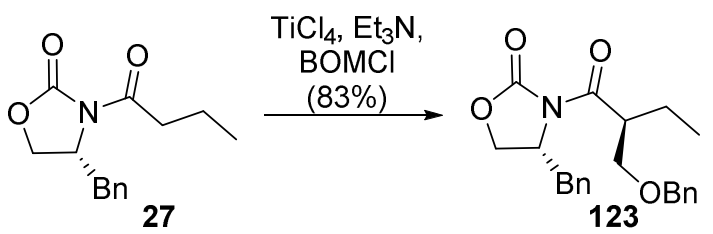


<smiles>CCC(CO)C(=O)N1C(=O)OCC1Br</smiles>

123 major product<smiles>CC[C@H](CO)C(=O)N1C(=O)OC[C@H]1Br</smiles>

130 minor product
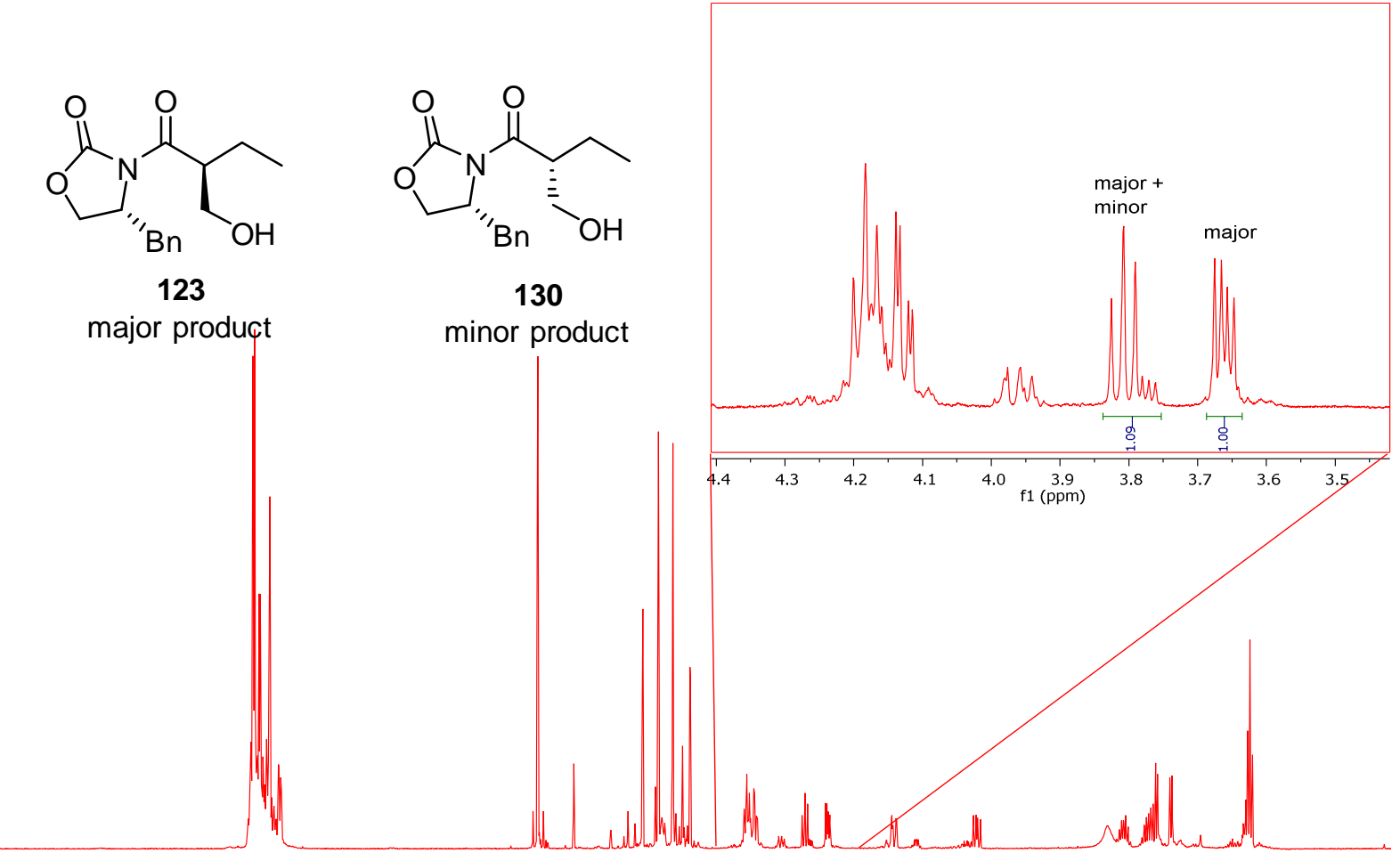

\section{0}

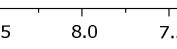

Figure 2.4: ${ }^{1} \mathrm{H}$ NMR of the crude mixture containing the major (123) and the minor (130) diastereomer

\subsubsection{The synthesis of methyl ester 125}

The oxazolidinone in $\mathbf{1 2 3}$ was reductively removed using lithium borohydride to give the alcohol 124 (Scheme 2.14). This alcohol was then oxidised using DMP and the aldehyde 131 (Experimental) was used in the next step without purification. Because of the low yields obtained previously with the Ando olefination using the diphenyl phosphonate $\mathbf{1 2 2}$, the trifluoroethyl phosphonate 133 was synthesised (Scheme 2.15) for use in the Still-Gennari olefination. Taylor et al. ${ }^{64}$ had also employed this method in their side-chain synthesis.<smiles>CCC(C[18O]Br)C(=O)N1C(=O)OCC1Br</smiles>

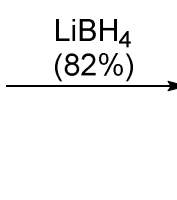

1. DMP

2. 133, KHMDS, 18-crown-6 $(71 \%)$<smiles>CCC(C=C(C)C(=O)OC)COC</smiles>

Scheme 2.14: The synthesis of methyl ester 125

The phosphonate 133 was synthesised in one step from the commercially available methyl $P, P$-bis(2,2,2-trifluoroethyl)phosphonoacetate 132 (Scheme 2.15). ${ }^{114}$ The methylation of the 
phosphonoacetate always led to a mixture of mono- and di-methylated products and the separation of the three compounds (di-methylated, mono-methylated, starting material) on silica gel column was more difficult than for the diphenyl phosphonate $\mathbf{1 2 2}$.

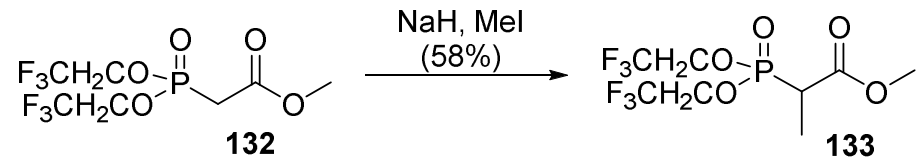

Scheme 2.15: The synthesis of phosphonate 133

The Still-Gennari olefination reaction was carried out at $-78{ }^{\circ} \mathrm{C}$ using potassium bis(trimethylsilyl)amide (KHMDS) as base to deprotonate the phosphonate. The corresponding crown ether (18-crown-6) was used in excess (3.5 equiv.) to sequester the potassium ions and prevent chelation with the two oxygens. With the implemented changes in methodology, the yield of the olefination reaction was significantly improved, giving $\mathbf{1 2 5}$ in yields of $51-71 \%$ over the two steps. The improvement in the yield could also have been due to increased stability of aldehyde 131. In addition, the selectivity of the reaction had improved with the Still-Gennari method. The $E$-isomer was not observed in the ${ }^{1} \mathrm{H} N M R$ spectrum of the crude reaction mixture at any time.

\subsubsection{The synthesis of methyl ketone 128}

The next two steps were the same as for the synthesis of TBS protected fragment: the DIBAI-H reduction of the methyl ester 125 and the oxidation of the allylic alcohol (126) with DMP to give $\alpha, \beta$-unsaturated aldehyde 127 (Scheme 2.16).

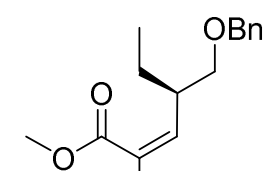

125

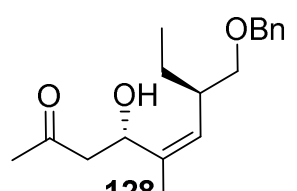

128

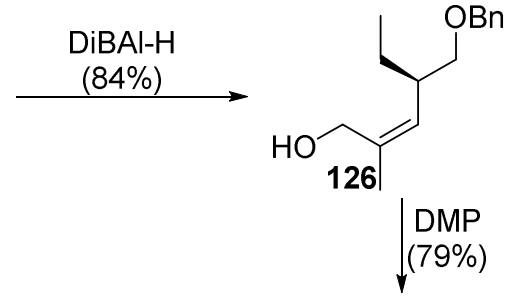

(+)-DIPCI, Et ${ }_{3} \mathrm{~N}$,
acetone (59\%)

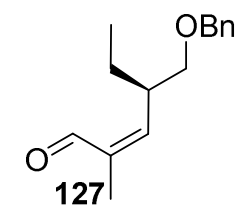


With the aldehyde 127 available, the proline catalysed acetone-aldol reaction was attempted. This reaction was tried under similar reaction conditions to those used for the TBS-protected fragment: on a small scale $(0.04 \mathrm{mmol})$ and with a higher catalyst loading (70 mol\%) in 4:1 DMSO:acetone. After 24 hours of stirring, TLC analysis indicated that much of starting material remained but, there were other more-polar faint spots so the reaction was continued. After 5 days of stirring at room temperature TLC analysis showed starting material spot was still the most intense but one of the new spots was now more visible. In order to determine if that component was aldol product, the reaction was quenched and the ${ }^{1} \mathrm{H}$ NMR spectrum of the crude reaction mixture obtained. However, there were no expected product peaks in the spectrum. Nevertheless, the crude material was subjected to silica gel chromatography and the product corresponding to the new TLC spot was isolated. This was not the desired aldol-addition product 128, but appeared to be the aldolcondensation product (134), a commonly observed by-product of these reactions. This stems from proline forming an iminium species with the aldehyde which reacts with the ketone to give a $\beta$-amino ketone (Scheme 2.17). ${ }^{110,115}$ This product can then undergo $\beta$ elimination to give the $\alpha, \beta$-unsaturated condensation product.

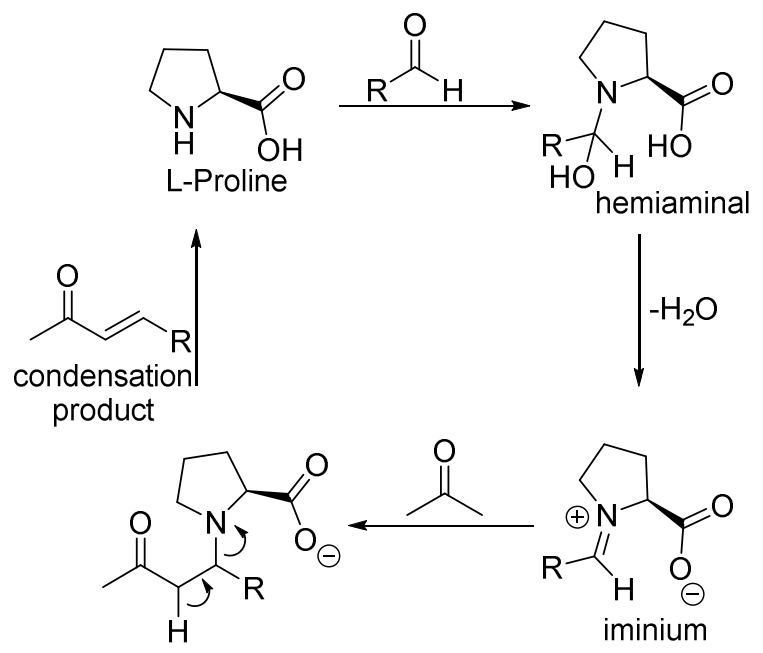

Scheme 2.17: Mechanism for the formation of the condensation product in the proline catalysed aldol reaction

Literature suggested that better aldol selectivity could be obtained with prolinol. ${ }^{115}$ The reaction was repeated on $0.12 \mathrm{mmol}$ scale $(27.7 \mathrm{mg}$ ) with D-prolinol as the catalyst $(60 \mathrm{~mol}$ $\%)$ and using acetone as the solvent, since prolinol, unlike proline, is soluble is acetone (Scheme 2.18a). The reaction was monitored by TLC daily for four days. After this time the reaction was worked up and the crude material subjected to column chromatography. 
a)

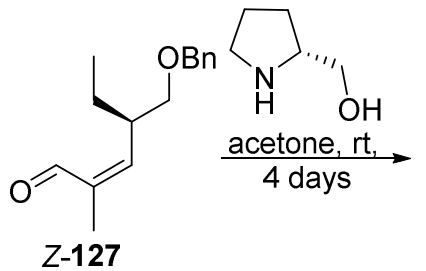

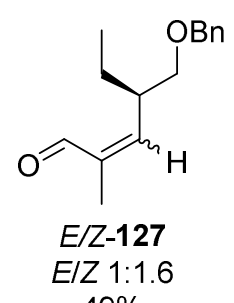

$49 \%$<smiles>CCC(C=C(C)C=CC(C)=O)Cc1ccccc1</smiles>

$E / Z-134$ $13 \%$<smiles>CCC(C=C(C)[C@@H](O)CC(C)=O)Cc1ccccc1</smiles>

$E / Z-128$ b)<smiles>[Z17]C(C=O)=CC(CC)COCc1ccccc1</smiles>

Scheme 2.18: a) D-Prolinol; b) (R)-(+)- $\alpha, \alpha$-diphenyl-2-pyrrolidinemethanol catalysed reactions

The major species isolated, once again was the condensation product 134 ( $2.4 \mathrm{mg}, 13 \%)$, but the desired addition-product 128 was also isolated albeit in $10 \%$ yield (3.3 mg). Aldehyde starting material 127 was recovered (13.5 mg, 49\%). Unfortunately, both the aldol product and the recovered aldehyde appeared to be mixtures of isomers.

The ${ }^{1} \mathrm{H}$ NMR spectrum of the recovered aldehyde (Figure 2.5) showed two distinct environments for the aldehydic proton ( $\mathrm{H} 15)$, as well as for the olefinic proton $(\mathrm{H} 17)$ and the methine adjacent to the double bond $(\mathrm{H} 18)$. These are the chemical shifts expected to change the most with the change in geometry of the double bond. The isomerisation of the aldehyde was confirmed by NOESY experiment (Figure 2.6). For the Z-isomer, through-space correlations were observed between $\mathrm{H} 15$ (aldehyde proton) and $\mathrm{H} 18$ methine and between $\mathrm{H} 17$ (olefinic proton) and the $\mathrm{H} 21$ methyl protons. The $E$-isomer had only one significant correlation and that is between $\mathrm{H} 15$ and $\mathrm{H} 17$. No NOESY experiment was attempted on the aldol-product due to insufficient sample; it is assumed that the isomers are also geometrical. 


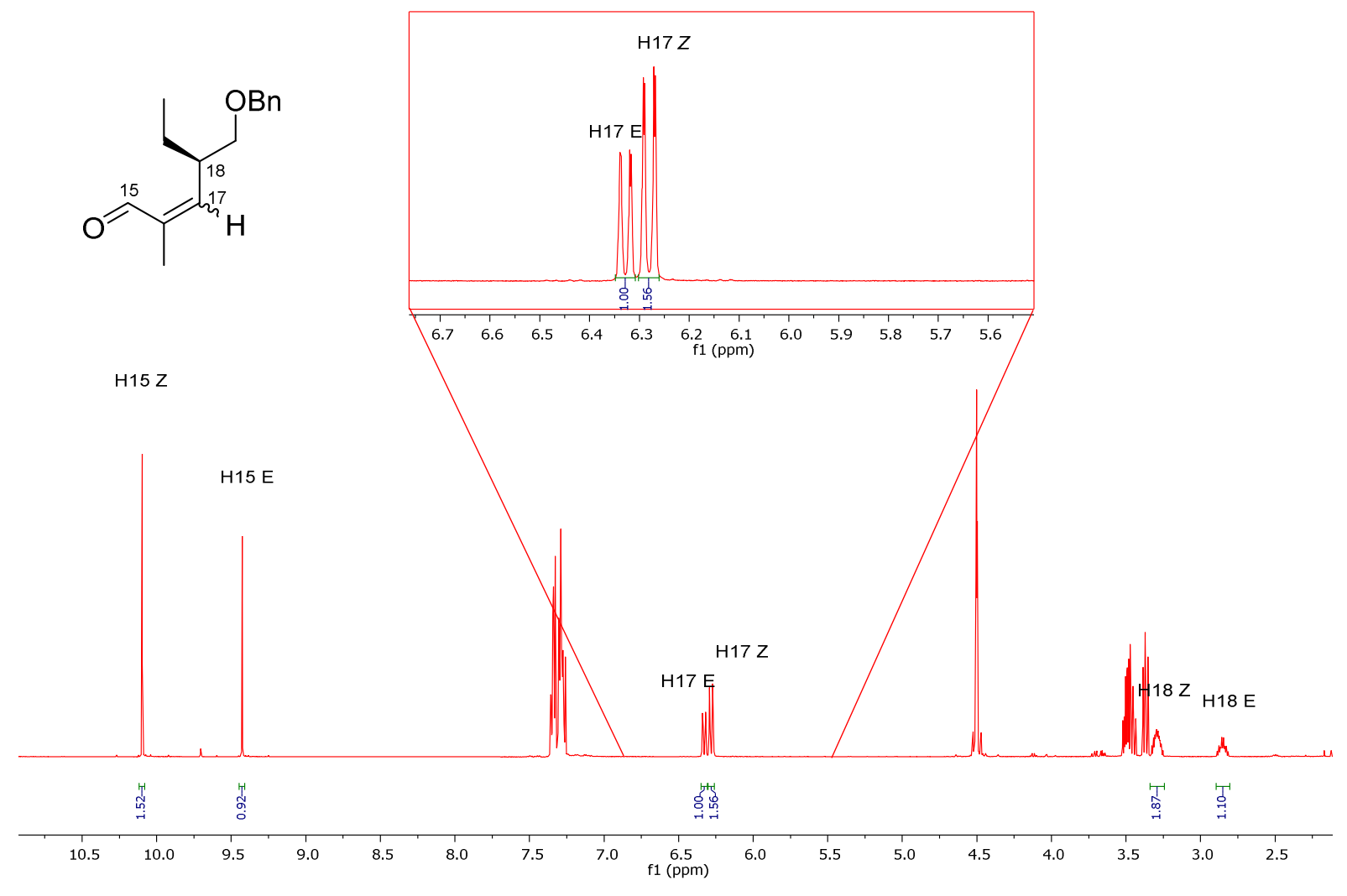

Figure 2.5: ${ }^{1} \mathrm{H}$ NMR spectrum of the recovered aldehyde 127 from the D-prolinol reaction 


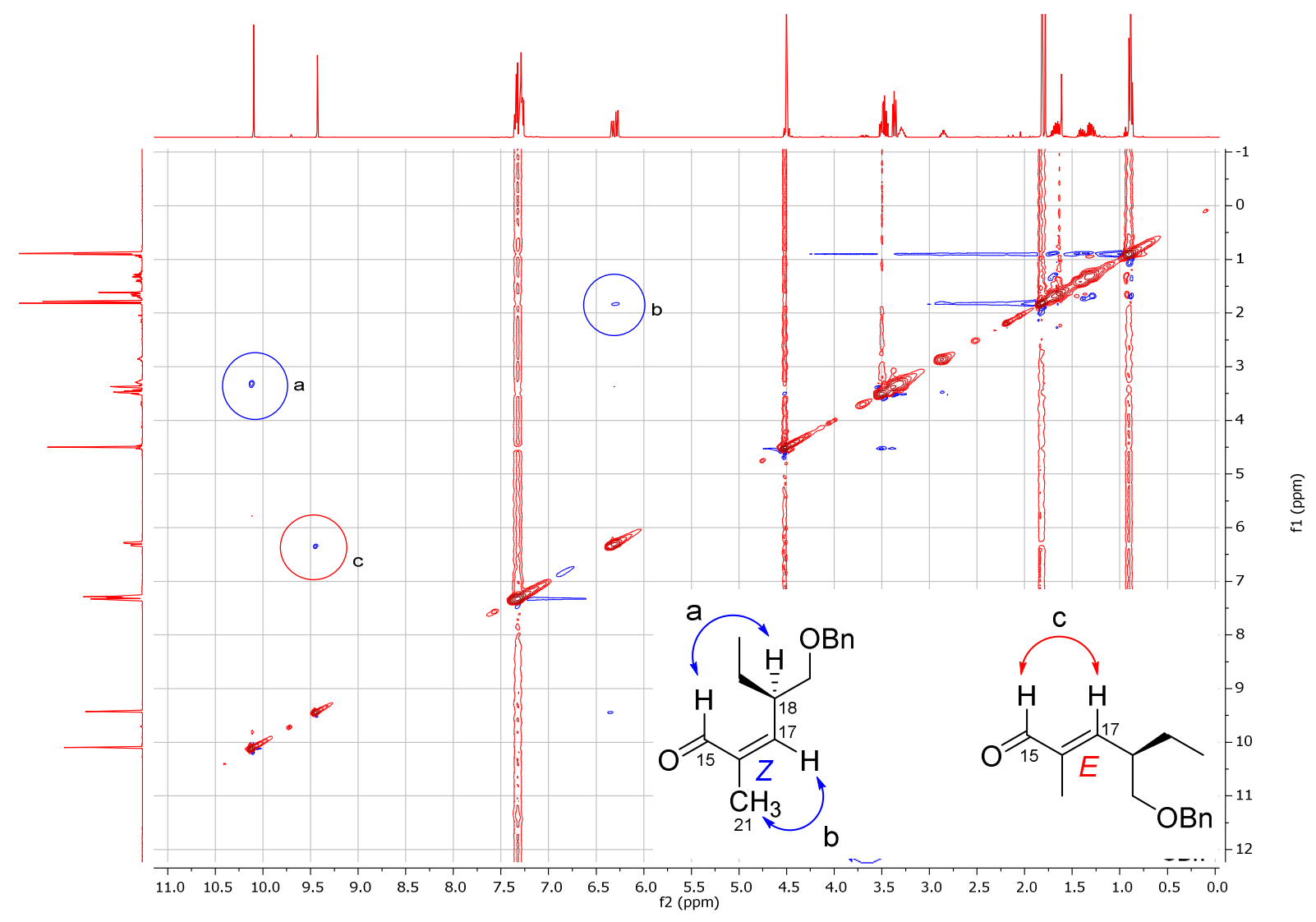

Figure 2.6: 2D NOESY spectrum of the isomerised aldehyde $\mathbf{1 2 7}$

The reaction with $(R)-(+)-\alpha, \alpha$-diphenyl-2-pyrrolidinemethanol (Scheme 2.18b) did not give any identifiable products (addition or condensation). The ${ }^{1} \mathrm{H}$ NMR spectrum of the crude reaction mixture showed that the isomerisation of the aldehyde 127 occured (Z/E 8:1). However, the isomerisation with this catalyst under the same reaction conditions and time was significantly slower. In a reinvestigation of the ${ }^{1} \mathrm{H}$ NMR spectrum of the crude reaction mixture from the D-proline catalysed reaction, it was found that the aldehyde isomerisation also occurred under those conditions (Z/E 3.5:1).

The mechanism of the observed isomerisation is unclear. It could result from an addition/elimination sequence with prolinol, being the most nucleophilic species involved, giving most isomerisation (Scheme 2.19a) and the most sterically hindered $\alpha, \alpha$-diphenyl-2pyrrolidinemethanol giving least isomerisation. Some isomerisation could also occur at the iminium stage, where the configurational equilibration can happen in the resonance structure (Scheme 2.19b). ${ }^{116}$ 
a)

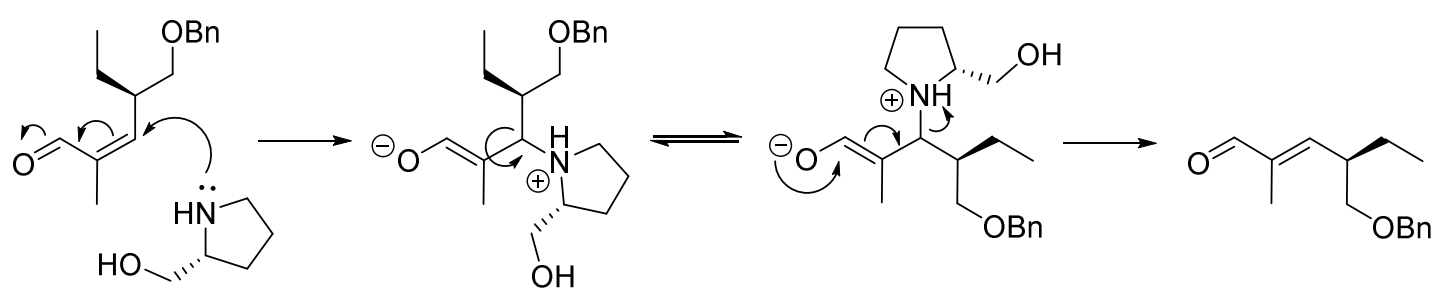

b)

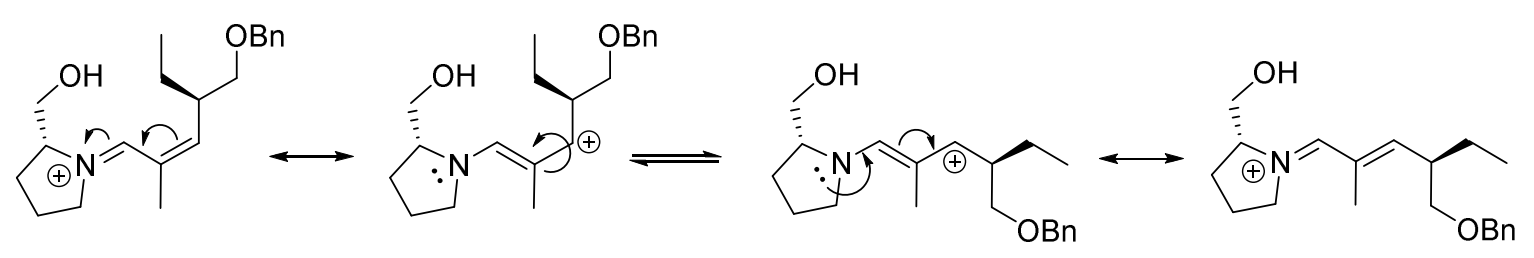

Scheme 2.19: Proposed isomerisation mechanisms

Faced with such unpromising results, we turned to Paterson's aldol reaction that was employed in his synthesis of ent-C12-C20 fragment of peloruside A. ${ }^{117}$ This reaction uses triethylamine as the base and diisopinocampheylboron chloride (DIPCl) to generate a boron enolate with a ketone. As shown by Paterson, aldol reactions of diisopinocampheylborinates from methyl ketones pass through a twist-boat transition state, which minimises the steric interaction between the methyl group (of the methyl ketone) and the bulky isopinocampheyl (Ipc) group (Scheme 2.20). ${ }^{80,81}$ A chair-like transtion state, would place these groups in closer proximity. Analogously, the aldehyde is oriented so its R group is in the pseudo-axial position whereas in the chair transition state it would assume a pseudoequatorial position.

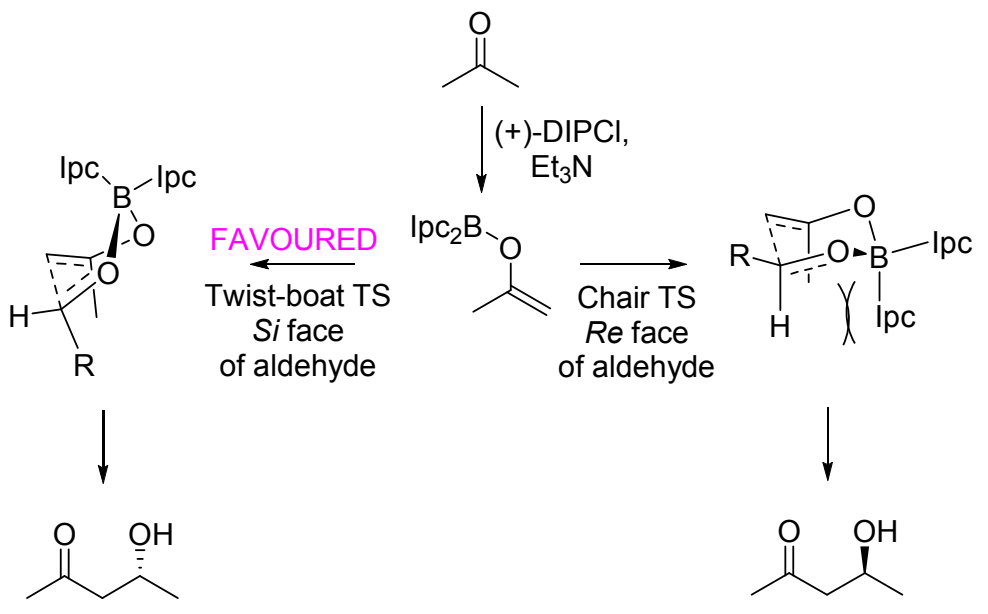


Thus, in order to obtain the desired stereochemistry (Scheme 2.16) we used (+)diisopinocampheylboron chloride as the boron enolate of acetone formed with (+)-DIPCl, would react with the si face of aldehyde $127\left((+)\right.$-DIPCl, Figure 2.7). ${ }^{118,119}$ This enolate formation was allowed to proceed for 2 hours at $0{ }^{\circ} \mathrm{C}$, followed by the addition of aldehyde at $-78{ }^{\circ} \mathrm{C}$ then the reaction was kept at $-18{ }^{\circ} \mathrm{C}$ (freezer) for $18-20$ hours for completion. ${ }^{80}$ The aldol reaction was followed by an oxidative workup $\left(\mathrm{H}_{2} \mathrm{O}_{2}, \mathrm{MeOH} / \mathrm{pH} 7\right.$ buffer) and chromatographic purification, which gave two diastereomers in 8:1 ratio (isolated) in good yield (59\% major 128, 7\% minor 135 diastereomer, 66\% total, Experimental).

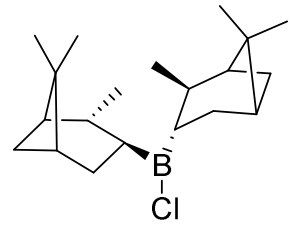

$(+)$-DIPCl

Figure 2.7: The structure of (+)-DIPCl

\subsubsection{Determination of stereochemistry and the PMB protection}

In order to determine the C15 stereochemistry of the major product $\mathbf{1 2 8}$ of the aldol reaction, a Mosher ester analysis was conducted. The alcohol 128 was reacted with both $R$ and $S \alpha$-methoxy- $\alpha$-(trifluoromethyl)phenylacetic acid (MTPA) (Scheme 2.21), under Steglich esterification conditions (DCC, DMAP, acid). ${ }^{75}$
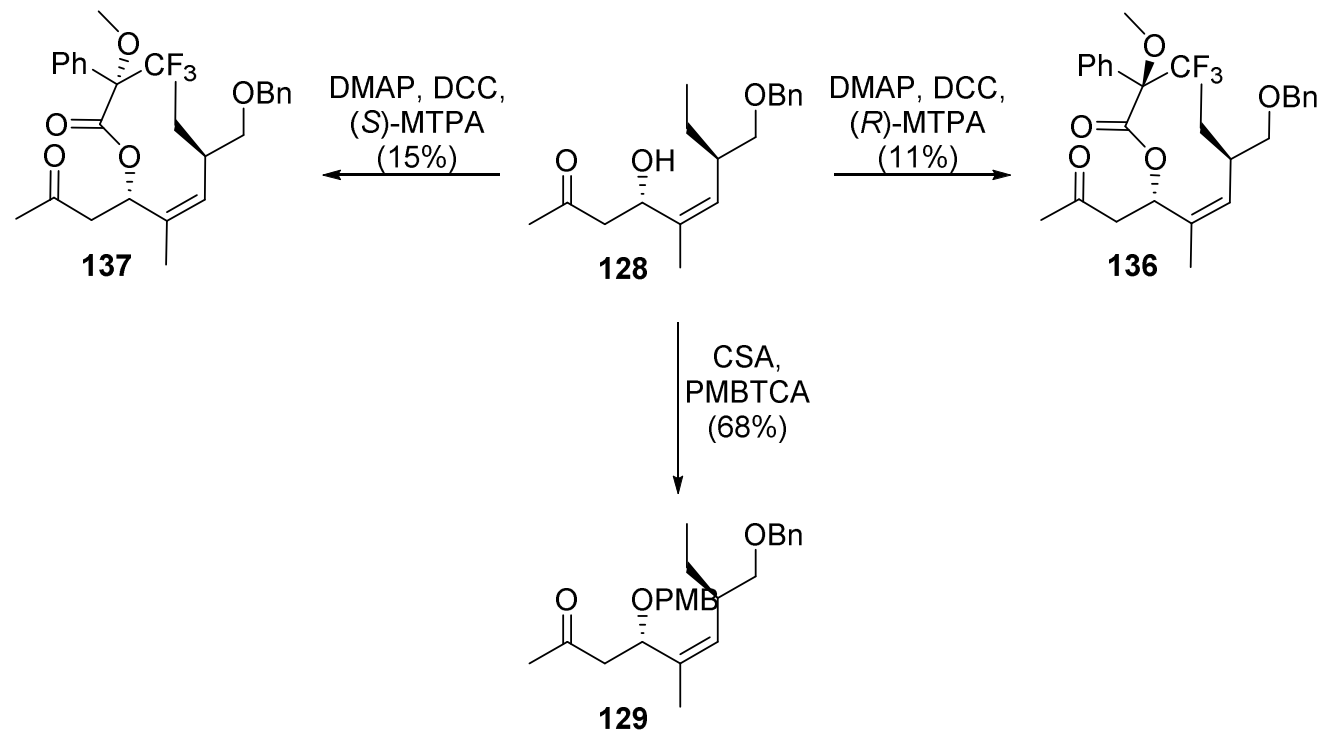
The yields for the resulting esters were low, 11\% for the R-MTP ester (136) and 15\% for the S-MTP ester (137). These yields are most likely due to a competing base-induced E1cB elimination which gives the already familiar conjugated product $\mathbf{1 3 4}$. However, enough product was isolated to obtain ${ }^{1} \mathrm{H}$ NMR spectra.

These esters are used for determining stereochemistry because their chiral substituents affect the proton chemical shifts each side of the stereocentre differently (Figure 2.8). ${ }^{120}$ The methyl ether substituent, being an electron-withdrawing, deshields the protons on one side of the stereocentre, so their chemical shifts are moved downfield (to higher ppm). On the other side of the stereocentre, the phenyl group is shielding, moving the chemical shifts upfield (to low ppm). So, if we calculate $\Delta \delta=\delta_{S}-\delta_{R}$ for the protons on either side of the stereocentre one side will have values of $\Delta \delta>0$ and the other will have values of $\Delta \delta<0$. The side with positive $\Delta \delta$ value must be $R_{2}$ and the side with a negative $\Delta \delta$ value must be $R_{1}$. For compounds 136 and 137, we looked at the chemical shifts of protons at $\alpha, \beta$, and $\gamma$ positions to the stereocentre and this analysis confirmed the stereochemistry at C15 as $S$ (Figure 2.8).
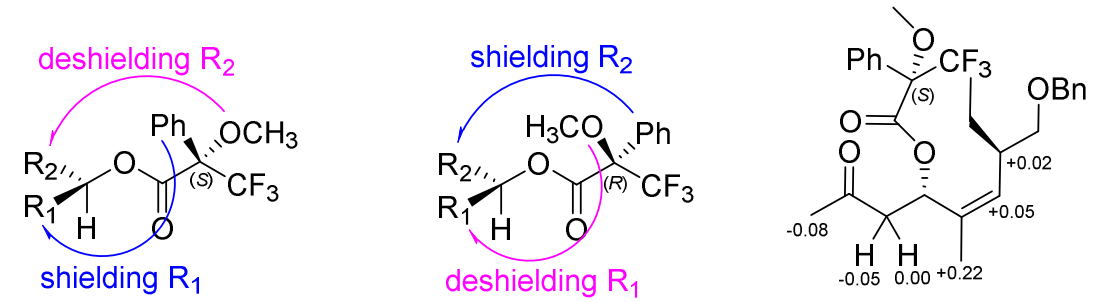

Figure 2.8: Mosher's ester analysis and the $\Delta \delta$ values obtained for compound $\mathbf{1 2 8}$

The last step in the synthesis of $\mathrm{C} 12-\mathrm{C} 20$ fragment, the PMB protection of the $\mathrm{C} 15$ hydroxyl group, proved to be more challenging than expected. The most commonly used conditions involving a base (usually $\mathrm{NaH}$ ) and para-methoxybenzyl chloride (PMBCl) led to the $\mathrm{E} 1 \mathrm{cB}$ elimination sequence, yielding once again the compound $\mathbf{1 3 4}$. Therefore, it was necessary to achieve PMB protection under acidic conditions, using para-methoxybenzyl-2,2,2trichloroacetimidate (PMBTCA, 138, Scheme 2.22). ${ }^{121}$ PMBTCA was synthesised previously by Dr. Claire Cuyamendous, a postdoctoral researcher in the group. However, even under acidic conditions the protection reaction did not always proceed smoothly. The elimination still occurred, albeit to a lesser degree, and the product co-eluted with the by-product of the reaction, PMB-trichloroacetamide $(139$, Scheme 2.22$)$. This resulted from the rearrangement of the PMB-trichloroacetimidate (Scheme 2.22). Literature suggests this 
rearrangement to be a cationic $\left(\mathrm{S}_{N} 1\right.$ type) process. ${ }^{122-124}$ The assignment of structure to the impurity was based on the change in the ${ }^{13} \mathrm{C}$ NMR shift of the benzylic carbon (45.1 ppm) which is much lower than the one observed for the acetimidate (70.7 ppm) suggesting a different heteroatom affecting the carbon. The associated HMBC correlation of the benzylic protons (4.49 ppm) to an unprotonated carbon at $161.9 \mathrm{ppm}$ is congruent with the values observed in the literature for this type of acetamides cabonyl carbons.

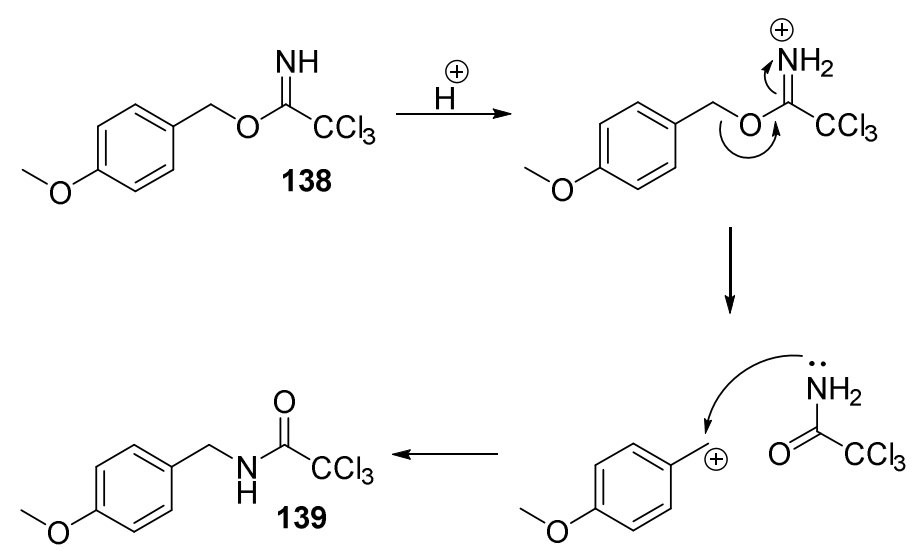

Scheme 2.22: Acetimidate to acetamide: the proposed rearrangement mechanism

Out of the acidic conditions used (PPTS, PTSA and CSA), the best results were obtained with camphorsulfonic acid (CSA), giving the highest yield of $129(68 \%){ }^{125}$ CSA was used catalytically (30 mol\%) and a decrease in number of equivalents of PMBTCA from 3 equiv. to 2 equiv. did not affect the yield significantly.

Having the $\mathrm{C} 15$ hydroxyl protected as a PMB ether, affected the ${ }^{1} \mathrm{H}$ NMR shift of $\mathrm{H} 15$ shifting it upfield to $4.78 \mathrm{ppm}$, and it no longer overlapped with the olefinic proton $\mathrm{H} 17$ (5.19 ppm). Despite the presence of acetamide impurity, it was possible to fully assign the product NMR peaks (Experimental). With this PMB protection, the synthesis of C12-C20 fragment (129) was complete.

\subsection{Scalability}

The overall yield for the synthesis of the side-chain fragment was $12 \%$ over nine linear steps (Scheme 2.23). The lowest yielding step was the acetone aldol reaction but this may be further optimised. A further change with this reaction would be to use (+)-DIPCl as a solution in hexane (available as a $58 \%$ solution from $\mathrm{TCl}$ ) instead of the pure, very hygroscopic, solid. This has already been used in the large scale industrial synthesis of a 
marine natural product. ${ }^{126}$ Using the reagents as solutions, especially if they are obtained in sealed bottles, makes it easier to dispense the amounts needed under an inert atmosphere.<smiles>O=C1NC(Br)CO1</smiles><smiles></smiles><smiles>CCCC(=O)N1C(=O)OC[C@H]1Br</smiles><smiles>C[13CH3]</smiles><smiles>CCC(COc1ccccc1)C(=O)N1C(=O)OC[C@H]1Br</smiles>
$82 \%$<smiles>CCC(/C=C(/C)CO)COCc1ccccc1</smiles><smiles>CCCCCCC</smiles><smiles>CCC(/C=C(/C)C(=O)OC)COc1ccccc1</smiles>

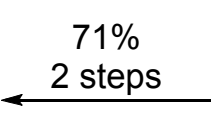<smiles>CCC(CO)COc1ccccc1</smiles>
$79 \%$<smiles>CCC(/C=C(/C)C=O)COCc1ccccc1</smiles><smiles>C[13CH3]</smiles><smiles>CC[C@H](/C=C(/C)[C@H](O)CC(C)=O)COc1ccccc1</smiles>

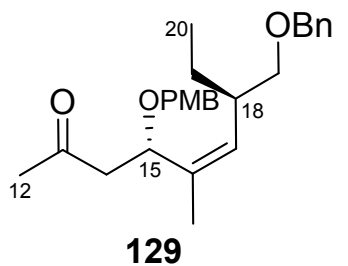

Scheme 2.23: Yields for each step in the synthesis of the side-chain fragment C12-C20 129 with 12\% overall

Another potential issue in assessing scalability is with phosphonate 133 . Methyl $P, P$ bis(2,2,2-trifluoroethyl)phosphonoacetate is expensive and the methylation unreliable. It would be worth investigating a better way to obtain 133 .

To further improve the overall yield some of the chromatographic purifications could be omitted, especially the purification of the $\mathrm{N}$-acylated oxazolidinone $\mathbf{2 7}$ and the allylic alcohol 126. For these compounds the ${ }^{1} \mathrm{H}$ NMR spectra of the crude reaction mixtures after workup indicate appear to be of adequate purity to be used directly in the next step.

Lastly, the purification of the product after the PMB protection reaction should be addressed as PMB-trichloroacetamide elutes with the product 129 on silica gel chromatography. This impurity could impact the yield of the next step (Chapter 3) so it would be beneficial to remove it. Alternative workup procedures could be explored, e.g. 
washing the organic layer several times with $2 \mathrm{M}$ aq. $\mathrm{NaOH}$ solution. ${ }^{123}$ Another way to separate the product from this impurity could be by use of size-exclusion chromatography but this remains to be attempted. 


\section{Synthesis of C8-C20 fragments}

\subsection{The two planned C8-C20 fragments}

With the synthesis of the side-chain fragment 129 completed, it was used for the synthesis of the two versions of the $\mathrm{C} 8-\mathrm{C} 20$ fragment (Figure 3.1) that were needed for two different approaches to PelA.

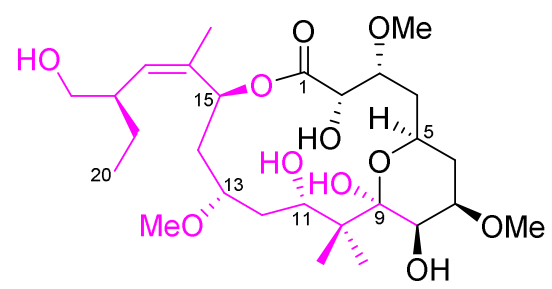

Figure 3.1: PelA with C8-C20 fragment highlighted

The methyl ketone version of fragment C8-C20 (140, Figure 3.2) would be coupled to the aldehyde C1-C7 fragment using an enantioselective aldol reaction [(+)-DIPCl] to form a C7C8 bond (Chapter 1). Then, the macrocycle would be closed using Yamaguchi macrolactonisation, which was the method used in the majority of previous PelA syntheses. ${ }^{1,3,4,55}$ In the alternative approach, the vinyl ketone variant (141, Figure 3.2) would be joined to the $\mathrm{C} 1-\mathrm{C} 7$ acid fragment through an esterification reaction (Yamaguchi ${ }^{57,58}$ or Steglich ${ }^{75}$ ) and the macrocycle would then be closed using a RCM (Chapter 1 ).
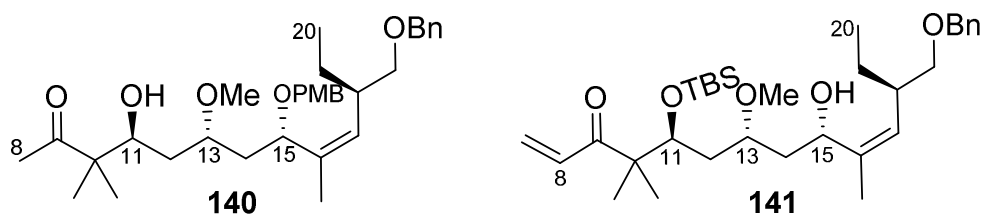

Figure 3.2: Two variants of $\mathrm{C} 8-\mathrm{C} 20$ fragment

The approach for the synthesis of the two C8-C20 fragments (140 and 141) is adopted mostly from Evans' synthesis. ${ }^{1}$ In the key coupling step, the side-chain fragment $\mathbf{1 2 9}$ will be coupled to two versions of the C8-C11 fragment (56 and 57) using the aldol reaction employed by Evans (Scheme 3.1) to give the intermediates 142 and 143, respectively. The C8-C11 fragment 56 will be synthesised using de Brabander's established methodology from 2,2-dimethylpropane-1,3-diol (Scheme 3.1). ${ }^{127}$ This approach would then be extended to the synthesis of $\mathbf{5 7}$. 


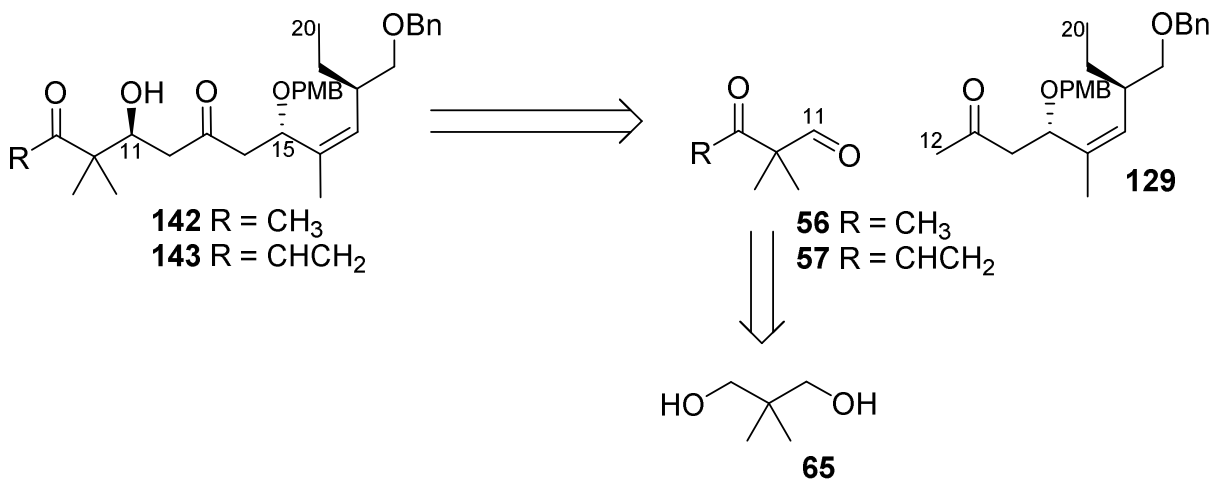

Scheme 3.1: Strategy for the synthesis of the C8-C20 intermediates 142 and 143

\subsection{The C8-C20 methyl ketone 140}

\subsubsection{The synthesis of C8-C11 $\beta$-hydroxyketone 71}

Benzyl protected $\beta$-hydroxyketone 24 (Scheme 3.2) was employed by De Brabander in the first total synthesis of PelA. ${ }^{54}$ This compound was synthesised in four high-yielding steps starting from 2,2-dimethylpropane-1,3-diol (65).

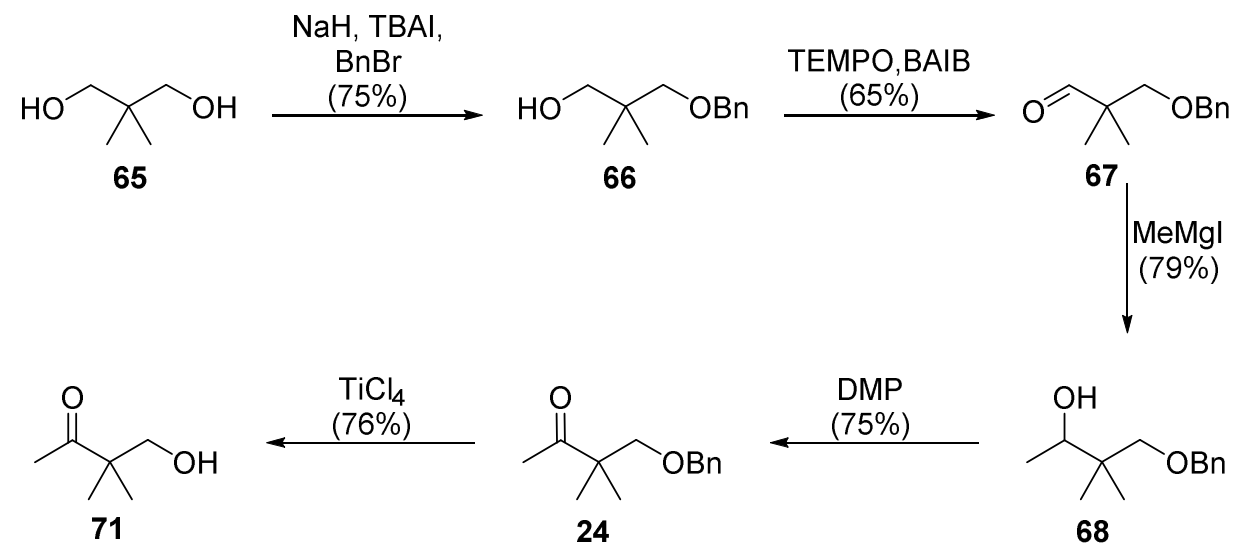

Scheme 3.2: The synthesis of $\beta$-hydroxyketone 71

The first step in this sequence was the mono-benzyl protection of the 2,2-dimethylpropane1,3-diol using Williamson ether synthesis $(\mathrm{NaH}, \mathrm{BnBr}, \mathrm{TBAI})$ and the reaction proceeded with $75 \%$ yield. ${ }^{128}$ The resulting primary alcohol (66) was then oxidised using TEMPO/BAIB and the aldehyde (67) reacted with methylmagnesium iodide (MeMgl) in a Grignard reaction to give the secondary alcohol $\mathbf{6 8} .^{79,127}$ This alcohol was oxidised to the methyl ketone $\mathbf{2 4}$ using Dess-Martin periodinane (DMP). ${ }^{107}$ Lewis acid $\left(\mathrm{TiCl}_{4}\right)$ mediated benzyl ether cleavage gave the $\beta$-hydroxyketone (71) in $22 \%$ yield over 5 steps. ${ }^{129}$ 


\subsubsection{The aldol coupling and determination of configuration at $C 11$}

As mentioned earlier, an aldol reaction was intended to connect the C8-C11 aldehyde fragment (56) to the side-chain fragment 129 (Scheme 3.3). For this reaction, the primary alcohol in $\mathbf{7 1}$ had to first be oxidised to the corresponding aldehyde. The oxidation was achieved using DMP, and the resulting aldehyde used in the aldol reaction without purification. Because this aldehyde was assumed to be volatile and prone to polymerisation, special care was taken during the reaction workup. After the reaction was quenched the aqueous layer was extracted with diethyl ether and the solvent was evaporated at room temperature. Additional drying of this compound using a high vacuum pump was omitted.

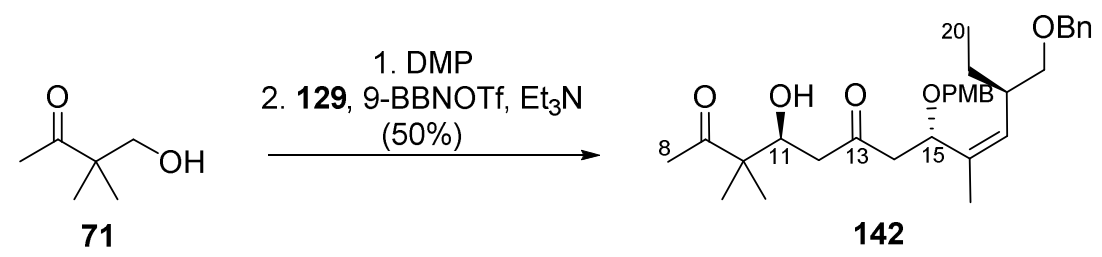

Scheme 3.3: The synthesis of the C8-C20 intermediate 142 through an aldol reaction

The aldehyde was then added to the pre-formed boron enolate of the $\mathrm{C} 12-\mathrm{C} 20$ fragment (129). The same reaction conditions were used by Evans for the formation of $\mathrm{C} 11-\mathrm{C} 12$ bond in order to obtain the complete carbon skeleton of peloruside A. ${ }^{1}$ The aldol reaction was allowed to proceed for 4.5 hours at $-78^{\circ} \mathrm{C}$. After the oxidative workup and silica gel column purification, a single product $\mathbf{1 4 2}$ was obtained in $50 \%$ yield. The limiting reagent in this reaction was 129 which, as discussed in the previous chapter, was contaminated with an inseparable impurity (PMB-acetamide). This impurity could have affected the yield of the reaction.

This reaction is expected to preferentially give a 1,5-anti relationship between the $\beta$-alkoxy substituent on the ketone fragment (C15) and the new stereocentre (C11). ${ }^{1}$ Remote 1,5-stereoinduction was first observed by Masamune et al. ${ }^{130}$ in 1989 and was subsequently independently developed by Paterson and Evans in $1996 .{ }^{131,132}$ Since then, numerous contributing factors have been identified. Early on, it was suggested the induction is due to electrostatic rather than steric effects. ${ }^{131,132}$ The electronic nature of the $\beta$-substituent appears to be one of the most important factors, more specifically the nature of the $\beta$ hydroxyl protecting group. High levels of anti-induction have been observed with a PMB 
protecting group whereas a TBS group gave very low syn-induction. ${ }^{131,132}$ Hoberg et al. ${ }^{133}$ suggested that a $\pi$ orbital interaction between the enolate double bond and a single phenyl ring on the protecting group might be responsible for the observed induction. ${ }^{133}$ Their findings however cannot explain why the $\mathrm{CH}_{2} \mathrm{Bn} \beta$-substituent had no inductive effect despite its capacity for $\pi$-stacking or the high inductive effect of the tetrahydropyran protecting group even though it does not possess an aromatic moiety (Scheme 3.4). ${ }^{132,134-}$ 139
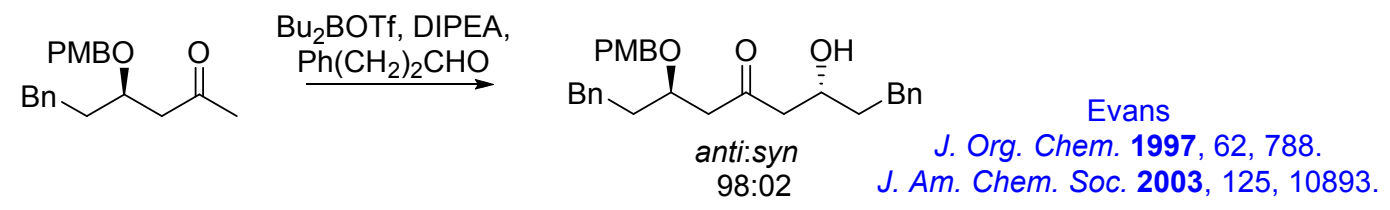<smiles>C=CC[C@@H]1C[C@@H](O[R15](F)(F)C(C)(C)C)O[C@@H](CC(C)=O)C1</smiles>

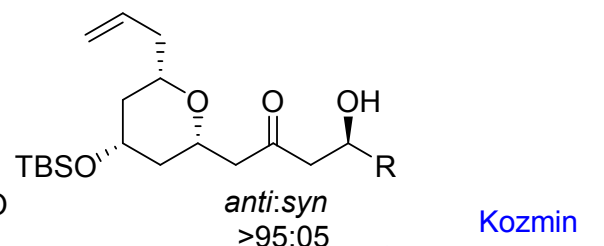

Org. Lett. 2001, 3, 755

J. Am. Chem. Soc. 2002, 124,13670.
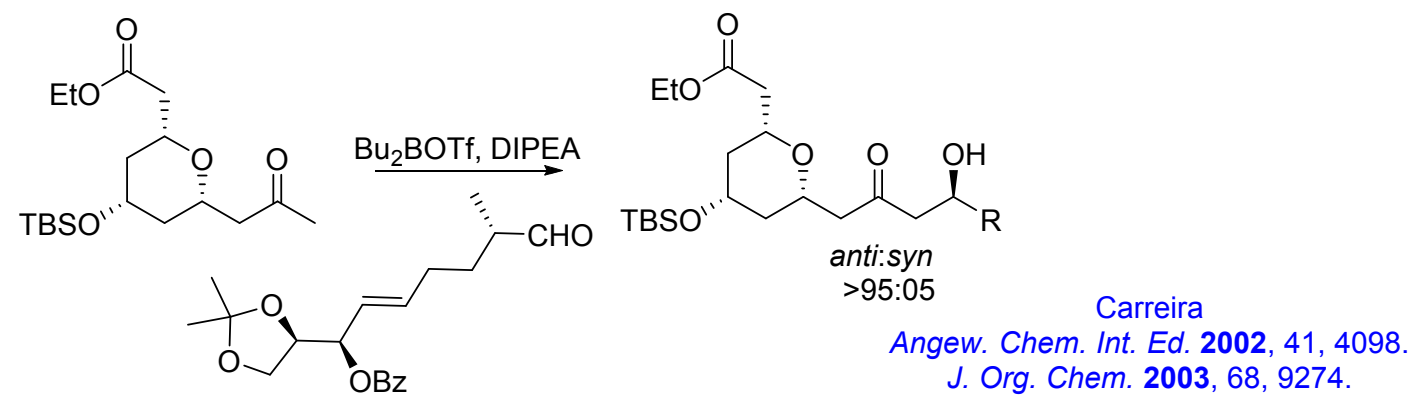

Scheme 3.4: Examples from the literature highlighting the importance of $\beta$-oxygenation in aldol stereo-selectivity

These results further emphasised the influence of the electrostatic over a steric effect as the compared substituents, $\mathrm{OPMB}$ and $\mathrm{CH}_{2} \mathrm{Bn}$, possess similar steric bulk but are electronically very different. Because of these observations it was suggested that $\beta$-oxygenation was necessary for the observed inductive effect. The nature of the enolate was also observed to be contributing to the selectivity of the induction. Low asymmetric induction was observed with Lewis acids $\left(\mathrm{TMS} / \mathrm{BF}_{3} . \mathrm{OEt}, \mathrm{TiCl}_{4}, \mathrm{Ti}(\mathrm{OiPr})_{2} \mathrm{Cl}_{2}, \mathrm{SnCl}_{4}, \mathrm{ZnCl}_{2}\right.$ etc.) and metals capable of chelation with the $\beta$-oxygen ( $\mathrm{Li}, \mathrm{Mg}$, $\mathrm{Ti}$ and $\mathrm{Sn}){ }^{132,134}$ Alkylborinates $\left(\mathrm{Bu}_{2} \mathrm{BOTf}, \mathrm{Chx}_{2} \mathrm{BCl}, 9\right.$ BBNOTf) gave high levels of stereoinduction which was attributed to the relatively short bond length between oxygen and boron in the enolate which results in formation of quite tight transition state structures. In 2008, Goodman et al. ${ }^{139}$ proposed a boat-like transition 
state, based on their computational calculations. ${ }^{139}$ The boat like transition state (Figure 3.3) is favourable as it minimises the 1,3-axial interactions between the enolate side-chain and the ligands on the boron. ${ }^{139}$ The enantioselectivity is explained through an advantageous hydrogen bonding between the $\beta$-oxygen and the aldehyde formyl hydrogen. ${ }^{139}$ Their calculations can also explain the low level of stereoinduction observed with silyl ethers. The delocalisation of electrons into silicon's $d$ orbital makes the $\beta$-oxygen electron-deficient so it cannot form a hydrogen bond with the formyl hydrogen. ${ }^{139}$ In the absence of electrostatic stabilisation the steric effect of the silyl ether takes over with the alkyl chain oriented away from the reaction site resulting in the observed negligible diastereoselectivity. ${ }^{139}$

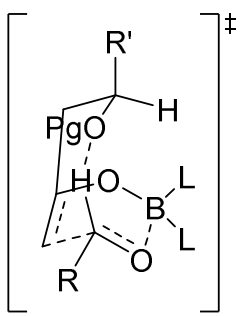

1,5-anti

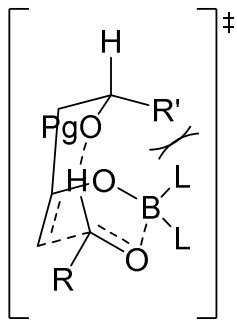

$1,5-s y n$

Figure 3.3: Goodman TS explaining the observed 1,5-anti induction ${ }^{139}$

The ${ }^{1} \mathrm{H}$ NMR spectrum of the aldol product (142) showed a new oxymethine peak (H11) as a doublet of doublets of doublets $(J=1.9,3.6,10.2 \mathrm{~Hz})$ at $4.23 \mathrm{ppm}$ attached to a carbon (HSQC) at $\delta$ 71.9. In the COSY spectrum (Figure 3.4), this proton peak correlated to the two methylene protons, an ABX system consisting of two doublets of doublets at $\delta 2.44(\mathrm{H} 12 \mathrm{~b})$ and $2.60 \mathrm{ppm}$ ( $\mathrm{H} 12 \mathrm{a})$. There was also a COSY correlation to a doublet at $3.22 \mathrm{ppm}$, assigned as the proton of the hydroxyl group because it lacked an HSQC correlation. The rest of the structural assignment was made mostly through HMBC correlations (Figure 3.4). Oxymethine $\mathrm{H} 11$ showed only one $\mathrm{HMBC}$ correlation and that is to a carbon at $19.5 \mathrm{ppm}$ assigned as one of the gem-dimethyl carbons (C21). Proton $\mathrm{H} 12 \mathrm{~b}$ had HMBC correlations to two unprotonated carbons at $\delta 51.2$ and 209.9. The carbon at $51.2 \mathrm{ppm}$ had additional $\mathrm{HMBC}$ correlations from the $\mathrm{OH}$ proton and from a singlet at 2.14 corresponding to a methyl group (H8). Because of these correlations and its chemical shift it was assigned as the C10 quaternary carbon. The carbonyl carbon at $209.9 \mathrm{ppm}$ had correlations from another set of methylene protons at $2.97 \mathrm{ppm}(\mathrm{H} 14 \mathrm{a})$ and $2.26 \mathrm{ppm}(\mathrm{H} 14 \mathrm{~b})$ so it was assigned as the $\mathrm{C} 13$ 
carbon. The second carbonyl carbon (C9) at $213.7 \mathrm{ppm}$ had HMBC correlations from the two gem-dimethyl singlets at 1.07 and 1.04 ppm.

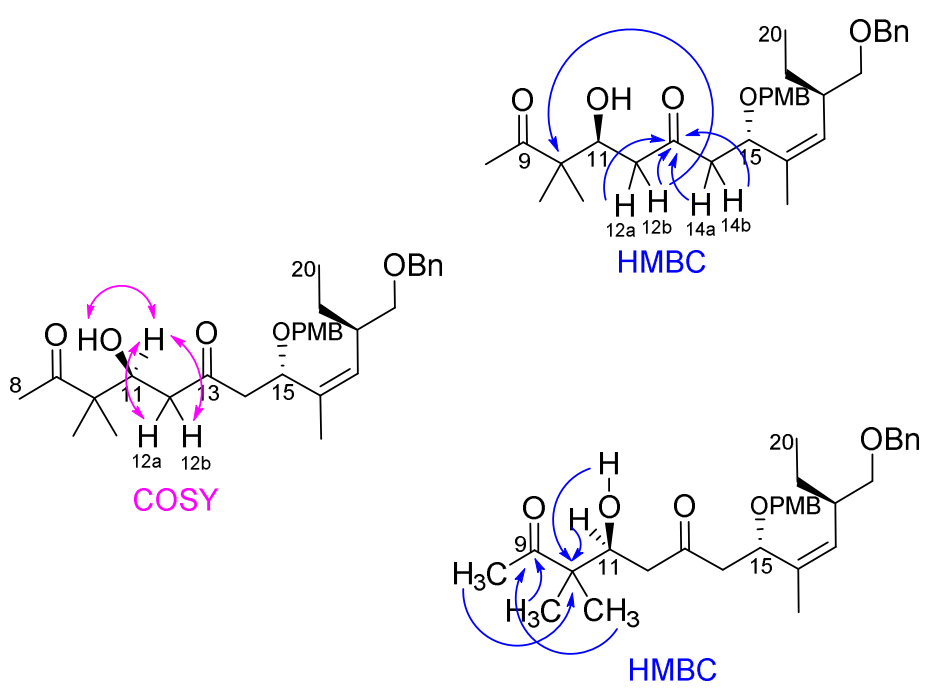

Figure 3.4: Selected COSY and HMBC correlations observed for 142

Once again, Mosher ester analysis was used to determine the stereochemical configuration at the new stereocentre C11. S-MTP ester 144 (Scheme 3.5) was made using standard Steglich esterification conditions (DCC, DMAP) but the reaction was low yielding $(0.3 \mathrm{mg}$, $4 \%) .{ }^{75}$ After 5 days of stirring and with high excess of reagents, starting material was still the most prominent component of the crude mixture as determined by ${ }^{1} \mathrm{H}$ NMR spectroscopy.

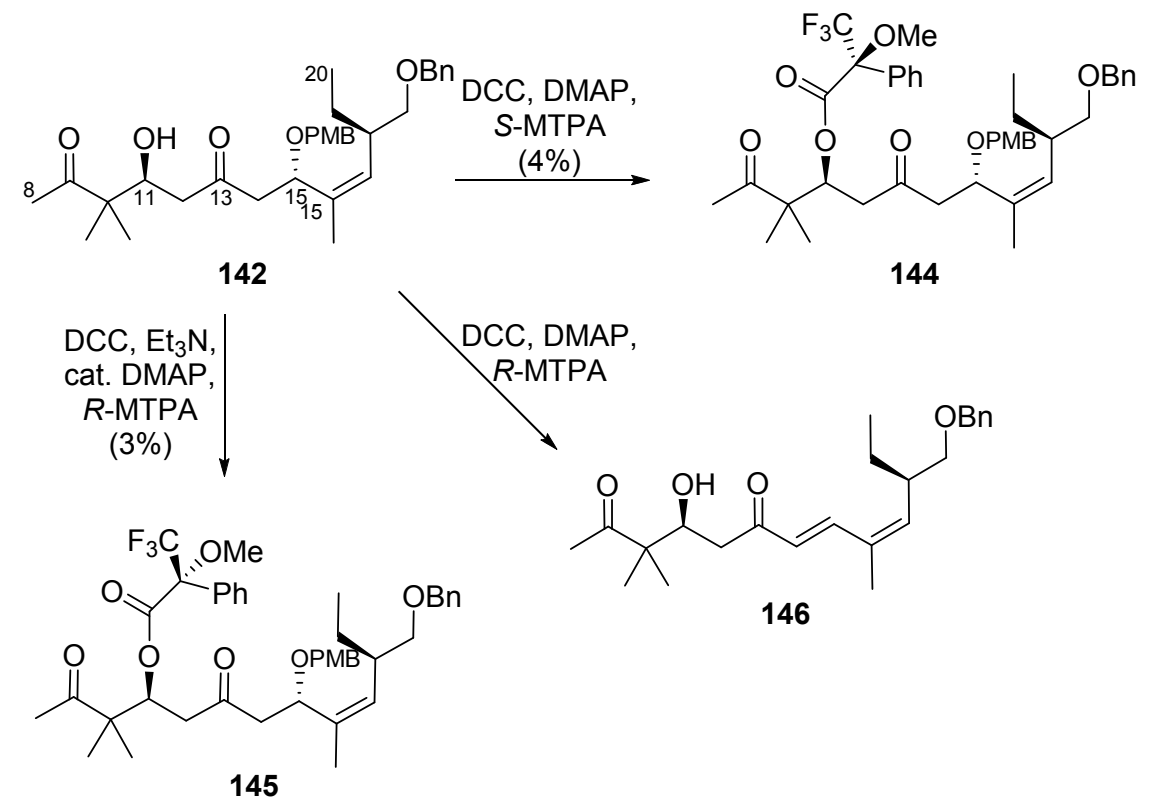

Scheme 3.5: The synthesis of Mosher's esters 144 and 145 
It was attempted to make the $R$-ester (145) under the same conditions but after 5 days of stirring only what is believed to be the elimination product (146) was observed. This byproduct was not fully characterised but the ${ }^{1} \mathrm{H}$ NMR spectrum shows the absence of PMB related peaks $(7.11,6.81,4.29,4.08$ and $3.78 \mathrm{ppm}$ in the starting material) and the presence of two new peaks in the olefinic region at $7.67 \mathrm{ppm}(\mathrm{d}, J=15.7 \mathrm{~Hz}, 1 \mathrm{H})$ and $6.20 \mathrm{ppm}(\mathrm{dd}, J=$ 15.7, $0.7 \mathrm{~Hz}, 1 \mathrm{H}$ ), which each integrated for one proton (Figure 3.5). Similar E1cB elimination was also observed with the side-chain fragment under these conditions (Chapter 2). It is proposed that the presence of a ketone at C13 increases the acidity of the $\alpha$-protons on either side, making them more prone to deprotonation. Since ${ }^{-} \mathrm{OPMB}$ is a better leaving group than ${ }^{-} \mathrm{OH}$, and because of the presence of a double bond $\gamma$ to the ketone, the elimination occurs predominantly on that side, bringing the ketone and the Z-olefin into conjugation.

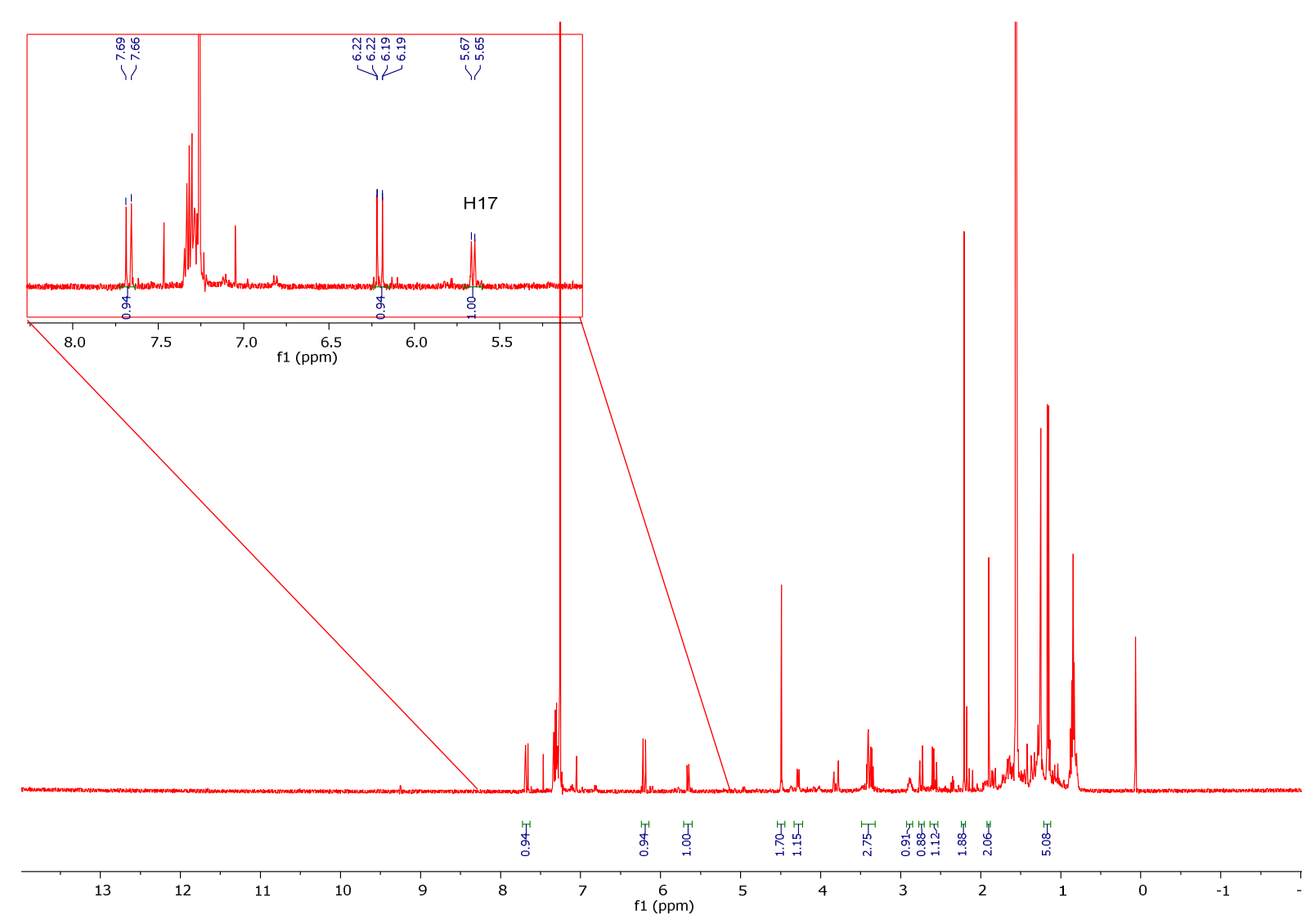

Figure 3.5: ${ }^{1} \mathrm{H}$ NMR spectrum of the elimination by-product 146

Therefore, it was necessary to modify the procedure for the synthesis of $R$-MTP ester. The successful esterification was achieved using a non-nucleophilic base (triethylamine) and catalytic DMAP with the same coupling agent (DCC). ${ }^{120}$ Even under these conditions the 
yield of 145 was very low $(0.3 \mathrm{mg}, 3 \%)$ but this was enough to obtain a ${ }^{1} \mathrm{H}$ NMR spectrum. Mosher ester analysis was conducted in the same manner as described in the previous chapter (Chapter 2). ${ }^{120,140}$ The calculated $\Delta \delta$ values (for $\alpha, \beta, \gamma$ and $\delta$ protons) are shown in the Figure 3.6. These values confirmed the $S$ stereochemical configuration at $\mathrm{C} 11$ and therefore the 11,15-anti relationship.

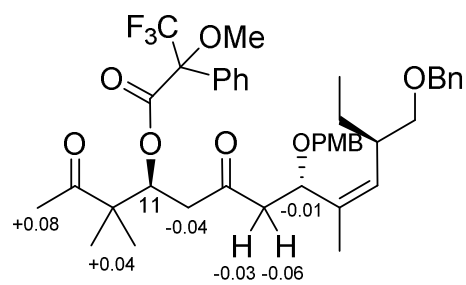

Figure 3.6: $\Delta \delta$ values obtained from ${ }^{1} H$ NMR spectra of the two Mosher's esters 144 and $145\left(\Delta \delta=\delta_{s}-\delta_{R}\right)$

\subsubsection{The final steps in the synthesis of C8-C20 methyl ketone 140}

The overall goal of the following steps was to reduce the ketone at C13, both regioselectively over the $\mathrm{C} 9$ ketone and stereoselectively, then methylate the resulting C13 hydroxyl group. To achieve this Evans' methodology was chosen again. ${ }^{1}$ First, the alcohol at C11 was converted into a silyl ether using chlorodiisopropylsilane (Scheme 3.6). This reaction proceeded to give the hydrosilane 147 in $63 \%$ yield. A characteristic Si-H proton peak was observed in the ${ }^{1} \mathrm{H}$ NMR spectrum of the product as a singlet at $4.07 \mathrm{ppm}$.

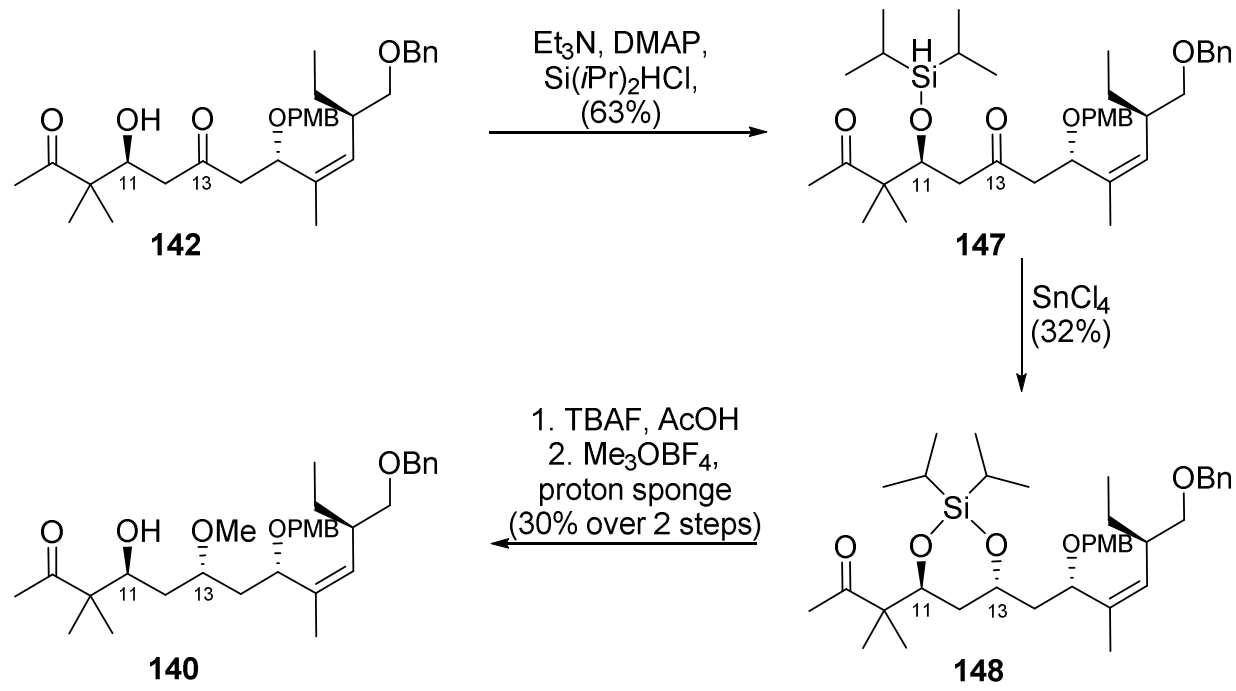

Scheme 3.6: The synthesis of the methyl ketone C8-C20 fragment 140

This silane (147) then underwent a Lewis acid-assisted reduction of the C13 ketone. Tin(IV) chloride was used to activate the ketone, allowing the silane to deliver the hydride to the 
carbonyl carbon. The regioselectivity was due to sterics of the gem-dimethyl group hindering the C9 carbonyl carbon. The stereoselectivity of the reaction was due to the configuration at $\mathrm{C} 11$ as the silane would deliver the hydride from the near face.

Usually the product from this reaction was used in the next step without chromatographic purification. On the occasion when it was subjected to silica gel column, for the purpose of obtaining NMR data (vide infra), the product, dioxasilane 148, was isolated in $32 \%$ yield. The low yield of this reaction was attributed to the quality of tin chloride solution. It is postulated that the presence of $\mathrm{HCl}$ in the tin chloride solution led to silyl deprotection as the deprotected starting material 142 was also recovered (14\%).

The structural rigidity imposed by the presence of the silyl acetal protecting group for the 11,13-diol made it possible to use ${ }^{3} J_{H H}$ coupling values to determine the relative 11,13 stereochemistry of $148 .{ }^{141}$ In the ${ }^{1} \mathrm{H}$ NMR spectrum of the product, proton $\mathrm{H} 11$ appears as a doublet of doublets at $4.30 \mathrm{ppm}$ with $J$ values of 11.2 and $1.6 \mathrm{~Hz}$ (Table 3.1, Figure 3.7) which are due to coupling with the $\mathrm{H} 12$ protons. The $\mathrm{H} 12 \mathrm{a}(\delta 1.81)$ proton presents as a doublet of doublets of doublets with $J$ values of $14.3,11.1$ and $5.8 \mathrm{~Hz}$ and $\mathrm{H} 12 \mathrm{~b}(\delta 1.39)$ is a doublet of doublets with the coupling constants of 14.4 and $1.6 \mathrm{~Hz}$. The largest coupling constant for the $\mathrm{H} 12$ protons, $14.4 \mathrm{~Hz}$, results from ${ }^{2} \mathrm{~J}_{\mathrm{HH}}$ coupling of protons $\mathrm{H} 12 \mathrm{a}$ and $\mathrm{H} 12 \mathrm{~b}$ with each other. The coupling of proton $\mathrm{H} 11$ with $\mathrm{H} 12 \mathrm{a}$ gave a rather large coupling constant $(11.2 \mathrm{~Hz})$ whereas the coupling constant for $\mathrm{H} 12 \mathrm{~b}$ is significantly smaller $(1.6 \mathrm{~Hz})$. The large coupling constant would imply an axial/axial interaction between $\mathrm{H} 11$ and $\mathrm{H} 12 \mathrm{a}$ whereas a smaller coupling constant between $\mathrm{H} 11$ and $\mathrm{H} 12 \mathrm{~b}$ would result from an axialequatorial interaction. In determining the stereochemistry at $\mathrm{C} 13(\delta 4.16$, dddd with $J=9.9$, $5.9,4.2,1.1 \mathrm{~Hz}$ ) the $J$ value for the coupling with the axial C12a will be the most important. This coupling constant was $5.9 \mathrm{~Hz}$, which is significantly smaller than the value observed for an axial/axial interaction ( $\mathrm{H} 11$ to $\mathrm{H} 12 \mathrm{a}$ ) and would imply an axial/equatorial interaction and a non-perfect chair conformation. The axial/equatorial interaction between $\mathrm{H} 12 \mathrm{a}$ and $\mathrm{H} 13$ would confirm the relative 1,3-anti relationship between the substituents at C11 and C13. It was assumed the coupling value of $1.1 \mathrm{~Hz}$ is due to $\mathrm{H} 13$ coupling with $\mathrm{H} 12 \mathrm{~b}$ as the coupling constants of $9.9 \mathrm{~Hz}$ and $4.2 \mathrm{~Hz}$ are due to $\mathrm{H} 13$ coupling to $\mathrm{H} 14 \mathrm{a}$ and $\mathrm{H} 14 \mathrm{~b}$ respectively (Experimental). This value $(1.1 \mathrm{~Hz})$ in the expected range for the equatorial/equatorial interaction. While these results are fairly conclusive that the 1,3-anti-relationship was 
obtained, additional assurances were obtained by using molecular modelling and Karplus calculations.

Table 3.1: Chemichal shifts and coupling values observed for the silyl acetal 148

\begin{tabular}{l|c|c} 
Proton \# & Chemical shift (ppm) & $\begin{array}{c}\text { Coupling constants } \\
(\mathrm{Hz})\end{array}$ \\
\hline $\mathrm{H} 11$ & 4.30 & $11.2,1.4$ \\
\hline $\mathrm{H} 12 \mathrm{a}$ & 1.81 & $14.3,11.1,5.8$ \\
\hline $\mathrm{H} 12 \mathrm{~b}$ & 1.39 & $14.4,1.6$ \\
\hline $\mathrm{H} 13$ & 4.16 & $9.9,5.9,4.2,1.1$
\end{tabular}

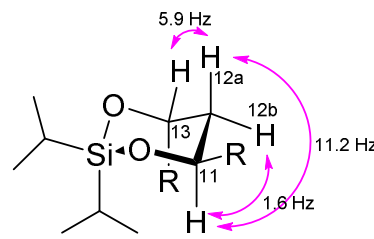

Figure 3.7: Diagrammatic representation of coupling constants and inferred relationships

The MM2 function in Chem3D Pro 14.0 was used for structural energy minimisation. The full structure of the C8-C20 intermediate 148 after minimisation gave a chair-like conformation of the silyl acetal (a truncated version of the minimised structure was used to simplify analysis and is shown in Figure 3.8). When a simplified structure was used for the calculation, where the C15 OPMB was replaced with OMe and the rest of the side chain (C16-C20) was replaced by a methyl group (Figure 3.9), the minimised structure was in a slightly twisted chair conformation. The dihedral angles obtained for the two structures are shown in Table 3.2. Haasnoot's modification of the Karplus dihedral angle calculation ${ }^{142,143}$ was also used to calculate the values of the dihedral angles based on the nature of the substituents and the measured ${ }^{3} J_{\mathrm{HH}}$ values (Table 3.2). The calculation gives the H11-C11$\mathrm{C} 12-\mathrm{H} 12 \mathrm{a}$ angle as $175^{\circ}$ based on the coupling constant of $11.1 \mathrm{~Hz}$ which closely matches the values obtained from energy minimisation for both the full $\left(179^{\circ}\right)$ and the simplified structure $\left(172^{\circ}\right)$. This torsion value further supports the proposed diaxial relationship between the two protons (Figure 3.7, Figure 3.10). The calculated torsion angles for the smaller coupling constant of $1.6 \mathrm{~Hz}$ for $\mathrm{H} 11-\mathrm{C} 11-\mathrm{C} 12-\mathrm{H} 12 \mathrm{~b}$ are listed in the Table $\mathbf{3 . 2}$ and again both values obtained from the minimised structures were similar in magnitude and in the range for ax/eq dihedral angles. For the $\mathrm{H} 13-\mathrm{C} 13-\mathrm{C} 12-\mathrm{H} 12 \mathrm{a}$, the coupling constant of 5.9 $\mathrm{Hz}$ gives the calculated torsion angle of $47^{\circ}$. This value strongly supports the earlier claim 
that the relationship between $\mathrm{H} 13$ and $\mathrm{H} 12 \mathrm{a}$ is equatorial/axial rather than axial/axial. The angle value would also imply a distortion of the chair configuration which was suggested previously based on the magnitude of the coupling constant $(5.9 \mathrm{~Hz})$. To further support the distorted-chair argument, the angle value obtained from the simplified structure (twistedchair, Figure 3.9) matched the calculated one better $\left(48^{\circ}\right)$. Similarly, the calculated torsion angle for $\mathrm{H} 13-\mathrm{C} 13-\mathrm{C} 12-\mathrm{H} 12 \mathrm{~b}$ (Table 3.2) was well within the expected range for an equatorial/equatorial interaction and the calculated value fitted better with the simplified structure value. All these calculations together with the measured $J$ values confirm the 11,13-anti relationship and suggest the dioxasilane assumes a twisted-chair conformation.

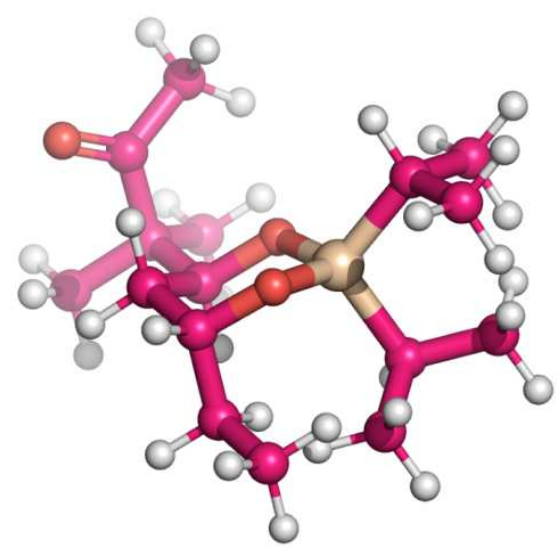

Figure 3.8: Full structure after energy minimisation (truncated)

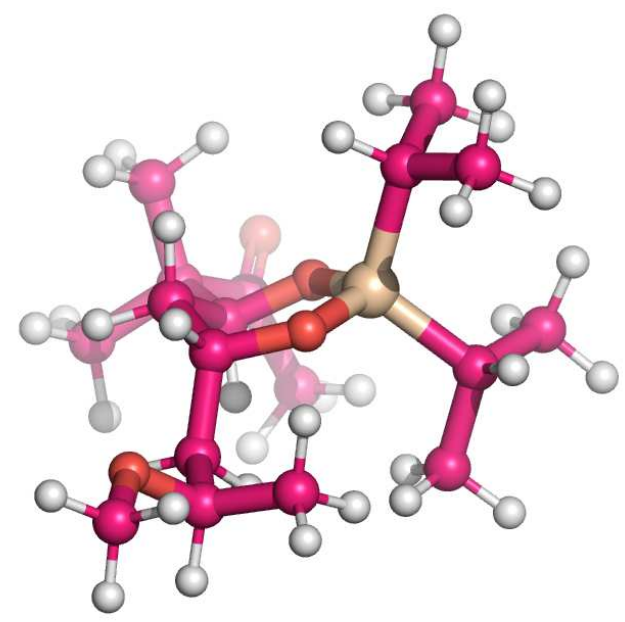

Figure 3.9: Simplified structure after energy minimisation 
Table 3.2: Calculated dihedral angle values for the silyl acetal 148

\begin{tabular}{l|c|c|c|c}
$\begin{array}{l}\text { Torsion angle for } \\
\text { H11-C11-C12- } \\
\text { H12a }\end{array}$ & $\begin{array}{c}{ }^{3} \mathrm{~J}_{\mathrm{HH}} \\
11.2 \mathrm{~Hz}\end{array}$ & $\begin{array}{c}\text { Haasnoot } \\
\text { values }^{\mathrm{a}}\end{array}$ & $\begin{array}{l}\text { Chem 3D MM2 full } \\
\text { structure }\end{array}$ & $\begin{array}{c}\text { Chem 3D MM2 } \\
\text { simplified }\end{array}$ \\
\hline $\begin{array}{l}\mathrm{H} 11-\mathrm{C} 11-\mathrm{C} 12- \\
\mathrm{C} 12 \mathrm{~b}\end{array}$ & $1.6 \mathrm{~Hz}$ & $\begin{array}{c}82^{\circ}, 104^{\circ}, 260^{\circ} \\
293^{\circ}\end{array}$ & $179^{\circ}$ & $172^{\circ}$ \\
\hline $\mathrm{H} 13-\mathrm{C} 13-\mathrm{C} 12-$ & $5.6 \mathrm{~Hz}$ & $\begin{array}{c}47^{\circ}, 137^{\circ}, 235^{\circ}, \\
327^{\circ}\end{array}$ & $-61^{\circ}\left(299^{\circ}\right)$ & $-72^{\circ}\left(288^{\circ}\right)$ \\
$\mathrm{H} 12 \mathrm{a}$ & $1.1 \mathrm{~Hz}$ & $272^{\circ}, 290^{\circ}$ & $-62^{\circ}\left(298^{\circ}\right)$ & $48^{\circ}$ \\
\hline $\mathrm{H} 13-\mathrm{C} 13-\mathrm{C} 12-$ & & & $-67^{\circ}\left(293^{\circ}\right)$
\end{tabular}

${ }^{\mathrm{a}}$ Haasnoot values are based on $J$ values
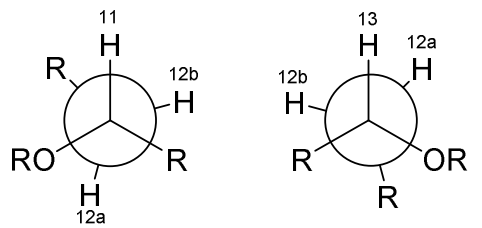

Figure 3.10: Newman projections based on the calculated angles

The silane 148 was cleaved under standard silyl deprotection conditions (TBAF, AcOH) and the crude reaction mixture used directly in the next step, the $O$-methylation of the C13 hydroxyl. The methylation was achieved using Meerwein's salt $\left(\mathrm{Me}_{3} \mathrm{OBF}_{4}\right)$ and 1,8bis(dimethylamino)naphthalene (proton sponge). ${ }^{144}$ The product (140) was isolated in $30 \%$ yield (1.1 mg) along with some unreacted diol 149 (1.1 mg, 31\%).

The methyl ether singlet (3.34 ppm) present in the ${ }^{1} \mathrm{H}$ NMR spectrum of 140 showed HMBC correlation to a carbon at 77.1, assigned as $\mathrm{C} 13$. The regioselectivity of this reaction is attributed again to steric hindrance imposed on the C11 hydroxyl by the vicinal gemdimethyl group and C11 methyl ether product was not isolated.

The diol 149 was not fully characterised due to its immediate methylation but its ${ }^{1} \mathrm{H}$ NMR spectrum was assigned based on comparison with the methyl ether product. The absence of a methyl singlet and the up-field shift of the $\mathrm{H} 13$ oxymethine in the methyl ether ( $3.65 \mathrm{ppm}$ ) compared to the diol $(4.16 \mathrm{ppm})$ were the major differences between the two spectra. The large chamical shift difference was also observed for $\mathrm{H} 11$ and $\mathrm{H} 15$ protons (Figure 3.11). This difference was attributed to the $\mathrm{H}$-bonding in the diol which strongly influences the chemical shifts moving them further downfield than the electron-withdrawing methyl ether group. 

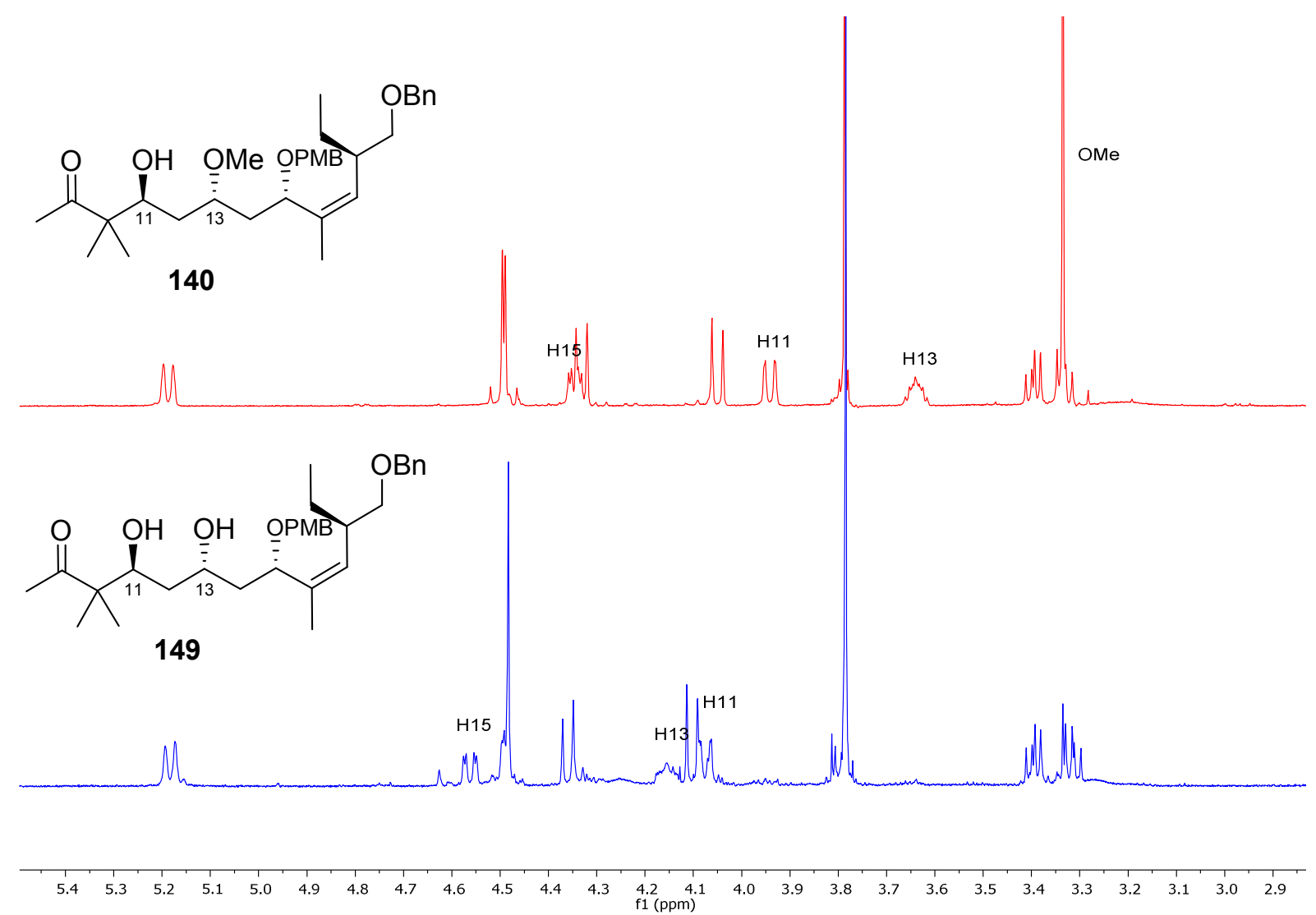

Figure 3.11: Comparison of the proton spectra of the methyl ether 140 and diol 149

\subsection{The C8-C20 vinyl ketone 141}

\subsubsection{The synthesis of C8-C11 vinyl ketone 72}

The aldehyde 67 was also used to synthesise the vinyl ketone 72 (Scheme 3.7). The aldehyde was vinylated under Grignard conditions and the reaction proceeded with 69\% yield to give the secondary alcohol $69 .{ }^{145}$ The alcohol was then oxidised using DMP to give the $\alpha, \beta$-unsaturated ketone $\mathbf{7 0}$ in $85 \%$ yield. 


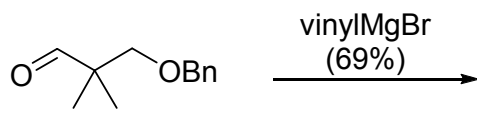

67

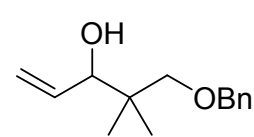

69
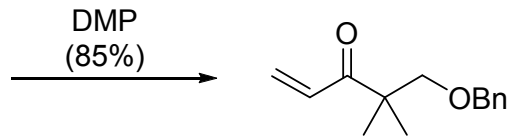

70
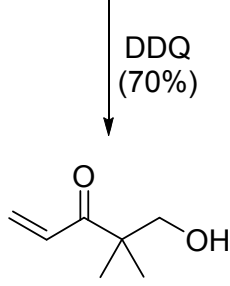

72

Scheme 3.7: The synthesis of vinyl ketone intermediate $\mathbf{7 2}$

An attempt to remove the benzyl protecting group in 70 using $\mathrm{TiCl}_{4}$, as was successfully employed for the methyl ketone, resulted in formation of an unsymmetrical dimer which was assigned the structure 150 (Figure 3.12) based on the NMR data. The ${ }^{1} \mathrm{H}$ NMR spectrum of this product (Figure 3.13) showed an absence of both aromatic and olefinic peaks present in the starting material. Evidently, in addition to the benzyl deprotection, addition of the resulting alcohol to the electrophilic acryloyl double bond of another molecule had taken place. There appeared to be four different proton environments all of them doubled, with the integration ratio between the two sets of peaks being 1:1. These characteristics suggested a dimer that lacks symmetry, hence the observed doubling of the peaks. It was postulated that the lack of symmetry is a result of a different heteroatom being added to the double bond of the second acryloyl moiety. Other than oxygen present in the starting material, the only other heteroatom in the reaction capable of acting as a nucleophile was chlorine. Since chlorine is less electronegative than oxygen, the carbon attached to the chlorine would be shifted upfield from the carbon attached to an oxygen. This is indeed what was observed in the ${ }^{13} \mathrm{C}$ NMR spectrum of $\mathbf{1 5 0}$ (Table 3.3). Even though protons $\mathrm{H} 1$ (3.72 ppm) and $\mathrm{H}^{\prime}$ (3.68 ppm) had very similar chemical shifts, carbons $\mathrm{C} 1$ (38.8 ppm) and C1' (66.9 ppm) were clearly in very different chemical environments. Based on the magnitude of their chemical shifts it was suggested $\mathrm{C} 1$ is attached to a chlorine and $\mathrm{C} 1$ ' to an oxygen. The analysis of the $\mathrm{HMBC}$ spectrum confirmed the ether link between the two units showing $\mathrm{H}^{\prime}$ proton correlating to carbon $\mathrm{C} 5$ (77.9 ppm) and the $\mathrm{H} 5$ proton (3.42 ppm) correlating to $\mathrm{C1}^{\prime}$. The other "terminus" of the dimer $\mathrm{C5}^{\prime}(\delta 69.6)$ had no extra HMBC correlations so it was assumed the substituent on this carbon is a hydroxyl group. 
<smiles>CC(C)(CO)C(=O)CCOCC(C)(C)C(=O)CCCl</smiles>

Figure 3.12: The proposed structure of the by-product 150

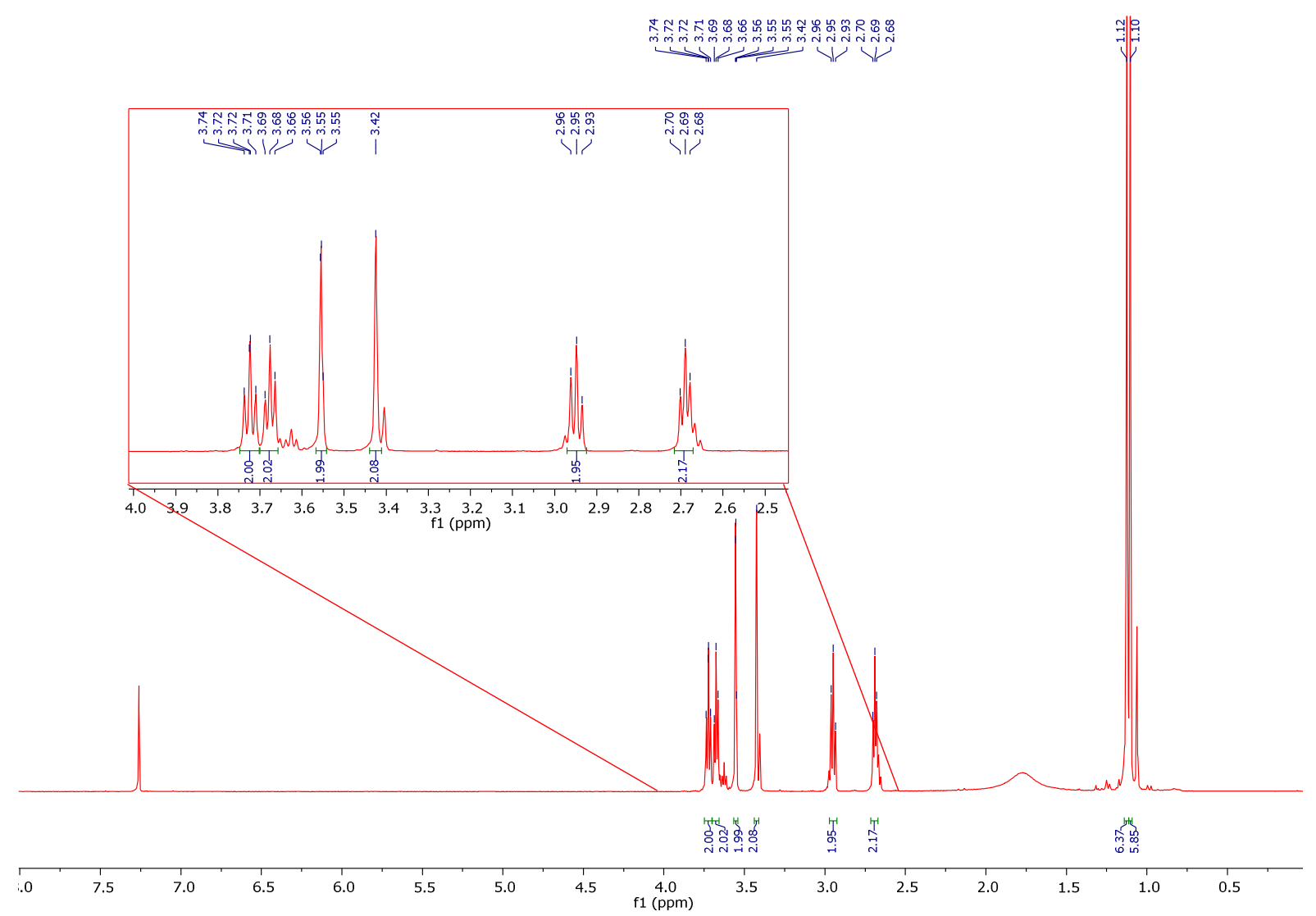

Figure 3.13: ${ }^{1} \mathrm{H}$ NMR spectrum of the by-product 150

Table 3.3: ${ }^{1} \mathrm{H}$ and ${ }^{13} \mathrm{C}$ chemical shifts of by-product 150

\begin{tabular}{c|c|c} 
C\# & ${ }^{1} \mathrm{H}(\mathrm{ppm})$ & ${ }^{13} \mathrm{C}(\mathrm{ppm})$ \\
\hline 1 & 3.72 & 38.8 \\
\hline 2 & 2.95 & 40.6 \\
\hline 3 & - & 211.0 \\
\hline 4 & - & 48.8 \\
\hline 5 & 3.42 & 77.9 \\
\hline $6 \mathrm{a} / 6 \mathrm{~b}$ & 1.10 & 21.7 \\
\hline $1^{\prime}$ & 3.68 & 66.9 \\
\hline $2^{\prime}$ & 2.69 & 37.3 \\
\hline $3^{\prime}$ & - & 214.9 \\
\hline $4^{\prime}$ & - & 49.7 \\
\hline $5^{\prime}$ & 3.55 & 69.6 \\
\hline 6'a/6'b & 1.12 & 21.2
\end{tabular}


The proposed mechanism for the formation of the by-product $\mathbf{1 5 0}$ involves, after the expected benzyl deprotection (Scheme 3.8a), the activation of the double bond through titanium coordination with the oxygen of the ketone (Scheme $\mathbf{3 . 8 b}$ ). This would make the $\beta$ position more electron deficient and therefore prone to a nucleophilic attack by either an oxygen, which results in polymerisation, or chlorine, which ends the polymerisation.

a) Benzyl deprotection

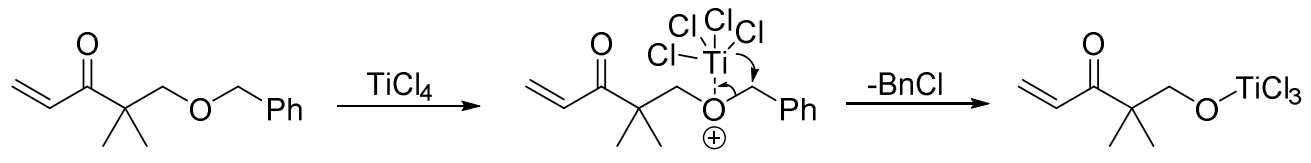

b) Proposed mechanism for the formation of 150
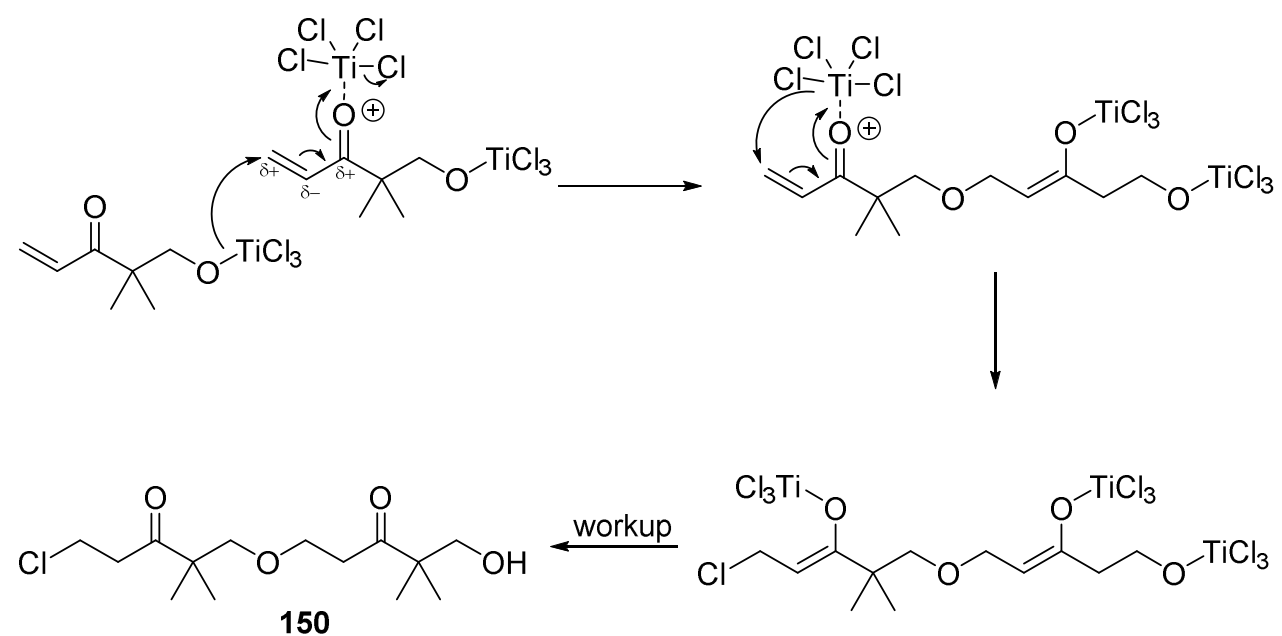

Scheme 3.8: a) benzyl deprotection; b) the proposed mechanism for the formation of $\mathbf{1 5 0}$

Although the $\mathrm{TiCl}_{4}$ reaction was successful in removing the benzyl ether protecting group, the observed polymerisation made it necessary to find a different cleavage method. The most common deprotection method, hydrogenolysis, was deemed not suitable due to the presence of unsaturation in the molecule. Therefore, cleavage of the benzylic ether by oxidative means, using 2,3-dichloro-5,6-dicyano-1,4-benzoquinone (DDQ), was considered. ${ }^{146}$ The deprotection is generally thought to proceed through a single electron transfer process, followed by hydride abstraction from the benzylic methylene group. ${ }^{147-149}$ This reaction was done in 5\% aq. DCM and took 4 days to proceed to completion at room temperature. After purification, the product $\mathbf{7 2}$ was isolated in $70 \%$ yield. 
The overall yield for the synthesis of this fragment was $20 \%$ over five steps from 2,2dimethylpropane-1,3-diol.

\subsubsection{The attempted synthesis of C8-C20 vinyl ketone intermediate 143}

The alcohol 72 was oxidised to the corresponding aldehyde 57 using DMP (Scheme 3.9). The aldehyde was then reacted with the boron enolate of the side-chain fragment 129 in the same manner as described before for the methyl ketone. However, under these conditions, the reaction yielded no product.

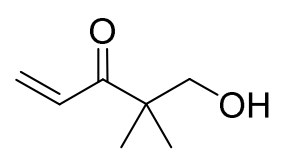

72
1. DMP 2. 129, 9-BBNOTf, $\mathrm{Et}_{3} \mathrm{~N}$

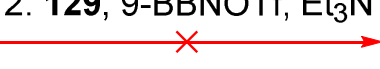

143

Scheme 3.9: Attempted aldol reaction for the formation of C8-C20 vinyl ketone 143

The ${ }^{1} \mathrm{H}$ NMR spectrum of the crude reaction mixture (Figure 3.14) showed that both starting materials were still present, based on the aldehyde peak at $9.65 \mathrm{ppm}$ and the methyl ketone methyl peak at $2.14 \mathrm{ppm}$. The lack of reaction was attributed to the quality of the 9-BBNOTf reagent and it was planned to repeat the reaction using $\mathrm{Bu}_{2} \mathrm{BOTf}$ but due to lack of available materials and the time constraints this was not investigated. 


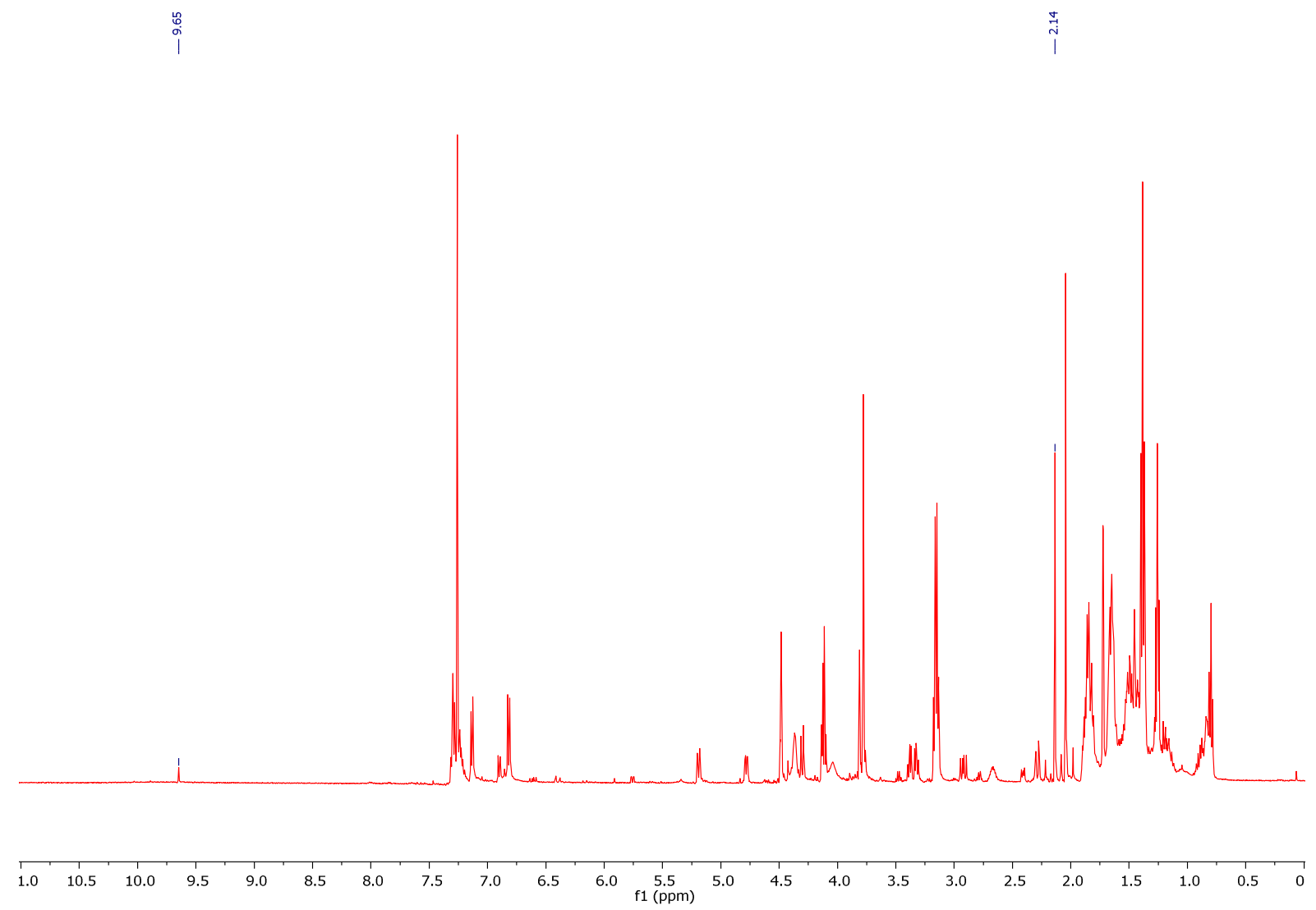

Figure 3.14: ${ }^{1} \mathrm{H}$ NMR spectrum of the crude reaction mixture after the attempted aldol addition

\subsection{Scalability}

The scalability will only be discussed for the reactions and processes involved in the synthesis of the methyl ketone C8-C20 fragment 140 . The synthesis of the C8-C11 methyl ketone fragment is well established and high yielding. The yield of the aldol reaction with the side-chain (C12-C20) fragment needs to be improved. In achieving this, removing the persistent impurity form the side-chain fragment could be essential. Another option would involve using a different dialkylboron reagent $\left(\mathrm{Bu}_{2} \mathrm{BOTf}\right)$.

The sequence of four steps from the aldol product $\mathbf{1 4 2}$ to the methyl ether $\mathbf{1 4 0}$ also leaves a lot of room for improvement. In his total synthesis of PelA, Evans had tried to shorten this sequence by using $\mathrm{NaBH}(\mathrm{OAc})_{3}$ but the regioselectivity was poor. ${ }^{1}$ Another option would be using the Evans-Tishchenko reduction. ${ }^{150}$ This would involve the formation of an $\mathrm{C} 11$ ester which would be followed by $\mathrm{Sml}_{2}$ mediated stereoselective reduction of $\mathrm{C} 13$ ketone. The 1,3-anti stereoselectivity of this reaction has been well documented. ${ }^{151}$ The reaction has even been utilised in Paterson's partial PelA synthesis and Smiths's synthesis of 2-epi- 
PelA. ${ }^{117,152}$ However it is hard to predict whether in our diketone structure there will be enough steric differentiation to assure the selective reduction of C13 ketone over the C9 ketone. It was observed that with gem-dimethyl substituents the Evans-Tishchenko reduction gives lower yields $(45-60 \%)$ at the standard reaction conditions $\left(-10{ }^{\circ} \mathrm{C} / \mathrm{THF}\right) .{ }^{153}$ Clearly the presence of the gem-dimethyl substituent is impeding the reaction which is something that could be further explored in order to obtain the desired differentiation.

Furthermore, the recovery of the diol 149 indicated the 013 methylation reaction had not gone to completion and excess of reagents, both the Meerwein's salt and the proton sponge, might be necessary for future attempts to ensure the full conversion.

Evidently, a few points need to be addressed in order to make the synthesis of $\mathrm{C} 8-\mathrm{C} 20$ methyl ketone fragment more efficient and the optimisation of these steps will be an important part of our future efforts. 


\section{Synthesis of C1-C7 fragments}

\subsection{Overview}

The synthesis of the $\mathrm{C} 1-\mathrm{C} 7$ fragments (Figure 4.1) proved to be the most synthetically challenging. The difficulty arises from the presence of three stereocentres in the fragment with 2,3-syn and 3,5-syn relative stereochemistry. Previous syntheses of the C1-C7 fragment vary greatly in the choice of starting materials, methodology, protecting group strategy and oxygenation state at $\mathrm{C} 1$ (Chapter 1 )..$^{1-4,54,55}$ The main consistency is the apparent requirement for a MOM protecting group at the C2 hydroxyl group for successful elaboration to the natural product. As all the syntheses involve a macrolactonisation step, it becomes essential to have a small protecting group such as MOM at this position so it does not impede the cyclisation.

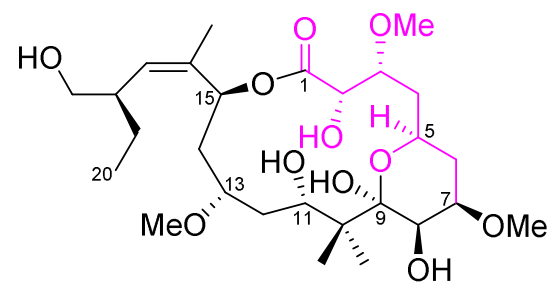

Figure 4.1: $\mathrm{C} 1-\mathrm{C} 7$ fragment of PelA highlighted

This chapter will mainly focus on describing our efforts to synthesise the last common intermediate which can be derivatised into the required $\mathrm{C} 1-\mathrm{C} 7$ fragments (Chapter $\mathbf{1}$ ). Initially, it was planned to make the two C1-C7 fragments 52 and 53 (Figure 2) using a novel approach from the same starting material employed by Ghosh, (-)-2,3-O-isopropylidene-Dthreitol (32), as it contains two out of the three desired stereocentres (vide infra). ${ }^{2}$

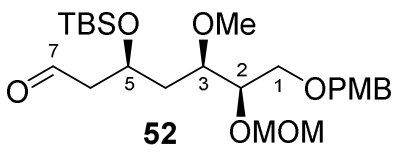<smiles>C=C([Te])C[C@H](C[C@@H](OC)[C@@H](OC)C(=O)O)OC</smiles><smiles>CO[C@@H](CC(C)=O)C[C@@H](OC)[C@@H](C[OH+])OC</smiles>

151 OMOM

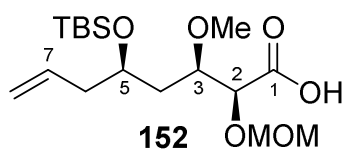<smiles>CO[C@H](CC(C)=O)C[C@@H](OC)[C@@H](OC)C(=O)N1C(=O)OC[C@H]1Br</smiles><smiles>C=C(C)C[C@H](C[C@@H](OC)[C@H](OC)C(=O)O)OC(C)(C)C</smiles> 
Unfortunately, many issues were encountered when exploring this synthetic plan, which ultimately led to this protocol being abandoned. Subsequently, a new plan was devised that borrowed elements from the Jacobsen synthesis (vide infra). ${ }^{3}$ However, the Jacobsen-based plan was also unsuccessful in delivering the C1-C7 fragments 151 and 152 (Figure 4.2). Eventually, the last common intermediate was successfully synthesised using Taylor methodology (vide infra), but its elaboration into the two C1-C7 fragments 153 and 154 (Figure 4.2) has not yet been completed. ${ }^{4}$

\subsection{Ghosh-inspired approach: from (-)-2,3-0-isopropylidene- D-threitol}

\subsubsection{The strategy}

In devising the synthesis of the last common intermediate $\mathbf{8 1}$ (Scheme 4.1) that would be further elaborated into the two C1-C7 fragments, the presence of the $\mathrm{C} 7$ alkene was especially important as it allowed the fragment to be useful in both end-game approaches (Chapter 1).

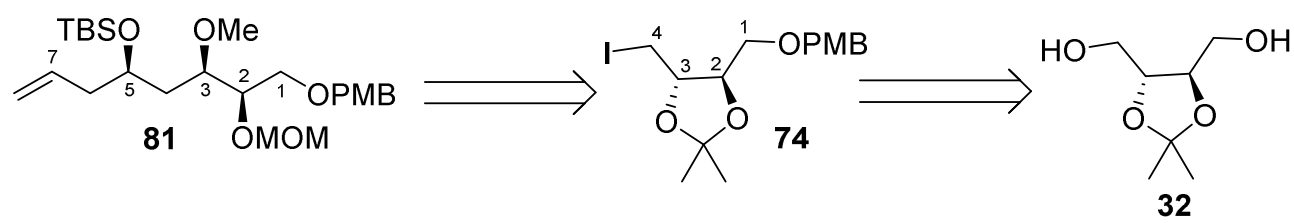

Scheme 4.1: The C1-C7 intermediate 81

The alkene functionality is necessary for the RCM route while ozonolytic cleavage of the terminal olefin would give the C7 aldehyde fragment 52 (Scheme 4.2) required for the macrolactonisation route. Further elaboration of the intermediate 81 to the $\mathrm{C} 1-\mathrm{C} 7$ acid fragment (53, Scheme 4.2) would involve PMB deprotection, oxidation of the primary alcohol to the aldehyde and lastly the oxidation of the aldehyde to the carboxylic acid. These modifications of the common intermediate $\mathbf{8 1}$ would give the two $\mathrm{C} 1-\mathrm{C} 7$ fragments $\mathbf{5 2}$ and $\mathbf{5 3}$ in 10 and 12 steps, respectively, from the threitol starting material. 


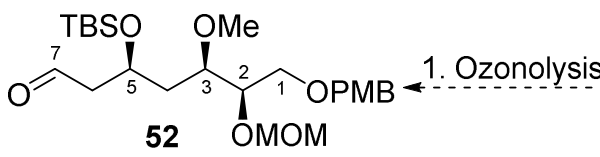<smiles>C=CCC(CC(OC)C(COC)OC)OC</smiles>

1. PMB
cleavage
$\begin{aligned} & \text { 3. Oxidation } \\ & \text { Pinnick }\end{aligned}$

Scheme 4.2: The synthesis of the two C1-C7 fragments 52 and 53

Intermediate $\mathbf{7 4}$ was prepared by Ghosh et al. in the synthesis of the C1-C10 enone fragment 31 (Scheme 1.3). ${ }^{2}$ In this synthesis (Scheme 4.3), the threitol-derived diol 32 was mono-protected with PMB first, followed by an Appel iodination reaction to give the iodide 74. Another $S_{N} 2$ reaction between the iodide and vinylmagnesium bromide gave the alkene 155. The acetonide was then removed under acidic conditions and subsequent iodoetherification yielded the iodide 156. After the $\mathrm{O} 3$ methylation, the terminal alkene was obtained by reductive cleavage of the iodoether using zinc and the remaining hydroxyl protected as a MOM acetal (157). The terminal double bond was then dihydroxylated and the diol oxidatively cleaved to give the C5 aldehyde (158). Stereoselective Brown allylation and TBS protection of the 05 gave 81 in $26 \%$ yield over 12 steps. Even though the overall yield for the synthesis of the intermediate $\mathbf{8 1}$ is good, because of the number of steps and in order to introduce novelty, we decided not to use Ghosh's methodology. Still, some elements were taken from his synthesis and these will be discussed in the following paragraph.

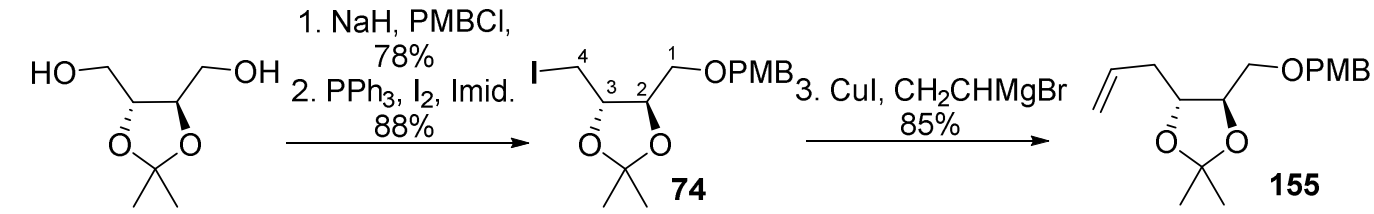

32

. $\mathrm{Me}_{3} \mathrm{OBF}_{4}$ 7. Zn, aq. EtOH

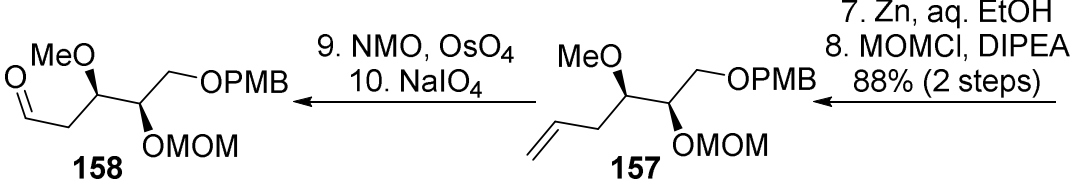
157

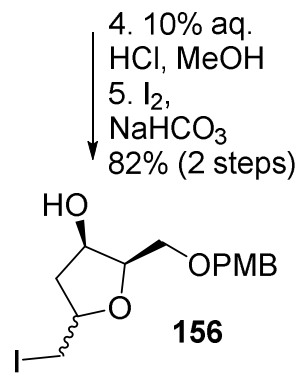

11. $\left|p c_{2} B-A l l y\right|$

$74 \%$ (3 steps)

12. TBSCI, Imid.

$\checkmark 6 \%$

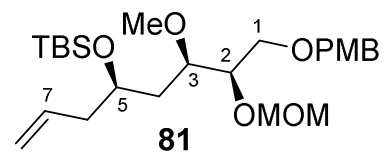


The first two steps of the synthetic plan would be the same as Ghosh's: the mono-PMB protection and subsequent conversion of the primary alcohol (73) into iodide 74 (Scheme 4.4). The iodide would be reacted with 2-allyl-1,3-dithiane to complete the seven-carbon skeleton of the fragment (75). This approach is highly convergent and was initially preferred to the alternative stepwise process involving the addition of unsubstituted 1,3-dithiane followed by allylation. However, the coupling with allyl dithiane is not well precedented in the literature and it was expected to require some effort to optimise this key step. Next, the acetonide protecting group would be removed.

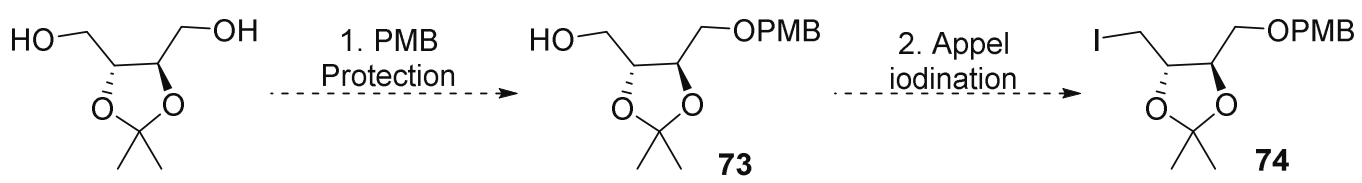

32

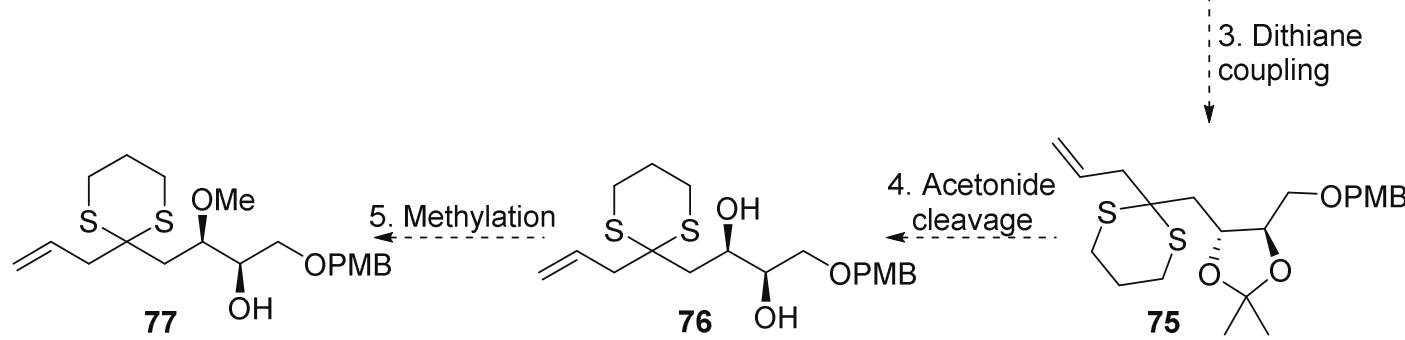

6. MOM

protection

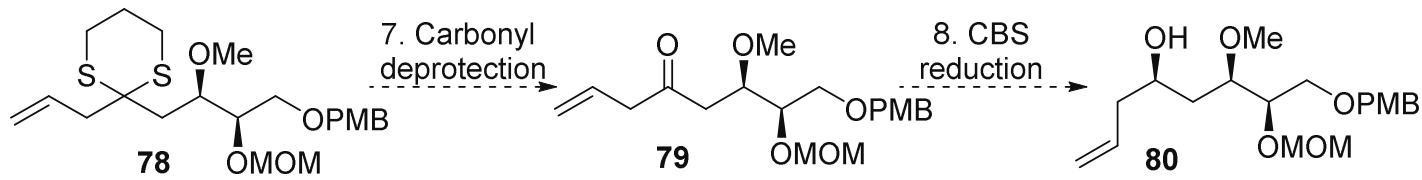

9. TBS

protection

$\checkmark$

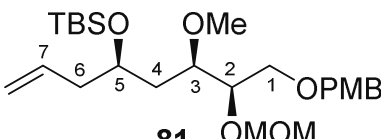

81

Scheme 4.4: Strategy for the synthesis of the C1-C7 intermediate 81

The selective methylation of $\mathrm{O} 3$ over $\mathrm{O} 2$ was anticipated to be challenging, as we relied solely on the differentiation based on the sterics imposed by the $\mathrm{O} 1$ protecting group. After the dithiane protecting group is removed, the ketone at C5 (79) was going to be reduced in a stereoselective manner (Corey-Bakshi-Shibata reduction) to give the alcohol $80 .{ }^{154}$ The 
synthesis of the fragment would conclude with the TBS protection of the C5 hydroxyl group (81). The choice of protecting groups, PMB at 01 and TBS at 05, was adopted from Ghosh. ${ }^{2}$ Using this approach the required $\mathrm{C} 1-\mathrm{C} 7$ intermediate $\mathbf{8 1}$ would be synthesised in nine linear steps.

\subsubsection{Dithiane coupling}

The first step in the synthesis was the mono-PMB protection of (-)-2,3-O-isopropylidene-Dthreitol (Scheme 4.5). The reaction proceeded to give 73 in $69 \%$ yield and the best results were obtained when 1:1 v/v THF/DMF was used as a solvent. ${ }^{155}$ The orthogonality of the 01 protecting group is important, especially for the macrolactonisation route. At a later stage in the synthesis, it will be necessary to selectively deprotect at this position (over O24), then oxidise the primary alcohol to the corresponding acid. PMB ethers are relatively stable to acidic and basic conditions and can be cleaved selectively using DDQ. Despite the presence of another PMB protecting group in the molecule (at 015 ), which will also be cleaved in this step, it should be possible to oxidise the resulting primary alcohol (C1) selectively over the secondary alcohol (C15). The selective oxidation at C1 (over C15) was already achieved by Ghosh using tetrapropylammonium perruthenate (TPAP) to first selectively oxidise the primary alcohol to the aldehyde which was subsequently oxidised to the carboxylic acid under standard Pinnick reaction ${ }^{67}$ conditions $\left(\mathrm{NaClO}_{2}, \mathrm{NaH}_{2} \mathrm{PO}_{4}\right) .{ }^{2}$ Selective primary alcohol oxidation (over a secondary C11 hydroxyl) was also achieved by Jacobsen, employing TEMPO/BAIB., ${ }^{3,79}$

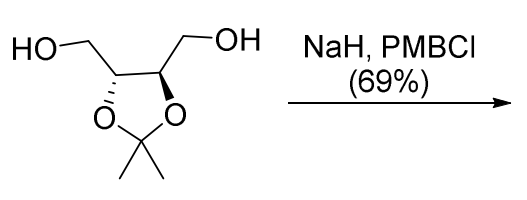

32

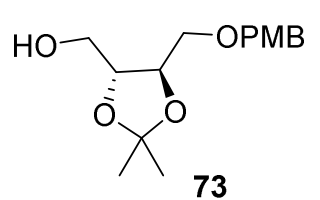

73

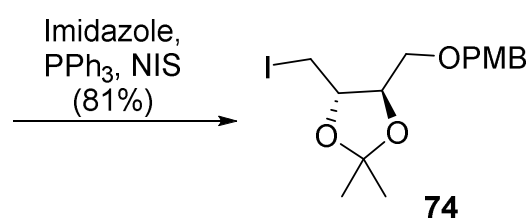

74

Scheme 4.5: The synthesis of the C1-C4 iodide 74

The mono-protection was followed by an Appel iodination reaction which proceeded with $81 \%$ yield to give the iodide 74 (Scheme 4.5). ${ }^{156}$ Mechanistically, triphenylphosphine reacts with the electrophilic iodine of $\mathrm{N}$-iodosuccinimide to give a phosphonium species 159 (Scheme 4.6). The nucleophile (OH group) can then attack the phosphonium and displace the iodide to give an oxophosphonium intermediate 160. Now, the hydroxyl is converted 
into a better leaving group and undergoes an $\mathrm{S}_{\mathrm{N}} 2$ reaction with iodide to give the product $\mathbf{7 4}$ accompanied by triphenylphosphine oxide as a readily-separable by-product.

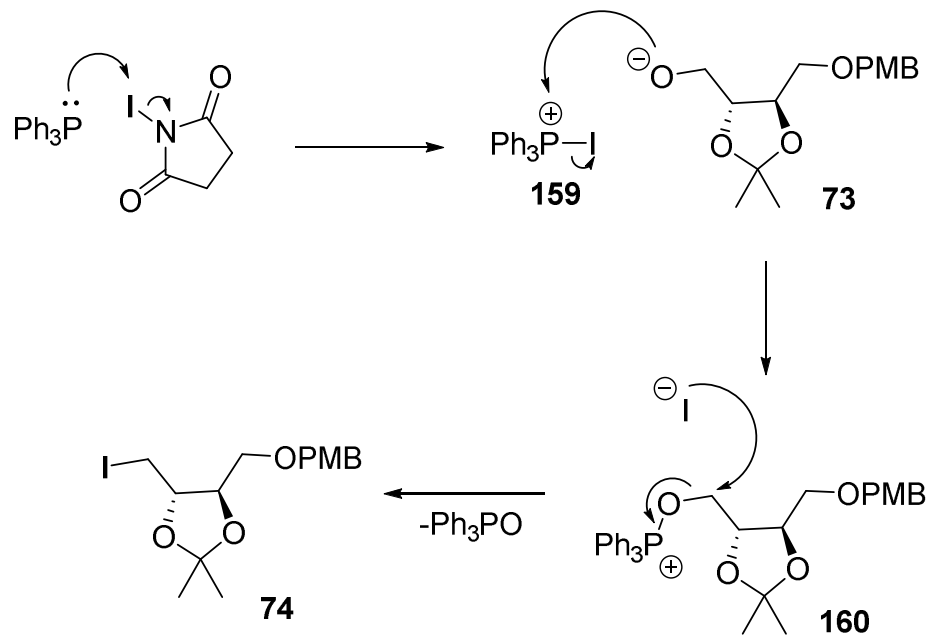

Scheme 4.6: Mechanism of the Appel reaction

The next step involved the preparation of the dithiane coupling partner. Seminal work by Corey and Seebach in the 1960's and 1970's revealed the usefulness of 1,3-dithianes in constructing carbon-carbon double bonds. ${ }^{157-159}$ Since then, this method has been employed numerous times for natural product synthesis. ${ }^{160}$ These compounds represent carbonyl umpolung synthons (Scheme 4.7) due to their nucleophilic reactivity that is revealed upon base treatment.

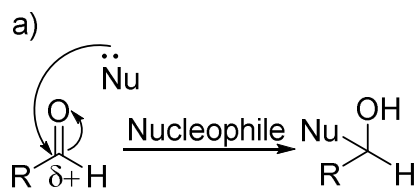

b)

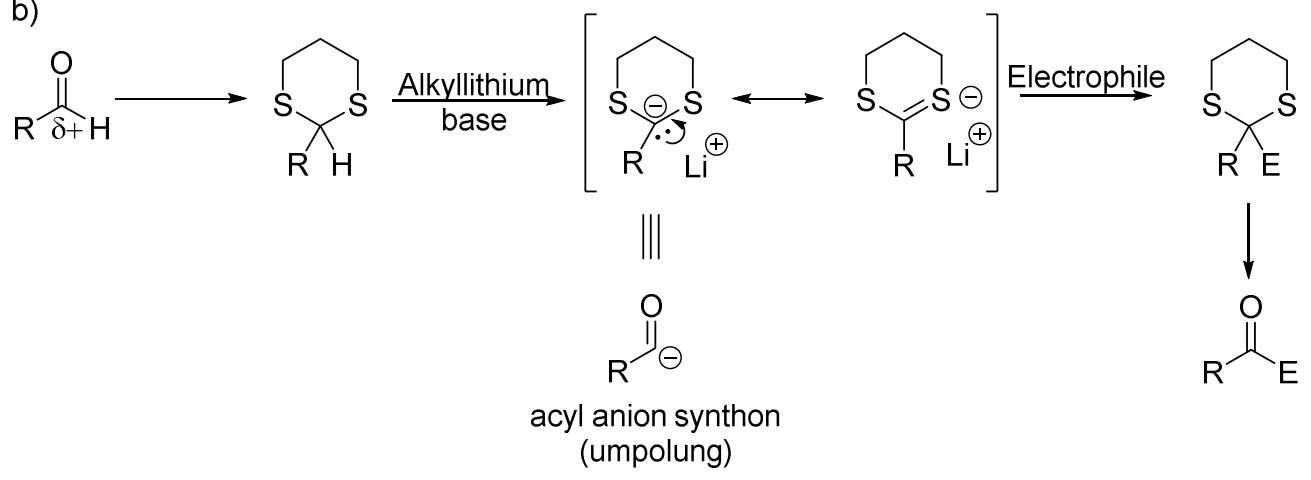

Scheme 4.7: a) Normal carbonyl reactivity and b) reverse reactivity achieved by using dithiane

1,3-Dithianes can be easily deprotonated by alkyllithium reagents to give a lithiated species where the carbanion is stabilised by sulfur's empty $d$ orbital. ${ }^{161}$ This species is an equivalent 
of an acyl anion and reacts readily with many types of electrophiles (alkyl halides, sulfonates, triflates, epoxides, carbonyl compounds etc.). ${ }^{160}$

The 2-allyl-1,3-dithiane 161 was synthesised in a single step from 1,3-dithiane and allyl bromide, providing the product in 78\% yield (Scheme 4.8). ${ }^{162}$ The dithiane was first lithiated using $n$-BuLi then allyl bromide was added, upon which the bromide was displaced by dithiane.

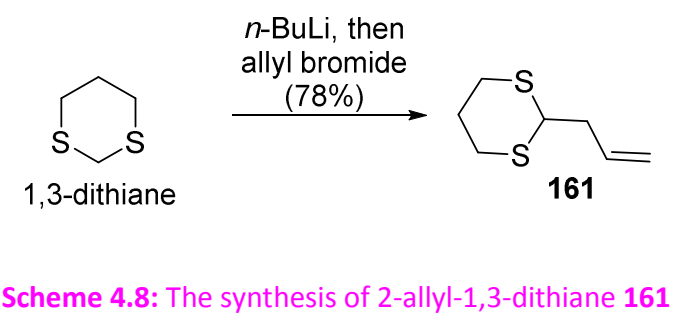

With the successful synthesis of both the dithiane 161 and iodide 74, the key coupling step could be attempted. Even though 2-substituted-1,3-dithianes are sometimes difficult to deprotonate, ${ }^{163,164}$ based on the few literature examples where 2-allyl-1,3-dithiane was used, ${ }^{165,166}$ we were persuaded that it could be deprotonated by $n$-BuLi.

In the first attempt at the coupling (Scheme 4.9), allyl dithiane 161 (1.3 equiv.) was dissolved in dry THF and treated with $n$-BuLi (1.3 equiv.) at $-78{ }^{\circ} \mathrm{C}$. The reaction was allowed to gradually warm up to $-20{ }^{\circ} \mathrm{C}$ then kept at this temperature for 1 hour to complete the deprotonation. Then, the reaction was cooled back to $-78{ }^{\circ} \mathrm{C}$ and the iodide 74 (1 equiv.) was added as a solution in THF. The reaction was allowed to warm to $-30{ }^{\circ} \mathrm{C}$, then stirred at this temperature for 2 hours. At this point, TLC analysis indicated almost full consumption of the dithiane starting material and the presence of a new spot more polar than both starting materials. Oddly, the iodide spot was still very intense. After silica gel purification, the compound corresponding to the new spot was isolated. Unfortunately, the ${ }^{1} \mathrm{H} N M R$ spectrum of this compound had insufficient types of proton environments to be the expected dithiane product 75 (Scheme 4.9).

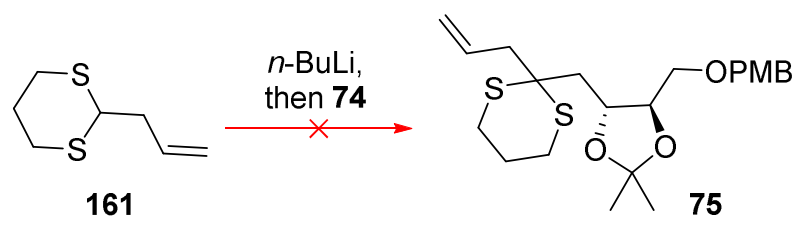


The ${ }^{1} \mathrm{H}$ NMR spectrum of this compound (Figure 4.3) showed the PMB related peaks (7.26, $6.89,4.51,3.81 \mathrm{ppm})$, the terminal double bond peaks [ $\delta 5.82(\mathrm{H} 3), 5.35(\mathrm{H} 4 \mathrm{a})$ and 5.19 $(\mathrm{H} 4 \mathrm{~b})]$, a single oxymethine proton peak at $4.33 \mathrm{ppm}(\mathrm{H} 2)$ and the $\mathrm{C} 1$ methylene protons at $3.52(\mathrm{H} 1 \mathrm{a})$ and $3.34(\mathrm{H} 1 \mathrm{~b}) \mathrm{ppm}$. The connectivity was confirmed through the analysis of the COSY spectrum. Based on the NMR data, the new compound was assigned the structure 162 (Figure 4.3). ${ }^{167}$
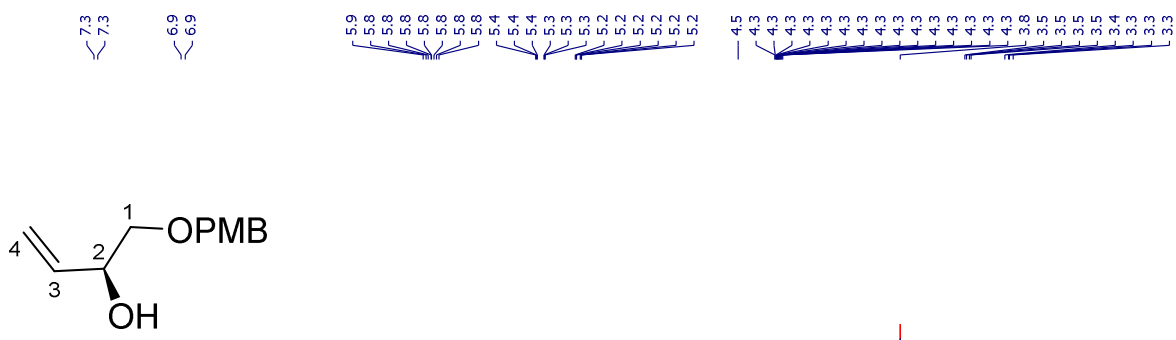

162

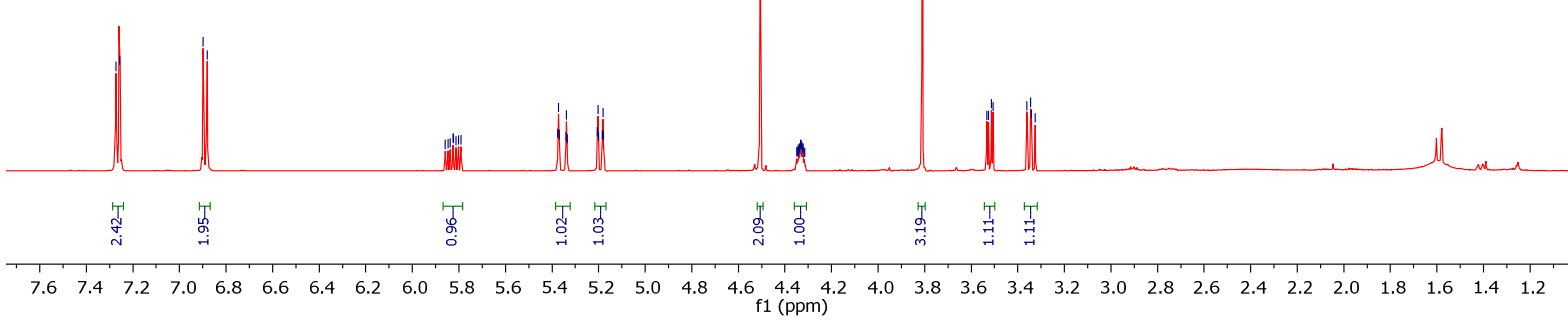

Figure 4.3: ${ }^{1} \mathrm{H}$ NMR spectrum of the isolated by-product 162

It is thought that compound $\mathbf{1 6 2}$ resulted from an elimination process in the iodide starting material. Based on a similar example from the literature ${ }^{168}$, which involved iodide-for-zinc exchange, it was postulated that this elimination was a result of iodide-for-lithium exchange followed by expulsion of acetone (Scheme 4.10 ). This by-product accounted for $63 \%$ of the iodide starting material.
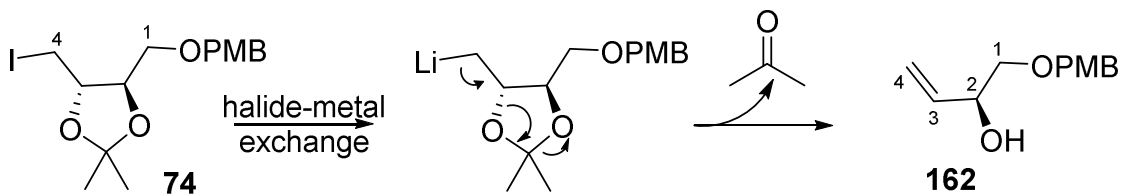

Scheme 4.10: Proposed mechanism for the formation of by-product 162 
Surprisingly, the ${ }^{1} \mathrm{H}$ NMR spectrum (Figure 4.4a) of the sample that was assumed to be only the remaining iodide $\mathbf{7 4}$ starting material (Figure $\mathbf{4 . 4 b}$ ), showed also the presence of another compound related to the allyl dithiane. The most notable features of the spectrum were the allyl peaks (olefinic protons at 6.18 and $5.15 \mathrm{ppm}$ and allylic protons as part of the 2.94-2.79 ppm multiplet), the absence of the allyl dithiane methine peak (in the starting material 161 this appeared as a triplet at $4.10 \mathrm{ppm}$, Experimental). The presence of a new singlet peak at $1.43 \mathrm{ppm}$, integrating for six protons, indicated the presence of two equivalent methyl groups. Given the reaction conditions, the observed elimination product and these spectral features, we proposed the unknown compound 163 was a result of a reaction of the dithiane carbanion with the acetone that formed in the elimination of $\mathbf{7 4}$ (Scheme 4.11).

a)

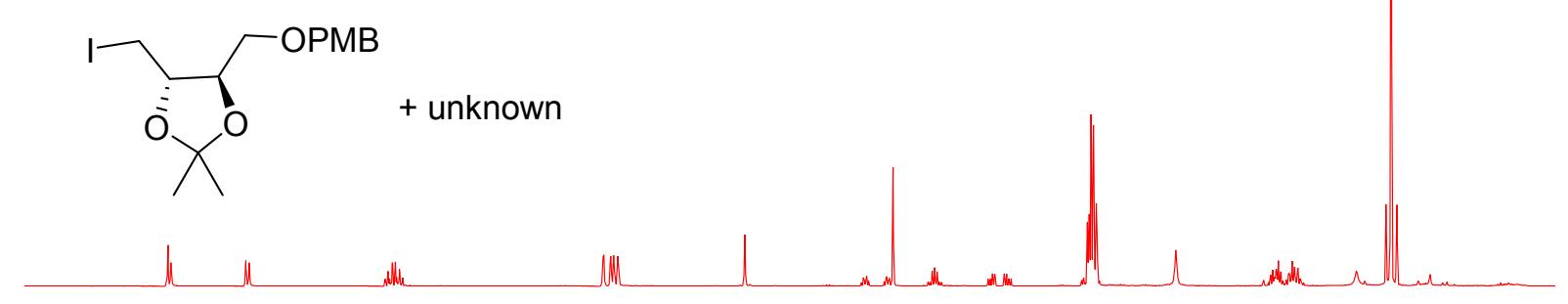

b)

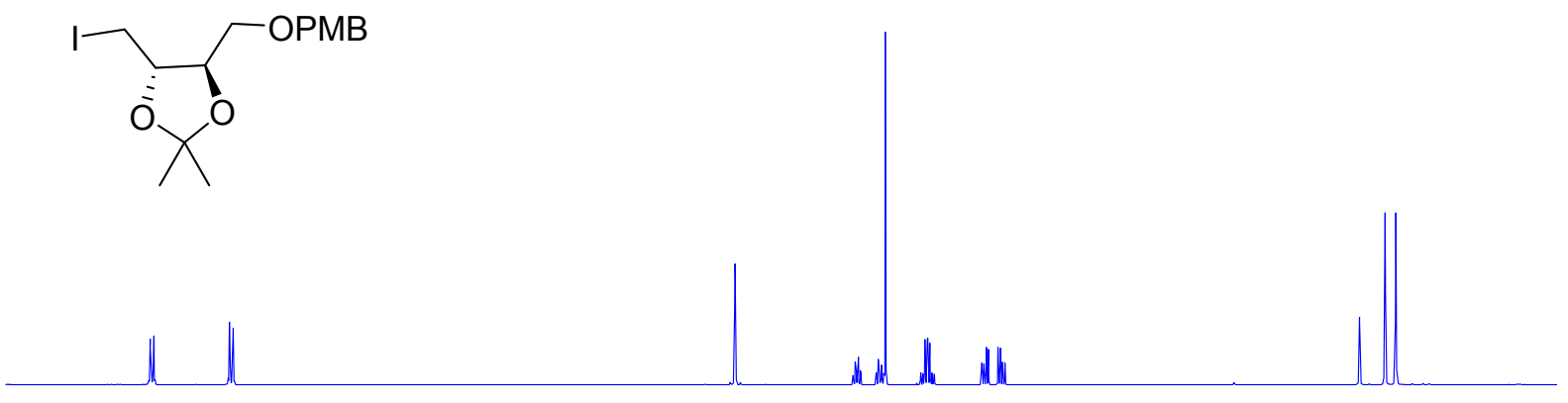

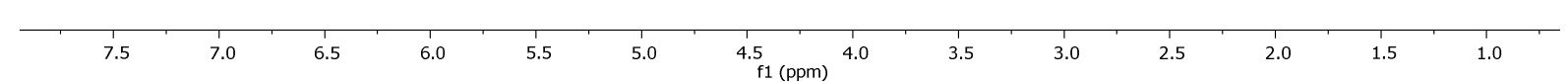

Figure 4.4: ${ }^{1} \mathrm{H}$ NMR spectra of a) sample containing by-product 163 and iodide $\mathbf{7 4}$ b) iodide 74

After the assignment of the carbon peaks corresponding to the iodide starting material and the protonated carbons of the new dithiane compound, there were two unassigned unprotonated carbons at 78.4 and $63.0 \mathrm{ppm}$, presumably $\mathrm{C} 5$ and $\mathrm{C} 4$ in the ${ }^{13} \mathrm{C} \mathrm{NMR}$ spectrum. The assignment of these peaks was based on the observed HMBC correlations 
from the $\mathrm{H} 2$ and $\mathrm{OH}$ protons to the carbon at $63.0 \mathrm{ppm}$ which was therefore assigned as $\mathrm{C} 4$. Additionally, with the oxygen being more electronegative, the C5 carbon is expected to be further downfield than the $\mathrm{C} 4$ carbon. Based on integration of the ${ }^{1} \mathrm{H}$ NMR spectrum, it was possible to determine the mass contribution of the two components, and the integration ratio $(0.30: 1.0074: 163)$ shows the molar ratio of $23: 77$. This was used to calculate the mass contribution of each compound. The starting material $\mathbf{7 4}$ accounted for $35 \%$ of the isolated sample mass ( $4.87 \mathrm{mg}, 12 \%$ recovery) whereas the by-product 163 accounted for $65 \%$ of the isolated sample mass ( $9.04 \mathrm{mg}, 40 \%$ yield).

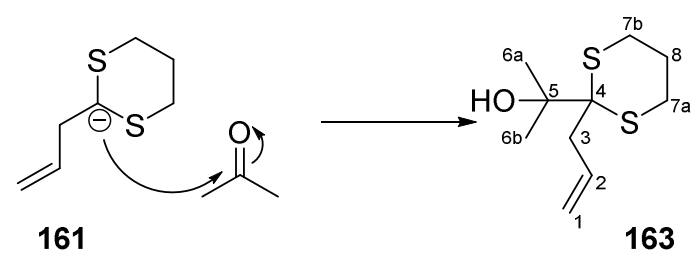

Scheme 4.11: Mechanism for the formation of by-product 163

Even though the desired product was not observed, the formation of by-product $\mathbf{1 6 3}$ clearly showed that the dithiane carbanion was indeed formed upon treatment of 161 with $n$-BuLi. In order to encourage the desired reaction to take place, efforts were made towards preventing the lithium for halogen exchange and the subsequent elimination. When a coordinating solvent, $\mathrm{N}, \mathrm{N}$-dimethylpropyleneurea (DMPU), was used as a co-solvent (5:1 THF:DMPU), no by-products were observed but again the desired reaction did not occur. Next we repeated the reaction using a lithium coordinating additive, tetramethylethylenediamine (TMEDA), that is commonly used with $n$-BuLi as it is known to increase its basicity. ${ }^{169}$ However, with TMEDA, the by-products 162 and 163 were observed again. Lastly, the reaction was attempted using a potassium base (KHMDS) and again gave no by-products but also no product. Given the lack of success in optimising the reaction conditions, we decided to take a closer look at the electrophile and the nucleophile.

To determine whether the problem lay with the electrophilic starting material, the bromide variant was made. With bromide being less reactive than iodide, it was postulated the bromide-for-lithium exchange might not occur as rapidly, which would slow down the elimination pathway. Bromide $\mathbf{1 6 4}$ was prepared by an Appel reaction from the previously made alcohol 73 (Scheme 4.12 ) in $43 \%$ yield. 


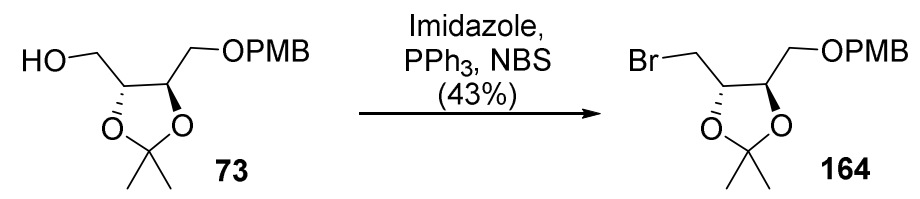

Scheme 4.12: The synthesis of the bromide 164

The coupling reaction of 2-allyl-1,3-dithiane with the bromide 164 was unfortunately also unsuccessful and resulted solely in the recovery of starting materials. Next, we decided to exchange the nucleophile, 2-allyl-1,3-dithiane, for the unsubstituted, 1,3-dithiane, as there were more literature examples of the reaction of various iodides and bromides with 1,3dithiane itself than with substituted dithianes. ${ }^{156,160,170}$ If this reaction was successful the resulting product could be allylated in the next step.

Accordingly, the iodide $\mathbf{7 4}$ was reacted with the lithiated 1,3-dithiane, and a small amount of product 165 (Scheme 4.13) was isolated from the reaction mixture. ${ }^{156}$ The ${ }^{1} \mathrm{H}$ NMR spectrum of the product showed the new H5 methine at $4.20 \mathrm{ppm}$ as a doublet of doublets $(J=8.2,6.4 \mathrm{~Hz})$ and a significant change in the $\mathrm{H} 4$ methylene chemical shift $(\delta 1.99)$ compared to the iodide starting material ( $\delta 3.34$ for $\mathrm{H} 4 \mathrm{a}$ and $\delta 3.26$ for $\mathrm{H} 4 \mathrm{~b}$ ). The $\mathrm{H} 4$ position showed COSY correlations to two methine environments, the dithiomethine (H5) at $4.20 \mathrm{ppm}$ and the oxymethine $(\mathrm{H} 3)$ at $4.09 \mathrm{ppm}$. The rest of the dithiane proton environments, $\mathrm{H} 8 \mathrm{a}$ and $\mathrm{H} 8 \mathrm{~b}$ (4 protons at $2.83 \mathrm{ppm}$ ), and $\mathrm{H} 9$ protons ( $\mathrm{H} 9 \mathrm{a}$ at $2.10 \mathrm{ppm}$ and $\mathrm{H}-9 \mathrm{~b}$ at $1.87 \mathrm{ppm})$, were readily discernible in the ${ }^{1} \mathrm{H}$ NMR spectrum.

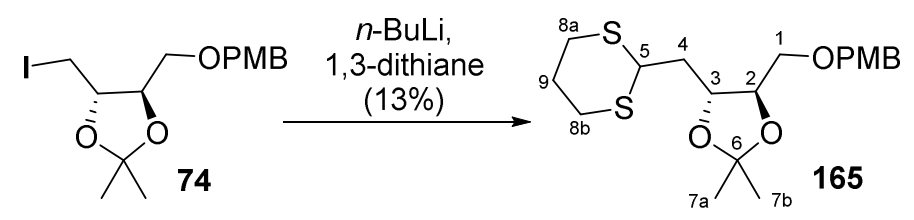

Scheme 4.13: The synthesis of the dithiane 165

Despite this positive identification of the desired product, the elimination pathway was typically preferred over the $S_{N} 2$ pathway. Through our efforts to optimise the reaction, it was observed that the reaction temperature was important in suppressing the formation of 162. The reaction had to be kept at temperatures below $-60^{\circ} \mathrm{C}$, but at these temperatures the desired $S_{N} 2$ reaction was also slow. The best obtained yield of the dithiane 165 was 13\% (Scheme 4.13 and Experimental). 
Aside from the by-product 162 resulting from elimination, another by-product was sometimes observed. The ${ }^{1} \mathrm{H}$ NMR spectrum of the isolated compound had some similarities with the elimination product; a single oxymethine peak and the absence of acetonide methyl peaks (Figure 4.5). All the PMB related peaks were still present. A distinctive new feature in the proton spectrum was a methyl singlet at $2.20 \mathrm{ppm}$. This peak had two correlations in the $\mathrm{HMBC}$ spectrum. One of the correlations was to an unprotonated carbon at $208.0 \mathrm{ppm}$, which is a typical environment for ketone carbonyl carbons. The second correlation was to the carbon of the oxymethine (C2) at $77.0 \mathrm{ppm}$. In the COSY spectrum, oxymethine $(\mathrm{H} 2)$ at $4.25 \mathrm{ppm}$ showed correlations to the two $\mathrm{C} 1$ methylene protons $(\mathrm{H} 1 \mathrm{a}$ at 3.80, partly obstructed the PMB methoxy methyl peak, and $\mathrm{H} 1 \mathrm{~b}$ at $3.68 \mathrm{ppm}$ ), as well as to a proton at $3.63 \mathrm{ppm}$, assigned as a hydroxyl proton due to the absence of an HSQC correlation. Based on these correlations the structure was determined to be $\mathbf{1 6 6}$ (Figure 4.5).

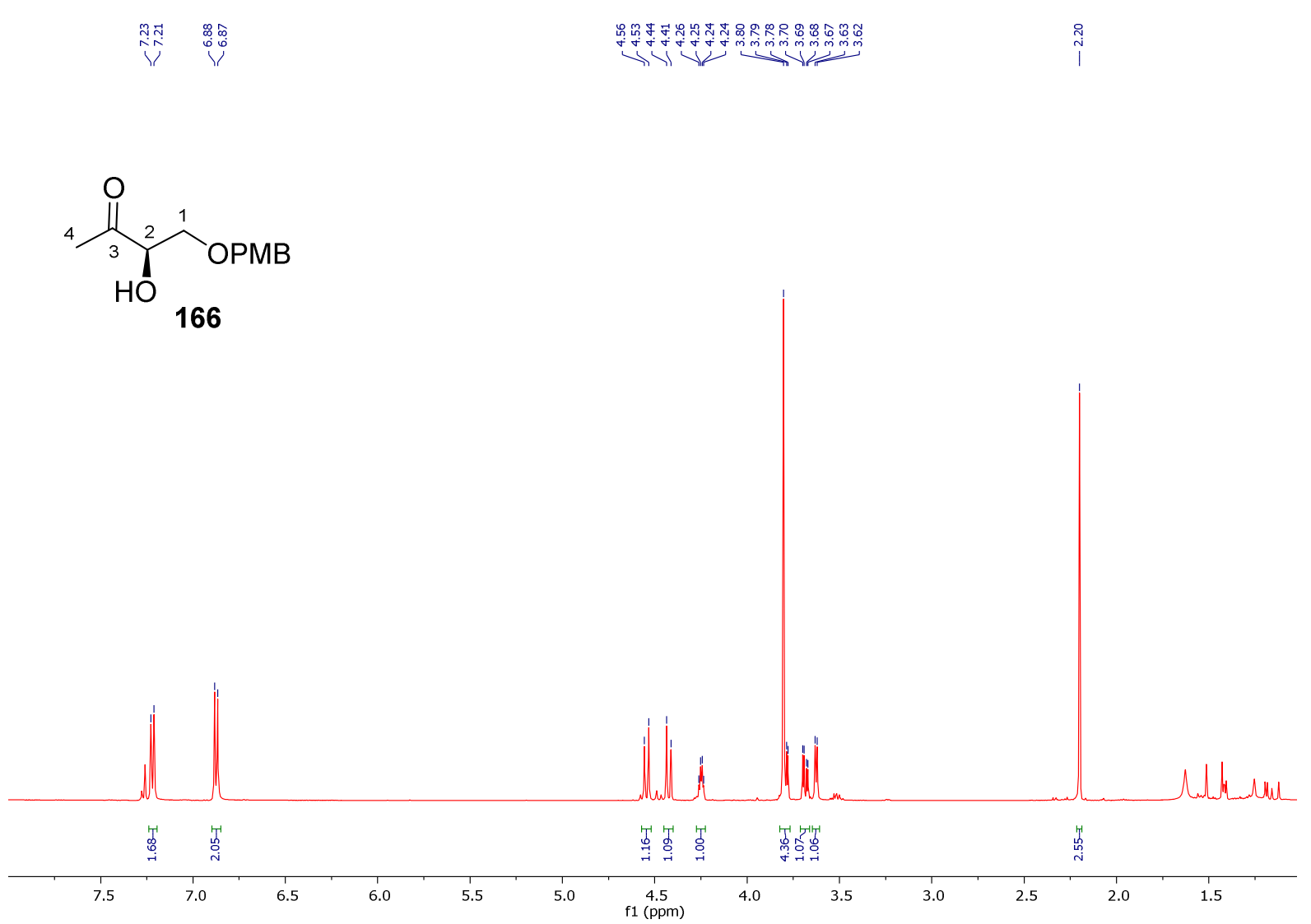

Figure 4.5: ${ }^{1} \mathrm{H}$ NMR spectrum of the by-product 166

Even though by-product $\mathbf{1 6 6}$ was not consistently produced in the reaction, on the occasion when it was isolated, it accounted for $43 \%$ of the conversion of starting material iodide $\mathbf{7 4 .}$ 
Previous observations with primary alkyl-iodides showed that, when the $S_{N} 2$ reaction site is hindered, a dithiane-initiated SET type reaction can take place. ${ }^{171}$ Based on this, the following mechanism for the formation of 166 was proposed (Scheme 4.14), although the corresponding heterolytic process is also plausible.
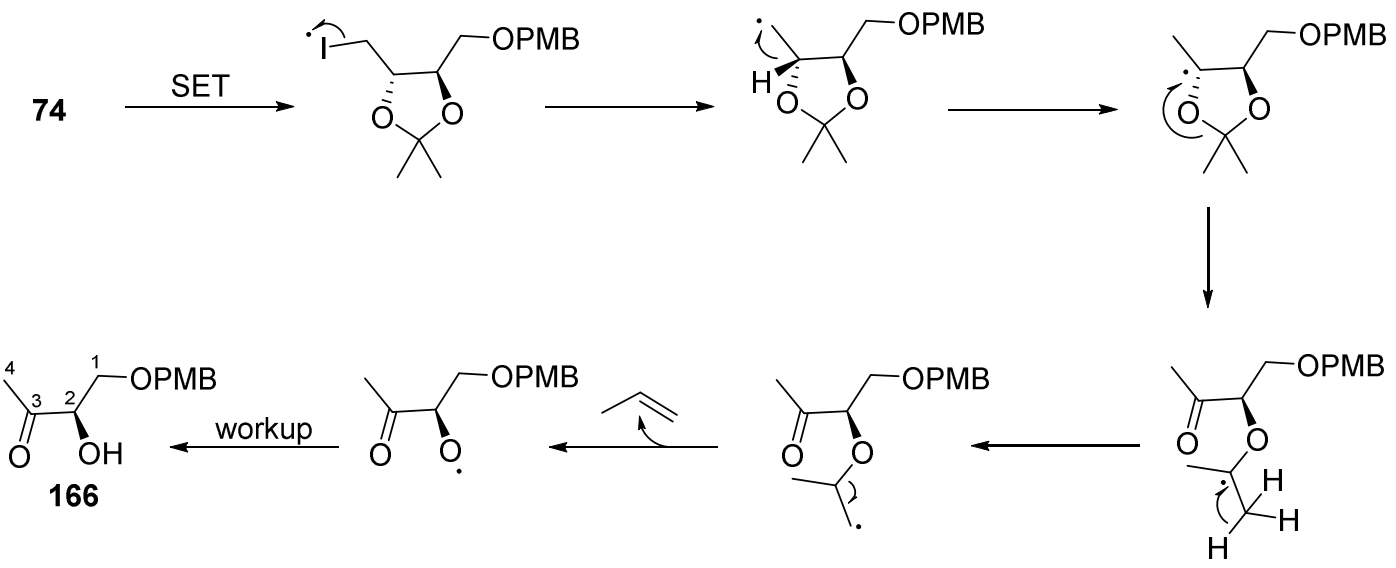

Scheme 4.14: Proposed mechanism for the synthesis of by-product 166

The coupling reactions of the 1,3-dithiane with bromide 164, both with and without TBAI, were unsuccessful and only the peaks corresponding to the two starting materials were observed in the ${ }^{1} \mathrm{H}$ NMR spectrum of the crude reaction mixtures. The tert-butyldiphenylsilyl (TBDPS) 01 protected iodide $(\mathbf{1 6 8}$, Scheme $\mathbf{4 . 1 5})$ was also synthesised. Mono-TBDPS protection of $\mathbf{3 2}$ to give $\mathbf{1 6 7}$ was followed by an Appel reaction to give the iodide $\mathbf{1 6 8}$ in $\mathbf{7 2 \%}$ yield over the two steps. However, the dithiane coupling with 168 gave the dithiane 169 only in trace amounts.

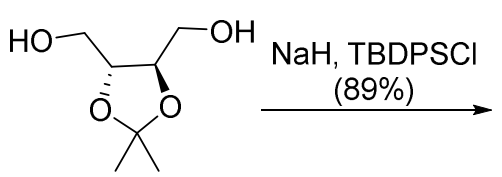

32
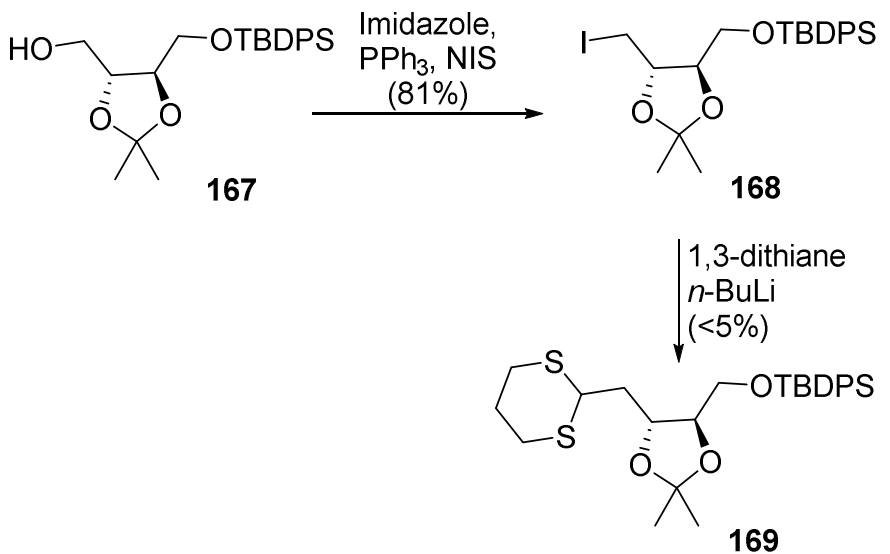
Despite the poor yield of the dithiane homologation reaction, the small quantity of the dithiane (165) obtained was used to evaluate the subsequent steps (Scheme 4.16). The acetonide protecting group had to be removed first and this was achieved under mild acidic conditions using $p$-toluenesulfonic acid in methanol. The diol 170 (not shown, Experimental) was then subjected to methylation using Meerwein's salt. ${ }^{2,144}$ Only a single product was obtained from this reaction in $86 \%$ yield. While the methylation occurred regioselectively, it was unfortunately $\mathrm{O} 2$ that was methylated (171). This was confirmed through a COSY correlation between the $\mathrm{OH}$ proton $(\delta 2.63)$ and the $\mathrm{H} 3$ oxymethine $(\delta 4.04)$, and the latter correlated with the $\mathrm{H} 2$ proton at $3.22 \mathrm{ppm}$. Additionally, oxymethine $\mathrm{H} 2$ correlated to the $\mathrm{H} 1$ methylene protons ( $\delta 3.62$ and 3.55). The COSY correlations could also be followed from the $\mathrm{H} 3$ oxymethine to the $\mathrm{H} 5$ oxymethine. It was postulated that simply by changing the order of the two reactions (MOM protection first, then methylation), it would be possible to obtain the correct fragment. However, due to the failure to further optimise the dithiane coupling reaction this postulate was not tested and the subsequent steps were explored on the $\mathrm{O} 2$ methylated product of this reaction.

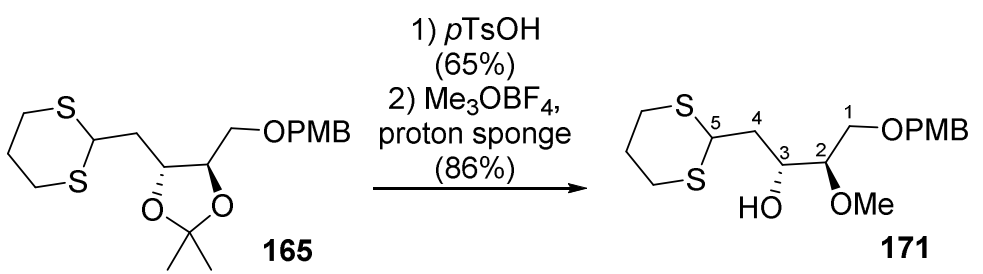

Scheme 4.16: Regioselectivity of the methylation to give the $\mathrm{O} 2$ methylated product 171

The alcohol in 171 was then protected as MOM acetal $(\mathbf{1 7 2}$, Scheme $\mathbf{4 . 1 7})$ and the crude reaction material was used in the next step without purification. An attempt to allylate the resulting dithiane was unsuccessful and the starting material 172 was recovered in $49 \%$ yield as the only identifiable product. 


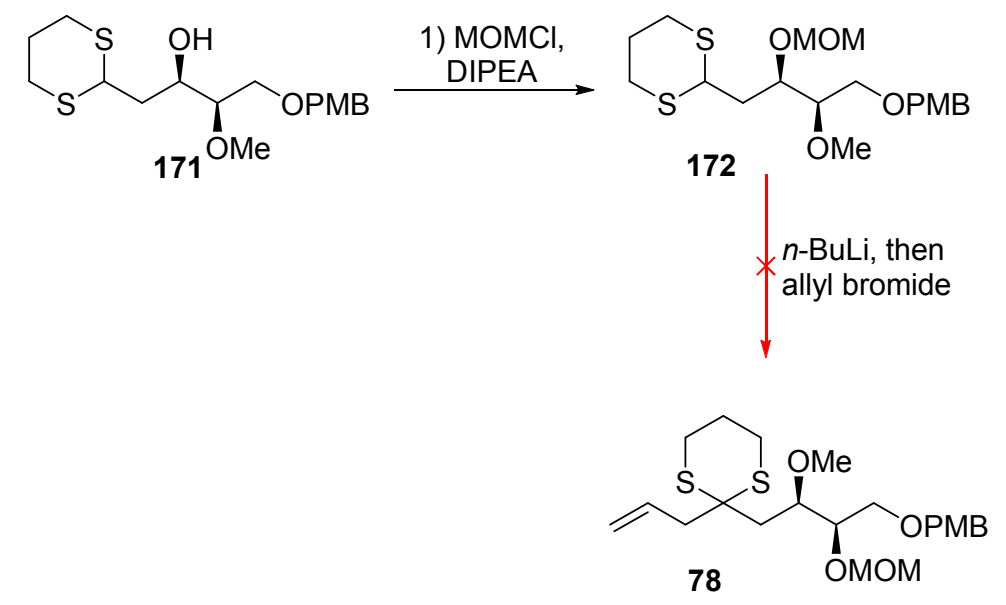

Scheme 4.17: Attempted synthesis of the dithiane $\mathbf{7 8}$

However, the allylation was only attempted once, and the chemistry was not explored further as this route was abandoned due to the poor yielding coupling reaction with 1,3dithiane.

\subsubsection{Wittig homologation}

Given the lack of success with the dithiane homologation, it was decided to attempt a Wittig olefination to form an enol ether as a masked aldehyde (Scheme 4.18). This change necessitated some alterations to the overall synthetic pathway. After mono-protection of the diol to give 167, the alcohol was to be oxidised to the aldehyde, which would then undergo olefination with the (methoxymethyl)triphenylphosphonium ylide to afford 173 . This sequence was tried with the mono-TBDPS alcohol 167 in order to investigate the effect of an even bulkier Pg on the selectivity of the methylation reaction. After the removal of the methyl enol ether protecting group, the resulting aldehyde $\mathbf{1 7 4}$ would be allylated in a stereoselective manner using a Brown allylation (175), as this reaction had already been successfully employed by Ghosh (Scheme 4.3). ${ }^{68,69,72}$ The acetonide would be removed after the C5 hydroxyl was protected. It was decided to keep the same protecting group at 05 (TBS) but the change in strategy implied that the acetonide would have to be cleaved in the presence of this TBS ether. Here we relied on the secondary TBS to be more stable to mild acidic conditions than the acetonide. The diol would again be subjected to methylation using Meerwein's salt ${ }^{144}$ and the selectivity in this step would direct the order of methylation/MOM protection steps. This plan for the synthesis of the intermediate $\mathbf{1 7 6}$ had the same number of steps as the original allyl dithiane route. 

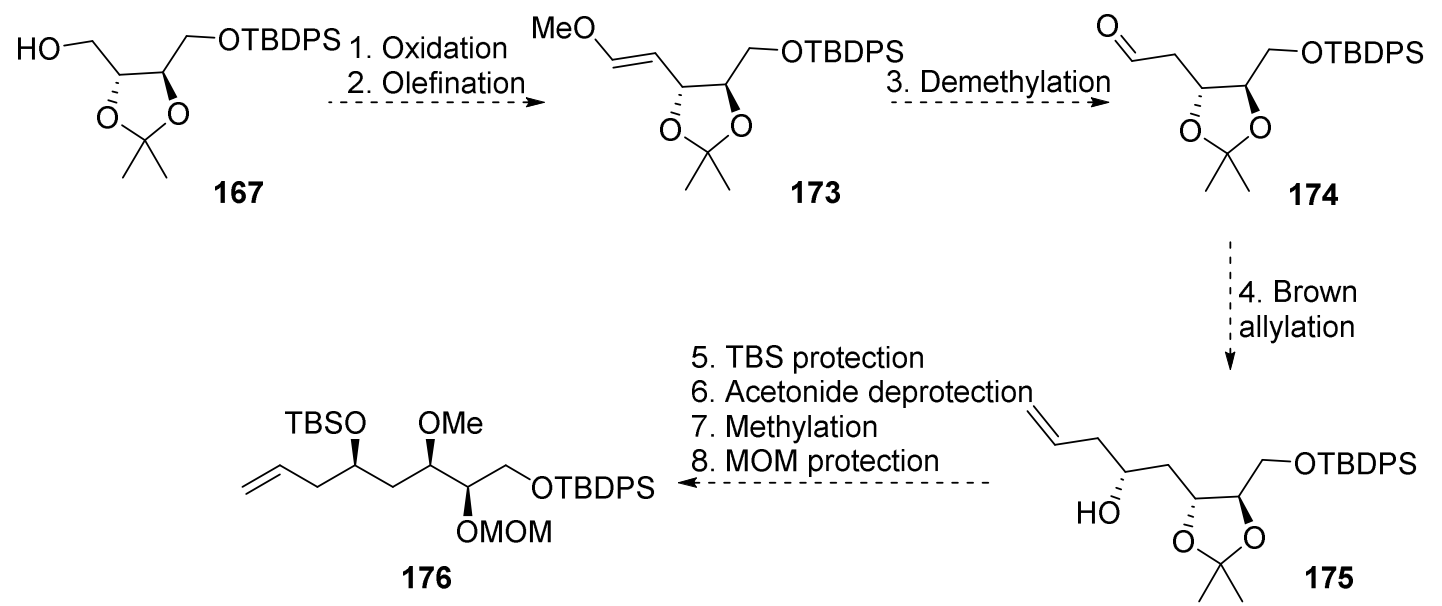

Scheme 4.18: Synthesis of the C1-C7 intermediate 176 through the Wittig homologation

The Wittig reaction was discovered and subsequently developed by Georg Wittig in 1950s. ${ }^{172,173}$ Early on, a two-step mechanism involving formation of a betaine was postulated. ${ }^{174}$ However, it is now widely believed the formation of oxaphosphetane occurs as the direct result of a $2+2$ cycloaddition reaction between a phosphonium ylide and an aldehyde or ketone (Scheme 4.19). ${ }^{174,175}$ The oxaphosphetane then rearranges to give the olefin product and triphenylphosphine oxide.

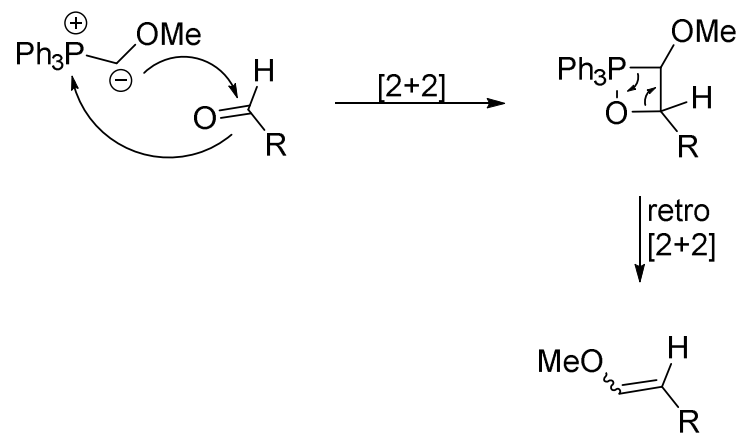

Scheme 4.19: Mechanism of the Wittig reaction using the (methoxymethyl)triphenylphosphonium bromide

In accordance with the new plan, the alcohol 167 was oxidised using Dess-Martin periodinane $^{107}$ and then reacted without purification with the preformed phosphonium ylide (Scheme 4.20) derived from (methoxymethyl)triphenylphosphonium bromide in a MOM-Wittig olefination reaction. ${ }^{176,177}$ 


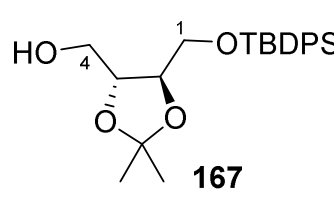

167

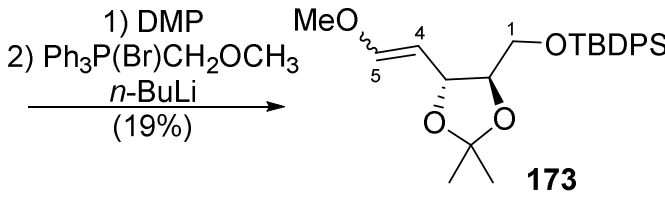

$Z: E 3: 2$

Scheme 4.20: Synthesis of the enol methyl ether 173

The product (173) was obtained as a mixture of geometrical isomers with a 3:2 ratio of $Z$ and $E$ isomers. The ${ }^{1} \mathrm{H}$ NMR (Figure 4.6) showed two sets of olefinic protons: Peaks at $6.56 \mathrm{ppm}$ $(\mathrm{H} 5)$ and $4.70 \mathrm{ppm}(\mathrm{H} 4)$ with coupling constants of $12.9 \mathrm{~Hz}$ were assigned as belonging to the $E$ isomer, while those at $6.10 \mathrm{ppm}(\mathrm{H} 5)$ and $4.43 \mathrm{ppm}(\mathrm{H} 4)$ with a coupling of $6.2 \mathrm{~Hz}$ were determined to belong to the $Z$ isomer. Protons at the electron-rich, end of the double bond $(\mathrm{H} 4)$ had typically low alkene chemical shifts $(4.70$ and $4.43 \mathrm{ppm}$ ) resulting from the increased shielding due to resonance electron-donation. Two methyl ether singlets were also present in the spectrum at $\delta 3.59$ for the $Z$ isomer (assigned through HMBC correlation with the $Z C 5$ carbon at $150.9 \mathrm{ppm}$ ) and at $\delta 3.55$ for the $E$ isomer (assigned through $\mathrm{HMBC}$ correlation with the E C5 peak at $152.3 \mathrm{ppm})$. Some of the other proton environments were also doubled (Experimental). 


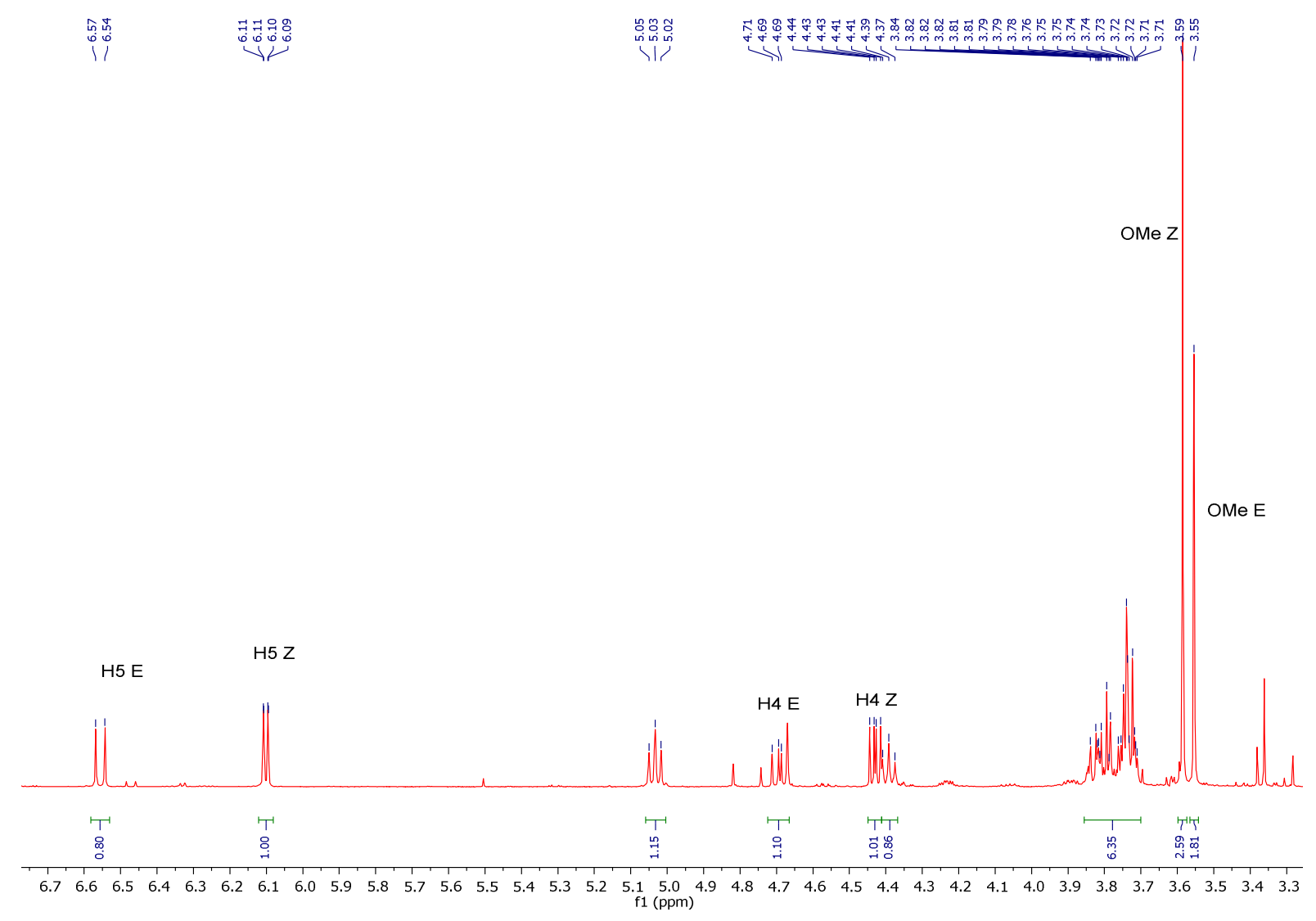

Figure 4.6: Part of the ${ }^{1}$ H NMR spectrum of the methyl enol ether 173

The yield of the reaction was low (19\% over two steps) but optimisation was planned after establishing whether removal of the methyl enol ether protecting group in the presence of the acetonide was possible. This was anticipated to be difficult to achieve as both groups are acid labile.

Treatment of the methyl enol ether 173 with CSA (0.13 equiv.) in acetone at $0-4{ }^{\circ} \mathrm{C}$ overnight gave the $\alpha, \beta$-unsaturated aldehyde $\mathbf{1 7 7}$ as the only identifiable product (Scheme 4.21) instead of the desired acetonide-containing product.
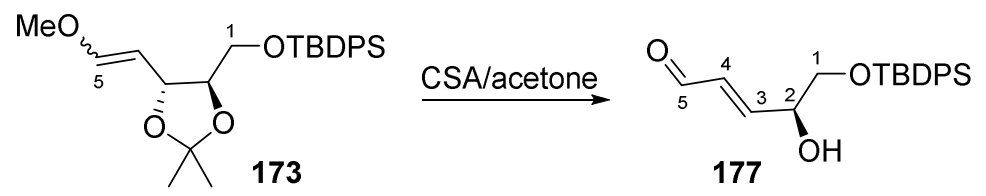

Scheme 4.21: Methyl enol ether conversion into aldehyde 177

The ${ }^{1} \mathrm{H}$ NMR spectrum (Figure 4.7) showed the aldehyde proton peak as a doublet at 9.52 $\operatorname{ppm}(J=7.8 \mathrm{~Hz}, \mathrm{H} 5)$. In the COSY spectrum this proton correlated to an olefinic proton at $\delta$ $6.37 \mathrm{ppm}(\mathrm{H} 4)$, a doublet of doublets of doublets $(J=15.7,7.8,1.8 \mathrm{~Hz})$, which had a 
correlation to another olefinic proton at $6.68 \mathrm{ppm}(\mathrm{dd}, J=15.7,4.2 \mathrm{~Hz}, \mathrm{H}-3)$. The ${ }^{3} J_{\mathrm{HH}}$ coupling constant for the two olefinic protons was $15.7 \mathrm{~Hz}$, suggesting an E-geometry of the double bond, as would be expected. The $\mathrm{H} 2$ oxymethine at $4.50 \mathrm{ppm}$ had COSY correlations to the olefinic proton at $6.68 \mathrm{ppm}$, the methylene protons at $3.82(\mathrm{H} 1 \mathrm{a})$ and $3.64 \mathrm{ppm}$ ( $\mathrm{H} 1 \mathrm{~b})$, as well as to a proton doublet at $2.71 \mathrm{ppm}$, assigned as the $\mathrm{OH}$ proton. Both phenyl and methyl proton peaks of the TBDPS protecting group were also present in the ${ }^{1} \mathrm{H}$ NMR spectrum.

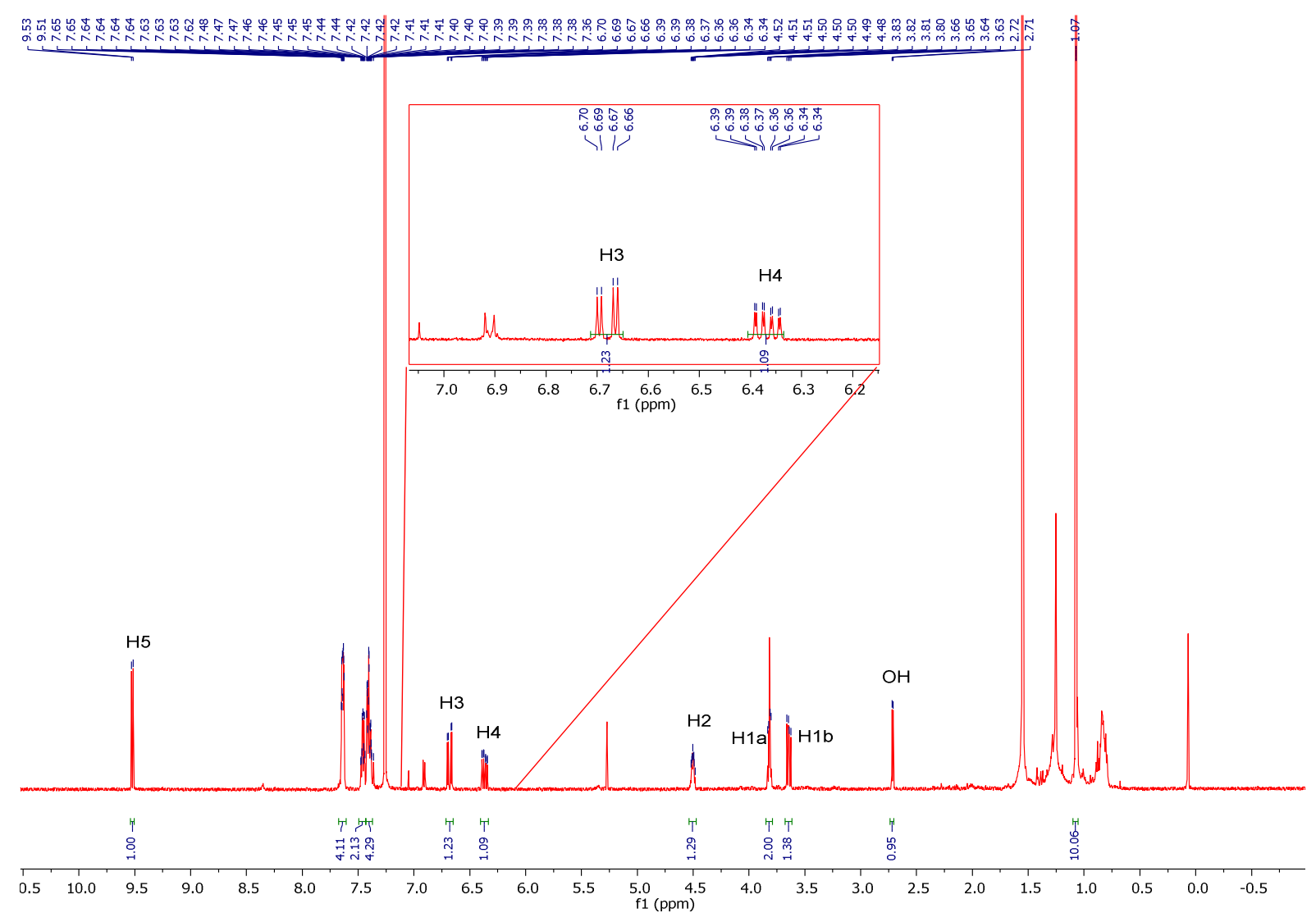

Figure 4.7: ${ }^{1} \mathrm{H}$ NMR spectrum of the aldehyde 177

The proposed mechanism (Scheme 4.22) involves the protonation of the acetonide oxygen that drives the formation of the aldehyde and the elimination of acetone to give the observed product 177. 


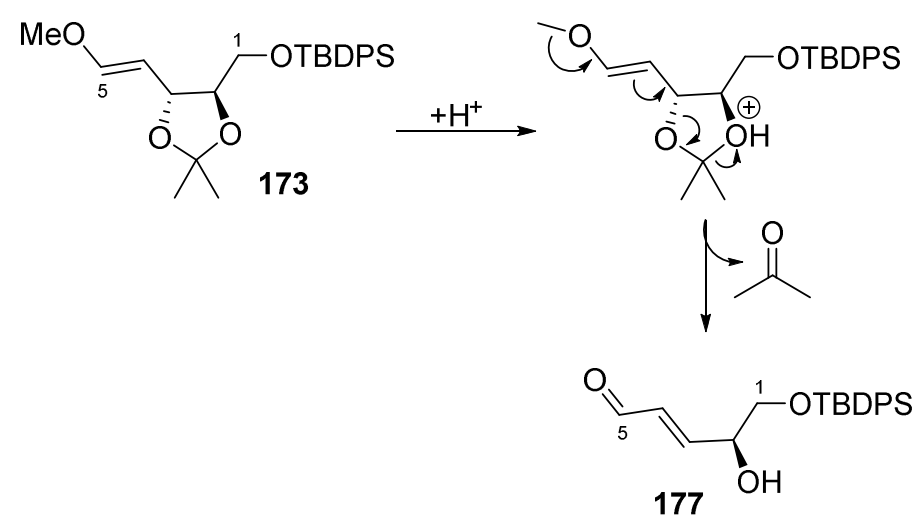

Scheme 4.22: The proposed mechanism for the formation of 177

An elimination reaction to give the $\alpha, \beta$-unsaturated aldehyde was previously reported for a similar methyl enol ether substrate by Du et al. ${ }^{178}$ They circumvented the elimination sidereaction by modifying their mercury acetate $\left(\mathrm{Hg}(\mathrm{OAc})_{2}\right)$ hydrolysis procedure by lowering the reaction temperature $\left(0^{\circ} \mathrm{C}\right)$ and buffering the reaction with $8 \% \mathrm{KI}$ solution. ${ }^{178}$ As an aim of this project was to produce a scalable approach employing non-toxic reagents wherever possible, the mercury acetate hydrolysis of the methyl enol ether $\mathbf{1 7 3}$ was not attempted.

A subsequent attempt to remove the methyl ether using $10 \%$ aq. $\mathrm{HCl} / \mathrm{MeOH}(1: 15 \mathrm{v} / \mathrm{v})$ resulted in the formation of the same $\alpha, \beta$-unsaturated aldehyde (177). Lastly, methyl cleavage was tried using trimethylsilyl iodide (TMSI), formed in situ from $\mathrm{Nal}$ and $\mathrm{TMSCl}$ in 10:1 v/v CH $3 \mathrm{CN}_{3} / \mathrm{H}_{2} \mathrm{O}$, to remove the methyl ether. ${ }^{179,180}$ These conditions led to degradation of the starting material.

\subsubsection{Ohira-Bestmann modification of the Seyferth-Gilbert homologation}

Because of the shortcomings of the methyl enol ether route, a different synthetic plan had to be devised (Scheme 4.23). This involved a different one-carbon homologation where the alcohol would again be oxidised, and the aldehyde converted into an alkyne using the OhiraBestman reagent in a Seyferth-Gilbert homologation reaction. ${ }^{181,182}$ The acetonide protecting group would then be removed and the selective $\mathrm{O} 3$ methylation of the diol evaluated. After selective methylation/MOM protection is achieved, a hydroboration/oxidation sequence on the alkyne should give the $C 5$ aldehyde. Then, the Brown allylation can be undertaken and the resulting C5 hydroxyl protected as a silyl ether. $^{68,69,72}$ 
<smiles>CC1(C)OC(CO)C(COP)O1</smiles>

1. Oxidation 2. Ohira-Bestman<smiles>C#CC1OC(C)(C)OC1COP</smiles>

3. Acetonide deprotection $\rightarrow$

$\mathrm{HO} \quad \mathrm{OH}$

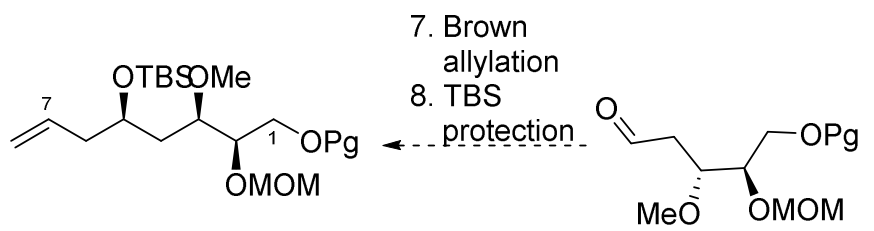
4. Methylation 5. MOM protection

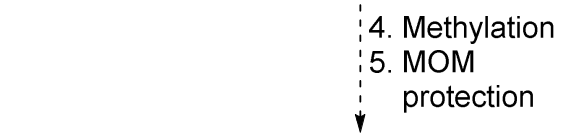

6. Hydroboration/ oxidation

Scheme 4.23: Synthesis of the C1-C7 intermediate through Seyferth-Gilbert homologation

The development of what is now known as the Seyferth-Gilbert homologation began in 1971 when Seyferth et al. ${ }^{183}$ published the synthesis and reactivity of a dimethylphosphonosubstituted diazoalkane, dimethyl $\alpha$-diazomethylphosphonate $\left(\mathbf{1 7 8}\right.$, Scheme 4.24a). ${ }^{183}$ This was followed by the work of Colvin and Hamill in 1973 and 1977 that showed the alkynes can be obtained by reacting aryl aldehydes and ketones with the anion generated upon treatment of dimethyl $\alpha$-diazomethylphosphonate (178) with base ( $n$-BuLi or $t$-BuOK). ${ }^{184,185}$ In 1982, Gilbert et al. ${ }^{186}$ expanded on the work of Colvin and Hamill and provided a mechanistic insight into the nature of this homologation reaction. ${ }^{186}$ They postulated that the reaction proceeds through a HWE-type transformation with an initial nucleophilic addition of the carbanion to the electrophilic carbonyl carbon (Scheme 4.24a). After the elimination of nitrogen, the resulting carbene spontaneously rearranges to give the alkyne. However, this reaction was limited to aldehydes and ketones with aryl groups. The first example of a non-aromatic aldehyde undergoing homologation to the alkyne came from Ohira in 1989. ${ }^{182}$ He employed dimethyl-1-diazo-2-oxopropylphosphonate (179) that under basic conditions underwent acyl cleavage resulting in the in situ formation of the anionic species (Scheme $\mathbf{4 . 2 4 b}$ ). The reaction was further explored by Bestmann et al. during late 1990s and early 2000s, and became known as the Ohira-Bestmann modification of the Seyferth-Gilbert homologation. ${ }^{181,187}$ 
a)

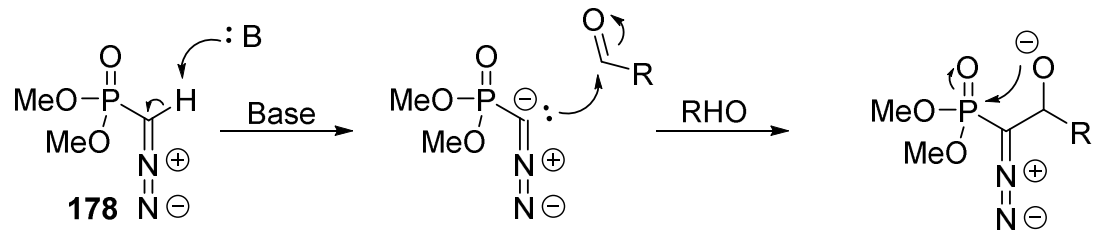

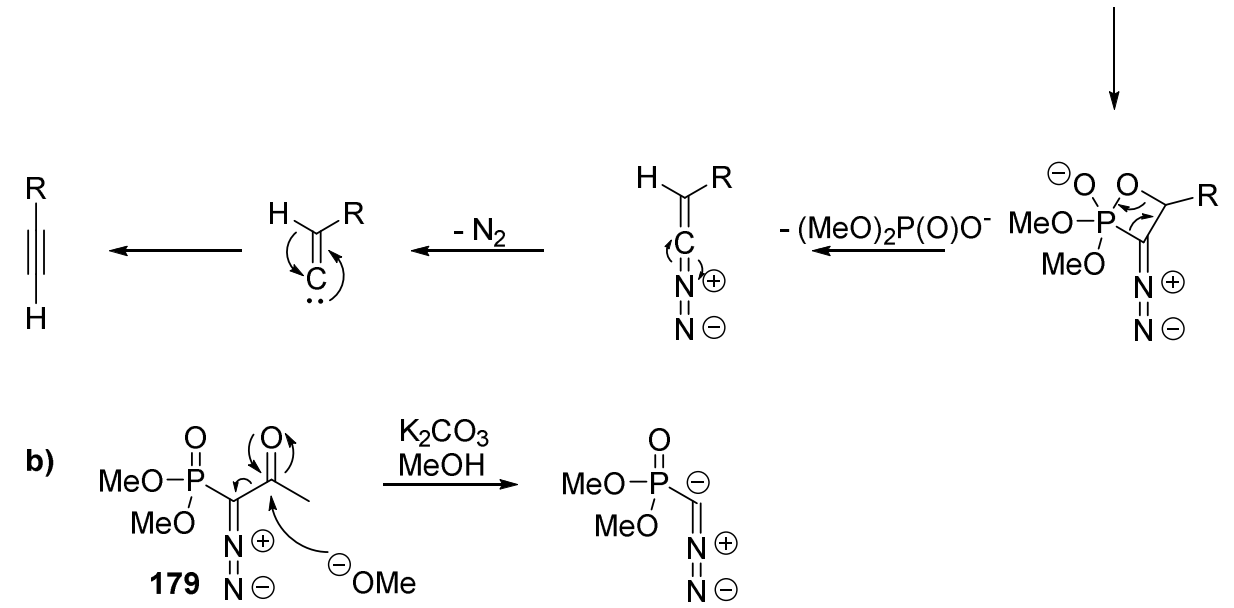

Scheme 4.24: a) Seyferth-Gilbert homologation mechanism; b) the Ohira-Bestmann reagent

The Seyferth-Gilbert reaction was tried on both the TBDPS and PMB protected starting materials 167 and 73 (Scheme 4.25). These alcohols were oxidised using Dess-Martin periodinane, then the resulting aldehydes reacted with Ohira-Bestmann reagent 179 under basic conditions. ${ }^{187}$ The reactions were consistently and similarly low yielding, with alkyne yields of $34 \%$ for 180 and $29 \%$ for $\mathbf{1 8 1}$. The ${ }^{1} \mathrm{H}$ NMR spectra showed the alkyne proton peaks as doublets at $2.53 \mathrm{ppm}$ in $\mathbf{1 8 0}$ and $2.52 \mathrm{ppm}$ in $\mathbf{1 8 1}$. The sp carbons in the ${ }^{13} \mathrm{C}$ NMR spectra were present at $74.6 \mathrm{ppm}$ (C5) and 81.4 ppm (C4) for 180 and at $74.8 \mathrm{ppm}$ (C5) and $80.9 \mathrm{pp}$ (C4) for 181.<smiles>CC1(C)OC(CO[SbH2])[C@H](CO)O1</smiles><smiles>CCCOC[C@H]1OC(C)(C)O[C@@H]1CO</smiles>

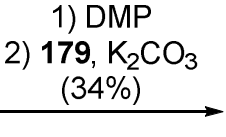

1) $\mathrm{DMP}$

2) $179, \mathrm{~K}_{2} \mathrm{CO}_{3}$ $(29 \%)$<smiles>C#C[C@H]1OC(C)(C)O[C@@H]1CO[PbH2]</smiles>

180<smiles>C#C[C@H]1OC(C)(C)O[C@@H]1C(C)O[Mg]</smiles>

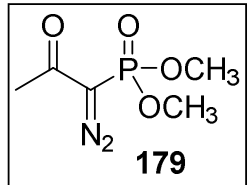

181

Scheme 4.25: The synthesis of alkynes 180 and 181

The next step, deprotection of the 2,3-acetonide, worked better with the TBDPS protecting group, probably due to PMB being less stable under the acidic deprotection conditions. 
Accordingly, it was decided to continue investigations with the diol $\mathbf{1 8 2}$. The removal of the acetonide was achieved using $80 \%$ acetic acid in water to give the diol in $58 \%$ yield (Scheme 4.26).

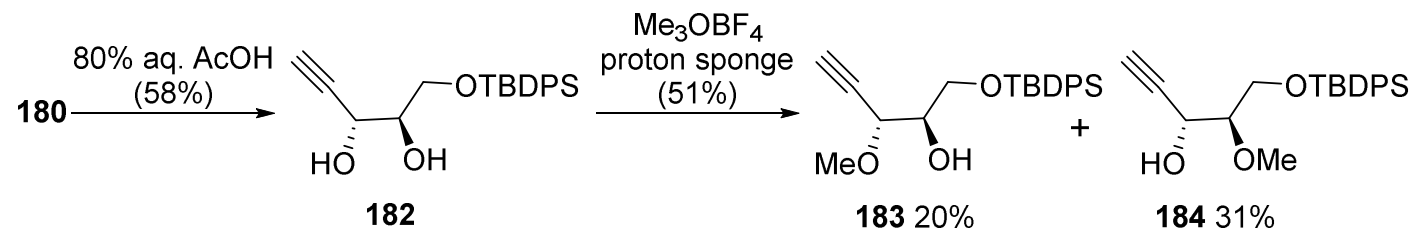

Scheme 4.26: The synthesis of the O-methylated products 183 and 184

The diol was then reacted with the methylating agent (Meerwein's salt, 1.5 equiv.) in the presence of proton sponge. The desired $\mathrm{O} 3$ and the undesired $\mathrm{O} 2$ methylated products, 183 and 184 , were obtained in a $0.7: 1$ ratio respectively. COSY was used to assign the two oxymethine protons ( $\mathrm{H} 2$ and $\mathrm{H} 3$ ) and the respective carbons were assigned from the HSQC correlations. Then, the HMBC correlations from the methyl protons of the methoxy group to either C2 or C3 (3 bonds apart) were used to determine whether the methylation occurred at $\mathrm{O} 2$ or $\mathrm{O} 3$. The corresponding reverse $\mathrm{HMBC}$ correlations (from either $\mathrm{H} 2$ or $\mathrm{H} 3$ to the carbon of the methoxy) were also observed. A TBDPS protecting group is usually considered significantly more bulky than an alkyne, yet here, methylation of the $\mathrm{O} 2$ is slightly favoured over the methylation of the 03 . To rationalise the selectivity of the reaction, conformational modelling of the starting material was performed by Dr Muhammad Ali Hashmi (at the time a fellow PhD student). He performed relaxed scans of the dihedral angles using Gaussian $09^{188}$ in pairs of two dihedrals using all the possible combinations of the dihedral angles in 24 steps of $15^{\circ}$ increments. ${ }^{189}$ Then, after selecting the lowest energy structures from all the scans, those structures were optimised without any constraints at the PBEO-D3BJ/def2-SVP level of theory, followed by the removal of duplicates. This generated eight lowest energy conformers of the diol 182 (Table 4.1) that represented nearly 100\% of the Boltzmann distribution. From his analysis, in the lowest energy conformation one of the phenyl groups of TBDPS is oriented towards the C3 hydroxyl group, apparently blocking the hydroxyl group sterically, whereas the $\mathrm{O} 2$ is significantly less hindered and thus more likely to react (Figure 4.8). This conformation accounted for $64 \%$ of the Boltzmann distribution. The next two conformations (Figure 4.9 and Figure 4.10), accounting together for $30 \%$ of the Boltzmann distribution, show the TBDPS phenyl ring away from the $\mathrm{O} 3$ leaving it free to react but the 
$\mathrm{O} 2$ is still relatively unhindered as well. These results together appear to explain the experimentally observed selectivity of the methylation reaction displaying preferential reactivity of $\mathrm{O} 2$ compared to $\mathrm{O} 3$.

Table 4.1:Boltzmann percentage of different conformers of the diol 182

\begin{tabular}{c|c|c|c|c|c} 
S.No. & $\begin{array}{c}\text { File/Conformers } \\
\text { Name }\end{array}$ & Abs. E (Hartree) & $\begin{array}{c}\text { Rel. E } \\
\text { (kcal/mol) }\end{array}$ & $\begin{array}{c}\text { Boltzmann } \\
\text { Factor }\end{array}$ & $\begin{array}{c}\text { Boltzmann } \\
\% \text { age }\end{array}$ \\
\hline 1 & AR-conf-01 & -1328.440603 & 0.00 & 1.0000 & 63.71 \\
\hline 2 & AR-conf-02 & -1328.439674 & 0.58 & 0.3738 & 23.82 \\
\hline 3 & AR-conf-03 & -1328.438398 & 1.38 & 0.0968 & 6.17 \\
\hline 4 & AR-conf-04 & -1328.437771 & 1.78 & 0.0498 & 3.17 \\
\hline 5 & AR-conf-05 & -1328.436845 & 2.36 & 0.0187 & 1.19 \\
\hline 6 & AR-conf-06 & -1328.436801 & 2.39 & 0.0178 & 1.14 \\
\hline 7 & AR-conf-07 & -1328.436474 & 2.59 & 0.0126 & 0.80 \\
\hline 8 & AR-conf-08 & -1328.431519 & 5.70 & 0.0001 & 0.00
\end{tabular}

$(\mathrm{T}=298.15 \mathrm{~K} ; \mathrm{k}($ in kCal$/ \mathrm{molK})=0.0019872041 ; \mathrm{kT}=0.592484902 ;$ partition function $\mathrm{Q}=1.5696)$
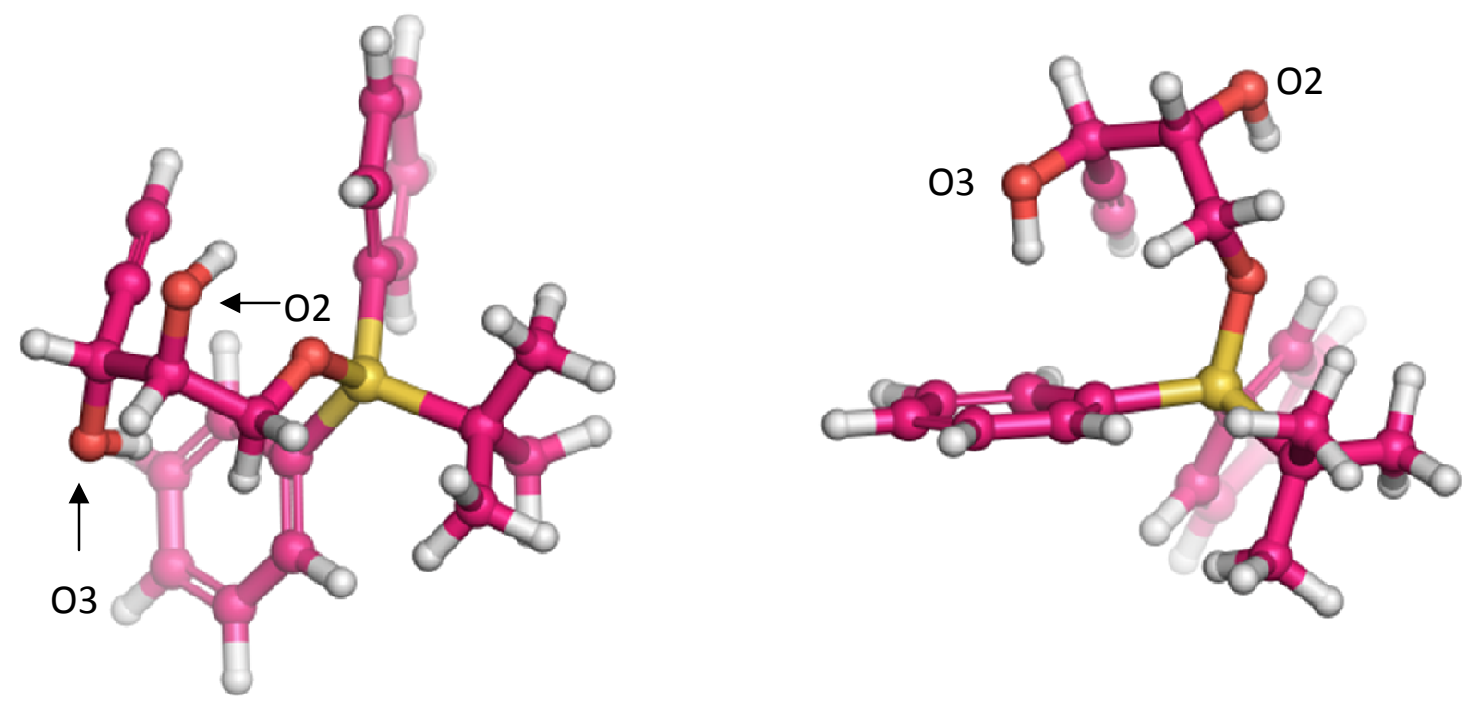

Figure 4.8: The lowest energy conformer of the diol 182 (conformer 1) 

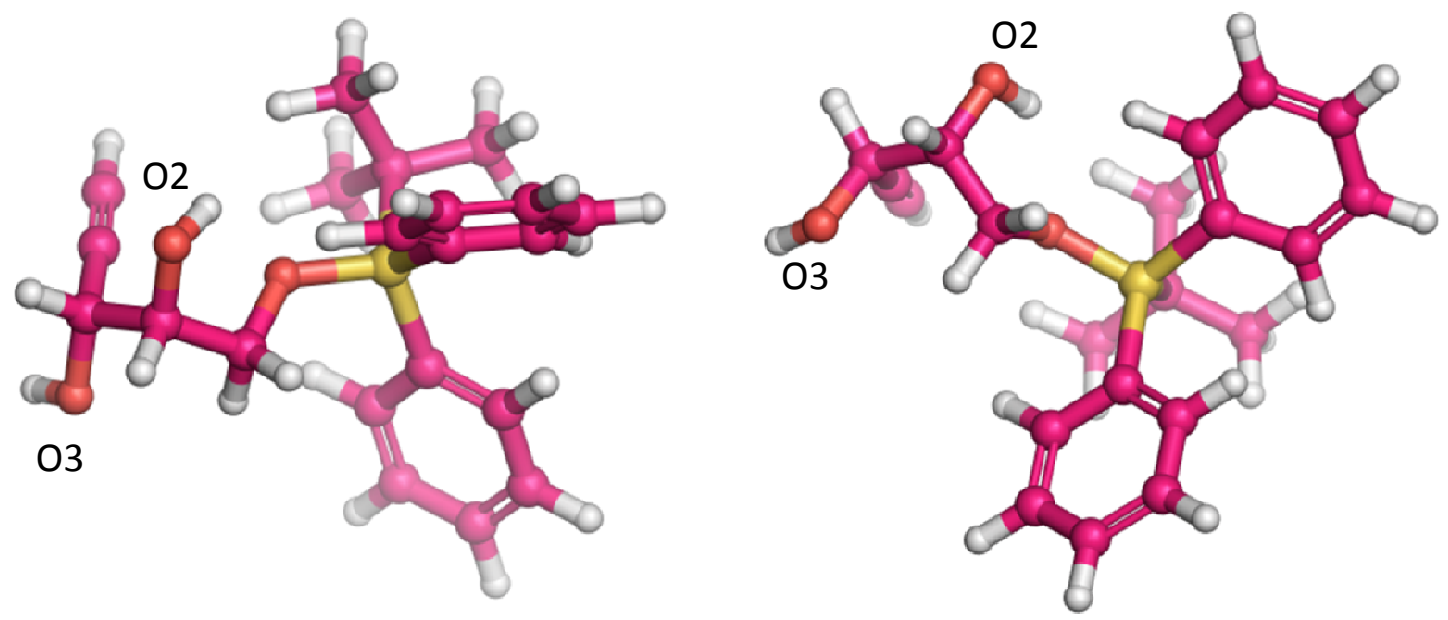

Figure 4.9: Conformer 2 of the diol 182

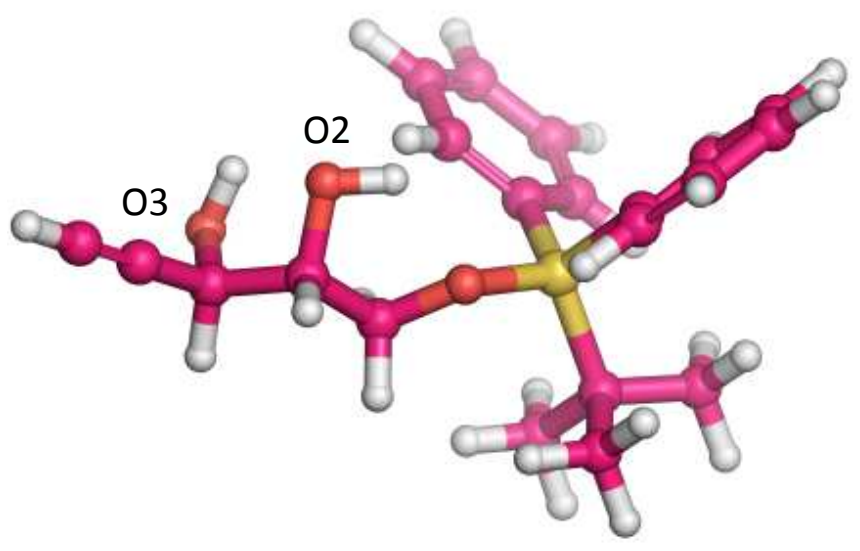

Figure 4.10: Conformer 3 of the diol 182

The desired $\mathrm{O} 3$ methylated product 183 underwent MOM protection of the $\mathrm{O} 2$ hydroxyl to give the alkyne 185 in $27 \%$ yield on a small scale (Scheme 4.27). Then the hydroboration/oxidation sequence was attempted (Scheme 4.27) to obtain the C5 aldehyde 
186. A solution of bicyclo(3.3.1)nonane (9-BBN 187, $0.5 \mathrm{M}$ in THF) was added to the starting material $\mathbf{1 8 5}$ in $\mathrm{THF}$ at $0{ }^{\circ} \mathrm{C}$ and the reaction allowed to proceed at room temperature overnight. ${ }^{190}$ At that point, TLC analysis indicated there was still a substantial amount of starting material present, but a more polar spot was also visible. It was therefore decided to continue with the oxidation step $\left(1: 1 \mathrm{v} / \mathrm{v} 30 \%\right.$ aq. $\mathrm{H}_{2} \mathrm{O}_{2} / 20 \%$ aq. $\left.\mathrm{NaOH}\right)$ and determine whether the compound corresponding to the new TLC spot would oxidise to the desired aldehyde. Unfortunately, the ${ }^{1} \mathrm{H}$ NMR spectrum of the crude reaction mixture did not show any peaks in the aldehyde region and only starting material peaks were identifiable in the complex spectrum.

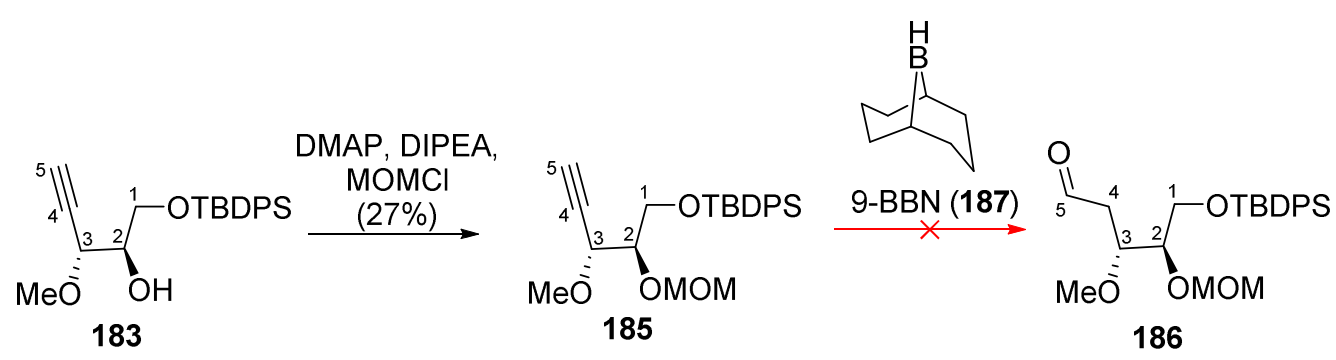

Scheme 4.27: The attempted synthesis of the C5 aldehyde 186

The hydroboration/oxidation was chosen because it should proceed with the desired antiMarkovnikov regioselectivity and would give the enol product (Scheme 4.28) that would tautomerise to the aldehyde. ${ }^{191}$ Therefore, this reaction sequence should result in the desired oxygenation at the C5 carbon. Usually with alkynes, dialkyl boranes with bulky alkyl substituents, such as 9-BBN (187), are used to prevent a second addition of the borane to the alkenylborane intermediate. ${ }^{192}$

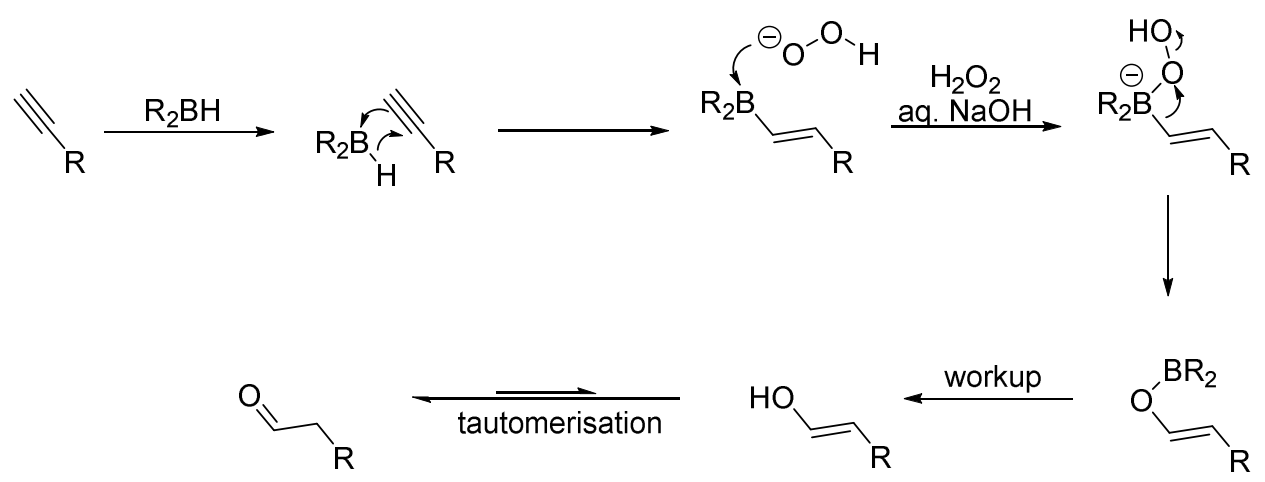

Scheme 4.28: Alkyne hydroboration/oxidation mechanism

The hydroboration/oxidation was further explored on the acetonide protected compounds 180 and 181 (Scheme 4.30). No product was observed when alkyne 180 was reacted with 
the 9-BBN dimer ${ }^{193}$ but a trace amount of aldehyde 188 (aldehydic proton triplet at 9.79 ppm, Experimental) was observed when 9-BBN monomer as a solution in THF (1.0 equiv.) was used at room temperature (Scheme 4.30a). All the efforts to optimise the reaction, such as increasing the number of equivalents of the borane ( 2 equiv.) and the reaction temperature (reflux in THF), did not improve the reaction yield.

a)

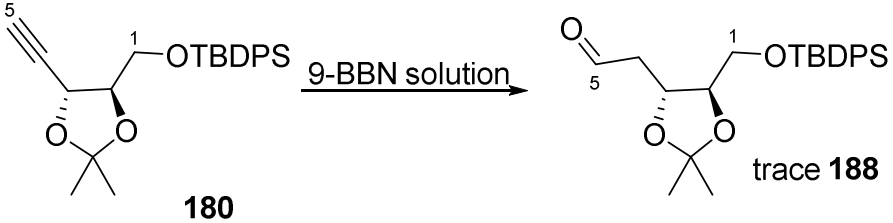

b)

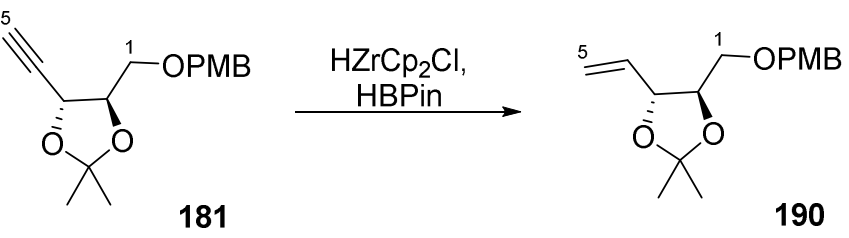

c)

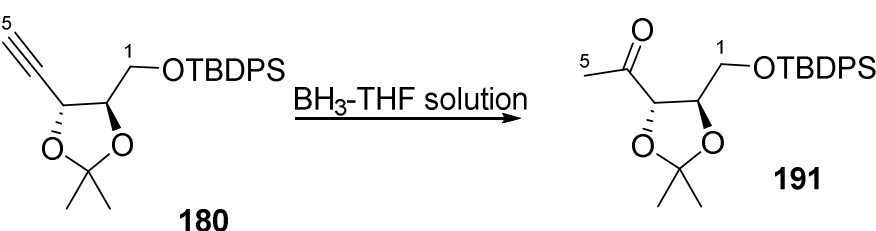

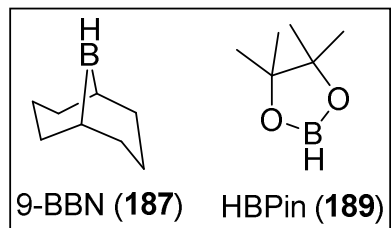

Scheme 4.29: Results from hydroboration/oxidation attempts using different boranes

For the oxidation step it was important to add the hydroboration reaction mixture to the basic solution of $\mathrm{H}_{2} \mathrm{O}_{2}$ as the reverse addition resulted in protonolysis. The protonolysis product (192) was present in the ${ }^{1} \mathrm{H}$ NMR spectrum (Figure 4.11) of the sample containing the recovered alkyne starting material (180) isolated after the first attempt at 9-BBN hydroboration. The two compounds have very similar $R_{f}$ values (0.64 in 10:1 PE/EA) and could not be separated by silica gel chromatography. The alkyne to olefin ratio in the sample was 1:0.6 (based on the relative integration of their $\mathrm{H} 3$ protons). The identification of the protonolysis product was based on the presence of the three olefinic proton peaks at $\delta 5.84$ 
(ddd, $J=17.2,10.3,6.9 \mathrm{~Hz}, 1 \mathrm{H}), 5.33(\mathrm{dt}, J=17.2,1.3 \mathrm{~Hz}, 1 \mathrm{H}$ ) and $5.21(\mathrm{ddd}, J=10.4,1.5,0.9$ $\mathrm{Hz}, 1 \mathrm{H})$. Based on the chemical shifts, the number of olefinic protons and the literature examples of protonolysis of alkylboranes occuring under these conditions, ${ }^{191}$ we were confident the observed by-product was the terminal olefin 192.

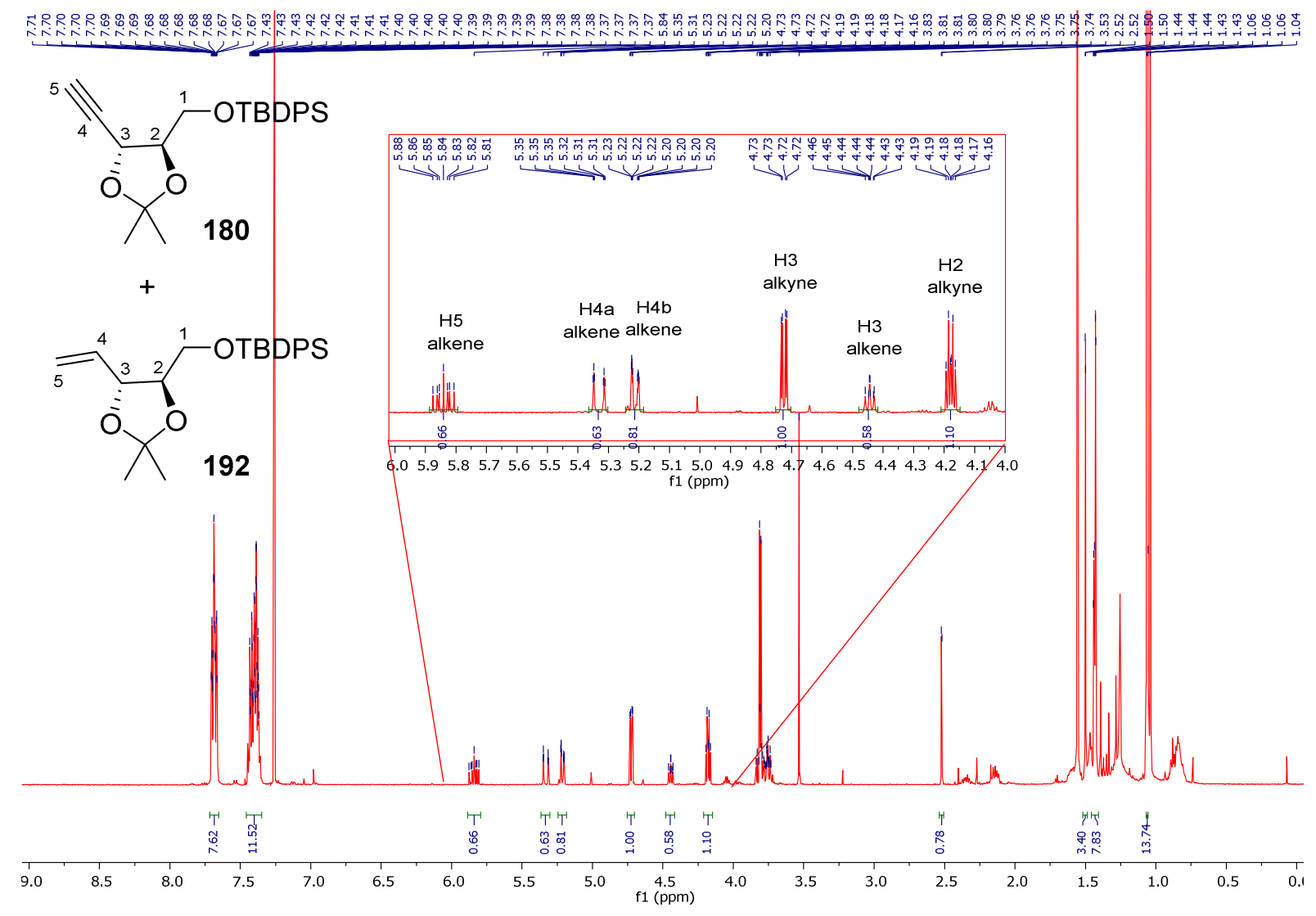

Figure 4.11: ${ }^{1} \mathrm{H}$ NMR spectrum containing alkyne starting material 180 and the protonolysis by-product 192

The reaction of 9-BBN with 01 PMB protected alkyne 181 resulted in full recovery of the starting material. Given the lack of reactivity between the alkynes 180 and 181 and 9-BBN, it was also attempted to make a pinacol boronic ester of $\mathbf{1 8 1}$. Thus, the starting material 181 was first reacted with bis(cyclopentadienyl)zirconium(IV) chloride hydride that was made in situ from bis(cyclopentadienyl)zirconium(IV)dichloride and DIBAL-H. ${ }^{194,195}$ The alkylzirconium intermediate would then undergo transmetallation when treated with pinacolborane (189). However, after the aqueous quench and chromatographic purification, only the protonolysis product was recovered $(190$, Scheme $4.29 b)$. Suspecting that the size of the alkyl substituents on these boranes is the reason for the observed lack of reactivity, hydroboration with $\mathrm{BH}_{3} \cdot \mathrm{THF}$ complex (0.35 equiv), was also attempted. The borane was added to the alkyne at $-78{ }^{\circ} \mathrm{C}$ and the reaction kept at this temperature for 1.5 hours at 
which point TLC analysis showed only the starting material spot. The reaction was then allowed to warm to $0{ }^{\circ} \mathrm{C}$ over 4.5 hours and kept at this temperature for another hour. During the course of warm up, a more polar spot started appearing on the TLC plate. After the oxidation step, only one new spot was present on the TLC plate (along with the unreacted starting material) with an $\mathrm{R}_{f}$ value of 0.56 (10:1 PE/EA) similar to the previously isolated aldehyde $(0.57,10: 1 \mathrm{PE} / \mathrm{EA})$. Interestingly, in the ${ }^{1} \mathrm{H}$ NMR spectrum of the isolated product, there was no aldehyde proton peak at $9.79 \mathrm{ppm}$. Instead, there was a new methyl peak at $2.27 \mathrm{ppm}$. It was predicted that the isolated product was a methyl ketone 191 (Scheme 4.29c). The structure of 191 was established by the presence of a ketone carbonyl carbon at $208.7 \mathrm{ppm}$ in the ${ }^{13} \mathrm{C}$ NMR spectrum and a HMBC correlation between the methyl protons ( $\delta$ 2.27) and this carbon, as well as the carbon at 81.6 assigned as C3. This result indicated that the reaction had proceeded with unexpected Markovinkov regioselectivity. The reasons for the observed Markovnikov regioselectivity are unclear. With that said, a possible explanation is that a combination of electronic and steric interactions between the alkyne and the aromatic groups on the TBDPS protecting group are making the alkyne end terminus less accessible and perhaps more electron deficient than the internal terminus and therefore more likely to receive the hydride from the borane. It is also possible that when bulkier boranes were used, this effect became secondary to the steric effect imposed by the borane alkyl substituents, so that no ketone product was ever observed. However, the terminus of the alkyne, still subjected to the same effects, remained unreactive, which is why only trace amount of aldehyde was only ever obtained.

\subsubsection{Johnson-Corey-Chaykovsky reaction}

In parallel with the hydroboration attempts, another type of one-carbon homologation was also explored with the PMB protected alcohol 73. The Johnson-Corey-Chaykovsky reaction is a homologation that proceeds through formation of an epoxide. ${ }^{196}$ The reaction mechanism is somewhat similar to that of the Wittig reaction and the related modifications (Scheme 4.30). ${ }^{197}$ Here, aldehyde is reacted with a preformed trimethylsulfonium ylide resulting in formation of a betaine. ${ }^{197}$ Unlike phosphonium ylide reactions that result in the formation of oxaphosphetane, the sulfonium acts as a leaving group, resulting in the formation of the epoxide. The epoxide formation was to be followed by the Meinwald rearrangement ${ }^{198}$ 
(Scheme 4.31), where the activation of the epoxide under acidic/Lewis acidic conditions leads to a 1,2-hydride shift to give the aldehyde.

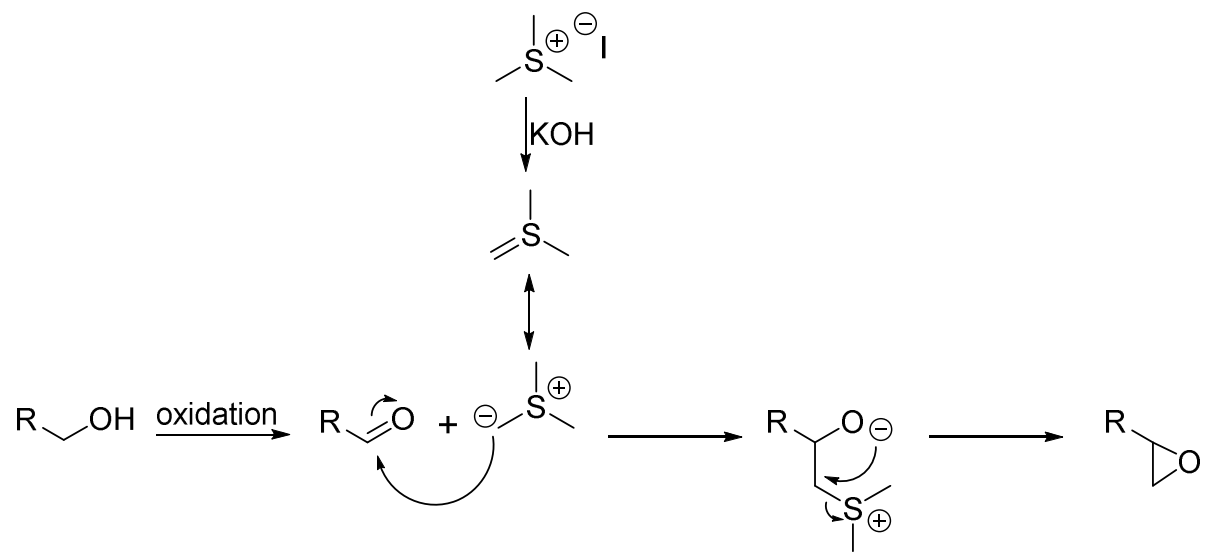

Scheme 4.30: Johnson-Corey-Chaykovsky reaction

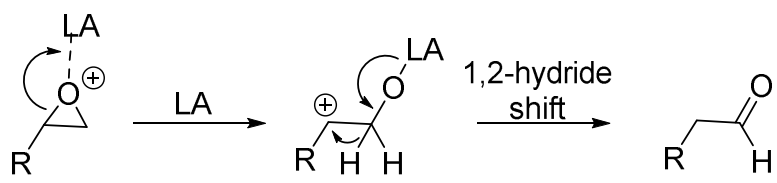

Scheme 4.31: Meinwald rearrangement mechanism

The reaction of the aldehyde derived from $\mathbf{7 3}$ gave the epoxide 193 in $7 \%$ yield over two steps $(7.8 \mathrm{mg})$, as a mixture of isomers (Scheme 4.32). It was postulated that the excess of base (6 equiv. $\mathrm{KOH}$ ) that was adopted from the literature procedure was probably unnecessary and responsible for the low yield. ${ }^{199,200}$ Before optimisation of the epoxidation, it was necessary to establish the feasibility of the next step, Meinwald rearrangement, on this acid-sensitive material. It was anticipated that the acetonide would be labile under most of the conditions usually required for the rearrangement (acidic/Lewis acidic). The mildest established method was tried first, namely stirring the starting material in EtOAc with $\mathrm{SiO}_{2}$. However, no rearrangement to the $\mathrm{C} 5$ aldehyde 194 occurred after 1.5 hours at room temperature. ${ }^{201}$ The same lack of reactivity was observed when the rearrangement was attempted using acidic Amberlite resin in a mixture of EtOAc/Acetone (2:1) at room temperature for 2 hours. When $10 \%$ aq. $\mathrm{HCl}$ was added to the Amberlite reaction mixture, a second, more polar spot appeared after $15 \mathrm{~min}$ at room temperature. The reaction was neutralised with $\mathrm{NaHCO}_{3}$ powder after 25 min and a ${ }^{1} \mathrm{H}$ NMR spectrum of the crude reaction mixture obtained. However, no aldehyde peak was present in the spectrum and the epoxide 193 (as a mixture of isomers) was still the major species. Due to the lack of an aldehyde 
peak and the small scale of the reaction, the crude reaction mixture was not subjected to chromatographic purification. Both CSA (in acetone) and $\mathrm{TiCl}_{4} \cdot 2 \mathrm{THF}$ complex (in DCM) led to the removal of the acetonide. Because of the previously observed susceptibility of the acetonide to other acidic conditions this methodology was not pursued further.
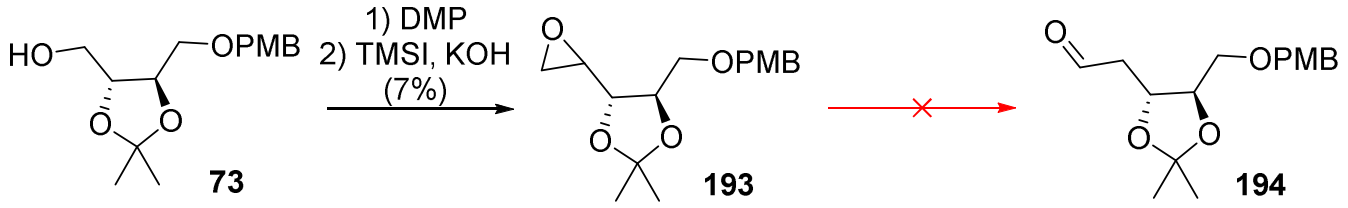

Scheme 4.32: Synthesis of the epoxide 193 and the attempted rearrangement to the aldehyde 194

Throughout all of these attempts at one-carbon homologations/oxidations (dithiane, methyl enol ether, alkyne and epoxidation), many insurmountable issues were encountered. leading us to abandon further exploration of the threitol-based route and consider a different approach to the synthesis of the $\mathrm{C} 1-\mathrm{C} 7$ fragments.

\subsection{Jacobsen-based approach: from cis-2,3-butenediol}

\subsubsection{The strategy}

The second synthetic plan to access the C1-C7 fragments was devised based on Jacobsen's methodology. ${ }^{3}$ Here, the C1-C4 epoxide intermediate 195 would be made from cis-2,3butenediol (Scheme 4.33) in 4 steps. This epoxide would be used for the synthesis of the C1-C7 intermediate 198 that would be derivatised, in the same manner as described before, into the two required $\mathrm{C} 1-\mathrm{C} 7$ fragments.

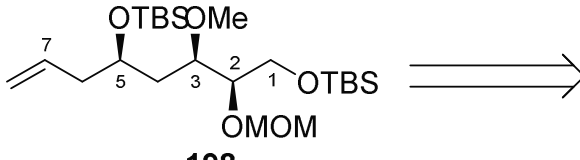

198
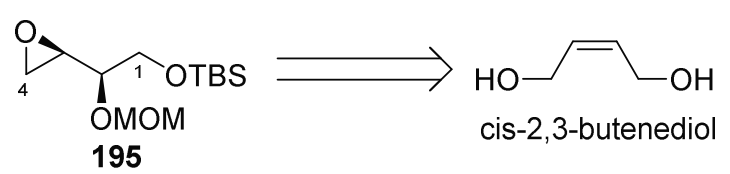

Scheme 4.33: Retrosynthetic analysis of the Jacobsen based plan for the synthesis of the C1-C7 intermediate 198

Ring opening of epoxide 195 with previously synthesised allyl dithiane (161) would be attempted (Scheme 4.34) in order to obtain dithiane 196. After O3 methylation the dithiane would be cleaved to reveal the C5 ketone (197). Then, a stereoselective reduction (CBS) should give the desired stereochemical configuration at C5. The last step would be the protection of the C5 alcohol with a TBS group to give the intermediate 198. The choice of 
protecting groups for $\mathrm{O} 1$ and $\mathrm{O} 5$ was also adopted from Jacobsen. The new synthesis required the same number of steps to complete (nine steps from cis-2,3-butenediol) as the original strategy.
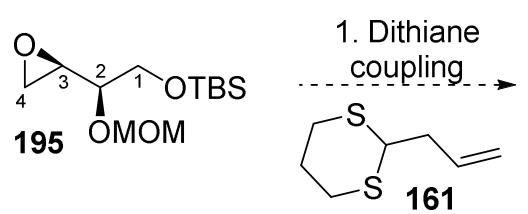

161

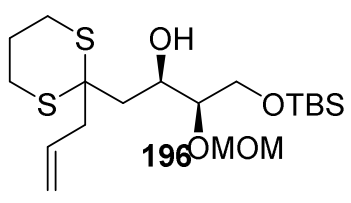

2. Methylation

3. Dithiane

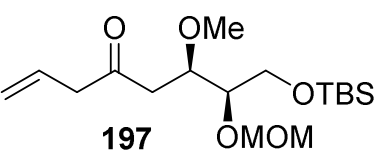

Scheme 4.34: Strategy for the synthesis of the C1-C7 intermediate 198 from Jacobsen's epoxide 195

However, changing the protecting group strategy led to further changes to the strategy for the side-chain. For the macrolactonsation route, having 01 TBS protecting group that needed to be removed selectively before the global deprotection, meant that the primary OTBS on the side-chain (O24) had to be replaced with an orthogonal protecting group, a benzyl ether (Chapter 2). Selective removal of the TBS from the primary hydroxyl in the presence of other acid-labile protecting groups had been already achieved successfully by Jacobsen. ${ }^{3}$

\subsubsection{The synthesis of $\mathrm{C1}-\mathrm{C} 4$ epoxide}

The synthesis of the C1-C4 epoxide started with a well-established epoxidation of the commercially available cis-2,3-butenediol using meta-chloroperbenzoic ( $m$ CPBA) acid to give the meso-epoxide 199 (Scheme 4.35). The purification of this epoxide, or, more specifically, separating it from the meta-chlorobenzoic acid by-product, was quite challenging. A common method involves dissolving the reaction mixture in diethyl ether and keeping it at $20^{\circ} \mathrm{C}$ for an hour, at which point the product should crystallise out leaving the benzoic acid to stay dissolved in solution. Subsequently, the resulting product and benzoic acid can be separated via filtration. It was, however, found that some of the benzoic acid was also crystallising out under those conditions and affecting the subsequent rearrangement step, therefore additional purification using silica gel chromatography (10\%-15\% MeOH in DCM) was necessary. ${ }^{202}$ This significantly improved the purity of the epoxide and improved the 
outcome of the next step. Because the epoxidation with $m$-CPBA is concerted the cis geometry is retained in the epoxide product (Scheme 4.36).

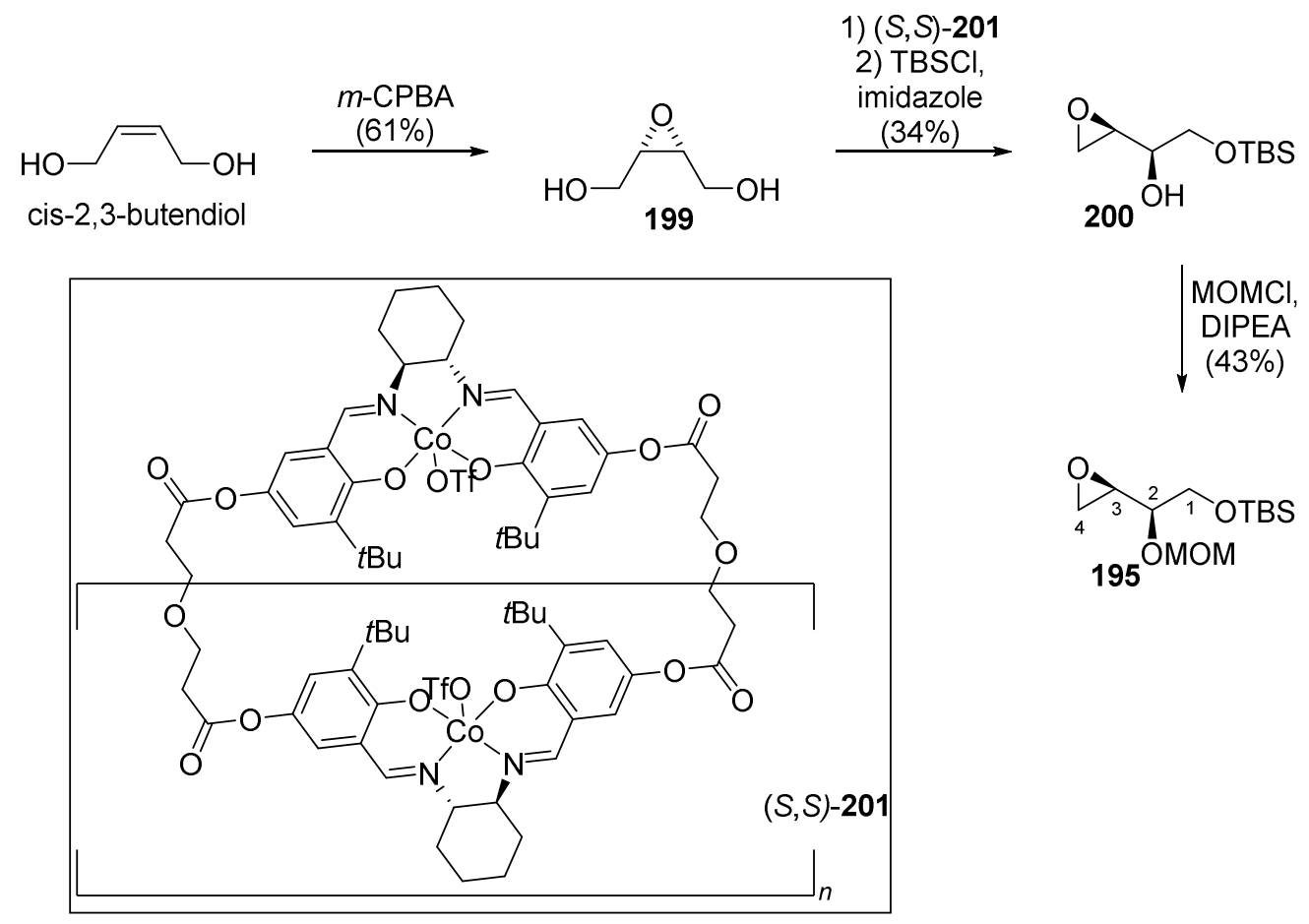

Scheme 4.35: The synthesis of the epoxide 195

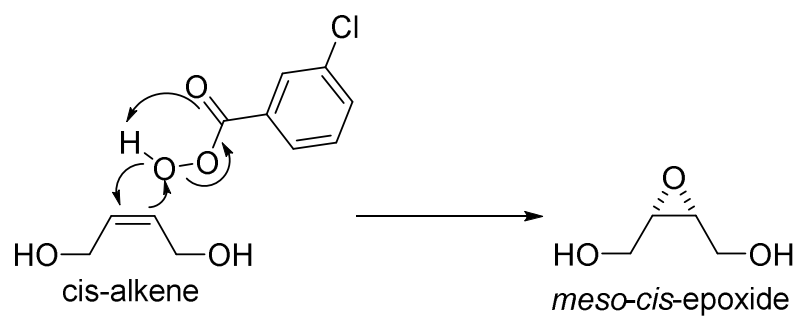

Scheme 4.36: $m$-CPBA epoxidation mechanism

The next step was the desymmetrisation of the meso-epoxide 199 through an enantioselective version of the Payne rearrangement ${ }^{3}$ that was followed by in situ TBS protection to give the epoxide 200 (Scheme 4.35). The Payne rearrangement is a baseinduced migration of the epoxide in 2,3-epoxy alcohols. ${ }^{203,204}$ The migration occurs in a stereospecific manner, resulting in the inversion of configuration at the C2 of the epoxide (Scheme 4.37a). ${ }^{203,204}$ Regio and enantioselective variants of the rearrangement have been developed by Jacobsen, where a chiral catalyst is used to activate both the electrophile (epoxide) and the nucleophile (alcohol). ${ }^{205}$ For our purpose, Jacobsen's oligomeric $(S, S)$ [(salen)Co"l] $O$ OTf catalyst 201 was synthesised by Prof. Peter Tyler (Ferrier Research Institute, 
VUW) according to the methodology published by Jacobsen in $2014 .{ }^{3,206}$ Due to the catalyst stereochemistry, the symmetric alcohol attacks the epoxide enantioselectively resulting in desymmetrisation (Scheme 4.37b). Despite the reaction only requiring a low catalyst loading of $0.02 \mathrm{~mol} \%$ (with respect to Co), due to the small reaction scale and the issues associated with accurately weighing the sub-milligram amounts, a catalyst loading of 3.5 mol\% was used instead. The catalyst activates the epoxide for the nucleophilic attack by the alcohol.

a)

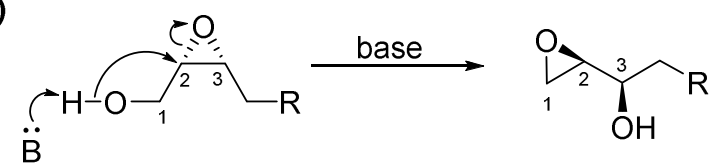

b)

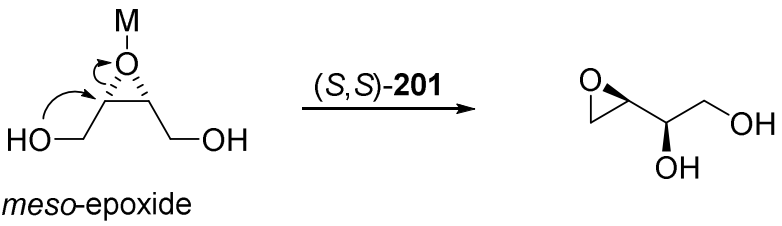

Scheme 4.37: a) the classic and b) the enantioselective versions of the Payne rearrangement

The rearrangement was allowed to proceed for about 14 hours, then the reaction was diluted with DMF and treated with TBSCl and imidazole. The TBS-protected product $\mathbf{2 0 0}$ was obtained in $38 \%$ yield after purification. The characteristic terminal epoxide proton chemical shifts were observed in the isolated product: $\mathrm{H} 3$ triplet of doublets at $3.09 \mathrm{ppm}(J=4.1,2.8$ $\mathrm{Hz})$ and $\mathrm{H} 4 \mathrm{a}, \mathrm{H} 4 \mathrm{~b}$ doublets of doublets at $2.78(J=5.0,4.2 \mathrm{~Hz})$ and $2.74 \mathrm{ppm}(J=5.1,2.8 \mathrm{~Hz})$, respectively. A correlation was observed in the COSY spectrum between the H3 proton and a proton at $3.70 \mathrm{ppm}$ assigned as the $\mathrm{H} 2$ oxymethine. The oxymethine peak at $\delta 3.70$ had a COSY correlation to a doublet at $2.39 \mathrm{ppm}$ assigned as the proton of the $\mathrm{C} 2 \mathrm{OH}$ group. This was confirmed by the absence of an HSQC correlation. Two methyl singlets at 0.89 and 0.07 ppm, integrating for 9 and 6 protons respectively, are consistent with a TBS group.

The reasons for the observed low yield are unclear. As mentioned earlier, it was observed that the reaction did not proceed if the starting material was contaminated with $\mathrm{m}$ chlorobenzoic acid. However, the chromatographic purification of the meso-epoxide starting material resolved this issue. Occasionally some mono and di-TBS protected 2,3-epoxide was isolated but not in sufficient quantity to account for the loss of mass. The subsequent step, MOM protection of the secondary hydroxyl also gave a relatively poor yield $(48 \%$, Scheme 
4.35). The optical rotation of the $O 2 \mathrm{MOM}$ protected compound $195+10.4\left(c 0.68, \mathrm{CHCl}_{3}\right)$, which matches the value reported by Jacobsen $\left(+10.4, c 1.3, \mathrm{CHCl}_{3}, 98 \%\right.$ e.e.). ${ }^{3}$

\subsubsection{Epoxide-opening attempts}

Despite the low yields of the reactions forming the epoxide 195, enough material was obtained to explore the dithiane chemistry (Scheme 4.38). The plan was to optimise the reactions once the methodology for preparing the C1-C7 intermediate (198) was fully established. The reactions of epoxides with 1,3-dithianes are well precedented in the literature ${ }^{160}$ as they represent an alternative to aldol chemistry. Additionally, our previous research on allyl dithiane (4.2.2.) showed that it can be successfully deprotonated with $n$ BuLi. There are also several reports of 2-allyl-1,3-dithiane being used to open an epoxide. ${ }^{165,207,208}$ Since the epoxides can be obtained in enantiomerically enriched form and their reaction with dithianes proceeds in a stereocontrolled manner, the resulting masked aldol product can be obtained with high enantiomeric purity. ${ }^{209}$

The deprotonation of the dithiane 161 was allowed to proceed for $45 \mathrm{~min}$ to $1 \mathrm{~h}$ and then the epoxide was added as a solution in THF at $-78^{\circ} \mathrm{C}$. The reaction was allowed to gradually warm up to $0{ }^{\circ} \mathrm{C}$. During that time the reaction was monitored by TLC analysis and only starting material spots were observed. ${ }^{1} \mathrm{H}$ NMR spectrum of the crude reaction mixture also showed only starting material peaks. After the purification, the epoxide (195) starting material was recovered ( $75 \%$ recovered yield).

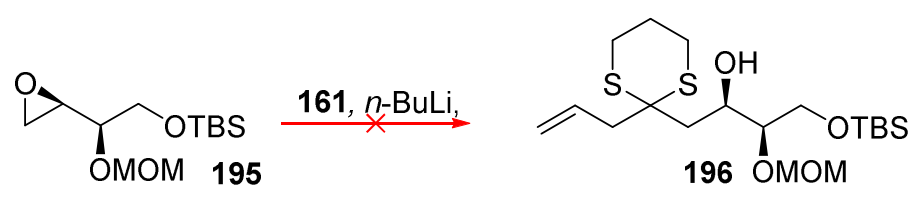

Scheme 4.38: Attempted synthesis of dithiane 196

As an alternative, a Smith-inspired linchpin reaction ${ }^{209}$ with the commercially available 2-(trimethylsilyl)-1,3-dithiane (TMS-dithiane 202, Scheme 4.39) and an allyl halide was also attempted. In these type of reactions, the epoxide is first reacted with the lithiated TMSdithiane, then the negative charge resulting from the epoxide opening is relayed, throughspace (via a 1,4-Brook rearrangement), back to the 2-position of the dithiane (Scheme 4.39). Typically, the first part of the reaction is performed in THF or ether, as these solvents suppress the rearrangement. A more polar solvent (HMPA or DMPU) is added after the first 
reaction has completed (usually after one hour) to facilitate the anion relay and, at the same time, the second electrophilic reagent is also added to the reaction.
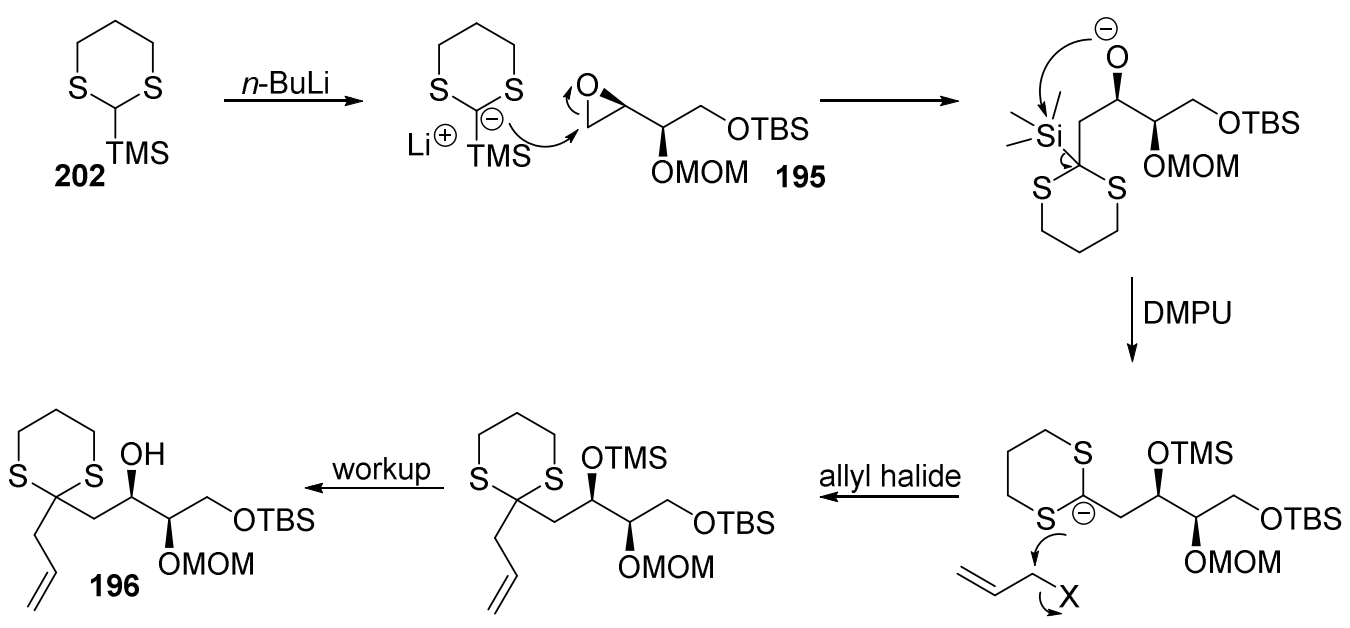

Scheme 4.39: Dithiane linchpin reaction mechanism

In accordance with this, 2-TMS-1,3-dithiane solution in dry diethyl ether was treated with $n$ BuLi at $0{ }^{\circ} \mathrm{C}$. The deprotonation was allowed to proceed for $20 \mathrm{~min}$ at room temperature after which the reaction was cooled to $-78{ }^{\circ} \mathrm{C}$ and a solution of the epoxide in ether added dropwise. The reaction was then allowed to warm up to $-30{ }^{\circ} \mathrm{C}$ and kept at this temperature for 1.5 hours. Then, the reaction temperature was lowered to $-40{ }^{\circ} \mathrm{C}$ and to the reaction added a solution of allyl bromide in 5:1 $\mathrm{Et}_{2} \mathrm{O} / \mathrm{DMPU}$. The reaction was allowed to warm up to room temperature gradually, then stirred for another 2 hours. Unfortunately, the dithiane-linchpin reaction did not give 196 and resulted only in a complicated mixture of dithiane related products that could not be entirely separated using silica chromatography. It was fairly obvious that these complex mixtures had no features related to the epoxide fragment (no TBS or MOM peaks) even though only $10 \%$ of the epoxide starting material (195) was recovered. Some of the products appeared to be singly and doubly allylated dithiane.

The low yields for the epoxide formation and the observed lack of reactivity of the epoxide made it necessary to change the synthetic strategy again. In order to save time, it was decided to use a previously published synthetic methodology to access the C1-C7 fragments and we chose Taylor's for the reasons explained in the next paragraph. 


\subsection{Taylor approach: from (S)-glycidyl tosylate}

\subsubsection{The strategy}

The appeal of Taylor's methodology is in its convergence, short sequence and step efficiency. The stereochemistry at C2 and C3 is introduced in a single step (Scheme 4.40), through an Evans aldol reaction, employing a chiral oxazolidinone auxiliary (Aux). ${ }^{4}$ Another advantage of this methodology is that the $\mathrm{C} 1$ oxazolidinone can be hydrolysed under basic conditions to give the acid directly, eliminating the need for oxidation steps, thus making the overall synthesis shorter.
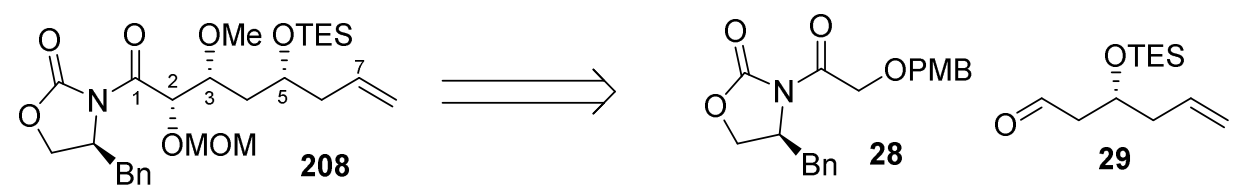

Scheme 4.40: Taylor's methodology for introducing the stereochemistry at C2 and C3 in a single step using an Evans aldol reaction

Following Taylor's methodology, the common intermediate 208 (Scheme 4.41) can be synthesised starting from commercially available (S)-glycidyl tosylate (Scheme 4.41) in eight steps. ${ }^{4}$ In a first approach to aldehyde $29,(S)$-glycidyl tosylate would first be reacted with dithiane and the resulting migrated epoxide (203) opened with a vinyl group to give the secondary alcohol 204. The resulting C5 hydroxyl would be protected as a triethylsilyl ether (205, not shown in this scheme). The dithiane would then be cleaved to reveal the aldehyde 29, which would react with oxazolidinone $\mathbf{2 8}$ in an Evans aldol reaction that will install the 2,3-syn stereochemistry in 206. 


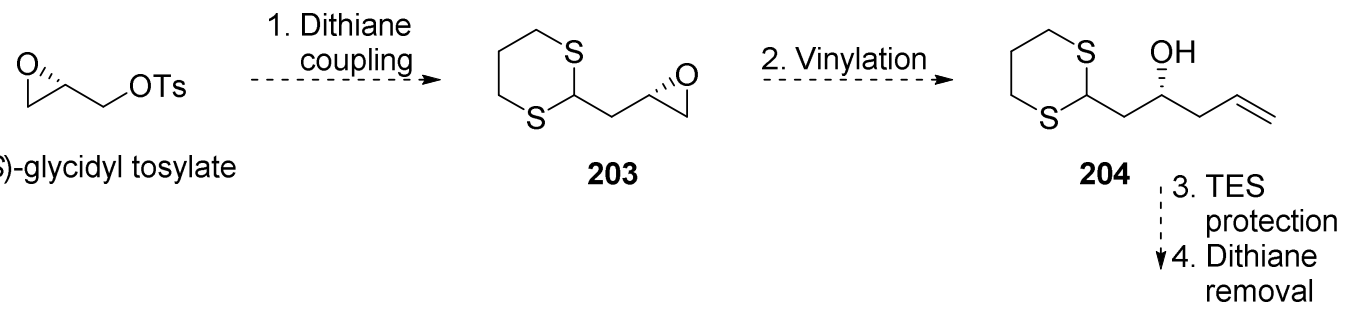

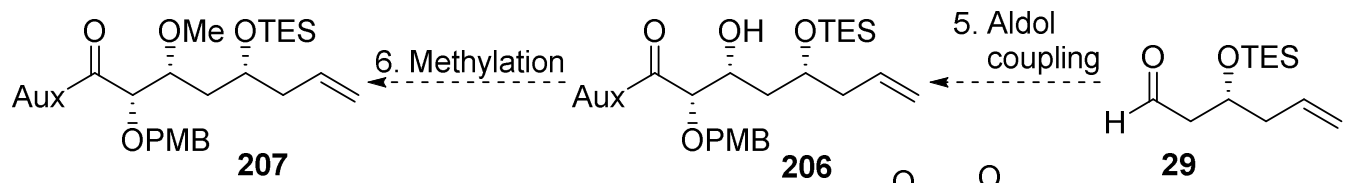

7. PMB

cleavage

8. MOM

protection

206<smiles>CCCCC(=O)OCC(=O)N1C(=O)OCC1Br</smiles>

29<smiles>C=C(C)CCC[C@H](OC)C(=O)N1C(=O)OCC1Br</smiles>

Scheme 4.41: Taylor's synthesis of the C1-C7 intermediate 208

After methylation at $\mathrm{O} 3$ (207), the $\mathrm{O} 2 \mathrm{PMB}$ protecting would be replaced with a MOM group in a two-step sequence to give the intermediate 208. As discussed earlier, previous work indicates that an $\mathrm{O} 2 \mathrm{MOM}$ protecting group is necessary for the macrolactonisation route as it provides the least hindrance to the nearby reaction site.

From the intermediate 208, a single step, base hydrolysis of the oxazolidionone would give the C1-C7 acid fragment 209 needed for the RCM approach (Scheme 4.42). For the elaboration of the Taylor's C1-C7 aldehyde fragment 26 (Scheme 4.42), the oxidative cleavage of the terminal alkene in $\mathbf{2 0 8}$ would give the desired C7 aldehyde functionality. With this approach the two C1-C7 fragments 26 and 209 could both be obtained in nine steps.
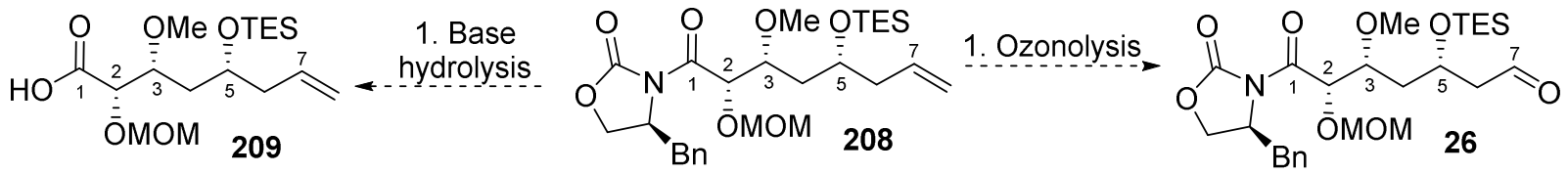


\subsubsection{The synthesis of C1-C2 oxazolidionone}

The synthesis of C1-C2 oxazolidinone started from bromoacetic acid that was converted into PMB-protected glycolic acid 210 in the first step (Scheme 4.43). ${ }^{210,211}$ First, deprotonation with $\mathrm{NaH}$ (2 equiv.) gave a sodium carboxylate of the bromoacetic acid. After the deprotonation of the acid was completed (determined by observing the evolution of $\mathrm{H}_{2}$ to cease), the $p$-methoxybenzyl alcohol (PMBOH) was added at $0{ }^{\circ} \mathrm{C}$ and was deprotonated by the second $\mathrm{NaH}$ equivalent. This caused a nucleophilic attack by the resulting alkoxide to displace the bromine from the $\alpha$-carbon in an $\mathrm{S}_{\mathrm{N}} 2$ fashion. After an acid-base workup, the product (210) was obtained as a solid in $91 \%$ yield and in sufficient purity that it did not require further purification. The next step was the coupling of the acid to the (S)-4-benzyl-2oxazolidinone (37). The acid $\mathbf{2 1 0}$ was converted into an activated ester using a mild activating reagent, $\quad \mathrm{N}, N, N^{\prime}, N^{\prime}$-tetramethyl-O-(1H-benzotriazol-1-yl)uronium hexafluorophosphate (HBTU), which was then reacted with the lithiated oxazolidinone $\mathbf{3 7}$ to give the C1-C2 fragment 28 in 61\% yield after purification (Scheme 4.43). ${ }^{212}$

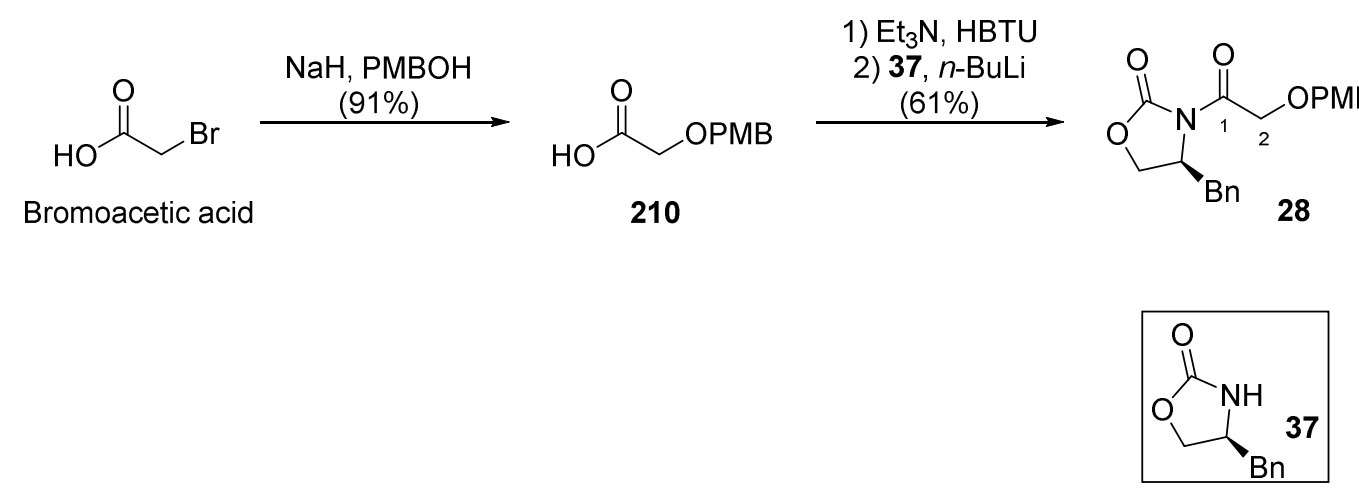

Scheme 4.43: The synthesis of the oxazolidinone $\mathbf{2 8}$

\subsubsection{The synthesis of $\mathrm{C3}-\mathrm{C7}$ dithiane}

The synthesis of C3-C7 dithiane 205 started from (S)-glycidyl tosylate (Scheme 4.44) and, again following Taylor's methodology ${ }^{4}$ the epoxide was first reacted with the lithiated 1,3dithiane that opened the epoxide, attacking from the less hindered end and with inversion of configuration. This immediately caused an intramolecular $\mathrm{S}_{\mathrm{N}} 2$ reaction to form the migrated epoxide $\mathbf{2 0 3}$ in $\mathbf{5 0 \%}$ yield. The epoxide was then opened with a vinyl nucleophile and the resulting alcohol (204) protected as a triethylsilyl ether (TES) to give the dithiane 205. 


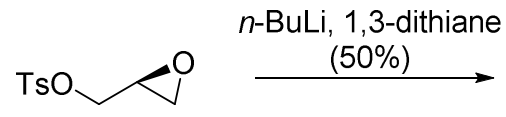

(S)-glycidyl tosylate

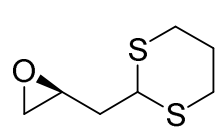

203
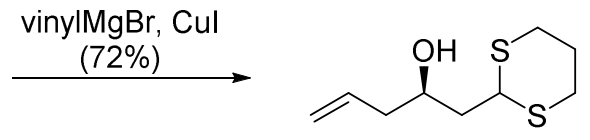

204

$$
\begin{aligned}
& \text { TESCI, } \\
& \text { DMAP, } \\
& \mathrm{Et} \mathrm{t}_{3} \mathrm{~N} \\
& (76 \%)
\end{aligned}
$$

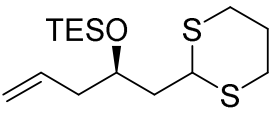

205

Scheme 4.44: The synthesis of the dithiane 205

\subsubsection{Coupling attempts}

The dithiane functionality in $\mathbf{2 0 5}$ was then removed through methylation to give the aldehyde 29 (aldehyde proton observed in the ${ }^{1} \mathrm{H}$ NMR spectrum of the crude reaction mixture as a triplet at $9.81 \mathrm{ppm}$ ), which was used in the next step (the Evans aldol reaction, Scheme 4.45) without further purification. The aldehyde was added to the preformed boron enolate of $\mathrm{C} 1-\mathrm{C} 2$ fragment $\mathbf{2 8}$ but no product was obtained and the oxazolidinone starting material was recovered.

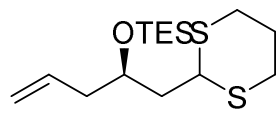

205
1) $\mathrm{Mel}, \mathrm{CaCO}_{3}$

2) $28, \mathrm{Bu}_{2} \mathrm{BOTf}, \mathrm{Et}_{3} \mathrm{~N}$<smiles>C=CC[C@H](C[C@@H](O)[C@@H](O[Na])C(=O)N1C(=O)OC[C@H]1Br)O[S-]</smiles>

206

Scheme 4.45: Attempted synthesis of 206

The dithiane removal using $\mathrm{Mel} / \mathrm{CaCO}_{3}$ was not a clean transformation judging by TLC and NMR, and it was postulated that the impurities present in the crude reaction mixture might be affecting the aldol addition. This deprotection is initiated by a nucleophilic attack of the sulfur on the methyl iodide resulting in the loss of iodide (Scheme 4.46). This leads to the opening of the dithiane to give the sulfonium cation that reacts with water to afford the hemthioacetal. The subsequent elimination of the thiol (211) results in the formation of the aldehyde. 


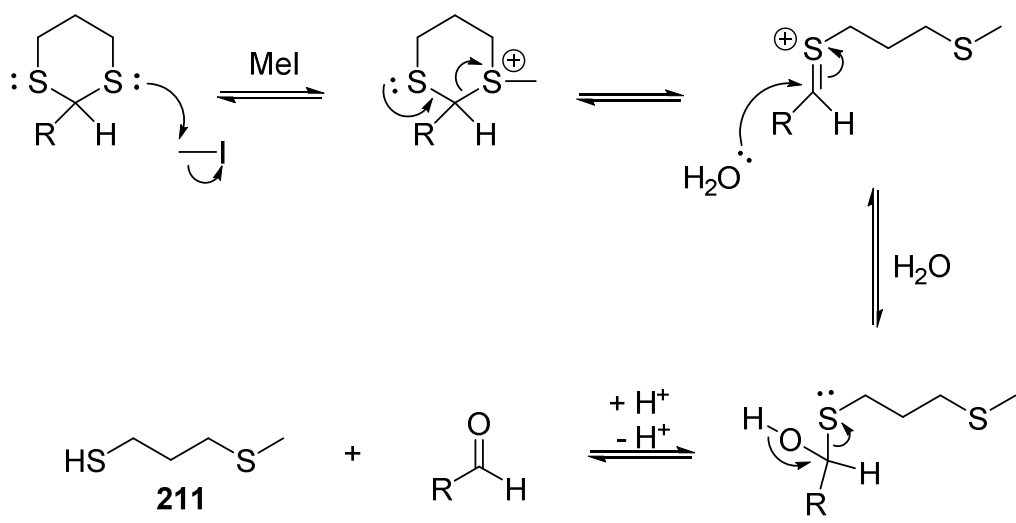

Scheme 4.46: Methylative removal of the dithiane

A range of other dithiane deprotection conditions were tried. Several oxidative methods $\left(\mathrm{I}_{2}{ }^{213} \mathrm{NBS},{ }^{214,215} \mathrm{NBS} / \mathrm{AgClO}_{4},{ }^{216} \mathrm{DMP} / \mathrm{NaHCO}_{3},{ }^{217} \mathrm{DDQ}^{218}\right)$ and a mercury-based method $\left(\mathrm{Hg}\left(\mathrm{CF}_{3} \mathrm{COO}\right)_{2} / \mathrm{CaCO}_{3}\right)^{219}$ were explored but due to the presence of a terminal double bond and an acid-labile protecting group (TES), most methods resulted in unwanted sidereactions or decomposition of the material.

Given the persistent lack of reactivity between the aldehyde and the enolate, it was decided to change the protecting groups in order to improve the stability of the aldehyde. PMBprotected aldehyde 213 (Scheme 4.47a) had been previously synthesised by $\mathrm{Dr}$ Joanne Harvey (unpublished work) and had shown good stability and reactivity in the Evans aldol reaction, encouraging its use in this project also. For this purpose, the PMB protected $\mathrm{C} 3-\mathrm{C} 7$ aldehyde $\mathbf{2 1 3}$ was synthesised from secondary alcohol 204. Accordingly, the alcohol 204 was protected as a $p$-methoxybenzyl ether (212), then the dithiane was cleaved to give the aldehyde $\mathbf{2 1 3}$ in a $\mathbf{4 7 \%}$ yield over two steps. This aldehyde was significantly more stable to than 29 and could be easily purified on silica gel. 
a)<smiles>C=CC[C@@H](O)CC1SCCCS1</smiles>

b)<smiles>O=C(CO[18OH])N1C(=O)OC[C@H]1Br</smiles>
1) $\mathrm{DDQ}$

2) DIPEA, MOMCI TBAI<smiles>C1CC2CC1[As]2</smiles><smiles></smiles>

Scheme 4.47: The synthesis of the a) aldehyde $\mathbf{2 1 3}$ and b) the oxazolidionone $\mathbf{2 1 4}$

In order to achieve orthogonal protection of the C2 and C5 hydroxyl groups, it became necessary to change the $\mathrm{C} 2$ protecting group. For this it was decided to use MOM protection from the beginning, as the MOM was needed at a later stage. The removal of the PMB protecting group from the $\mathrm{C} 1-\mathrm{C} 2$ fragment $\mathbf{2 8}$ and the subsequent methoxymethyl ether protection proceeded in $69 \%$ yield over two steps to give the oxazolidinone $\mathbf{2 1 4}$ (Scheme 4.47b).

However, the attempted coupling of these two fragments was unsuccessful and resulted solely in the recovery of the two starting materials (Scheme 4.48). This result, although disappointing, was not entirely unexpected, as Taylor also commented on the lack of reactivity between the MOM-oxazolidinone (214) and the TES-protected aldehyde (29). ${ }^{4}$

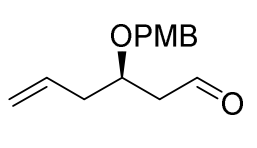

213

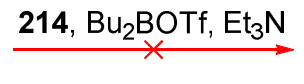

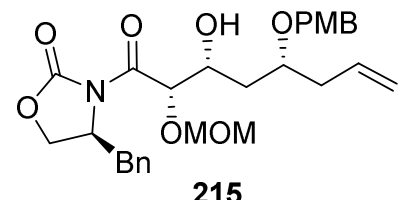

215

Scheme 4.48: Attempted coupling of the aldehyde $\mathbf{2 1 3}$ and the oxazolidinone $\mathbf{2 1 4}$

Lastly, coupling of the aldehyde $\mathbf{2 1 3}$ and the already prepared PMB-protected oxazolidinone 28 (Scheme 4.49) was attempted even though the product, if obtained, would have the same (PMB) protecting groups at $\mathrm{O} 2$ and 05 . To our delight, the coupling between these two fragments worked to give $\mathbf{2 1 6}$ as a single product in $\mathbf{7 2} \%$ yield. It must be noted that the yield of the reaction largely depended on the quality of the $\mathrm{Bu}_{2} \mathrm{BOTf}$ reagent and that the reagent is very sensitive to moisture. 


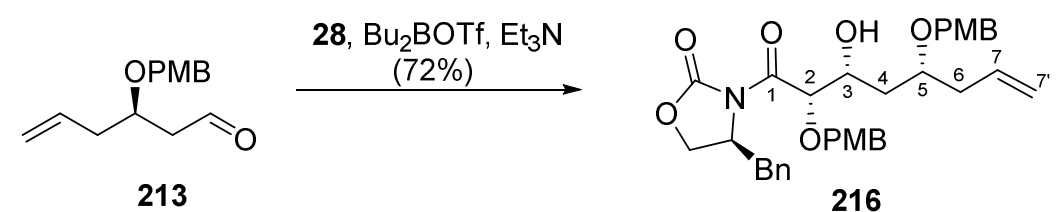

Scheme 4.49: The synthesis of the C1-C7 oxazolidinone 216

Two new oxymethine peaks featured in the ${ }^{1} \mathrm{H}$ NMR spectrum of 216: a doublet at $5.18 \mathrm{ppm}$ $(J=3.0 \mathrm{~Hz})$ and a doublet of triplets at $4.09 \mathrm{ppm}(J=9.1,3.2 \mathrm{~Hz})$. Based on the multiplicity and $\mathrm{HMBC}$ correlations to one of the PMB oxymethylene carbons $(\delta 72.9)$ as well as a correlation to the carbonyl carbon at 170.8 (C1), the doublet at $5.18 \mathrm{ppm}$ was assigned as $\mathrm{H} 2$. In the COSY spectrum the oxymethine at $4.09 \mathrm{ppm}$ correlated to the one at $5.18 \mathrm{ppm}$ as well as the methylene protons at $\delta 1.91$ and $\delta 1.76$ assigned as $\mathrm{H} 4 \mathrm{a}$ and $\mathrm{H} 4 \mathrm{~b}$ respectively. This oxymethine was therefore assigned as $\mathrm{H} 3$. In the COSY spectrum it was possible to follow the correlations from $\mathrm{H} 2(\delta 5.18)$ all the way to the double bond terminus $H 7^{\prime}(\delta$ 5.81). All the other product features were present in the spectrum: the terminal alkene, the oxazolidinone and the two distinct PMB environments. The product was also confirmed by mass spectrometry, where the measured mass of 590.2734 matched the calculated value of 590.2748 for the $[\mathrm{M}+\mathrm{H}]^{+}$with $\Delta=2.49 \mathrm{ppm}$ (Experimental).

In the 1980s, Evans pioneered the use of chiral oxazolidinone auxiliaries to control the diastereoselectivity and enantioselectivity of propionate aldol additions. ${ }^{65,220}$ Expanding on the findings of Dubois ${ }^{221}$ and Heathcock ${ }^{222}$ that the product stereochemistry is related to the enolate geometry, he observed that propionyl oxazolidinones, when treated with either lithium or sodium bases (LiHMDS or NaHMDS) or with dialkylboron triflates $\left(\mathrm{Bu}_{2} \mathrm{BOTf}\right)$ selectively form $Z$ enolates $(Z / E>100: 1)$ in order to minimise allylic strain ${ }^{220}$ with the bulky oxazolidinone substituent $R_{1}$ (Scheme 4.50).
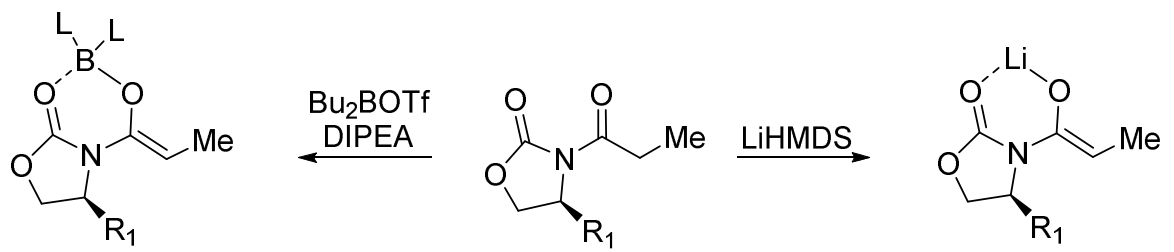

Scheme 4.50: Z-enolate formation

Next, it was noticed that lithium-promoted alkylations and boron aldol additions of these enolates exhibit an opposite chirality transfer, which was evident from the product 
structures (Scheme 4.51). ${ }^{223}$ In the lithium (or sodium) alkylations (Scheme 4.51a) the asymmetric induction in a metal-chelated transition state is directed by the stereochemistry at the chiral centre of the oxazolidinone such that the electrophile approaches from the opposite face to the oxazolidinone substituent. In boron aldol reactions, boron complexation of the aldehyde electrophile in conjunction with other factors such as carbonyl-carbonyl dipole minimisation and the positioning of the oxazolidinone chiral substituent in the chair transition state direct the diastereofacial selectivity in the opposite way (Scheme 4.51b). ${ }^{65,66}$

a)<smiles>[R1]C1COC(=O)N1C(=O)CC</smiles><smiles>[R]C(C)C</smiles><smiles>[R]C(C)C(=O)N1C(=O)OCC1[R]</smiles>

b)

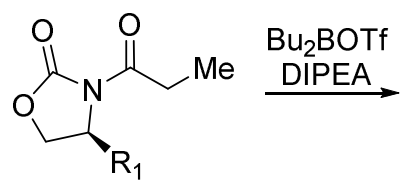<smiles></smiles>
(see $\underset{\text { Scheme }}{\stackrel{\mathrm{RCHO}}{\longrightarrow}}$<smiles>C1CCOCC1</smiles><smiles>[R]C=COB([13CH3])O/C(=C\C)N1C(=O)OC[C@H]1[R]</smiles><smiles>[R]C(O)[C@H](C)C(=O)N1C(=O)OCC1[R1]</smiles>

Scheme 4.51: Chirality transfer in reactions of metal enolates with a) alkyl electrophile b) aldehyde

In the favoured transition state, the oxazolidinone is oriented to minimise the imide dipoledipole interactions with the enolate $\mathrm{O}$, and the chiral substituent is oriented exo with regard to the transition state (Scheme 4.52). ${ }^{66,220}$ In these boron aldol reactions, the diastereoselectivity arises from minimisation of 1,3-axial interactions so that high levels of syn-selectivity and "Evans"-type enantioselectivity are generally obtained (typically around $99 \%$ d.e. and e.e.). ${ }^{65,66,220}$ 

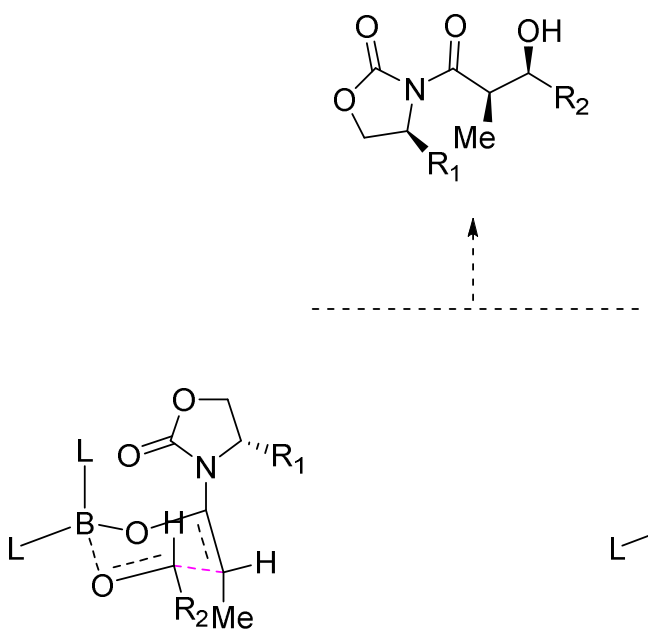

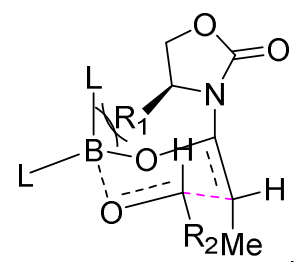

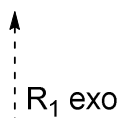

dipole<smiles>[R16]OC(=C[14CH3])N1C(=O)OCC1[R7]</smiles>

minimisation

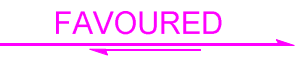<smiles>[Y16]C=C(OCCC)N1C(=O)OC[C@@H]1[R7]</smiles>
$\mathrm{R}_{1}$ endo high steric
encumberance $R_{1}$ endo 
3,5-syn. It was attempted to confirm the relative stereochemistry using the coupling values and NOESY correlations for the acetate $\mathbf{2 2 1}$ (vide infra) but due to insufficient material and the poor resolution of the peaks this was not possible.

\subsubsection{Final modifications}

The aldol product was methylated at $\mathrm{O} 3$ using Meerwein's salt and proton sponge. This reaction also required some optimisation. When Taylor's procedure ${ }^{4}$ was followed (15 equiv. $\mathrm{Me}_{3} \mathrm{OBF}_{4}, 15$ equiv. proton sponge), whereby the salt and the base were added sequentially to a cooled $\left(0^{\circ} \mathrm{C}\right)$ solution of the starting material in $\mathrm{DCM}$, the product $\mathbf{2 1 7}$ was isolated as an inseparable mixture with the retro-aldol product, oxazolidinone $\mathbf{2 8}$ (Scheme 4.53).

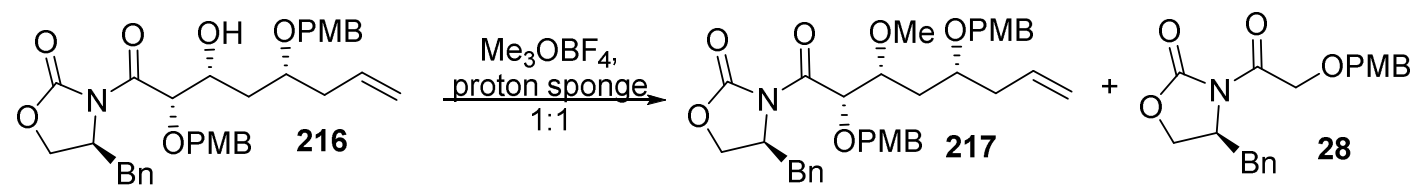

Scheme 4.53: Result from the first attempt at the 03 methylation reaction

Since this is a result of a deprotonation of the $\mathrm{OH}$ (Scheme 4.54), which is competing with the methylation, it was postulated that this could be circumvented by having excess of methylating reagent over the base.

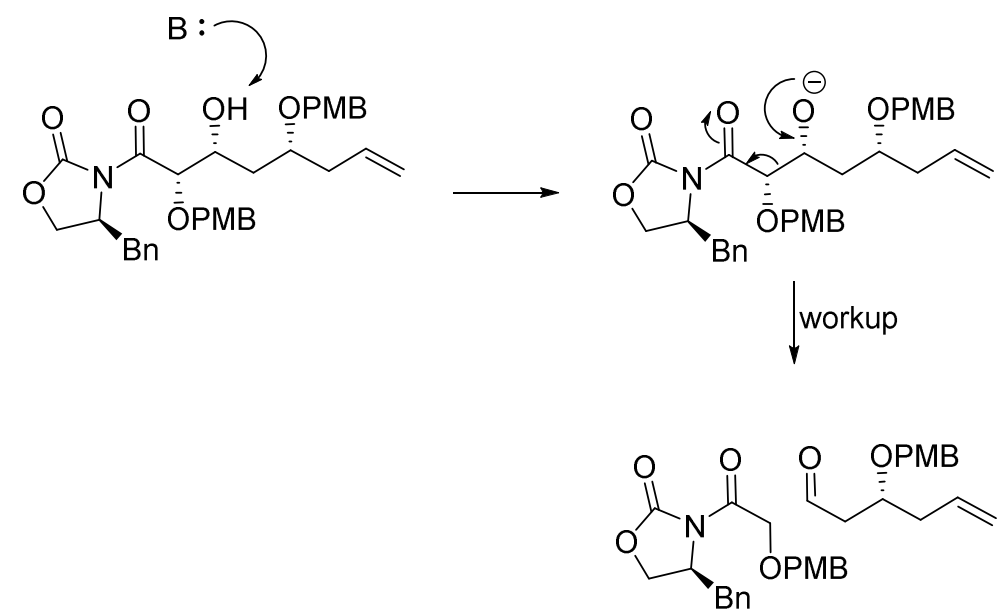

Scheme 4.54: Base-induced retro-aldol reaction of 216

However, when the number of equivalents of proton sponge was decreased (salt/base 5:3) the reaction resulted in the formation of the 2,3,5-tri-O-methylated oxazolidinone 218 (Scheme 4.55). The ${ }^{1} \mathrm{H}$ NMR spectrum of the isolated compound had some unidentifiable 
impurities but it showed the absence of all PMB-related peaks and instead three new singlets at $3.48,3.35$ and $3.34 \mathrm{ppm}$ (integrating for $3 \mathrm{H}$ each), corresponding to three methyl ether proton environments. All other structural features were also discernible: the oxazolidinone protons, terminal alkene, three oxymethine protons and the two methylene sets of protons. The mass spectroscopy analysis confirmed the presence of the tri-2,3,5-Omethylated compound with the detected molecular ion peak at $\mathrm{m} / \mathrm{z} 414.1894$ and the calculated value for $\mathrm{C}_{21} \mathrm{H}_{29} \mathrm{NNaO}_{6}{ }^{+}[\mathrm{M}+\mathrm{Na}]^{+} 414.1887$ with $\Delta=1.7 \mathrm{ppm}$.

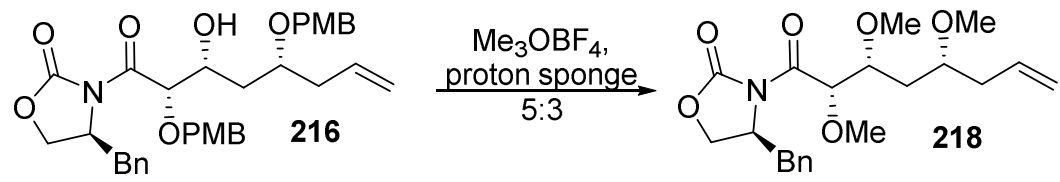

Scheme 4.55: The formation of the 2,3,5-tri-O-methylated product 218 with excess $\mathrm{Me}_{3} \mathrm{OBF}_{4}$

A postulated mechanism for the formation of 218 (Scheme 4.56) involves 02 and 05 reacting with the electrophilic trimethyloxonium species, which initiates an electron cascade from the PMB methoxy. This eventually leads to the elimination of the oxonium species (proposed to be quenched with water in the workup if not before) to give the methyl ether in place of the PMB ether.

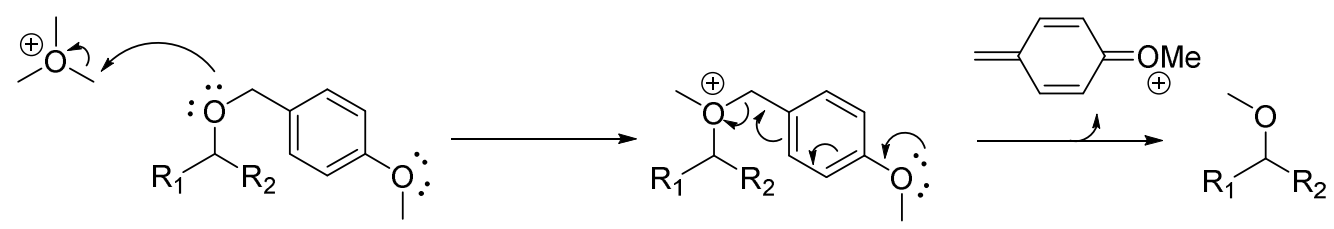

Scheme 4.56: Proposed mechanism for the deprotection of PMB ethers in the formation of $\mathbf{2 1 8}$

It was therefore assumed that the buffering effect provided by the proton sponge was important in preventing this side-reaction in the previous attempts. Yet having excess base would lead to deprotonation and the retro-aldol product. Therefore, equimolar amounts of proton sponge and Meerwein's reagent was determined to be optimal, as per the original procedure. It then became necessary to consider the order of addition of reagents and the reaction time. After further optimisation, it was established that the best results were obtained when the starting material was added dropwise, as a solution in DCM, to a cooled solution $\left(0{ }^{\circ} \mathrm{C}\right)$ of the salt and the base (1:1 ratio, 15 equiv. each) in DCM. TLC analysis showed the reaction was completed after 5 hours at room temperature. With this protocol the formation of the previously observed by-products was suppressed. The $\mathrm{O} 3$ methylated 
product 217 (Scheme 4.57), although stable on silica gel, was usually used in the next step with minimal purification (silica plug) in order to expedite the synthesis of the two fragments. Mass spectrometry analysis found $\mathrm{m} / \mathrm{z}$ 604.2918, corresponding to the calculated value for $\mathrm{C}_{35} \mathrm{H}_{42} \mathrm{NO}_{8}{ }^{+}[\mathrm{M}+\mathrm{H}]^{+}$604.2905. $\Delta=2.2 \mathrm{ppm}$.
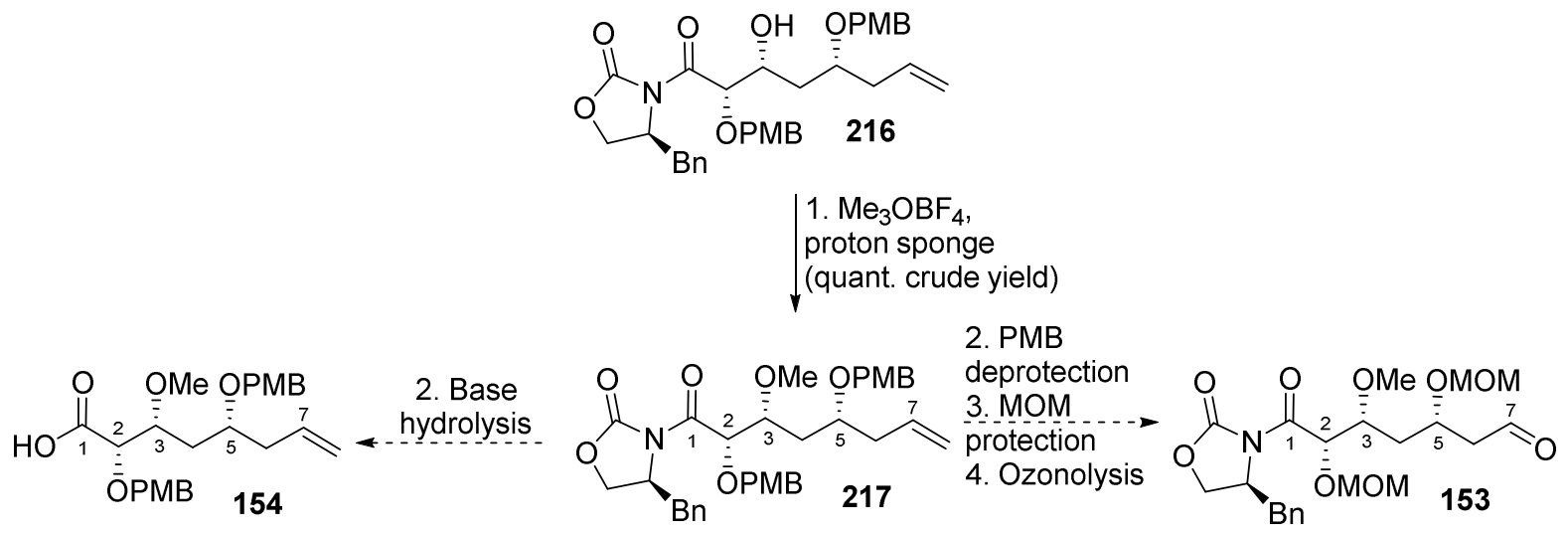

Scheme 4.57: The synthesis of the 03-methylated oxazolidinone 217 and the plan for the completion of synthesis of the two C1-C7 fragments

The successful 03-methylation gives the C1-C7 intermediate $\mathbf{2 1 7}$ as a common precursor for the synthesis of the two C1-C7 fragments 153 and 154 (Scheme 4.57). To synthesise the C1C7 acid fragment 154 needed for the RCM route, the auxiliary needs to be hydrolysed under basic conditions. For the synthesis of the $\mathrm{C} 1-\mathrm{C} 7$ aldehyde fragment $\mathbf{1 5 3}$ that is necessary for the macrolactonisation approach, the PMB ethers need to be replaced with MOM acetals, as an $\mathrm{O} 2 \mathrm{MOM}$ protecting was already observed to be suitable for macrocyclisation due to its small size. ${ }^{1-4,55}$ Then, the ozonolysis of the terminal olefin would give the desired aldehyde functionality at C7. Because C8-C20 methyl ketone 140 was already synthesised (Chapter 3), it was decided to prioritise the synthesis of the C1-C7 aldehyde 153 so that the two fragments can be coupled.

Again, DDQ (6 equiv.) in 5\% aq. DCM was used to cleave the PMB ethers and the resulting diol $(\mathbf{2 1 9}$, Scheme 4.58$)$ reacted with bromomethyl methyl ether ( $\mathrm{MOMBr}$ ) (10 equiv.) in the presence of DIPEA (10 equiv.). None of the expected di-2,5-MOM-protected product (220) was obtained but instead a cyclic acetal species 221 was isolated (Scheme 4.58). The proton spectrum showed the presence of only one methyl peak in the methyl ether region, assigned as the $\mathrm{O} 3$ methyl group. An additional methylene group was present in the acetal region with the two doublets at $5.04(J=5.6 \mathrm{~Hz})$ and $5.00 \mathrm{ppm}(J=5.6 \mathrm{~Hz})$ correlating (HSQC) to a carbon at $93.6 \mathrm{ppm}$. These two protons had HMBC correlations to two carbons: 
at $72.2 \mathrm{ppm}$ and $77.5 \mathrm{ppm}$, assigned as C5 and C2, respectively. Additionally, mass spectrometry analysis confirmed the structure with the found mass of 376.1754 matching the calculated value for $\mathrm{C}_{19} \mathrm{H}_{20} \mathrm{NO}_{8}{ }^{+}[\mathrm{M}+\mathrm{H}]^{+} 376.1755$ with $\Delta=0.3 \mathrm{ppm}$.
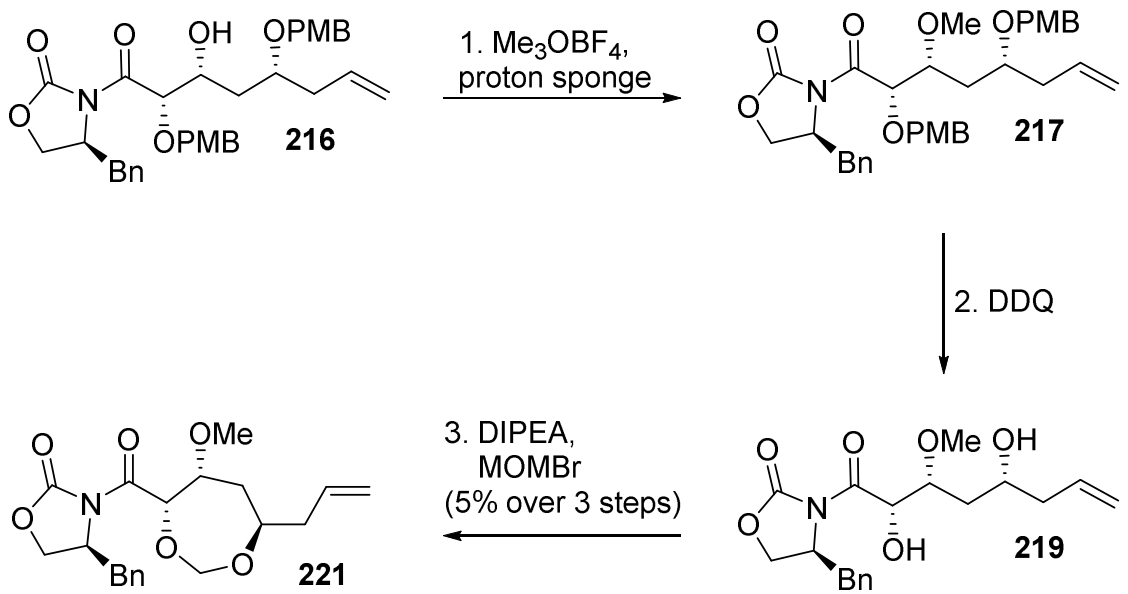

Scheme 4.58: The formation of the acetal 221 under MOM protection conditions

The proposed mechanism (Scheme 4.59) involves one of the oxygens reacting with the $\mathrm{MOMBr}$ in the usual way to displace the bromide, although these steps may be reversed. The other oxygen, instead of reacting with another molecule of $\mathrm{MOMBr}$ attacks the acetal carbon of the attached MOM and a methoxy group gets displaced.

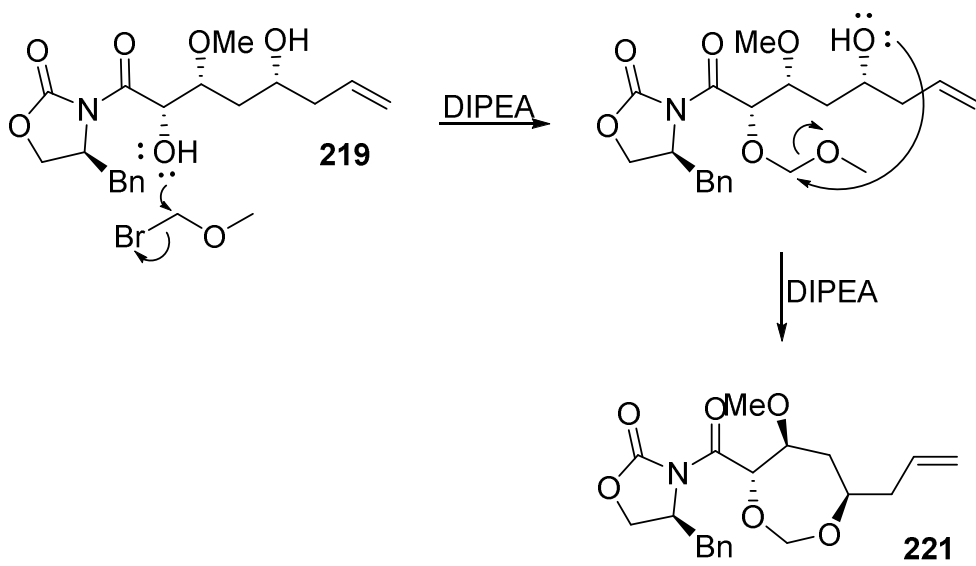

Scheme 4.59: Proposed mechanism for the formation of the acetal 221

The reaction was performed on a small scale $(0.016 \mathrm{mmol})$ and the higher dilution might have favoured intramolecular reaction $(0.04 \mathrm{M}$, excess DCM was used to prevent complete evaporation of the solvent over the $20 \mathrm{~h}$ course of the reaction), despite bromide being a better leaving group then methoxide. However, the excess of $\mathrm{MOMBr}$ (10 equiv.) should 
have compensated for the high dilution and the observed result, the formation of the acetal $\mathbf{2 2 1}$, led us to doubt the quality of the reagent.

Therefore, in the second attempt at this reaction, $\mathrm{MOMCl}$ was used instead. This time some product $\mathbf{2 2 0}$ was isolated but mostly it was inseparable from a by-product resulting from a partial PMB deprotection and the yield of the reaction could not be determined. However, a small fraction containing 220 (Figure 4.12) was obtained with a minimal amount of impurity and was used for characterisation (Experimental).

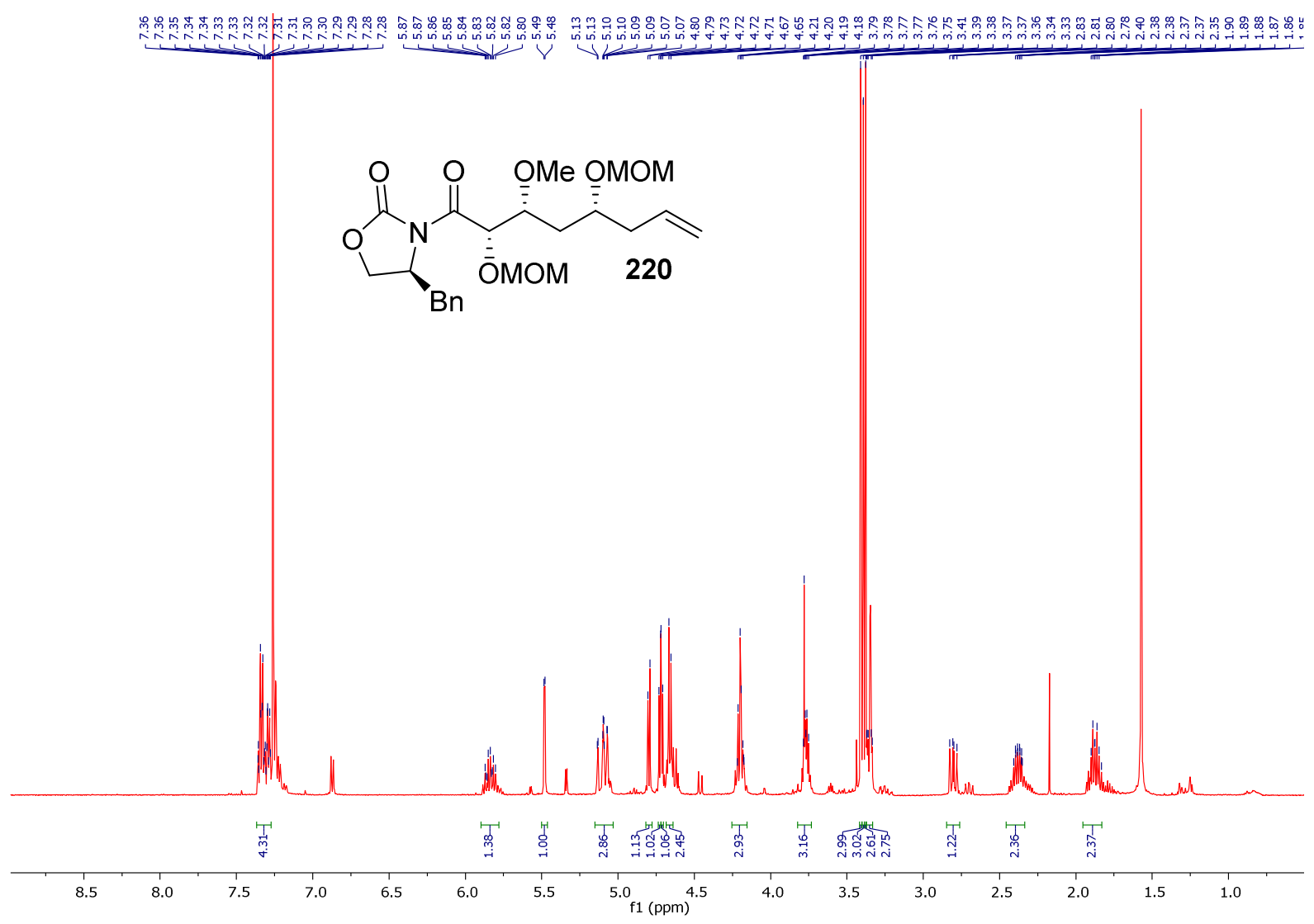

Figure 4.12: ${ }^{1} \mathrm{H}$ NMR spectrum of the oxazolidinone $\mathbf{2 2 0}$

The product 220 had three distinct methyl ether singlets at $\delta 3.41,3.39$ and 3.38 in the ${ }^{1} \mathrm{H}$ NMR spectrum, as well as the four new MOM acetal environments (four doublets): 4.80 ( $J=$ $6.8 \mathrm{~Hz}), 4.73(J=5.6 \mathrm{~Hz}), 4.71(J=5.3 \mathrm{~Hz})$ and $4.66(J=7.0 \mathrm{~Hz}) \mathrm{ppm}$ corresponding to two MOM groups. HSQC correlation showed that protons at 4.80 and $4.71 \mathrm{ppm}$ are on the same carbon at $97.7 \mathrm{ppm}$. There is an HMBC correlation between the $5.48 \mathrm{ppm}$ doublet $(J=4.3$ $\mathrm{Hz}, \mathrm{H} 2$ ) and the carbon at $97.7 \mathrm{ppm}$ making this MOM group the one protecting $\mathrm{O} 2$. The two other protons at 4.73 and $4.66 \mathrm{ppm}$ correlate to a carbon at $96.2 \mathrm{ppm}$ in the HSQC. In the 
HMBC spectrum, there are correlations between these protons and a carbon at 74.7 (assigned as C5), which confirms the presence of the 05 MOM protecting group.

\subsection{Scalability}

Taylor's methodology offers quick access (six steps) to the C1-C7 intermediate $\mathbf{2 1 7}$ (Scheme 4.60). The yield over six steps was however not determined as all of the $\mathrm{O} 3$ methylated oxazolidinone obtained was used directly, after a silica plug (quant. crude yield), in the subsequent attempts to obtain the C1-C7 fragment 153 necessary for the macrolactonisation route (namely the swapping of protecting groups). Due to time constrains and the unexpected difficulties with both the $\mathrm{O} 3$ methylation and the subsequent PMB deprotection/MOM protection sequence of steps, the last step, ozonolysis of the terminal alkene to give the $\mathrm{C} 1-\mathrm{C} 7$ aldehyde fragment (153), was not attempted. The base hydrolysis of the intermediate $\mathbf{2 1 7}$ to give the C1-C7 acid fragment $\mathbf{1 5 4}$ needed for the RCM route has also not been attempted yet. These reactions, as well as optimisation of the PMB deprotection/MOM protection sequence are part of our future plans as discussed in

\section{Chapter 5.}

The six reactions leading to the formation of $\mathrm{C} 1-\mathrm{C} 7$ alkene fragment are reliable, relatively high yielding (Scheme 4.60) and the materials and reagents required are commercially available and affordable. One of the things to consider when scaling up is the use of large excess of iodomethane in the dithiane removal. Due to the presence of the double bond and the PMB protecting group, some of the methods tried on the O5 TES compound 205 (earlier text) would still be unsuitable ( $\left.I_{2}, N B S, D D Q\right)$. An alternative would be to explore a DMP method $^{217}$ for removing the dithiane whilst buffering the reaction to avoid any acidmediated PMB deprotection.

As mentioned earlier, the boron reagent $\left(\mathrm{Bu}_{2} \mathrm{BOTf}, 1 \mathrm{M}\right.$ in $\left.\mathrm{DCM}\right)$ used for the Evan aldol reaction was very moisture sensitive and even when all precautions were taken, after three uses the reaction yield dropped from $72 \%$ to $10 \%$. Given that only $100 \mathrm{~mL}$ bottles of the reagent are available commercially (from Sigma-Aldrich and Acros), it would be more suitable for a large scale synthesis than small scale, as the scale of the reaction can be 
adjusted so that all or close to $100 \mathrm{~mL}$ is used in a single reaction. This would decrease the cost of the synthesis as less reagent would be wasted.<smiles>CC[18O][Se-]</smiles>

(S)-glycidyl tosylate<smiles>C1CSC(CC2CO2)CO1</smiles>

203
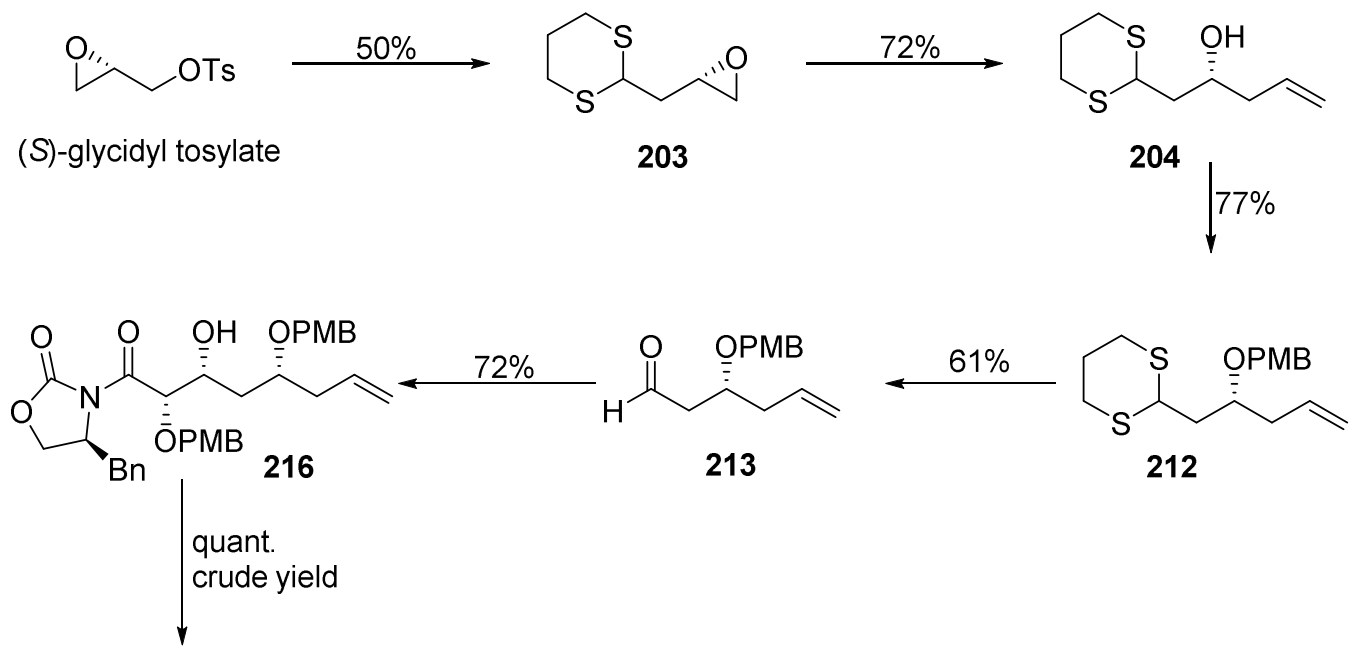

2 steps, yield not determined
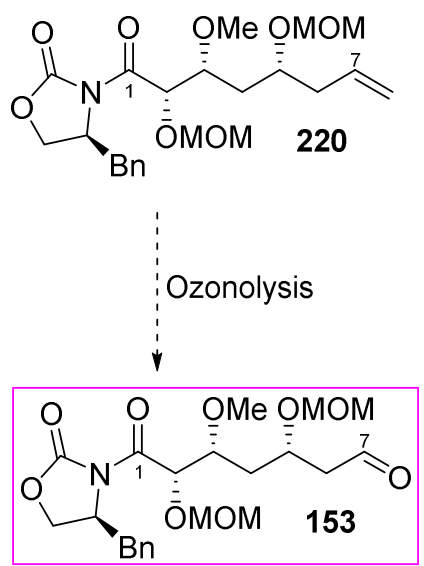

Scheme 4.60: Efforts towards the synthesis of the C1-C7 fragments 153 and 154 


\section{Summary and future work}

\subsection{Summary}

In developing a scalable synthesis of PelA, the idea was to evaluate two different end-game approaches, a macrolactonisation approach and a RCM approach. Following our initial synthetic plan (Chapter 1), we set out to make the required fragments. With some modifications to Evans' methodology, ${ }^{1}$ and drawing on Taylor's and Paterson's methods, ${ }^{4,117}$ fragment C12-C20 (129) was synthesised in nine high-yielding steps (Scheme 5.1). This fragment was then successfully coupled with the C8-C11 aldehyde fragment 56 and subsequent modifications provided the complete C8-C20 methyl ketone fragment 140 (Scheme 5.1). Unfortunately, an attempt to couple the C12-C20 fragment with the C8-C11 aldehyde fragment $\mathbf{5 7}$ did not give the desired product.

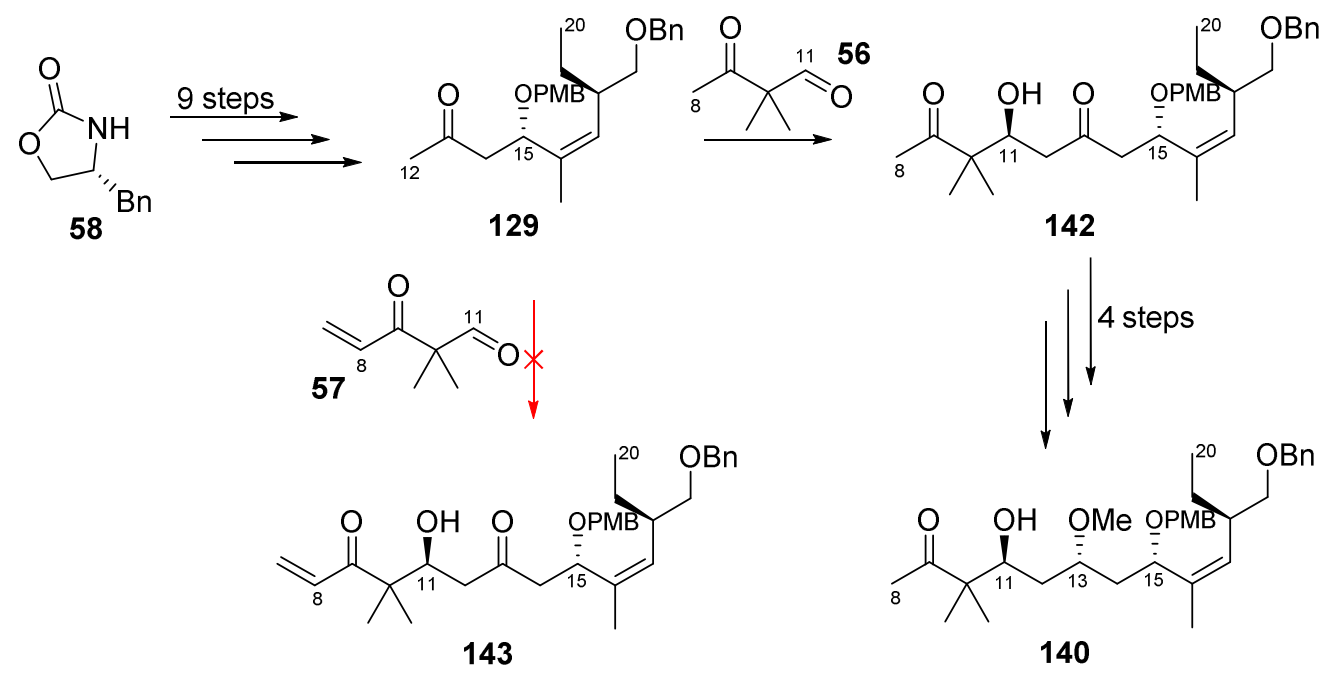

Scheme 5.1: Summary of the efforts towards the synthesis of two C8-C20 fragments

In an effort to make the C1-C7 fragments, several iterations and modifications of the original Ghosh-inspired ${ }^{2}$ strategy starting from diol 32 (Scheme 5.2) and a Jacobsen-based ${ }^{3}$ approach (Scheme 5.3) were explored, albeit unsuccessfully (Chapter 4). Eventually, using Taylor's methodology, ${ }^{4}$ the 03-methylated C1-C7 intermediate $\mathbf{2 1 7}$ was successfully synthesised in just six steps (Scheme 5.4). The removal of the chiral auxiliary in this intermediate would give the complete $\mathrm{C} 1-\mathrm{C} 7$ fragment 154 , possessing the required $\mathrm{C} 1$ acid and C7 alkene functionalities for the RCM strategy (Scheme 5.4). The final modifications needed for the establishment of the C1-C7 aldehyde fragment 153 require further 
optimisation of the PMB deprotection and MOM protection steps (Chapter 4) before the final step, the ozonolysis, is attempted in the future (Scheme 5.4).

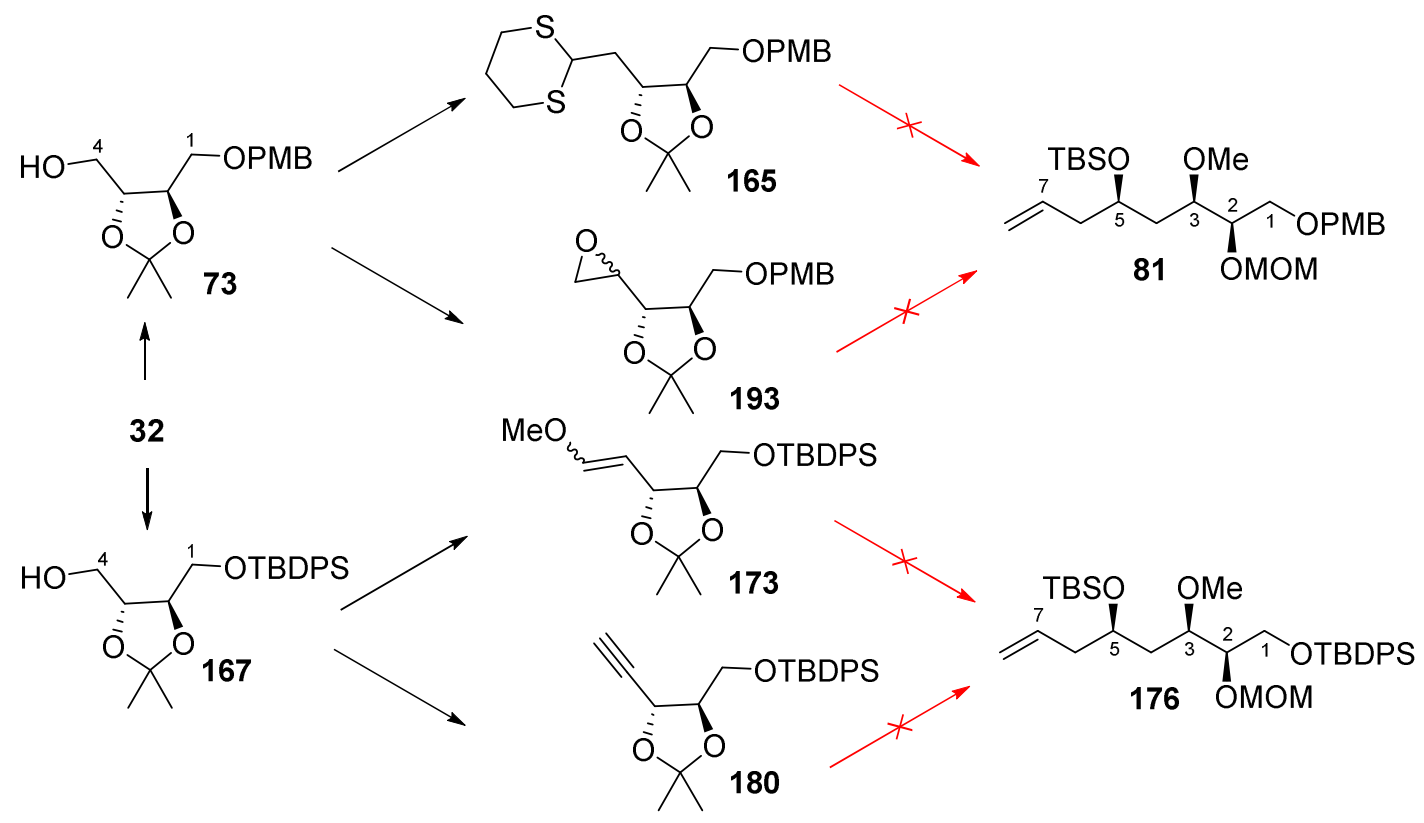

Scheme 5.2: Ghosh-inspired attempts at the synthesis of the C1-C7 fragments

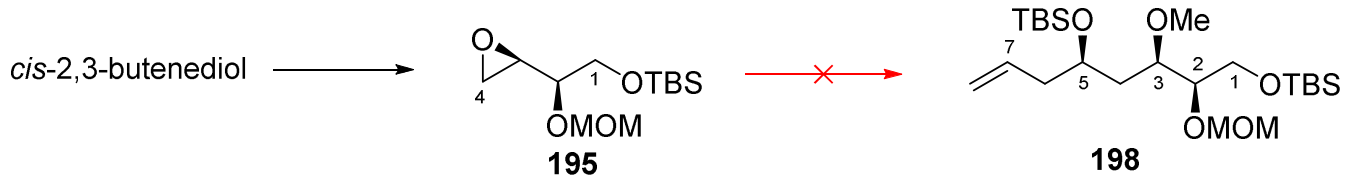

Scheme 5.3: Jacobsen-based attempt at the C1-C7 fragments synthesis

(S)-glycidyl tosylate
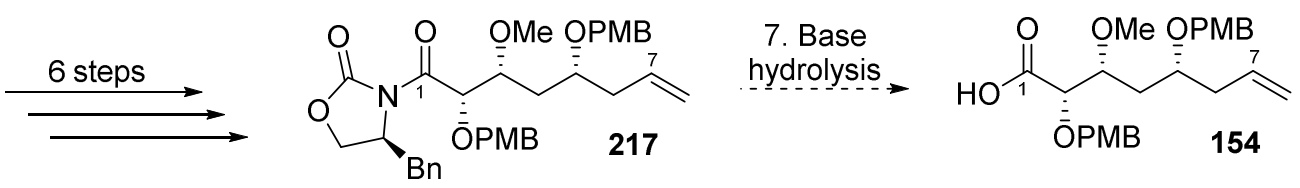
7. PMB deprotection
8. MOM protection
9. Ozonolysis

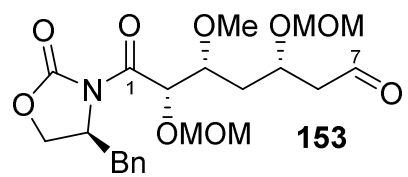


In conclusion, two of the three envisioned fragments for the RCM route, the C8-C11 aldehyde 57 and the C12-C20 methyl ketone 129, have been successfully synthesised and their syntheses optimised. The synthesis of the C1-C7 acid fragment (154) requires the completion of one more step, the base hydrolysis of the Evans' auxiliary (Scheme 5.4). For the macrolactonisation route, the synthesis of the $\mathrm{C} 8-\mathrm{C} 20$ methyl ketone fragment $\mathbf{1 4 0}$ has been achieved but it requires some optimisation (Chapter 3 ). The $\mathrm{C} 1-\mathrm{C} 7$ aldehyde fragment (153) synthesis is almost completed, with the unexpected difficulties in PMB deprotection/MOM protection sequence delaying the attempt at the last step-the ozonolysis of the terminal double bond.

Additionally, the changes to the initially planned side-chain methodology reduced the longest linear sequence of the RCM approach from 22 steps (Chapter 1 ) to 21 steps. For the macrolactonisation approach, the shorter side-chain synthesis together with the change in the $\mathrm{C} 1-\mathrm{C} 7$ fragment synthesis (the use of the chiral auxiliary) also reduced the number of steps in the longest linear sequence to 21 from the originally anticipated 23 (Chapter 1 ).

\subsection{Future work}

The future work will first focus on completing the synthesis of the $\mathrm{C} 1-\mathrm{C} 7$ fragments and then on establishing and optimising the fragment coupling methodologies. For the RCM route, the coupling of the side-chain fragment C12-C20 (129) and the C8-C11 vinylketoaldehyde fragment (57) needs to be further explored (Scheme 5.5). As proposed in Chapter 3 , the coupling will be attempted using a different boron reagent $\left(\mathrm{Bu}_{2} \mathrm{BOTf}\right)$ but it might also require exploration of different solvents, such as THF or toluene. After this coupling has been achieved, the C8-C20 vinyl ketone intermediate 143 will be functionalised (Scheme 5.5) to give the 013 methylated fragment 224. Then, the C11 hydroxyl would be protected as a TBS ether and the O15 PMB group would be removed in the subsequent step to give the complete C8-C20 vinyl ketone fragment 141. 


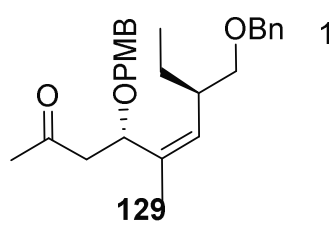<smiles>C=CC(=O)C(C=O)(C=O)C(=O)C=C</smiles><smiles>C=CC(=O)C(C)(C)[C@H](O)CC(=O)C[C@@H](O[C@H](C)CCc1ccccc1)C(=C)C</smiles>
143 2. $\mathrm{Et}_{3} \mathrm{~N}, \mathrm{DMAP}$<smiles>C=CC(=O)C(C)(C)[C@H]1C[C@@H](C[C@H](OC)/C(C)=C\C(CCc2ccccc2)COC)O[Si](C(C)C)(C(C)C)O1</smiles><smiles></smiles>

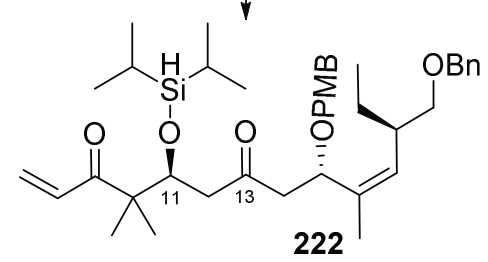

4. TBAF, AcOH

5. $\mathrm{Me}_{3} \mathrm{OBF}_{4}$ iproton sponge

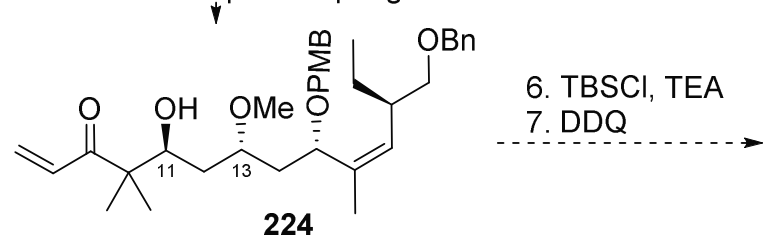

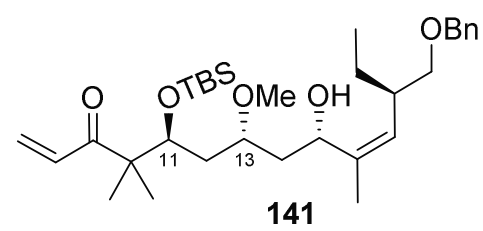

Scheme 5.5: The planned elaboration to the C8-C20 vinyl ketone fragment 141

After the chiral auxiliary is removed from the $\mathrm{C} 1-\mathrm{C} 7$ intermediate $\mathbf{2 1 7}$ to give the $\mathrm{C} 1-\mathrm{C} 7$ acid 154, the two fragments will be coupled in an esterification (Yamaguchi ${ }^{5}$ or Steglich ${ }^{75}$ ) reaction, which would be followed by an RCM to close the macrocycle with a Z-selective Grubbs catalyst ${ }^{90}$ (Scheme 5.6). The 7,8-olefin 226 would then be asymmetrically dihydroxylated (Sharpless $A D^{71,91}$ ) and the methylation at 07 attempted. Here we would be relying on the steric bulk imposed by the $\mathrm{C} 10 \mathrm{gem}$-dimethyl on the $\mathrm{O} 8$ hydroxyl to ensure the selectivity of the methylation. Then, global deprotection would give PelA in 21 steps (IIs). 


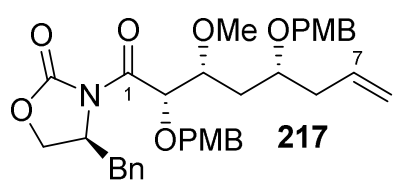

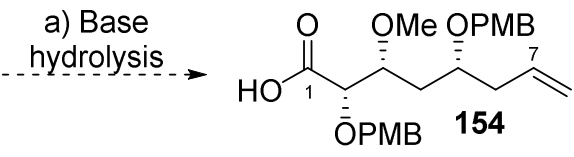

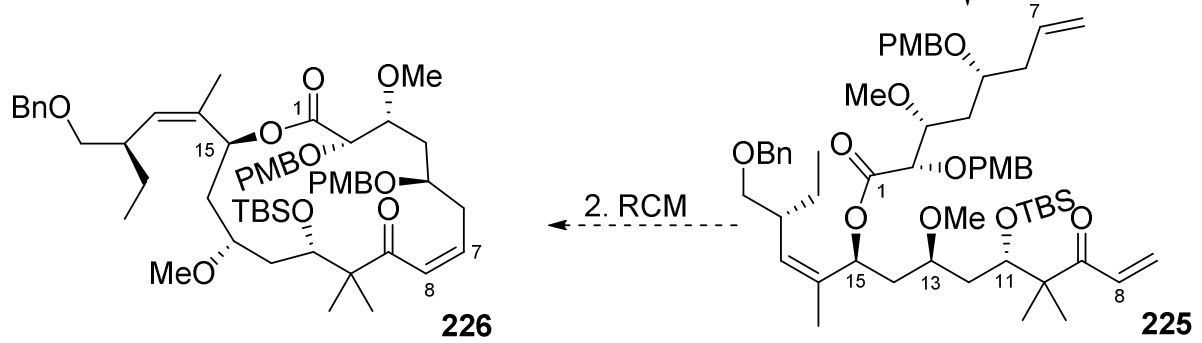

3. Sharpless AD

4. Methylation

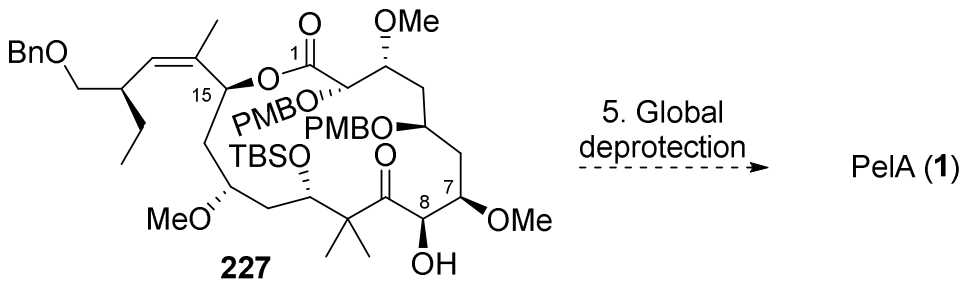

Scheme 5.6: Macrocycle formation through RCM and final modifications to PelA

For the macrolactonisation approach, the 2,5-di-O-MOM-protected variant of the $\mathrm{C} 1-\mathrm{C} 7$ fragment (220) will be used (Scheme 5.7). It is has been observed in previous total syntheses that this relatively small $\mathrm{Pg}$ does not impede the macrocyclisation. ${ }^{1-4,55}$ The ozonolysis of the alkene double bond would give the aldehyde (153) needed for the aldol coupling to the previously synthesised C8-C20 methyl ketone $\mathbf{1 4 0}$. This will be followed by 07 methylation, which should occur selectively at 07 as the 011 is sterically hindered by the C10 gem-dimethyl group. 


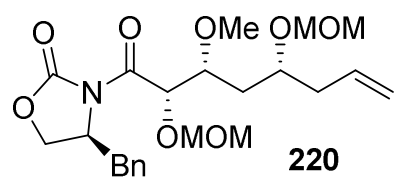

a) Ozonolysis
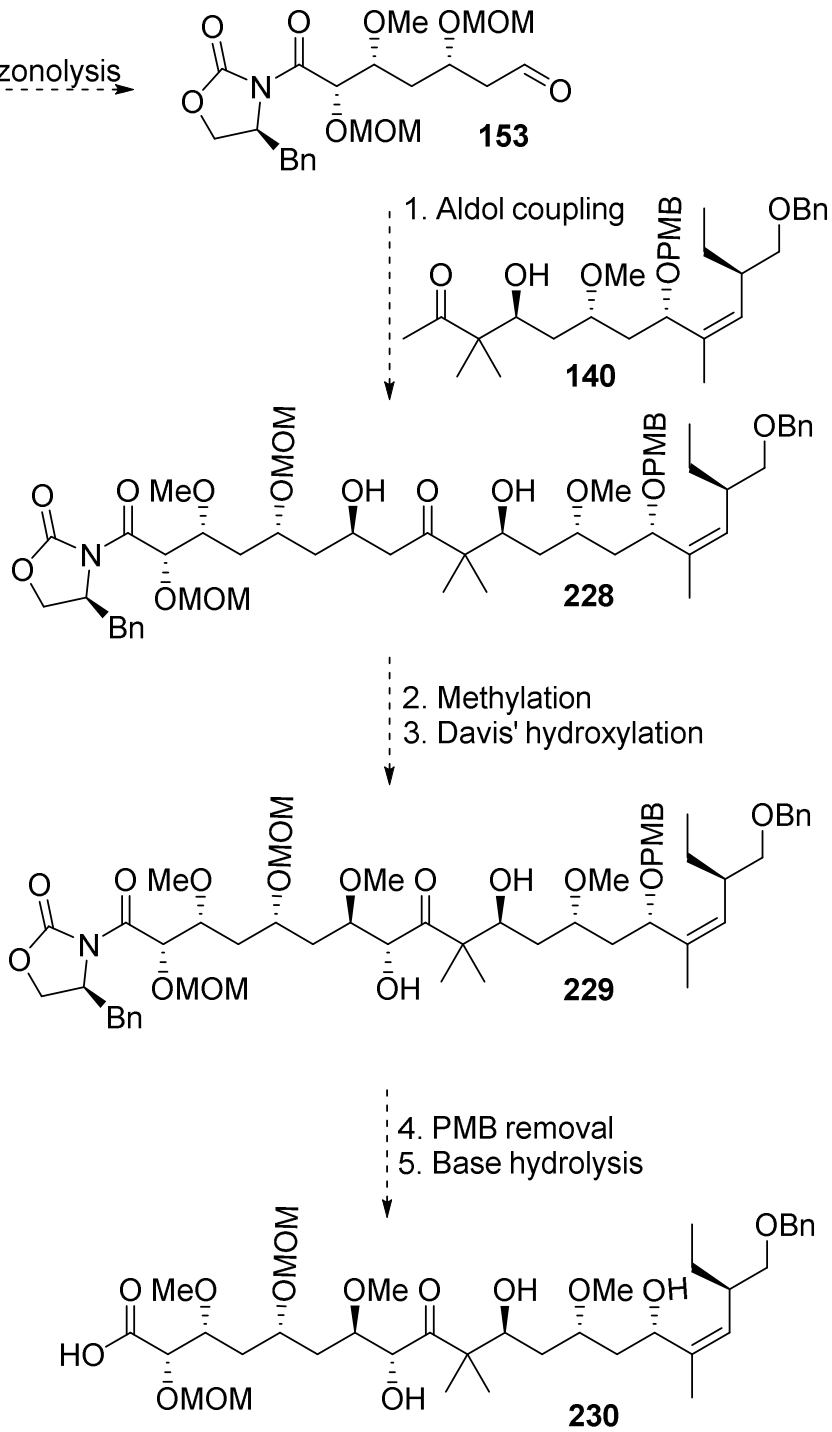

6. Yamaguchi

$$
\text { macrolactonisation }
$$

7. Global deprotection

PelA (1)

Scheme 5.7: Plan for the completion of the synthesis of PelA through macrolactonisation

After the methylation, $\alpha$-hydroxylation using Davis' methodology ${ }^{82,83}$ should give the C8 hydroxyl (229). The PMB group at O15 and the auxiliary would then be removed to give the seco-acid (230). Macrolactonisation using the Yamaguchi protocol ${ }^{57,58}$ and global deprotection should give peloruside A after 21 steps (Ils). Incomplete selectivity in the Davis' hydroxylation would also allow access to 8-epi-PelA that would be used to determine the effect of a configurational change at that position on the binding and activity. 
In both approaches, the implemented changes to the original synthetic plan (Chapter 1) have reduced the number of steps in the longest linear sequence from 22 and 23 (for the RCM and macrolactonisation, respectively) to 21 steps. In our future efforts we aim to further decrease this number and improve the overall yield. Additional work on assessing and improving the scalability of the two synthetic approaches will hopefully lead to an efficient route that will be able to deliver PelA in large quantities. Access to PelA will enable the manufacture of a drug and subsequent testing in vivo and in clinical trials. 


\section{Experimental}

\section{General experimental}

Unless otherwise stated, the following conditions were applied. All reactions were performed under an inert atmosphere (nitrogen or argon) delivered through a Schlenk line or from a balloon. The glassware was kept in an oven at $110{ }^{\circ} \mathrm{C}$ for at least 24 hours then vacuum-dried or flame-dried under vacuum before use. Standard syringe/cannulation techniques were applied. Commonly used solvents: tetrahydrofuran (THF), dichloromethane $\left(\mathrm{CH}_{2} \mathrm{Cl}_{2}\right)$, diethylether $\left(\mathrm{Et}_{2} \mathrm{O}\right)$, and toluene $\left(\mathrm{C}_{6} \mathrm{H}_{5} \mathrm{CH}_{3}\right)$ were either taken from solvent purification system (using Innovative Technology's PureSolv system) or freshly distilled from calcium hydride (for $\mathrm{CH}_{2} \mathrm{Cl}_{2}$ and toluene) or sodium metal, benzoquinone (for THF). Methanol $(\mathrm{MeOH})$ was distilled from sodium then dried over activated $3 \AA$ molecular sieves. Acetonitrile was distilled from $\mathrm{CaH}_{2}$ through a Vigreux column. Acetone was purified by first stirring overnight with boron trioxide $(5 \% \mathrm{w} / \mathrm{v})$ under an inert atmosphere followed by distillation. DMPU was distilled from $\mathrm{CaH}_{2}$ under reduced pressure. Triethylamine was used freshly distilled from $\mathrm{CaH}_{2}$. Anhydrous $\mathrm{N}, \mathrm{N}$-dimethylformamide (DMF) was used as received (Acros, $99.8 \%$, extra dry over molecular sieves). Sodium hydride $(\mathrm{NaH})$ was obtained as $60 \%$ $(w / w)$ dispersion in paraffin oil and was used as obtained. Other reagents were used as obtained from commercial suppliers without further purification. Reaction progress was monitored using thin layer chromatography (TLC) with silica $G / U_{254}$ pre-coated polyester plates and visualised by either UV fluorescence quenching $(254 \mathrm{~nm})$ or by staining. General purpose stains used were ceric ammonium molybdate, phosphomolybdic acid or $p$ anisaldehyde. For substrates with groups capable of oxidation, potassium permanganate stain was used. Retention factor $\left(R_{f}\right)$ values were calculated by dividing the distance run by compound with the distance run by specified solvent system. Purification of products via flash chromatography was conducted using a column filled with Silica Zeoprep 60 (40-63 microns) as the matrix, obtained from Pure Science Ltd, with solvent systems as indicated. ${ }^{1} \mathrm{H}$ and ${ }^{13} \mathrm{C}$ NMR spectra were recorded on a Varian Unity Inova 500. The spectrometer was operating at $500 \mathrm{MHz}$ for ${ }^{1} \mathrm{H}$ nuclei and $125 \mathrm{MHz}$ for ${ }^{13} \mathrm{C}$ nuclei and was also used for COSY, HSQC and HMBC experiments. Some spectra were obtained on Varian Unity Inova 600 (operating at $600 \mathrm{MHz}$ for ${ }^{1} \mathrm{H}$ nuclei and $151 \mathrm{MHz}$ for ${ }^{13} \mathrm{C}$ nuclei). NOESY spectra were 
recorded on a Varian Unity Inova $300\left(300 \mathrm{MHz}\right.$ for $\left.{ }^{1} \mathrm{H}\right)$. Spectra were recorded in the solvent indicated. Proto-deuterated solvent signals were used as internal references $\left(\mathrm{CDCl}_{3}\right.$ : $\left.\delta_{H} 7.26, \delta_{C} 77.16, \mathrm{CD}_{3} \mathrm{OD}: \delta_{H} 4.87,3.31, \delta_{C} 49.00\right)$. Chemical shifts are expressed in parts per million (ppm) relative to the residual non-deuterated solvent in ${ }^{1} \mathrm{H}$ NMR and to deuterated solvent in ${ }^{13} \mathrm{C}$ NMR. The signals from ${ }^{1} \mathrm{H}$ spectra were recorded as chemical shift $(\delta)$ in parts per million (ppm) relative to an internal standard, relative integral, multiplicity of the signal ( $\mathrm{s}=$ singlet, $\mathrm{d}=$ doublet, $\mathrm{t}=$ triplet, $\mathrm{m}=$ multiplet, $\mathrm{dd}=$ doublet of doublets, $\mathrm{dt}=$ doublet of triplets, etc.), coupling constants $(J, \mathrm{~Hz})$ and the assignment of the atom. Signals in the ${ }^{13} \mathrm{C}$ were recorded as chemical shifts $(\delta)$ in parts per million (ppm) relative to an internal standard and assignment of the atom. Optical rotations were measured on Autopol II polarimeter from Rudolph Research Analytical at $589 \mathrm{~nm}$, using a $1.5 \mathrm{~mL}$ optical rotation cell and the $[\alpha]_{D}$ values calculated according to equation: $[\alpha]_{D}=(100 \times \alpha) /(c \times I)$, where $\alpha$ is the measured absorbance, $\mathrm{c}$ is the concentration in $\mathrm{g} / 100 \mathrm{ml}$ and $\mathrm{I}$ is the path-length in $\mathrm{dm}$. Infrared spectra were obtained on a Bruker Tensor 27 FTIR spectrometer with ATR accessory and quoted as wavelengths $(v)$ in $\mathrm{cm}^{-1}$. Mass spectra were obtained in high resolution, using electrospray ionisation (ESI) technique on a 6530 Accurate Mass Q-TOF LC/MS instrument (Agilent Technologies). The mass spectral data are quoted as molecular ion peaks $\mathrm{m} / \mathrm{z}$. The structure of each compound is presented with the corresponding method of preparation and spectroscopic data.

\section{Experimental for Chapter 2}

\section{(R)-4-Benzyl-3-butryryloxazolidin-2-one (27)}

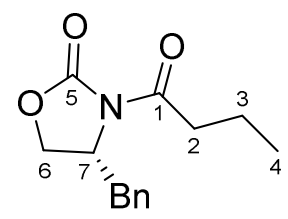

(R)-4-Benzyl-1,3-oxazolidin-2-one (1.048 g, $5.92 \mathrm{mmol})$ was dissolved in dry THF (15 mL) and cooled to $-78{ }^{\circ} \mathrm{C}$ under an atmosphere of argon. To the solution was then added $\mathrm{n}$-BuLi ( $2 \mathrm{M}$ in cyclohexane, $3.55 \mathrm{~mL}, 7.10 \mathrm{mmol}, 1.2$ equiv.) dropwise and the reaction became orange in colour. The addition of $n$-BuLi was followed by the addition of butyryl chloride $(0.68 \mathrm{~mL}$, $6.7 \mathrm{mmol}, 1.1$ equiv.) and the colour changed to pale yellow. The reaction was stirred for 30 
min at $-78^{\circ} \mathrm{C}$ then allowed to warm to room temperature before being quenched with sat. aq. $\mathrm{NH}_{4} \mathrm{Cl}(30 \mathrm{~mL})$. The aqueous layer was extracted with EtOAc $(3 \times 30 \mathrm{~mL})$, the combined organic layers were washed with aq. $1 \mathrm{M} \mathrm{NaOH}(30 \mathrm{~mL})$, then brine $(30 \mathrm{~mL})$, dried over anhydrous $\mathrm{MgSO}_{4}$ and concentrated. The resulting crude material was purified on silica column, the product 27 eluting with 5:1 PE/EtOAc as a colourless oil (1.3726 g, 94\% yield). Experimental spectroscopic data for $\mathbf{2 7}$ matched those previously reported. ${ }^{86}$

$\mathbf{R}_{f}=0.53(3: 1 \mathrm{PE} / \mathrm{EA}) ;[\boldsymbol{\alpha}]_{\mathrm{D}}{ }^{24}=-64.3\left(c 1.09, \mathrm{CHCl}_{3}\right)\left[\mathrm{lit}^{86}{ }^{86}[\boldsymbol{\alpha}]_{\mathrm{D}}{ }^{23}=-57.1\right.$ (c 1.14, $\left.\left.\mathrm{CHCl}_{3}\right)\right] ;{ }^{1} \mathbf{H} \mathrm{NMR}$ $\left(500 \mathrm{MHz}, \mathrm{CDCl}_{3}\right) \delta 7.37-7.31(\mathrm{~m}, 2 \mathrm{H}, \mathrm{Ph}), 7.28(\mathrm{~d}, J=7.3 \mathrm{~Hz}, 1 \mathrm{H}, \mathrm{Ph}), 7.24-7.19(\mathrm{~m}, 2 \mathrm{H}$, Ph), 4.68 (ddt, J = 9.6, 7.4, 3.2 Hz, 1H, H-7), $4.23-4.15$ (m, 2H, H-6), 3.30 (dd, $J=13.4,3.4$ $\mathrm{Hz}, 1 \mathrm{H}, \mathrm{CH}_{2} \mathrm{aPh}$ ), $3.00-2.83(\mathrm{~m}, 2 \mathrm{H}, \mathrm{H}-2), 2.77$ (dd, $J=13.4,9.6 \mathrm{~Hz}, 1 \mathrm{H}, \mathrm{CH}_{2} \mathrm{bPh}$ ), $1.78-1.68$ (m, 2H, H-3), 1.01 (t, J = 7.4 Hz, 3H, H-4).; ${ }^{13} \mathrm{C}$ NMR (125 MHz, CDCl 3 ) $\delta 173.4$ (C, C-1), 153.6 (C, C-5), 135.5 (C, Ph), $129.6(2 \mathrm{CH}, \mathrm{Ph}), 129.1(2 \mathrm{CH}, \mathrm{Ph}), 127.5(\mathrm{CH}, \mathrm{Ph}), 66.3\left(\mathrm{CH}_{2}, \mathrm{C}-6\right), 55.3$ (CH, C-7), $38.1\left(\mathrm{CH}_{2}, \mathrm{CH}_{2} \mathrm{Ph}\right), 37.5\left(\mathrm{CH}_{2}, \mathrm{C}-2\right), 17.9\left(\mathrm{CH}_{2}, \mathrm{C}-3\right), 13.8\left(\mathrm{CH}_{3}, \mathrm{C}-4\right)$; IR (film from $\left.\mathrm{CHCl}_{3}\right) v_{\max } 2965,2876,1778,1699,1388,1210,1126,703 \mathrm{~cm}^{-1}$.

(R)-4-Benzyl-3-((S)-2-(hydroxymethyl)butanoyl)oxazolidin-2-one (59)

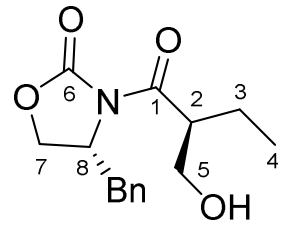

(R)-4-Benzyl-3-butyryloxazolidin-2-one 27 (1.096 g, $4.43 \mathrm{mmol}$ ) was dissolved in dry DCM $(15 \mathrm{~mL})$ under nitrogen and cooled to $0{ }^{\circ} \mathrm{C}$, then treated with a $1 \mathrm{M}$ solution of $\mathrm{TiCl}_{4}$ in dichloromethane (4.90 mL, 1.1 equiv.) dropwise. After 10 minutes of stirring at same temperature $\mathrm{Et}_{3} \mathrm{~N}$ was added $(0.68 \mathrm{~mL}, 4.87 \mathrm{mmol}, 1.1$ equiv.) and the reaction mixture colour changed to dark-red. After an hour of stirring at $0{ }^{\circ} \mathrm{C}, 1,3,5$-trioxane $(0.486 \mathrm{~g}, 5.32$ mmol, 1.2 equiv.) in DCM (4 mL) was added followed by another 1.1 equivalents of $\mathrm{TiCl}_{4}$ solution and the reaction left stirring at the same temperature for another 1.5 hours. The reaction was quenched with sat. $\mathrm{NH}_{4} \mathrm{Cl}$ solution $(20 \mathrm{~mL})$ and the aqueous phase extracted with DCM (2x $20 \mathrm{~mL})$. The combined organic fractions were washed with water $(30 \mathrm{~mL})$ then brine $(30 \mathrm{~mL})$, dried over anhydrous $\mathrm{MgSO}_{4}$ and the solvent removed under reduced pressure. The crude reaction mixture was purified by gradient chromatography (5:1 PE/EA 
then $3: 1$ then $1: 1$ ) on silica gel to yield the product 59 as a yellow oil ( $0.887 \mathrm{~g}, 72 \%)$. Spectral properties matched those previously reported for this compound. ${ }^{101,225}$

$\mathbf{R}_{f}=0.15(3: 1 \mathrm{PE} / \mathrm{EA}) ;[\boldsymbol{\alpha}]_{\mathrm{D}}{ }^{27}=-119.1$ (c 1.15, $\left.\mathrm{CHCl}_{3}\right)\left[\mathrm{lit}^{101}{ }^{101}[\boldsymbol{\alpha}]_{\mathrm{D}}{ }^{22.5}=-52.1\right.$ (c 1.00, $\left.\left.\mathrm{CHCl}_{3}\right)\right] ;{ }^{1} \mathrm{H}-$ NMR $\left(500 \mathrm{MHz} \mathrm{CDCl}_{3}\right): \delta 7.36-7.31(\mathrm{~m}, 2 \mathrm{H}, \mathrm{Ph}), 7.30-7.27(\mathrm{~m}, 1 \mathrm{H}, \mathrm{Ph}), 7.25-7.21(\mathrm{~m}$, $2 \mathrm{H}, \mathrm{Ph}), 4.75-4.66(\mathrm{~m}, 1 \mathrm{H}, \mathrm{H}-8), 4.26-4.15$ (m, 2H, H-7), $3.93-3.81$ (m, 3H, H-2, H-5), 3.30 (dd, $J=13.5,3.5 \mathrm{~Hz}, 1 \mathrm{H}, \mathrm{CH}_{2} \mathrm{aPh}$ ), 2.82 (ddd, $J=13.5,9.4,1.2 \mathrm{~Hz}, 1 \mathrm{H}, \mathrm{CH}_{2} \mathrm{bPh}$ ), 2.30 (t, $J=5.7$ $\mathrm{Hz}, 1 \mathrm{H}, \mathrm{OH}), 1.80-1.70$ (m, 1H, H-3a), $1.67-1.54$ (m, 1H, H-3b), 0.97 (t, J= 7.5 Hz, 3H, H-4); ${ }^{13}$ C-NMR (126 MHz, CDCl $)$ ): $\delta 175.9$ (C, C-1), 153.7 (C, C-6), 135.3 (C, Ph), 129.6 (2CH, Ph), $129.1(2 \mathrm{CH}, \mathrm{Ph}), 127.5(\mathrm{CH}, \mathrm{Ph}), 66.3\left(\mathrm{CH}_{2}, \mathrm{C}-7\right), 63.6\left(\mathrm{CH}_{2}, \mathrm{C}-5\right), 55.6(\mathrm{CH}, \mathrm{C}-8), 47.2(\mathrm{CH}, \mathrm{C}-$ 2), $38.0\left(\mathrm{CH}_{2}, \mathrm{CH}_{2} \mathrm{Ph}\right), 21.8\left(\mathrm{CH}_{2}, \mathrm{C}-3\right), 11.8\left(\mathrm{CH}_{3}, \mathrm{C}-4\right)$; HRMS (ESI) Found 278.1398. Calculated for $\mathrm{C}_{15} \mathrm{H}_{20} \mathrm{NO}_{4}{ }^{+}[\mathrm{M}+\mathrm{H}]^{+}$278.1387. $\Delta=3.95$ ppm; IR (film from $\mathrm{CHCl}_{3}$ ) $v_{\max } 3489$, $2967,2933,2878,1775,1695,1456,1390,1351,1211 \mathrm{~cm}^{-1}$.

\section{$(4 R, 4 ' R)-3,3^{\prime}-\left((2 S, 2 ' S)-2,2^{\prime}-((\right.$ Methylenebis(oxy))bis(methylene))bis(butanoyl))bis(4-}

\section{benzyloxazolidin-2-one) (119)}

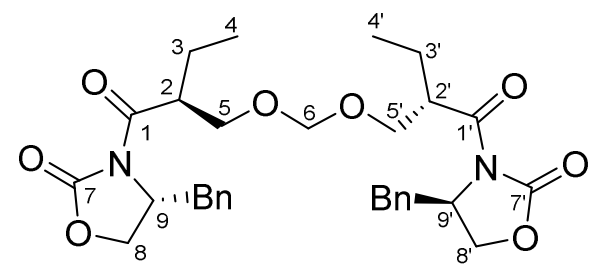

During certain attempts at the reaction, depending on the quality of the $\mathrm{TiCl} 4$ solution, variable amounts of a by-product were obtained, assigned as dimer 119.

${ }^{1}$ H NMR $\left(500 \mathrm{MHz}, \mathrm{CDCl}_{3}\right) \delta 7.34-7.29(\mathrm{~m}, 4 \mathrm{H}, \mathrm{Ph}), 7.28-7.22(\mathrm{~m}, 6 \mathrm{H}, \mathrm{Ph}), 4.75-4.70(\mathrm{~m}$, $\left.2 \mathrm{H}, \mathrm{H}-9 / \mathrm{H}^{-9}\right), 4.69$ (s, 2H, H-6), $4.22-4.12\left(\mathrm{~m}, 4 \mathrm{H}, \mathrm{H}-8 / \mathrm{H}-8^{\prime}\right), 4.13-4.05$ (m, 2H, H-2/H-2'), 3.86 (dd, J = 9.5, 8.0 Hz, 2H, H-5a/H-5'a), 3.74 (dd, J = 9.5, 4.9 Hz, 2H, H-5b/H-5'b), 3.26 (dd, J $=13.4,3.2 \mathrm{~Hz}, 2 \mathrm{H}, \mathrm{CH}_{2} \mathrm{aPh}$ ), $2.80\left(\mathrm{dd}, J=13.5,9.4 \mathrm{~Hz}, 2 \mathrm{H}, \mathrm{CH}_{2} \mathrm{bPh}\right), 1.81-1.69(\mathrm{~m}, 2 \mathrm{H}, \mathrm{H}-$ 3a/H-3'a), 1.65 - 1.51 (m, 2H, H-3b/H-3'b), 0.94 (t, J = $7.5 \mathrm{~Hz}, 6 \mathrm{H}, \mathrm{H}-4 / \mathrm{H}-4$ '); ${ }^{13} \mathrm{C}$ NMR (126 $\left.\mathrm{MHz}, \mathrm{CDCl}_{3}\right) \delta 174.9$ (2C, C-1/C-1'), 153.4 (2C, C-7/C-7'), 135.5 (2C, Ph), 129.6 (4CH, Ph), $129.0(4 \mathrm{CH}, \mathrm{Ph}), 127.4(2 \mathrm{CH}, \mathrm{Ph}), 95.5\left(\mathrm{CH}_{2}, \mathrm{C}-6\right), 68.4\left(2 \mathrm{CH}_{2}, \mathrm{C}-5 / \mathrm{C}-5^{\prime}\right), 66.0\left(2 \mathrm{CH}_{2}, \mathrm{C}-8 / \mathrm{C}-8^{\prime}\right)$, $55.5\left(2 \mathrm{CH}, \mathrm{C}-9 / \mathrm{C}-9^{\prime}\right), 44.8\left(2 \mathrm{CH}, \mathrm{C}-2 / \mathrm{C}-2^{\prime}\right), 37.9\left(2 \mathrm{CH}_{2}, \mathrm{CH}_{2} \mathrm{Ph}\right), 22.3\left(2 \mathrm{CH}_{2}, \mathrm{C}-3 / \mathrm{C}-3{ }^{\prime}\right), 11.6$ $\left(2 \mathrm{CH}_{3}, \mathrm{C}-4 / \mathrm{C}-4^{\prime}\right)$. HRMS (ESI) Found 567.2714. Calculated for $\mathrm{C}_{31} \mathrm{H}_{39} \mathrm{~N}_{2} \mathrm{O}_{8}^{+}[\mathrm{M}+\mathrm{H}]^{+}$567.2701. 
$\Delta=2.3$ ppm; IR (film from $\mathrm{CHCl}_{3}$ ) $v_{\max } 2968,2932,2878,1772,1692,1454,1388,1349,1209$, $1014,729,702 \mathrm{~cm}^{-1}$.

(R)-4-Benzyl-3-((S)-2-(((tert-butyldimethylsilyl)oxy)methyl)butanoyl)oxazolidin-2-one (60)

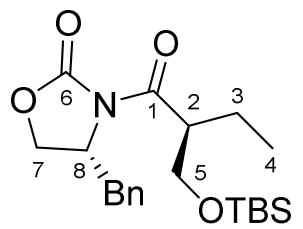

Alcohol 59 (0.383 g, $1.38 \mathrm{mmol})$ was dissolved in dry DCM $(6 \mathrm{~mL})$ under nitrogen, cooled to $0{ }^{\circ} \mathrm{C}$, then treated with tert-butyldimethylsilyl chloride (TBSCl) $(0.326 \mathrm{~g}, 2.10 \mathrm{mmol}, 1.5$ equiv.), 4-dimethylaminopyridine (DMAP) $\left(0.021 \mathrm{~g}, 0.17 \mathrm{mmol}, 0.1\right.$ equiv.) and $\mathrm{Et}_{3} \mathrm{~N}(0.40$ $\mathrm{mL}, 2.8 \mathrm{mmol}, 2$ equiv.). The reaction was allowed to warm to room temperature and stirred overnight. The reaction was quenched with sat. ammonium chloride solution (15 mL) at $0{ }^{\circ} \mathrm{C}$ and extracted with DCM (2x $20 \mathrm{~mL}$ ). The combined organic layers were dried over anhydrous magnesium sulfate and concentrated under reduced pressure. Purification by flash chromatography (9:1 petroleum ether/ethyl acetate) gave product 60 as a colourless oil $(0.474 \mathrm{~g}, 88 \%)$. Spectral data were in accordance with those reported in the literature. ${ }^{101}$

$\mathbf{R}_{f}=0.47(5: 1 \mathrm{PE} / \mathrm{EA}) ;{ }^{1} \mathrm{H}-\mathrm{NMR}\left(500 \mathrm{MHz}, \mathrm{CDCl}_{3}\right): \delta 7.34-7.29(\mathrm{~m}, 2 \mathrm{H}, \mathrm{Ph}), 7.28-7.24(\mathrm{~m}$, $1 \mathrm{H}, \mathrm{Ph}), 7.24-7.21(\mathrm{~m}, 2 \mathrm{H}, \mathrm{Ph}), 4.76-4.67(\mathrm{~m}, 1 \mathrm{H}, \mathrm{H}-8), 4.19-4.06(\mathrm{~m}, 2 \mathrm{H}, \mathrm{H}-7), 4.04-$ $3.96(\mathrm{~m}, 1 \mathrm{H}, \mathrm{H}-2), 3.92(\mathrm{td}, J=9.6,1.5 \mathrm{~Hz}, 1 \mathrm{H}, \mathrm{H}-5 \mathrm{a}$ ), 3.80 (dd, J=9.2, $5.2 \mathrm{~Hz}, 1 \mathrm{H}, \mathrm{H}-5 \mathrm{~b}), 3.28$ (dd, $J=13.4,3.2 \mathrm{~Hz}, 1 \mathrm{H}, \mathrm{CH}_{2} \mathrm{aPh}$ ), 2.71 (dd, $J=13.4,9.5 \mathrm{~Hz}, 1 \mathrm{H}, \mathrm{CH}_{2} \mathrm{bPh}$ ), $1.77-1.65(\mathrm{~m}, 1 \mathrm{H}$, $\mathrm{H}-3 \mathrm{a}$ ), $1.60-1.50$ (m, 1H, H-3b), 0.93 (t, J=7.5 Hz, 3H, H-4), 0.88 (d, J = 1.4 Hz, 9H, OTBS), $0.06\left(\mathrm{~d}, J=5.2 \mathrm{~Hz}, 6 \mathrm{H}\right.$, OTBS); ${ }^{13} \mathrm{C}-\mathrm{NMR}\left(126 \mathrm{MHz}, \mathrm{CDCl}_{3}\right): \delta 175.0$ (C, C-1), 153.3 (C, C-6), 135.6 (C, Ph), 129.5 (2CH, Ph), 129.0 (2CH, Ph), 127.3 (CH, Ph), $65.9\left(\mathrm{CH}_{2}, \mathrm{C}-7\right), 64.1$ ( $\mathrm{CH}_{2}, \mathrm{C}-$ 5), $55.3(\mathrm{CH}, \mathrm{C}-8), 47.3(\mathrm{CH}, \mathrm{C}-2), 38.1\left(\mathrm{CH}_{2}, \mathrm{CH}_{2} \mathrm{Ph}\right), 25.9\left(3 \mathrm{CH}_{3}, \mathrm{OTBS}\right), 21.8\left(\mathrm{CH}_{2}, \mathrm{C}-3\right), 18.3$ (C, OTBS), $11.6\left(\mathrm{CH}_{3}, \mathrm{C}-4\right),-5.39\left(\mathrm{CH}_{3}\right.$, OTBS $),-5.45\left(\mathrm{CH}_{3}, \mathrm{OTBS}\right)$. 


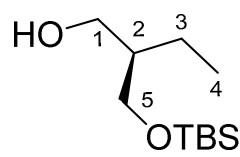

Acyl oxazolidinone 60 (0.370 g, $0.95 \mathrm{mmol})$ was dissolved in dry $\mathrm{Et}_{2} \mathrm{O}(10 \mathrm{~mL})$ and water $\left(0.02 \mathrm{~mL}, 1\right.$ equiv.), cooled to $0{ }^{\circ} \mathrm{C}$ and treated with lithium borohydride ( $2 \mathrm{M}$ in THF, 1.00 $\mathrm{mL}, 2.2$ equiv.) under a nitrogen atmosphere. The reaction was stirred at the same temperature for $2 \mathrm{~h}$, then quenched with brine $(10 \mathrm{~mL})$, warmed to room temperature and stirred for another hour. The layers were separated and the aqueous layer extracted with $\mathrm{Et}_{2} \mathrm{O}$. The combined organic layers were dried over anhydrous magnesium sulfate and concentrated. The crude reaction mixture was purified using flash chromatography (9:1 petroleum ether/ethyl acetate) to afford 61 as a colourless oil ( $0.150 \mathrm{~g}, 73 \%)$. Experimental properties matched those previously reported. ${ }^{1,102}$

$\mathbf{R}_{f}=0.31(5: 1 \mathrm{PE} / \mathrm{EA}) ;[\boldsymbol{\alpha}]_{\mathrm{D}}{ }^{24}=+14.8\left(c 0.23, \mathrm{CHCl}_{3}\right)\left[\mathrm{lit}^{102}[\boldsymbol{\alpha}]_{\mathrm{D}}{ }^{35}=+6.2\right.$ (c 1.00, $\left.\left.\mathrm{CHCl}_{3}\right)\right] ;{ }^{1} \mathbf{H}-$ NMR (500 MHz, CDCl 3 ): $\delta 3.82$ (ddd, $J=9.9,4.0,0.9$ Hz, 1H, H-5a), $3.77-3.72(\mathrm{~m}, 1 \mathrm{H}, \mathrm{H}-1 \mathrm{a})$, $3.67-3.63$ (m, 1H, H-1b), 3.60 (dd, $J=9.8,7.5 \mathrm{~Hz}, 1 \mathrm{H}, \mathrm{H}-5 \mathrm{~b}$ ), 2.93 (dd, $J=6.7,4.4 \mathrm{~Hz}, 1 \mathrm{H}$, $\mathrm{OH}), 1.67-1.61(\mathrm{~m}, 1 \mathrm{H}, \mathrm{H}-2), 1.33-1.22(\mathrm{~m}, 2 \mathrm{H}, \mathrm{H}-3), 0.93(\mathrm{t}, J=7.4 \mathrm{~Hz}, 3 \mathrm{H}, \mathrm{H}-4), 0.90(\mathrm{~s}$, 9H, OTBS), 0.07 (s, 6H, OTBS); ${ }^{13} \mathrm{C}$ NMR (126 MHz, CDCl $) \delta 67.5\left(\mathrm{CH}_{2}, \mathrm{C}-5\right), 66.8\left(\mathrm{CH}_{2}, \mathrm{C}-1\right)$, 43.7 (CH, C-2), 26.0 (3CH $\mathrm{CH}_{3}$ OTBS), 20.7 ( $\left.\mathrm{CH}_{2}, \mathrm{C}-3\right), 18.3$ (C, OTBS), $11.9\left(\mathrm{CH}_{3}, \mathrm{C}-4\right),-5.4\left(\mathrm{CH}_{3}\right.$, OTBS), -5.5 ( $\mathrm{CH}_{3}$, OTBS); IR (film from $\mathrm{CHCl}_{3}$ ) $v_{\max } 3375,2956,2929,2858,1461,1253,1090$, $837,775 \mathrm{~cm}^{-1}$.

Methyl (R,Z)-4-(((tert-butyldimethylsilyl)oxy)methyl)-2-methylhex-2-enoate (62)<smiles>CC[C@H](/C=C(/F)C(=O)OC)CO[Sb]</smiles>

To a solution of alcohol 61 (38.8 mg, $0.18 \mathrm{mmol}$ ) and TEMPO (3.0 mg, $0.02 \mathrm{mmol}, 0.1$ equiv.) in dry DCM (4 mL), was added BAIB (64.0 mg, $0.20 \mathrm{mmol}, 1.1$ equiv.) at room temperature under nitrogen atmosphere. After 2.5 hours of stirring at the same temperature (TLC analysis in 5:1 PE/EA showed the absence of alcohol 61 ) the reaction was diluted with DCM 
$(2 \mathrm{~mL})$ and the organic layer washed sequentially with sat. aq. $\mathrm{Na}_{2} \mathrm{~S}_{2} \mathrm{O}_{3}(5 \mathrm{~mL})$, sat. aq. $\mathrm{NaHCO}_{3}(10 \mathrm{~mL})$ and brine $(10 \mathrm{~mL})$, then dried with $\mathrm{MgSO}_{4}$ and concentrated under reduced pressure at room temperature. The crude mixture containing aldehyde was used in the next step without further purification.

Phosphonate 122 (0.137 g, $0.26 \mathrm{mmol}, 1.4$ equiv.) was dissolved in dry THF ( $3 \mathrm{~mL}$ ) and cooled to $0{ }^{\circ} \mathrm{C}$ then treated with $\mathrm{Nal}(0.042 \mathrm{mg}, 0.28 \mathrm{mmol}, 1.5$ equiv.) and DBU $(0.04 \mathrm{~mL}$, $0.28 \mathrm{mmol}, 1.5$ equiv.). The reaction was stirred for $10 \mathrm{~min}$ that temperature then cooled to $-78^{\circ} \mathrm{C}$ and the aldehyde in THF $(1 \mathrm{~mL})$ was added dropwise via cannula. The reaction was stirred at $-78{ }^{\circ} \mathrm{C}$ for $45 \mathrm{~min}$, then for another hour at $0{ }^{\circ} \mathrm{C}$. The reaction was quenched with sat. aq. $\mathrm{NH}_{4} \mathrm{Cl}$ solution $(5 \mathrm{~mL})$ and the aqueous layer extracted with $\mathrm{Et}_{2} \mathrm{O}(2 \times 10 \mathrm{~mL})$. The organic layer was washed with brine $(5 \mathrm{~mL})$, dried with anhydrous $\mathrm{MgSO}_{4}$ and concentrated. The crude mixture was purified using flash chromatography (40:1 PE/EA) to give the product 62 as a colourless oil $(0.026 \mathrm{~g}, 30 \%)$. The spectral data were similar to those previously reported for the corresponding ethyl ester. ${ }^{1}$

$\mathbf{R}_{f}=0.25(40: 1 \mathrm{PE} / \mathrm{EA}) ;{ }^{1} \mathrm{H}-\mathrm{NMR}\left(500 \mathrm{MHz}, \mathrm{CDCl}_{3}\right): \delta 5.73(\mathrm{dd}, J=10.2,1.5 \mathrm{~Hz}, 1 \mathrm{H}, \mathrm{H}-3), 3.73$ (s, 3H, H-9), $3.58-3.49$ (m, 2H, H-8), $3.15-3.07$ (m, 1H, H-4), 1.92 (d, J=1.4 Hz, 3H, H-7), $1.63-1.54$ (m, 1H, H-5b), $1.30-1.21$ (m, 1H, H-5a), 0.88 (s, 9H, OTBS), 0.85 (t, $J=7.5 \mathrm{~Hz}$, $3 \mathrm{H}, \mathrm{H}-6), 0.02$ (d, J = $2.9 \mathrm{~Hz}, 6 \mathrm{H}, \mathrm{OTBS})$.

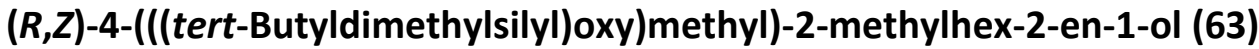

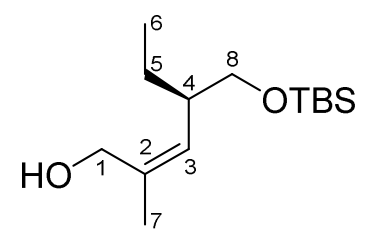

Methyl ester $62(25.7 \mathrm{mg}, 0.09 \mathrm{mmol})$ was dissolved in dry DCM $(2 \mathrm{~mL})$ and cooled to $-78^{\circ} \mathrm{C}$, then treated with a $1 \mathrm{M}$ solution of DIBAL-H in cyclohexane $(0.22 \mathrm{~mL}, 2.5$ equiv). The reaction was stirred at $-78^{\circ} \mathrm{C}$ for 10 min then quenched with sat. aq. Rochelle's salt solution at the same temperature and stirred for another 2 hours at room temperature. The aqueous layer was extracted with DCM $(3 \times 10 \mathrm{~mL})$ and the combined organic layers washed with brine, dried over anhydrous $\mathrm{MgSO}_{4}$ and concentrated under reduced pressure. Purification using flash chromatography (9:1 PE/EA) gave the alcohol product 63 as a colourless oil 
(0.015 g, 63\%). Experimental properties were in accordance with those published in literature. $^{1}$

$\mathbf{R}_{f}=0.27(9: 1 \mathrm{PE} / \mathrm{EA}) ;{ }^{1} \mathrm{H}-\mathrm{NMR}\left(500 \mathrm{MHz}, \mathrm{CDCl}_{3}\right): \delta 4.95$ (dq $\left.J=10.1,1.4 \mathrm{~Hz}, 1 \mathrm{H}, \mathrm{H}-3\right), 4.22$ (dd, $J=11.7,2.4 \mathrm{~Hz}, 1 \mathrm{H}, \mathrm{H}-1 \mathrm{a}$ ), 3.79 (dd, $J=11.6,8.0 \mathrm{~Hz}, 1 \mathrm{H}, \mathrm{H}-1 \mathrm{~b}$ ), 3.59 (dd, $J=9.4,4.6 \mathrm{~Hz}$, $1 \mathrm{H}, \mathrm{H}-8 \mathrm{a}), 3.25$ (t, J = 9.3 Hz, 1H, H-8b), 2.60 (dd, J = 8.4, 3.3 Hz, 1H, OH), $2.54-2.45(\mathrm{~m}, 1 \mathrm{H}$, $\mathrm{H}-4), 1.83$ (d, J = $1.4 \mathrm{~Hz}, 3 \mathrm{H}, \mathrm{H}-7), 1.44-1.34$ (m, 1H, H-5a), $1.17-1.06$ (m, 1H, H-5b), 0.89 (s, 9H, OTBS), 0.86 (t, $J=7.4 \mathrm{~Hz}, 3 \mathrm{H}, \mathrm{H}-6), 0.05$ (s, 6H, OTBS); ${ }^{13} \mathrm{C}-\mathrm{NMR}\left(126 \mathrm{MHz}^{\mathrm{C}} \mathrm{CDCl}_{3}\right): \delta$ 138.1 (C, C-2), $131.0(\mathrm{CH}, \mathrm{C}-3), 67.0\left(\mathrm{CH}_{2}, \mathrm{C}-8\right), 62.3\left(\mathrm{CH}_{2}, \mathrm{C}-1\right), 42.7(\mathrm{CH}, \mathrm{C}-4), 26.2\left(3 \mathrm{CH}_{3}\right.$, OTBS), $24.7\left(\mathrm{CH}_{2}, \mathrm{C}-5\right), 22.8\left(\mathrm{CH}_{3}, \mathrm{C}-7\right), 18.7$ (C, OTBS), $12.0\left(\mathrm{CH}_{3}, \mathrm{C}-6\right),-5.3\left(2 \mathrm{CH}_{3}, \mathrm{OTBS}\right)$.

$(4 S, 7 R, Z)-7-(((t e r t-B u t y l d i m e t h y l s i l y l) o x y)$ methyl)-4-hydroxy-5-methylnon-5-en-2-one (64)

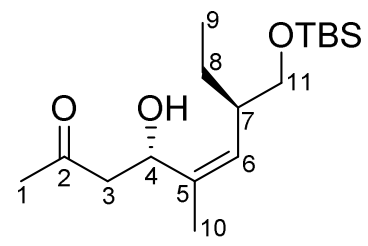

Alcohol 63 (15.8 mg, $0.06 \mathrm{mmol})$ in $0.5 \mathrm{~mL} \mathrm{DCM}$ was added to a solution of DMP $(54.1 \mathrm{mg}$, $0.12 \mathrm{mmol}, 2$ equiv.) and pyridine $(0.01 \mathrm{~mL}, 0.12 \mathrm{mmol}, 2$ equiv. $)$ in $\mathrm{DCM}(1 \mathrm{~mL})$ at $0{ }^{\circ} \mathrm{C}$. The reaction was stirred at the same temperature for 1 hour, after which TLC analysis in 9:1 $P E / E A$ indicated no starting material was present, then quenched with 1:1 v/v sat. aq. $\mathrm{Na}_{2} \mathrm{~S}_{2} \mathrm{O}_{3} /$ sat. aq. $\mathrm{NaHCO}_{3}$. After stirring vigorously for $30 \mathrm{~min}$ at room temperature, the aqueous layer was extracted with $\mathrm{Et}_{2} \mathrm{O}(2 \times 5 \mathrm{~mL})$ and the combined organic layers washed with brine, dried with $\mathrm{MgSO}_{4}$ and concentrated. The crude reaction mixture was used in the next step without purification.

D-proline (7.0 mg, 0.95 equiv.) was premixed with 4:1 DMSO:acetone $(0.25 \mathrm{~mL})$ for $10 \mathrm{~min}$ at room temperature. The aldehyde 118 was then added in $0.10 \mathrm{~mL}$ acetone. The reaction was stirred for 24 hours at room temperature. HRMS analysis of an aliquot indicated the presence of product. The reaction was quenched with sat. aq. $\mathrm{NH}_{4} \mathrm{Cl}(1 \mathrm{~mL})$ and the aqueous layer extracted with EtOAc $(3 \times 2 \mathrm{~mL})$, dried over $\mathrm{MgSO}_{4}$ and concentrated. The crude material was separated and purified on a silica column. The 9:1 PE/EA eluent delivered the aldehyde intermediate 118 as a colourless oil (2.1 mg, 13\%); EA flush delivered the product 64 as a colourless oil (6.8 $\mathrm{mg}, 35 \%)$. 
${ }^{1} \mathrm{H}-\mathrm{NMR}\left(500 \mathrm{MHz}, \mathrm{CDCl}_{3}\right.$ ): $\delta 4.98-4.94(\mathrm{~m}, 2 \mathrm{H}, \mathrm{H}-4, \mathrm{H}-6), 3.55(\mathrm{dd}, J=9.6,5.3 \mathrm{~Hz}, 1 \mathrm{H}, \mathrm{H}-$ 11a), 3.32 (dd, J = 9.6, 8.3 Hz, 1H, H-11b), 2.83 (dd, J = 16.5, $9.1 \mathrm{~Hz}, 1 \mathrm{H}, \mathrm{H}-3 \mathrm{a}$ ), $2.58-2.52$ (m, 1H, H-7), 2.49 (dd, J = 16.4, 4.1 Hz, 1H, H-3b), 2.21 (s, 3H, H-1), 1.72 (d, J=1.4 Hz, 3H, H10), $1.50-1.39$ (m, 1H, H-8a), $1.17-1.07$ (m, 1H, H-8b), 0.88 (s, 9H, OTBS), 0.85 (t, $J=7.5$ $\mathrm{Hz}, 3 \mathrm{H}, \mathrm{C}-9), 0.04$ (2 x s, 6H, OTBS); ${ }^{13} \mathrm{C}-\mathrm{NMR}\left(126 \mathrm{MHz}^{\mathrm{C} C D C l}\right.$ ): $\delta 208.6$ (C, C-2), 138.1 (C, C5), $131.3(\mathrm{CH}, \mathrm{C}-6), 67.0\left(\mathrm{CH}_{2}, \mathrm{C}-11\right), 65.5(\mathrm{CH}, \mathrm{C}-4), 47.9\left(\mathrm{CH}_{2}, \mathrm{C}-3\right), 42.1(\mathrm{CH}, \mathrm{C}-7), 31.0\left(\mathrm{CH}_{3}\right.$, C-1), $26.2\left(3 \mathrm{CH}_{3}, \mathrm{OTBS}\right), 24.7\left(\mathrm{CH}_{2}, \mathrm{C}-8\right), 18.7$ (C, OTBS), $18.5\left(\mathrm{CH}_{3}, \mathrm{C}-10\right), 12.0\left(\mathrm{CH}_{3}, \mathrm{C}-9\right)$, $5.24\left(2 \mathrm{CH}_{3}\right.$, OTBS); HRMS (ESI) Found 315.2349. Calculated for $\mathrm{C}_{17} \mathrm{H}_{35} \mathrm{O}_{3} \mathrm{Si}^{+}[\mathrm{M}+\mathrm{H}]^{+} 315.2350$. $\Delta=0.42 \mathrm{ppm}$.

(R,Z)-4-(((tert-Butyldimethylsilyl)oxy)methyl)-2-methylhex-2-enal (118)<smiles>CC[C@@H](C=C(C)C=O)CO[SbH3]</smiles>

$\mathbf{R}_{f}=0.53\left(10: 1\right.$ PE/EA); ${ }^{1} \mathrm{H}-\mathrm{NMR}\left(500 \mathrm{MHz}, \mathrm{CDCl}_{3}\right): \delta 10.08(\mathrm{~s}, 1 \mathrm{H}, \mathrm{H}-1), 6.24$ (dq, $J=10.8,1.5$ $\mathrm{Hz}, 1 \mathrm{H}, \mathrm{H}-3$ ), 3.64 (dd, J = 9.8, $5.2 \mathrm{~Hz}, 1 \mathrm{H}, \mathrm{H}-8 \mathrm{a}$ ), 3.47 (dd, J = 9.8, $7.3 \mathrm{~Hz}, 1 \mathrm{H}, \mathrm{H}-8 \mathrm{~b}$ ), $3.19-$ $3.10(\mathrm{~m}, 1 \mathrm{H}, \mathrm{H}-4), 1.81$ (d, $J=1.3 \mathrm{~Hz}, 3 \mathrm{H}, \mathrm{H}-7), 1.65-1.57$ (m, $1 \mathrm{H}, \mathrm{H}-5 \mathrm{a}), 1.33-1.23(\mathrm{~m}, 1 \mathrm{H}$, $\mathrm{H}-5 \mathrm{~b}), 0.90$ (t, $J=7.6 \mathrm{~Hz}, 3 \mathrm{H}, \mathrm{H}-6), 0.86$ (s, 9H, OTBS), 0.02 (s, 3H, OTBS), 0.01 (s, 3H, OTBS); ${ }^{13}$ C-NMR (126 MHz, CDCl $)$ ): $\delta 192.3(\mathrm{CH}, \mathrm{C}-1), 151.4$ (C, C-2), $137.9(\mathrm{CH}, \mathrm{C}-3), 66.1\left(\mathrm{CH}_{2}, \mathrm{C}-8\right)$, $41.2(\mathrm{CH}, \mathrm{C}-4), 26.0\left(3 \mathrm{CH}_{3}, \mathrm{OTBS}\right), 24.6\left(\mathrm{CH}_{2}, \mathrm{C}-5\right), 18.4$ (C, OTBS), $16.7\left(\mathrm{CH}_{3}, \mathrm{C}-7\right), 11.9\left(\mathrm{CH}_{3}\right.$, C-6), $-5.3\left(2 \mathrm{CH}_{3}, \mathrm{OTBS}\right)$.

Methyl 2-(diphenoxyphosphoryl)acetate (121)<smiles>COC(=O)CI(C)P(=O)(O)Oc1ccccc1</smiles>

Diphenylphosphite $(2.50 \mathrm{~mL}, 13.1 \mathrm{mmol})$ was dissolved in dry DCM $(30 \mathrm{~mL})$ and cooled to 0 ${ }^{\circ} \mathrm{C}$ then methyl bromoacetate $(1.38 \mathrm{~mL}, 14.1 \mathrm{mmol}, 1.1$ equiv.) was added followed by triethylamine $\left(2.50 \mathrm{~mL}, 17.8 \mathrm{mmol}, 1.4\right.$ equiv.). The reaction was stirred at $0{ }^{\circ} \mathrm{C}$ for 15 minutes then at room temperature for 2.5 hours. The reaction was quenched with water ( 30 $\mathrm{mL})$ then extracted with ethyl acetate $(3 \times 30 \mathrm{~mL})$. The organic layers were washed with 
brine $(50 \mathrm{~mL})$, then dried $\left(\mathrm{MgSO}_{4}\right)$, filtered and concentrated. The crude material was purified on silica gel column (5:1 then 2:1 PE/EA) to give the phosphonate 121 as a colourless oil (2.125 g, 53\%). Spectral data matched those previously reported. ${ }^{103}$

$\mathbf{R}_{f}=0.37(2: 1 \mathrm{PE} / \mathrm{EA}) ;{ }^{1} \mathbf{H}$ NMR $\left(500 \mathrm{MHz}, \mathrm{CDCl}_{3}\right): \delta 7.37-7.31(\mathrm{~m}, 4 \mathrm{H}, \mathrm{Ph}), 7.25-7.17(\mathrm{~m}$, $6 \mathrm{H}, \mathrm{Ph}), 3.77$ (s, 3H, H-3), 3.28 (d, J = $21.6 \mathrm{~Hz}, 2 \mathrm{H}, \mathrm{H}-1) ;{ }^{13} \mathrm{C} \mathrm{NMR}\left(126 \mathrm{MHz}, \mathrm{CDCl}_{3}\right): \delta 165.4$ (d, J = 6.2 Hz, C, C-2), 150.1 (d, J = 8.6 Hz, 2C, Ph), 130.0 (d, J = 1.0 Hz, 4CH, Ph), 125.7 (d, J = 1.4 Hz, 2CH, Ph), 120.8 (d, J=4.5 Hz, 4CH, Ph), $53.0\left(\mathrm{CH}_{3}, \mathrm{C}-3\right), 34.0$ (d, J=137.6 Hz, CH, $\mathrm{C}-$ 1).

\section{Methyl 2-(diphenoxyphosphoryl)propanoate (122)}

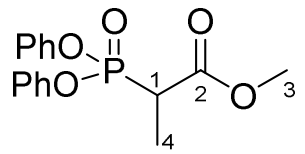

Phosphonate 121 (2.125 g, $6.9 \mathrm{mmol})$ was dissolved in dry DMF $(20 \mathrm{~mL})$, cooled to $0{ }^{\circ} \mathrm{C}$ and treated with $\mathrm{NaH}(0.251 \mathrm{~g}, 6.3 \mathrm{mmol}, 0.9$ equiv.). The reaction was stirred at room temperature for $30 \mathrm{~min}$ then $\mathrm{Mel}(0.48 \mathrm{~mL}, 7.6 \mathrm{mmol}, 1.1$ equiv.) was added and the reaction stirred at the same temperature for another hour. The reaction was then quenched with sat. aq. ammonium chloride solution $(30 \mathrm{~mL})$ and extracted with EtOAc $(2 \times 30 \mathrm{~mL})$. The combined organic layers were washed with water $(50 \mathrm{~mL})$ then brine $(50 \mathrm{~mL})$, dried $\left(\mathrm{MgSO}_{4}\right)$ and concentrated. The crude material was purified using gradient chromatography (PE:EA $5: 1,3: 1,2: 1,1: 1$ then $100 \%$ EA) with care being needed to separate the product from the dimethylated product and, especially, from the remaining starting material. Product 122 was isolated as a white solid (1.127 g, 51\%, 71\% BRSM) yield. The experimental properties were in accordance with those reported in literature. ${ }^{226}$

$\mathbf{R}_{f}=0.50(2: 1 \mathrm{PE} / \mathrm{EA}) ;{ }^{1} \mathrm{H}$ NMR $\left(500 \mathrm{MHz}, \mathrm{CDCl}_{3}\right): \delta 7.36-7.29(\mathrm{~m}, 4 \mathrm{H}, \mathrm{Ph}), 7.22-7.13(\mathrm{~m}$, $6 \mathrm{H}, \mathrm{Ph}$ ), 3.76 (s,3H, H-3), 3.39 (dq, J = 23.7, 7.3 Hz, 1H, H-1), 1.65 (dd, J = 19.2, 7.3 Hz, 3H, H4); ${ }^{13}$ C NMR (126 MHz, CDCl 3 ) $\delta 169.4$ (C, C-2), 129.9 (4CH, Ph), 128.4 (2C,Ph), 125.5 (d, $J=$ $1.9 \mathrm{~Hz}, 2 \mathrm{CH}, \mathrm{Ph}), 120.7$ (d, J = 4.4 Hz, 2CH, Ph), 120.6 (d, J = 4.4 Hz, 2CH, Ph), $53.0\left(\mathrm{CH}_{3}, \mathrm{C}-3\right.$ ), $39.6(\mathrm{~d}, J=135.8 \mathrm{~Hz}, \mathrm{CH}, \mathrm{C}-1), 12.0\left(\mathrm{~d}, J=6.3 \mathrm{~Hz}, \mathrm{CH}_{3}, \mathrm{C}-4\right)$; HRMS (ESI) Found 322.0908. Calculated for $\mathrm{C}_{16} \mathrm{H}_{18} \mathrm{O}_{5} \mathrm{P}^{+}[\mathrm{M}+\mathrm{H}]^{+}$322.0920. $\Delta=3.92 \mathrm{ppm}$. 


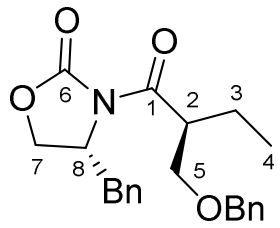

To a solution of $27(3.272 \mathrm{~g}, 13.2 \mathrm{mmol})$ in dry DCM $(40 \mathrm{~mL})$ was added $\mathrm{TiCl}_{4}(1 \mathrm{M}$ in DCM, 20 $\mathrm{mL}, 20.0 \mathrm{mmol}, 1.5$ equiv.) at $0{ }^{\circ} \mathrm{C}$ under argon. After stirring for $10 \mathrm{~min}$ the resulting yellow solution was treated with $\mathrm{NEt}_{3}(2.10 \mathrm{~mL}, 14.9 \mathrm{mmol}, 1.1$ equiv.) and the colour changed to dark red. The reaction was stirred at $0{ }^{\circ} \mathrm{C}$ for another $30 \mathrm{~min}$ before benzyl chloromethyl ether (technical grade, $3.80 \mathrm{~mL}, 14.6 \mathrm{mmol}, 1.1$ equiv.) was added dropwise and the reaction stirred for $4 \mathrm{~h}$ at the same temperature. After 4 hours the reaction was quenched with sat. aq. $\mathrm{NH}_{4} \mathrm{Cl}(50 \mathrm{~mL})$ and extracted with $\mathrm{DCM}(3 \times 50 \mathrm{~mL})$. The organic fractions were combined, dried over anhydrous $\mathrm{MgSO}_{4}$ and the solvent removed under reduced pressure. The resulting crude reaction mixture was purified by flash chromatography (9:1 then 5:1 $\mathrm{PE} / \mathrm{EtOAC})$ to give the major product $(\mathbf{1 2 3})$ as a yellow oil $(4.035 \mathrm{~g}, 83 \%)$ and the minor product (130) as a colourless oil $(0.241 \mathrm{~g}, 4 \%)$. Experimental properties for 123 matched those previously reported. ${ }^{86}$

Major diastereomer 123:

$\mathbf{R}_{f}=0.47(3: 1 \mathrm{PE} / \mathrm{EA}) ;[\boldsymbol{\alpha}]_{\mathrm{D}}{ }^{22}=-37.3$ (c 1.05, $\mathrm{CHCl}_{3}$ ) $\left[\right.$ lit. $^{86}[\boldsymbol{\alpha}]_{\mathrm{D}}{ }^{24}=-39.1$ (c $\left.\left.0.96, \mathrm{CHCl}_{3}\right)\right] ;{ }^{1} \mathbf{H}$ NMR $\left(500 \mathrm{MHz}, \mathrm{CDCl}_{3}\right) \delta 7.36-7.15(\mathrm{~m}, 10 \mathrm{H}, 2 \mathrm{Ph}), 4.77-4.70(\mathrm{~m}, 1 \mathrm{H}, \mathrm{H}-8), 4.55(\mathrm{~m}, 2 \mathrm{H}$, $\mathrm{OCH}_{2} \mathrm{Ph}$ ), $4.22-4.10(\mathrm{~m}, 3 \mathrm{H}, \mathrm{H}-2, \mathrm{H}-7), 3.81$ (td, $\left.J=8.7,1.4 \mathrm{~Hz}, 1 \mathrm{H}, \mathrm{H}-5 \mathrm{a}\right), 3.67$ (ddd, J = 9.1, 5.0, 1.4 Hz, 1H, H-5b), 3.23 (dd, J=13.6, 3.3 Hz, 1H, $\mathrm{CH}_{2} \mathrm{aPh}$ ), 2.71 (ddd, J= 13.5, 9.3, $1.4 \mathrm{~Hz}$, $\left.1 \mathrm{H}, \mathrm{CH}_{2} \mathrm{bPh}\right), 1.76(\mathrm{~m}, 1 \mathrm{H}, \mathrm{H}-3 \mathrm{a}), 1.59(\mathrm{~m}, 1 \mathrm{H}, \mathrm{H}-3 \mathrm{~b}), 0.94$ (td, J = 7.5, $\left.1.4 \mathrm{~Hz}, 3 \mathrm{H}, \mathrm{H}-4\right) ;{ }^{13} \mathrm{C}$ NMR (125 MHz, CDCl $)_{3} \delta 175.0$ (C, C-1), 153.2 (C, C-6), 138.2 (C, OCH $\left.\mathrm{Ph}\right) 135.3$ (C, CH $\mathrm{Ph}$ ), 129.5 (2CH, Ph), 128.9 (2CH, Ph), 128.3 (2CH, Ph), 127.6 (2CH, Ph), 127.56 ( $\mathrm{CH}, \mathrm{Ph}), 127.2$ $(\mathrm{CH}, \mathrm{Ph}), 73.1\left(\mathrm{CH}_{2}, \mathrm{OCH} 2 \mathrm{Ph}\right), 71.0\left(\mathrm{CH}_{2}, \mathrm{C}-5\right), 65.8\left(\mathrm{CH}_{2}, \mathrm{C}-7\right), 55.2(\mathrm{CH}, \mathrm{C}-8), 44.8(\mathrm{CH}, \mathrm{C}-2)$, $37.7\left(\mathrm{CH}_{2}, \mathrm{CH}_{2} \mathrm{Ph}\right), 22.1\left(\mathrm{CH}_{2}, \mathrm{C}-3\right), 11.5\left(\mathrm{CH}_{3}, \mathrm{C}-4\right)$; IR (film from $\left.\mathrm{CHCl}_{3}\right) v_{\max } 2966,2931$, $2874,1776,1697,1454,1388,1350,1211,1010 \mathrm{~cm}^{-1}$. 
Minor diastereomer (contaminated with $\mathrm{BnOCH}_{2} \mathrm{Cl}$ ):

(R)-4-Benzyl-3-((R)-2-((benzyloxy)methyl)butanoyl)oxazolidin-2-one (130)

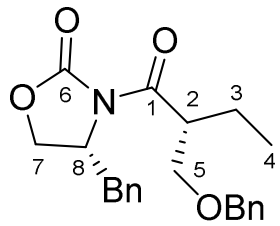

$\mathbf{R}_{f}=0.39(3: 1 \mathrm{PE} / \mathrm{EA}) ;{ }^{1} \mathbf{H}$ NMR $\left(500 \mathrm{MHz}, \mathrm{CDCl}_{3}\right) \delta 7.40-7.20(\mathrm{~m}, 10 \mathrm{H}, 2 \mathrm{Ph}), 4.75-4.73(\mathrm{~m}$, $1 \mathrm{H}, \mathrm{H}-8), 4.63\left(\mathrm{~d}, J=11.7 \mathrm{~Hz}, 1 \mathrm{H}, \mathrm{OCH}_{2 \mathrm{a}} \mathrm{Ph}\right), 4.58\left(\mathrm{~d}, J=11.8 \mathrm{~Hz}, 1 \mathrm{H}, \mathrm{OCH}_{2 \mathrm{~b}} \mathrm{Ph}\right), 4.22-4.12$ (m, 3H, H-2, H-7), 3.96 (dd, J = 9.4, 8.4 Hz, 1H, H-5a), 3.78 (dd, J = 9.4, $4.7 \mathrm{~Hz}, 1 \mathrm{H}, \mathrm{H}-5 \mathrm{~b}$ ), 3.27 (dd, $J=13.5,3.3 \mathrm{~Hz}, 1 \mathrm{H}, \mathrm{CH}_{2} \mathrm{aPh}$ ), 2.76 (dd, $J=13.5,9.3 \mathrm{~Hz}, 1 \mathrm{H}, \mathrm{CH}_{2} \mathrm{bPh}$ ), 1.78-1.73 (m, $1 \mathrm{H}$, $\mathrm{H}-3 \mathrm{a}), 1.64-1.57(\mathrm{~m}, 1 \mathrm{H}, \mathrm{H}-3 \mathrm{~b}), 0.97$ (t, J = $7.5 \mathrm{~Hz}, 3 \mathrm{H}, \mathrm{H}-4) ;{ }^{13} \mathrm{C}$ NMR $\left(126 \mathrm{MHz} \mathrm{CDCl}_{3}\right) \delta$ 175.0(C, C-1), 153.4 (C, C-6), 137.9 (C, OCH $\left.{ }_{2} P h\right), 135.4$ (C, $\left.\mathrm{CH}_{2} P h\right), 129.6\left(2 \mathrm{CH}_{1} \mathrm{CH}_{2} \mathrm{Ph}\right.$ ), 129.0 $\left(2 \mathrm{CH}, \mathrm{CH}_{2} \mathrm{Ph}\right), 128.6\left(2 \mathrm{CH}, \mathrm{OCH}_{2} \mathrm{Ph}\right), 128.1\left(2 \mathrm{CH}, \mathrm{OCH}_{2} \mathrm{Ph}\right), 127.9\left(\mathrm{CH}, \mathrm{OCH}_{2} P h\right), 127.4(\mathrm{CH}$, $\left.\mathrm{CH}_{2} \mathrm{Ph}\right), 69.5\left(\mathrm{CH}_{2}, \mathrm{OCH}_{2} \mathrm{Ph}\right), 68.5\left(\mathrm{CH}_{2}, \mathrm{C}-5\right), 66.0\left(\mathrm{CH}_{2}, \mathrm{C}-7\right), 55.4(\mathrm{CH}, \mathrm{C}-8), 44.9(\mathrm{CH}, \mathrm{C}-2)$, $37.9\left(\mathrm{CH}_{2}, \mathrm{CH}_{2} \mathrm{Ph}\right), 22.4\left(\mathrm{CH}_{2}, \mathrm{C}-3\right), 11.6\left(\mathrm{CH}_{3}, \mathrm{C}-4\right)$.

(R)-2-((Benzyloxy)methyl)butan-1-ol (124)<smiles>CCC(C=CBr)C(C)O</smiles>

Oxazolidinone 123 (3.793 g, $10.3 \mathrm{mmol}$ ) was dissolved in dry diethyl ether $(40 \mathrm{~mL}$ ) and ethanol (1.40 mL, 2 equiv.). The solution was cooled to $0{ }^{\circ} \mathrm{C}$ under argon, then treated dropwise with a solution of lithium borohydride $(2.0 \mathrm{M}$ in THF, $10.10 \mathrm{~mL}, 20.7 \mathrm{mmol}, 2.0$ equiv.). The reaction was stirred at the same temperature for $1 \mathrm{~h}$ then quenched with sat. aq. sodium bicarbonate $(40 \mathrm{~mL})$ and left stirring for 2 hours before the layers were separated. The aqueous layer was extracted with $\mathrm{Et}_{2} \mathrm{O}(3 \times 40 \mathrm{~mL})$, the organic layers combined, dried over anhydrous magnesium sulfate and the solvent removed under reduced pressure. Product was purified on silica gel column (5:1 PE/EA), to give product 124 as a colourless oil (1.643 g, 82\%). Experimental properties were in accordance with those previously reported. ${ }^{86}$ 
$\mathbf{R}_{f}=0.25(5: 1 \mathrm{PE} / \mathrm{EA}) ;[\alpha]_{\mathrm{D}}{ }^{22}=+13.1$ (c 1.10, $\mathrm{CHCl}_{3}$ ) $\left[\mathrm{lit}^{86}{ }^{8 \alpha}\right]_{\mathrm{D}}{ }^{24}=+19.1$ (c 3.67, $\left.\left.\mathrm{CHCl}_{3}\right)\right] ;{ }^{1} \mathbf{H}$ NMR (500 MHz, $\left.\mathrm{CDCl}_{3}\right) \delta 7.38-7.28(\mathrm{~m}, 5 \mathrm{H}, \mathrm{Ph}), 4.54$ (d, J=11.9 Hz, 1H, OCH $\mathrm{OPPh}_{2}, 4.50$ (d, $J=12.0 \mathrm{~Hz}, 1 \mathrm{H}, \mathrm{OCH}_{2} \mathrm{bPh}$ ), 3.73 (ddd, $\left.J=10.9,3.6,0.8 \mathrm{~Hz}, 1 \mathrm{H}, \mathrm{H}-1 \mathrm{a}\right), 3.66-3.61(\mathrm{~m}, 2 \mathrm{H}, \mathrm{H}-$ 1b, H-5a), 3.48 (dd, J = 9.0, 7.8 Hz, 1H, H-5b), $2.60(\mathrm{~m}, 1 \mathrm{H}, \mathrm{OH}), 1.80(\mathrm{~m}, 1 \mathrm{H}, \mathrm{H}-2), 1.38-1.25$ (m, 2H, H-3), $0.93(\mathrm{t}, J=7.5 \mathrm{~Hz}, 3 \mathrm{H}, \mathrm{H}-4) ;{ }^{13} \mathrm{C}$ NMR (125 MHz, CDCl 3 ) $\delta 138.0(\mathrm{C}, \mathrm{Ph}), 128.5$ $(2 \mathrm{CH}, \mathrm{Ph}), 127.7(\mathrm{CH}, \mathrm{Ph}), 127.6(2 \mathrm{CH}, \mathrm{Ph}), 74.0\left(\mathrm{CH}_{2}, \mathrm{C}-5\right), 73.5\left(\mathrm{CH}_{2}, \mathrm{OCH}_{2} \mathrm{Ph}\right), 66.1\left(\mathrm{CH}_{2}, \mathrm{C}-\right.$ 1), $42.2(\mathrm{CH}, \mathrm{C}-2), 21.0\left(\mathrm{CH}_{2}, \mathrm{C}-3\right), 11.7\left(\mathrm{CH}_{3}, \mathrm{C}-4\right)$; IR (film from $\left.\mathrm{CHCl}_{3}\right) v_{\max } 3421,2961,2927$, 2875, 1454, 1364, 1206, 1097, 1029, $736 \mathrm{~cm}^{-1}$; HRMS (ESI) Found 195.1372. Calculated for $\mathrm{C}_{12} \mathrm{H}_{19} \mathrm{O}_{2}^{+}[\mathrm{M}+\mathrm{H}]^{+}$195.1380. $\Delta=4.18 \mathrm{ppm}$.

\section{(S)-2-((Benzyloxy)methyl)butanal (131)}

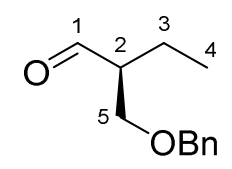

Alcohol 124 (1.003 g, $5.2 \mathrm{mmol})$ in dichloromethane $(25 \mathrm{~mL})$ was treated with Dess-Martin periodinane $\left(2.671 \mathrm{~g}, 6.1 \mathrm{mmol}, 1.2\right.$ equiv.) at $0{ }^{\circ} \mathrm{C}$ under air. The reaction was stirred at the same temperature for 1 hour then quenched with 1:1 v/v sat. aq. sodium thiosulfate/sat. aq. sodium bicarbonate $(50 \mathrm{~mL})$ and stirred for another $30 \mathrm{~min}$ at room temperature. The aqueous layer was then extracted with diethyl ether $(2 \times 25 \mathrm{~mL})$. The organic layers were combined, dried over anhydrous $\mathrm{MgSO}_{4}$ and concentrated. The product 131 was used in the next step without further purification and within $30 \mathrm{~min}$. Spectral data matched those reported in the literature. ${ }^{86}$

$\mathbf{R}_{f}=0.63(5: 1 \mathrm{PE} / \mathrm{EA}) ;{ }^{1} \mathrm{H}$ NMR $\left(500 \mathrm{MHz}, \mathrm{CDCl}_{3}\right) \delta 9.71(\mathrm{~d}, \mathrm{~J}=2.3 \mathrm{~Hz}, \mathrm{H}-4)$ 7.36-7.28 (m, 5H, $\mathrm{Ph}$ ), 4.55 (d, J=12.4 Hz, 1H, OCH $2 \mathrm{aPh}$ ), 4.50 (d, J = 12.4 Hz, 1H, OCH $\mathrm{CPPh}_{2}, 3.71$ (dd, J=9.5, $7.1 \mathrm{~Hz}, 1 \mathrm{H}, \mathrm{H}-5 \mathrm{a}), 3.66$ (dd, $J=9.5,4.9 \mathrm{~Hz}, 1 \mathrm{H}, \mathrm{H}-5 \mathrm{~b}), 2.53-2.47(\mathrm{~m}, 1 \mathrm{H}, \mathrm{H}-2), 1.78-1.70(\mathrm{~m}$, $1 \mathrm{H}, \mathrm{H}-3 \mathrm{a}, \mathrm{H}-3 \mathrm{~b}), 1.61-1.530 .94$ (t, $J=7.6 \mathrm{~Hz}, 3 \mathrm{H}, \mathrm{H}-4) ;{ }^{13} \mathrm{C}$ NMR $\left(125 \mathrm{MHz}, \mathrm{CDCl}_{3}\right) \delta 204.1$ (C, C-1), 137.9 (C, Ph), 128.4 (2CH, Ph), 127.7 ( $\mathrm{CH}, \mathrm{Ph}), 127.6(2 \mathrm{CH}, \mathrm{Ph}), 73.3\left(\mathrm{CH}_{2}, \mathrm{OCH}_{2} \mathrm{Ph}\right), 68.3$ $\left(\mathrm{CH}_{2}, \mathrm{C}-5\right), 53.7(\mathrm{CH}, \mathrm{C}-2), 19.0\left(\mathrm{CH}_{2}, \mathrm{C}-3\right), 11.4\left(\mathrm{CH}_{3}, \mathrm{C}-4\right)$. 


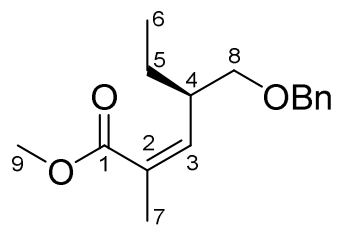

A solution of phosphonate 133 ( $2.057 \mathrm{~g}, 6.2 \mathrm{mmol}, 1.2$ equiv.) and 18-crown-6 (4.760 g, 17.8 mmol, 3.5 equiv.) in dry THF (26 mL) was cooled to $-78{ }^{\circ} \mathrm{C}$ under argon and treated dropwise with a $0.5 \mathrm{M}$ solution of KHMDS in THF (10 mL, $5.0 \mathrm{mmol}, 0.9$ equiv.). After stirring at the same temperature for another $10 \mathrm{~min}$, the crude mixture containing aldehyde 131 (in $5 \mathrm{~mL}$ THF) was added via cannula. The reaction was stirred at $-78{ }^{\circ} \mathrm{C}$ for $2 \mathrm{~h}$ then allowed to warm to $-30{ }^{\circ} \mathrm{C}$ and quenched with sat. aq. $\mathrm{NH}_{4} \mathrm{Cl}$ solution $(50 \mathrm{~mL})$. The aqueous layer was extracted with EtOAc $(2 \times 50 \mathrm{~mL})$, washed with brine, dried $\left(\mathrm{MgSO}_{4}\right)$ and concentrated. The crude reaction material was purified on a silica gel column, eluting with 9:1 PE/EA, to give the product 125 as a colourless oil $(0.904 \mathrm{~g}, 71 \%)$.

$\mathbf{R}_{f}=0.69(5: 1 \mathrm{PE} / \mathrm{EA}) ;[\boldsymbol{\alpha}]_{\mathrm{D}}{ }^{\mathbf{2 2}}=-61.4\left(c 0.98, \mathrm{CH}_{2} \mathrm{Cl}_{2}\right) ;{ }^{1} \mathbf{H} \mathbf{N M R}\left(500 \mathrm{MHz}, \mathrm{CDCl}_{3}\right) \delta$ 7.36-7.27 (m, 5H, Ph), 5.77 (dd, J = 9.9, $1.5 \mathrm{~Hz}, 1 \mathrm{H}, \mathrm{H}-3), 4.54-4.46\left(\mathrm{~m}, 2 \mathrm{H}, \mathrm{OCH}_{2} \mathrm{Ph}\right), 3.72$ (s, 3H, $\mathrm{OCH}_{3}$ ), $3.41(\mathrm{~d}, J=6.3 \mathrm{~Hz}, 2 \mathrm{H}, \mathrm{H}-8), 3.38-3.30(\mathrm{~m}, 1 \mathrm{H}, \mathrm{H}-4), 1.93(\mathrm{~d}, J=1.4 \mathrm{~Hz}, 3 \mathrm{H}, \mathrm{H}-7), 1.61$ (dqd, $J=13.1,7.5,5.4 \mathrm{~Hz}, 1 \mathrm{H}, \mathrm{H}-5 \mathrm{a}$ ), $1.33-1.27$ (m, $1 \mathrm{H}, \mathrm{H}-5 \mathrm{~b}), 0.87$ (t, $J=7.5 \mathrm{~Hz}, 3 \mathrm{H}, \mathrm{H}-6) ;{ }^{13} \mathrm{C}$ NMR (125 MHz, CDCl $) \delta 168.6$ (C, C-1), 144.8 (CH, C-3), 138.8 (C, C-2), 128.4 (2CH, Ph), 128.3 (C, Ph), 127.7 (2CH, Ph), $127.6(\mathrm{CH}, \mathrm{Ph}), 73.4\left(\mathrm{CH}_{2}, \mathrm{C}-8\right), 72.9\left(\mathrm{CH}_{2}, \mathrm{OCH}_{2} \mathrm{Ph}\right), 51.4\left(\mathrm{CH}_{3}\right.$, $\left.\mathrm{OCH}_{3}\right), 40.7(\mathrm{CH}, \mathrm{C}-4), 25.0\left(\mathrm{CH}_{2}, \mathrm{C}-5\right), 21.1\left(\mathrm{CH}_{3}, \mathrm{C}-7\right), 11.7\left(\mathrm{CH}_{3}, \mathrm{C}-6\right)$; IR (film from $\left.\mathrm{CH}_{2} \mathrm{Cl}_{2}\right)$ $V_{\max } 3384,3185,2962,2037,1713,1593,1399,1223,1091,1072 \mathrm{~cm}^{-1}$; HRMS (ESI) Found 263.1641. Calculated for $\mathrm{C}_{16} \mathrm{H}_{23} \mathrm{O}_{3}{ }^{+}[\mathrm{M}+\mathrm{H}]^{+}$263.1642. $\Delta=0.27 \mathrm{ppm}$.

$(R, Z)-4-((B e n z y l o x y) m e t h y l)-2-m e t h y l h e x-2-e n-1-o l ~(126)$<smiles>CC[C@H](C=C(C)CO)COCc1ccccc1</smiles>

Ester 125 (0.904 g, $3.45 \mathrm{mmol})$ was dissolved in dry DCM (40 mL) and cooled to $-78{ }^{\circ} \mathrm{C}$ under argon atmosphere then treated with $17.4 \mathrm{~mL}$ of DIBALH solution $(1 \mathrm{M}$ in cyclohexane, 17.4 
mmol, 5 equiv.). After 2 hours at $-78{ }^{\circ} \mathrm{C}$ the reaction was allowed to warm to $0{ }^{\circ} \mathrm{C}$ and quenched with Rochelle's salt $(20 \mathrm{~mL})$ and stirred for $2 \mathrm{~h}$ at room temperature. After $2 \mathrm{~h}$ the layers were separated and aqueous layer extracted with DCM (3x $40 \mathrm{~mL})$. The combined organic layers were washed with brine $(30 \mathrm{~mL})$, dried $\left(\mathrm{MgSO}_{4}\right)$ and concentrated. Purification on silica gel column (5:1 PE/EA) gave compound 126 as a colourless oil ( $0.680 \mathrm{~g}$, $84 \%$ yield).

$\mathbf{R}_{f}=0.35(5: 1 \mathrm{PE} / \mathrm{EA}) ;[\boldsymbol{\alpha}]_{\mathrm{D}}{ }^{22}=+8.7\left(c 1.05, \mathrm{CH}_{2} \mathrm{Cl}_{2}\right) ;{ }^{1} \mathrm{H}$ NMR $\left(500 \mathrm{MHz}, \mathrm{CDCl}_{3}\right) \delta 7.37-7.27$ (m, 5H, Ph), 5.00 (dq, J = 9.9, $1.4 \mathrm{~Hz}, 1 \mathrm{H}, \mathrm{H}-3), 4.50\left(\mathrm{~m}, 2 \mathrm{H}, \mathrm{OCH}_{2} \mathrm{Ph}\right), 4.25$ (dd, $J=11.5,1.0$ $\mathrm{Hz}, 1 \mathrm{H}, \mathrm{H}-1 \mathrm{a}$ ), 3.73 (d, J=11.5 Hz, 1H, H-1b), 3.44 (dd, J=8.6, $4.4 \mathrm{~Hz}, 1 \mathrm{H}, \mathrm{H}-8 \mathrm{a}$ ), 3.13 (dd, J = 9.5, 8.6 Hz, 1H, H-8b), $2.64-2.53(\mathrm{~m}, 1 \mathrm{H}, \mathrm{H}-4), 1.85$ (d, J = $1.5 \mathrm{~Hz}, 3 \mathrm{H}, \mathrm{H}-7), 1.46-1.35$ (m, $1 \mathrm{H}, \mathrm{H}-5 \mathrm{a}), 1.22-1.10(\mathrm{~m}, 1 \mathrm{H}, \mathrm{H}-5 \mathrm{~b}), 0.85(\mathrm{t}, J=7.5 \mathrm{~Hz}, 3 \mathrm{H}, \mathrm{H}-6) ;{ }^{13} \mathrm{C}$ NMR $\left(126 \mathrm{MHz}, \mathrm{CDCl}_{3}\right) \delta$ 138.0 (C, C-2), 137.8 (C, Ph), 130.8 (CH, C-3), 128.6 (2CH, Ph), $128.0(2 \mathrm{CH}, \mathrm{Ph}), 127.9$ (CH, Ph), $73.7\left(\mathrm{CH}_{2}, \mathrm{C}-8\right), 73.4\left(\mathrm{CH}_{2}, \mathrm{OCH}_{2} \mathrm{Ph}\right), 62.0\left(\mathrm{CH}_{2}, \mathrm{C}-1\right), 40.4(\mathrm{CH}, \mathrm{C}-4), 25.0\left(\mathrm{CH}_{2}, \mathrm{C}-5\right), 22.8$ $\left(\mathrm{CH}_{3}, \mathrm{C}-7\right), 11.9\left(\mathrm{CH}_{3}, \mathrm{C}-6\right)$; IR (film from $\left.\mathrm{CH}_{2} \mathrm{Cl}_{2}\right) v_{\max }$ 2963, 2930, 2860, 1454, 1364, 1265, $1075,1002,734,699 \mathrm{~cm}^{-1}$; HRMS (ESI) Found 257.1514. Calculated for $\mathrm{C}_{15} \mathrm{H}_{22} \mathrm{NaO}_{2}{ }^{+}[\mathrm{M}+\mathrm{Na}]^{+}$ 257.1512. $\Delta=0.76 \mathrm{ppm}$.

$(R, Z)-4-((B e n z y l o x y) m e t h y l)-2-m e t h y l h e x-2-e n a l ~(127)$<smiles>CC[C@H](C=C(C)C=O)COc1ccccc1</smiles>

Alcohol $126(0.680 \mathrm{~g}, 2.90 \mathrm{mmol})$ was dissolved in wet DCM $(12 \mathrm{~mL})$ and cooled to $0{ }^{\circ} \mathrm{C}$ under air then treated with DMP (1.826 g, $4.12 \mathrm{mmol}, 1.5$ equiv.). The reaction was stirred at the same temperature for 1.5 hours, by which time TLC analysis indicated all starting material had been consumed, then quenched with $1: 1 \mathrm{v} / \mathrm{v}$ sat. aq. $\mathrm{Na}_{2} \mathrm{~S}_{2} \mathrm{O}_{3} /$ sat. aq. $\mathrm{NaHCO}_{3}$ $(30 \mathrm{~mL})$ and stirred at room temperature for an hour. The aqueous layer was then extracted with DCM $(3 \times 30 \mathrm{~mL})$ and the combined organic layers washed with brine $(40 \mathrm{~mL})$, dried over anhydrous $\mathrm{MgSO}_{4}$ and concentrated. The crude material was purified using flash chromatography (10:1 PE/EA) and the product 127 was isolated as a pale yellow oil (0.532 $\mathrm{g}$, $79 \%$ yield). 
$\mathbf{R}_{f}=0.59(5: 1 \mathrm{PE} / \mathrm{EA}) ;[\boldsymbol{\alpha}]_{\mathrm{D}}{ }^{21}=-49.3\left(c 0.52, \mathrm{CHCl}_{3}\right) ;{ }^{1} \mathbf{H} \mathbf{N M R}\left(500 \mathrm{MHz}, \mathrm{CDCl}_{3}\right) \delta 10.10(\mathrm{~s}, 1 \mathrm{H}$, $\mathrm{H}-1), 7.37-7.27(\mathrm{~m}, 5 \mathrm{H}, \mathrm{Ph}), 6.28(\mathrm{dq}, J=10.7,1.4 \mathrm{~Hz}, 1 \mathrm{H}, \mathrm{H}-3), 4.51(\mathrm{~d}, J=12.2 \mathrm{~Hz}, 1 \mathrm{H}$, $\mathrm{OCH}_{2} \mathrm{aPh}$ ), 4.48 (d, J = $12.2 \mathrm{~Hz}, 1 \mathrm{H}, \mathrm{OCH}_{2} \mathrm{bPh}$ ), 3.49 (dd, J = 9.1, $5.3 \mathrm{~Hz}, 1 \mathrm{H}, \mathrm{H}-8 \mathrm{a}$ ), 3.37 (dd, $J$ = 9.1, 7.2 Hz, 1H, H-8b), $3.33-3.25(\mathrm{~m}, 1 \mathrm{H}, \mathrm{H}-4), 1.82$ (d, J = $1.4 \mathrm{~Hz}, 3 \mathrm{H}, \mathrm{H}-7), 1.70$ - 1.62 (m, $1 \mathrm{H}, \mathrm{H}-5 \mathrm{a}), 1.36-1.25$ (m, 2H, H-5b), $0.89(\mathrm{t}, J=7.5 \mathrm{~Hz}, 3 \mathrm{H}, \mathrm{H}-6) ;{ }^{13} \mathrm{C}$ NMR $\left(125 \mathrm{MHz}, \mathrm{CDCl}_{3}\right) \delta$ 192.0 (CH, C-1), 150.9 (CH, C-3), 138.3 (C, Ph), 137.7 (C, C-2), 128.5 (2CH, Ph), 127.8 (CH, Ph), $127.6(2 \mathrm{CH}, \mathrm{Ph}), 73.3\left(2 \mathrm{CH}_{2}, \mathrm{C}-8, \mathrm{OCH}{ }_{2} \mathrm{Ph}\right), 38.9(\mathrm{CH}, \mathrm{C}-4), 25.0\left(\mathrm{CH}_{2}, \mathrm{C}-5\right), 16.8\left(\mathrm{CH}_{3}, \mathrm{C}-7\right)$, $11.8\left(\mathrm{CH}_{3}, \mathrm{C}-6\right)$; IR (film from $\left.\mathrm{CHCl}_{3}\right) \mathrm{V}_{\max }$ 2962, 2928, 2858, 1678, 1454, 1362, 1207, 1099, 737, $698 \mathrm{~cm}^{-1}$; HRMS (ESI) Found 233.1535. Calculated for $\mathrm{C}_{15} \mathrm{H}_{21} \mathrm{O}_{2}{ }^{+}[\mathrm{M}+\mathrm{H}]^{+}$233.1536. $\Delta=$ $0.64 \mathrm{ppm}$.

$(4 S, 7 R, Z)-7-((B e n z y l o x y) m e t h y l)-4-h y d r o x y-5-m e t h y l n o n-5-e n-2-o n e ~(128)$

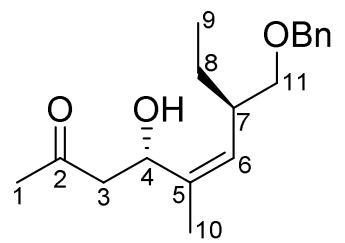

(+)-DIPCl (1.375 g, $4.23 \mathrm{mmol}, 2.0$ equiv.) was dissolved in dry $\mathrm{Et}_{2} \mathrm{O}(11 \mathrm{~mL})$ under Ar and cooled down to $0{ }^{\circ} \mathrm{C}$, then treated with $\mathrm{Et}_{3} \mathrm{~N}(0.62 \mathrm{~mL}, 4.40 \mathrm{mmol}, 2.1$ equiv.) dropwise to yield a white suspension. After 5 min of stirring, distilled acetone $(0.35 \mathrm{~mL}, 4.70 \mathrm{mmol}, 2.3$ equiv.) was added dropwise and the reaction stirred for another $2 \mathrm{~h}$ at $0^{\circ} \mathrm{C}$. After $2 \mathrm{hrs}$ the reaction was cooled to $-78{ }^{\circ} \mathrm{C}$ and aldehyde 127 (0.493 g, $\left.2.12 \mathrm{mmol}\right)$ in $1 \mathrm{~mL}$ of dry Et $\mathrm{E}_{2} \mathrm{O}$ (+ $0.5 \mathrm{~mL}$ rinse) was added dropwise via cannula. The reaction was stirred at the same temperature for $1 \mathrm{~h}$ then allowed to warm to $-20{ }^{\circ} \mathrm{C}$ and stirred at this temperature for 2 hours. The reaction flask was then put into a freezer $\left(-18^{\circ} \mathrm{C}\right)$ overnight. The reaction was then quenched with $\mathrm{MeOH}(11 \mathrm{~mL})$ and the solvent removed under reduced pressure. The residue was then dissolved in $\mathrm{DCM}(15 \mathrm{~mL})$ and $\mathrm{pH} 7$ buffer $(15 \mathrm{~mL})$, cooled to $0{ }^{\circ} \mathrm{C}$ and then treated with $30 \%$ aq. $\mathrm{H}_{2} \mathrm{O}_{2}(3.20 \mathrm{~mL}, 31 \mathrm{mmol}, 0.75 \mathrm{~mL} / \mathrm{mmol} \mathrm{DIPCl})$. After stirring at room temperature for 1 hour, the solution was poured into $100 \mathrm{~mL} \mathrm{H} \mathrm{H}_{2} \mathrm{O}$ and extracted with DCM (3x $50 \mathrm{~mL})$. The combined organic layers were then dried $\left(\mathrm{MgSO}_{4}\right)$ and concentrated. The crude reaction mixture was subjected to gradient chromatography $(10: 1 \mathrm{PE} / \mathrm{EA}$ then $4: 1$ $\mathrm{PE} / \mathrm{EA}$ then 2:1 PE/EA) to afford the two diastereomers as colourless oils: 128 (0.358 g, 59\%) and 135 (0.041 g, 7\%). Isolated d.r. 8:1. 


\section{Major diastereomer (128):}

$\mathbf{R}_{f}=0.26(3: 1 \mathrm{PE} / \mathrm{EA}) ;[\boldsymbol{\alpha}]_{\mathrm{D}}{ }^{21}=-15.63\left(c\right.$ 1.28, $\left.\mathrm{CHCl}_{3}\right) ;{ }^{1} \mathbf{H} \mathbf{N M R}\left(500 \mathrm{MHz}, \mathrm{CDCl}_{3}\right) \delta 7.36-7.27$ (m, 5H, Ph), $5.01-4.93(\mathrm{~m}, 2 \mathrm{H}, \mathrm{H}-4, \mathrm{H}-6), 4.51$ (d, J = $\left.12.1 \mathrm{~Hz}, 1 \mathrm{H}, \mathrm{OCH}_{2} \mathrm{aPh}\right), 4.47$ (d, J = 12.1 $\mathrm{Hz}, 1 \mathrm{H}, \mathrm{OCH}_{2} \mathrm{bPh}$ ), 3.42 (dd, J=8.8, $4.9 \mathrm{~Hz}, 1 \mathrm{H}, \mathrm{H}-11 \mathrm{a}$ ), 3.16 (t, J=8.9 Hz, 1H, H-11b), 2.82 (dd, $J=16.2,9.0 \mathrm{~Hz}, 1 \mathrm{H}, \mathrm{H}-3 \mathrm{a}), 2.74-2.64(\mathrm{~m}, 1 \mathrm{H}, \mathrm{H}-7), 2.47$ (dd, $J=16.3,4.3 \mathrm{~Hz}, 1 \mathrm{H}, \mathrm{H}-3 \mathrm{~b}$ ), $2.19(\mathrm{~s}, 3 \mathrm{H}, \mathrm{H}-1), 1.73$ (d, J = $1.4 \mathrm{~Hz}, 3 \mathrm{H}, \mathrm{H}-10), 1.48-1.39(\mathrm{~m}, 1 \mathrm{H}, \mathrm{H}-8 \mathrm{a}), 1.21-1.11(\mathrm{~m}, 1 \mathrm{H}$, $\mathrm{H}-8 \mathrm{~b}), 0.84$ (t, J = 7.4 Hz, 3H, H-9).; ${ }^{13} \mathrm{C}$ NMR (125 MHz, CDCl $) \delta 208.5$ (C, C-2), 138.3 (C, C-5), 137.9 (C, Ph), 131.2 (C-6), 128.6 (2CH, Ph), 127.9 (2CH, Ph), 127.9 (CH, Ph), $73.8\left(\mathrm{CH}_{2}, \mathrm{C}-11\right)$, $73.4\left(\mathrm{CH}_{2}, \mathrm{OCH}_{2} \mathrm{Ph}\right), 65.3(\mathrm{CH}, \mathrm{C}-4), 47.7\left(\mathrm{CH}_{2}, \mathrm{C}-3\right), 39.7(\mathrm{CH}, \mathrm{C}-7), 31.0\left(\mathrm{CH}_{3}, \mathrm{C}-1\right), 25.0\left(\mathrm{CH}_{2}\right.$, C-8), $18.5\left(\mathrm{CH}_{3}, \mathrm{C}-10\right), 11.9\left(\mathrm{CH}_{3}, \mathrm{C}-9\right)$; IR (film from $\left.\mathrm{CHCl}_{3}\right) \mathrm{v}_{\max } 3430,2961,2928,2859$, 1711, 1454, 1359, 1074, 738, $699 \mathrm{~cm}^{-1}$; HRMS (ESI) Found 313.1782. Calculated for $\mathrm{C}_{18} \mathrm{H}_{26} \mathrm{NaO}_{3}{ }^{+}[\mathrm{M}+\mathrm{Na}]^{+}$313.1774. $\Delta=2.48 \mathrm{ppm}$.

\section{Minor diastereomer (135):}

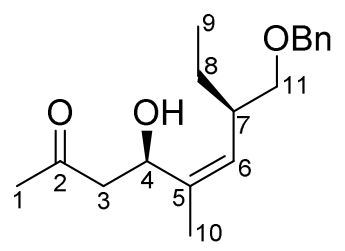

$\mathbf{R}_{f}=0.26(3: 1 \mathrm{PE} / \mathrm{EA}) ;{ }^{1} \mathbf{H}$ NMR $\left(500 \mathrm{MHz}, \mathrm{CDCl}_{3}\right) \delta 7.35-7.27(\mathrm{~m}, 5 \mathrm{H}, \mathrm{Ph}), 4.98-4.91(\mathrm{~m}, 2 \mathrm{H}$, $\mathrm{H}-4, \mathrm{H}-6), 4.45$ (m, 2H, OCH $2 \mathrm{Ph}$ ), 3.40 (dd, J=8.9, 5.0 Hz, 1H, H-11a), 3.19 (t, J = 8.7 Hz, 1H, $\mathrm{H}-11 \mathrm{~b}), 2.71-2.61$ (m, 2H, H-3a, H-7), 2.52 (dd, J = 17.4, $2.6 \mathrm{~Hz}, 1 \mathrm{H}, \mathrm{H}-3 \mathrm{~b}), 1.99$ (s, 3H, H-1), $1.74(\mathrm{~d}, J=1.4 \mathrm{~Hz}, 3 \mathrm{H}, \mathrm{H}-10), 1.50-1.41(\mathrm{~m}, 1 \mathrm{H}, \mathrm{H}-8 \mathrm{a}), 1.17-1.11(\mathrm{~m}, 1 \mathrm{H}, \mathrm{H}-8 \mathrm{~b}), 0.88$ (t, $J=$ $7.4 \mathrm{~Hz}, 3 \mathrm{H}, \mathrm{H}-9$ ).; ${ }^{13} \mathrm{C}$ NMR (125 MHz, CDCl $) \delta 210.0$ (C, C-2), 138.6 (C, Ph), 137.5 (C, C-5), 129.8 (C-6), $128.5(2 \mathrm{CH}, \mathrm{Ph}), 127.8(2 \mathrm{CH}, \mathrm{Ph}), 127.7(\mathrm{CH}, \mathrm{Ph}), 74.4\left(\mathrm{CH}_{2}, \mathrm{C}-11\right), 73.3\left(\mathrm{CH}_{2}\right.$ $\left.\mathrm{OCH}_{2} \mathrm{Ph}\right), 66.9(\mathrm{CH}, \mathrm{C}-4), 48.9\left(\mathrm{CH}_{2}, \mathrm{C}-3\right), 39.9(\mathrm{CH}, \mathrm{C}-7), 30.6\left(\mathrm{CH}_{3}, \mathrm{C}-1\right), 25.2\left(\mathrm{CH}_{2}, \mathrm{C}-8\right), 18.9$ $\left(\mathrm{CH}_{3}, \mathrm{C}-10\right), 11.8\left(\mathrm{CH}_{3}, \mathrm{C}-9\right)$. 
(4S,7R,Z)-7-((Benzyloxy)methyl)-5-methyl-2-oxonon-5-en-4-yl(R)-3,3,3-trifluoro-2methoxy-2-phenylpropanoate (136)<smiles>COC(C(=O)OC(CC(C)=O)C(C)Cc1ccccc1)(c1ccccc1)C(F)(F)c1ccccc1</smiles>

Alcohol 128 (25.2 mg, $0.09 \mathrm{mmol}$ ) was dissolved in $0.6 \mathrm{~mL}$ dry DCM under argon and treated sequentially with 4-(dimethylamino)pyridine (DMAP) $\left(27.4 \mathrm{mg}, 0.22 \mathrm{mmol}, 2.6\right.$ equiv.), $N, N^{\prime}$ dicyclohexylcarbodiimide (DCC) $(62.1 \mathrm{mg}, 0.30 \mathrm{mmol}, 3.4$ equiv.) and (R)-(-)- $\alpha$-methoxy- $\alpha-$ (trifluoromethyl)phenylacetic acid ((R)-MTPA) (34.2 mg, $0.15 \mathrm{mmol}, 1.7$ equiv.). The reaction was stirred for 3 days then quenched with water $(8 \mathrm{~mL})$ and the aqueous phase extracted with DCM (3x $10 \mathrm{~mL})$. The combined organic layers were dried and concentrated, then purified on a silica gel column (9:1 PE/EA) to give the ester product 136 as a colourless oil (4.7 mg, 11\%).

$\mathbf{R}_{f}=0.57(5: 1 \mathrm{PE} / \mathrm{EA}) ;{ }^{1} \mathbf{H}$ NMR $\left(500 \mathrm{MHz}, \mathrm{CDCl}_{3}\right) \delta 7.47-7.42(\mathrm{~m}, 3 \mathrm{H}, \mathrm{Ph}), 7.40-7.28(\mathrm{~m}, 6 \mathrm{H}$, Ph), $7.25-7.23(\mathrm{~m}, 1 \mathrm{H}, \mathrm{Ph}) 6.34$ (dd, J = 10.2, $2.7 \mathrm{~Hz}, 1 \mathrm{H}, \mathrm{H}-4), 5.23(\mathrm{dq}, J=10.5,1.6 \mathrm{~Hz}, 1 \mathrm{H}$, $\mathrm{H}-6), 4.54\left(\mathrm{~m}, 2 \mathrm{H}, \mathrm{OCH}_{2} \mathrm{Ph}\right), 3.53(\mathrm{dd}, J=9.4,4.9 \mathrm{~Hz}, 1 \mathrm{H}, \mathrm{H}-11 \mathrm{a}), 3.48\left(\mathrm{~m}, 3 \mathrm{H}, \mathrm{OCH}_{3}\right), 3.40$ (dd, J = 9.4, $6.5 \mathrm{~Hz}, 1 \mathrm{H}, \mathrm{H}-11 \mathrm{~b}), 3.11$ (dd, $J=17.4,10.2 \mathrm{~Hz}, 1 \mathrm{H}, \mathrm{H}-3 \mathrm{a}), 2.95-2.85(\mathrm{~m}, 1 \mathrm{H}, \mathrm{H}-$ 7), $2.46(\mathrm{dd}, J=17.4,2.7 \mathrm{~Hz}, 1 \mathrm{H}, \mathrm{H}-3 \mathrm{~b}), 2.14(\mathrm{~s}, 3 \mathrm{H}, \mathrm{H}-1), 1.74-1.63(\mathrm{~m}, 1 \mathrm{H}, \mathrm{H}-8 \mathrm{a}), 1.50$ (d, J $=1.4 \mathrm{~Hz}, 3 \mathrm{H}, \mathrm{H}-10), 1.28-1.18(\mathrm{~m}, 1 \mathrm{H}, \mathrm{H}-8 \mathrm{~b}), 0.80(\mathrm{t}, J=7.5 \mathrm{~Hz}, 3 \mathrm{H}, \mathrm{H}-9) ;{ }^{13} \mathrm{C}$ NMR (125 $\mathrm{MHz}, \mathrm{CDCl}_{3}$ ) $\delta 204.4$ (C, C-2), 165.3 (C, MTPA), 139.0 (C, $\mathrm{OCH}_{2} \mathrm{Ph}$ ), 133.2 (CH, C-6), 132.7 (C, Ph) 131.4 (C, C-5), 129.6 (CH, Ph), 128.4 (4CH, Ph, OCH $\mathrm{OCh}_{2}, 127.7\left(2 \mathrm{CH}, \mathrm{OCH}_{2} \mathrm{Ph}\right), 127.5(\mathrm{CH}$, $\left.\mathrm{OCH}_{2} \mathrm{Ph}\right), 127.44(\mathrm{CH}, \mathrm{Ph}), 127.43(\mathrm{CH}, \mathrm{Ph}), 84.6$ (C, MTP, determined from HMBC), 73.4 $\left(\mathrm{CH}_{2}, \mathrm{C}-11\right), 72.9\left(\mathrm{CH}_{2}, \mathrm{OCH} 2 \mathrm{Ph}\right), 70.6(\mathrm{CH}, \mathrm{C}-4), 55.7\left(\mathrm{CH}_{3}, \mathrm{OCH}_{3}\right), 46.0\left(\mathrm{CH}_{2}, \mathrm{C}-3\right), 39.7(\mathrm{CH}, \mathrm{C}-$ 7), $30.7\left(\mathrm{CH}_{3}, \mathrm{C}-1\right), 25.0\left(\mathrm{CH}_{2}, \mathrm{C}-8\right), 18.0\left(\mathrm{CH}_{3}, \mathrm{C}-10\right), 12.0\left(\mathrm{CH}_{3}, \mathrm{C}-9\right), \mathrm{CF}_{3}$ signal obscured or missing (too weak). 


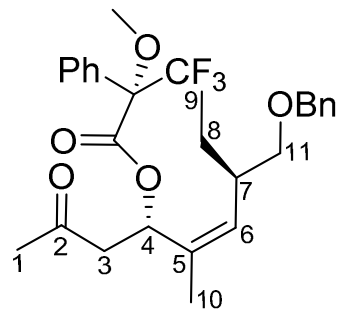

Alcohol 128 (11 mg, $0.04 \mathrm{mmol})$ was dissolved in dry DCM (0.5 mL) under argon and treated sequentially with 4-(dimethylamino)pyridine (DMAP) $\left(15.1 \mathrm{mg}, 0.12 \mathrm{mmol}, 3.2\right.$ equiv.), $N, N^{\prime}$ dicyclohexylcarbodiimide (DCC) (25.9 mg, $0.12 \mathrm{mmol}, 3.3$ equiv.) and (S)-(-)- $\alpha$-methoxy- $\alpha-$ (trifluoromethyl)phenylacetic acid ((S)-MTPA) (17.1 mg, $0.07 \mathrm{mmol}, 1.9$ equiv.). The reaction was stirred for 8 days with more reagents being added to it on the second and on the fifth day. After 8 days the reaction was quenched with water $(5 \mathrm{~mL})$ and the aqueous phase extracted with DCM (3x $5 \mathrm{~mL})$. Organic layers were dried and concentrated then purified on silica gel column (9:1 PE/EA) to yield the product 137 as a colourless oil (2.9 mg, 15\%).

$\mathbf{R}_{f}=0.44(5: 1 \mathrm{PE} / \mathrm{EA}) ;{ }^{1} \mathbf{H}$ NMR $\left(500 \mathrm{MHz}, \mathrm{CDCl}_{3}\right) \delta 7.48-7.42(\mathrm{~m}, 2 \mathrm{H}), 7.39-7.27(\mathrm{~m}, 7 \mathrm{H})$, $7.26-7.22(\mathrm{~m}, 1 \mathrm{H}), 6.44$ (dd, J=9.8, 3.2 Hz, 1H, H-4), 5.28 (dq, J = 10.5, $1.6 \mathrm{~Hz}, 1 \mathrm{H}, \mathrm{H}-6)$, $4.50\left(\mathrm{~d}, J=12.2 \mathrm{~Hz}, 1 \mathrm{H}, \mathrm{OCH}_{2} \mathrm{aPh}\right), 4.46$ (d, $J=12.3 \mathrm{~Hz}, 1 \mathrm{H}, \mathrm{OCH}_{2} \mathrm{bPh}$ ), $3.48\left(\mathrm{~s}, 3 \mathrm{H}, \mathrm{OCH}_{3}\right.$ ), 3.46 (dd, J = 9.5, $5.2 \mathrm{~Hz}, 1 \mathrm{H}, \mathrm{H}-11 \mathrm{a}$ ), 3.36 (dd, $J=9.4,6.2 \mathrm{~Hz}, 1 \mathrm{H}, \mathrm{H}-11 \mathrm{~b}$ ), 3.06 (dd, J = 17.0, $9.8 \mathrm{~Hz}, 1 \mathrm{H}, \mathrm{H}-3 \mathrm{a}), 2.92(\mathrm{~m}, 1 \mathrm{H}, \mathrm{H}-7), 2.46$ (dd, J = 17.0, $3.2 \mathrm{~Hz}, 1 \mathrm{H}, \mathrm{H}-3 \mathrm{~b}), 2.06(\mathrm{~s}, 3 \mathrm{H}, \mathrm{H}-1)$, $1.72(\mathrm{~d}, J=1.4 \mathrm{~Hz}, 3 \mathrm{H}, \mathrm{H}-10), 1.70-1.61(\mathrm{~m}, 1 \mathrm{H}, \mathrm{H}-8 \mathrm{a}), 1.30-1.20(\mathrm{~m}, 1 \mathrm{H}, \mathrm{H}-8 \mathrm{~b}), 0.82$ (t, $J=$ $7.4 \mathrm{~Hz}, 3 \mathrm{H}, \mathrm{H}-9)$.

(4S,7R,Z)-7-((Benzyloxy)methyl)-4-((4-methoxybenzyl)oxy)-5-methylnon-5-en-2-one (129)

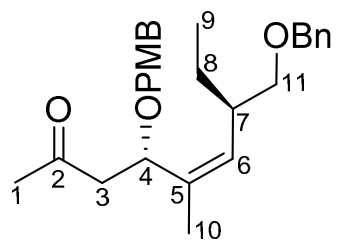

Secondary alcohol $128(22.8 \mathrm{mg}, 0.08 \mathrm{mmol})$ was dissolved in dry DCM (1 mL) and cooled to $0{ }^{\circ} \mathrm{C}$ then treated with CSA ( $8.1 \mathrm{mg}, 0.03 \mathrm{mmol}, 0.4$ equiv.) and, dropwise, with PMBTCA ( $0.06 \mathrm{~mL}, 0.3 \mathrm{mmol}, 4$ equiv.) which was added dropwise. The reaction was allowed to warm 
to room temperature and stirred overnight then quenched with $1: 1 \mathrm{v} / \mathrm{v} \mathrm{H}_{2} \mathrm{O} / \mathrm{sat}$. aq. $\mathrm{NaHCO}_{3}(5 \mathrm{~mL})$. The aqueous layer was extracted with DCM $(3 \times 5 \mathrm{~mL})$ and the combined organic layers dried $\left(\mathrm{MgSO}_{4}\right)$ and concentrated in vacuo. The crude reaction mixture was purified using flash chromatography (9:1 PE/EA) to give the product 129 contaminated with an inseparable impurity, $p$-methoxybenzyl-2,2,2-trichloroacetamide (the product was $73 \%$ of the $30.3 \mathrm{mg}$ sample, $22.1 \mathrm{mg}, 68 \%)$.

$\mathbf{R}_{f}=0.72(3: 1 \mathrm{PE} / \mathrm{EA}) ;{ }^{1} \mathrm{H}$ NMR $\left(500 \mathrm{MHz}, \mathrm{CDCl}_{3}\right) \delta 7.33-7.26(\mathrm{~m}, 5 \mathrm{H}, \mathrm{Ph}), 7.14(\mathrm{~d}, \mathrm{~J}=8.6 \mathrm{~Hz}$, 1H, OPMB), 6.82 (d, $J=8.6 \mathrm{~Hz}, 1 \mathrm{H}, \mathrm{OPMB}), 5.19$ (dq, $J=10.6,1.9 \mathrm{~Hz}, 1 \mathrm{H}, \mathrm{H}-6), 4.78$ (dd, $J=$ 9.8, 3.2 Hz, 1H, H-4), $4.52-4.46\left(\mathrm{~m}, 2 \mathrm{H}, \mathrm{OCH}_{2} \mathrm{Ph}\right.$ ), 4.30 (d, J = $\left.11.1 \mathrm{~Hz}, 1 \mathrm{H}, \mathrm{OPMB}\right), 4.13$ (d, J $=11.1 \mathrm{~Hz}, 1 \mathrm{H}, \mathrm{OPMB}), 3.78$ (s, 3H, OPMB), 3.38 (dd, $J=9.1,6.1 \mathrm{~Hz}, 1 \mathrm{H}, \mathrm{H}-11 \mathrm{a}), 3.32$ (dd, $J=$ 9.1, 6.6 Hz, 1H, H-11b), 2.92 (dd, J = 15.5, $9.8 \mathrm{~Hz}, 1 \mathrm{H}, \mathrm{H}-3 \mathrm{a}), 2.72-2.62(\mathrm{~m}, 1 \mathrm{H}, \mathrm{H}-7), 2.29$ (dd, $J=15.5,3.2 \mathrm{~Hz}, 1 \mathrm{H}, \mathrm{H}-3 \mathrm{~b}$ ), 2.14 (s, 3H, H-1), 1.73 (d, J=1.5 Hz, 3H, H-10), $1.60-1.54$ (m, 1H, H-8a), $1.21-1.12$ (m, 1H, H-8b), 0.80 (t, J = $7.4 \mathrm{~Hz}, 3 \mathrm{H}, \mathrm{H}-9) ;{ }^{13} \mathrm{C}$ NMR (125 MHz, $\mathrm{CDCl}_{3}$ ) $\delta 207.0$ (C, C-2), 159.1 (C, OPMB), 138.7 (C, Ph), 135.2 (C, C-5), 132.1 (CH, C-6), 130.8 (C, OPMB), 129.5 (2CH, OPMB), 128.4 (2CH, Ph), 127.7 (2CH, Ph), $127.6(\mathrm{CH}, \mathrm{Ph}), 113.8(2 \mathrm{CH}$, OPMB), $74.2\left(\mathrm{CH}_{2}, \mathrm{C}-11\right), 73.0\left(\mathrm{CH}, \mathrm{C}-4\right.$ and $\left.\mathrm{CH}_{2}, \mathrm{OCH}_{2} \mathrm{Ph}\right), 70.0\left(\mathrm{CH}_{2}, \mathrm{OPMB}\right), 55.4\left(\mathrm{CH}_{3}\right.$, OPMB), $48.1\left(\mathrm{CH}_{2}, \mathrm{C}-3\right), 39.5(\mathrm{CH}, \mathrm{C}-7), 31.2\left(\mathrm{CH}_{3}, \mathrm{C}-1\right), 25.2\left(\mathrm{CH}_{2}, \mathrm{C}-8\right), 18.1\left(\mathrm{CH}_{3}, \mathrm{C}-10\right), 11.9$ $\left(\mathrm{CH}_{3}, \mathrm{C}-9\right)$; HRMS (ESI) Found 428.2808. Calculated for $\mathrm{C}_{26} \mathrm{H}_{38} \mathrm{NO}_{4}{ }^{+}\left[\mathrm{M}+\mathrm{NH}_{4}\right]^{+}$428.2795. $\Delta=$ $2.86 \mathrm{ppm}$.

Methyl 2-(bis(2,2,2-trifluoroethoxy)phosphoryl)propanoate (133)<smiles>CCOP(=O)(OCC(F)(F)F)C(C)C(=O)OC</smiles>

Methyl P,P-bis(2,2,2-trifluoroethyl)phosphonoacetate [Methyl 2-(bis(2,2,2trifluoroethoxy)phosphoryl)acetate] $(0.65 \mathrm{~mL}, 3.1 \mathrm{mmol})$ was added dropwise to a suspension of $\mathrm{NaH}(0.149 \mathrm{~g}, 3.7 \mathrm{mmol}, 1.2$ equiv.) in dry DMF (6 mL) at room temperature under an atmosphere of argon. After $10 \mathrm{~min}$ of stirring, Mel (0.68 mL, $11 \mathrm{mmol}, 3.5$ equiv.) was added dropwise to the yellow solution. The reaction was stirred at room temperature for 48 hours then quenched with sat. aq. $\mathrm{NH}_{4} \mathrm{Cl}(10 \mathrm{~mL})$ and diluted with $50 \mathrm{~mL}$ water. The aqueous layer was extracted with EtOAc ( $3 \times 15 \mathrm{~mL})$ and the combined organic layers were 
washed with sat. aq. $\mathrm{Na}_{2} \mathrm{~S}_{2} \mathrm{O}_{3}(30 \mathrm{~mL})$, brine $(30 \mathrm{~mL})$ then water $(30 \mathrm{~mL})$, dried over anhydrous $\mathrm{MgSO}_{4}$ and concentrated. Crude reaction mixture was purified by flash chromatography (2:1 PE/EA) to give the product 133 as a colourless oil $(0.597 \mathrm{~g}, 58 \%, 76 \%$ BRSM).

$\mathbf{R}_{f}=0.37(2: 1 \mathrm{PE} / \mathrm{EA}) ;{ }^{1} \mathrm{H}$ NMR $\left(500 \mathrm{MHz}, \mathrm{CDCl}_{3}\right): \delta 4.51-4.36(\mathrm{~m}, 4 \mathrm{H}, \mathrm{H}-5), 3.78(\mathrm{~s}, 3 \mathrm{H}, \mathrm{H}-4)$, $3.20(\mathrm{dq}, J=22.5,7.4 \mathrm{~Hz}, 1 \mathrm{H}, \mathrm{H}-2), 1.52$ (dd, $J=19.3,7.4 \mathrm{~Hz}, 3 \mathrm{H}, \mathrm{H}-3) ;{ }^{13} \mathrm{C}$ NMR $(126 \mathrm{MHz}$, $\left.\mathrm{CDCl}_{3}\right): \delta 169.1(\mathrm{C}, \mathrm{d}, J=3.2 \mathrm{~Hz}, \mathrm{C}-1), 122.62\left(\mathrm{CF}_{3}, \mathrm{qd}, J=277.2,2.5 \mathrm{~Hz}, \mathrm{C}-6 \mathrm{a}\right), 122.55\left(\mathrm{CF}_{3}\right.$, qd, $J=277.2,2.5 \mathrm{~Hz}, \mathrm{C}-6 \mathrm{~b}), 62.8\left(\mathrm{CH}_{2}, \mathrm{qd}, J=38.7,2.2 \mathrm{~Hz}, \mathrm{C}-5 \mathrm{a}\right), 62.7\left(\mathrm{CH}_{2}, \mathrm{qd}, J=38.3,1.7\right.$ $\mathrm{Hz}, \mathrm{C}-5 \mathrm{~b}), 53.2\left(\mathrm{CH}_{3}, \mathrm{C}-4\right), 39.48(\mathrm{CH}, \mathrm{d}, J=141.2 \mathrm{~Hz}, \mathrm{C}-2), 11.73\left(\mathrm{CH}_{3}, \mathrm{~d}, J=6.6 \mathrm{~Hz}, \mathrm{C}-3\right)$; HRMS (ESI) Found 333.032345. Calculated for $\mathrm{C}_{8} \mathrm{H}_{12} \mathrm{~F}_{6} \mathrm{O}_{5} \mathrm{P}^{+}[\mathrm{M}+\mathrm{H}]^{+} 333.03211 . \Delta=2.59 \mathrm{ppm}$.

\section{Experimental for Chapter 3}

\section{3-(Benzyloxy)-2,2-dimethylpropan-1-ol (66)}

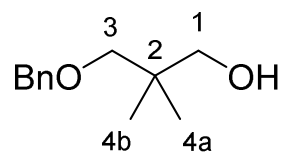

2,2-Dimethylpropane-1,3-diol $(1.092 \mathrm{~g}, 10.48 \mathrm{mmol})$ in dry THF $(27 \mathrm{~mL})$ was cooled to $0{ }^{\circ} \mathrm{C}$ under an argon atmosphere then treated with $\mathrm{NaH}(0.422 \mathrm{~g}, 10.56 \mathrm{mmol}, 1$ equiv.), TBAI (0.386 g, $1.02 \mathrm{mmol}, 0.1$ equiv.) and $\mathrm{BnBr}(1.30 \mathrm{~mL}, 10.70 \mathrm{mmol}, 1$ equiv.). The reaction was then allowed to warm up to room temperature and stirred overnight. After stirring overnight, the reaction was quenched with sat. aq. $\mathrm{NH}_{4} \mathrm{Cl}$ solution $(30 \mathrm{~mL})$ and the aqueous layer extracted with DCM $(3 \times 30 \mathrm{~mL})$. The organic layers were combined and dried with anhydrous $\mathrm{MgSO}_{4}$, then the solvent removed under reduced pressure. The crude material was purified using silica gel chromatography (10:1 PE/EA, then 5:1 PE:EA) to give the product 66 as a colourless oil (1.534 g, 75\%). Experimental data matched those reported. ${ }^{128}$

$\mathbf{R}_{f}=0.39(5: 1 \mathrm{PE} / \mathrm{EA}) ;{ }^{1} \mathrm{H}-\mathrm{NMR}\left(500 \mathrm{MHz}, \mathrm{CDCl}_{3}\right): \delta 7.38-7.27(\mathrm{~m}, 5 \mathrm{H}, \mathrm{Ph}), 4.52(\mathrm{~s}, 2 \mathrm{H}$, $\mathrm{CH}_{2} \mathrm{Ph}$ ), 3.46 (s, 2H, H-1), 3.33 (s, 2H, H-3), 0.93 (s, 6H, H-4a, H-4b); ${ }^{13} \mathrm{C}-\mathrm{NMR}(100 \mathrm{MHz}$, $\left.\mathrm{CDCl}_{3}\right): \delta 138.3(\mathrm{C}, \mathrm{Ph}), 128.6(2 \mathrm{CH}, \mathrm{Ph}), 127.8(\mathrm{CH}, \mathrm{Ph}), 127.6(2 \mathrm{CH}, \mathrm{Ph}), 79.6\left(\mathrm{CH}_{2}, \mathrm{C}-3\right)$, $73.7\left(\mathrm{CH}_{2}, \mathrm{OCH}_{2} \mathrm{Ph}\right), 72.0\left(\mathrm{CH}_{2}, \mathrm{C}-1\right), 36.4$ (C, $\left.\mathrm{C} 2\right), 22.0\left(2 \mathrm{CH}_{3}, \mathrm{C}-4 \mathrm{a}, \mathrm{C}-4 \mathrm{~b}\right)$. (AB3.155.1). HRMS (ESI) Found 195.1382. Calculated for $\mathrm{C}_{12} \mathrm{H}_{19} \mathrm{O}_{2}{ }^{+}[\mathrm{M}+\mathrm{H}]^{+}$195.1380. $\Delta=1.23 \mathrm{ppm}$; 


\section{3-(Benzyloxy)-2,2-dimethylpropanal (67)}

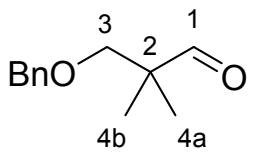

To a solution of alcohol $66(2.571 \mathrm{~g}, 13.23 \mathrm{mmol})$ and TEMPO $(0.211 \mathrm{~g}, 1.35 \mathrm{mmol}, 0.1$ eqiuiv.) in dry DCM (15 mL), was added BAIB (4.785 g, $14.86 \mathrm{mmol}, 1.1$ equiv.) under air at room temperature. After 2.5 hours, the reaction had completed (based on TLC analysis, 3:1 PE:EA) and was diluted with $25 \mathrm{~mL} \mathrm{DCM}$ and the reaction mixture washed with sat. aq. sodium thiosulfate $(25 \mathrm{~mL})$ then extracted with DCM (2x $25 \mathrm{~mL})$. The combined organic extracts were washed with sat. aq. $\mathrm{NaHCO}_{3}(25 \mathrm{~mL})$ and brine $(25 \mathrm{~mL})$, dried with anhydrous $\mathrm{MgSO}_{4}$, and concentrated under reduced pressure to give a reddish-yellow residue which was purified using silica gel chromatography $(10: 1 \mathrm{PE} / \mathrm{EA})$ to afford the product 67 as a colourless oil ( $1.664 \mathrm{~g}, 65 \%$ yield). Spectral data matched those reported. ${ }^{128}$

$\mathbf{R}_{f}=0.68(10: 1 \mathrm{PE} / \mathrm{EA}) ;{ }^{1} \mathrm{H}-\mathrm{NMR}\left(500 \mathrm{MHz}, \mathrm{CDCl}_{3}\right): \delta 9.58(\mathrm{~s}, 1 \mathrm{H}, \mathrm{H}-1), 7.36-7.29(\mathrm{~m}, 5 \mathrm{H}, \mathrm{Ph})$, $4.52\left(\mathrm{~s}, 2 \mathrm{H}, \mathrm{CH}_{2} \mathrm{Ph}\right), 3.46$ (s, 2H, H-3), 1.10 (s, 6H, H-4a, H-4b); ${ }^{13} \mathrm{C}-\mathrm{NMR}\left(100 \mathrm{MHz}, \mathrm{CDCl}_{3}\right): \delta$ 205.3 (CH, C-1), 138.2 (C, Ph), $128.4(2 \mathrm{CH}, \mathrm{Ph}), 127.7$ (CH, Ph), $127.5(2 \mathrm{CH}, \mathrm{Ph}), 75.2\left(\mathrm{CH}_{2}, \mathrm{C}-\right.$ 3), $73.5\left(\mathrm{CH}_{2}, \mathrm{CH}_{2} \mathrm{Ph}\right), 47.2$ (C, C-2), $19.1\left(2 \mathrm{CH}_{3}, \mathrm{C}-4 \mathrm{a}, \mathrm{C}-4 \mathrm{~b}\right)$; IR (film from $\left.\mathrm{CHCl}_{3}\right) \mathrm{v}_{\max } 3369$, 2971, 2858, 1730, 1098, 737, $698 \mathrm{~cm}^{-1}$.

\section{4-(Benzyloxy)-3,3-dimethylbutan-2-ol (68)}

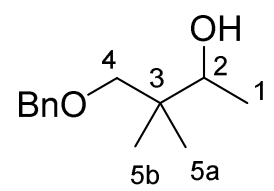

Starting material aldehyde $67(2.047 \mathrm{~g}, 10.65 \mathrm{mmol})$ was dissolved in $10 \mathrm{~mL}$ dry $\mathrm{Et}_{2} \mathrm{O}$ and treated with $\mathrm{MeMgl}\left(3 \mathrm{M}\right.$ in $\mathrm{Et}_{2} \mathrm{O}, 5.30 \mathrm{~mL}, 15.90 \mathrm{mmol}, 1.5$ equiv.) dropwise at room temperature under argon. After 1 hour of stirring at room temperature, the reaction was quenched with water and acidified with $10 \%$ aq. $\mathrm{HCl}$ until a $\mathrm{pH}$ of 4 was reached. The aqueous phase was then extracted with $\mathrm{Et}_{2} \mathrm{O}(3 \times 20 \mathrm{~mL})$. Purification on silica gel column (9:1 PE/EA) gave the alcohol 68 as a colourless oil (1.759 g, 79\%). Spectral data matched those reported. ${ }^{227}$ 
$\mathbf{R}_{f}=0.50(10: 1 \mathrm{PE} / \mathrm{EA}) ;{ }^{1} \mathrm{H}-\mathrm{NMR}\left(500 \mathrm{MHz}, \mathrm{CDCl}_{3}\right): \delta 7.37-7.28(\mathrm{~m}, 5 \mathrm{H}, \mathrm{Ph}), 4.52(\mathrm{~s}, 2 \mathrm{H}$, $\mathrm{CH}_{2} \mathrm{Ph}$ ), 3.70-3.68 (m, 1H, H-2), 3.39 (d, J=8.8 Hz, 1H, H-4a), 3.31 (d, J=8.8 Hz, 1H, H-4b), $3.27(\mathrm{~d}, J=4.0 \mathrm{~Hz}, 1 \mathrm{H}, \mathrm{OH}), 1.11$ (d, J = 6.4 Hz, 3H, H-1), 0.92 (s, 3H, H-5a), 0.88 (s, 3H, H-5b); ${ }^{13} \mathrm{C}-\mathrm{NMR}\left(100 \mathrm{MHz}, \mathrm{CDCl}_{3}\right): \delta 138.0(\mathrm{C}, \mathrm{Ph}), 128.6(2 \mathrm{CH}, \mathrm{Ph}), 127.8(\mathrm{CH}, \mathrm{Ph}), 127.6(2 \mathrm{CH}, \mathrm{Ph})$, $80.2\left(\mathrm{CH}_{2}, \mathrm{C}-4\right), 74.7(\mathrm{CH}, \mathrm{C}-2), 73.7\left(\mathrm{CH}_{2}, \mathrm{CH}_{2} \mathrm{Ph}\right), 38.5$ (C, $\left.\mathrm{C}-3\right), 22.8\left(\mathrm{CH}_{3}, \mathrm{C}-5 \mathrm{a}\right.$ or $\left.\mathrm{C}-5 \mathrm{~b}\right), 19.1$ $\left(\mathrm{CH}_{3}, \mathrm{C}-5 \mathrm{a}\right.$ or $\left.\mathrm{C}-5 \mathrm{~b}\right), 17.9\left(\mathrm{CH}_{3}, \mathrm{C}-1\right)$.

\section{4-(Benzyloxy)-3,3-dimethylbutan-2-one (24)}

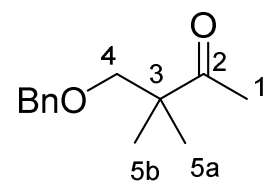

Secondary alcohol 68 (1.451 g, $6.97 \mathrm{mmol})$ was dissolved in $25 \mathrm{~mL}$ DCM and treated with $\operatorname{DMP}$ (3.656 g, $8.62 \mathrm{mmol}, 1.2$ equiv.) at room temperature under air. After stirring for $1.5 \mathrm{~h}$ at the same temperature (by which time TLC analysis in 9:1 PE/EA indicated starting material was consumed), the reaction was quenched with $15 \mathrm{~mL}$ of sat. aq. sodium thiosulfate and $15 \mathrm{~mL}$ sat. aq. sodium bicarbonate. Quenched reaction was stirred for 10 min then the two layers were separated and the aqueous layer extracted two more times with DCM (2x $15 \mathrm{~mL})$. The combined organic fractions were washed with brine $(20 \mathrm{~mL})$, dried with anhydrous $\mathrm{MgSO}_{4}$, and concentrated under reduced pressure. Purification on a silica gel column (9:1 PE/EA) yielded the product 24 as a colourless oil (1.0759 g, 75\%).

${ }^{1}$ H-NMR (500 MHz, CDCl $): \delta 7.35-7.32(\mathrm{~m}, 2 \mathrm{H}, \mathrm{Ph}), 7.30-7.28(\mathrm{~m}, 3 \mathrm{H}, \mathrm{Ph}), 4.50(\mathrm{~s}, 2 \mathrm{H}$, $\mathrm{CH}_{2} \mathrm{Ph}$ ), 3.45 (s, 2H, H-4), 2.15 (s, 3H, H-1), 1.15 (s, 6H, H-5a, H-5b); ${ }^{13} \mathrm{C}-\mathrm{NMR}(100 \mathrm{MHz}$, $\left.\mathrm{CDCl}_{3}\right): \delta 212.9$ (C, C-2), 138.3 (C, Ph), 128.5 (2CH, Ph), 127.7 (CH, Ph), $127.6(2 \mathrm{CH}, \mathrm{Ph}), 77.2$ $\left(\mathrm{CH}_{2}, \mathrm{C}-4\right), 73.5\left(\mathrm{CH}_{2}, \mathrm{CH}_{2} \mathrm{Ph}\right), 48.8(\mathrm{C}, \mathrm{C}-3), 25.9\left(\mathrm{CH}_{3}, \mathrm{C}-1\right), 22.1\left(2 \mathrm{CH}_{3}, \mathrm{C}-5 \mathrm{a}, \mathrm{C}-5 \mathrm{~b}\right)$.

\section{4-Hydroxy-3,3-dimethylbutan-2-one (71)}

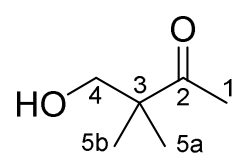

Benzyl ether 24 (0.142 g, $0.69 \mathrm{mmol})$ was dissolved in dry DCM (3 mL) under Ar atmosphere and cooled to $0{ }^{\circ} \mathrm{C}$ then treated dropwise with $\mathrm{TiCl}_{4}$ solution $(1 \mathrm{M}$ in $\mathrm{DCM}, 2.10 \mathrm{~mL}, 2.10$ 
mmol, 3 equiv.). The reaction was stirred at the same temperature for $15 \mathrm{~min}$, at which point TLC analysis (5:1 PE/EA) showed complete consumption of starting material. The reaction was quenched with brine $(10 \mathrm{~mL})$ and extracted with $\mathrm{DCM}(3 \times 10 \mathrm{~mL})$. The combined organic layers were dried over anhydrous $\mathrm{MgSO}_{4}$ and concentrated under reduced pressure. Crude material was purified on silica column eluting with 3:1 PE/EA to give a product 71 as a colourless oil $(60.5 \mathrm{mg}, 76 \%)$. Spectral data matched those previously reported. $^{228}$

$\mathbf{R}_{f}=0.16(3: 1 \mathrm{PE} / \mathrm{EA}) ;{ }^{1} \mathrm{H}-\mathrm{NMR}\left(500 \mathrm{MHz}, \mathrm{CDCl}_{3}\right): \delta 3.54(\mathrm{~s}, 2 \mathrm{H}, \mathrm{H}-4), 2.15(\mathrm{~s}, 3 \mathrm{H}, \mathrm{H}-1), 1.15(\mathrm{~s}$, 6H, H-5a, H-5b); ${ }^{13} \mathrm{C}-\mathrm{NMR}\left(100 \mathrm{MHz}, \mathrm{CDCl}_{3}\right): \delta 215.5$ (C, C-2), $69.5\left(\mathrm{CH}_{2}, \mathrm{C}-4\right), 49.3$ (C, C-3) , $25.7\left(\mathrm{CH}_{3}, \mathrm{C}-1\right), 21.8\left(2 \mathrm{CH}_{3}, \mathrm{C}-5 \mathrm{a}, \mathrm{C}-5 \mathrm{~b}\right)$.

\section{2,2-Dimethyl-3-oxobutanal (56)}

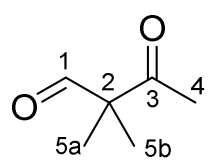

Alcohol 71 (4.5 mg, $0.04 \mathrm{mmol})$ was dissolved in $\mathrm{DCM}(0.4 \mathrm{~mL})$ at room temperature under air and treated with DMP $(29.0 \mathrm{mg}, 0.07 \mathrm{mmol}, 1.7$ equiv) and stirred at the same temperature for 1 hour. The reaction was quenched with 1:1 v/v sat. aq. $\mathrm{NaHCO}_{3} / \mathrm{sat}$. aq. $\mathrm{Na}_{2} \mathrm{~S}_{2} \mathrm{O}_{3}(4 \mathrm{~mL})$ and stirred for 30 min then extracted with $\mathrm{Et}_{2} \mathrm{O}(3 \times 4 \mathrm{~mL})$. The combined organic fractions were dried with anhydrous $\mathrm{MgSO}_{4}$ and concentrated under reduced pressure while keeping the rotavap water-bath temperature bellow $30^{\circ} \mathrm{C}$. The crude sample containing aldehyde 56 was used in the next step without purification. ${ }^{1} \mathrm{H}$ NMR data matched those reported. ${ }^{229}$

${ }^{1} \mathrm{H}-\mathrm{NMR}\left(500 \mathrm{MHz}, \mathrm{CDCl}_{3}\right) \delta 9.62(\mathrm{~s}, 1 \mathrm{H}, \mathrm{H}-1), 2.18(\mathrm{~s}, 3 \mathrm{H}, \mathrm{H}-4), 1.34$ (s, 6H, H-5a,H-5b). 
(4S,8S,11R,Z)-11-((Benzyloxy)methyl)-4-hydroxy-8-((4-methoxybenzyl)oxy)-3,3,9-

trimethyltridec-9-ene-2,6-dione (142)

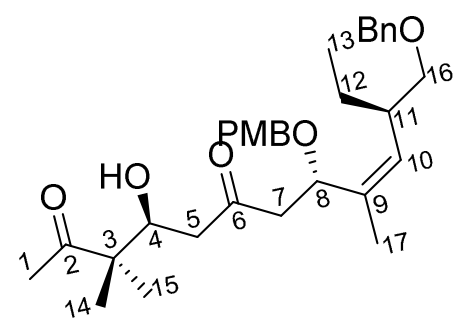

Alcohol 71 (31.3 mg, $0.27 \mathrm{mmol})$ was dissolved in $0.1 \mathrm{~mL} \mathrm{DCM}$ and treated with DMP (0.189 g, $0.43 \mathrm{mmol}$ ) at room temperature. The reaction was stirred for 1.5 hours then quenched with $10 \mathrm{~mL}$ v/v sat. aq. $\mathrm{Na}_{2} \mathrm{~S}_{2} \mathrm{O}_{3} / \mathrm{sat}$. aq. $\mathrm{NaHCO}_{3}$ and stirred for $30 \mathrm{~min}$. The aqueous layer was then extracted with $\mathrm{Et}_{2} \mathrm{O}(3 \times 10 \mathrm{~mL})$. The combined organic layers were dried over anhydrous $\mathrm{MgSO}_{4}$ and the solvent removed under reduced pressure (Rotavap water bath temperature kept at $30^{\circ} \mathrm{C}$ ). The crude reaction mixture containing aldehyde $\mathbf{5 6}$ was used in the next step without further purification.

Ketone 129 ( $65.8 \mathrm{~g}, 74 \%$ purity by NMR, $0.11 \mathrm{mmol})$ was dissolved in dry $\mathrm{Et}_{2} \mathrm{O}(3 \mathrm{~mL})$ and cooled to $-78{ }^{\circ} \mathrm{C}$ then treated sequentially with $\mathrm{Et}_{3} \mathrm{~N}(0.05 \mathrm{~mL}, 0.36 \mathrm{mmol})$ and 9-BBN triflate (0.5 $\mathrm{M}$ in hexane, $0.41 \mathrm{~mL}, 0.21 \mathrm{mmol}$ ) and stirred at the same temperature for 1.25 hours. The aldehyde 56 in $1 \mathrm{~mL} \mathrm{Et}{ }_{2} \mathrm{O}$ was then added dropwise and the reaction stirred at the same temperature for 4.5 hours. The reaction was quenched at $-70{ }^{\circ} \mathrm{C}$ with methanol $(2 \mathrm{~mL})$ and $\mathrm{pH} 7$ phosphate buffer $(2 \mathrm{~mL})$ then allowed to warm up to $0{ }^{\circ} \mathrm{C}$ and treated with $\mathrm{H}_{2} \mathrm{O}_{2}(0.50$ $\mathrm{mL}, 37 \%$ aq.) After stirring for 1 hour at room temperature the reaction was diluted with EtOAc $(10 \mathrm{~mL})$.The layers were then separated and the organic layer washed with sat. aq. $\mathrm{Na}_{2} \mathrm{~S}_{2} \mathrm{O}_{3}(10 \mathrm{~mL})$ and brine $(10 \mathrm{~mL})$, dried with $\mathrm{MgSO}_{4}$ and concentrated. Concentrated reaction mixture was purified using silica chromatography (5:1 PE/EA) to give the product 142 as colourless oil ( $28.8 \mathrm{mg}, 50 \%)$.

$\mathbf{R}_{f}=0.28(3: 1 \mathrm{PE} / \mathrm{EA}) ;[\boldsymbol{\alpha}]_{\mathrm{D}}{ }^{21}=-56.4\left(c\right.$ 1.49, $\left.\mathrm{CHCl}_{3}\right) ;{ }^{1} \mathrm{H}-\mathrm{NMR}\left(500 \mathrm{MHz}, \mathrm{CDCl}_{3}\right): \delta 7.33-7.21$ (m, 5H, OCH $\mathrm{O}_{2} \mathrm{Ph}$ ), 7.11 (d, $\left.J=8.6 \mathrm{~Hz}, 2 \mathrm{H}, \mathrm{OPMB}\right), 6.81$ (d, $\left.J=8.6 \mathrm{~Hz}, 1 \mathrm{H}, \mathrm{OPMB}\right), 5.20$ (dq, $J=$ 11.3, $2.5 \mathrm{~Hz}, 1 \mathrm{H}, \mathrm{H}-10), 4.79(\mathrm{dd}, J=10.2,2.9 \mathrm{~Hz}, 1 \mathrm{H}, \mathrm{H}-8), 4.48\left(\mathrm{~m}, 2 \mathrm{H}, \mathrm{OCH}_{2} \mathrm{Ph}\right), 4.29$ (d, J = $11.0 \mathrm{~Hz}, 1 \mathrm{H}, \mathrm{OPMB}$ ), 4.23 (ddd, $J=10.2,3.6,1.9 \mathrm{~Hz}, 1 \mathrm{H}, \mathrm{H}-4), 4.08(\mathrm{~d}, J=11.1 \mathrm{~Hz}, 1 \mathrm{H}$, OPMB), 3.78 (s, 3H, OPMB), 3.39 (dd, $J=9.1,6.0 \mathrm{~Hz}, 1 \mathrm{H}, \mathrm{H}-16 \mathrm{a}), 3.32$ (dd, $J=9.1,6.8 \mathrm{~Hz}, 1 \mathrm{H}$, 
$\mathrm{H}-16 \mathrm{~b}), 3.22$ (d, J = 3.6 Hz, 1H, OH), 2.97 (dd, J = 15.0, $10.3 \mathrm{~Hz}, 1 \mathrm{H}, \mathrm{H}-7 \mathrm{a}), 2.72-2.64(\mathrm{~m}, 1 \mathrm{H}$, $\mathrm{H}-11$ ), 2.60 (dd, J = 17.3, 1.9 Hz, 1H, H-5a), 2.44 (dd, J = 17.2, $10.2 \mathrm{~Hz}, 1 \mathrm{H}, \mathrm{H}-5 \mathrm{~b}$ ), 2.26 (dd, $J=$ 15.1, 3.0 Hz, 1H, H-7b), $2.14(\mathrm{~s}, 3 \mathrm{H}, \mathrm{H}-1), 1.73$ (d, J = $1.4 \mathrm{~Hz}, 3 \mathrm{H}, \mathrm{H}-17), 1.60-1.51(\mathrm{~m}, 1 \mathrm{H}, \mathrm{H}-$ 12a), $1.20-1.12$ (m, 1H, H-12b), 1.07 (s, 3H, H-14), 1.04 (s, 3H, H-15), 0.80 (t, J = 7.4 Hz, 3H, $\mathrm{H}-13$ ); ${ }^{13} \mathrm{C}$ NMR (126 MHz, CDCl $)$ ): $\delta 213.7$ (C, C-2), 209.9 (C, C-6), 159.2 (C, OPMB), 138.6 (C, $\mathrm{OCH}_{2} \mathrm{Ph}$ ), 134.8 (C, C-9), 132.5 (CH, C-10), 130.6 (C, OPMB), 129.6 (2CH, OPMB), 128.4 (2CH, $\left.\mathrm{OCH}_{2} \mathrm{Ph}\right), 127.7\left(2 \mathrm{CH}, \mathrm{OCH}_{2} P h\right), 127.6\left(\mathrm{CH}, \mathrm{OCH}_{2} P h\right), 113.8(2 \mathrm{CH}, \mathrm{OPMB}), 74.2\left(\mathrm{CH}_{2}, \mathrm{C}-16\right)$, 73.5 ( $\mathrm{CH}, \mathrm{C}-8), 73.2\left(\mathrm{CH}_{2}, \mathrm{OCH}_{2} \mathrm{Ph}\right), 71.9$ ( $\left.\mathrm{CH}, \mathrm{C}-4\right), 70.1$ ( $\left.\mathrm{CH}_{2}, \mathrm{OPMB}\right), 55.4\left(\mathrm{CH}_{3}, \mathrm{OPMB}\right), 51.2$ (C, C-3), $47.7\left(\mathrm{CH}_{2}, \mathrm{C}-7\right), 45.9\left(\mathrm{CH}_{2}, \mathrm{C}-5\right), 39.6(\mathrm{CH}, \mathrm{C}-11), 26.5\left(\mathrm{CH}_{3}, \mathrm{C}-1\right), 25.1\left(\mathrm{CH}_{2}, \mathrm{C}-12\right)$, $21.5\left(\mathrm{CH}_{3}, \mathrm{C}-15\right), 19.5\left(\mathrm{CH}_{3}, \mathrm{C}-14\right), 18.0\left(\mathrm{CH}_{3}, \mathrm{C}-17\right), 11.9\left(\mathrm{CH}_{3}, \mathrm{C}-13\right)$; IR (film from $\left.\mathrm{CHCl}_{3}\right) v_{\max }$ 3499, 2960, 2923, 2857, 1703, 1514, 1247, 1074, 822, $699 \mathrm{~cm}^{-1}$; HRMS (ESI) Found 525.3227. Calculated for $\mathrm{C}_{32} \mathrm{H}_{45} \mathrm{O}_{6}{ }^{+}[\mathrm{M}+\mathrm{H}]^{+}$525.3211. $\Delta=3.1 \mathrm{ppm}$; IR (film from $\mathrm{CHCl}_{3}$ ) $\mathrm{v}_{\max }$ $3499,2960,2923,2857,1703,1613,1514,1247,1074,1035,822,699 \mathrm{~cm}^{-1}$.

(4S,8S,11R,Z)-11-((Benzyloxy)methyl)-8-((4-methoxybenzyl)oxy)-3,3,9-trimethyl-2,6dioxotridec-9-en-4-yl (S)-3,3,3-trifluoro-2-methoxy-2-phenylpropanoate (144)

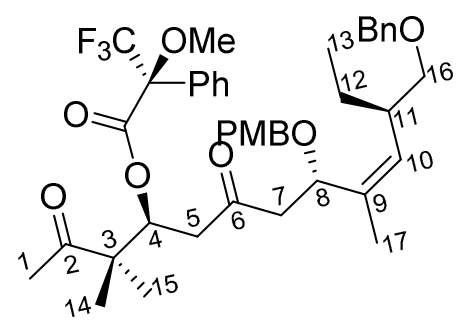

Alcohol 142 (5.3 mg, $0.01 \mathrm{mmol})$ was dissolved in dry DCM $(0.20 \mathrm{~mL})$ under argon then treated with DMAP (4.7 mg, $0.04 \mathrm{mmol}, 4$ equiv.), DCC (7.9 mg, $0.04 \mathrm{mmol}, 4$ equiv.), and SMTPA ( $3.9 \mathrm{mg}, 0.02 \mathrm{mmol}, 2$ equiv.) at room temperature. The reaction was stirred for 24 hours at which point TLC analysis indicated there was still starting material left and the same amount of each reagent was added to the reaction. After 5 days, the reaction was quenched with water $(1 \mathrm{~mL})$, then diluted with DCM $(5 \mathrm{~mL})$ and water $(5 \mathrm{~mL})$. The two layers were separated and aqueous layer extracted with DCM $(2 \times 5 \mathrm{~mL})$. The combined organic layers were washed with brine $(5 \mathrm{~mL})$, dried $\left(\mathrm{MgSO}_{4}\right)$ and concentrated. Crude material was purified on silica gel column (5:1 PE/EA) to give the ester 144 as a colourless oil $(0.3 \mathrm{mg}$, $4 \%)$. 
$\mathbf{R}_{f}=0.20$ (5:1 PE/EA); ${ }^{1} \mathbf{H}-\mathbf{N M R}\left(500 \mathrm{MHz}, \mathrm{CDCl}_{3}\right) \delta 7.51-7.46$ (m, 2H, Ph-MTP), $7.40-7.36$ (m, 3H, Ph-MTP), $7.33-7.27$ (m, 4H, OCH 2 Ph), $7.25-7.19\left(\mathrm{~m}, 1 \mathrm{H}, \mathrm{OCH}_{2} P h\right), 7.10$ (d, J = 8.6 $\mathrm{Hz}, 2 \mathrm{H}, \mathrm{OPMB}), 6.80$ (d, J=8.7 Hz, 2H, OPMB), 5.88 (t, J=5.5 Hz, 1H, H-4), 5.19 (d, J=10.1 $\mathrm{Hz}, 1 \mathrm{H}, \mathrm{H}-10), 4.76$ (dd, J = 10.4, $2.5 \mathrm{~Hz}, 1 \mathrm{H}, \mathrm{H}-8$ ), 4.49 (d, J = $12.3 \mathrm{~Hz}, 1 \mathrm{H}, \mathrm{OCH}_{2} \mathrm{bPh}$ ), 4.46 (d, $J=12.2 \mathrm{~Hz}, 1 \mathrm{H}, \mathrm{OCH}_{2} \mathrm{bPh}$ ), 4.26 (d, $\left.J=10.9 \mathrm{~Hz}, 1 \mathrm{H}, \mathrm{OPMB}\right), 4.07$ (d, $\left.J=11.0 \mathrm{~Hz}, 1 \mathrm{H}, \mathrm{OPMB}\right)$, $3.78(\mathrm{~s}, 3 \mathrm{H}, \mathrm{OPMB}), 3.44\left(\mathrm{~s}, 3 \mathrm{H}, \mathrm{OCH}_{3}\right), 3.39$ (dd, $\left.J=9.1,6.0 \mathrm{~Hz}, 1 \mathrm{H}, \mathrm{H}-16 \mathrm{a}\right), 3.31$ (dd, $J=9.1$, $6.8 \mathrm{~Hz}, 1 \mathrm{H}, \mathrm{H}-16 \mathrm{~b}), 2.88$ (dd, J = 15.1, $10.4 \mathrm{~Hz}, 1 \mathrm{H}, \mathrm{H}-7 \mathrm{a}), 2.71-2.65$ (m, 1H, H-11), 2.63 (d, J $=5.6 \mathrm{~Hz}, 2 \mathrm{H}, \mathrm{H}-5), 2.15(\mathrm{dd}, J=15.1,2.6 \mathrm{~Hz}, 1 \mathrm{H}, \mathrm{H}-7 \mathrm{~b}), 2.10(\mathrm{~s}, 3 \mathrm{H}, \mathrm{H}-1), 1.70(\mathrm{~d}, J=1.3 \mathrm{~Hz}$, $3 \mathrm{H}, \mathrm{H}-17$ ), $1.55-1.50$ (obscured $\mathrm{m}, 1 \mathrm{H}, \mathrm{H}-12 \mathrm{a}$, seen in COSY and HSQC), $1.20-1.11$ (m, 1H, H-12b), 1.05 (d, J = 5.9 Hz, 6H, H-14, H-15), 0.79 (t, J = $7.4 \mathrm{~Hz}, 3 \mathrm{H}, \mathrm{H}-13) ;{ }^{13} \mathrm{C}$ NMR (151 MHz, $\mathrm{CDCl}_{3}$ ) $\delta 210.7$ (C, C-2), 204.6 (C, C-6), 165.6 (C, CO-MTP), 159.2 (C, OPMB), 138.8 (C, OCH ${ }_{2} \mathrm{Ph}$ ), 134.9 (C, C-9), 132.4 (CH, C-10), 131.8 (C, Ph-MTP), 130.7 (C, OPMB), 129.7 (CH, Ph-MTP), 129.6 (2CH, OPMB), 128.5 (2CH, Ph-MTP), 128.4 (2CH, OCH $\left.{ }_{2} \mathrm{Ph}\right), 127.9$ (2CH, PhMTP), 127.7 (2CH, OCH $2 \mathrm{Ph}), 127.6\left(\mathrm{CH}, \mathrm{OCH}_{2} \mathrm{Ph}\right), 113.8$ (2CH, OPMB), $75.4(\mathrm{CH}, \mathrm{C}-4), 74.2$ $\left(\mathrm{CH}_{2}, \mathrm{C}-16\right), 73.7(\mathrm{CH}, \mathrm{C}-8), 73.2\left(\mathrm{CH}_{2}, \mathrm{OCH}_{2} \mathrm{Ph}\right), 70.1\left(\mathrm{CH}_{2}, \mathrm{OPMB}\right), 55.4\left(2 \mathrm{CH}_{3}, \mathrm{OCH}_{3}-\mathrm{MTP}\right.$, OPMB), 51.2 (C, C-3), $47.3\left(\mathrm{CH}_{2}, \mathrm{C}-7\right), 45.0\left(\mathrm{CH}_{2}, \mathrm{C}-5\right), 39.5(\mathrm{CH}, \mathrm{C}-11), 26.1\left(\mathrm{CH}_{3}, \mathrm{C}-1\right), 25.1$ $\left(\mathrm{CH}_{2}, \mathrm{C}-12\right), 20.7\left(\mathrm{CH}_{3}, \mathrm{C}-14\right), 20.5\left(\mathrm{CH}_{3}, \mathrm{C}-15\right), 18.0\left(\mathrm{CH}_{3}, \mathrm{C}-17\right), 11.8\left(\mathrm{CH}_{3}, \mathrm{C}-13\right)$, Two $\mathrm{C}$ signals obscured or missing (too weak); HRMS (ESI) Found 758.3888. Calculated for $\mathrm{C}_{42} \mathrm{H}_{55} \mathrm{~F}_{3} \mathrm{NO}_{8}^{+}\left[\mathrm{M}+\mathrm{NH}_{4}\right]^{+}$758.3874. $\Delta=1.75 \mathrm{ppm} ;$

(4S,8S,11R,Z)-11-((Benzyloxy)methyl)-8-((4-methoxybenzyl)oxy)-3,3,9-trimethyl-2,6dioxotridec-9-en-4-yl (R)-3,3,3-trifluoro-2-methoxy-2-phenylpropanoate (145)

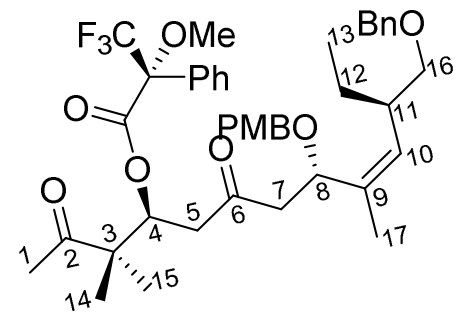

Secondary alcohol 142 (7.6 mg, $0.014 \mathrm{mmol})$ was dissolved in dry DCM (0.5 mL) under argon then treated with TEA ( $7 \mu \mathrm{L}, 0.043 \mathrm{mmol}, 3$ equiv., DMAP (1.8 mg, $0.015 \mathrm{mmol}, 1$ equiv.), DCC (12.1 mg, $0.059 \mathrm{mmol}, 4$ equiv.), and R-MTPA ( $8.5 \mathrm{mg}, 0.036 \mathrm{mmol}, 2.6$ equiv.) at room temperature. After 1 day of stirring the reaction was quenched with $4 \mathrm{~mL}$ water then 
extracted with DCM $(3 \times 5 \mathrm{~mL})$, dried $\left(\mathrm{MgSO}_{4}\right)$ and concentrated. Crude material was purified on silica gel column (5:1 PE/EA) to give the ester 145 (0.3 mg, 3\%) as a colourless oil.

$\mathbf{R}_{f}=0.34$ (5:1 PE/EA); ${ }^{1} \mathbf{H}-\mathbf{N M R}\left(500 \mathrm{MHz}, \mathrm{CDCl}_{3}\right) \delta 7.53-7.48(\mathrm{~m}, 2 \mathrm{H}, \mathrm{Ph}-\mathrm{MTP}), 7.39-7.34$ (m, 3H, Ph-MTP), $7.33-7.27\left(\mathrm{~m}, 4 \mathrm{H}, \mathrm{OCH}_{2} P h\right), 7.25-7.19\left(\mathrm{~m}, 1 \mathrm{H}, \mathrm{OCH}_{2} P h\right), 7.10(\mathrm{~d}, J=8.6$ $\mathrm{Hz}, 2 \mathrm{H}, \mathrm{OPMB}), 6.80$ (d, J = 8.7 Hz, 2H, OPMB), 5.85 (dd, J = 6.8, 4.2 Hz, 1H, H-4), 5.20 (d, $J=$ $10.3 \mathrm{~Hz}, 1 \mathrm{H}, \mathrm{H}-10), 4.77$ (dd, $J=10.5,2.7 \mathrm{~Hz}, 1 \mathrm{H}, \mathrm{H}-8$ ), 4.49 (d, J=12.5 Hz, 1H, OCH $\mathrm{aPh}$ ), $4.47\left(\mathrm{~d}, J=12.7 \mathrm{~Hz}, 1 \mathrm{H}, \mathrm{OCH}_{2} \mathrm{bPh}\right.$ ), 4.27 (d, J = $\left.11.0 \mathrm{~Hz}, 1 \mathrm{H}, \mathrm{OPMB}\right), 4.07$ (d, J = $11.0 \mathrm{~Hz}, 1 \mathrm{H}$, OPMB), 3.77 (s, 3H, OPMB), 3.46 (d, $J=0.7 \mathrm{~Hz}, 3 \mathrm{H}, \mathrm{OCH}_{3}$ ), 3.39 (dd, $J=9.1,5.9 \mathrm{~Hz}, 1 \mathrm{H}, \mathrm{H}-$ 16a), 3.31 (dd, $J=9.1,6.8 \mathrm{~Hz}, 1 \mathrm{H}, \mathrm{H}-16 \mathrm{~b}), 2.91$ (dd, $J=15.0,10.5 \mathrm{~Hz}, 1 \mathrm{H}, \mathrm{H}-7 \mathrm{a}$ ), $2.71-2.68$ (m, 1H, H-11), $2.68-2.65(m, 2 \mathrm{H}, \mathrm{H}-5), 2.21$ (dd, J = 15.1, $2.7 \mathrm{~Hz}, 1 \mathrm{H}, \mathrm{H}-7 \mathrm{~b}), 2.02(\mathrm{~s}, 3 \mathrm{H}, \mathrm{H}-1)$, $1.71(\mathrm{~d}, J=1.4 \mathrm{~Hz}, 3 \mathrm{H}, \mathrm{H}-17$ ), $1.56-1.51$ (obscured $\mathrm{m}, 1 \mathrm{H}, \mathrm{H}-12 \mathrm{a}$, seen in COSY and HSQC), $1.20-1.12$ (m, 1H, H-12b), 1.01 (d, $J=10.3 \mathrm{~Hz}, 6 \mathrm{H}, \mathrm{H}-14 / \mathrm{H}-15), 0.78$ (t, J = 7.4 Hz, 3H, H-13); HRMS (ESI) Found 758.3907. Calculated for $\mathrm{C}_{42} \mathrm{H}_{55} \mathrm{~F}_{3} \mathrm{NO}_{8}{ }^{+}\left[\mathrm{M}+\mathrm{NH}_{4}\right]^{+} 758.3874 . \Delta=4.29 \mathrm{ppm}$.

(4S,8S,11R,Z)-11-((Benzyloxy)methyl)-4-((diisopropylsilyl)oxy)-8-((4-methoxybenzyl)oxy)-

\section{3,3,9-trimethyltridec-9-ene-2,6-dione (147)}

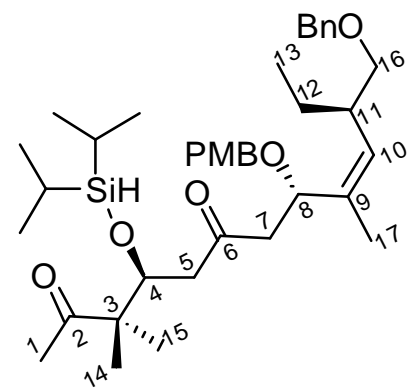

Diketone starting material $142(18.4 \mathrm{mg}, 0.035 \mathrm{mmol})$ in dry DMF $(1 \mathrm{~mL})$ was cooled to $0{ }^{\circ} \mathrm{C}$ under argon then treated with DMAP (21.5 mg, $0.174 \mathrm{mmol}, 5$ equiv.), TEA (0.03 mL, 0.175 mmol, 5 equiv.) and $\mathrm{Si}(i \mathrm{Pr})_{2} \mathrm{HCl}(0.02 \mathrm{~mL}, 0.11 \mathrm{mmol}, 3$ equiv.). After $30 \mathrm{~min}$ TLC analysis showed that all starting material has been consumed so the reaction was quenched by pouring into sat. aq. $\mathrm{NaHCO}_{3}$ solution $(3 \mathrm{~mL})$. The aqueous layer was extracted with EtOAc (3x $5 \mathrm{~mL}$ ). The combined organic layers were washed with brine, dried with anhydrous $\mathrm{MgSO}_{4}$ and concentrated under reduced pressure. The crude material was purified on silica gel column (9:1 PE/EA) to give the product 147 as a colourless oil (14.0 mg, 63\%). 
$\mathbf{R}_{f}=0.56(5: 1 \mathrm{PE} / \mathrm{EA}) ;[\alpha]_{\mathrm{D}}{ }^{21}=-39.7\left(c 0.70, \mathrm{CHCl}_{3}\right) ;{ }^{1} \mathbf{H} \mathbf{N M R}\left(500 \mathrm{MHz}, \mathrm{CDCl}_{3}\right): \delta 7.33-7.27$ (m, 4H, OCH 2 Ph), $7.25-7.20\left(\mathrm{~m}, 1 \mathrm{H}, \mathrm{OCH}_{2} P h\right), 7.11$ (d, $\left.J=8.6 \mathrm{~Hz}, 2 \mathrm{H}, \mathrm{OPMB}\right), 6.80$ (d, $J=8.7$ $\mathrm{Hz}, 2 \mathrm{H}, \mathrm{OPMB}$ ), 5.19 (dd, J = 10.5, $1.6 \mathrm{~Hz}, 1 \mathrm{H}, \mathrm{H}-10$ ), 4.82 (dd, J=10.2, $2.5 \mathrm{~Hz}, 1 \mathrm{H}, \mathrm{H}-8$ ), 4.65 (dd, $J=6.1,3.9 \mathrm{~Hz}, 1 \mathrm{H}, \mathrm{H}-4$ ), 4.49 (d, $J=12.1 \mathrm{~Hz}, 1 \mathrm{H}, \mathrm{OCH}_{2} \mathrm{aPh}$ ), 4.47 (d, $J=12.3 \mathrm{~Hz}, 1 \mathrm{H}$, $\mathrm{OCH}_{2} \mathrm{bPh}$ ), 4.27 (d, $\left.J=10.9 \mathrm{~Hz}, 1 \mathrm{H}, \mathrm{OPMB}\right), 4.11$ (d, $\left.J=10.9 \mathrm{~Hz}, 1 \mathrm{H}, \mathrm{OPMB}\right), 4.07$ (bs, $1 \mathrm{H}$, $\mathrm{SiH}$ ), 3.77 (s, 3H, OPMB), 3.38 (dd, $J=9.1,6.1 \mathrm{~Hz}, 1 \mathrm{H}, \mathrm{H}-16 \mathrm{a}$ ), 3.32 (dd, $J=9.1,6.6 \mathrm{~Hz}, 1 \mathrm{H}, \mathrm{H}-$ 16b), 2.96 (dd, J = 15.8, 10.2 Hz, 1H, H-7a), $2.76-2.65(\mathrm{~m}, 1 \mathrm{H}, \mathrm{H}-11), 2.58-2.53(\mathrm{~m}, 2 \mathrm{H}, \mathrm{H}-$ 5), 2.24 (dd, J = 15.8, 2.5 Hz, 1H, H-7b), 2.14 (s, 3H, H-1), 1.72 (d, J=1.4 Hz, 3H, H-17), $1.59-$ 1.52 (m, 1H, H-12a), $1.22-1.12$ (m, 1H, H-12b), 1.06 (s, 3H, H-14), 1.03 (s, 3H, H-15), $1.02-$ $0.98(\mathrm{~m}, 7 \mathrm{H}, i \mathrm{Pr}), 0.98-0.92(\mathrm{~m}, 7 \mathrm{H}, i \mathrm{Pr}), 0.81(\mathrm{t}, J=7.4 \mathrm{~Hz}, 3 \mathrm{H}, \mathrm{H}-13) ;{ }^{13} \mathrm{C}$ NMR $(126 \mathrm{MHz}$, $\mathrm{CDCl}_{3}$ ) $\delta 212.8$ (C, C-2), 206.4 (C, C-6), 159.1 (C, OPMB), 138.7 (C, OCH $\left.{ }_{2} P h\right), 135.1$ (C, C-9), 132.1 ( $\mathrm{CH}, \mathrm{C}-10), 130.8$ (C, OPMB), $129.5(2 \mathrm{CH}, \mathrm{OPMB}), 128.4\left(2 \mathrm{CH}, \mathrm{OCH}_{2} \mathrm{Ph}\right), 127.6(2 \mathrm{CH}$, $\left.\mathrm{OCH}_{2} \mathrm{Ph}\right), 127.6\left(\mathrm{CH}, \mathrm{OCH}_{2} \mathrm{Ph}\right), 113.7(2 \mathrm{CH}, \mathrm{OPMB}), 74.2\left(\mathrm{CH}_{2}, \mathrm{C}-16\right), 73.3(\mathrm{CH}, \mathrm{C}-4), 73.2(\mathrm{CH}$, $\mathrm{C}-8), 73.1\left(\mathrm{CH}_{2}, \mathrm{OCH}_{2} \mathrm{Ph}\right), 70.2\left(\mathrm{CH}_{2}, \mathrm{OPMB}\right), 55.4\left(\mathrm{CH}_{3}, \mathrm{OPMB}\right), 52.9$ (C, $\left.\mathrm{C}-3\right), 48.2\left(\mathrm{CH}_{2}, \mathrm{C}-5\right)$, $48.0\left(\mathrm{CH}_{2}, \mathrm{C}-7\right), 39.5(\mathrm{CH}, \mathrm{C}-11), 26.9\left(\mathrm{CH}_{3}, \mathrm{C}-1\right), 25.2\left(\mathrm{CH}_{2}, \mathrm{C}-12\right), 20.9\left(\mathrm{CH}_{3}, \mathrm{C}-15\right), 20.5\left(\mathrm{CH}_{3}\right.$, C-14), $18.1\left(\mathrm{CH}_{3}, \mathrm{C}-17\right), 17.6\left(\mathrm{CH}_{3}, i \operatorname{Pr}\right), 17.6\left(\mathrm{CH}_{3}, i \operatorname{Pr}\right), 17.6\left(\mathrm{CH}_{3}, i \operatorname{Pr}\right), 17.6\left(\mathrm{CH}_{3}, i \operatorname{Pr}\right), 12.5$ $(\mathrm{CH}, i \mathrm{Pr}), 12.4(\mathrm{CH}, i \mathrm{Pr}), 11.9\left(\mathrm{CH}_{3}, \mathrm{C}-13\right)$; HRMS (ESI) Found 639.4081. Calculated for $\mathrm{C}_{38} \mathrm{H}_{59} \mathrm{O}_{6} \mathrm{Si}^{+}[\mathrm{M}+\mathrm{H}]^{+}$639.4075. $\Delta=0.82$ ppm; IR (film from $\mathrm{CHCl}_{3}$ ) $v_{\max } 2958,2941,2864$, $2144,2095,1704,1613,1514,1248,1085,822,808 \mathrm{~cm}^{-1}$.

\section{3-((4S,6R)-6-((2S,5R,Z)-5-((Benzyloxy)methyl)-2-((4-methoxybenzyl)oxy)-3-methylhept-3-} en-1-yl)-2,2-diisopropyl-1,3,2-dioxasilinan-4-yl)-3-methylbutan-2-one (148)

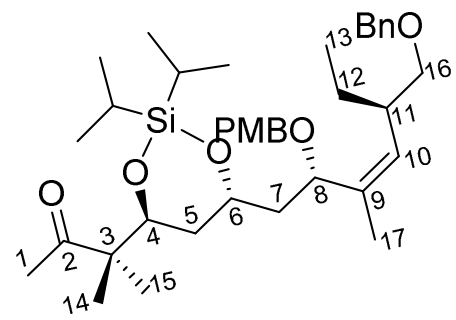

Hydrosilane 147 (13.9 mg, $0.022 \mathrm{mmol})$ in dry DCM (1 mL) under an argon atmosphere was cooled to $-78{ }^{\circ} \mathrm{C}$ and treated dropwise with $\mathrm{SnCl}_{4}$ solution (1 M in DCM, $0.05 \mathrm{~mL} 0.05 \mathrm{mmol}$, 2 equiv.). The reaction was stirred for $45 \mathrm{~min}$ at the same temperature (TLC analysis indicated complete consumption of the starting material) then quenched with sat. aq. $\mathrm{NaHCO}_{3}(2 \mathrm{~mL})$. The aqueous layer was extracted with EtOAc $(3 \times 5 \mathrm{~mL})$. The combined 
organic layer was dried with anhydrous $\mathrm{MgSO}_{4}$ and the solvent removed under reduced pressure. Usually, the crude reaction mixture was used in the next step without purification. On one occasion the crude reaction material was purified on silica gel column (9:1 PE/EA) to give the product 148 as a colourless oil ( $4.4 \mathrm{mg}, 32 \%$ ) that was used to confirm the 1,3-anti outcome of the reduction.

$\mathbf{R}_{f}=0.57(5: 1 \mathrm{PE} / \mathrm{EA}) ;{ }^{1} \mathbf{H}$ NMR (500 MHz, $\left.\mathrm{CDCl}_{3}\right): \delta 7.32-7.27\left(\mathrm{~m}, 4 \mathrm{H}, \mathrm{OCH}_{2} P h\right), 7.25-7.20$ (m, 1H, OCH $\left.{ }_{2} P h\right), 7.17$ (d, J = 8.6 Hz, 2H, OPMB), 6.82 (d, J=8.6 Hz, 2H, OPMB), 5.33 (dd, $J=$ 10.3, $1.7 \mathrm{~Hz}, 1 \mathrm{H}, \mathrm{H}-10), 4.52-4.43\left(\mathrm{~m}, 3 \mathrm{H}, \mathrm{H}-8, \mathrm{OCH}_{2} \mathrm{Ph}\right.$ ), 4.35 (d, J = $\left.11.4 \mathrm{~Hz}, 1 \mathrm{H}, \mathrm{OPMB}\right)$, 4.30 (dd, $J=11.2,1.4 \mathrm{~Hz}, 1 \mathrm{H}, \mathrm{H}-4$ ), 4.16 (dddd, $J=9.9,5.9,4.2,1.1 \mathrm{~Hz}, 1 \mathrm{H}, \mathrm{H}-6$ ), 4.07 (d, $J=$ $11.3 \mathrm{~Hz}, 1 \mathrm{H}, \mathrm{OPMB}$ ), 3.78 (s, 3H, OPMB), 3.39 (dd, $J=9.2,6.3 \mathrm{~Hz}, 1 \mathrm{H}, \mathrm{H}-16 \mathrm{a}$ ), 3.32 (dd, $J=$ 9.2, 6.4 Hz, 1H, H-16b), $2.76-2.67$ (m, 1H, H-11), 2.19 (ddd, J = 13.7, 9.9, 5.9 Hz, 1H, H-7a), 2.15 (s, 3H, H-1), 1.81 (ddd, J = 14.3, 11.1, $5.8 \mathrm{~Hz}, 1 \mathrm{H}, \mathrm{H}-5 \mathrm{a}$ ), 1.70 (d, J=1.3 Hz, 3H, H-17), 1.63 (ddd, $J=13.3,8.9,4.3 \mathrm{~Hz}, 1 \mathrm{H}, \mathrm{H}-7 \mathrm{~b}), 1.57-1.50$ (m, 1H, H-12a), 1.39 (dd, J = 14.4, 1.6 $\mathrm{Hz}, 1 \mathrm{H}, \mathrm{H}-5 \mathrm{~b}$ ), 1.31 - 1.25 (m, 1H, H-12b), 1.08 (s, 3H, H-14), 0.99 (s, 3H, H-15), $0.98-0.94$ $\left(\mathrm{m}, 6 \mathrm{H}, 2 \mathrm{CH}\left(\mathrm{CH}_{3}\right)_{2}\right), 0.92-0.82\left(\mathrm{~m}, 2 \mathrm{H}, 2 \mathrm{CH}\left(\mathrm{CH}_{3}\right)_{2}\right), 0.86(\mathrm{t}, J=7.5 \mathrm{~Hz}, 3 \mathrm{H}, \mathrm{H}-13) ;{ }^{13} \mathrm{C}$ NMR (126 MHz, CDCl ${ }_{3}$ ) $\delta 213.0$ (C, C-2), 159.1 (C, OPMB), 138.8 (C, OCH $\mathrm{O}_{2} \mathrm{Ph}$ ), 134.6 (C, C-9), 133.8 (CH, C-10), 131.2 (C, OPMB), $129.5(2 \mathrm{CH}, \mathrm{OPMB}), 128.4\left(2 \mathrm{CH}, \mathrm{OCH}_{2} \mathrm{Ph}\right), 127.6(2 \mathrm{CH}$, $\left.\mathrm{OCH}_{2} \mathrm{Ph}\right), 127.6\left(\mathrm{CH}, \mathrm{OCH}_{2} \mathrm{Ph}\right), 113.8(2 \mathrm{CH}, \mathrm{OPMB}), 74.0\left(\mathrm{CH}_{2}, \mathrm{C}-16\right), 73.9(\mathrm{CH}, \mathrm{C}-8), 73.4(\mathrm{CH}$, $\mathrm{C}-4), 73.0\left(\mathrm{CH}_{2}, \mathrm{OCH}_{2} \mathrm{Ph}\right), 69.6\left(\mathrm{CH}_{2}, \mathrm{OPMB}\right), 68.3(\mathrm{CH}, \mathrm{C}-6), 55.4\left(\mathrm{CH}_{3}, \mathrm{OPMB}\right), 52.4$ (C, $\left.\mathrm{C}-3\right)$, $40.2\left(\mathrm{CH}_{2}, \mathrm{C}-7\right), 38.8(\mathrm{CH}, \mathrm{C}-11), 34.4\left(\mathrm{CH}_{2}, \mathrm{C}-5\right), 26.6\left(\mathrm{CH}_{3}, \mathrm{C}-1\right), 25.4\left(\mathrm{CH}_{2}, \mathrm{C}-12\right), 21.0\left(\mathrm{CH}_{3}\right.$, C-15), $18.8\left(\mathrm{CH}_{3}, \mathrm{C}-14\right), 17.7\left(\mathrm{CH}_{3}, \mathrm{C}-17\right), 17.0\left(\mathrm{CH}_{3}, i \mathrm{Pr}\right), 16.9\left(\mathrm{CH}_{3}, i \operatorname{Pr}\right), 16.9\left(\mathrm{CH}_{3}, \mathrm{PPr}\right), 16.8$ $\left(\mathrm{CH}_{3}, i \mathrm{Pr}\right), 13.7(\mathrm{CH}, i \mathrm{Pr}), 13.1(\mathrm{CH}, i \mathrm{Pr}), 11.5\left(\mathrm{CH}_{3}, \mathrm{C}-13\right)$.

(4S,6S,8S,11R,Z)-11-((Benzyloxy)methyl)-4-hydroxy-6-methoxy-8-((4-methoxybenzyl)oxy)3,3,9-trimethyltridec-9-en-2-one (140)

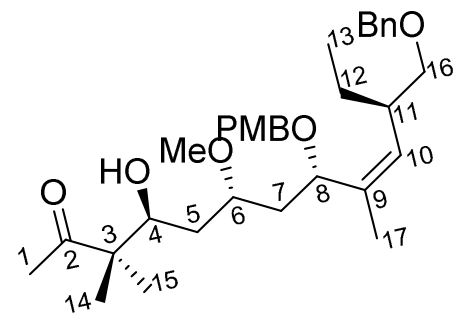


Starting material $148(4.4 \mathrm{mg}, 6.9 \mu \mathrm{mol})$ was dissolved in dry THF $(0.20 \mathrm{~mL})$ then cooled to 0 ${ }^{\circ} \mathrm{C}$ under argon atmosphere and treated with $\mathrm{AcOH}(1 \mathrm{M}$ in THF, $0.04 \mathrm{~mL}, 0.04 \mathrm{mmol}, 6$ equiv.) and TBAF ( $1 \mathrm{M}$ in THF, $0.04 \mathrm{~mL}, 0.04 \mathrm{mmol}, 6$ equiv.). After $2 \mathrm{~h} 45 \mathrm{~min}$ hours the reaction was quenched with $5 \mathrm{~mL}$ sat. aq. $\mathrm{NH}_{4} \mathrm{Cl}$ solution and diluted with $15 \mathrm{~mL}$ EtOAc. The two layers were separated and the organic layer washed with sat. aq. $\mathrm{NH} 4 \mathrm{Cl}(2 \times 5 \mathrm{~mL})$ then brine $(1 \times 10 \mathrm{~mL})$, dried $\left(\mathrm{MgSO}_{4}\right)$ and concentrated. The crude material was used in the next step without purification.

The crude diol mixture from the previous step was dissolved in dry DCM $(0.20 \mathrm{~mL})$ under argon in a flask wrapped with aluminium foil then treated with proton sponge $(0.024 \mathrm{~g}, 0.1$ mmol, 15 equiv.) and $\mathrm{Me}_{3} \mathrm{OBF}_{4}(0.011 \mathrm{~g}, 0.07 \mathrm{mmol}, 10$ equiv.) at room temperature. The reaction was stirred at for 1 hour then filtered through celite into sat. aq. $\mathrm{NaHCO}_{3}$ solution $(2 \mathrm{~mL})$. Celite was further washed with EtOAc $(10 \mathrm{~mL})$. The organic layer was separated then washed with sat. aq. $\mathrm{NaHCO}_{3}(5 \mathrm{~mL})$ then brine $(5 \mathrm{~mL})$ dried over $\mathrm{MgSO}_{4}$ and concentrated under reduced pressure. Crude reaction mixture was purified on silica gel column $(5: 1$ then 3:1 PE/EA) to give the product 140 as a colourless oil (1.1 $\mathrm{mg}, 30 \%)$.

$\mathbf{R}_{f}=0.24(3: 1 \mathrm{PE} / \mathrm{EA}) ;{ }^{1} \mathbf{H}$ NMR $\left(500 \mathrm{MHz}, \mathrm{CDCl}_{3}\right): \delta 7.34-7.27\left(\mathrm{~m}, 4 \mathrm{H}, \mathrm{OCH}_{2} \mathrm{Ph}\right), 7.25-7.20$ (m, $\left.1 \mathrm{H}, \mathrm{OCH}_{2} P h\right), 7.17$ (d, J=8.6 Hz, 2H, OPMB), 6.82 (d, $\left.J=8.6 \mathrm{~Hz}, 2 \mathrm{H}, \mathrm{OPMB}\right), 5.19$ (d, $J=$ $9.7 \mathrm{~Hz}, 1 \mathrm{H}, \mathrm{H}-10$ ), 4.51 (d, J = $12.2 \mathrm{~Hz}, 1 \mathrm{H}, \mathrm{OCH}_{2} \mathrm{aPh}$ ), 4.48 (d, J=12.2 Hz, 1H, OCH $\mathrm{OCPh}, 4.37$ - 4.30 (m, 2H, H-8, OPMB), 4.05 (d, J = 11.3 Hz, 1H, 1H, OPMB), 3.94 (dd, J = 10.7, 1.6 Hz, 1H, H-4), 3.79 (s, 3H, OPMB), $3.68-3.60$ (m, 1H, H-6), 3.40 (dd, J=9.1, $6.2 \mathrm{~Hz}, 1 \mathrm{H}, \mathrm{H}-16 \mathrm{a}), 3.37-$ $3.30\left(\mathrm{~m}, 4 \mathrm{H}, \mathrm{H}-16 \mathrm{~b}, \mathrm{OCH}_{3}\right), 2.67-2.58(\mathrm{~m}, 1 \mathrm{H}, \mathrm{H}-11), 2.19$ (ddd, J = 14.3, 10.2, $4.1 \mathrm{~Hz}, 1 \mathrm{H}, \mathrm{H}-$ 7a), 2.13 (s, 3H, H-1), 1.73 (d, J = $1.3 \mathrm{~Hz}, 3 \mathrm{H}, \mathrm{H}-17$ ), $1.63-1.50$ (m, 3H, H-5a, H-7b, H-12a, obscured by the water peak but observed in COSY and HSQC), 1.41 (ddd, $J=14.4,6.3,1.7$ $\mathrm{Hz}, 1 \mathrm{H}, \mathrm{H}-5 \mathrm{~b}), 1.24-1.16(\mathrm{~m}, 1 \mathrm{H}, \mathrm{H}-12 \mathrm{~b}), 1.03$ (s, 3H, H-14), 1.00 (s, 3H, H-15), 0.84 (t, J = $7.4 \mathrm{~Hz}, 3 \mathrm{H}, \mathrm{H}-13$ ); ${ }^{13} \mathrm{C}$ NMR (151 MHz, CDCl 3 ) $\delta 214.5$ (C, C-2), 159.2 (C, OPMB), 138.7 (C, OCH ${ }_{2} \mathrm{Ph}$ ), 136.1 (C, C-9), 131.6 (CH, C-10), 130.8 (C, OPMB), 129.7 (2CH, OPMB), 128.4 (2CH, $\left.\mathrm{OCH}_{2} \mathrm{Ph}\right), 127.7\left(2 \mathrm{CH}, \mathrm{OCH}_{2} \mathrm{Ph}\right), 127.6\left(\mathrm{CH}, \mathrm{OCH}_{2} \mathrm{Ph}\right), 113.8(2 \mathrm{CH}, \mathrm{OPMB}), 77.1(\mathrm{CH}, \mathrm{C}-6$, obscured by the solvent peak but observed in $\mathrm{HSQC}), 74.2\left(\mathrm{CH}_{2}, \mathrm{C}-16\right), 73.2\left(\mathrm{CH}_{2}, \mathrm{OCH}_{2} \mathrm{Ph}\right)$, $73.1(\mathrm{CH}, \mathrm{C}-4), 72.9(\mathrm{CH}, \mathrm{C}-8), 69.7\left(\mathrm{CH}_{2}, \mathrm{OPMB}\right), 56.8\left(\mathrm{CH}_{3}, \mathrm{OCH}_{3}\right), 55.4\left(\mathrm{CH}_{3}, \mathrm{OPMB}\right), 52.0$ (C, C-3), $39.5(\mathrm{CH}, \mathrm{C}-11), 36.3\left(\mathrm{CH}_{2}, \mathrm{C}-7\right), 33.9\left(\mathrm{CH}_{2}, \mathrm{C}-5\right), 26.5\left(\mathrm{CH}_{3}, \mathrm{C}-1\right), 25.3\left(\mathrm{CH}_{2}, \mathrm{C}-12\right)$, $21.5\left(\mathrm{CH}_{3}, \mathrm{C}-15\right), 19.4\left(\mathrm{CH}_{3}, \mathrm{C}-14\right), 18.1\left(\mathrm{CH}_{3}, \mathrm{C}-17\right), 11.9\left(\mathrm{CH}_{3}, \mathrm{C}-13\right)$; HRMS (ESI) Found 
563.3365. Calculated for $\mathrm{C}_{33} \mathrm{H}_{48} \mathrm{NaO}_{6}{ }^{+}[\mathrm{M}+\mathrm{Na}]^{+}$563.3343. $\Delta=3.84 \mathrm{ppm}$; IR (film from $\mathrm{CH}_{2} \mathrm{Cl}_{2}$ ) $v_{\max } 3475,2960,2931,2858,1719,1513,1207,1083,1036,821,737,698 \mathrm{~cm}^{-1}$.

\section{5-(Benzyloxy)-4,4-dimethylpent-1-en-3-ol (69)}

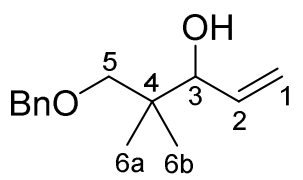

Aldehyde 67 (0.350 g, $1.82 \mathrm{mmol})$ was dissolved in dry THF $(5 \mathrm{~mL})$ and treated with vinyl $\mathrm{MgBr}\left(1 \mathrm{M}\right.$ in THF, $3.60 \mathrm{~mL}, 3.60 \mathrm{mmol}, 2$ equiv.) dropwise at $0{ }^{\circ} \mathrm{C}$ under argon. The reaction was stirred for $20 \mathrm{~min}$ at room temperature then quenched with sat. aq. $\mathrm{NH}_{4} \mathrm{Cl}(10$ $\mathrm{mL})$. The aqueous phase was extracted with EtOAc $(3 \times 10 \mathrm{~mL})$ and the combined organic layers washed sequentially with sat. aq. $\mathrm{NH}_{4} \mathrm{Cl}(20 \mathrm{~mL})$, water $(20 \mathrm{~mL})$ and brine $(20 \mathrm{~mL})$ then dried over anhydrous $\mathrm{MgSO}_{4}$ and concentrated. Purification on a silica gel column (10:1 PE/EA) gave the alcohol 69 as a colourless oil (0.277 g, 69\%). Experimental data matched those reported. ${ }^{230}$

$\mathbf{R}_{f}=0.36(10: 1 \mathrm{PE} / \mathrm{EA}) ;{ }^{1} \mathrm{H}-\mathrm{NMR}\left(500 \mathrm{MHz}, \mathrm{CDCl}_{3}\right): \delta$ 7.37-7.28 (m, 5H, Ph), 5.89 (ddd, J = 17.1, 10.5, $6.5 \mathrm{~Hz}, 1 \mathrm{H}, \mathrm{H}-2$ ), 5.27 (ddd, $J=17.1,2.0,1.4 \mathrm{~Hz}, 1 \mathrm{H}, \mathrm{H}-1 \mathrm{a}$ ), 5.18 (ddd, $J=10.5,2.0,1.2$ $\mathrm{Hz}, 1 \mathrm{H}, \mathrm{H}-1 \mathrm{~b}), 4.51\left(\mathrm{~s}, 2 \mathrm{H}, \mathrm{CH}_{2} \mathrm{Ph}\right), 3.96(\mathrm{dt}, J=6.5,1.2 \mathrm{~Hz}, 1 \mathrm{H}, \mathrm{H}-3), 3.41(\mathrm{~d}, J=8.8 \mathrm{~Hz}, 1 \mathrm{H}, \mathrm{H}-$ 5a), 3.29 (d, J = 8.8 Hz, 1H, H-5b), 0.96 (s, 3H, H-6a), 0.90 (s, 3H, H-6b).; ${ }^{13}$ C-NMR (100 MHz, $\left.\mathrm{CDCl}_{3}\right): \delta$ 138.0(C, Ph), $137.7(\mathrm{CH}, \mathrm{C}-2), 128.6(2 \mathrm{CH}, \mathrm{Ph}), 127.9(\mathrm{CH}, \mathrm{Ph}), 127.7(2 \mathrm{CH}, \mathrm{Ph})$, $116.5\left(\mathrm{CH}_{2}, \mathrm{C}-1\right), 80.2(\mathrm{CH}, \mathrm{C}-3), 79.4\left(\mathrm{CH}_{2}, \mathrm{C}-5\right), 73.7\left(\mathrm{CH}_{2}, \mathrm{CH}_{2} \mathrm{Ph}\right), 38.4(\mathrm{C}, \mathrm{C}-4), 22.9\left(\mathrm{CH}_{3}, \mathrm{C}-\right.$ 6a), $20.1\left(\mathrm{CH}_{3}, \mathrm{C}-6 \mathrm{~b}\right)$; HRMS (ESI) Found 221.1536. Calculated for $\mathrm{C}_{14} \mathrm{H}_{21} \mathrm{O}_{2}^{+}[\mathrm{M}+\mathrm{H}]^{+}$ 221.1536. $\Delta=0.05$ ppm; IR (film from $\mathrm{CHCl}_{3}$ ) $v_{\max } 3448,2961,2871,1477,1091,1075,992$, $921,734,696 \mathrm{~cm}^{-1}$.

\section{5-(Benzyloxy)-4,4-dimethylpent-1-en-3-one (70)}

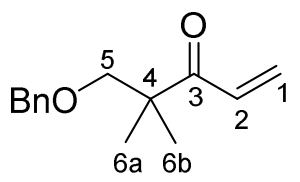

Secondary alcohol $69(0.277 \mathrm{~g}, 1.26 \mathrm{mmol})$ was dissolved in DCM $(5 \mathrm{~mL})$ and treated with DMP (0.695 g, $1.59 \mathrm{mmol}, 1.3$ equiv.) at room temperature under air. After stirring for $1.5 \mathrm{~h}$ 
at the same temperature (TLC analysis in 10:1 PE/EA indicated starting material was consumed), the reaction was quenched with $20 \mathrm{~mL}$ of $1: 1 \mathrm{v} / \mathrm{v}$ sat. aq. sodium thiosulfate/sat. aq. sodium bicarbonate. The quenched reaction was stirred for 1 hour then the two layers were separated and the aqueous layer extracted two more times with DCM $(2 x 15 \mathrm{~mL})$. The combined organic fraction were washed with brine $(20 \mathrm{~mL})$, dried with anhydrous $\mathrm{MgSO}_{4}$, and concentrated under reduced pressure. Purification on silica gel column (10:1 PE/EA) yielded the ketone 70 as a colourless oil $(0.233 \mathrm{~g}, 85 \%)$.

$\mathbf{R}_{\boldsymbol{f}}=0.61(10: 1 \mathrm{PE} / \mathrm{EA}) ;{ }^{1} \mathrm{H}-\mathrm{NMR}\left(500 \mathrm{MHz}, \mathrm{CDCl}_{3}\right): \delta 7.35-7.27(\mathrm{~m}, 5 \mathrm{H}, \mathrm{Ph}), 6.79$ (dd, $J=$ 16.9, 10.4 Hz, 1H, H-2), 6.34 (dd, J = 16.9, $2.0 \mathrm{~Hz}, 1 \mathrm{H}, \mathrm{H}-1 \mathrm{a}), 5.63$ (dd, J = 10.4, $2.1 \mathrm{~Hz}, 1 \mathrm{H}, \mathrm{H}-$ 1b), 4.50 (s, 2H, CH $2 \mathrm{Ph}$ ), 3.49 (s, 2H, H-5), 1.18 (s, 6H, H-6a, H-6b); ${ }^{13} \mathrm{C}-\mathrm{NMR}(100 \mathrm{MHz}$, $\left.\mathrm{CDCl}_{3}\right): \delta 203.0(\mathrm{C}, \mathrm{C}-3), 138.4(\mathrm{C}, \mathrm{Ph}), 131.4(\mathrm{CH}, \mathrm{C}-2), 128.4(2 \mathrm{CH}, \mathrm{Ph}), 128.3\left(\mathrm{CH}_{2}, \mathrm{C}-1\right)$, $127.7(\mathrm{CH}, \mathrm{Ph}), 127.6(2 \mathrm{CH}, \mathrm{Ph}), 76.6\left(\mathrm{CH}_{2}, \mathrm{C}-5\right), 73.5\left(\mathrm{CH}_{2}, \mathrm{CH}_{2} \mathrm{Ph}\right), 47.7(\mathrm{C}, \mathrm{C}-4), 21.8\left(2 \mathrm{CH}_{3}\right.$, C-6a, C-6b); HRMS (ESI) Found 219.1381. Calculated for $\mathrm{C}_{14} \mathrm{H}_{19} \mathrm{O}_{2}{ }^{+}[\mathrm{M}+\mathrm{H}]^{+}$219.138. $\Delta=0.78$ ppm; IR (film from $\mathrm{CHCl}_{3}$ ) $v_{\max }$ 2969, 2864, 1693, 1610, 1400, 1098, 1057, 736, $697 \mathrm{~cm}^{-1}$.

\section{5-Hydroxy-4,4-dimethylpent-1-en-3-one (72)}

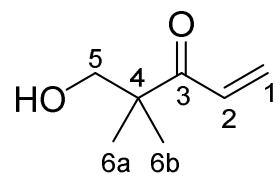

Benzyl ether 70 (0.056 g, $0.26 \mathrm{mmol})$ was dissolved in 5\% aq. DCM (5 mL) and treated with $\operatorname{DDQ}\left(0.213 \mathrm{~g}, 0.93 \mathrm{mmol}, 3.7\right.$ equiv.) at $0{ }^{\circ} \mathrm{C}$ under air. The reaction was allowed to warm to room temperature and stirred for 3 days. When the consumption of starting material was complete, as indicated by TLC analysis (5:1 PE/EA), the reaction was quenched with sat. aq. $\mathrm{NaHCO}_{3}(10 \mathrm{~mL})$ and the aqueous layer extracted with $\mathrm{DCM}(3 \times 10 \mathrm{~mL})$. The combined organic layers were washed with sat. aq. $\mathrm{NaHCO}_{3}(10 \mathrm{~mL})$, then dried over anhydrous $\mathrm{MgSO}_{4}$, filtered and concentrated under reduced pressure. The crude material was purified on a silica gel column (2:1 PE/EA) to give the $\beta$-hydroxy ketone 72 as a yellow oil $(0.023 \mathrm{mg}$, $70 \%)$.

$\mathbf{R}_{f}=0.26(3: 1 \mathrm{PE} / \mathrm{EA})^{1} \mathbf{H}$ NMR $\left(500 \mathrm{MHz}, \mathrm{CDCl}_{3}\right) \delta 6.77(\mathrm{dd}, J=16.9,10.4 \mathrm{~Hz}, 1 \mathrm{H}, \mathrm{H}-2), 6.39$ (dd, $J=17.0,1.9 \mathrm{~Hz}, 1 \mathrm{H}, \mathrm{H}-1 \mathrm{a}$ ), 5.72 (dd, J = 10.4, 1.9 Hz, 1H, H-1b), 3.60 (s, 2H, H-5), 1.18 (s, 
6H, H-6a, H-6b); ${ }^{13} \mathrm{C}$ NMR (126 MHz, CDCl 3 ) $\delta 205.1$ (C, C-3), 130.9 (CH, C-2), $129.5\left(\mathrm{CH}_{2}, \mathrm{C}-\right.$ 1), $69.4\left(\mathrm{CH}_{2}, \mathrm{C}-5\right), 48.1$ (C, C-4), $21.3\left(2 \mathrm{CH}_{3}, \mathrm{C}-6 \mathrm{a}, \mathrm{C}-6 \mathrm{~b}\right)$.

\section{Experimental for Chapter 4}

((4R,5R)-5-(((4-Methoxybenzyl)oxy)methyl)-2,2-dimethyl-1,3-dioxolan-4-yl)methanol (73)

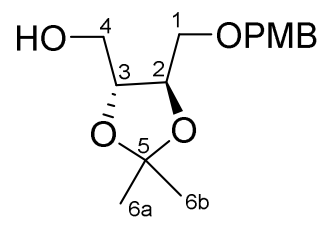

(-)-2,3-O-Isopropylidene-D-threitol (0.520 g, $3.14 \mathrm{mmol})$ was dissolved in 1:1 THF/DMF (6 $\mathrm{mL}$ ) then cooled to $0{ }^{\circ} \mathrm{C}$ under argon and treated with $\mathrm{NaH}(0.140 \mathrm{~g}, 3.51 \mathrm{mmol}, 1.12$ equiv.). The reaction was stirred for $10 \mathrm{~min}$ at $0{ }^{\circ} \mathrm{C}$, then 1 hour at room temperature. After 1 hour $\mathrm{PMBCl}(0.50 \mathrm{~mL}, 3.50 \mathrm{mmol}, 1.11$ equiv.,) was added dropwise and the reaction stirred at room temperature for 4 hours then quenched with sat. aq. $\mathrm{NH}_{4} \mathrm{Cl}(10 \mathrm{~mL})$ and diluted with water $(50 \mathrm{~mL})$. The aqueous layer was extracted with EtOAc $(3 \times 30 \mathrm{~mL})$. The combined organic layers were washed with brine $(50 \mathrm{~mL})$ then water $(50 \mathrm{~mL})$, dried over anhydrous $\mathrm{MgSO}_{4}$ and the solvent removed under reduced pressure. The crude reaction mixture was purified on silica gel column (2:1 PE/EA) to give the product 73 as a colourless oil $(0.616 \mathrm{~g}, 69 \%)$. Experimental data matched those previously reported. ${ }^{231}$

$\mathbf{R}_{f}=0.23(2: 1 \mathrm{PE} / \mathrm{EA}) ;[\alpha]_{\mathrm{D}}{ }^{23}=-9.95$ (c 1.03, $\left.\mathrm{CHCl}_{3}\right)\left[\mathrm{lit}^{232}[\alpha]_{\mathrm{D}}{ }^{23}=-8.44\right.$ (c 1.08, $\left.\left.\mathrm{CHCl}_{3}\right)\right] ;{ }^{1} \mathrm{H}-$ NMR (500 MHz, CDCl 3 ): $\delta 7.25$ (d, J=8.6 Hz, 2H OPMB), 6.88 (d, J=8.6 Hz, 2H, OPMB), 4.51 (m, 2H, OPMB), 4.03 (ddd, $J=8.2,6.0,5.0 \mathrm{~Hz}, 1 \mathrm{H}, \mathrm{H}-2), 3.92(\mathrm{~m}, 1 \mathrm{H}, \mathrm{H}-3), 3.81(\mathrm{~s}, 3 \mathrm{H}$, OPMB), 3.75 (dt, J=11.6, 4.4 Hz, 1H, H-4), $3.71-3.64$ (m, 2H, H-4, H-1), 3.52 (dd, J = 9.7, 6.1 $\mathrm{Hz}, 1 \mathrm{H}, \mathrm{H}-1), 2.26$ (dd, J = 8.4, 4.3 Hz, 1H, OH), $1.44-1.39$ (d, $2.0 \mathrm{~Hz}, 6 \mathrm{H}, \mathrm{H}-6 \mathrm{a}, \mathrm{H}-6 \mathrm{~b}$ ); ${ }^{13} \mathrm{C}-$ NMR (100 MHz, CDCl $)$ : $\delta 159.5$ (C, OPMB), 129.7 (C, OPMB), 129.6 (2CH, OPMB), 114.0 (2CH, OPMB), 109.4 (C, C-5), 80.0 ( $\mathrm{CH}, \mathrm{C}-3) 76.9$ (CH, C-2), $73.5\left(\mathrm{CH}_{2}, \mathrm{OPMB}\right), 70.1\left(\mathrm{CH}_{2}, \mathrm{C}-1\right)$, $62.6\left(\mathrm{CH}_{2}, \mathrm{C}-4\right)$, $55.4\left(\mathrm{CH}_{3}, \mathrm{OPMB}\right), 27.1\left(2 \mathrm{CH}_{3}, \mathrm{C}-6 \mathrm{a}, \mathrm{C}-6 \mathrm{~b}\right)$; HRMS (ESI) Found 305.136148. Calculated for $\mathrm{C}_{15} \mathrm{H}_{22} \mathrm{NaO}_{5}{ }^{+}[\mathrm{M}+\mathrm{Na}]^{+}$305.1360. $\Delta=0.65$ ppm; IR (film from $\mathrm{CHCl}_{3}$ ) $\mathrm{V}_{\max }$ 3464, $2987,2934,2869,1613,1514,1371,1248,1081,1035,846,820 \mathrm{~cm}^{-1}$. 


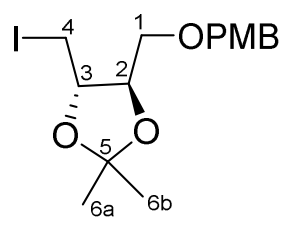

Alcohol 73 (0.500 g, $1.77 \mathrm{mmol})$ was dissolved in dry toluene $(11 \mathrm{~mL})$ and heated to reflux under argon then, when the refluxing started, the heating was stopped. When the boiling ceased, imidazole (0.367 g, $5.33 \mathrm{mmol}, 3$ equiv.), $\mathrm{PPh}_{3}(0.701 \mathrm{~g}, 2.67 \mathrm{mmol}, 1.5$ equiv.) and NIS ( $0.650 \mathrm{~g}, 2.80 \mathrm{mmol}, 1.6$ equiv.) were added sequentially. The reaction was heated again and refluxed for $20 \mathrm{~min}$ then allowed to cool down to room temperature. The reaction was quenched at room temperature with sat. aq. $\mathrm{Na}_{2} \mathrm{~S}_{2} \mathrm{O}_{3}(10 \mathrm{~mL})$ and the aqueous layer extracted with diethyl ether $(3 \times 20 \mathrm{~mL})$. Combined organic layers were dried with anhydrous $\mathrm{MgSO}_{4}$ then the solvent was removed under reduced pressure. The concentrated crude reaction mixture was purified on silica gel column (PE/EA 5:1) to give the iodide 74 as a colourless oil $(0.565 \mathrm{~g}, 81 \%)$. Experimental data matched those previously reported. ${ }^{2}$

$\mathbf{R}_{f}=0.55(5: 1 \mathrm{PE} / \mathrm{EA}) ;[\alpha]_{\mathrm{D}}{ }^{22}=+8.3^{\circ}$ (c 2.05, $\left.\mathrm{CHCl}_{3}\right)\left[\right.$ lit. $[\alpha]_{\mathrm{D}}{ }^{23}=+12.42$ (c 2.21, $\left.\left.\mathrm{CHCl}_{3}\right)\right]^{2} ;{ }^{1} \mathrm{H}-$ NMR (500 MHz, CDCl 3 ): $\delta 7.26$ (d, $9.1 \mathrm{~Hz}, 2 \mathrm{H}, \mathrm{OPMB}), 6.88$ (d, J=8.6 Hz, 2H, OPMB), 4.52 (m, 2H, OPMB), $3.94(\mathrm{dt}, J=7.4,5.1 \mathrm{~Hz}, 1 \mathrm{H}, \mathrm{H}-2), 3.86-3.82(\mathrm{~m}, 1 \mathrm{H}, \mathrm{H}-3), 3.81(\mathrm{~s}, 3 \mathrm{H}$, OPMB), $3.65-3.57$ (m, 2H, H-1), 3.34 (dd, $J=10.6,5.1 \mathrm{~Hz}, 1 \mathrm{H}, \mathrm{H}-4 \mathrm{a}$ ), 3.26 (dd, $J=10.6,5.4$ $\mathrm{Hz}, 1 \mathrm{H}, \mathrm{H}-4 \mathrm{~b}), 1.46$ (s, 3H, H-6a), 1.41 (s, 3H, H-6b); ${ }^{13} \mathrm{C}-\mathrm{NMR}$ (126 MHz, CDCl 3 ): $\delta 159.4$ (C, OPMB), 130.0 (C, OPMB), 129.5 (2CH, OPMB), 114.0 (2CH, OPMB), 109.9 (C, C-5) 80.2 (CH, C2), $77.9(\mathrm{CH}, \mathrm{C}-3), 73.4\left(\mathrm{CH}_{2}, \mathrm{OPMB}\right), 70.3\left(\mathrm{CH}_{2}, \mathrm{C}-1\right), 55.4\left(\mathrm{CH}_{3}, \mathrm{OPMB}\right), 27.5\left(\mathrm{CH}_{3}, \mathrm{C}-6 \mathrm{a}\right) 27.4$ $\left(\mathrm{CH}_{3}, \mathrm{C}-6 \mathrm{~b}\right), 6.6\left(\mathrm{CH}_{2}, \mathrm{C}-4\right)$; HRMS (ESI) Found 415.0361. Calculated for $\mathrm{C}_{15} \mathrm{H}_{21} \mathrm{INaO}_{4}{ }^{+}[\mathrm{M}+\mathrm{Na}]^{+}$ 415.0377. $\Delta=3.82$ ppm; IR (film from $\mathrm{CHCl}_{3}$ ) $v_{\max } 2986,2932,2861,1612,1513,1246,1088$, $1034,818 \mathrm{~cm}^{-1}$. 


\section{2-Allyl-1,3-dithiane (161)}

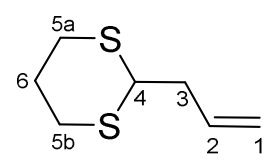

1,3-Dithiane (0.293 g, $2.4 \mathrm{mmol})$ was dissolved in dry THF $(6 \mathrm{~mL})$ and cooled to $-30{ }^{\circ} \mathrm{C}$ under argon. n-Butyllithium ( $2 \mathrm{M}$ in cyclohexane, $1.50 \mathrm{~mL}, 3 \mathrm{mmol}, 1.2$ equiv.) was then added dropwise and the solution was kept between -10 and $-20{ }^{\circ} \mathrm{C}$ for $40 \mathrm{~min}$. The reaction was then cooled to $-70{ }^{\circ} \mathrm{C}$ and allyl bromide $(0.43 \mathrm{~mL}, 4.9 \mathrm{mmol}, 2$ equiv.) was added dropwise. Reaction was allowed to warm up and kept between -50 and $-30{ }^{\circ} \mathrm{C}$ for $1 \mathrm{~h}$ at which point TLC analysis in 9:1 PE/EA indicated that all of the dithiane starting material was consumed. The reaction was allowed to warm to $0{ }^{\circ} \mathrm{C}$ then quenched with sat. aq. ammonium chloride solution $(10 \mathrm{~mL})$. The aqueous phase was extracted with diethyl ether $(3 \times 10 \mathrm{~mL})$ dried with $\mathrm{MgSO}_{4}$ and concentrated. The crude material was purified on silica gel (20:1 PE/EA) to give the product 161 as a colourless oil $(0.304 \mathrm{~g}, 78 \%)$. Analytical data matched those reported. ${ }^{162}$

$\mathbf{R}_{f}=0.68(9: 1 \mathrm{PE} / \mathrm{EA}) ;{ }^{1} \mathbf{H}$ NMR $\left(500 \mathrm{MHz}, \mathrm{CDCl}_{3}\right) \delta 5.86(\mathrm{ddt}, J=17.0,10.0,7.0 \mathrm{~Hz}, 1 \mathrm{H}, \mathrm{H}-2)$, $5.19-5.09(\mathrm{~m}, 2 \mathrm{H}, \mathrm{H}-1), 4.10(\mathrm{t}, J=6.9 \mathrm{~Hz}, 1 \mathrm{H}, \mathrm{H}-4), 2.94-2.79(\mathrm{~m}, 4 \mathrm{H}, \mathrm{H}-5 \mathrm{a}, \mathrm{H}-5 \mathrm{~b}), 2.51$ (tt, $J=7.0,1.3 \mathrm{~Hz}, 2 \mathrm{H}, \mathrm{H}-3), 2.16-2.07(\mathrm{~m}, 1 \mathrm{H}, \mathrm{H}-6 \mathrm{a}), 1.91-1.79(\mathrm{~m}, 1 \mathrm{H}, \mathrm{H}-6 \mathrm{~b}) ;{ }^{13} \mathrm{C}$ NMR (126 $\left.\mathrm{MHz}, \mathrm{CDCl}_{3}\right) \delta 133.8(\mathrm{CH}, \mathrm{C}-2), 118.1\left(\mathrm{CH}_{2}, \mathrm{C}-1\right), 47.2(\mathrm{CH}, \mathrm{C}-4), 39.9\left(\mathrm{CH}_{2}, \mathrm{C}-3\right), 30.6\left(2 \mathrm{CH}_{2}, \mathrm{C}-\right.$ 5a, C-5b), $25.9\left(\mathrm{CH}_{2}, \mathrm{C}-6\right)$.

(S)-1-((4-Methoxybenzyl)oxy)but-3-en-2-ol (162)<smiles>[R16]OC[C@@H](O)[C@@H](O)C=C</smiles>

$n$-BuLi (2 $\mathrm{M}$ in cyclohexane, $0.08 \mathrm{~mL}, 0.16 \mathrm{mmol}, 1.6$ equiv.) was added dropwise to a solution of dithiane $161(0.021 \mathrm{~g}, 0.13 \mathrm{mmol}, 1.3$ equiv. $))$ in THF $(0.38 \mathrm{ml})$ at $-78{ }^{\circ} \mathrm{C}$. The reaction quickly became yellow in colour and was allowed to warm to $-20{ }^{\circ} \mathrm{C}$ and kept at this temperature for $1 \mathrm{~h}$. Then, the reaction was cooled back to $-78{ }^{\circ} \mathrm{C}$ and iodide $74(0.040 \mathrm{~g}$, $0.10 \mathrm{mmol})$ in THF (0.1 mL plus $0.1 \mathrm{~mL}$ syringe rinse) was added dropwise. The reaction was allowed to warm to $-30^{\circ} \mathrm{C}$ over 1.5 hour, then kept at this temperature for another hour at 
which point TLC analysis (5:1 PE/EA) indicated very little starting material iodide was left. The reaction was allowed to warm up to $0{ }^{\circ} \mathrm{C}$ then quenched with water $(5 \mathrm{~mL})$ and the aqueous layer extracted with ether $(3 \times 5 \mathrm{~mL})$. After the solvent was removed under reduced pressure, the crude reaction mixture was purified on a silica gel column (9:1 PE/EA, then 5:1 PE/EA, then $100 \%$ EA). The title elimination product 162 was isolated as a colourless oil (13.3 mg, 63\%).

$\mathbf{R}_{f}=0.17(5: 1 \mathrm{PE} / \mathrm{EA}) ;{ }^{1} \mathrm{H}-\mathrm{NMR}\left(500 \mathrm{MHz}, \mathrm{CDCl}_{3}\right): \delta 7.26(\mathrm{~d}, J=8.6 \mathrm{~Hz}, 2 \mathrm{H}, \mathrm{OPMB}), 6.89$ (d, $J=$ 8.6 Hz, 2H, OPMB), 5.82 (ddd, $J=17.2,10.6,5.6 \mathrm{~Hz}, 1 \mathrm{H}, \mathrm{H}-2$ ), 5.35 (dt, J = 17.2, $1.5 \mathrm{~Hz}, 1 \mathrm{H}, \mathrm{H}-$ 1a), 5.19 (dt, $J=10.6,1.5 \mathrm{~Hz}, 1 \mathrm{H}, \mathrm{H}-1 \mathrm{~b}), 4.51$ (m, 2H, OPMB), $4.36-4.30$ (m, 1H, H-3), 3.81 (s, 3H, OPMB), 3.52 (dd, J = 9.6, 3.3 Hz, 1H, H-4a), 3.34 (dd, J = 9.6, 8.1 Hz, $1 \mathrm{H}, \mathrm{H}-4 \mathrm{~b}$ ); ${ }^{13} \mathrm{C}$ NMR (126 MHz, CDCl $)_{3}$ : $\delta 159.5$ (C, OPMB), 136.7 (CH, C-2), 130.0 (C, OPMB), 129.6 (2CH, OPMB), $116.6\left(\mathrm{CH}_{2}, \mathrm{C}-1\right), 114.0(2 \mathrm{CH}, \mathrm{OPMB}), 73.8\left(\mathrm{CH}_{2}, \mathrm{C}-4\right), 73.2\left(\mathrm{CH}_{2}, \mathrm{OPMB}\right), 71.7(\mathrm{CH}, \mathrm{C}-$ 3), $55.4\left(\mathrm{CH}_{3}, \mathrm{OPMB}\right)$; IR (film from $\left.\mathrm{CHCl}_{3}\right) v_{\max } 3449,2907,2858,1612,1512,1245,1099$, $1033,924,758 \mathrm{~cm}^{-1}$. Experimental data matched those reported. ${ }^{167}$

\section{1-(2-Allyl-1,3-dithian-2-yl)ethan-1-ol (163)}

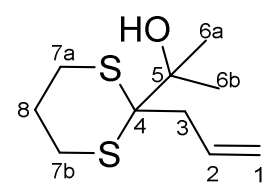

By-product 163 was also isolated as an inseparable mixture with starting material iodide $\mathbf{7 4}$ (13.9 mg total sample: $9.04 \mathrm{mg} 163,40 \%$ yield; $4.87 \mathrm{mg} \mathrm{74,} \mathrm{12 \%} \mathrm{recovered).}$

$\mathbf{R}_{f}=0.53(5: 1 \mathrm{PE} / \mathrm{EA}) ;{ }^{1} \mathrm{H}$ NMR $\left(500 \mathrm{MHz}, \mathrm{CDCl}_{3}\right) \delta 6.18(\mathrm{ddt}, J=17.1,10.2,6.9 \mathrm{~Hz}, 1 \mathrm{H}, \mathrm{H}-2)$, $5.19-5.11(\mathrm{~m}, 2 \mathrm{H}, \mathrm{H}-1), 2.94-2.79(\mathrm{~m}, 6 \mathrm{H}, \mathrm{H}-3, \mathrm{H}-7 \mathrm{a}, \mathrm{H}-7 \mathrm{~b}), 2.46(\mathrm{~s}, 1 \mathrm{H}, \mathrm{OH}), 2.03-1.94$ (m, 1H, H-8a), $1.94-1.85$ (m, 1H, H-8b), 1.43 (s, 6H, H-6a,H-6b); ${ }^{13} \mathrm{C}$ NMR (126 MHz, CDCl 3 ) $\delta 136.3(\mathrm{CH}, \mathrm{C}-2), 117.3\left(\mathrm{CH}_{2}, \mathrm{C}-1\right), 78.4$ (C, C-5), 63.0 (C, C-4), $41.7\left(\mathrm{CH}_{2}, \mathrm{C}-3\right), 26.9\left(2 \mathrm{CH}_{2}, \mathrm{C}-\right.$ 7a, C-7b), 26.2 (2CH $3, \mathrm{C}-6 \mathrm{a}, \mathrm{C}-6 \mathrm{~b}), 24.2\left(\mathrm{CH}_{2}, \mathrm{C}-8\right)$; IR (film from $\mathrm{CHCl}_{3}$ ) v $\max 3490,3073,2979$, 2931, 1636, 1612, 1515, 1368, 1247, 1175, 1113, $911 \mathrm{~cm}^{-1}$. 


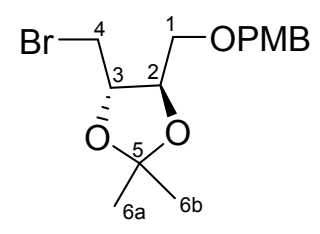

Alcohol $73(0.261 \mathrm{~g}, 0.93 \mathrm{mmol})$ was dissolved in dry toluene $(6 \mathrm{~mL})$ and heated to reflux under argon then when the refluxing started the heating was stopped. When the boiling ceased, imidazole (0.194 g, $2.82 \mathrm{mmol}, 3$ equiv.), $\mathrm{PPh}_{3}(0.367 \mathrm{~g}, 1.40 \mathrm{mmol}, 1.5$ equiv.) and NBS (0.254 g, $1.40 \mathrm{mmol}, 1.5$ equiv.) were added sequentially. The reaction was heated again and refluxed for 2.5 hours then allowed to cool down to room temperature. The reaction was quenched at room temperature with sat. aq. $\mathrm{Na}_{2} \mathrm{~S}_{2} \mathrm{O}_{3}(10 \mathrm{~mL})$ and the aqueous layer extracted with diethyl ether $(3 \times 20 \mathrm{~mL})$. The combined organic layers were dried with anhydrous $\mathrm{MgSO}_{4}$ then the solvent was removed under reduced pressure. Concentrated crude reaction mixture was purified on silica gel column (PE/EA 5:1) to give the bromide 164 as a colourless oil ( $0.137 \mathrm{~g}, 43 \%$ yield, $88 \%$ BRSM). Experimental data matched those previously reported.

$\mathbf{R}_{f}=0.29(10: 1 \mathrm{PE} / \mathrm{EA}) ;{ }^{1} \mathbf{H}$ NMR $\left(500 \mathrm{MHz}, \mathrm{CDCl}_{3}\right) \delta 7.28-7.23(\mathrm{~m}, 2 \mathrm{H}, \mathrm{OPMB}), 6.88$ (d, $J=$ $8.7 \mathrm{~Hz}, 1 \mathrm{H}, \mathrm{OPMB}), 4.52(\mathrm{~m}, 2 \mathrm{H}, \mathrm{OPMB}), 4.10-4.01$ (m, 2H, H-2, H-3), 3.81 (s, 3H, OPMB), $3.66-3.57(\mathrm{~m}, 2 \mathrm{H}, \mathrm{H}-1), 3.51$ (dd, J = 10.7, $4.7 \mathrm{~Hz}, 1 \mathrm{H}, \mathrm{H}-4 \mathrm{a}), 3.46$ (dd, J = 10.7, $5.1 \mathrm{~Hz}, 1 \mathrm{H}$, $\mathrm{H}-4 \mathrm{~b}), 1.44$ (s, 3H, H-6a), 1.42 (s, 3H, H-6b); ${ }^{13} \mathrm{C}$ NMR (126 MHz, CDCl 3 ) $\delta 159.4$ (C, OPMB), 130.0 (C, OPMB), 129.5 (2C, OPMB), 113.97 (2C, OPMB), 110.1 (C, C-5), 78.9 (CH, C-2 or C-3), $78.0(\mathrm{CH}, \mathrm{C}-2$ or $\mathrm{C}-3) 73.4\left(\mathrm{CH}_{2}, \mathrm{C}-1\right.$ or $\left.\mathrm{C}-4\right), 70.3\left(\mathrm{CH}_{2}, \mathrm{C}-1\right.$ or $\left.\mathrm{C}-4\right), 55.4\left(\mathrm{CH}_{3}\right.$, $\left.\mathrm{OCH}_{2} \mathrm{PhOCH}_{3}\right), 27.3\left(\mathrm{CH}_{3}, \mathrm{C}-6 \mathrm{a}\right), 27.7\left(\mathrm{CH}_{3}, \mathrm{C}-6 \mathrm{~b}\right)$; HRMS (ESI) Found 362.0972. Calculated for $\mathrm{C}_{15} \mathrm{H}_{25} \mathrm{BrNO}_{4}^{+}\left[\mathrm{M}+\mathrm{NH}_{4}\right]^{+}$362.0962. $\Delta=2.80 \mathrm{ppm}$; 
(4R,5R)-4-((1,3-Dithian-2-yl)methyl)-5-(((4-methoxybenzyl)oxy)methyl)-2,2-dimethyl-1,3dioxolane (165)

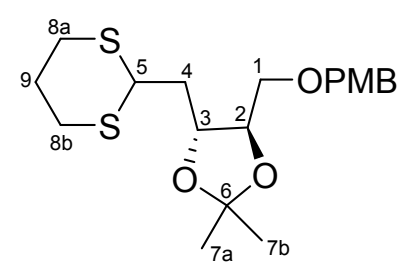

1,3-Dithiane (0.031 g, $0.25 \mathrm{mmol}, 1.9$ equiv.) was dissolved in 5:1 v/v THF/DMPU (0.80 mL) and cooled to $-30{ }^{\circ} \mathrm{C}$ then treated dropwise with a solution of $n$-BuLi $(0.13 \mathrm{~mL}, 2.60 \mathrm{mmol}, 2$ equiv., $2 \mathrm{M}$ in cyclohexane). The colour of the solution changed to pink during the addition. The reaction was then cooled to $-78{ }^{\circ} \mathrm{C}$ and treated dropswise with a solution of iodide $\mathbf{7 4}$ $(0.051 \mathrm{~g}, 0.13 \mathrm{mmol})$ in 5:1 THF/DMPU $(0.20 \mathrm{~mL})$. The reaction was then allowed to warm to $-60{ }^{\circ} \mathrm{C}$ and kept at this temperature for $5 \mathrm{hrs}$. After 5 hours the reaction was quenched with water $(2 \mathrm{~mL})$ at the same temperature to minimise the formation of by-products, then allowed to warm to room temperature. The aqueous layer was extracted with ether $(3 \times 10$ $\mathrm{mL}$ ) and the combined organic layers dried over $\mathrm{MgSO}_{4}$ and concentrated. The crude material was purified on a silica gel column (10:1 PE/EA then 5:1) to give the dithiane $\mathbf{1 6 5}$ as a colourless oil ( $6.4 \mathrm{mg}, 13 \%$ yield). lodide 74 was also recovered ( $25.9 \mathrm{mg}, 51 \%)$.

$\mathbf{R}_{f}=0.36(5: 1 \mathrm{PE} / \mathrm{EA}) ;{ }^{1} \mathbf{H}-\mathrm{NMR}\left(500 \mathrm{MHz}, \mathrm{CDCl}_{3}\right): \delta 7.29-7.25(\mathrm{~m}, 2 \mathrm{H}, \mathrm{OPMB}), 6.88(\mathrm{~d}, J=8.6$ $\mathrm{Hz}, 2 \mathrm{H}, \mathrm{OPMB}$ ), 4.54 (d, $J=11.8 \mathrm{~Hz}, 1 \mathrm{H}, \mathrm{OPMB}), 4.48$ (d, $J=11.8 \mathrm{~Hz}, 1 \mathrm{H}, \mathrm{OPMB}), 4.20$ (dd, $J=$ 8.2, $6.4 \mathrm{~Hz}, 1 \mathrm{H}, \mathrm{H}-5), 4.13-4.06(\mathrm{~m}, 1 \mathrm{H}, \mathrm{H}-3), 3.84$ (ddd, J = 7.9, 5.7, $4.8 \mathrm{~Hz}, 1 \mathrm{H}, \mathrm{H}-2$ ), 3.81 (s, $3 \mathrm{H}, \mathrm{OPMB}$ ), 3.56 (dd, $J=10.0,5.7 \mathrm{~Hz}, 1 \mathrm{H}, \mathrm{H}-1 \mathrm{a}), 3.49$ (dd, $J=10.1,4.8 \mathrm{~Hz}, 1 \mathrm{H}, \mathrm{H}-1 \mathrm{~b}), 2.91-$ $2.76(m, 4 \mathrm{H}, \mathrm{H}-8 \mathrm{a}, \mathrm{H}-8 \mathrm{~b}), 2.14-2.06$ (m, 1H, H-9a), $2.01-1.96$ (m, 2H, H-4), $1.92-1.82$ (m, 1H, H-9b), 1.40 (d, $2.4 \mathrm{~Hz}, 6 \mathrm{H}, \mathrm{H}-7 \mathrm{a}, \mathrm{H}-7 \mathrm{~b}) ;{ }^{13} \mathrm{C}-\mathrm{NMR}$ (126 MHz, CDCl 3 ): $\delta 159.4$ (C, OPMB), 130.1 (C, OPMB), 129.5 (2CH, OPMB), 114.0 (2CH, OPMB), 109.5 (C, C-6) 80.0 (CH, C-2), 75.4 ( $\mathrm{CH}, \mathrm{C}-3), 73.3\left(\mathrm{CH}_{2}, \mathrm{OPMB}\right), 70.3\left(\mathrm{CH}_{2}, \mathrm{C}-1\right), 55.4\left(\mathrm{CH}_{3}, \mathrm{OPMB}\right), 43.9(\mathrm{CH}, \mathrm{C}-5), 39.8\left(\mathrm{CH}_{2}, \mathrm{C}-\right.$ 4), $30.4\left(\mathrm{CH}_{2}, \mathrm{C}-8 \mathrm{a}\right), 29.9\left(\mathrm{CH}_{2}, \mathrm{C}-8 \mathrm{~b}\right), 27.4\left(\mathrm{CH}_{3}, \mathrm{C}-7 \mathrm{a}\right) 27.2\left(\mathrm{CH}_{3}, \mathrm{C}-7 \mathrm{~b}\right), 26.1\left(\mathrm{CH}_{2}, \mathrm{C}-9\right.$; HRMS (ESI) Found 385.1514. Calculated for $\mathrm{C}_{19} \mathrm{H}_{29} \mathrm{O}_{4} \mathrm{~S}_{2}{ }^{+}[\mathrm{M}+\mathrm{H}]^{+}$385.1502. $\Delta=3.21 \mathrm{ppm}$; IR (film from $\left.\mathrm{CHCl}_{3}\right) v_{\max } 2985,2931,2900,1612,1513,1247,1088,1035,843,821 \mathrm{~cm}^{-1}$. 
<smiles>CC(=O)OCC(C)O[Mg]</smiles>

Occasionally, by-product 166 was also isolated from the reaction.

$\mathbf{R}_{f}=0.66(5: 1 \mathrm{PE} / \mathrm{EA}) ;{ }^{1} \mathrm{H}-\mathrm{NMR}\left(500 \mathrm{MHz}, \mathrm{CDCl}_{3}\right): \delta 7.22(\mathrm{~d}, J=8.6 \mathrm{~Hz}, 2 \mathrm{H}, \mathrm{OPMB}), 6.87$ (d, $J=$ 8.6 Hz, 2H, OPMB), 4.54 (d, J = 11.7 Hz, 1H, OPMB), 4.42 (d, J = 11.7 Hz, 1H, OPMB), 4.25 (q, $J=4.2 \mathrm{~Hz}, 1 \mathrm{H}, \mathrm{H}-3$ ), 3.80 (m, 4H, H-4a, OPMB), 3.68 (dd, J = 10.4, 3.8 Hz, 1H, H-4b), 3.63 (d, J $=5.1 \mathrm{~Hz}, 1 \mathrm{H}, \mathrm{OH}), 2.20(\mathrm{~s}, 3 \mathrm{H}, \mathrm{H}-1) ;{ }^{13} \mathrm{C}-\mathrm{NMR}(126 \mathrm{MHz}, \mathrm{CDCl}) \delta 208.0\left(\mathrm{CH}_{3}, \mathrm{C}-1\right), 159.5(\mathrm{C}$, OPMB), 129.6 (C, OPMB), 129.6 (2CH, OPMB), 114.0 (2CH, OPMB), 77.0 (CH, C-3), $73.5\left(\mathrm{CH}_{2}\right.$, OPMB), $70.6\left(\mathrm{CH}_{2}, \mathrm{C}-4\right), 55.4\left(\mathrm{CH}_{3}, \mathrm{OPMB}\right), 25.9\left(\mathrm{CH}_{3}, \mathrm{C}-1\right)$; IR (film from $\left.\mathrm{CHCl}_{3}\right) \mathrm{V}_{\max }$ 3467, $2910,2862,1718,1612,1513,1246,1096,1032,820 \mathrm{~cm}^{-1}$.

(2R,3R)-1-(1,3-Dithian-2-yl)-4-((4-methoxybenzyl)oxy)butane-2,3-diol (170)

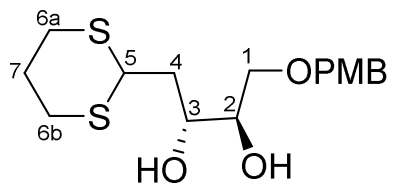

Acetonide 165 (9.0 mg, $0.023 \mathrm{mmol})$ was dissolved in $\mathrm{MeOH}(0.1 \mathrm{~mL})$ and treated with a small spatula of $p \mathrm{TSOH}$ then stirred at room temperature overnight. The reaction was quenched with sat. aq. $\mathrm{NaHCO}_{3}(5 \mathrm{~mL})$ and the aqueous layer extracted with EtOAc (3x $5 \mathrm{~mL}$ ). Purification on silica gel column (2:1 PE/EA, then 100\% EA) gave diol 170 (5.2 mg, 65\% yield).

$\mathbf{R}_{f}=0.31$ (1:1 PE/EA); ${ }^{1} \mathbf{H}-\mathrm{NMR}\left(300 \mathrm{MHz}, \mathrm{CDCl}_{3}\right.$, assigned based on COSY): $\delta 7.24$ (d, $J=8.9$ $\mathrm{Hz}, 2 \mathrm{H}, \mathrm{OPMB}$ ), 6.88 (d, J = 8.6 Hz, 2H, OPMB), 4.48 (s, 2H, OPMB), 4.27 (dd, J = 9.8, $4.6 \mathrm{~Hz}$, $1 \mathrm{H}, \mathrm{H}-5), 4.04-3.93(\mathrm{~m}, 1 \mathrm{H}, \mathrm{H}-3), 3.81$ (d, J = $1.0 \mathrm{~Hz}, 3 \mathrm{H}, \mathrm{OPMB}), 3.66-3.53(\mathrm{~m}, 3 \mathrm{H}, \mathrm{H}-1, \mathrm{H}-$ 2), $2.99-2.78(\mathrm{~m}, 4 \mathrm{H}, \mathrm{H}-6 \mathrm{a}, \mathrm{H}-6 \mathrm{~b}), 2.76(\mathrm{~d}, J=4.7 \mathrm{~Hz}, 1 \mathrm{H}, \mathrm{OH}), 2.64(\mathrm{~d}, J=5.3 \mathrm{~Hz}, 1 \mathrm{H}, \mathrm{OH})$, $2.19-1.99$ (m, 2H, H-4a, H-7a), $1.96-1.76$ (m, 2H, H-4b, H-7b). 


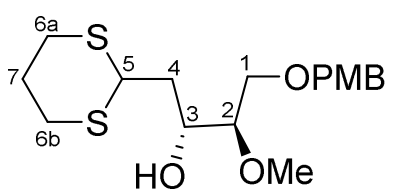

Diol 170 (5.2 mg, $0.015 \mathrm{mmol})$ was dissolved in dry DCM $(0.4 \mathrm{~mL})$ and cooled to $0{ }^{\circ} \mathrm{C}$ under an argon atmosphere, then treated with $\mathrm{Me}_{3} \mathrm{OBF}_{4}(4.6 \mathrm{mg}, 0.03 \mathrm{mmol}, 2$ equiv.) and 1,8Bis(dimethylamino)naphthalene (5.7 $\mathrm{mg}, 0.027 \mathrm{mmol}, 1.8$ equiv.). The reaction was allowed to warm to room temperature and stirred overnight. After overnight stirring, the reaction was quenched with sat. aq. $\mathrm{NaHCO}_{3}(5 \mathrm{~mL})$, extracted with $\mathrm{DCM}(3 \times 5 \mathrm{~mL})$ and the combined organic layers washed with brine $(5 \mathrm{~mL})$. The organic layer was then dried over anhydrous $\mathrm{MgSO}_{4}$ and concentrated. The crude reaction mixture was purified using silica gel chromatography (1:1 PE/EA) to give a single product 171 (4.5 mg, 86\%).

$\mathbf{R}_{f}=0.56(1: 1 \mathrm{PE} / \mathrm{EA}) ;{ }^{1} \mathrm{H}-\mathrm{NMR}\left(300 \mathrm{MHz}, \mathrm{CDCl}_{3}\right): \delta 7.25$ (d, $\left.J=9.1 \mathrm{~Hz}, 2 \mathrm{H}, \mathrm{OPMB}\right), 6.88(\mathrm{~d}, J=$ 8.7 Hz, 2H, OPMB), 4.49 (d, J = 11.6 Hz, 1H, OPMB), 4.46 (d, J=11.6 Hz, 1H, OPMB), 4.30 (dd, $J=10.1,4.2 \mathrm{~Hz}, 1 \mathrm{H}, \mathrm{H}-5), 4.06-3.99(\mathrm{~m}, 1 \mathrm{H}, \mathrm{H}-3), 3.81$ (s, 3H, OPMB), 3.62 (dd, J = 10.2, 5.0 $\mathrm{Hz}, 1 \mathrm{H}, \mathrm{H}-1 \mathrm{a}$ ), 3.55 (dd, $J=10.2,4.8 \mathrm{~Hz}, 1 \mathrm{H}, \mathrm{H}-1 \mathrm{~b}$ ), 3.45 (s, 3H, OCH ${ }_{3}$ ), 3.22 (dd, $J=9.1,4.8$ $\mathrm{Hz}, 1 \mathrm{H}, \mathrm{H}-2$ ), $2.97-2.79$ (m, 4H, H-6a, H-6b), 2.63 (d, J = 5.7 Hz, 1H, OH), 2.11 (m, 1H, H-7a), 1.97 (ddd, $J=14.3,10.1,4.3 \mathrm{~Hz}, 1 \mathrm{H}, \mathrm{H}-4 \mathrm{a}), 1.93-1.83$ (m, 2H, H-4b, H-7b); ${ }^{13} \mathrm{C}$ NMR (126 $\mathrm{MHz}, \mathrm{CDCl}_{3}$ ) $\delta 159.4$ (C, OPMB), 130.0 (C, OPMB), 129.5 (2CH, OPMB), 114.0 (2CH, OPMB), $82.1(\mathrm{CH}, \mathrm{C}-2), 73.4\left(\mathrm{CH}_{2}, \mathrm{OPMB}\right), 69.2\left(\mathrm{CH}_{2}, \mathrm{C}-1\right), 68.6(\mathrm{CH}, \mathrm{C}-3), 58.8\left(\mathrm{CH}_{3}, \mathrm{OCH}_{3}\right), 55.4\left(\mathrm{CH}_{3}\right.$, OPMB), 44.0 ( $\mathrm{CH}, \mathrm{C}-5), 39.2\left(\mathrm{CH}_{2}, \mathrm{C}-4\right), 30.6\left(\mathrm{CH}_{2}, \mathrm{C}-6 \mathrm{a}\right), 30.1\left(\mathrm{CH}_{2}, \mathrm{C}-6 \mathrm{~b}\right), 26.2\left(\mathrm{CH}_{2}, \mathrm{C}-7\right)$.

\section{2-((2R,3R)-3-Methoxy-4-((4-methoxybenzyl)oxy)-2-(methoxymethoxy)butyl)-1,3-dithiane} (172)

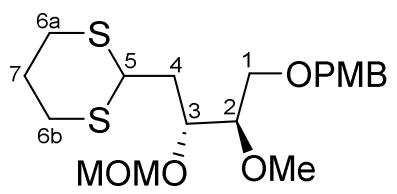

Alcohol $171(4.5 \mathrm{mg}, 0.013 \mathrm{mmol})$ in $\mathrm{DCM}(0.30 \mathrm{~mL})$ was cooled to $0^{\circ} \mathrm{C}$ under argon then treated with DIPEA (0.01 mL, $0.06 \mathrm{mmol}, 5$ equiv.) and $\mathrm{MOMCl}(0.01 \mathrm{~mL}, 0.10 \mathrm{mmol}, 10$ equiv.). The reaction was allowed to warm to room temperature and stirred overnight. The 
reaction was quenched with water $(2 \mathrm{~mL})$ extracted with DCM $(3 \times 3 \mathrm{~mL})$. The crude material was subjected to the next step without purification, but no reaction occurred. Therefore, the title product 172 was obtained after flash chromatography (3:1 PE/EA), as a colourless oil ( $2.5 \mathrm{mg}, 49 \%$ yield).

$\mathbf{R}_{f}=0.34(3: 1 \mathrm{PE} / \mathrm{EA}) ;{ }^{1} \mathrm{H}$ NMR $\left(500 \mathrm{MHz}, \mathrm{CDCl}_{3}\right) \delta 7.27(\mathrm{~d}, J=6.9 \mathrm{~Hz}, 2 \mathrm{H}, \mathrm{OPMB}), 6.87(\mathrm{~d}, J=$ $8.6 \mathrm{~Hz}, 2 \mathrm{H}, \mathrm{OPMB}), 4.71\left(\mathrm{~d}, J=6.8 \mathrm{~Hz}, 1 \mathrm{H}, \mathrm{OCH}_{2} \mathrm{OCH}_{3}\right), 4.65\left(\mathrm{~d}, J=6.8 \mathrm{~Hz}, 1 \mathrm{H}, \mathrm{OCH}_{2} \mathrm{OCH}_{3}\right)$, $4.50(\mathrm{~d}, J=11.6 \mathrm{~Hz}, 1 \mathrm{H}, \mathrm{OPMB}), 4.45(\mathrm{~d}, J=11.6 \mathrm{~Hz}, 1 \mathrm{H}, \mathrm{OPMB}), 4.11(\mathrm{dd}, J=9.4,5.0 \mathrm{~Hz}, 1 \mathrm{H}$, H-5), $4.04-3.98$ (m, 1H, H-3), 3.80 (s, 3H, OPMB), 3.64 (dd, J=8.8, 2.5 Hz, 1H, H-1a), $3.56-$ $3.49(\mathrm{~m}, 2 \mathrm{H}, \mathrm{H}-1 \mathrm{~b}, \mathrm{H}-2), 3.47\left(\mathrm{~s}, 3 \mathrm{H}, \mathrm{OCH}_{3}\right), 3.37\left(\mathrm{~s}, 3 \mathrm{H}, \mathrm{OCH}_{2} \mathrm{OCH}_{3}\right), 2.90-2.77(\mathrm{~m}, 4 \mathrm{H}, \mathrm{H}-6 \mathrm{a}$, H-6b), 2.14 - 2.04 (m, 2H, H-4a, H-7a), 1.93 - 1.84 (m, 2H, H-4b, H-7b); ${ }^{13}$ C NMR (126 MHz, $\left.\mathrm{CDCl}_{3}\right) \delta 159.3$ (C, OPMB), 130.4 (C, OPMB), 129.5 (2CH, OPMB), 113.9 (2CH, OPMB), 97.6 $\left(\mathrm{CH}_{2}, \mathrm{OCH}_{2} \mathrm{OCH}_{3}\right), 81.6(\mathrm{CH}, \mathrm{C}-2), 74.8(\mathrm{CH}, \mathrm{C}-3), 73.3\left(\mathrm{CH}_{2}, \mathrm{OPMB}\right), 69.4\left(\mathrm{CH}_{2}, \mathrm{C}-1\right), 58.9\left(\mathrm{CH}_{3}\right.$, $\left.\mathrm{OCH}_{3}\right), 56.1\left(\mathrm{CH}_{3}, \mathrm{OCH}_{2} \mathrm{OCH}_{3}\right), 55.4\left(\mathrm{CH}_{3}, \mathrm{OPMB}\right), 43.8(\mathrm{CH}, \mathrm{C}-5), 36.5\left(\mathrm{CH}_{2}, \mathrm{C}-4\right), 30.2\left(\mathrm{CH}_{2}, \mathrm{C}-\right.$ 6a), $29.9\left(\mathrm{CH}_{2}, \mathrm{C}-6 \mathrm{~b}\right), 26.1\left(\mathrm{CH}_{2}, \mathrm{C}-7\right)$.

((4R,5R)-5-(((tert-Butyldiphenylsilyl)oxy)methyl)-2,2-dimethyl-1,3-dioxolan-4-yl)methanol (167)

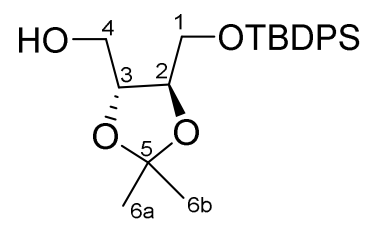

(-)-2,3-O-Isopropylidene-D-threitol $(1.054 \mathrm{~g}, 6.37 \mathrm{mmol})$ dissolved in dry THF (30 $\mathrm{mL})$ was cooled to $0{ }^{\circ} \mathrm{C}$ under argon and treated with $\mathrm{NaH}(0.265 \mathrm{~g}, 6.63 \mathrm{mmol}, 1.04$ equiv.). The reaction was stirred for $30 \mathrm{~min}$ at $0{ }^{\circ} \mathrm{C}$, then TBDPSCI $(1.60 \mathrm{~mL}, 6.05 \mathrm{mmol}, 0.95$ equiv.) in 12 $\mathrm{mL}$ THF was added dropwise and the reaction stirred at room temperature over night then quenched water $(50 \mathrm{~mL})$. The aqueous layer was extracted with DCM (3x $50 \mathrm{~mL})$. The combined organic layers were dried over anhydrous $\mathrm{MgSO}_{4}$ and the solvent removed under reduced pressure. The crude reaction mixture was purified on a silica gel column (5:1 PE/EA) to give the product 167 as a colourless oil $(2.261 \mathrm{~g}, 89 \%)$. Experimental data matched those reported for the enantiomer. ${ }^{233}$ 
$\mathbf{R}_{f}=0.31(5: 1 \mathrm{PE} / \mathrm{EA}) ;[\boldsymbol{\alpha}]_{\mathrm{D}}{ }^{22}=+16.4\left(c 1.10, \mathrm{CHCl}_{3}\right)\left[\mathrm{lit}^{233}[\boldsymbol{\alpha}]_{\mathrm{D}}{ }^{25}=-1.5\right.$ (c 14.5, $\left.\mathrm{CHCl}_{3}\right)$ for ent 167]; ${ }^{1} \mathrm{H}-\mathrm{NMR}$ (500 MHz, $\mathrm{CDCl}_{3}$ ): $\delta$ 7.69-7.65 (m, 4H, OTBDPS), $7.47-7.36$ (m, 6H, OTBDPS), 4.08 (dt, $J=8.3,4.2 \mathrm{~Hz}, 1 \mathrm{H}, \mathrm{H}-3$ ), 3.97 (ddd, $J=8.1,6.2,4.0 \mathrm{~Hz}, 1 \mathrm{H}, \mathrm{H}-2), 3.85-3.79(\mathrm{~m}, 2 \mathrm{H}$, $\mathrm{H}-1, \mathrm{H}-4$ ), 3.74 (dd, J = 10.7, $6.3 \mathrm{~Hz}, 1 \mathrm{H}, \mathrm{H}-1$ ), 3.66 (ddd, J=11.9, 7.6, $4.5 \mathrm{~Hz}, 1 \mathrm{H}, \mathrm{H}-4$ ), 2.16 (dd, $J=8.0,4.7 \mathrm{~Hz}, 1 \mathrm{H}, \mathrm{OH}), 1.41$ (d, $J=12.2 \mathrm{~Hz}, 6 \mathrm{H}, \mathrm{H}-6 \mathrm{a}, \mathrm{H}-6 \mathrm{~b}), 1.06$ (s, 9H, OTBDPS).; ${ }^{13} \mathrm{C}-$ NMR (100 MHz, $\mathrm{CDCl}_{3}$ ): $\delta 135.7$ (4CH, OTBDPS), 133.0 (C, OTBDPS), 132.9 (C, OTBDPS), 130.04 (CH, OTBDPS), 130.00 (CH, OTBDPS), 127.94 (2CH, OTBDPS), 127.92 (2CH, OTBDPS), 109.3 (C, C-5), 79.7 ( $\mathrm{CH}, \mathrm{C}-3), 77.6$ (C-2), $64.3\left(\mathrm{CH}_{2}, \mathrm{C}-1\right), 62.7\left(\mathrm{CH}_{2}, \mathrm{C}-4\right), 27.2\left(\mathrm{CH}_{3}, \mathrm{C}-6 \mathrm{a}\right)$, 27.1 ( $\left.\mathrm{CH}_{3}, \mathrm{C}-6 \mathrm{~b}\right), 27.0\left(3 \mathrm{CH}_{3}, \mathrm{OTBDPS}\right), 19.3$ (C, OTBDPS); IR (film from $\left.\mathrm{CHCl}_{3}\right) \mathrm{v}_{\max }$ 3436, $3071,2932,2859,1628,1428,1245,1112,1078,702,505 \mathrm{~cm}^{-1}$.

tert-Butyl(((4R,5S)-5-(iodomethyl)-2,2-dimethyl-1,3-dioxolan-4-yl)methoxy)diphenylsilane (168)

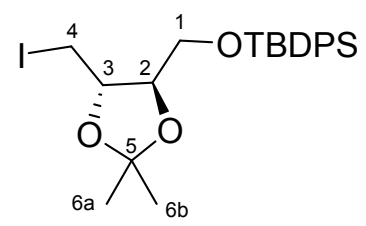

Primary alcohol $167(0.916 \mathrm{~g}, 2.3 \mathrm{mmol})$ was dissolved in dry toluene $(14 \mathrm{~mL})$ and heated to reflux, then stoppped heating. When the boiling ceased, the solution was treated with imidazole (0.496 g, $7.2 \mathrm{mmol}, 3.2$ equiv.), $\mathrm{PPh}_{3}(0.912 \mathrm{~g}, 3.5 \mathrm{mmol}, 1.5$ equiv.) and NIS (0.822 $\mathrm{g}, 3.5 \mathrm{mmol}, 1.5$ equiv.). The reaction was heated at reflux for 1 hour, then allowed to cool down to room temperature and quenched with sat. aq. $\mathrm{Na}_{2} \mathrm{~S}_{2} \mathrm{O}_{3}(30 \mathrm{~mL})$. The aqueous layer was extracted with diethyl ether $(3 \times 30 \mathrm{~mL})$ and the combined organic layers dried $\left(\mathrm{MgSO}_{4}\right)$ and concentrated. The crude reaction matreial was purified on a silica gel column (PE $100 \%$ then 9:1 PE/EA) and the product 168 isolated as a colourless oil. ${ }^{1} \mathrm{H}$ NMR spectrum showed the product to be contaminated with $\mathrm{PPh}_{3}$. The yield was determined from the integration of peaks in the ${ }^{1} \mathrm{H}$ NMR $(0.942 \mathrm{~g}, 81 \%$, accounting for $88 \%$ of the sample containing $1.069 \mathrm{~g})$.

${ }^{1}$ H NMR (500 MHz, CDCl 3 ) $\delta 7.47-7.37$ (m, 6H OTBDPS), 7.36 - 7.28 (m, 4H, OTBDPS), 3.99 - $3.93(\mathrm{~m}, 1 \mathrm{H}, \mathrm{H}-3), 3.90-3.81(\mathrm{~m}, 2 \mathrm{H}, \mathrm{H}-2, \mathrm{H}-1 \mathrm{a}), 3.77$ (dd, J=10.5, $5.5 \mathrm{~Hz}, 1 \mathrm{H}, \mathrm{H}-1 \mathrm{~b}), 3.39$ (dd, $J=10.6,4.7 \mathrm{~Hz}, 1 \mathrm{H}$ ), 3.29 (dd, $J=10.6,5.3 \mathrm{~Hz}, 1 \mathrm{H}, \mathrm{H}-4 \mathrm{a}$ ), 1.46 (d, J=0.9 Hz, 3H, H-5a), $1.39(\mathrm{t}, J=0.7 \mathrm{~Hz}, 3 \mathrm{H}, \mathrm{H}-5 \mathrm{~b}), 1.07$ (s, 9H, OTBDPS). ${ }^{13} \mathrm{C}$ NMR $\left(126 \mathrm{MHz}, \mathrm{CDCl}_{3}\right) \delta 135.8(4 \mathrm{CH}$, OTBDPS), 133.1 (2C, OTBDPS), 130.0 (2CH, OTBDPS), 128.0 (4CH, OTBDPS), 109.7 (C, C-5), 
$81.3(\mathrm{CH}, \mathrm{C}-3), 77.8(\mathrm{CH}, \mathrm{C}-2) 64.3\left(\mathrm{CH}_{2}, \mathrm{C}-1\right), 27.6\left(\mathrm{CH}_{3}, \mathrm{C}-6 \mathrm{a}\right), 27.0\left(\mathrm{CH}_{3}, \mathrm{C}-6 \mathrm{~b}\right), 19.4(\mathrm{C}$, OTBDPS), $7.0\left(\mathrm{CH}_{2}, \mathrm{C}-4\right)$.

tert-Butyl(((4R,5R)-5-(2-methoxyvinyl)-2,2-dimethyl-1,3-dioxolan-4-yl)methoxy) diphenylsilane (173)

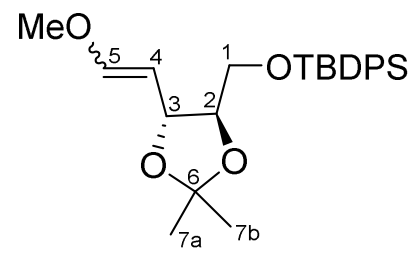

Alcohol 167 (0.056 g, $0.14 \mathrm{mmol})$ was dissolved in DCM $(1.0 \mathrm{~mL})$ and treated with pyridine (0.02 mL, $0.21 \mathrm{mmol}, 1.5$ equiv.) and DMP (0.094 g, $0.21 \mathrm{mmol}, 1.5$ equiv.) at $0{ }^{\circ} \mathrm{C}$. After 2 hours of stirring at the same temperature, reaction was quenched with $1: 1 \mathrm{v} / \mathrm{v}$ sat. aq. $\mathrm{Na}_{2} \mathrm{~S}_{2} \mathrm{O}_{3} /$ sat. aq. $\mathrm{NaHCO}_{3}(10 \mathrm{~mL})$ and stirred for $30 \mathrm{~min}$ at room temperature. The aqueous phase was extracted with $\mathrm{Et}_{2} \mathrm{O}(3 \times 10 \mathrm{~mL})$, and the combined organic layers washed with brine, dried $\left(\mathrm{MgSO}_{4}\right)$ then concentrated. The crude mixture was used in the next step without purification.

A solution of (methoxymethyl)triphenylphosphonium bromide $(0.140 \mathrm{~g}, 0.35 \mathrm{mmol}, 2.5$ equiv.) in dry THF $(1.0 \mathrm{~mL}$ ) under argon was treated with $n$-BuLi (2 $\mathrm{M}$ in cyclohexane, 0.18 $\mathrm{mL}, 0.33 \mathrm{mmol}, 2.4$ equiv.) at $-40{ }^{\circ} \mathrm{C}$ and then stirred at room temperature for 15 minutes. Then the solution was cooled to $-40{ }^{\circ} \mathrm{C}$ again and the crude mixture containing the aldehyde $167 \mathrm{a}$ in THF $(0.5 \mathrm{~mL})$ was added dropwise. The reaction was allowed to warm to room temperature gradually and stirred for 2 hours. The reaction was quenched with sat. aq. NaHCO3 $(10 \mathrm{~mL})$ and extracted with EtOAc (3x $10 \mathrm{~mL})$. The combined organic layer was dried over $\mathrm{MgSO}_{4}$ and concentrated under reduced pressure. Crude material was purified on silica gel column (9:1 PE/EA) to give the product 173 as a colourless oil and as an inseparable mixture of isomers (Z/E 1:0.8, $11.6 \mathrm{mg}, 19 \%)$.

$\mathbf{R}_{f}=0.39,0.41$ (9:1 PE/EA); ${ }^{1} \mathbf{H}$ NMR (500 MHz, $\mathrm{CDCl}_{3}$, mixture of isomers) $\delta 7.75-7.64(\mathrm{~m}$, 4H, OTBDPS), $7.46-7.33$ (m, 6H, OTBDPS), 6.56 (d, $J=12.6 \mathrm{~Hz}, 0.4 \mathrm{H}, \mathrm{H}-5 \mathrm{E}), 6.10$ (dd, $J=$ $6.2,0.9 \mathrm{~Hz}, 0.6 \mathrm{H}, \mathrm{H}-5 \mathrm{Z}), 5.03(\mathrm{t}, J=8.2 \mathrm{~Hz}, 0.6 \mathrm{H}, \mathrm{H}-3 \mathrm{Z}$ ), 4.70 (dd, J=12.9, 8.9 Hz, 0.4H, H-4 E), $4.43(\mathrm{dd}, J=9.0,6.2 \mathrm{~Hz}, 0.6 \mathrm{H}, \mathrm{H}-4 \mathrm{Z}), 4.39(\mathrm{t}, J=8.6 \mathrm{~Hz}, 0.4 \mathrm{H}, \mathrm{H}-3 \mathrm{E}), 3.86-3.70(\mathrm{~m}, 3 \mathrm{H}$, $\mathrm{H}-2, \mathrm{H}-1$ ), 3.59 (s, 1.8H, OMe Z), 3.55 (s, 1.2H, OMe E), 1.44 (d, J = 2.1 Hz, 6H, H-7a, H-7b), 
$1.06\left(\mathrm{~d}, J=1.3 \mathrm{~Hz}, 9 \mathrm{H}\right.$, OTBDPS); ${ }^{13} \mathrm{C}$ NMR (126 MHz, CDCl 3 ) $\delta 152.3(\mathrm{CH}, \mathrm{C}-5 E), 150.9$ (CH, C5Z), 135.9 (2CH, OTBDPS), 135.8 (2CH, OTBDPS), 135.8 (2CH, OTBDPS), 135.8 (2CH, OTBDPS), 133.6 (C, OTBDPS), 133.6 (C, OTBDPS), 133.4 (C, OTBDPS), 133.4 (C, OTBDPS), 129.9 (CH, OTBDPS), 129.8 (CH, OTBDPS), 129.7 (CH, OTBDPS), 129.7 (CH, OTBDPS), 127.8 (2CH, OTBDPS), 127.8 (2CH, OTBDPS), 127.7 (2CH, OTBDPS), 127.7 (2CH, OTBDPS), 108.7 (C, C-6), 108.5 (C, C-6), 103.3 (CH, C-4Z), 99.6 (CH, C-4E), 82.0 (CH, C-2Z), 81.8 (CH, C-2E), 76.7 (CH, C3E), $71.4(\mathrm{CH}, \mathrm{C}-3 Z), 63.2\left(\mathrm{CH}_{2}, \mathrm{C}-1 Z\right), 62.7(\mathrm{CH} 2, \mathrm{C}-1 E), 60.1\left(\mathrm{CH}_{3}, \mathrm{OMe} Z\right), 56.1(\mathrm{CH}$, OMe E), $27.5\left(2 \mathrm{CH}_{3}, \mathrm{C}\left(\mathrm{CH}_{3}\right)_{2}\right), 27.1\left(2 \mathrm{CH}_{3}, \mathrm{C}\left(\mathrm{CH}_{3}\right)_{2}\right), 26.9\left(3 \mathrm{CH}_{3}, \mathrm{OTBDPS}\right), 26.8\left(3 \mathrm{CH}_{3}, \mathrm{OTBDPS}\right)$, 19.4 (2C, 2x OTBDPS).

tert-Butyl(((4R,5R)-5-ethynyl-2,2-dimethyl-1,3-dioxolan-4-yl)methoxy)diphenylsilane (180)

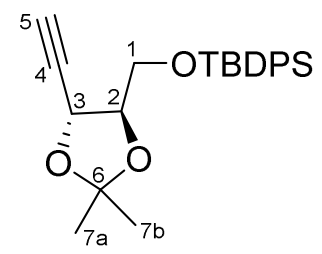

Alcohol 167 (0.342 g, $0.86 \mathrm{mmol})$ was dissolved in DCM $(3.0 \mathrm{~mL})$ at room temperature under air then treated with $\mathrm{CaCO}_{3}(0.172 \mathrm{~g}, 1.72 \mathrm{mmol}, 2$ equiv.) and DMP $(0.742 \mathrm{~g}, 1.70 \mathrm{mmol}, 2$ equiv.). The reaction was quenched after $2 \mathrm{~h}$ with $1: 1 \mathrm{v} / \mathrm{v}$ sat. aq. $\mathrm{Na}_{2} \mathrm{~S}_{2} \mathrm{O}_{3} /$ sat. aq. $\mathrm{NaHCO}_{3}$ $(10 \mathrm{~mL})$ and stirred for $30 \mathrm{~min}$. The aqueous layer was then extracted with $\mathrm{Et}_{2} \mathrm{O}(3 \times 10 \mathrm{~mL})$, and the combined organic layers washed with brine $(10 \mathrm{~mL})$, dried $\left(\mathrm{MgSO}_{4}\right)$ then concentrated. The crude reaction mixture was used in the next step without purification.

The crude mixture containing aldehyde in dry $\mathrm{MeOH}(3.5 \mathrm{~mL})$ under argon was treated with dried $\mathrm{K}_{2} \mathrm{CO}_{3}(0.244 \mathrm{~g}, 1.71 \mathrm{mmol}, 2$ equiv.) and dimethyl(1-diazo-2-oxopropyl)phosphonate (0.18 mL, $1.12 \mathrm{mmol}, 1.3$ equiv.) at room temperature. After 10 minutes the colour of reaction changed from pale yellow to red. The reaction was stirred overnight then quenched with sat. aq. $\mathrm{NH}_{4} \mathrm{Cl}(1.5 \mathrm{~mL})$. Most of methanol was removed under reduced pressure then, the reaction was diluted with EtOAc $(20 \mathrm{~mL})$ and sat. aq. $\mathrm{NH}_{4} \mathrm{Cl}(20 \mathrm{~mL})$. The aqueous layer was extracted with EtOAc $(3 \times 20 \mathrm{~mL})$, the combined organic layers dried over anhydrous $\mathrm{MgSO}_{4}$ and concentrated. The crude reaction mixture was purified using flash chromatography (10:1 PE/EA) to give the alkyne 180 as a yellow oil $(0.071 \mathrm{~g}, 34 \%$ yield). Experimental data matched those previously reported for the $S, S$ isomer with optical rotation value of the similar magnitude and opposite sign. ${ }^{234}$ 
$\mathbf{R}_{f}=0.64(10: 1 \mathrm{PE} / \mathrm{EA}) ;[\boldsymbol{\alpha}]_{\mathrm{D}}{ }^{23}=+18.4\left(c\right.$ 0.76, $\left.\mathrm{CH}_{2} \mathrm{Cl}_{2}\right)\left[{ }^{2}\right.$ it. ${ }^{234}[\boldsymbol{\alpha}]_{\mathrm{D}}=-16.1$ (c 1.27, $\left.\mathrm{CH}_{2} \mathrm{Cl}_{2}\right)$ for ent-180]; ${ }^{1} \mathrm{H}$ NMR (500 MHz, $\mathrm{CDCl}_{3}$ ) $\delta 7.72$ - 7.66 (m, 4H, OTBDPS), $7.46-7.35(\mathrm{~m}, 6 \mathrm{H}$, OTBDPS), 4.73 (dd, $J=7.2,2.1 \mathrm{~Hz}, 1 \mathrm{H}, \mathrm{H}-3$ ), 4.18 (dt, $J=7.2,4.2 \mathrm{~Hz}, 1 \mathrm{H}, \mathrm{H}-2$ ), 3.81 (d, $J=4.1$ $\mathrm{Hz}, 2 \mathrm{H}, \mathrm{H}-1$ ), 2.53 (d, J = $2.1 \mathrm{~Hz}, 1 \mathrm{H}, \mathrm{H}-5$ ), 1.51 (s, 3H, H-7a), 1.44 (s, 3H, H-7b), 1.07 (s, 9H, OTBDPS); ${ }^{13} \mathrm{C}$ NMR (126 MHz, CDCl 3 ) $\delta 135.8$ (2CH, OTBDPS), 135.7 (2CH, OTBDPS), 133.3 (C, OTBDPS), 133.2 (C, OTBDPS), 129.9 (CH, OTBDPS), 129.9 (CH, OTBDPS), 127.9 (2CH, OTBDPS), 127.9 (2CH, OTBDPS), 110.8 (C, C-6), 82.1 (CH, C-2), 81.4 (C, C-4), 74.6 (CH, C-5), $67.2(\mathrm{CH}, \mathrm{C}-3), 62.8\left(\mathrm{CH}_{2}, \mathrm{C}-1\right), 27.0\left(\mathrm{CH}_{3}, \mathrm{C}-7 \mathrm{~b}\right), 26.9$ (3CH $\mathrm{CH}_{3}$ OTBDPS), $26.4\left(\mathrm{CH}_{3}, \mathrm{C}-7 \mathrm{a}\right), 19.4$ (C, OTBDPS); IR (film from $\mathrm{CH}_{2} \mathrm{Cl}_{2}$ ) $v_{\max } 3293,3071,2934,2858,1473,1428,1144,822,702$, $504 \mathrm{~cm}^{-1}$; HRMS (ESI) Found 412.2285. Calculated for $\mathrm{C}_{24} \mathrm{H}_{34} \mathrm{NO}_{3} \mathrm{Si}^{+}\left[\mathrm{M}+\mathrm{NH}_{4}\right]^{+} 412.2302$. $\Delta=4.23 \mathrm{ppm}$.

(4R,5R)-4-Ethynyl-5-(((4-methoxybenzyl)oxy)methyl)-2,2-dimethyl-1,3-dioxolane (181)

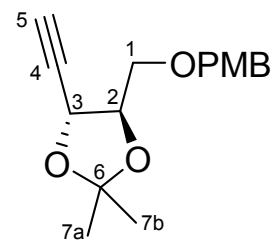

Alcohol 73 (0.346 g, $1.23 \mathrm{mmol})$ was dissolved in DCM $(5.0 \mathrm{~mL})$ at room temperature under air then treated with $\mathrm{CaCO}_{3}(0.145 \mathrm{~g}, 1.43 \mathrm{mmol}, 1.2$ equiv.) and DMP (1.00 g, $2.3 \mathrm{mmol}, 1.9$ equiv.). The reaction was quenched after 1.75 hours with $1: 1 \mathrm{v} / \mathrm{v}$ sat. aq. $\mathrm{Na}_{2} \mathrm{~S}_{2} \mathrm{O}_{3} / \mathrm{sat}$. aq. $\mathrm{NaHCO}_{3}(15 \mathrm{~mL})$ and stirred for $30 \mathrm{~min}$. The aqueous layer was then extracted with $\mathrm{Et}_{2} \mathrm{O}(3 \mathrm{x}$ $15 \mathrm{~mL})$, and combined organic layers dried $\left(\mathrm{MgSO}_{4}\right)$ then concentrated. The crude reaction mixture was used in the next step without purification.

The crude mixture containing aldehyde in dry $\mathrm{MeOH}(5 \mathrm{~mL})$ under argon was treated with dried $\mathrm{K}_{2} \mathrm{CO}_{3}$ (0.368 g, $2.7 \mathrm{mmol}, 2.2$ equiv.) and dimethyl(1-diazo-2-oxopropyl)phosphonate (0.25 mL, $1.6 \mathrm{mmol}, 1.3$ equiv.) at room temperature. After 10 minutes the colour of the reaction changed from pale yellow to red. The reaction was stirred overnight then quenched with sat. aq. $\mathrm{NH}_{4} \mathrm{Cl}(1.5 \mathrm{~mL})$. Most of methanol was removed under reduced pressure then diluted with EtOAc $(20 \mathrm{~mL})$ and sat. aq. $\mathrm{NH}_{4} \mathrm{Cl}(20 \mathrm{~mL})$. The aqueous layer was extracted with EtOAc $(3 \times 20 \mathrm{~mL})$ and the combined organic layers dried over anhydrous $\mathrm{MgSO}_{4}$ and concentrated. The crude reaction mixture was purified using flash chromatography (10:1 
PE/EA) to give the alkyne 181 as a yellow oil (0.101 g, 29\%). Experimental data was in accordance to what was previously reported. ${ }^{235}$

$\mathbf{R}_{f}=0.58(5: 1 \mathrm{PE} / \mathrm{EA}) ;[\boldsymbol{\alpha}]_{\mathrm{D}}{ }^{22}=+29.6\left(c\right.$ 0.98, $\left.\mathrm{CHCl}_{3}\right)\left[\mathrm{lit}^{235}[\boldsymbol{\alpha}]_{\mathrm{D}}{ }^{20}=+4.37\right.$ (c 1.16, $\left.\left.\mathrm{CHCl}_{3}\right)\right] ;{ }^{1} \mathbf{H}$ NMR (500 MHz, CDCl $) \delta 7.27$ (d, J=8.2 Hz, 2H, OPMB), 6.88 (d, J=8.6 Hz, 2H, OPMB), 4.54 (s, 2H, OPMB), 4.49 (dd, J = 7.4, 2.1 Hz, 1H, H-3), 4.27 (ddd, J = 7.4, 5.2, $4.0 \mathrm{~Hz}, 1 \mathrm{H}, \mathrm{H}-2$ ), 3.81 (s, 3H, OPMB), 3.62 (dd, J=10.6, 4.0 Hz, 1H, H-1a), 3.58 (dd, J=10.6, 5.2 Hz, 1H, H-1b), 2.52 $(\mathrm{d}, J=2.1 \mathrm{~Hz}, 1 \mathrm{H}, \mathrm{H}-5), 1.50(\mathrm{~d}, J=0.8 \mathrm{~Hz}, 3 \mathrm{H}, \mathrm{H}-7 \mathrm{a}), 1.43$ (d, J = 0.7 Hz, 3H, H-7b); ${ }^{13} \mathrm{C}$ NMR (126 MHz, CDCl ${ }_{3}$ ) $\delta 159.4$ (C, OPMB), 130.0 (C, OPMB), 129.5 (2CH, OPMB), 113.9 (2CH, OPMB), 111.0 (C, C-6), 81.0 ( $\mathrm{CH}, \mathrm{C}-2), 80.9$ (C, C-4), 74.8 (CH, C-5), $73.4\left(\mathrm{CH}_{2}, \mathrm{OPMB}\right), 68.9$ ( $\left.\mathrm{CH}_{2}, \mathrm{C}-1\right), 67.3(\mathrm{CH}, \mathrm{C}-3)$, $55.4\left(\mathrm{CH}_{3}, \mathrm{OPMB}\right), 27.1\left(\mathrm{CH}_{3}, \mathrm{C}-7 \mathrm{~b}\right), 26.3\left(\mathrm{CH}_{3}, \mathrm{C}-7 \mathrm{a}\right)$; IR (film from $\left.\mathrm{CHCl}_{3}\right) v_{\max } 3285,2988,2935,2905,2856,1612,1586,1382,1372,1247,1085,1035,849$, $818 \mathrm{~cm}^{-1}$.

(2R,3R)-1-((tert-Butyldiphenylsilyl)oxy)pent-4-yne-2,3-diol (182)

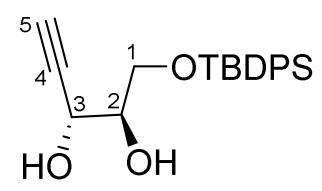

Acetonide 180 (0.052 g, $0.13 \mathrm{mmol})$ was dissolved in $70 \%$ aq. AcOH $(7 \mathrm{~mL})$, heated to $70{ }^{\circ} \mathrm{C}$ and stirred at this temperature for 2.5 hours. The reaction was then allowed to cool down to room temperature and neutralised with $\mathrm{NaHCO}_{3}$. The aqueous solution was diluted with EtOAc ( $2 \mathrm{~mL})$, the organic layer separated and the solvent removed under reduced pressure. The crude material was purified on a silica gel column (3:1 PE/EA) to give the diol $\mathbf{1 8 2}$ as a pale yellow oil $(0.027 \mathrm{~g}, 58 \%)$.

$\mathbf{R}_{f}=0.31(3: 1 \mathrm{PE} / \mathrm{EA}) ;[\boldsymbol{\alpha}]_{\mathrm{D}}{ }^{24}=+9.0\left(c\right.$ 1.00, $\left.\mathrm{CH}_{2} \mathrm{Cl}_{2}\right) ;{ }^{\mathbf{1}} \mathbf{H} \mathbf{N M R}\left(500 \mathrm{MHz}, \mathrm{CDCl}_{3}\right) \delta 7.69-7.64$ (m, 4H, OTBDPS), $7.48-7.36$ (m, 6H, OTBDPS), 4.51 (dd, J=5.6, 2.2 Hz, 1H, H-3), $3.88-3.73$ (m, 3H, H-1, H-2), 2.46 (d, J = 2.2 Hz, 1H, H-5), 1.07 (s, 9H, OTBDPS); ${ }^{13} \mathrm{C}$ NMR (126 MHz, $\mathrm{CDCl}_{3}$ ) $\delta 135.7$ (2CH, OTBDPS), 135.7 (2CH, OTBDPS), 132.7 (C, OTBDPS), 132.7 (C, OTBDPS), 130.1 (2CH, OTBDPS), 128.0 (2CH, OTBDPS), 128.0 (2CH, OTBDPS), 81.8 (C, C-4), 74.5 (CH, C2), 74.3 ( $\mathrm{CH}, \mathrm{C}-5), 64.2$ ( $\left.\mathrm{CH}_{2}, \mathrm{C}-1\right), 63.5$ ( $\left.\mathrm{CH}, \mathrm{C}-3\right), 27.0\left(6 \mathrm{CH}_{3}\right.$, OTBDPS), 19.4 (2C, OTBDPS); IR (film from $\mathrm{CH}_{2} \mathrm{Cl}_{2}$ ) $v_{\max }$ 3403, 3302, 3071, 2931, 2858, 1472, 1428, 1113, 702, $505 \mathrm{~cm}^{-1}$; HRMS (ESI) Found 377.154. Calculated for $\mathrm{C}_{21} \mathrm{H}_{26} \mathrm{NaO}_{3} \mathrm{Si}^{+}[\mathrm{M}+\mathrm{Na}]^{+}$377.1543. $\Delta=0.79 \mathrm{ppm}$. 


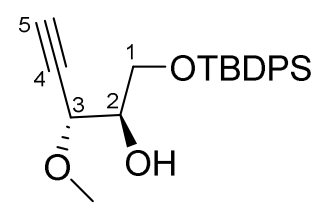

Diol 182 (0.041 g, $0.12 \mathrm{mmol})$ was dissolved in dry DCM $(2 \mathrm{~mL})$ under argon at room temparature in a flask wrapped with foil then treated with proton sponge $(0.037 \mathrm{~g}, 0.17$ mmol, 1.5 equiv.) and $\mathrm{Me}_{3} \mathrm{OBF}_{4}(0.026 \mathrm{~g}, 0.17 \mathrm{mmol}, 1.5$ equiv.). The reaction was stirred overnight then filtered through celite into with sat. aq. $\mathrm{NaHCO}_{3}(5 \mathrm{~mL})$ and the celite washed with EtOAc $(3 \times 5 \mathrm{~mL})$. The two layers were separated and the aqueous layer extracted again with EtOAc (1x $20 \mathrm{~mL}$ ). The organic layer was dried with $\mathrm{MgSO}_{4}$ and the solvent removed in vacuo. Purification of the crude material on a silica gel column (10:1 PE/EA) gave the desired product $183(8.7 \mathrm{mg}, 20 \%)$ as a minor product and the $\mathrm{O} 2$ methyl product $184(13.3 \mathrm{mg}$, $31 \%)$ as the major product of the reaction.

$\mathbf{R}_{f}=0.23(10: 1 \mathrm{PE} / \mathrm{EA}) ;{ }^{1} \mathbf{H}$ NMR $\left(500 \mathrm{MHz}, \mathrm{CDCl}_{3}\right) \delta 7.71-7.66(\mathrm{~m}, 4 \mathrm{H}$, OTBDPS), $7.46-7.35$ (m, 6H, OTBDPS), 4.18 (dd, $J=5.9,2.1 \mathrm{~Hz}, 1 \mathrm{H}, \mathrm{H}-3), 3.86-3.84(\mathrm{~m}, 2 \mathrm{H}, \mathrm{H}-1), 3.84-3.80(\mathrm{~m}$, $1 \mathrm{H}, \mathrm{H}-2), 3.47\left(\mathrm{~s}, 3 \mathrm{H}, \mathrm{OCH}_{3}\right), 2.45$ (d, $\left.J=2.1 \mathrm{~Hz}, 1 \mathrm{H}, \mathrm{H}-5\right), 1.07$ (s, 9H, OTBDPS); ${ }^{13} \mathrm{C}$ NMR (126 $\mathrm{MHz}, \mathrm{CDCl}_{3}$ ) $\delta 135.7$ (2CH, OTBDPS), 135.7 (2CH, OTBDPS), 133.3 (C, OTBDPS), 133.2 (C, OTBDPS), 129.9 (2CH, OTBDPS), 127.9 (2CH, OTBDPS), 127.9 (2CH, OTBDPS), 79.9 (C, C-4), $75.8(\mathrm{CH}, \mathrm{C}-5), 73.9(\mathrm{CH}, \mathrm{C}-2), 71.8(\mathrm{CH}, \mathrm{C}-3), 64.0\left(\mathrm{CH}_{2}, \mathrm{C}-1\right), 57.1\left(\mathrm{CH}_{3}, \mathrm{OCH}_{3}\right), 27.0\left(6 \mathrm{CH}_{3}\right.$, OTBDPS), 19.4 (2C, OTBDPS).

(3R,4R)-5-((tert-Butyldiphenylsilyl)oxy)-4-methoxypent-1-yn-3-ol (184)

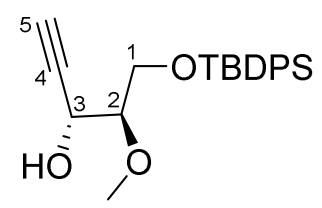

$\mathbf{R}_{f}=0.36$ (10:1 PE/EA); ${ }^{1} \mathbf{H}$ NMR (500 MHz, CDCl $)$ $\delta 7.71-7.67$ (m, 4H, OTBDPS), 7.47 - 7.36 (m, 6H, OTBDPS), 4.60 (dd, $J=4.8,2.2 \mathrm{~Hz}, 1 \mathrm{H}, \mathrm{H}-3$ ), 3.89 (dd, $J=10.8,6.0 \mathrm{~Hz}, 1 \mathrm{H}, \mathrm{H}-1 \mathrm{a}$ ), 3.79 (dd, $J=10.8,4.7 \mathrm{~Hz}, 1 \mathrm{H}, \mathrm{H}-1 \mathrm{~b}), 3.46\left(\mathrm{~s}, 3 \mathrm{H}, \mathrm{OCH}_{3}\right.$ ), 3.41 (dd, $\left.J=10.6,4.9 \mathrm{~Hz}, 1 \mathrm{H}, \mathrm{H}-2\right), 2.46$ $(\mathrm{d}, J=2.2 \mathrm{~Hz}, 1 \mathrm{H}, \mathrm{H}-5), 1.06$ (s, 9H, OTBDPS); ${ }^{13} \mathrm{C}$ NMR $\left(126 \mathrm{MHz}, \mathrm{CDCl}_{3}\right) \delta 135.8(2 \mathrm{CH}$, OTBDPS), 135.7 (2CH, OTBDPS), 133.1 (C, OTBDPS), 133.0 (C, OTBDPS), 130.0 (2CH, 
OTBDPS), 130.0 (2CH, OTBDPS), 127.9 (2CH, OTBDPS), 127.9 (2CH, OTBDPS), 83.2 (CH, C-2), $82.6(\mathrm{C}, \mathrm{C}-4), 73.9(\mathrm{CH}, \mathrm{C}-5), 73.9(\mathrm{CH}, \mathrm{C}-2), 62.4(\mathrm{CH}, \mathrm{C}-3), 62.3\left(\mathrm{CH}_{2}, \mathrm{C}-1\right), 59.2\left(\mathrm{CH}_{3}, \mathrm{OCH}_{3}\right)$, $26.9\left(6 \mathrm{CH}_{3}\right.$, OTBDPS), 19.3 (2C, OTBDPS).

\section{(R)-5-((R)-1-Methoxyprop-2-yn-1-yl)-9,9-dimethyl-8,8-diphenyl-2,4,7-trioxa-8-siladecane} (185)

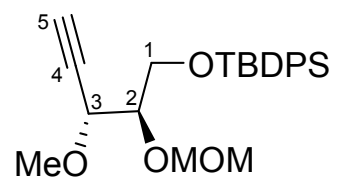

Secondary alcohol $183(7.0 \mathrm{mg}, 0.02 \mathrm{mmol})$ was dissolved in DCM $(1 \mathrm{~mL})$ and cooled to $0^{\circ} \mathrm{C}$ under an argon atmosphere, then treated with DMAP $(0.8 \mathrm{mg}, 6 \mu \mathrm{mol}, 0.3$ equiv.), DIPEA (0.01 mL, $0.06 \mathrm{mmol}, 3$ equiv.) and $\mathrm{MOMCl}(0.01 \mathrm{~mL}, 0.12 \mathrm{mmol}, 6$ equiv.). The reaction was allowed to warm to room temperature and stirred overnight then quenched with water (1 $\mathrm{mL}$ ) and extracted with DCM $(3 \times 3 \mathrm{~mL})$. The combined organic layers were dried over anhydrous $\mathrm{MgSO}_{4}$ and concentrated. The crude reaction material was purified using silica gel column (10:1 PE/EA) to give the product 185 (2.1 mg, 27\%).

$\mathbf{R}_{f}=0.41(10: 1 \mathrm{PE} / \mathrm{EA}) ;{ }^{1} \mathrm{H}$ NMR $\left(500 \mathrm{MHz}, \mathrm{CDCl}_{3}\right) \delta 7.72-7.65(\mathrm{~m}, 4 \mathrm{H}$, OTBDPS), $7.46-7.34$ (m, 6H, OTBDPS), 4.78 (dd, $\left.J=6.9,0.9 \mathrm{~Hz}, 1 \mathrm{H}, \mathrm{OCH}_{2} \mathrm{OCH}_{3}\right), 4.75$ (dd, $J=6.8,0.9 \mathrm{~Hz}, 1 \mathrm{H}$, $\left.\mathrm{OCH}_{2} \mathrm{OCH}_{3}\right), 4.31-4.28(\mathrm{~m}, 1 \mathrm{H}, \mathrm{H}-3), 3.92-3.82(\mathrm{~m}, 3 \mathrm{H}, \mathrm{H}-1 / \mathrm{H}-2), 3.44(\mathrm{~d}, J=0.9 \mathrm{~Hz}, 3 \mathrm{H}$, $\left.\mathrm{OCH}_{3}\right), 3.35\left(\mathrm{~d}, J=0.9 \mathrm{~Hz}, 3 \mathrm{H}, \mathrm{OCH}_{2} \mathrm{OCH}_{3}\right), 2.43(\mathrm{dd}, J=2.2,1.0 \mathrm{~Hz}, 1 \mathrm{H}, \mathrm{H}-5), 1.05(\mathrm{~d}, J=1.0$ $\mathrm{Hz}, 9 \mathrm{H}$, OTBDPS); ${ }^{13} \mathrm{C}$ NMR (126 MHz, $\left.\mathrm{CDCl}_{3}\right) \delta 135.8$ (2CH, OTBDPS), 135.7 (2CH, OTBDPS), 133.5 (C, OTBDPS), 133.4 (C, OTBDPS), 129.8 (2CH, OTBDPS), 127.8 (2CH, OTBDPS), 127.8 (2CH, OTBDPS), $97.1\left(\mathrm{CH}_{2}, \mathrm{OCH}_{2} \mathrm{OCH}_{3}\right), 80.5$ (C, C-4), 79.0 (CH, C-2), $75.2(\mathrm{CH}, \mathrm{C}-5), 71.1(\mathrm{CH}$, $\mathrm{C}-3)$, , $63.1\left(\mathrm{CH}_{2}, \mathrm{C}-1\right), 57.3\left(\mathrm{CH}_{3}, \mathrm{OCH}_{3}\right), 55.8\left(\mathrm{CH}_{3}, \mathrm{OCH}_{2} \mathrm{OCH}_{3}\right), 26.9\left(6 \mathrm{CH}_{3}, \mathrm{OTBDPS}\right), 19.4$ (2C, OTBDPS); IR (film from $\mathrm{CHCl}_{3}$ ) $v_{\max } 3284,3071,2930,2890,2857,1472,1428,1112$, 703, $504 \mathrm{~cm}^{-1}$; HRMS (ESI) Found 435.1970. Calculated for $\mathrm{C}_{24} \mathrm{H}_{32} \mathrm{NaO}_{4} \mathrm{Si}^{+}[\mathrm{M}+\mathrm{Na}]^{+} 435.1962$. $\Delta=1.79 \mathrm{ppm}$. 


\section{2-((4R,5R)-5-(((tert-Butyldiphenylsilyl)oxy)methyl)-2,2-dimethyl-1,3-dioxolan-4-}

yl)acetaldehyde (188)

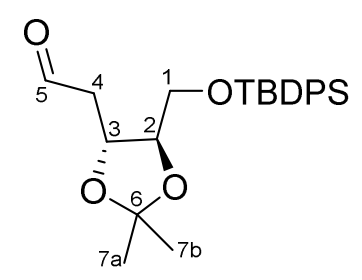

9-BBN solution ( $0.5 \mathrm{M}$ in THF, $0.11 \mathrm{~mL}, 1$ equiv.) was added dropwise to a solution of alkyne $180(0.023 \mathrm{~g}, 0.06 \mathrm{mmol})$ in dry $\operatorname{THF}(0.20 \mathrm{~mL})$ at $0{ }^{\circ} \mathrm{C}$. The reaction was allowed to warm to room temperature. After stirring overnight, the reaction was cooled to $0{ }^{\circ} \mathrm{C}$ and treated with $20 \%$ aq. $\mathrm{NaOH}(0.10 \mathrm{~mL})$ and $37 \%$ aq $\mathrm{H}_{2} \mathrm{O}_{2}(0.10 \mathrm{~mL})$ then stirred at room temperature for 5.5 hours. The aqueous solution was extracted with ether $(3 \times 2 \mathrm{~mL})$ and the solvent from the organic layer removed under reduced pressure. Purification of the crude material on a silica gel column gave trace amount of impure aldehyde 188.

$\mathbf{R}_{f}=0.57(10: 1 \mathrm{PE} / \mathrm{EA}) ;{ }^{1} \mathbf{H}$ NMR $\left(500 \mathrm{MHz}, \mathrm{CDCl}_{3}\right) \delta 9.79(\mathrm{t}, J=2.1 \mathrm{~Hz}, 1 \mathrm{H}, \mathrm{H}-5), 7.73-7.62$ ( $\mathrm{m}, 4 \mathrm{H}$, OTBDPS), $7.46-7.33$ (m, 6H, OTBDPS), 4.41 (td, $J=7.6,4.2 \mathrm{~Hz}, 1 \mathrm{H}, \mathrm{H}-3$ ), $3.87-3.72$ (m, 3H, H-1, H-2), $2.69-2.63(m, 2 \mathrm{H}, \mathrm{H}-4)$ ), 1.39 (d, J = $5.3 \mathrm{~Hz}, 6 \mathrm{H}, \mathrm{H}-7 \mathrm{a}, \mathrm{H}-7 \mathrm{~b}), 1.09-1.03$ (m, 9H, OTBDPS).

1-((4S,5R)-5-(((tert-Butyldiphenylsilyl)oxy)methyl)-2,2-dimethyl-1,3-dioxolan-4-yl)ethan-1one (191)

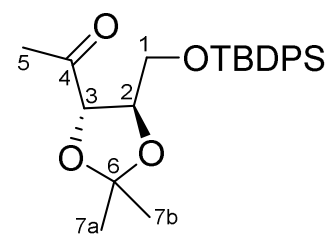

$\mathrm{BH}_{3} \cdot \mathrm{THF}$ complex solution ( $1 \mathrm{M}$ in THF, $0.02 \mathrm{~mL}, 0.35$ equiv.) was added dropwise to a solution of alkyne $180(0.022 \mathrm{~g}, 0.06 \mathrm{mmol})$ in dry THF $(0.15 \mathrm{~mL})$ at $-78{ }^{\circ} \mathrm{C}$. The reaction was stirred at this temperature for 1.5 hours then allowed to warm to $0^{\circ} \mathrm{C}$ slowly over 4.5 hours and kept at $0{ }^{\circ} \mathrm{C}$ for another hour. The reaction was then added to a cooled to $\left(0^{\circ} \mathrm{C}\right)$ solution of $20 \%$ aq. $\mathrm{NaOH}(0.10 \mathrm{~mL})$ and $37 \%$ aq $\mathrm{H}_{2} \mathrm{O}_{2}(0.10 \mathrm{~mL})$ then stirred at room temperature for 1 hour at which point TLC analysis showed only two spots (starting material and a new spot similar $R_{f}$ value to the aldehyde). The aqueous solution was diluted with EtOAc $(5 \mathrm{~mL})$, 
then the layers were separated and the solvent from the organic layer removed under reduced pressure. Crude reaction mixture purification on a silica gel column (10:1 PE/EA) gave methyl ketone 191.

$\mathbf{R}_{\boldsymbol{f}}=0.56(10: 1 \mathrm{PE} / \mathrm{EA}) ;{ }^{1} \mathrm{H}$ NMR $\left(500 \mathrm{MHz}, \mathrm{CDCl}_{3}\right) \delta 7.73-7.66(\mathrm{~m}, 4 \mathrm{H}$, OTBDPS), $7.46-7.35$ (m, 6H, OTBDPS), 4.43 (d, $J=7.4 \mathrm{~Hz}, 1 \mathrm{H}, \mathrm{H}-3), 4.15-4.10$ (m, 1H, H-2), 3.92 (dd, J = 11.4, 3.4 $\mathrm{Hz}, 1 \mathrm{H}, \mathrm{H}-1 \mathrm{a}$ ), 3.79 (dd, J = 11.4, 3.9 Hz, 1H, H-1b), 2.27 (s, 3H, H-5), 1.48 (s, 3H, H-7a), 1.43 (s, 4H, H-7b), 1.06 (s, 9H, OTBDPS); ${ }^{13}$ C NMR (126 MHz, CDCl 3 ) $\delta 208.7$ (C, C-4), 135.8 (2CH, OTBDPS), 133.3 (C, OTBDPS), 129.9 (CH, OTBDPS), 129.9 (CH, OTBDPS), 127.9 (2CH, OTBDPS), 127.8 (2CH, OTBDPS), 110.9 (C, C-6), 81.6 ( $\mathrm{CH}, \mathrm{C}-3), 78.8(\mathrm{CH}, \mathrm{C}-2), 63.6\left(\mathrm{CH}_{2}, \mathrm{C}-1\right)$, $27.1\left(\mathrm{CH}_{3}, \mathrm{C}-7 \mathrm{a}\right), 26.9\left(3 \mathrm{CH}_{3}, \mathrm{OTBDPS}\right), 26.6\left(\mathrm{CH}_{3}, \mathrm{C}-5\right), 26.5\left(\mathrm{CH}_{3}, \mathrm{C}-7 \mathrm{~b}\right), 19.4$ (C, OTBDPS).

(4R,5R)-4-(((4-Methoxybenzyl)oxy)methyl)-2,2-dimethyl-5-(oxiran-2-yl)-1,3-dioxolane (193)

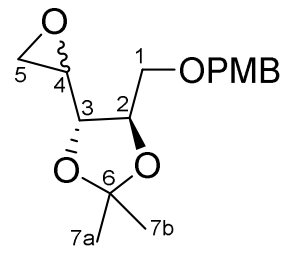

Alcohol $73(0.105 \mathrm{~g}, 0.37 \mathrm{mmol})$ was dissolved in DCM $(1.45 \mathrm{~mL})$ at room temperature under air then treated with $\mathrm{CaCO}_{3}(0.077 \mathrm{~g}, 0.77 \mathrm{mmol}, 2.1$ equiv. $)$ and DMP $(0.383 \mathrm{~g}, 0.88 \mathrm{mmol}$, 2.4 equiv.). The reaction was quenched after 2 hours with $1: 1 \mathrm{v} / \mathrm{v}$ sat. aq. $\mathrm{Na}_{2} \mathrm{~S}_{2} \mathrm{O}_{3} /$ sat. aq. $\mathrm{NaHCO}_{3}(10 \mathrm{~mL})$ and stirred for $30 \mathrm{~min}$. The aqueous layer was then extracted with DCM $(3 \mathrm{x}$ $10 \mathrm{~mL})$, and combined organic layers washed with brine $(10 \mathrm{~mL})$, dried $\left(\mathrm{MgSO}_{4}\right)$, filtered, then concentrated. The crude mixture containing aldehyde was used in the next step without purification.

$\mathrm{KOH}(0.118 \mathrm{~g}, 1.79 \mathrm{mmol}, 4.8$ equiv. $)$ was added to a solution of TMSI $(0.080 \mathrm{~g}, 0.39 \mathrm{mmol}, 1$ equiv.) in wet $\mathrm{ACN}(2 \mathrm{~mL})$. The solution was stirred at $60^{\circ} \mathrm{C}$ for 20 min then added aldehyde 73a in ACN $(0.5 \mathrm{~mL})$. The reaction was stirred at the same temperature for 3 hours then allowed to cool down to room temperature and diluted with $\mathrm{Et}_{2} \mathrm{O}$. $\mathrm{KI}$ precipitate was filtered off and the filtrate concentrated under reduced pressure. The crude reaction mixture was purified using flash chromatography (10:1 PE/EA) to give the epoxide 193 as a 4:1 mixture of isomers $(7.8 \mathrm{mg}, 7 \%)$. 
$\mathbf{R}_{\boldsymbol{f}}=0.42(5: 1 \mathrm{PE} / \mathrm{EA}) ;{ }^{1} \mathbf{H} \mathrm{NMR}\left(500 \mathrm{MHz}, \mathrm{CDCl}_{3}\right)$ (mixture of isomers) $\delta 7.28-7.24(\mathrm{~m}, 2 \mathrm{H}$, OPMB), $6.90-6.86$ (m, 2H, OPMB), $4.54-4.50$ (m, 2H, OPMB), $4.18-4.13(\mathrm{~m}, 1 \mathrm{H}, \mathrm{H}-2)$, $3.81(\mathrm{~s}, 2.4 \mathrm{H}, \mathrm{OPMB}), 3.80$ (s, 0.6H, OPMB), 3.69 (dd, $J=8.2,5.3 \mathrm{~Hz}, 0.2 \mathrm{H}, \mathrm{H}-3), 3.65$ (dd, $J=$ 7.8, $5.3 \mathrm{~Hz}, 0.8 \mathrm{H}, \mathrm{H}-3$ ), $3.63-3.53(\mathrm{~m}, 2 \mathrm{H}, \mathrm{H}-1$ ), 3.08 (ddd, J = 5.3, 3.9, $2.6 \mathrm{~Hz}, 0.8 \mathrm{H}, \mathrm{H}-4$ ), 3.03 (ddd, $J=5.3,4.2,2.6 \mathrm{~Hz}, 0.2 \mathrm{H}, \mathrm{H}-4$ ), 2.81 (dd, $J=5.0,4.0 \mathrm{~Hz}, 0.8 \mathrm{H}, \mathrm{H}-5 \mathrm{a}$ ), 2.76 (dd, $J=$ 5.2, 4.2 Hz, 0.2H, H-5a), 2.69 (dd, J = 5.0, 2.6 Hz, 0.8H, H-5b), 2.64 (dd, J = 5.2, 2.6 Hz, 0.2H, $\mathrm{H}-5 \mathrm{~b}$ ), 1.43 (d, J = 3.6 Hz, 4.8H, H-7a, H-7b), 1.41 (d, J=4.3 Hz, 1.2H, H-7a, H-7b); ${ }^{13} \mathrm{C} \mathrm{NMR}$ (126 MHz, CDCl $)_{3} \delta 159.4$ (2C, OPMB), 130.1 (C, OPMB), 129.9 (C, OPMB) 129.5 (CH, OPMB), 129.5 (CH, OPMB 114.0 (CH, OPMB), 113.9 (CH, OPMB), 110.2 (C, C-6), 110.2 (C, C-6), 79.4 ( $\mathrm{CH}, \mathrm{C}-3), 78.3(\mathrm{CH}, \mathrm{C}-2), 78.2(\mathrm{CH}, \mathrm{C}-3), 76.9(\mathrm{CH}, \mathrm{C}-2), 73.4\left(\mathrm{CH}_{2}, \mathrm{OPMB}\right), 73.4\left(\mathrm{CH}_{2}, \mathrm{OPMB}\right)$, $70.3\left(\mathrm{CH}_{2}, \mathrm{C}-1\right), 70.0\left(\mathrm{CH}_{2}, \mathrm{C}-1\right), 55.4\left(\mathrm{CH}_{3}, \mathrm{OPMB}\right), 55.4\left(\mathrm{CH}_{3}, \mathrm{OPMB}\right), 51.9(\mathrm{CH}, \mathrm{C}-4), 51.8$ $(\mathrm{CH}, \mathrm{C}-4), 45.1\left(\mathrm{CH}_{2}, \mathrm{C}-5\right), 44.2\left(\mathrm{CH}_{2}, \mathrm{C}-5\right), 27.1\left(\mathrm{CH}_{3}, \mathrm{C}-7 \mathrm{a}\right), 27.1\left(\mathrm{CH}_{3}, \mathrm{C}-7 \mathrm{a}\right), 26.8\left(\mathrm{CH}_{3}, \mathrm{C}-7 \mathrm{~b}\right)$, $26.8\left(\mathrm{CH}_{3}, \mathrm{C}-7 \mathrm{~b}\right)$.

\section{2,3-Oxirane-2,3-diyl)dimethanol (199)}

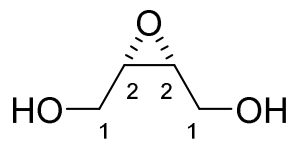

m-Chloroperbenzoic acid (75\%, $1.991 \mathrm{~g}, 8.7 \mathrm{mmol}, 1.3$ equiv.) was dissolved in of dry acetonitrile $(8.0 \mathrm{~mL})$ under an argon atmosphere at $0{ }^{\circ} \mathrm{C}$. After $10 \mathrm{~min},(Z)$-2-butene-1,4-diol $(0.54 \mathrm{~mL}, 6.6 \mathrm{mmol})$ was added at $0{ }^{\circ} \mathrm{C}$ and the reaction stirred for another hour at the same temperature during which time the solution became milky white. The reaction was allowed to warm to room temperature and stirred for another $16 \mathrm{~h}$. The solvent was then removed under reduced pressure and the residual white solid stirred in $4.0 \mathrm{~mL}$ of dry diethyl ether for $3 \mathrm{~h}$ at room temperature then kept in freezer $\left(-18^{\circ} \mathrm{C}\right)$ overnight. The solution was filtered the white solid washed with cold diethyl ether $(3 \times 5 \mathrm{~mL})$. The filtrate was concentrated and the compound further purified on silica column using 5-10\% MeOH/DCM to give 199 as an amorphous white solid $(0.384 \mathrm{~g}, 61 \%$ yield). Experimental data matched those previously reported. $^{202}$

$\mathbf{R}_{f}=0.33$ (9:1 DCM/MeOH); ${ }^{1} \mathbf{H}$ NMR (500 MHz, CD $\left.{ }_{3} \mathrm{OD}\right) \delta 3.76-3.70(\mathrm{~m}, 2 \mathrm{H}, \mathrm{H}-1 \mathrm{a}), 3.59$ (dd, $J=12.3,6.7 \mathrm{~Hz}, 2 \mathrm{H}, \mathrm{H}-1 \mathrm{~b}), 3.15-3.11(\mathrm{~m}, 2 \mathrm{H}, \mathrm{H}-2) ;{ }^{13} \mathrm{C} \mathrm{NMR}\left(125 \mathrm{MHz}, \mathrm{CD}_{3} \mathrm{OD}\right) \delta 61.2$ 
$\left(2 \mathrm{CH}_{2}, \mathrm{C}-1\right), 57.8(2 \mathrm{CH}, \mathrm{C}-2)$; HRMS (ESI) Found 105.0545. Calculated for $\mathrm{C}_{4} \mathrm{H}_{9} \mathrm{O}_{3}{ }^{+}[\mathrm{M}+\mathrm{H}]^{+}$ 105.0546. $\Delta=1.39 \mathrm{ppm}$.

(R)-2-((tert-Butyldimethylsilyl)oxy)-1-((R)-oxiran-2-yl)ethan-1-ol (200)<smiles>CC(C(O)CO[SbH3])C1CO1</smiles>

Meso epoxide 199 (0.147 g, $1.41 \mathrm{mmol})$ was dissolved in dry acetonitrile $(1.5 \mathrm{~mL})$ and treated with oligomeric (S,S)-(Salen)-Co(III)OTf catalyst $(0.0021 \mathrm{~g}, 3.5 \mathrm{~mol} \%$ in Co), The reaction was stirred under air for $14 \mathrm{~h} .{ }^{1} \mathrm{H}$ NMR spectrum of an aliquot taken at this point indicated 2:1 ratio of terminal to meso epoxide. The reaction was diluted with $3.5 \mathrm{~mL} \mathrm{~N}, \mathrm{~N}$ dimethylformamide and cooled to $-20{ }^{\circ} \mathrm{C}$ then treated with imidazole $(0.150 \mathrm{~g}, 2.1 \mathrm{mmol}$, 1.5 equiv.) and tert-butyldimethylsilyl chloride ( $0.340 \mathrm{~g}, 2.1 \mathrm{mmol}, 1.5$ equiv.). The reaction mixture was stirred at $-20^{\circ} \mathrm{C}$ for $10 \mathrm{~min}$ then left in the freezer $\left(-18^{\circ} \mathrm{C}\right)$ for $3 \mathrm{~h}$. After $3 \mathrm{~h}$, the reaction was quenched with water $(5 \mathrm{~mL})$ then diluted with another $25 \mathrm{~mL}$ water and the aqueous phase extracted with ethyl acetate $(3 \times 10 \mathrm{~mL})$. The combined organic layers were washed with brine $(20 \mathrm{~mL})$ and water $(20 \mathrm{~mL})$ then dried over anhydrous $\mathrm{MgSO}_{4}$, filtered and concentrated under reduced pressure. The crude reaction mixture was purified using silica gel chromatography (5:1 EA/PE) to afford the terminal epoxide $\mathbf{2 0 0}$ as colourless oil (0.1054 g, 34\%, over 2 steps).

$\mathbf{R}_{f}=0.46(3: 1 \mathrm{PE} / \mathrm{EA}) ;{ }^{1} \mathrm{H}$ NMR $\left(500 \mathrm{MHz}, \mathrm{CDCl}_{3}\right) \delta 3.70(\mathrm{~d}, J=5.7 \mathrm{~Hz}, 2 \mathrm{H}, \mathrm{H}-1), 3.63-3.58(\mathrm{~m}$, $1 \mathrm{H}, \mathrm{H}-2$ ), 3.09 (td, J = 4.1, 2.8 Hz, 1H, H-3), 2.78 (dd, J=5.0, 4.2 Hz, 1H, H-4a), 2.74 (dd, $J=$ 5.1, 2.8 Hz, 1H, H-4b), 2.39 (d, J = 6.3 Hz, 1H, OH), 0.89 (s, 9H, OTBS), 0.07 (s, 6H, OTBS); ${ }^{13} \mathrm{C}$ NMR $\left(125 \mathrm{MHz}, \mathrm{CDCl}_{3}\right) \delta 70.8(\mathrm{CH}, \mathrm{C}-2), 64.6\left(\mathrm{CH}_{2}, \mathrm{C}-1\right), 52.6(\mathrm{CH}, \mathrm{C}-3), 43.9\left(\mathrm{CH}_{2}, \mathrm{C}-4\right), 25.8$ (3CH $\left.\mathrm{CH}_{3}, \mathrm{OTBS}\right), 18.2$ (C, OTBS), $-5.5\left(2 \mathrm{CH}_{3}, \mathrm{OTBS}\right)$.

(R)-8,8,9,9-Tetramethyl-5-((R)-oxiran-2-yl)-2,4,7-trioxa-8-siladecane (195)<smiles>COC(CO[SnH3])C1CO1</smiles>

Epoxide $200(0.038 \mathrm{~g}, 0.17 \mathrm{mmol})$ was dissolved in dry toluene $(0.6 \mathrm{~mL})$ under an atmosphere of argon at room temperature and treated sequentially with DIPEA $(0.10 \mathrm{~mL}$, 
$0.52 \mathrm{mmol}, 3$ equiv.) and $\mathrm{MOMCl}(0.05 \mathrm{~mL}, 0.52 \mathrm{mmol}, 3$ equiv.), both added dropwise. The reaction was stirred at room temperature overnight then quenched with $1: 1 \mathrm{v} / \mathrm{v}$ water/EtOAc $(5 \mathrm{~mL})$. The layers were separated and the aqueous layer extracted two more times with EtOAc $(5 \mathrm{~mL})$. The combined organic layers were washed with brine, dried over anhydrous $\mathrm{MgSO}_{4}$ and concentrated. The crude material was purified using flash chromatography (9:1 PE/EtOAc) to give the epoxide 195 as a colourless oil (19.8 mg, 43\% yield). Experimental data matched those previously reported. ${ }^{3}$

$\mathbf{R}_{f}=0.59(5: 1 \mathrm{PE} / \mathrm{EA}) ;[\boldsymbol{\alpha}]_{\mathrm{D}}{ }^{20}=+10.4\left(c 0.68, \mathrm{CHCl}_{3}\right)\left[\mathrm{lit}^{3}{ }^{3}[\boldsymbol{\alpha}]_{\mathrm{D}}{ }^{23}=+10.4\left(c 1.3, \mathrm{CHCl}_{3}\right)\right] ;{ }^{1} \mathbf{H ~ N M R}$ $\left(500 \mathrm{MHz}, \mathrm{CDCl}_{3}\right) \delta 4.81\left(\mathrm{~d}, J=6.6 \mathrm{~Hz}, 1 \mathrm{H}, \mathrm{OCH}_{2} \mathrm{OCH}_{3}\right), 4.72\left(\mathrm{~d}, J=6.6 \mathrm{~Hz}, 1 \mathrm{H}, \mathrm{OCH}_{2} \mathrm{OCH}_{3}\right)$, 3.79-3.67 (m, $2 \mathrm{H}, \mathrm{H}-1), 3.42-3.38\left(\mathrm{~m}, 3 \mathrm{H}, \mathrm{H}-2 / \mathrm{OCH}_{2} \mathrm{OCH}_{3}\right), 3.08-3.04(\mathrm{~m}, 1 \mathrm{H}, \mathrm{H}-3), 2.80$ (dd, $J=5.1,4.2 \mathrm{~Hz}, 1 \mathrm{H}, \mathrm{H}-4), 2.66(\mathrm{dd}, J=5.0,2.7 \mathrm{~Hz}, 1 \mathrm{H}, \mathrm{H}-4), 0.89\left(\mathrm{~s}, 9 \mathrm{H}, \mathrm{OSi}\left(\mathrm{CH}_{3}\right)_{2} \mathrm{C}\left(\mathrm{CH}_{3}\right)_{3}\right), 0.07$ (s, 6H, OSi $\left.\left(\mathrm{CH}_{3}\right)_{2} \mathrm{C}\left(\mathrm{CH}_{3}\right)_{3}\right) ;{ }^{13} \mathrm{C} \mathrm{NMR}\left(125 \mathrm{MHz}, \mathrm{CDCl}_{3}\right) \delta 96.0\left(\mathrm{OCH}_{2} \mathrm{OCH}_{3}\right), 78.3$ (C-2), 63.6 (C1), $55.7\left(\mathrm{OCH}_{2} \mathrm{OCH}_{3}\right), \quad 53.0$ (C-3), 44.2 (C-4), 26.0 (3C, OSi $\left.\left(\mathrm{CH}_{3}\right)_{2} \mathrm{C}_{(}\left(\mathrm{CH}_{3}\right)_{3}\right), \quad 18.4$ (OSi $\left.\left(\mathrm{CH}_{3}\right)_{2} \mathrm{C}\left(\mathrm{CH}_{3}\right)_{3}\right),-5.3\left(\mathrm{C}, \mathrm{OSi}\left(\mathrm{CH}_{3}\right)_{2} \mathrm{C}\left(\mathrm{CH}_{3}\right)_{3}\right),-5.4$ (C, OSi $\left.\left(\mathrm{CH}_{3}\right)_{2} \mathrm{C}\left(\mathrm{CH}_{3}\right)_{3}\right)$; HRMS (ESI) Found 263.1682. Calculated for $\mathrm{C}_{12} \mathrm{H}_{27} \mathrm{O}_{4} \mathrm{Si}^{+}[\mathrm{M}+\mathrm{H}]^{+}$263.1673. $\Delta=3.28 \mathrm{ppm}$.

\section{2-((4-Methoxybenzyl)oxy)acetic acid (210)}<smiles>[13NH2]OCC(=O)O</smiles>

Bromoacetic acid ( $2.00 \mathrm{~g}, 14.5 \mathrm{mmol}, 1$ equiv.) was added to a suspension of $\mathrm{NaH}$ (1.16 g, 29 mmol, 2 equiv.) in dry THF $(25 \mathrm{~mL})$ at room temperature. After the evolution of $\mathrm{H}_{2}$ has ceased the reaction was cooled to $0^{\circ} \mathrm{C}$ and treated with $\mathrm{PMBOH}(1.80 \mathrm{~mL}, 14.5 \mathrm{mmol})$ then stirred at room temperature for another $5 \mathrm{~min}$. The reaction was then heated to reflux and stirred for $4 \mathrm{~h}$ then allowed to cool down to room temperature, then cooled to $0{ }^{\circ} \mathrm{C}$, hydrolised with EtOH $(10 \mathrm{~mL})$ and concentrated under reduced pressure. Crude material was diluted with $\mathrm{Et}_{2} \mathrm{O}(20 \mathrm{~mL})$ and extracted with sat. aq. $\mathrm{NaHCO}_{3}$ solution $(40 \mathrm{~mL})$. The aqueous layer was then acidified to $\mathrm{pH} 1$ by slow addition of $10 \%$ aq. $\mathrm{HCl}$. The aqueous layer was then extracted with EtOAc $(2 \times 50 \mathrm{~mL})$, dried and concentrated to obtain the product 210 as an off white solid (2.5811g, 91\%). The product was used without further purification. Experimental data matched those previously reported. ${ }^{210}$ 
${ }^{1}$ H NMR $\left(500 \mathrm{MHz}, \mathrm{CDCl}_{3}\right): \delta 7.29(\mathrm{~d}, J=8.6 \mathrm{~Hz}, 2 \mathrm{H}, 2 \mathrm{H}, \mathrm{OPMB}), 6.89$ (d, J=8.6 Hz, $2 \mathrm{H}$, OPMB), 4.58 (s, 2H, OPMB), 4.11 (s, 2H, H-2), 3.81 (s, 3H, OPMB); ${ }^{13} \mathrm{C}$ NMR (126 MHz, $\mathrm{CDCl}_{3}$ ): $\delta 175.1$ (C, C-1), 159.8 (C, OPMB), 130.0 (2CH, OPMB), 128.7 (C, OPMB), 114.1 (2CH, OPMB), $73.2\left(\mathrm{CH}_{2}, \mathrm{OPMB}\right), 66.3\left(\mathrm{CH}_{2}, \mathrm{C}-2\right), 55.4\left(\mathrm{CH}_{3}, \mathrm{OPMB}\right)$.

(S)-4-Benzyl-3-(2-((4-methoxybenzyl)oxy)acetyl)oxazolidin-2-one (28)<smiles>CCCOCC(=O)N1C(=O)OC[C@H]1Cc1ccccc1</smiles>

Carboxylic acid 210 (1.066 g, $5.4 \mathrm{mmol})$ was dissolved in dry ACN $(20 \mathrm{~mL})$ then treated with $\mathrm{Et}_{3} \mathrm{~N}(0.40 \mathrm{~mL}, 9.9 \mathrm{mmol}, 1.8$ equiv.) and HBTU (3.298 g, $8.5 \mathrm{mmol}, 1.6$ equiv.) at room temperature and stirred for 30 minutes. In a separate flask, $(S)$-4-benzyl-2-oxazolidinone (1.381 g, $7.7 \mathrm{mmol}, 1.4$ equiv.) was dissolved in dry THF $(20 \mathrm{~mL})$ and cooled to $-78{ }^{\circ} \mathrm{C}$ then treated with $n$-BuLi (4.25 mL, $8.5 \mathrm{mmol}, 1.6$ equiv.) and stirred for $15 \mathrm{~min}$ at the same temperature then, the acid mixture was added to it via cannula. The reaction mixture was allowed to warm to room temperature and stirred for 2.5 hours. The reaction was quenched with brine $(20 \mathrm{~mL})$ and extracted with $\mathrm{DCM}(2 \times 30 \mathrm{~mL})$. The combined organic layers were washed sequentially with $10 \%$ aq. $\mathrm{HCl}$ solution, sat. aq. $\mathrm{NaHCO}_{3}$ solution and water, dried over $\mathrm{MgSO}_{4}$ and the solvent removed under reduced pressure. The crude reaction material was purified using gradient chromatography $(5: 1,3: 1$ then $2: 1 \mathrm{PE} / \mathrm{EA})$ to give the oxazolidinone 28 as a colourless oil (1.169 g, 61\%). Experimental data matched those previously reported. ${ }^{212,236}$

$\mathbf{R}_{f}=0.19(3: 1 \mathrm{PE} / \mathrm{EA}) ;[\boldsymbol{\alpha}]_{\mathrm{D}}{ }^{24}=+175.2\left(c\right.$ 2.12, $\left.\mathrm{CH}_{2} \mathrm{Cl}_{2}\right)\left[\right.$ lit. ${ }^{236}[\boldsymbol{\alpha}]_{\mathrm{D}}=-69.2$ (c 1.32, $\left.\mathrm{CH}_{2} \mathrm{Cl}_{2}\right)$ for ent-28); ${ }^{1} \mathrm{H}$ NMR $\left(500 \mathrm{MHz}, \mathrm{CDCl}_{3}\right): \delta 7.38-7.27\left(\mathrm{~m}, 5 \mathrm{H}, \mathrm{CH}_{2} P h\right), 7.20(\mathrm{~d}, J=8.1 \mathrm{~Hz}, 2 \mathrm{H}$, OPMB), 6.90 (d, J = 8.7 Hz, 2H, OPMB), $4.72-4.66(\mathrm{~m}, 3 \mathrm{H}, \mathrm{H}-2, \mathrm{H}-5), 4.63(\mathrm{~d}, J=2.7 \mathrm{~Hz}, 2 \mathrm{H}$, OPMB), 4.27 (dd, J = 9.2, 7.8 Hz, 1H, H-4a), 4.22 (dd, J = 9.2, 3.0 Hz, 1H, H-4b), 3.81 (s, 3H, OPMB), 3.33 (dd, $J=13.4,3.3 \mathrm{~Hz}, 1 \mathrm{H}, \mathrm{CH}_{2} \mathrm{Ph}$ ), 2.81 (dd, $J=13.5,9.5 \mathrm{~Hz}, 1 \mathrm{H}, \mathrm{CH}_{2} \mathrm{Ph}$ ); ${ }^{13} \mathrm{C} \mathrm{NMR}$ (126 MHz, CDCl $): \delta 170.4$ (C, C-10), 159.6 (C, OPMB), 153.5 (C, C-3), 135.1 (C, $\left.\mathrm{CH}_{2} P h\right), 129.9$ (2CH, $\left.\mathrm{CH}_{2} P h\right), 129.5$ (2CH, OPMB), 129.4 (C, OPMB), $129.2\left(2 \mathrm{CH}, \mathrm{CH}_{2} P h\right), 127.6\left(\mathrm{CH}, \mathrm{CH}_{2} P h\right)$, 114.0 (2CH, OPMB), $73.3\left(\mathrm{CH}_{2}, \mathrm{OPMB}\right), 69.5\left(\mathrm{CH}_{2}, \mathrm{C}-2\right), 67.4\left(\mathrm{CH}_{2}, \mathrm{C}-4\right), 55.4\left(\mathrm{CH}_{3}, \mathrm{OPMB}\right)$, $54.9(\mathrm{CH}, \mathrm{C}-5), 37.9\left(\mathrm{CH}_{2}, \mathrm{CH}_{2} \mathrm{Ph}\right)$; HRMS (ESI) Found 373.1766. Calculated for $\mathrm{C}_{20} \mathrm{H}_{25} \mathrm{~N}_{2} \mathrm{O}_{5}{ }^{+}$ 
$\left[\mathrm{M}+\mathrm{NH}_{4}{ }^{+}\right]^{+}$373.1758. $\Delta=2.24$ ppm; IR (film from $\mathrm{CH}_{2} \mathrm{Cl}_{2}$ ) $\mathrm{v}_{\max } 2923,2860,1718,1715,1513$, $1300,1248,1074,1036,821 \mathrm{~cm}^{-1}$.

(S)-2-((1,3-Dithian-2-yl)methyl)oxirane (203)

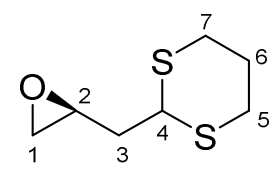

A solution of 1,3-dithiane (1.999 g, $16.1 \mathrm{mmol}, 1.2$ equiv.) in dry THF (9 mL) was cooled to $10{ }^{\circ} \mathrm{C}$ then treated with $n$-BuLi (2M in cyclohexane, $7.20 \mathrm{~mL}, 14.0 \mathrm{mmol}, 1.1$ equiv.). The solution was stirred at $-10{ }^{\circ} \mathrm{C}$ for 2 hours then cooled to $-78{ }^{\circ} \mathrm{C}$. The solution of S-glycidyl tosylate $(3.023 \mathrm{~g}, 13.2 \mathrm{mmol})$ in THF $(3 \mathrm{~mL})$ was then added dropwise via cannula. The reaction was kept at $-78{ }^{\circ} \mathrm{C}$ for 2 hours then allowed to warm to $-50{ }^{\circ} \mathrm{C}$ and kept at this temperature for 1.5 hours. After that time the reaction was allowed to gradually warm up to room temperature over 2 hours and quenched with sat. aq. $\mathrm{NaHCO}_{3}(10 \mathrm{~mL})$. The aqueous layer was extracted with $\mathrm{Et}_{2} \mathrm{O}(3 \times 10 \mathrm{~mL})$, dried over anhydrous $\mathrm{MgSO}_{4}$ and concentrated. Purification on a silica gel column (9:1 PE/EA) gave the epoxide 203 as a colourless oil (1.196 g, 50\%). Experimental data matched those previously reported. ${ }^{237}$

$\left.\mathbf{R}_{f}=0.62(3: 1 \mathrm{PE} / \mathrm{EA}) ;[\boldsymbol{\alpha}]_{\mathrm{D}}{ }^{27}=-9.9(c) 1.21, \mathrm{CHCl}_{3}\right)\left[\mathrm{lit}^{237}[\boldsymbol{\alpha}]_{\mathrm{D}}{ }^{24}=+4.6\right.$ (c 1.5, $\left.\mathrm{CHCl}_{3}\right)$ for ent 203]; ${ }^{1} \mathrm{H}-\mathrm{NMR}\left(500 \mathrm{MHz}, \mathrm{CDCl}_{3}\right): \delta 4.26(\mathrm{t}, J=7.0 \mathrm{~Hz}, 1 \mathrm{H}, \mathrm{H}-4), 3.19-3.13(\mathrm{~m}, 1 \mathrm{H}, \mathrm{H}-2), 2.97$ - 2.80 (m, 5H, H-1b/H-5/H-7), 2.55 (dd, J = 5.0, $2.6 \mathrm{~Hz}, 1 \mathrm{H}, \mathrm{H}-1 \mathrm{a}), 2.18-2.09$ (m, 1H, H-6a), 1.97 (dd, $J=6.9,5.8 \mathrm{~Hz}, 2 \mathrm{H}, \mathrm{H}-3$ ), 1.89 (dtt, $J=14.7,11.5,3.3 \mathrm{~Hz}, 1 \mathrm{H}, \mathrm{H}-6 \mathrm{~b}) . ;{ }^{13} \mathrm{C}-\mathrm{NMR}(126$ $\left.\mathrm{MHz}, \mathrm{CDCl}_{3}\right): \delta 49.8(\mathrm{CH}, \mathrm{C}-2), 47.6\left(\mathrm{CH}_{2}, \mathrm{C}-1\right), 44.9(\mathrm{CH}, \mathrm{C}-4), 38.8\left(\mathrm{CH}_{2}, \mathrm{C}-3\right), 30.6\left(\mathrm{CH}_{2}, \mathrm{C}-5\right)$, $30.4\left(\mathrm{CH}_{2}, \mathrm{C}-7\right), 25.8\left(\mathrm{CH}_{2}, \mathrm{C}-6\right)$; HRMS (ESI) Found 177.04038. Calculated for $\mathrm{C}_{7} \mathrm{H}_{13} \mathrm{OS}_{2}{ }^{+}$ $[\mathrm{M}+\mathrm{H}]^{+}$177.04023. $\Delta=-0.84 \mathrm{ppm}$; IR (film from $\mathrm{CHCl}_{3}$ ) $\mathrm{v}_{\max } 3048,2992,2931,2826,1686$, $1422,1277,1185,911 \mathrm{~cm}^{-1}$.

(R)-1-(1,3-Dithian-2-yl)pent-4-en-2-ol (204)

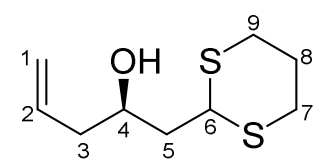

Vinylmagnesium bromide ( $1 \mathrm{M}$ in THF, $10 \mathrm{~mL}, 10 \mathrm{mmol}, 1.5$ equiv.) was added to a solution of Cul $(0.265 \mathrm{~g}, 1.4 \mathrm{mmol}, 0.21$ equiv. $)$ in dry THF $(30 \mathrm{~mL})$ at $-50{ }^{\circ} \mathrm{C}$ under argon then stirred 
at the same temperature for $30 \mathrm{~min}$. Then, the solution of epoxide 203 (1.170 g, $6.6 \mathrm{mmol})$ in THF ( $5 \mathrm{~mL}$ ) added via cannula dropwise. The reaction was stirred at $-40{ }^{\circ} \mathrm{C}$ for 40 minutes then allowed to warm to $-10{ }^{\circ} \mathrm{C}$ over $30 \mathrm{~min}$. The reaction was quenched at $-10{ }^{\circ} \mathrm{C}$ with sat. aq. $\mathrm{NH}_{4} \mathrm{Cl}(30 \mathrm{~mL})$ and the THF removed under reduced pressure. The aqueous layer was extracted with ether $\left(4 \times 30 \mathrm{~mL}\right.$ ) and the combined organic layers dried with $\mathrm{MgSO}_{4}$ then concentrated. The crude reaction mixture was purified using flash chromatography (9:1 PE/EA) to give secondary alcohol 204 as a yellow oil (0.982 g, 72\%). Experimental data matched those reported. ${ }^{105}$

$\mathbf{R}_{f}=0.47(3: 1 \mathrm{PE} / \mathrm{EA}) ;[\boldsymbol{\alpha}]_{\mathrm{D}}{ }^{24}=-27.1$ (c 1.00, $\mathrm{CHCl}_{3}$ ) $\left[\right.$ lit. ${ }^{105}[\boldsymbol{\alpha}]_{\mathrm{D}}{ }^{26}=+24.2$ (c 1.0, $\left.\mathrm{CHCl}_{3}\right)$ for ent 204]; ${ }^{1} \mathrm{H}-\mathrm{NMR}\left(500 \mathrm{MHz}, \mathrm{CDCl}_{3}\right): \delta 5.87-5.76(\mathrm{~m}, 1 \mathrm{H}, \mathrm{H}-2), 5.19-5.11(\mathrm{~m}, 2 \mathrm{H}, \mathrm{H}-1), 4.27$ (dd, J = 7.9, $6.5 \mathrm{~Hz}, 1 \mathrm{H}, \mathrm{H}-6), 4.03-3.96$ (m, 1H, H-4), $2.97-2.80$ (m, 4H, H-7, H-9), $2.34-$ 2.25 (m, 1H, H-3a), $2.25-2.18$ (m, 1H, H-3b), $2.16-2.09$ (m, 1H, H-8a), 1.97 (d, J = $4.1 \mathrm{~Hz}$, 1H, OH), $1.94-1.83$ (m, 3H, H-5, H-8b); ${ }^{13}$ C-NMR (126 MHz, CDCl 3 ) $\delta 134.2(\mathrm{CH}, \mathrm{C}-2), 118.7$ $\left(\mathrm{CH}_{2}, \mathrm{C}-1\right), 67.7(\mathrm{CH}, \mathrm{C}-4), 44.4(\mathrm{CH}, \mathrm{C}-6), 42.2\left(\mathrm{CH}_{2}, \mathrm{C}-5\right), 42.1\left(\mathrm{CH}_{2}, \mathrm{C}-3\right), 30.5\left(\mathrm{CH}_{2}, \mathrm{C}-7\right), 30.2$ $\left(\mathrm{CH}_{2}, \mathrm{C}-9\right), 26.1\left(\mathrm{CH}_{2}, \mathrm{C}-8\right)$; HRMS (ESI) Found 205.0708. Calculated for $\mathrm{C}_{9} \mathrm{H}_{17} \mathrm{OS}_{2}{ }^{+}[\mathrm{M}+\mathrm{H}]^{+}$ 205.0715. $\Delta=3.45$ ppm; IR (film from $\mathrm{CHCl}_{3}$ ) $v_{\max } 3409,2931,2900,1639,1422,1275,1243$, $1063,1030 \mathrm{~cm}^{-1}$.

\section{(R)-((1-(1,3-Dithian-2-yl)pent-4-en-2-yl)oxy)triethylsilane (205)}

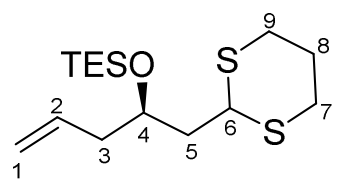

Secondary alcohol 204 (0.330 g, $1.6 \mathrm{mmol})$ was dissolved in dry DCM (10 mL) and cooled to $0^{\circ} \mathrm{C}$ then treated with TESCl $(0.41 \mathrm{~mL}, 2.4 \mathrm{mmol}, 1.5$ equiv.), DMAP $(0.022 \mathrm{~g}, 0.16 \mathrm{~mol}, 0.1$ equiv.) and $\mathrm{Et}_{3} \mathrm{~N}$ (0.45 mL, $3.2 \mathrm{mmol}, 2$ equiv). The reaction was allowed to warm to room temperature and stirred overnight. After overnight stirring, TLC analysis (5:1 PE/EA) indicated that all of the alcohol starting material has been consumed. The reaction was cooled to $0{ }^{\circ} \mathrm{C}$ and quenched by addition of sat. aq. $\mathrm{NH}_{4} \mathrm{Cl}$ solution $(10 \mathrm{~mL})$. The aqueous layer was extracted with DCM (2x $10 \mathrm{~mL})$, dried over $\mathrm{MgSO}_{4}$ and concentrated. The crude material was purified using flash chromatography (20:1 PE/EA). The product 205 was 
isolated inseparable from a small amount of 1,1,1,3,3,3-hexaethyldisiloxane ( $71 \%$ of 0.5434 g, 0.386 g, 76\%). Experimental data matched those previously reported. ${ }^{4}$

${ }^{1} \mathrm{H}-\mathrm{NMR}\left(500 \mathrm{MHz}, \mathrm{CDCl}_{3}\right): \delta 5.85-5.74(\mathrm{~m}, 1 \mathrm{H}, \mathrm{H}-2), 5.10-5.04(\mathrm{~m}, 2 \mathrm{H}, \mathrm{H}-1), 4.12$ (dd, J = 9.0, $5.6 \mathrm{~Hz}, 1 \mathrm{H}, \mathrm{H}-6), 4.07-3.98(\mathrm{~m}, 1 \mathrm{H}, \mathrm{H}-4), 2.94-2.75(\mathrm{~m}, 4 \mathrm{H}, \mathrm{H}-7, \mathrm{H}-9), 2.29-2.22(\mathrm{~m}$, $2 \mathrm{H}, \mathrm{H}-3), 2.16-2.06(\mathrm{~m}, 1 \mathrm{H}, \mathrm{H}-8 \mathrm{a}), 1.93-1.77$ (m, 3H, H-5, H-8b), 0.98 (t, J = $7.9 \mathrm{~Hz}, 9 \mathrm{H}$, OTES), 0.64 (q, $J=7.9 \mathrm{~Hz}, 6 \mathrm{H}$, OTES); ${ }^{13} \mathrm{C}-\mathrm{NMR}\left(126 \mathrm{MHz}^{\mathrm{C}} \mathrm{CDCl}_{3}\right): \delta 134.4$ (CH, C-2), 117.7 $\left(\mathrm{CH}_{2}, \mathrm{C}-1\right), 68.3(\mathrm{CH}, \mathrm{C}-4), 44.1(\mathrm{CH}, \mathrm{C}-6), 42.5\left(2 \mathrm{CH}_{2}, \mathrm{C}-3, \mathrm{C}-5\right), 30.7\left(\mathrm{CH}_{2}, \mathrm{C}-7\right), 30.2\left(\mathrm{CH}_{2}, \mathrm{C}-\right.$ 9), $26.2\left(\mathrm{CH}_{2}, \mathrm{C}-8\right), 7.1\left(3 \mathrm{CH}_{3}, \mathrm{OTES}\right), 5.2\left(3 \mathrm{CH}_{2}, \mathrm{OTES}\right)$.

(R)-2-(2-((4-Methoxybenzyl)oxy)pent-4-en-1-yl)-1,3-dithiane (212)

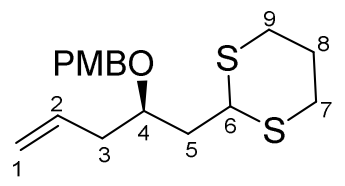

Alcohol 204 (0.867 g, $4.2 \mathrm{mmol})$ in dry DMF $(8 \mathrm{~mL})$ was added dropwise via cannula to a solution of $\mathrm{NaH}\left(0.260 \mathrm{~g}, 6.5 \mathrm{mmol}, 1.5\right.$ equiv.) in $\mathrm{DMF}(32 \mathrm{~mL})$ at $0{ }^{\circ} \mathrm{C}$. The reaction was stirred at the same temperature for an hour then treated with TBAI $(0.167 \mathrm{~g}, 0.44 \mathrm{mmol}, 0.1$ equiv.) and $\mathrm{PMBCl}(0.93 \mathrm{~mL}, 6.5 \mathrm{mmol}, 1.5$ equiv.). The reaction was allowed to warm to room temperature and stirred overnight then quenched with water $(10 \mathrm{~mL})$ and diluted with another $100 \mathrm{~mL}$ of water. The aqueous layer was extracted with extracted with $\mathrm{Et}_{2} \mathrm{O}(3 \times 50$ $\mathrm{mL}$ ) and the combined organic layers washed with brine $(100 \mathrm{~mL})$, dried $\left(\mathrm{MgSO}_{4}\right)$ and concentrated. The crude reaction material was purified on a silica gel column (10:1 PE/EA) to give the product 212 as a colourless oil (1.056 g, 77\%). Experimental data matched those previously reported. ${ }^{105}$

$\mathbf{R}_{f}=0.38(10: 1 \mathrm{PE} / \mathrm{EA}) ;[\alpha]_{\mathrm{D}}{ }^{25}=-38.7$ (c 1.24, $\left.\mathrm{CHCl}_{3}\right)\left[\mathrm{lit}^{105}{ }^{10}[\alpha]_{\mathrm{D}}{ }^{25}=+35.9\right.$ (c 1.0, $\left.\mathrm{CHCl}_{3}\right)$ for ent 212]; ${ }^{1} \mathrm{H}$ NMR (500 MHz, $\mathrm{CDCl}_{3}$ ): $\delta 7.29$ (d, $\left.J=8.7 \mathrm{~Hz}, 2 \mathrm{H}, \mathrm{OPMB}\right), 6.88(\mathrm{~d}, J=8.7 \mathrm{~Hz}, 2 \mathrm{H}$, OPMB), $5.86-5.76(m, 1 \mathrm{H}, \mathrm{H}-2), 5.16-5.06(\mathrm{~m}, 2 \mathrm{H}, \mathrm{H}-1), 4.56$ (d, J= $10.9 \mathrm{~Hz}, 1 \mathrm{H}, \mathrm{OPMB})$, $4.44(\mathrm{~d}, J=11.0 \mathrm{~Hz}, 1 \mathrm{H}, \mathrm{OPMB}), 4.16$ (dd, $J=9.7,4.8 \mathrm{~Hz}, 1 \mathrm{H}, \mathrm{H}-6), 3.81$ (s, 3H, OPMB), 3.80 $3.74(\mathrm{~m}, 1 \mathrm{H}, \mathrm{H}-4), 2.91-2.74(\mathrm{~m}, 4 \mathrm{H}, \mathrm{H}-7, \mathrm{H}-9), 2.41-2.27(\mathrm{~m}, 2 \mathrm{H}, \mathrm{H}-3), 2.14-2.06(\mathrm{~m}, 1 \mathrm{H}$, $\mathrm{H}-8 \mathrm{a}), 2.00-1.91$ (m, 1H, H-5a), $1.91-1.81$ (m, 2H, H-5b, H-8b); ${ }^{13} \mathrm{C}$ NMR (126 MHz, CDCl $\left.{ }_{3}\right)$ ठ 159.3 (C, OPMB), 134.3 (CH, C-2), 130.9 (C, OPMB), 129.6 (2CH, OPMB), $117.8\left(\mathrm{CH}_{2}, \mathrm{C}-1\right)$, 113.9 (2CH, OPMB), 74.9 ( $\mathrm{CH}, \mathrm{C}-4), 71.4\left(\mathrm{CH}_{2}, \mathrm{OPMB}\right), 55.5\left(\mathrm{CH}_{3}, \mathrm{OPMB}\right), 44.1(\mathrm{CH}, \mathrm{C}-6), 40.2$ 
$\left(\mathrm{CH}_{2}, \mathrm{C}-5\right), 38.7\left(\mathrm{CH}_{2}, \mathrm{C}-3\right), 30.5\left(\mathrm{CH}_{2}, \mathrm{C}-7\right), 30.1\left(\mathrm{CH}_{2}, \mathrm{C}-9\right), 26.2\left(\mathrm{CH}_{2}, \mathrm{C}-8\right) ;$ HRMS (ESI) Found 325.1302. Calculated for $\mathrm{C}_{17} \mathrm{H}_{25} \mathrm{O}_{2} \mathrm{~S}_{2}{ }^{+}[\mathrm{M}+\mathrm{H}]^{+}$325.1291. $\Delta=3.56 \mathrm{ppm}$; IR (film from $\mathrm{CHCl}_{3}$ ) $\mathrm{V}_{\max }$ 2933, 2900, 2834, 1612, 1513, 1246, 1074, 1034, 910, $820 \mathrm{~cm}^{-1}$.

\section{(R)-3-((4-Methoxybenzyl)oxy)hex-5-enal (213)}

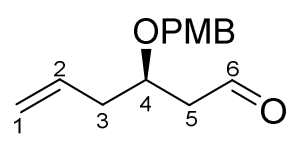

Dithiane $212(0.899 \mathrm{~g}, 2.8 \mathrm{mmol})$ was dissolved in $10 \%$ aq. ACN (40 mL) at room temperature then treated with $\mathrm{CaCO}_{3}(2.97 \mathrm{~g}, 29.4 \mathrm{mmol}, 11$ equiv.) and $\mathrm{Mel}(1.80 \mathrm{~mL}, 28.6$ mmol, 10 equiv.). The reaction was stirred at room temperature overnight then quenched with sat. aq. $\mathrm{NaHCO}_{3}$ solution $(30 \mathrm{~mL})$ and extracted with diethyl ether $(3 \mathrm{x} 30 \mathrm{~mL})$. The organic layers were combined, dried $\left(\mathrm{MgSO}_{4}\right)$ and concentrated. The crude material was purified on silica gel column (10:1 PE/EA) to give the aldehyde $\mathbf{2 1 3}$ as colourless oil (0.394 g, $61 \%)$. Experimental data matched those previously reported. ${ }^{238}$

$\left.\mathbf{R}_{f}=0.22(10: 1 \mathrm{PE} / \mathrm{EA}) ;[\boldsymbol{\alpha}]_{\mathrm{D}}{ }^{25}=-37.9(c) 1.24, \mathrm{CHCl}_{3}\right)\left[{ }^{25 i t}{ }^{105}[\boldsymbol{\alpha}]_{\mathrm{D}}{ }^{26}=+39.6\right.$ (c 1.0, $\left.\mathrm{CHCl}_{3}\right)$ for ent 213]; ${ }^{1} \mathrm{H}$ NMR (500 MHz, CDCl 3 ): $\delta 9.76(\mathrm{t}, J=2.1 \mathrm{~Hz}, 1 \mathrm{H}, \mathrm{H}-6), 7.24$ (d, $\left.J=8.6 \mathrm{~Hz}, 2 \mathrm{H}, \mathrm{OPMB}\right)$, $6.87(\mathrm{~d}, J=8.6 \mathrm{~Hz}, 2 \mathrm{H}, \mathrm{OPMB}), 5.86-5.75(\mathrm{~m}, 1 \mathrm{H}, \mathrm{H}-2), 5.16-5.08(\mathrm{~m}, 2 \mathrm{H}, \mathrm{H}-1), 4.55$ (d, J = $11.1 \mathrm{~Hz}, 1 \mathrm{H}, \mathrm{OPMB}), 4.45$ (d, J = $11.0 \mathrm{~Hz}, 1 \mathrm{H}, \mathrm{OPMB}), 4.05-3.98(\mathrm{~m}, 1 \mathrm{H}, \mathrm{H}-4), 3.80$ (s, 3H, OPMB), 2.66 (ddd, $J=16.6,7.8,2.4 \mathrm{~Hz}, 1 \mathrm{H}, \mathrm{H}-5 \mathrm{a}$ ), 2.55 (ddd, $J=16.6,4.5,1.7 \mathrm{~Hz}, 1 \mathrm{H}, \mathrm{H}-5 \mathrm{~b}$ ), $2.47-2.40(\mathrm{~m}, 1 \mathrm{H}, \mathrm{H}-3 \mathrm{a}), 2.40-2.32(\mathrm{~m}, 1 \mathrm{H}, \mathrm{H}-3 \mathrm{~b}) ;{ }^{13} \mathrm{C}$ NMR $\left(126 \mathrm{MHz}^{\mathrm{C} C D C l}\right): \delta 201.7$ (C, C-6), 159.4 (C, OPMB), 133.7 (CH, C-2), 130.3 (C, OPMB), 129.6 (2CH, OPMB), $118.4\left(\mathrm{CH}_{2}, \mathrm{C}-\right.$ 1), 114.0 (2CH, OPMB), 73.4 ( $\mathrm{CH}, \mathrm{C}-4), 71.1\left(\mathrm{CH}_{2}, \mathrm{OPMB}\right), 55.4\left(\mathrm{CH}_{3}, \mathrm{OPMB}\right), 48.1\left(\mathrm{CH}_{2}, \mathrm{C}-5\right)$, $38.4\left(\mathrm{CH}_{2}, \mathrm{C}-3\right)$; HRMS (ESI) Found 252.1592. Calculated for $\mathrm{C}_{14} \mathrm{H}_{22} \mathrm{NO}_{3}{ }^{+}[\mathrm{M}+\mathrm{H}]^{+} 252.1594$. $\Delta=0.75$ ppm; IR (film from $\mathrm{CHCl}_{3}$ ) $v_{\max } 2915,2837,1723,1613,1514,1248,1081,1034,919$, $822 \mathrm{~cm}^{-1}$. 
(S)-4-Benzyl-3-(2-(methoxymethoxy)acetyl)oxazolidin-2-one (214)<smiles>COCC(=O)N1C(=O)OC[C@H]1Br</smiles>

Oxazolidinone $28(0.506 \mathrm{~g}, 1.4 \mathrm{mmol})$ was dissolved in $5 \%$ aq. DCM $(15 \mathrm{~mL})$, cooled to $0{ }^{\circ} \mathrm{C}$ and treated with DDQ (1.00 g, $4.3 \mathrm{mmol}, 3$ equiv.). The reaction was allowed to warm to room temperature over $30 \mathrm{~min}$ and then stirred for $4 \mathrm{~h}$. Then, the reaction was quenched with sat. aq. $\mathrm{NaHCO}_{3}$ solution $(150 \mathrm{~mL})$ and extracted with $\mathrm{DCM}(3 \times 30 \mathrm{~mL})$. The combined organic layers were dried with anhydrous $\mathrm{MgSO}_{4}$ and concentrated. The crude reaction mixture was used in the next step without purification.

The crude reaction mixture from the previous step was dissolved in dry DCM $(7 \mathrm{~mL})$, cooled to $0{ }^{\circ} \mathrm{C}$ and treated with TBAI $(0.018 \mathrm{~g}, 0.05 \mathrm{mmol}, 0.03$ equiv.), DIPEA ( $1.00 \mathrm{~mL}, 5.7 \mathrm{mmol}, 4$ equiv.) and $\mathrm{MOMCl}(0.30,4.0 \mathrm{mmol}, 3$ equiv.). The reaction was allowed to warm to room temperature overnight then quenched with sat. aq. $\mathrm{NaHCO}_{3}$ solution $(10 \mathrm{~mL})$ and extracted with ethyl acetate $(2 \times 20 \mathrm{~mL})$. The combined organic layers were dried with anhydrous $\mathrm{MgSO}_{4}$ and concentrated under reduced pressure. The crude material was purified on a silica gel column using gradient chromatography (3:1 then 1:1 PE/EA, then $100 \% \mathrm{EA})$ to give the oxazolidinone 214 as yellow oil (0.273 g, 69\%). Experimental data were in accordance with those previously reported. ${ }^{239}$

${ }^{1} \mathbf{H}$ NMR $\left(500 \mathrm{MHz}, \mathrm{CDCl}_{3}\right): \delta 7.37-7.27\left(\mathrm{~m}, 3 \mathrm{H}, \mathrm{CH}_{2} P h\right), 7.24-7.19\left(\mathrm{~m}, 2 \mathrm{H}, \mathrm{CH}_{2} P h\right), 4.80-$ $4.76\left(\mathrm{~m}, 4 \mathrm{H}, \mathrm{H}-2 / \mathrm{OCH}_{2} \mathrm{OCH}_{3}\right), 4.74-4.65(\mathrm{~m}, 1 \mathrm{H}, \mathrm{H}-5), 4.34-4.20(\mathrm{~m}, 2 \mathrm{H}, \mathrm{H}-4), 3.44(\mathrm{~s}, 3 \mathrm{H}$, $\mathrm{OCH}_{2} \mathrm{OCH}_{3}$ ), 3.33 (dd, $J=13.4,3.3 \mathrm{~Hz}, 1 \mathrm{H}, \mathrm{CH}_{2} \mathrm{Ph}$ ), 2.82 (dd, $J=13.4,9.5 \mathrm{~Hz}, 1 \mathrm{H}, \mathrm{CH}_{2} \mathrm{Ph}$ ); ${ }^{13} \mathrm{C}$ NMR (126 MHz, CDCl $)$ : $\delta 170.1$ (C, C-1), 153.5 (C, C-3), 135.1 (C, $\left.\mathrm{CH}_{2} P h\right), 129.5\left(2 \mathrm{CH}, \mathrm{CH}_{2} P h\right)$, $129.2\left(2 \mathrm{CH}, \mathrm{CH}_{2} \mathrm{Ph}\right), 127.6\left(\mathrm{CH}, \mathrm{CH}_{2} \mathrm{Ph}\right), 96.6\left(\mathrm{CH}_{2}, \mathrm{OCH}_{2} \mathrm{CH}_{3}\right), 67.4\left(\mathrm{CH}_{2}, \mathrm{C}-4\right), 66.8\left(\mathrm{CH}_{2}, \mathrm{C}-2\right)$, $56.1\left(\mathrm{CH}_{3}, \mathrm{OCH}_{2} \mathrm{CH}_{3}\right), 55.0(\mathrm{CH}, \mathrm{C}-5), 37.9\left(\mathrm{CH}_{2}, \mathrm{CH}_{2} \mathrm{Ph}\right)$. 
(S)-4-Benzyl-3-((2S,3R,5R)-3-hydroxy-2,5-bis((4-methoxybenzyl)oxy)oct-7enoyl)oxazolidin-2-one (216)<smiles>[R16]O[C@H](O[Na])[C@H](O)C[C@H](CC=C)C(=O)N1C(=O)OC[C@@H]1Br</smiles>

Oxazolidinone $28(0.258 \mathrm{~g}, 0.73 \mathrm{mmol})$ was dissolved in dry toluene $(4 \mathrm{~mL})$ and the solution cooled down to $-50{ }^{\circ} \mathrm{C}$. Then, $\mathrm{Et}_{3} \mathrm{~N}$ (freshly distilled over $\mathrm{CaH}_{2}, 0.12 \mathrm{~mL}, 0.85 \mathrm{mmol}, 1.2$ equiv.) and $\mathrm{Bu}_{2} \mathrm{BOTf}(1 \mathrm{M}$ in $\mathrm{DCM}, 0.85 \mathrm{~mL}, 0.85 \mathrm{mmol}, 1.2$ equiv.) were added sequentially dropwise and the enolate formation proceeded for 1.5 hours at the same temperature. The reaction was then allowed to warm to $-30{ }^{\circ} \mathrm{C}$ and aldehyde $213(0.195 \mathrm{~g}, 0.83 \mathrm{mmol}, 1.15$ equiv.) in $1 \mathrm{~mL}$ toluene added dropwise via cannula. The reaction was kept at $-30{ }^{\circ} \mathrm{C}$ for 2 hours then quenched with $4 \mathrm{~mL} \mathrm{H}_{2} \mathrm{O}$. The aqueous layer was extracted with ethyl acetate ( $3 \times 15 \mathrm{~mL}$ ), the combined organic layers dried over anhydrous $\mathrm{MgSO}_{4}$ and the solvent removed under reduced pressure. The crude reaction mixture was purified on a silica gel column (5:1 then 3:1 PE/EA) to give the product 216 as a colourless oil $(0.306 \mathrm{~g}, 72 \%)$.

$\mathbf{R}_{f}=0.35(1: 1 \mathrm{PE} / \mathrm{EA}) ;[\boldsymbol{\alpha}]_{\mathrm{D}}{ }^{24}=+3.70\left(c 0.81, \mathrm{CHCl}_{3}\right) ;{ }^{1} \mathbf{H}$ NMR $\left(500 \mathrm{MHz}, \mathrm{CDCl}_{3}\right) \delta 7.36-7.27$ (m, 5H, $\left.\mathrm{CH}_{2} P h\right), 7.25-7.16(\mathrm{~m}, 4 \mathrm{H}, \mathrm{OPMB}), 6.88$ (d, $\left.J=8.6 \mathrm{~Hz}, 2 \mathrm{H}, \mathrm{OPMB}\right), 6.83$ (d, J = 8.7 Hz, $2 \mathrm{H}, \mathrm{OPMB}), 5.86-5.75(\mathrm{~m}, 1 \mathrm{H}, \mathrm{H}-7), 5.18$ (d, J = 3.0 Hz, 1H, H-2), $5.14-5.06(\mathrm{~m}, 2 \mathrm{H}, \mathrm{H}-8)$, $4.70-4.61$ (m, 2H, H-11, OPMB), 4.52 (d, $J=10.9 \mathrm{~Hz}, 1 \mathrm{H}, \mathrm{OPMB}$ ), 4.45 (d, J= $11.4 \mathrm{~Hz}, 1 \mathrm{H}$, OPMB), 4.39 (d, J = 10.9 Hz, 1H, OPMB), 4.20 (dd, $J=9.0,7.4 \mathrm{~Hz}, 1 \mathrm{H}, \mathrm{H}-10 \mathrm{a}), 4.15$ (dd, $J=$ 9.0, $2.2 \mathrm{~Hz}, 1 \mathrm{H}, \mathrm{H}-10 \mathrm{~b}), 4.09$ (dt, J = 9.1, 3.2 Hz, 1H, H-3), 3.78 (s, 6H, 2x OPMB), $3.69-3.62$ (m, $1 \mathrm{H}, \mathrm{H}-5$ ), 3.24 (dd, $J=13.5,3.3 \mathrm{~Hz}, 1 \mathrm{H}, \mathrm{CH}_{2} \mathrm{aPh}$ ), 2.70 (dd, $J=13.5,9.7 \mathrm{~Hz}, 1 \mathrm{H}, \mathrm{CH}_{2} \mathrm{bPh}$ ), $2.39-2.33$ (m, 2H, H-6), $1.96-1.86$ (m, 1H, H-4a), 1.76 (ddd, J = 14.9, 4.6, 3.5 Hz, 1H, H-4b); ${ }^{13} \mathrm{C}$ NMR (126 MHz, CDCl $) \delta 170.8$ (C, C-1), 159.7 (C, OPMB), 159.3 (C, OPMB), 153.5 (C, C9), 135.3 (C, $\left.\mathrm{CH}_{2} \mathrm{Ph}\right), 134.2$ ( $\left.\mathrm{CH}, \mathrm{C}-7\right), 130.4\left(2 \mathrm{CH}, \mathrm{CH}_{2} \mathrm{Ph}\right), 129.6$ (2CH, OPMB), 129.5 (2CH, OPMB), 129.3 (C, OPMB), 129.2 (C, O OPMB), 129.1 (2CH, $\left.\mathrm{CH}_{2} P h\right), 127.5$ ( $\left.\mathrm{CH}, \mathrm{CH}_{2} P h\right), 117.8$ $\left(\mathrm{CH}_{2}, \mathrm{C}-8\right), 114.0$ (2CH, OPMB), 113.9 (2CH, OPMB), $78.8(\mathrm{CH}, \mathrm{C}-2), 77.6(\mathrm{CH}, \mathrm{C}-5), 72.9\left(\mathrm{CH}_{2}\right.$, OPMB), $71.3(\mathrm{CH}, \mathrm{C}-3), 70.6\left(\mathrm{CH}_{2}, \mathrm{OPMB}\right), 66.9\left(\mathrm{CH}_{2}, \mathrm{C}-10\right), 55.8(\mathrm{CH}, \mathrm{C}-11), 55.4\left(\mathrm{CH}_{2}\right.$, OPMB), $55.4\left(\mathrm{CH}_{2}, \mathrm{OPMB}\right), 38.1\left(\mathrm{CH}_{2}, \mathrm{C}-6\right), 37.9\left(\mathrm{CH}_{2}, \mathrm{CH}_{2} \mathrm{Ph}\right), 37.2\left(\mathrm{CH}_{2}, \mathrm{C}-4\right)$; HRMS (ESI) 
Found 590.2734. Calculated for $\mathrm{C}_{34} \mathrm{H}_{40} \mathrm{NO}_{8}{ }^{+}[\mathrm{M}+\mathrm{H}]^{+}$590.2748. $\Delta=2.49$ ppm; IR (film from $\left.\mathrm{CHCl}_{3}\right) v_{\max } 3318,2916,1746,1612,1513,1245,1029,702 \mathrm{~cm}^{-1}$.

\section{(S)-3-((4S,5R,7R)-7-allyl-5-methoxy-1,3-dioxepane-4-carbonyl)-4-benzyloxazolidin-2-one}

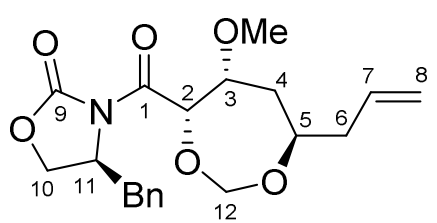

Oxazolidinone $216(9.2 \mathrm{mg}, 0.016 \mathrm{mmol})$ was dissolved in dry DCM $(0.5 \mathrm{~mL})$, cooled down to $0{ }^{\circ} \mathrm{C}$ then treated with $\mathrm{Me}_{3} \mathrm{OBF}_{4}(32.4 \mathrm{mg}, 0.24 \mathrm{mmol}, 15$ equiv.) and proton sponge (53.3 $\mathrm{mg}, 0.24 \mathrm{mmol}, 15$ equiv.). The reaction was allowed to warm to room temperature and stirred overnight then filtered through a pad of celite into sat. aq. $\mathrm{NaHCO}_{3}(5 \mathrm{~mL})$. Celite was washed with ethyl acetate $(3 \times 5 \mathrm{~mL})$ and the two layers separated. The organic layer was dried $\left(\mathrm{MgSO}_{4}\right)$, concentrated and passed through a silica plug (2:1 $\left.\mathrm{PE} / \mathrm{EA}\right)$ to give $4.7 \mathrm{mg}$ (50\% crude yield) of the slightly impure product $\mathbf{2 1 7}$ which was used directly in the next step.

$\mathbf{R}_{f}=0.73(1: 1 \mathrm{PE} / \mathrm{EA})$; HRMS (ESI) Found 604.2918. Calculated for $\mathrm{C}_{35} \mathrm{H}_{42} \mathrm{NO}_{8}{ }^{+}[\mathrm{M}+\mathrm{H}]^{+}$ 604.2905. $\Delta=2.20$ ppm.

The material obtained from the previous step was dissolved in $0.2 \mathrm{~mL} 5 \%$ aq. DCM and the solution cooled to $0{ }^{\circ} \mathrm{C}$, then $\mathrm{DDQ}(11.0 \mathrm{mg}, 0.05 \mathrm{mmol}, 6$ equiv.) was added. The reaction was stirred at the same temperature for 20 min then another 2 hours at room temperature at which point TLC analysis (1:1 PE/EA) indicated complete consumption of the starting material. The reaction was then quenched with $5 \mathrm{~mL}$ sat. aq. $\mathrm{NaHCO}_{3}$ and the aqueous layer extracted with DCM $(3 \times 5 \mathrm{~mL})$. The combined organic layers were dried $\left(\mathrm{MgSO}_{4}\right)$, filtered and concentrated. The crude reaction mixture containing diol 219 was dissolved in $0.2 \mathrm{~mL}$ dry DCM and treated at $0{ }^{\circ} \mathrm{C}$ with DIPEA $(0.02 \mathrm{~mL}, 0.08 \mathrm{mmol}, 10$ equiv.) then $\mathrm{MOMBr}(7 \mu \mathrm{L}$, $0.08 \mathrm{mmol}, 10$ equiv.). The reaction was allowed to gradually warm to room temperature and stirred overnight, then quenched by the addition of sat. aq. $\mathrm{NaHCO}_{3}(5 \mathrm{~mL})$. The aqueous layer was extracted with DCM $(3 \times 5 \mathrm{~mL})$, the organic layers dried over anhydrous 
$\mathrm{MgSO}_{4}$ and the solvent removed under reduced pressure. Purification of the crude reaction mixture on silica column gave the acetate $\mathbf{2 2 1}$ as a colourless oil ( $0.3 \mathrm{mg}, 5 \%$ over 3 steps).

$\mathbf{R}_{f}=0.14(3: 1 \mathrm{PE} / \mathrm{EA}) ;{ }^{1} \mathbf{H}$ NMR $\left(600 \mathrm{MHz}, \mathrm{CDCl}_{3}\right) \delta 7.36-7.31\left(\mathrm{~m}, 2 \mathrm{H}, \mathrm{CH}_{2} \mathrm{Ph}\right), 7.31-7.26(\mathrm{~m}$, $\left.1 \mathrm{H}, \mathrm{CH}_{2} P h\right), 7.25-7.21\left(\mathrm{~m}, 2 \mathrm{H}, \mathrm{CH}_{2} P h\right), 5.88-5.80(\mathrm{~m}, 1 \mathrm{H}, \mathrm{H}-7), 5.49$ (d, J = 2.6 Hz, 1H, H-2), $5.14-5.07(\mathrm{~m}, 2 \mathrm{H}, \mathrm{H}-8), 5.04(\mathrm{~d}, J=5.6 \mathrm{~Hz}, 1 \mathrm{H}, \mathrm{H}-12 \mathrm{a}), 5.00$ (d, J = 5.6 Hz, 1H, H-12b), $4.72-$ $4.67(\mathrm{~m}, 1 \mathrm{H}, \mathrm{H}-11), 4.24-4.21(\mathrm{~m}, 2 \mathrm{H}, \mathrm{H}-10), 4.02(\mathrm{dt}, J=4.8,2.6 \mathrm{~Hz}, 1 \mathrm{H}, \mathrm{H}-3), 3.98(\mathrm{dt}, J=$ 11.6, 6.4 Hz, 1H, H-5), 3.43 (dd, J=13.3, 3.2 Hz, 1H, $\mathrm{CH}_{2} \mathrm{Ph}$ ), 3.31 (s, 3H, $\mathrm{OCH}_{3}$ ), 2.77 (dd, $J=$ 13.5, 9.9 Hz, 1H, CH $2 \mathrm{Ph}$ ), $2.42-2.36$ (m, 1H, H-6a), 2.26 (ddd, J=13.8, 7.3, $5.8 \mathrm{~Hz}, 1 \mathrm{H}, \mathrm{H}-6 \mathrm{~b}$ ), 2.16 (ddd, $J=14.9,4.3,1.3 \mathrm{~Hz}, 1 \mathrm{H}, \mathrm{H}-4 \mathrm{a}$ ), 1.72 (ddd, $J=15.0,10.6,2.6 \mathrm{~Hz}, 1 \mathrm{H}, \mathrm{H}-4 \mathrm{~b}$ ); ${ }^{13} \mathrm{C}$ NMR (151 MHz, CDCl $\left.{ }_{3}\right) \delta 170.1$ (C, C-1), 153.5 (C, C-9), 135.3 (C, Ph), 134.7 (CH, C-7), 129.6 (2CH, Ph), 129.2 (2CH, Ph), $127.6(\mathrm{CH}, \mathrm{Ph}), 117.5\left(\mathrm{CH}_{2}, \mathrm{C}-8\right), 93.6\left(\mathrm{CH}_{2}, \mathrm{C}-12\right), 78.0(\mathrm{CH}, \mathrm{C}-3)$, 77.5 (underneath the solvent peak, $\mathrm{CH}, \mathrm{C}-2), 72.2(\mathrm{CH}, \mathrm{C}-5), 67.1\left(\mathrm{CH}_{2}, \mathrm{C}-10\right), 57.4\left(\mathrm{CH}_{3}\right.$, $\left.\mathrm{OCH}_{3}\right), 56.0(\mathrm{CH}, \mathrm{C}-11), 40.5\left(\mathrm{CH}_{2}, \mathrm{C}-6\right), 37.7\left(\mathrm{CH}_{2}, \mathrm{CH}_{2} \mathrm{Ph}\right), 36.4\left(\mathrm{CH}_{2}, \mathrm{C}-4\right)$; HRMS (ESI) Found 376.1754. Calculated for $\mathrm{C}_{19} \mathrm{H}_{20} \mathrm{NO}_{8}{ }^{+}[\mathrm{M}+\mathrm{H}]^{+}$376.1755. $\Delta=0.3 \mathrm{ppm}$; IR (film from $\mathrm{CH}_{2} \mathrm{Cl}_{2}$ ) $\mathrm{V}_{\max }$ $2920,2854,1777,1719,1389,1212,1105,702 \mathrm{~cm}^{-1}$.

\section{(S)-4-Benzyl-3-((2S,3R,5R)-3-hydroxy-2,5-bis(methoxymethoxy)oct-7-enoyl)oxazolidin-2-} one (220)

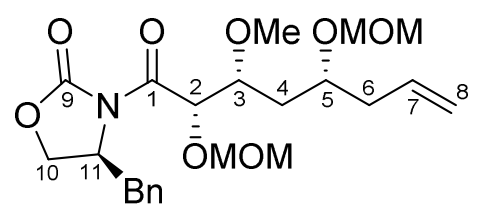

Oxazolidinone $216(45.7 \mathrm{mg}, 0.08 \mathrm{mmol})$ in $\mathrm{DCM}(0.5 \mathrm{~mL})$ was added at $0{ }^{\circ} \mathrm{C}$ to a solution of $\mathrm{Me}_{3} \mathrm{OBF}_{4}(0.180 \mathrm{~g}, 1.16 \mathrm{mmol}, 15$ equiv.) and proton sponge (0.254 g, $1.16 \mathrm{mmol}, 15$ equiv.) in DCM $(1.5 \mathrm{~mL})$. The reaction was stirred at the room temperature for 4.5 hours (at which point TLC analysis indicated all starting material was consumed) then filtered through celite into $5 \mathrm{~mL}$ sat. aq. $\mathrm{NaHCO}_{3}$. The celite was washed with EtOAc $(10 \mathrm{~mL})$ and the layers separated. The aqueous layer was extracted two more times with EtOAc $(5 \mathrm{~mL})$ and the organic layers combined, then dried over anhydrous $\mathrm{MgSO}_{4}$ and concentrated in vacuo. Purification through a silica plug (2:1 PE/EA) gave $47.0 \mathrm{mg}$ (quant. crude yield) of slightly impure product that was used in the next step without further purification. 
The product from the methylation reaction was dissolved in $5 \%$ aq. DCM $(2 \mathrm{~mL})$ and treated with DDQ (105.4 mg, $0.46 \mathrm{mmol}, 6$ equiv.) at $0{ }^{\circ} \mathrm{C}$. The reaction was stirred at the same temperature for $20 \mathrm{~min}$ then at room temperature for 3 hours. TLC analysis (1:1 PE/EA) indicated all starting material was consumed. The reaction was then quenched with sat.aq. $\mathrm{NaHCO}_{3}(15 \mathrm{~mL})$ and extracted with DCM $(3 \times 5 \mathrm{~mL})$. The combined organic layers were dried $\left(\mathrm{MgSO}_{4}\right)$ and the solvent removed under reduced pressure. The residue was dissolved in dry DCM (1.5 mL) and cooled to $0{ }^{\circ} \mathrm{C}$ then treated with DIPEA $(0.15 \mathrm{~mL}, 0.86 \mathrm{mmol}, 11$ equiv.) and $\mathrm{MOMCl}(0.07 \mathrm{~mL}, 0.9 \mathrm{mmol}, 11$ equiv.) sequentially. The reaction was allowed to gradually warm to room temperature and stirred overnight. After stirring overnight, the reaction was quenched with sat. aq. $\mathrm{NaHCO}_{3}(5 \mathrm{~mL})$ and the aqueous layer extracted with EtOAc ( $3 \times 5 \mathrm{~mL}$ ). Purification on a silica gel column (solvent) gave the oxazolidinone $\mathbf{2 2 0}$ as an inseparable mixture with a partially PMB protected by-product (at either $\mathrm{O} 2$ or $\mathrm{O5}$ ) and a fraction $(2.7 \mathrm{mg}, 8 \%$ yield) with the least impurity was used for characterisation.

$\mathbf{R}_{f}=0.69(1: 1 \mathrm{PE} / \mathrm{EA}) ;{ }^{1} \mathbf{H}$ NMR $\left(500 \mathrm{MHz}, \mathrm{CDCl}_{3}\right) \delta 7.37-7.27\left(\mathrm{~m}, 5 \mathrm{H}, \mathrm{CH}_{2} P h\right), 5.89-5.79(\mathrm{~m}$, $1 \mathrm{H}, \mathrm{H}-7), 5.48(\mathrm{~d}, J=4.3 \mathrm{~Hz}, 1 \mathrm{H}, \mathrm{H}-2), 5.15-5.06(\mathrm{~m}, 2 \mathrm{H}, \mathrm{H}-8), 4.80(\mathrm{~d}, J=6.8 \mathrm{~Hz}, 1 \mathrm{H}$, $\left.\mathrm{OCH}_{2} \mathrm{OCH}_{3}\right), 4.73\left(\mathrm{~d}, J=5.6 \mathrm{~Hz}, 1 \mathrm{H}, \mathrm{OCH}_{2} \mathrm{OCH}_{3}\right), 4.71\left(\mathrm{~d}, J=5.3 \mathrm{~Hz}, 1 \mathrm{H}, \mathrm{OCH}_{2} \mathrm{OCH}_{3}\right), 4.66(\mathrm{~m}$, $\left.2 \mathrm{H}, \mathrm{H}-11 / \mathrm{OCH}_{2} \mathrm{OCH}_{3}\right), 4.24-4.16(\mathrm{~m}, 2 \mathrm{H}, \mathrm{H}-10), 3.79-3.72(\mathrm{~m}, 2 \mathrm{H}, \mathrm{H}-3 / \mathrm{H}-5), 3.41(\mathrm{~s}, 3 \mathrm{H}$, $\mathrm{OCH}_{2} \mathrm{OCH}_{3}$ ), $3.39\left(\mathrm{~s}, \mathrm{OCH}_{3}\right) 3.38\left(\mathrm{~s}, 3 \mathrm{H}, \mathrm{OCH}_{2} \mathrm{OCH}_{3}\right), 3.35$ (dd, $\left.J=14.0,3.7 \mathrm{~Hz}, 1 \mathrm{H}, \mathrm{CH}_{2} \mathrm{Ph}\right)$, $2.80\left(\mathrm{dd}, J=13.4,9.6 \mathrm{~Hz}, 1 \mathrm{H}, \mathrm{CH}_{2} \mathrm{Ph}\right), 2.42-2.34(\mathrm{~m}, 2 \mathrm{H}, \mathrm{H}-6), 1.91-1.83(\mathrm{~m}, 2 \mathrm{H}, \mathrm{H}-4) ;{ }^{13} \mathrm{C}$ NMR (126 MHz, CDCl $) \delta 170.9$ (C, C-1), 153.4 (C, C-9, determined from HMBC), 135.3 (C, $\left.\mathrm{CH}_{2} \mathrm{Ph}\right), 134.6(\mathrm{CH}, \mathrm{C}-7), 129.6\left(2 \mathrm{CH}, \mathrm{CH}_{2} P h\right), 129.2\left(2 \mathrm{CH}, \mathrm{CH}_{2} P h\right), 127.5\left(\mathrm{CH}, \mathrm{CH}_{2} P h\right), 117.7$ $\left(\mathrm{CH}_{2}, \mathrm{C}-8\right), 97.7\left(\mathrm{CH}_{2}, \mathrm{OCH}_{2} \mathrm{OCH}_{3}\right), 96.2\left(\mathrm{CH}_{2}, \mathrm{OCH}_{2} \mathrm{OCH}_{3}\right), 78.4(\mathrm{CH}, \mathrm{C}-3), 76.8(\mathrm{CH}, \mathrm{C}-2), 74.7$ $(\mathrm{CH}, \mathrm{C}-5), 66.6\left(\mathrm{CH}_{2}, \mathrm{C}-10\right), 58.4\left(\mathrm{CH}_{3}, \mathrm{OCH}_{3}\right), 56.6\left(\mathrm{CH}_{3}, \mathrm{OCH}_{2} \mathrm{OCH}_{3}\right), 55.9(\mathrm{CH}, \mathrm{C}-11), 55.8$ $\left(\mathrm{CH}_{3}, \mathrm{OCH}_{2} \mathrm{OCH}_{3}\right), 39.4\left(\mathrm{CH}_{2}, \mathrm{C}-6\right), 37.6\left(\mathrm{CH}_{2}, \mathrm{CH}_{2} \mathrm{Ph}\right), 34.4\left(\mathrm{CH}_{2}, \mathrm{C}-4\right)$; HRMS (ESI) Found 474.2113. Calculated for $\mathrm{C}_{23} \mathrm{H}_{33} \mathrm{NNaO}_{8}{ }^{+}[\mathrm{M}+\mathrm{Na}]^{+}$474.2098. $\Delta=3.1 \mathrm{ppm}$; IR (film from $\mathrm{CH}_{2} \mathrm{Cl}_{2}$ ) $v_{\max } 2934,2826,1778,1719,1453,1388,1211,1037 \mathrm{~cm}^{-1}$. 


\section{References}

(1) Evans, D. A.; Welch, D. S.; Speed, A. W. H.; Moniz, G. A.; Reichelt, A.; Ho, S. J. Am. Chem. Soc. 2009, 131, 3840.

(2) Ghosh, A. K.; Xu, X.; Kim, J.; Xu, C. Org. Lett. 2008, 10, 1001.

(3) McGowan, M. A.; Stevenson, C. P.; Schiffler, M. A.; Jacobsen, E. N. Angew. Chemie Int. Ed. 2010, 49, 6147.

(4) Jin, M.; Taylor, R. E. Org. Lett. 2005, 7, 1303.

(5) Butler, M. S.; Robertson, A. a B.; Cooper, M. A. Nat. Prod. Rep. 2014, 31, 1612.

(6) Newman, D. J.; Cragg, G. M. J. Nat. Prod. 2016, 79, 629.

(7) Fusetani, N. Marine Toxins as Research Tools, in Progress in Molecular and Subcellular Biology, 2009th ed.; Fusetani, N., Kem, W., Eds.; Springer: Berlin, Heidelberg.

(8) Altmann, K. Chimia (Aarau). 2017, 71, 646.

(9) Miller, J. H.; Singh, A. J.; Northcote, P. T. Mar. Drugs 2010, 8, 1059.

(10) Bergmann, W.; Feeney, R. J. J. Org. Chem. 1951, 16, 981.

(11) Blunt, J. W.; Copp, B. R.; Keyzers, R. A.; Munro, M. H. G.; Prinsep, M. R. Nat. Prod. Rep. 2015, 32, 116.

(12) Hussain, S.; Fareed, S.; Ansari, S.; Khan, S. Indian J. Geo-Marine Sci. 2012, 4, 27.

(13) Newman, D. J.; Cragg, G. M. J. Nat. Prod. 2004, 67, 1216.

(14) Simmons, T. L.; Andrianasolo, E.; McPhail, K.; Flatt, P.; Gerwick, W. H. Mol. Cancer Ther. 2005, 4, 333.

(15) Rangel, M.; Falkenberg, M. J. Coast. Life Med. 2015, 3, 421.

(16) Rinehart, K. L.; Holt, T. G.; Fregeau, J. G.; Stroh, P. A.; Keifer, F.; Sun, L. H.; Li, D. G.; Martin, J. J. Org. Chem. 1990, 55, 4513. 
(17) Wright, A. E.; Forleo, D. A.; Gunawardana, P. G.; Gunasekera, S. P.; Koehn, F. E.; McConnell, O. J. J. Org. Chem. 1990, 55, 4508.

(18) Incalci, M. D.; Badri, N.; Galmarini, C. M.; Allavena, P. Br. J. Cancer 2014, 111, 646.

(19) Cortes, J.; O’Shaughnessy, J.; Loesch, D.; Blum, J. L.; Vahdat, L. T.; Petrakova, K.; Chollet, P.; Manikas, A.; Diéras, V.; Delozier, T.; Vladimirov, V.; Cardoso, F.; Koh, H.; Bougnoux, P.; Dutcus, C. E.; Seegobin, S.; Mir, D.; Meneses, N.; Wanders, J.; Twelves, C. Lancet (London, England) 2011, 377, 914.

(20) Dybdal-Hargreaves, N. F.; Risinger, A. L.; Mooberry, S. L. Clin. Cancer Reserch 2015, 21,1 .

(21) Towle, M. J.; Salvato, K. A.; Budrow, J.; Wels, B. F.; Kuznetsov, G.; Aalfs, K. K.; Welsh, S.; Zheng, W.; Seletsky, B. M.; Palme, M. H.; Habgood, G. J.; Singer, L. A.; Dipietro, L. V; Wang, Y.; Chen, J. J.; Quincy, D. A.; Davis, A.; Yoshimatsu, K.; Kishi, Y.; Yu, M. J.; Littlefield, B. A. Cancer Res. 2001, 61, 1013.

(22) Pettit, G. R.; Kamano, Y.; Herald, C. L.; Tuinman, A. A.; Boettner, F. E.; Kizu, H.; Schmidt, J. M.; Baczynskyj, L.; Tomer, K. B.; Bontems, R. J. J. Am. Chem. Soc. 1987, $109,6883$.

(23) Senter, P. D.; Sievers, E. L. Nat. Biotechnol. 2012, 30, 631.

(24) Pettit, G. R.; Srirangam, J. K.; Barkoczy, J.; Williams, M. D.; Boyd, M. R.; Hamel, M. E.; Pettit, R. K.; Hogan, F.; Bai, R.; Chapius, J.-C.; McAllister, S. C.; Schmidt, J. M. AntiCancer Drug Des. 1998, 13, 243.

(25) Bai, R.; Pettit, G. R.; Hamel, M. E. Biochem. Pharmacol. 1990, 39, 1941.

(26) West, L. M.; Northcote, P. T.; Battershill, C. N. J. Org. Chem. 2000, 65, 445.

(27) Hood, K. A.; West, L. M.; Rouwé, B.; Northcote, P. T.; Berridge, M. V.; Wakefield, S. J.; Miller, J. H. Cancer Res. 2002, 62, 3356.

(28) Gaitanos, T. N.; Buey, R. M.; Fernando Díaz, J.; Northcote, P. T.; Teesdale-Spittle, P.; Andreu, J. M.; Miller, J. H. Cancer Res. 2004, 64, 5063. 
(29) Prota, A. E.; Bargsten, K.; Northcote, P. T.; Marsh, M.; Altmann, K. H.; Miller, J. H.; Díaz, J. F.; Steinmetz, M. O. Angew. Chem. Int. Ed. 2014, 53, 1621.

(30) Kanakkanthara, A.; Rowe, M. R.; Field, J. J.; Northcote, P. T.; Teesdale-Spittle, P. H.; Miller, J. H. Cancer Lett. 2015, 365, 251.

(31) Ganguly, A.; Cabral, F.; Yang, H.; Patel, K. D. Oncoscience, Adv. Publ. 2015 2015, 1.

(32) Chan, A.; Singh, J. A.; Northcote, P. T.; Miller, J. H. Invest. New Drugs 2015, 33, 564.

(33) Meyer, C. J.; Krauth, M.; Wick, M. J.; Shay, J. W.; Gellert, G.; De Brabander, J. K.; Northcote, P. T.; Miller, J. H. Mol. Cancer Ther. 2015, 14, 1816.

(34) Chan, A.; Andreae, P. M.; Northcote, P. T.; Miller, J. H. Invest. New Drugs 2011, 29, 615.

(35) Das, V.; Miller, J. H. Eur. J. Neurosci. 2012, 35, 1705.

(36) Das, V.; Miller, J. H. Brain Res. 2012, 1489, 121.

(37) Das, V.; Sim, D. A.; Miller, J. H. J. Neurosci. Res. 2014, 92, 1155.

(38) Kanakkanthara, A.; Northcote, T.; Miller, J. H. Nat. Prod. Rep. 2016, 1.

(39) Miller, J. H.; Rouwe, B.; Gaitanos, T. N.; Hood, K. A.; Crume, K. P.; Backstrom, B. T.; La Flamme, A. C.; Berridge, M. V.; Northcote, P. T. Apoptosis 2004, 9, 785.

(40) Crume, K. P.; Miller, J. H.; La Flamme, A. C. Exp. Biol. Med. 2007, 232, 607.

(41) Crume, K. P.; O’Sullivan, D.; Miller, J. H.; Northcote, P. T.; La Flamme, A. C. J. Leukoc. Biol. 2009, 86, 949.

(42) O’Sullivan, D.; Miller, J. H.; Northcote, P. T.; La Flamme, A. C. Immunol. Cell Biol. 2013, 91, 583.

(43) Khrapunovich-Baine, M.; Menon, V.; Yang, C. P. H.; Northcote, P. T.; Miller, J. H.; Angeletti, R. H.; Fiser, A.; Horwitz, S. B.; Xiao, H. J. Biol. Chem. 2011, 286, 11765.

(44) Mukhtar, E.; Adhami, V. M.; Mukhtar, H. Mol. Cancer Ther. 2014, 13, 275. 
(45) Moudi, M.; Go, R.; Yien, C. Y.; Nazre, M. Int. J. Prev. Med. 2013, 4, 1231.

(46) Miller, J. H.; Field, J. J.; Kanakkanthara, A.; Owen, J. G.; Singh, A. J.; Northcote, P. T. J. Nat. Prod. 2018, 81, 691-702.

(47) Hamel, E.; Day, B. W.; Miller, J. H.; Jung, M. K.; Northcote, P. T.; Ghosh, A. K.; Curran, D. P.; Cushman, M.; Nicolaou, K. C.; Paterson, I.; Sorensen, E. J. Mol. Pharmacol. 2006, 70, 1555.

(48) Wilmes, A.; Sullivan, D. O.; Chan, A.; Chandrahasen, C.; Paterson, I.; Northcote, P. T.; La Flamme, A. C.; Miller, J. H. Cancer Chemother. Pharmacol. 2011, 68, 117.

(49) Castro-Alvarez, A.; Pineda, O.; Vilarrasa, J. ACS Omega 2018, 3, 1770.

(50) Kanakkanthara, A.; Wilmes, A.; O’Brate, A.; Escuin, D.; Chan, A.; Gjyrezi, A.; Crawford, J.; Rawson, P.; Kivell, B.; Northcote, P. T.; Hamel, E.; Giannakakou, P.; Miller, J. H. Mol. Cancer Ther. 2011, 8, 1419.

(51) Rowinsky, E. K.; Eisenhauer, E. A.; Chaudhry, V.; Arbuck, S. G.; Donehower, R. C. Semin. Oncol. 1993, No. 20, 1.

(52) Rowinsky, E. K.; Donehower, R. C. New Engl. J. Med. 1995, 332, 1004.

(53) Page, M. J.; Handley, S. J.; Northcote, P. T.; Cairney, D.; Willan, R. C. Aquaculture 2011, 312, 52 .

(54) Liao, X.; Wu, Y.; De Brabander, J. K. Angew. Chem. Int. Ed. 2003, 42, 1648.

(55) Hoye, T. R.; Jeon, J.; Kopel, L. C.; Ryba, T. D.; Tennakoon, M. A.; Wang, Y. Angew. Chem. Int. Ed. 2010, 49, 6151.

(56) Mitsunobu, O.; Yamada, M. Bull. Chem. Soc. Jpn. 1967, 40, 2380.

(57) Inanaga, J.; Hirata, K.; Saeki, H.; Katsuki, T.; Yamaguchi, M. Bull. Chem. Soc. Jpn. 1979, $52,1989$.

(58) Kawanami, Y.; Dainobu, Y.; Inanaga, J.; Katsuki, T.; Yamaguchi, M. Bull. Chem. Soc. Jpn. 1981, 54, 943. 
(59) Still, W. C.; Gennari, C. Tetrahedron Lett. 1983, 24, 4405.

(60) Reetz, M. T.; Kesseler, K.; Jung, A. Tetrahedron Lett. 1984, 25, 729.

(61) Trieselmann, T.; Hoffmann, R. W. Org. Lett. 2000, 2, 1209.

(62) Evans, D. A.; Dart, M. J.; Duffy, J. L.; Yang, M. G. J. Am. Chem. Soc. 1996, 118, 4322.

(63) Willson, T. M.; Kocrenski, P.; Jarowicki, K.; Isaac, K.; Hitchcock, P. M.; Faller, A.; Campbell, S. F. Tetrahedron 1990, 46, 1767.

(64) Taylor, R. E.; Jin, M. Org. Lett. 2003, 5, 4959.

(65) Evans, D. A.; Bartroli, J.; Shih, T. L. J. Am. Chem. Soc. 1981, 103, 2127.

(66) Evans, D. A.; Ng, H. P.; Clark, J. S.; Rieger, D. L. Tetrahedron 1992, 48, 2127.

(67) Bal, B. S.; Childers Jr., W. E.; Pinnick, H. W. Tetrahedron 1981, 37, 2091.

(68) Jadhav, P. K.; Bhat, K. S.; Perumal, P. T.; Brown, H. C. J. Org. Chem. 1986, 51, 432.

(69) Racherla, U. S.; Brown, H. C. J. Org. Chem. 1991, 56, 401.

(70) Ando, K. J. Org. Chem. 1998, 63, 8411.

(71) Jacobsen, E. N.; Marko, I.; Mungall, W. S.; Schroeder, G.; Sharpless, K. B. J. Am. Chem. Soc. 1988, 110, 1968.

(72) Ghosh, A. K.; Kim, J. 2003, 44, 3967.

(73) Hoye, T. R.; Peck, D. R.; Swanson, T. A. J. Am. Chem. Soc. 1984, 106, 2738.

(74) Hoye, T. R.; Jeffrey, C. S.; Tennakoon, M. A.; Wang, J.; Zhao, H. J. Am. Chem. Soc. 2004, 126, 10210.

(75) Neises, B.; Steglich, W. Angew. Chem. Int. Ed. 1978, 17, 522.

(76) Chen, Z.; Zhou, W. Tetrahedron Lett. 2006, 47, 5289.

(77) Smidt, J.; Hafner, W.; Jira, R.; Sedlmeier, J.; Sieber, R.; Ruttinger, R.; Kojer, H. Angew. Chem. Int. Ed. 1956, 71, 176. 
(78) Appel, R. Angew. Chem. Int. Ed. 1975, 14, 801.

(79) Mico, A. De; Margarita, R.; Parlanti, L.; Vescovi, A.; Piancatelli, G. J. Org. Chem. 1997, 62,6974 .

(80) Paterson, I.; Goodman, J. M.; Lister, M. A.; Schumann, R. C.; McClure, C. K.; Norcross, R. D. Tetrahedron 1990, 46, 4663.

(81) Paterson, I. Pure Appl. Chem. 1992, 64, 1821.

(82) Davis, F. A.; Jenkins Jr, R.; Yocklovich, S. G. Tetrahedron Lett. 1978, 52, 5171.

(83) Davis, F. A.; Kumar, A.; Chen, B. Tetrahedron Lett. 1991, 32, 867.

(84) Davis, F. A.; Sheppard, A. C.; Chen, B.; Haque, M. S. J. Am. Chem. Soc. 1990, 112, 6679.

(85) Davis, F. A.; Chen, B. Tetrahedron Lett. 1990, 31, 6823.

(86) Wullschleger, C. W.; Altmann, K.; Zu, C. Org. Lett. 2010, 12, 1120.

(87) Wullschleger, C. W.; Gertsch, J.; Altmann, K. Chem. Eur. J. 2013, 19, 13105.

(88) Zimmermann, N.; Pinard, P.; Carboni, B.; Gosselin, P.; Gaulon-Nourry, C.; Dujardin, G.; Collet, S.; Lebreton, J.; Mathé-Allainmat, M. Eur. J. Org. Chem. 2013, 2303.

(89) Zhao, Z.; Taylor, R. E. Org. Lett. 2012, 14, 669.

(90) Marx, V. M.; Herbert, M. B.; Keitz, B. K.; Grubbs, R. H. J. Am. Chem. Soc. 2013, 135, 94.

(91) Kolb, H. C.; Van Nieuwenhze, M. S. Sharpless, K. B. Chem. Rev. 1994, 94, 2483.

(92) Wang, L.; Sharpless, K. B. J. Am. Chem. Soc. 1992, 114, 7568.

(93) Brackovic, A.; Harvey, J. E. Chem. Commun. 2015, 51, 4750.

(94) Singh, A. J.; Razzak, M.; Teesdale-Spittle, P.; Gaitanos, T. N.; Wilmes, A.; Paterson, I.; Goodman, J. M.; Miller, J. H.; Northcote, P. T. Org. Biomol. Chem. 2011, 9, 4456.

(95) Katsuki, T.; Sharpless, K. B. J. Am. Chem. Soc. 1980, 102, 5974.

(96) Tu, Y.; Wang, Z.-X.; Shi, Y. J. Am. Chem. Soc. 1996, 118, 9806. 
(97) Wang, Z.-X.; Tu, W.; Frohn, M.; Zhang, J.-R.; Shi, Y. J. Am. Chem. Soc. 1997, 119, 11224.

(98) Zhang, W.; Loebach, J. L.; Wilson, S. R.; Jacobsen, E. N. J. Am. Chem. Soc. 1990, 112, 2801.

(99) Jacobsen, E. N.; Zhang, W.; Muci, A. R.; Ecker, J. R.; Deng, L. J. Am. Chem. Soc. 1991, $113,7063$.

(100) Irie, R.; Noda, K.; Ito, Y.; Matsumoto, N.; Katsuki, T. Tetrahedron Asymm. 1991, 2, 481.

(101) Ding, F.; William, R.; Leow, M. L.; Chai, H.; Zi, J.; Fong, M.; Liu, X. Org. Lett. 2014, 16, 26.

(102) Raghavan, S.; Kumar, V. V. Org. Biomol. Chem. 2013, 11, 2847.

(103) Ando, K. J. Org. Chem. 1999, 64, 8406.

(104) Blanchette, M. A.; Choy, W.; Davis, J. T.; Essenfeld, A. P.; Masamune, S.; Roush, W. R.; Sakai, T. Tetrahedron Lett. 1984, 25, 2183.

(105) Yokokawa, F.; Asano, T.; Shioiri, T. Tetrahedron 2001, 57, 6311.

(106) Siau, W.; Zhang, Y.; Zhao, Y. Top. Curr. Chem.; 2012; 327, 33-58.

(107) Dess, D. B.; Matrin, J. C. J. Org. Chem. 1983, 48, 4155.

(108) Clegg, W.; Harrington, R. W.; North, M.; Pizzato, F.; Villuendas, P. Tetrahedron: Asymmetry 2010, 21, 1262.

(109) Panday, S. K. Tetrahedron: Asymmetry 2011, 22, 1817.

(110) List, B.; Lerner, R. A.; Barbas, C. F. J. Am. Chem. Soc. 2000, 122, 2395.

(111) Hajos, Z. G.; Parrish, D. R. J. Org. Chem. 1974, 39, 1615.

(112) Eder, U.; Sauer, G.; Wiechert, R. Angew. Chem. Int. Ed. 1971, 10, 496.

(113) Sakthivel, K.; Notz, W.; Bui, T.; Barbas, C. F. J. Am. Chem. Soc. 2001, 123, 5260. 
(114) Lifchits, O.; Mahlau, M.; Reisinger, C. M.; Lee, A.; Fare, C.; Polyak, I.; Gopakumar, G.; Thiel, W.; List, B. J. Am. Chem. Soc. 2013, 135, 6677-6693.

(115) María, P. D. De; Bracco, P.; Castelhano, L. F.; Bargeman, G. ACS Catal. 2011, 1, 70.

(116) Kunz, R. K.; MacMillan, D. W. C. J. Am. Chem. Soc. 2005, 127, 3240.

(117) Paterson, I.; Di Francesco, M. E.; Kuhn, T. Org. Lett. 2003, 5, 599.

(118) Paterson, I.; Smith, J. D. Tetrahedron Lett. 1993, 34, 5351.

(119) Paterson, I.; Florence, G. J. Tetrahedron Lett. 2000, 41, 6935.

(120) Ohtani, I.; Kusumi, T.; Kashman, Y.; Kakisawa, H. J. Am. Chem. Soc. 1991, 113, 4092.

(121) Nakajima, N.; Horita, K.; Abe, R.; Yonemitsu, O. Tetrahedron 1988, 29, 4139.

(122) Cramer, F.; Pawelzik, K.; Lichtenthaler, F. W. Chem. Ber. 1958, 91, 1555.

(123) Howard, K. T.; Duffy, B. C.; Linaburg, M. R.; Chisholm, J. D. Org. Biomol. Chem. 2016, $14,1623$.

(124) Adhikari, A. A.; Suzuki, T.; Gilbert, R. T.; Linaburg, M. R.; Chisholm, J. D. J. Org. Chem. 2017, 82, 3982-3989.

(125) Green, R. A.; Jolley, K. E.; Al-hadedi, A. A. M.; Pletcher, D.; Harrowven, D. C.; Frutos, O. De; Mateos, C.; Klauber, D. J.; Rincon, J. A.; Brown, R. C. D. Org. Lett. 2017, 19, 2050.

(126) Mickel, S. J.; Niederer, D.; Daeffler, R.; Osmani, A.; Kuesters, E.; Schmid, E.; Schaer, K.; Gamboni, R.; Chen, W.; Loeser, E.; Kinder, F. R.; Konigsberger, K.; Prasad, K.; Ramsey, T. M.; Repic, O.; Wang, R.-M.; Florence, G.; Lyothier, I.; Paterson, I. Org. Process Res. Dev. 2004, 8, 122.

(127) Akeboshi, T.; Ohtsuka, Y.; Ishihara, T.; Sugai, T. Adv. Synth. Catal. 2001, 343 (6), 624.

(128) Kuranaga, T.; Sesoko, Y.; Sakata, K.; Maeda, N.; Hayata, A.; Inoue, M. J. Am. Chem. Soc. 2013, No. $135,5467-5474$.

(129) Surivet, J.-P.; Vatele, J.-M. Tetrahedron Lett. 1998, 39, 9681. 
(130) Short, R. P.; Kennedy, R. M.; Masamune, S. J. Org. Chem. 1989, 54, 1755.

(131) Paterson, I.; Gibson, K. R.; Oballa, R. M. Tetrahedron Lett. 1996, 37, 8585.

(132) Evans, D. A.; Coleman, P. J.; Cote, B. J. Org. Chem. 1997, 62, 788.

(133) Stocker, B. L.; Teesdale-spittle, P.; Hoberg, J. O. European J. Org. Chem. 2004, 330.

(134) Evans, D. A.; Cote, B.; Coleman, P. J.; Connell, B. T. J. Am. Chem. Soc. 2003, 125, 10893.

(135) Kozmin, S. A. Org. Lett. 2001, 3, 755.

(136) Wang, W.; Janjic, J.; A., K. S. J. Am. Chem. Soc. 2002, 124, 13670.

(137) Fettes, A.; Carreira, E. M. Angew. Chem. Int. Ed. 2002, 41, 4098.

(138) Fettes, A.; Carreira, E. M. J. Org. Chem. 2003, 68, 9274.

(139) Paton, R. S.; Goodman, J. M. J. Org. Chem. 2008, 73, 1253.

(140) Kusumi, T.; Hamada, T.; Ishitsuka, M. O.; Ohtani, I.; Kakisawa, H. J. Org. Chem. 1992, $57,1033$.

(141) Gvozdik, S. V; Shorin, S. M.; Makaeva, R. M.; Musavirov, R. R.; Spirikhin, L. V; Urazbaev, V. N.; Musavirov, R. S. Russ. J. Gen. Chem. 2001, 71, 724.

(142) Haasnoot, C. A. G. Generalized 3JHH calculation

http://www.stenutz.eu/conf/haasnoot.php.

(143) De Leeuw, F. A. A. M. De; Haasnoot, C. A. G.; Altona, C. J. Am. Chem. Soc. 1984, 106, 2299.

(144) Earle, M.; Fairhurst, R.; Giles, R.; Heaney, H. Synlett 1991, 10, 728.

(145) Fuwa, H.; Noto, K.; Sasaki, M. Org. Lett. 2010, 12, 1636.

(146) Ikemoto, N.; Schreiber, S. L. J. Am. Chem. Soc. 1992, 114, 2524.

(147) Kocienski, P. J. Protecting Groups, 3rd Editio.; Georg Thieme Verlag: Stuttgart, 2006. 
(148) Horita, K.; Yoshioka, T.; Tanaka, T.; Oikawa, Y.; Yonemitsu, O. Tetrahedron 1982, 42, 3021.

(149) Batista, V. S.; Crabtree, R. H.; Konezny, S. J.; Luca, O. R.; Praetorius, J. M. New J. Chem. 2012, No. 36, 1141.

(150) Evans, D. A.; Hoveyda, A. H. J. Am. Chem. Soc. 1990, 112, 6447.

(151) Ralston, K. J.; Hulme, A. N. Synthesis 2012, 44, 2310.2324.

(152) Smith III, A. B.; Cox, J. M.; Furuichi, N.; Kenesky, C. S.; Zheng, J.; Atasoylu, O.; Wuest, W. M. Org. Lett. 2008, 10, 5501.

(153) Blakemore, P. R.; Browder, C. C.; Hong, J.; Lincoln, C. M.; Nagornyy, P. A.; Robarge, L. A.; Wardrop, D. J.; White, J. D. J. Org. Chem. 2005, 70, 5449.

(154) Corey, E. J.; Shibata, S.; Bakshi, R. K. J. Org. Chem. 1988, 53, 2861.

(155) Zhang, Y.; Deng, L.; Zhao, G. Org. Biomol. Chem. 2011, 9, 4518.

(156) Enders, D.; Lenzen, A.; Backes, M.; Janeck, C.; Catlin, K.; Lannou, M.; Runsink, J.; Raabe, G. J. Org. Chem. 2005, 70, 10538.

(157) Corey, E. J.; Seebach, D. Angew. Chem. Int. Ed. 1965, 4, 1075.

(158) Corey, E. J.; Seebach, D. Angew. Chem. Int. Ed. 1965, 4, 1077.

(159) Seebach, D.; Corey, E. J. J. Org. Chem. 1975, 40, 231.

(160) Yus, M.; Najera, C.; Foubelo, F. Tetrahedron 2003, 59, 6147.

(161) Krief, A. Tetrahedron 1980, 36, 2531.

(162) Coulter, M. M.; Kou, K. G. M.; Galligan, B.; Dong, V. M. J. Am. Chem. Soc. 2010, 132, 16330.

(163) Lipshutz, B. H.; Garcia, E. Tetrahedron Lett. 1990, 31, 7261.

(164) Ide, M.; Nakata, M. Bull. Chem. Soc. Jpn. 1999, 72, 2491.

(165) Suenaga, K.; Hoshino, H.; Yoshii, T.; Mori, K.; Sone, H.; Bessho, Y.; Sakakura, A.; 
Hayakawa, I.; Yamada, K.; Kigoshi, H. Tetrahedron 2006, 62, 7687.

(166) Poornima, B.; Venkanna, A.; Swetha, B.; Kamireddy, K. reddy; Siva, B.; Phani, V. S.; Ummanni, K. R.; Babu, K. S. Tetrahedron 2016, 72, 4789.

(167) Haug, T. T.; Kirsch, S. F. Org. Biomol. Chem. 2010, No. 8, 991.

(168) Felzmann, W.; Castagnolo, D.; Rosenbeiger, D.; Mulzer, J. J. Org. Chem. 2007, 72, 2182.

(169) Collum, D. B. Acc. Chem. Res. 1992, 25, 448.

(170) Zang, Q.; Gulab, S.; Stocker, B. L.; Baars, S.; Hoberg, J. O.Eur. J. Org. Chem. 2011, 4465.

(171) Juaristi, E.; Jiménez-Vázquez, H. A. J. Org. Chem. 1991, 56, 1623.

(172) Wittig, G.; Geissler, G. Leibigs Ann. Chem. 1953, 44, 580.

(173) Wittig, G.; Schollkopf, U. Chem. Ber. 1954, 87, 1318.

(174) Maryanoff, B. E.; Reitz, A. B. Chem. Rev. 1989, 89, 863.

(175) Vadejs, E.; Meier, G. P.; Snoble, K. A. J. J. Am. Chem. Soc. 1981, 103, 2823.

(176) Thonhofer, M.; Santana, A. G.; Fischer, R.; Gomez, A. T.; Saf, R.; Schalli, M.; Stütz, A. E.; Withers, S. G. Carbohydr. Res. 2016, 420, 6.

(177) Pu, L.; Chen, J.; Li, M.; Li, Y.; Xie, J.; Zhou, Q. Adv. Synth. Catal. 2016, 358, 1229.

(178) Gao, Y.; Liu, J.; Wang, L.; Xiao, M.; Du, Y. Eur. J. Org. Chem. 2014, 2092.

(179) Garg, N. K.; Caspi, D. D.; Stoltz, B. M. J. Am. Chem. Soc. 2004, 126, 9552.

(180) Zutter, U.; Iding, H.; Spurr, P.; Wirz, B. J. Org. Chem. 2008, 73, 4895.

(181) Muller, S.; Liepold, B.; Roth, G. J.; Bestmann, H. J. Synlett 1996, 521.

(182) Ohira, S. Synth. Commun. 1989, 19, 561.

(183) Seyferth, D.; Marmor, R. S.; Hilbert, P. J. Org. Chem. 1971, 36, 1379.

(184) Colvin, E. W.; Hamill, B. J. J. Chem. Soc., Chem. Commun. 1973, 151. 
(185) Colvin, E. W.; Hamill, B. J. J. Chem. Soc., Perkin Trans. 1 1977, 869.

(186) Gilbert, J. C.; Weerasooriya, U. J. Org. Chem. 1982, 47, 1837.

(187) Roth, G. J.; Liepold, B.; Müller, S. G.; Bestmann, H. J. Synthesis 2004, 59.

(188) Frisch, M. J.; Trucks, G. W.; Schlegel, H. B.; Scuseria, G. E.; Robb, M. A.; Cheeseman, J. R. Scalmani, G.; Barone, V.; Mennucci, B.; Petersson, G. A.; Nakatsuji, H.; Caricato, M.; Li, X.; Hratchian, H. P.; Izmaylov, A. F.; Bloino, J.; Zheng, G.; Sonnenberg, J. L.; Hada, M.; Ehara, M.; Toyota, K.; Fukuda, R.; Hasegawa, J.; Ishida, M.; Nakajima, T.; Honda, Y.; Kitao, O.; Nakai, H.; Vreven, T.; Montgomery, J. A. J.; Peralta, J. E.; Ogliaro, F.; Bearpark, M.; Heyd, J. J.; Brothers, E.; Kudin, K. N.; Staroverov, V. N.; Kobayashi, R.; Normand, J.; Raghavachari, K.; Rendell, A.; Burant, J. C.; lyengar, S. S.; Tomasi, J.; Cossi, M.; Rega, N.; Millam, J. M.; Klene, M.; Knox, J. E.; Cross, J. B.; Bakken, V.; Adamo, C.; Jaramillo, J.; Gomperts, R.; Stratmann, R. E.; Yazyev, O.; Austin, A. J.; Cammi, R.; Pomelli, C.; Ochterski, J. W.; Martin, R. L.; Morokuma, K.; Zakrzewski, V. G.; Voth, G. A.; Salvador, P.; Dannenberg, J. J.; Dapprich, S.; Daniels, A. D.; Farkas, Ö.; Foresman, J. B.; Ortiz, J. V.; Cioslowski, J.; Fox, D. J. Gaussian Inc.; Wallingford CT 2010.

(189) Hashmi, M. A.; Andreassend, S. K.; Keyzers, A. Phys. Chem. Chem. Phys. 2016, 18, 24506.

(190) Pandey, G.; Kapur, M. Tetrahedron Lett. 2000, 41, 8821.

(191) Brown, H. C.; Scouten, C. G.; Liottaib, R. J. Am. Chem. Soc. 1979, 101, 96.

(192) Brown, H. C.; Gupta, S. K. J. Am. Chem. Soc. 1972, 94, 4370.

(193) Yadav, J. S.; Krishna, V. H.; Srilatha, A.; Somaiah, R.; Reddy, B. V. S. Synthesis 2010, 17, 3004.

(194) Huang, Z.; Negishi, E. Org. Lett. 2006, 8, 3675.

(195) Pereira, S.; Srebnik, M. Organometallics 1995, 14, 3127.

(196) Corey, E. J.; Chaykovsky, M. J. J. Am. Chem. Soc. 1962, 84, 867. 
(197) Aggarwal, V. K.; Richardson, J. Chem. Commun. 2003, 2644.

(198) Meinwald, J.; Labana, S. S.; Chadha, M. S. J. Am. Chem. Soc. 1963, 85, 582.

(199) Borredon, M. E.; Delmas, M.; A, G. Tetrahedron 1987, 43, 3945.

(200) Fei, Z.; Wu, Q.; Zhang, F.; Cao, Y.; Liu, C.; Shieh, W.; Xue, S.; McKenna, J.; Prasad, K.; Prashad, M.; Baeschlin, D.; Namoto, K. J. Org. Chem. 2008, 73, 9016.

(201) Lemini, C.; Ordoez, M.; Perez-Flores, J.; Cruz-Almanza, R. Synth. Commun. 1995, 25, 2695.

(202) Covell, D. J.; Vermeulen, N. A.; Labenz, N. A.; White, M. C. Angew. Chem. Int. Ed. 2006, 45, 8217 .

(203) Payne, G. B. J. Org. Chem. 1962, 27, 3819.

(204) Ibuka, T.; Nakai, K.; Habashita, H.; Hotta, Y.; Otaka, A.; Tamamura, H.; Fujii, N.; Mimura, N.; Miwa, Y.; Taga, T.; Chounan, Y.; Yamamoto, Y. J. Org. Chem. 1995, 60, 2044.

(205) Wu, M. H.; Hansen, K. B.; Jacobsen, E. N. Angew. Chem. Int. Ed. 1999, 38, 2012.

(206) White, D. E.; Tadross, P. M.; Lu, Z.; Jacobsen, E. N. Tetrahedron 2014, 70, 4165.

(207) Addada, R. R.; Regalla, V. R.; Vajja, M. R.; Vema, V. N.; Anna, V. R. Tetrahedron Lett. 2016, 57, 3933.

(208) Venkanna, A.; Siva, B.; Poornima, B.; Babu, K. S.; Rao, J. M. Tetrahedron Lett. 2014, 55, 403.

(209) Smith, A. B.; Pitram, S. M.; Boldi, A. M.; Gaunt, M. J.; Sfouggatakis, C.; Moser, W. H. J. Am. Chem. Soc. 2003, 125, 14435.

(210) Stockley, M.; Clegg, W.; Fontana, G.; Golding, B. T.; Martin, N.; Rigoreau, L. J. M.; Smith, C. M.; Griffin, R. J. Bioorg. Med. Chem. Lett. 2001, 11, 2837.

(211) Probst, N. P.; Haudrechy, A.; Ple, K. J. Org. Chem. 2008, No. 73, 4338.

(212) Casey, E. M.; Tho, F.; Harvey, J. E.; Teesdale-spittle, P. H. Tetrahedron 2011, 67, 9376. 
(213) Slade, M. C.; Johnson, J. S. Beilstein J. Org. Chem. 2013, 9, 166.

(214) Williams, D. R.; Jass, P. A.; Tse, H. A.; Gaston, R. D. J. Org. Chem. 1990, 112, 4552.

(215) Zengeya, T. T.; Kulkarni, R. A.; Meier, J. L. Org. Lett. 2015, 17, 2326-2329.

(216) Nicolaou, K. C.; Ajito, K.; Patron, A. P.; Khatuya, H.; Richter, P. K.; Bertinato, P. J. Am. Chem. Soc. 1996, 118, 3059.

(217) Langille, N. F.; Dakin, L. A.; Panek, J. S. Org. Lett. 2003, 5, 3667.

(218) Tanemura, K.; Dohya, H.; Imamura, M.; Suzuki, T.; Horaguchi, T. J. Chem. Soc., Perkin Trans. 1 1996, 453.

(219) Furneaux, R. H.; Schramm, V. L.; Tyler, P. C. Bioorg. Med. Chem. 1999, 7, 2599.

(220) Evans, D. A.; Takacs, J. M.; Mcgee, L. R.; Ennis, M. D.; Mathre, D. J.; Bartroli, J. Pure Appl. Chem. 1981, 53, 1109.

(221) Dubois, J. E.; Fellman, P. Tetrahedron Lett. 1975, 1225.

(222) Heathcock, C. H.; Buse, C. T.; Kleschick, W. A.; Pirrung, M. C.; Sohn, J. E.; Lampe, J. J. Org. Chem. 1980, 1066.

(223) Evans, D. A.; Ennis, M. D.; Mathre, D. J. J. Am. Chem. Soc. 1982, 104, 1737.

(224) Heravi, M. M.; Zadsirjan, V.Tetrahedron Asymm. 2013, 24 (144), 1149.

(225) Ihara, M.; Katsumata, A.; Setsu, F.; Tokunaga, Y.; Fukumoto, K. J. Org. Chem. 1996, 61, 677.

(226) Wu, D.; Forsyth, C. J. Org. Lett. 2013, 15, 1178.

(227) Cozzi, P. G.; Kotrusz, P. J. Am. Chem. Soc. 2006, 128, 4940.

(228) Trost, B. M.; Yang, H.; Thiel, O. R.; Frontier, A. J.; Brindle, C. S. J. Am. Chem. Soc. 2007, $129,2206$.

(229) Bezzenine-Lafollee, S.; Guibe, F.; Villar, H.; Zriba, R. Tetrahedron 2004, 60 (32), 6931.

(230) Yadav, J. S.; Aravind, S.; Gundluru, M. K.; Reddy, B. V. S. Synthesis 2012, 3077. 
(231) Roulland, E. Angew. Chem. Int. Ed. 2008, 47, 3762.

(232) Roulland, E. Angew. Chem. Int. Ed. 2008, 47, 3762.

(233) Perla, R.; Fadnavis, N. W. Eur. J. Chem. 2015, 6 (1), 93.

(234) Dimopoulos, P.; Athlan, A.; Manaviazar, S.; George, J.; Walters, M.; Lazarides, L.; Aliev, A. E.; Hale, K. J. Org. Lett. 2005, 7, 5369.

(235) Arthuis, M.; Beaud, R.; Gandon, V.; Roulland, E. Angew. Chem. Int. Ed. 2012, 51, 10510.

(236) Evans, D. A.; Gage, J. R.; Leighton, J. L.; Kim, A. S. J. Org. Chem. 1992, 57, 1961.

(237) Williams, D. R.; Plummer, S. V; Patnaik, S. Tetrahedron 2011, 67, 5083.

(238) Yokokawa, F.; Asano, T.; Shioiri, T. Tetrahedron 2001, 57, 6311.

(239) Owen, R. M.; Roush, W. R. Org. Lett. 2005, 7, 3941. 
Appendix 1: NMR spectra 
<smiles>CCCC(=O)N1CCOC1=O</smiles>

Bn 27
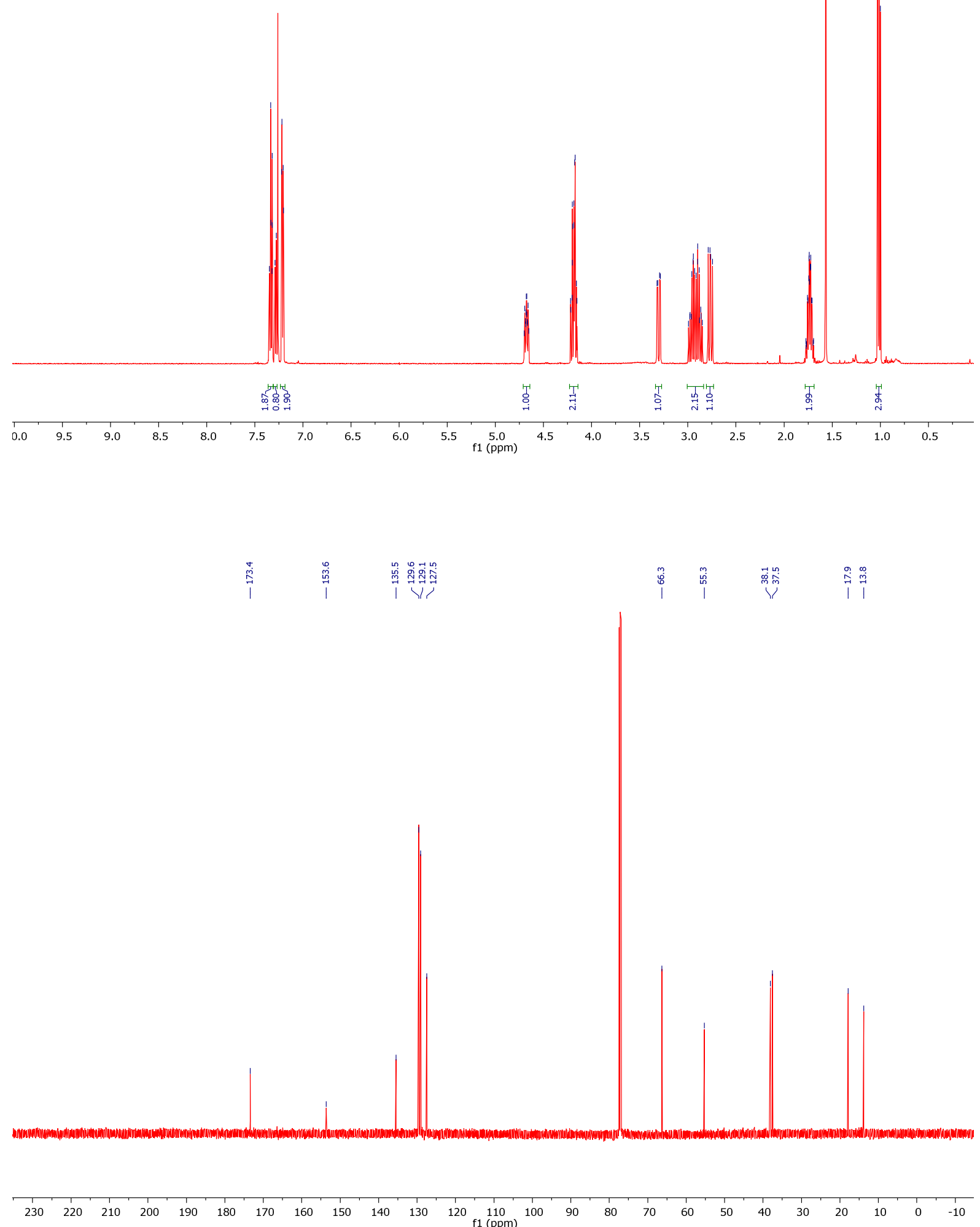
<smiles>CCC(CO)C(=O)N1C(=O)OCC1Br</smiles>

59
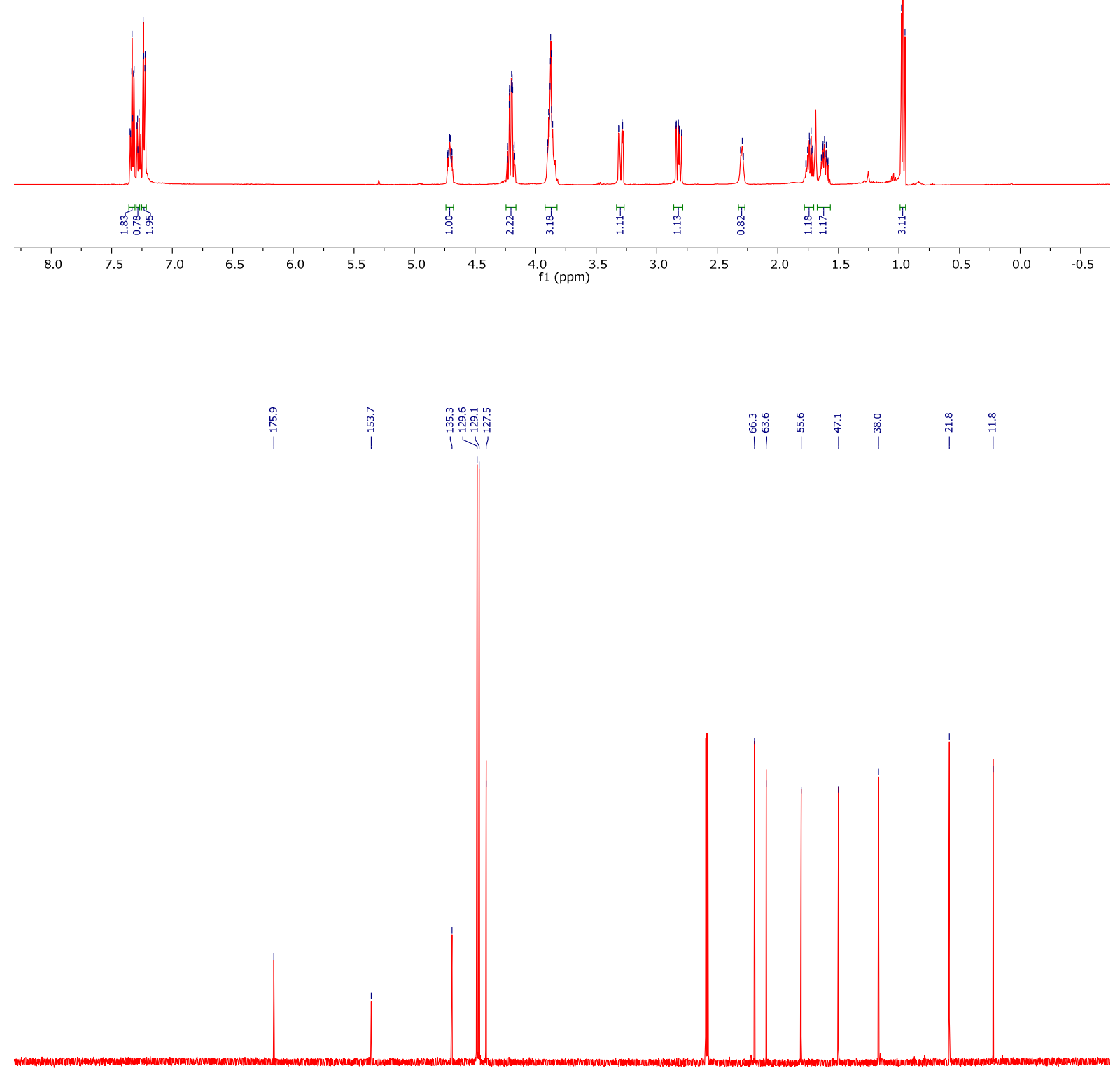

$\begin{array}{lllllllllllllllllllllllllll}230 & 220 & 210 & 200 & 190 & 180 & 170 & 160 & 150 & 140 & 130 & 120 & 110 & 100 & 90 & 80 & 70 & 60 & 50 & 40 & 30 & 20 & 10 & 0 & -10\end{array}$ 
<smiles>CCC(COCOCC(CC)C(=O)N1C(=O)OCC1Br)C(=O)N1C(=O)OCC1Br</smiles>

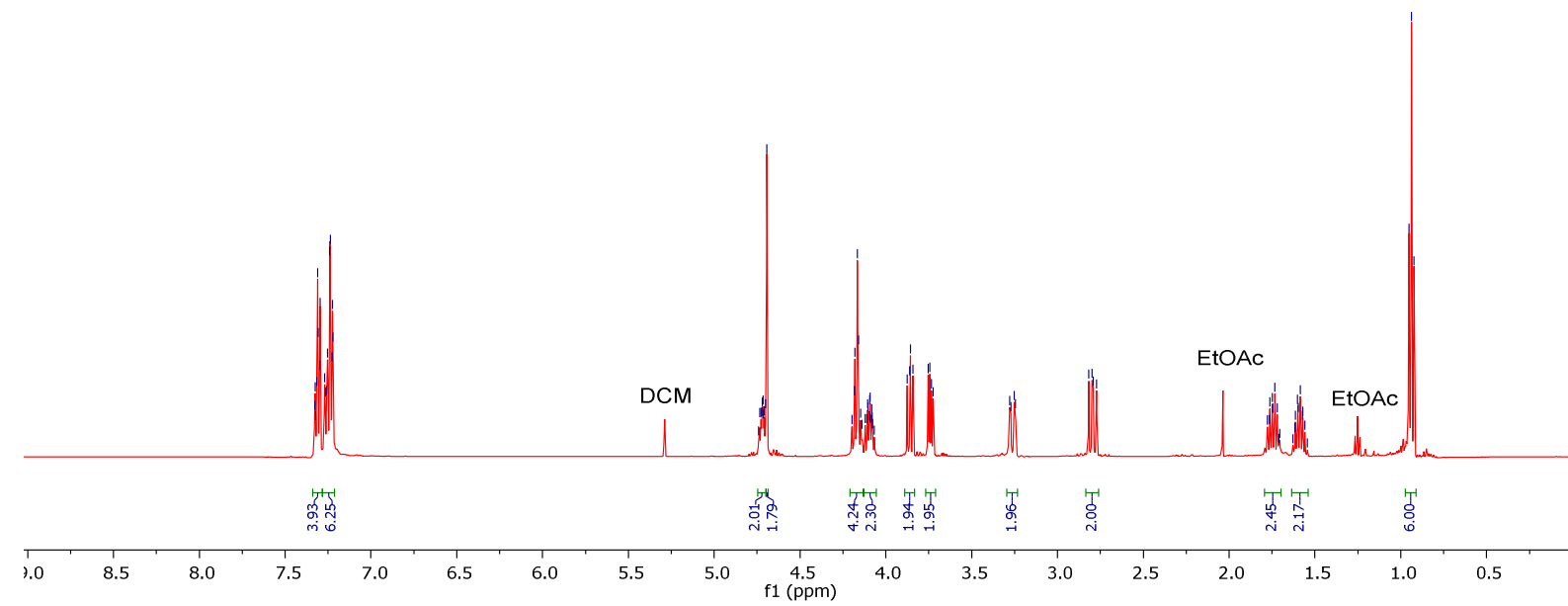

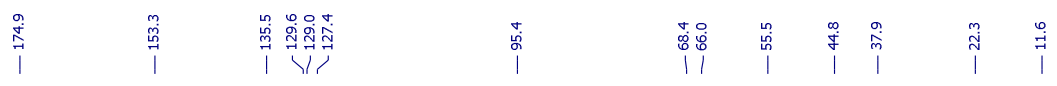

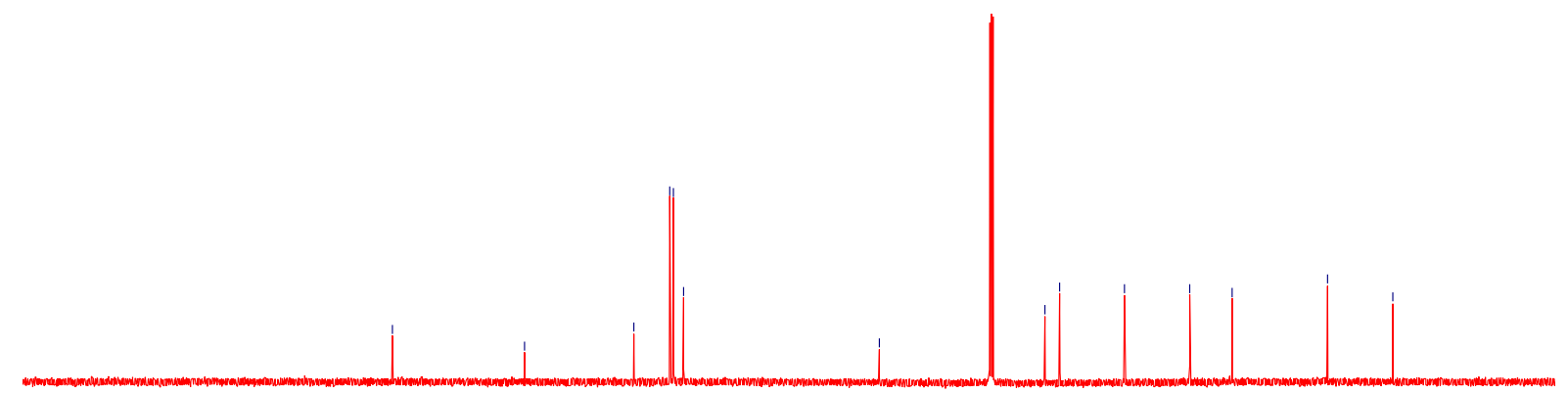

$\begin{array}{llllllllllllllllllllllllllllllll}230 & 220 & 210 & 200 & 190 & 180 & 170 & 160 & 150 & 140 & 130 & 120 & 110 & 100 & 90 & 80 & 70 & 60 & 50 & 40 & 30 & 20 & 10 & 0 & -10\end{array}$ 
<smiles>CCC(CO[SnH3])C(=O)N1C(=O)OC[C@H]1Br</smiles>

60
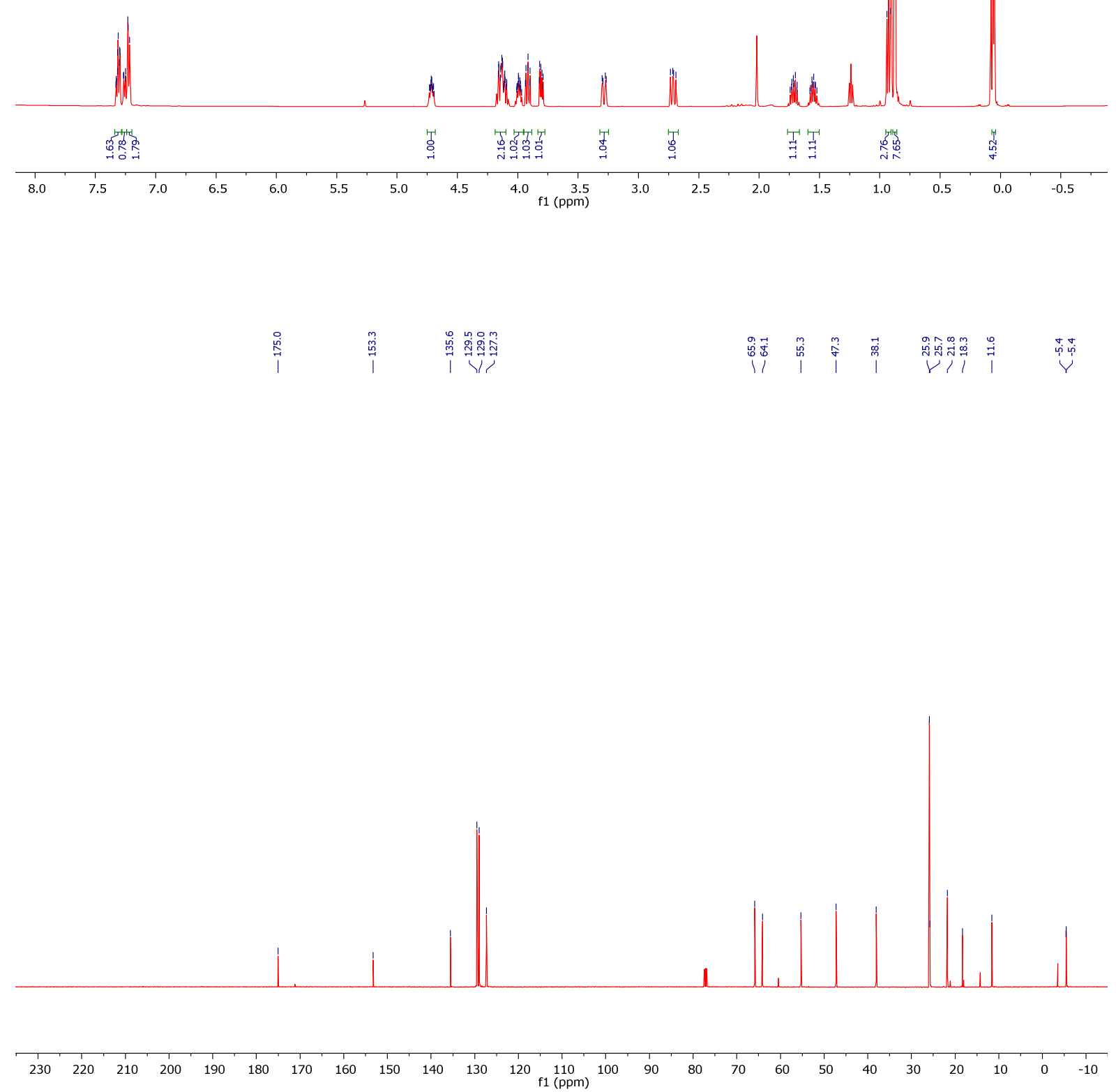

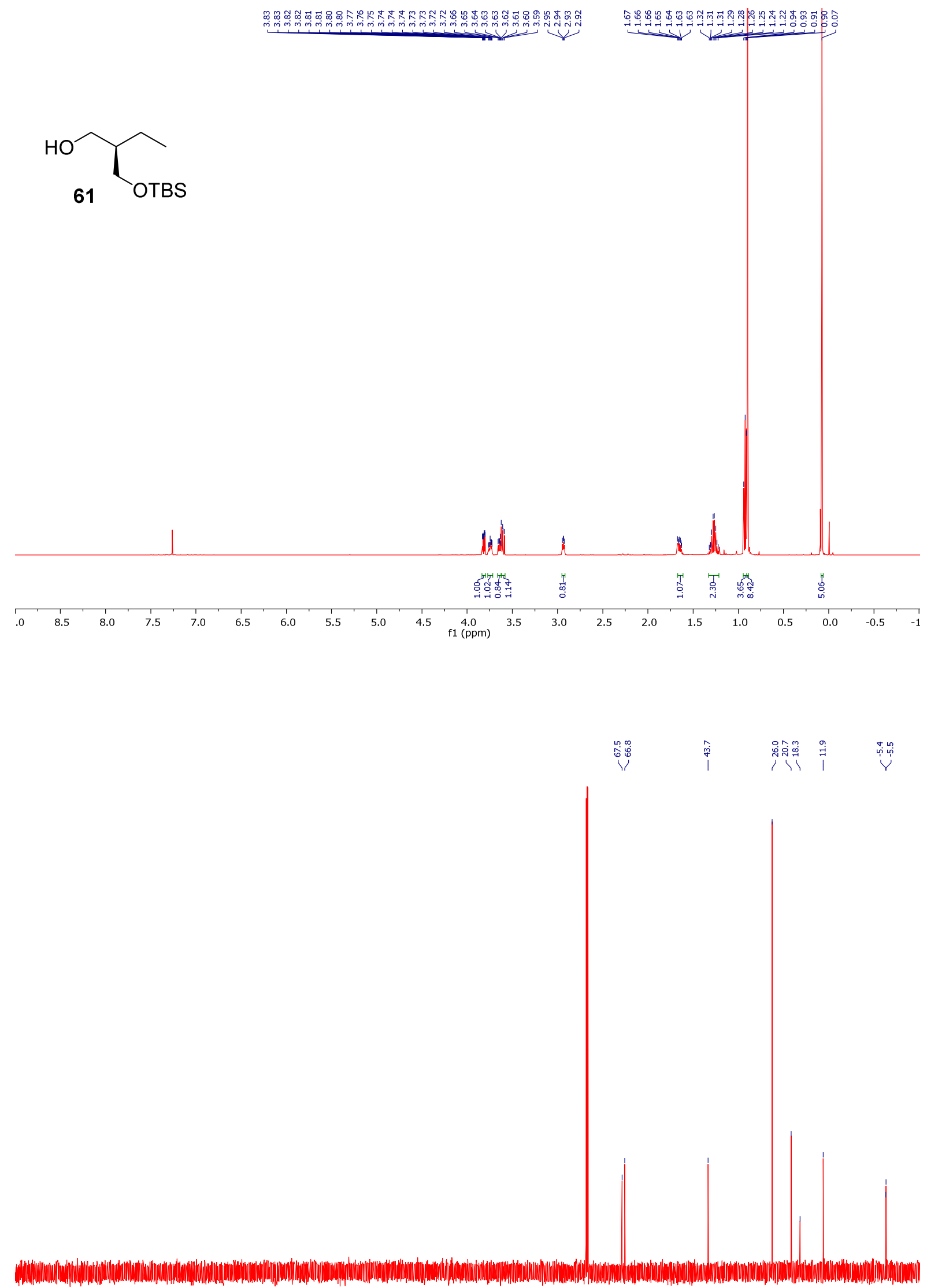

$\begin{array}{llllllllllllllllllllllllllll}230 & 220 & 210 & 200 & 190 & 180 & 170 & 160 & 150 & 140 & 130 & 120 & 110 & 100 & 90 & 80 & 70 & 60 & 50 & 40 & 30 & 20 & 10 & 0 & -10\end{array}$ 


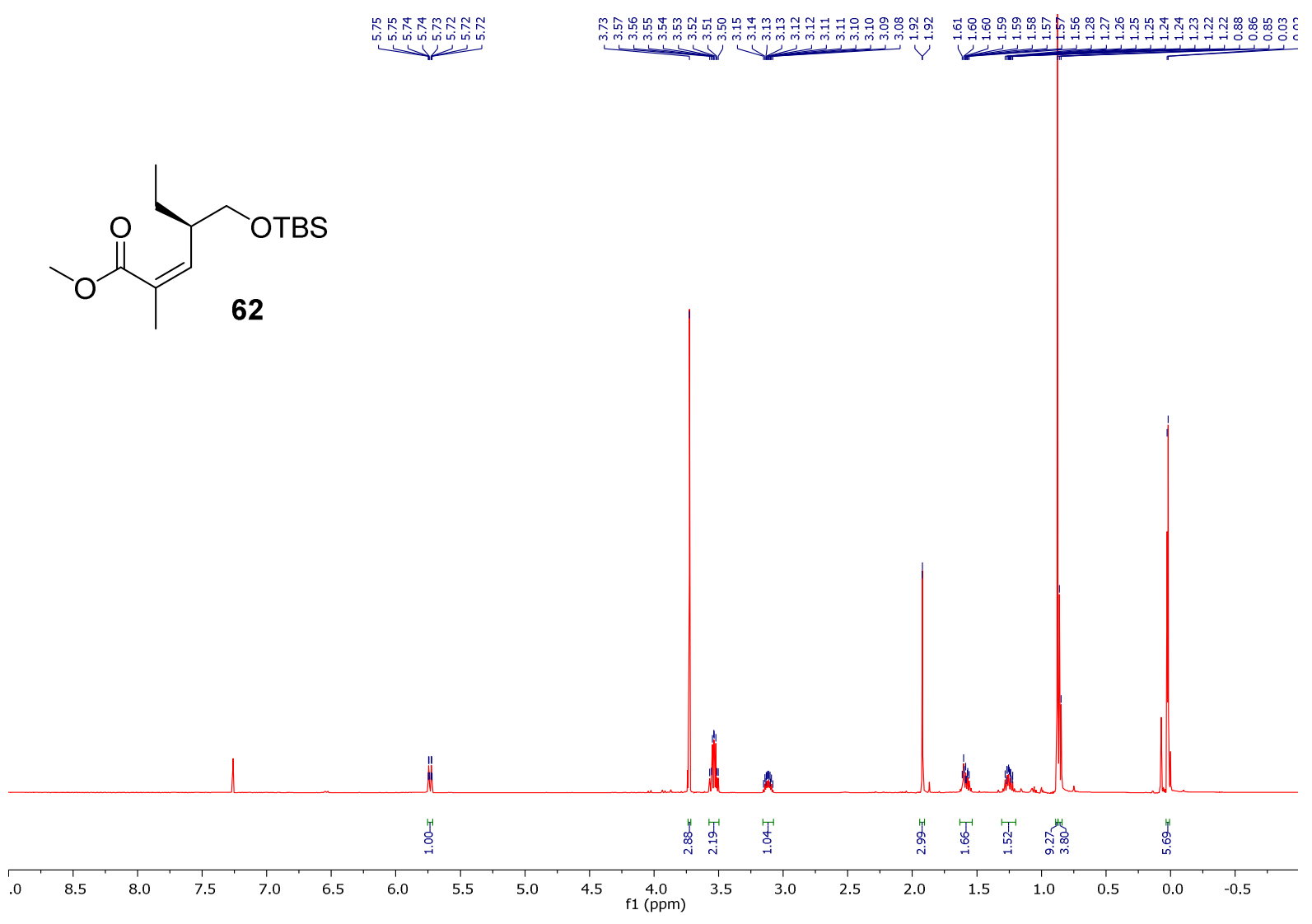




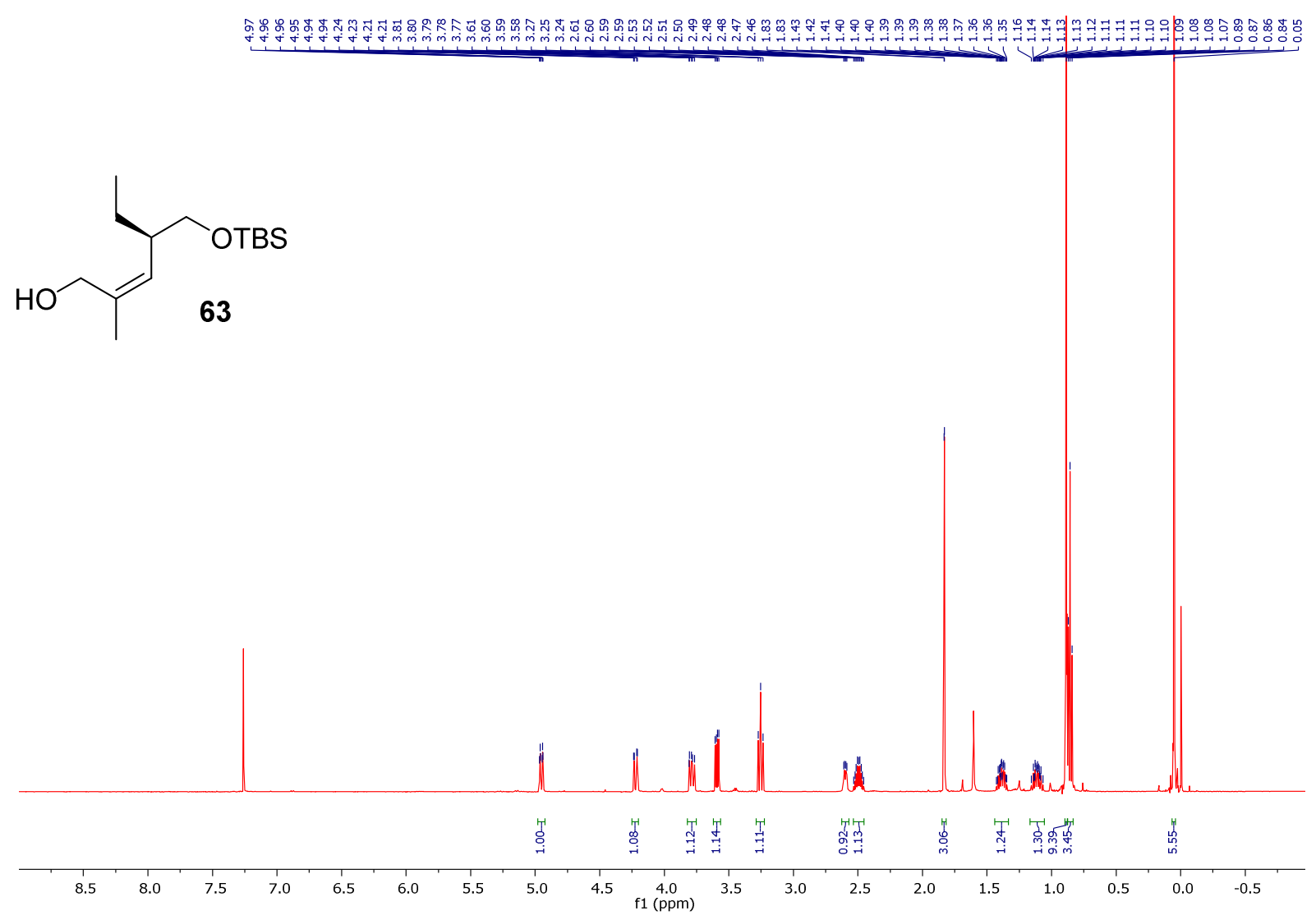

II

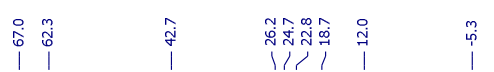

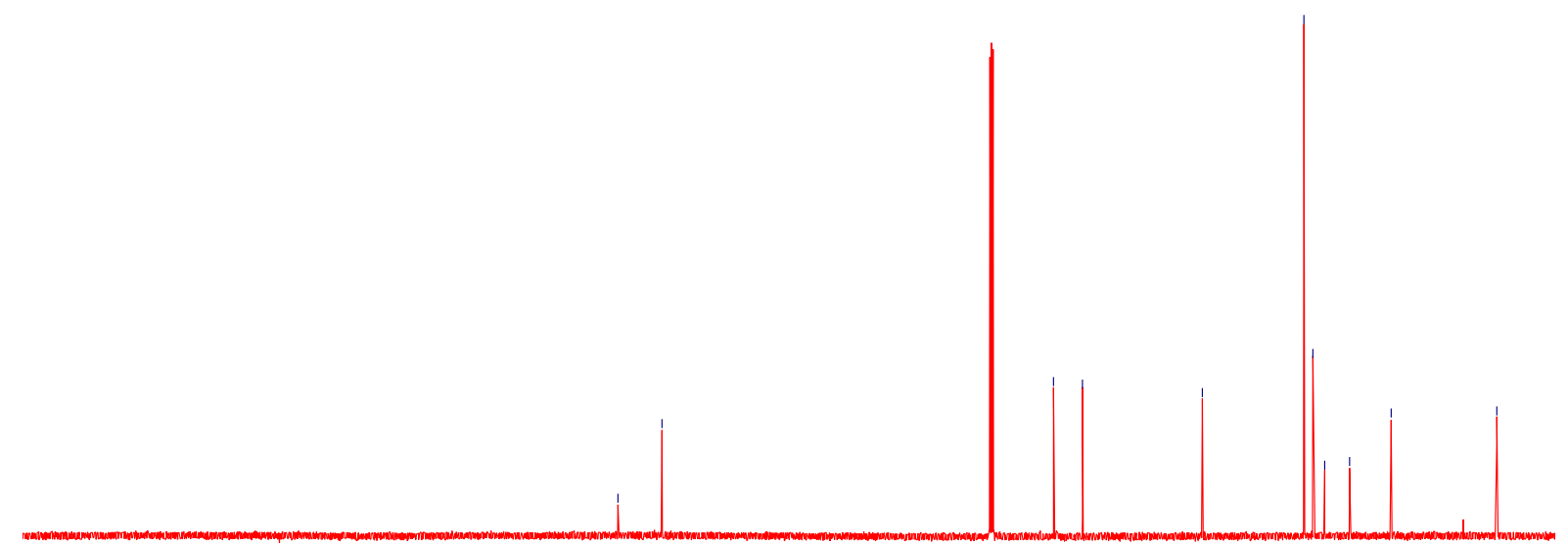

\begin{tabular}{lllllllllllllllllllllllllllllllll}
\hline 230 & 220 & 210 & 200 & 190 & 180 & 170 & 160 & 150 & 140 & 130 & 120 & 110 & 100 & 90 & 80 & 70 & 60 & 50 & 40 & 30 & 20 & 10 & 0 & -10
\end{tabular} 
<smiles>CCC(/C=C(/C)[C@@H](O)CC(C)=O)C[SeH2+]</smiles>

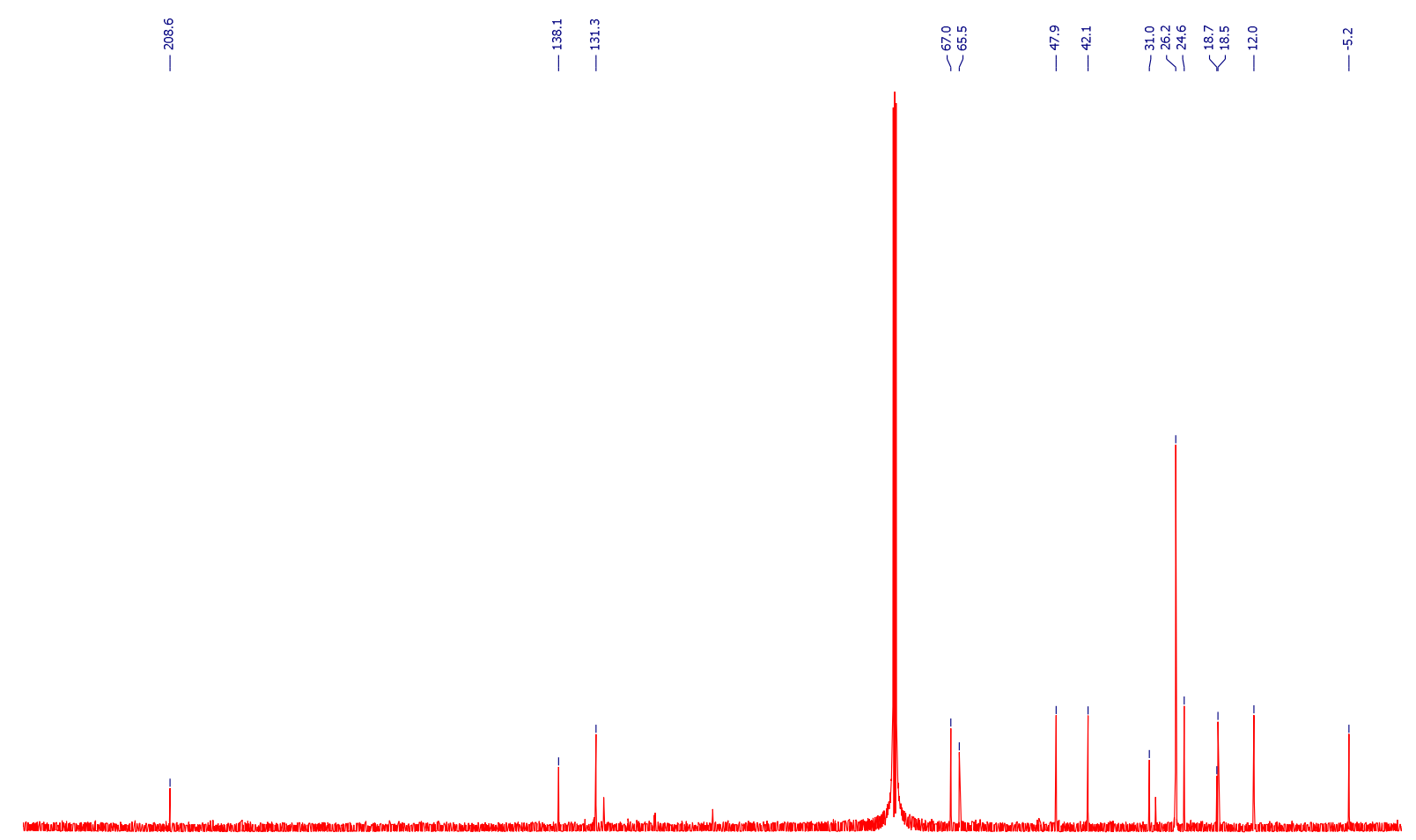

\begin{tabular}{llllllllllllllllllllllllllllllllll}
\hline 230 & 220 & 210 & 200 & 190 & 180 & 170 & 160 & 150 & 140 & 130 & 120 & 110 & 100 & 90 & 80 & 70 & 60 & 50 & 40 & 30 & 20 & 10 & 0 & -10
\end{tabular} 

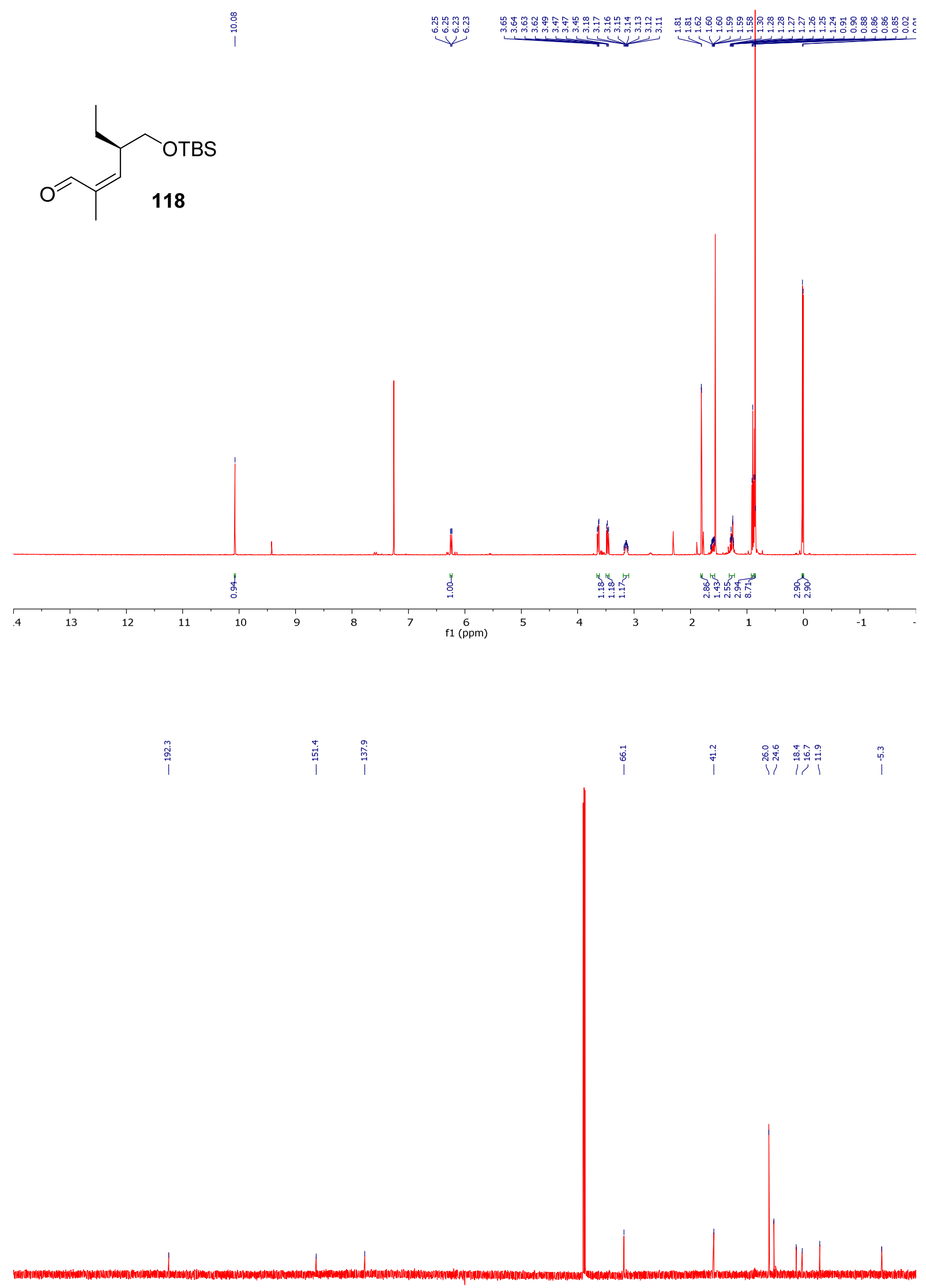

$\begin{array}{lllllllllllllllllllllllllllll}230 & 220 & 210 & 200 & 190 & 180 & 170 & 160 & 150 & 140 & 130 & 120 & 110 & 100 & 90 & 80 & 70 & 60 & 50 & 40 & 30 & 20 & 10 & 0 & -10\end{array}$ 


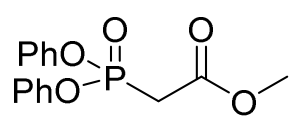

121

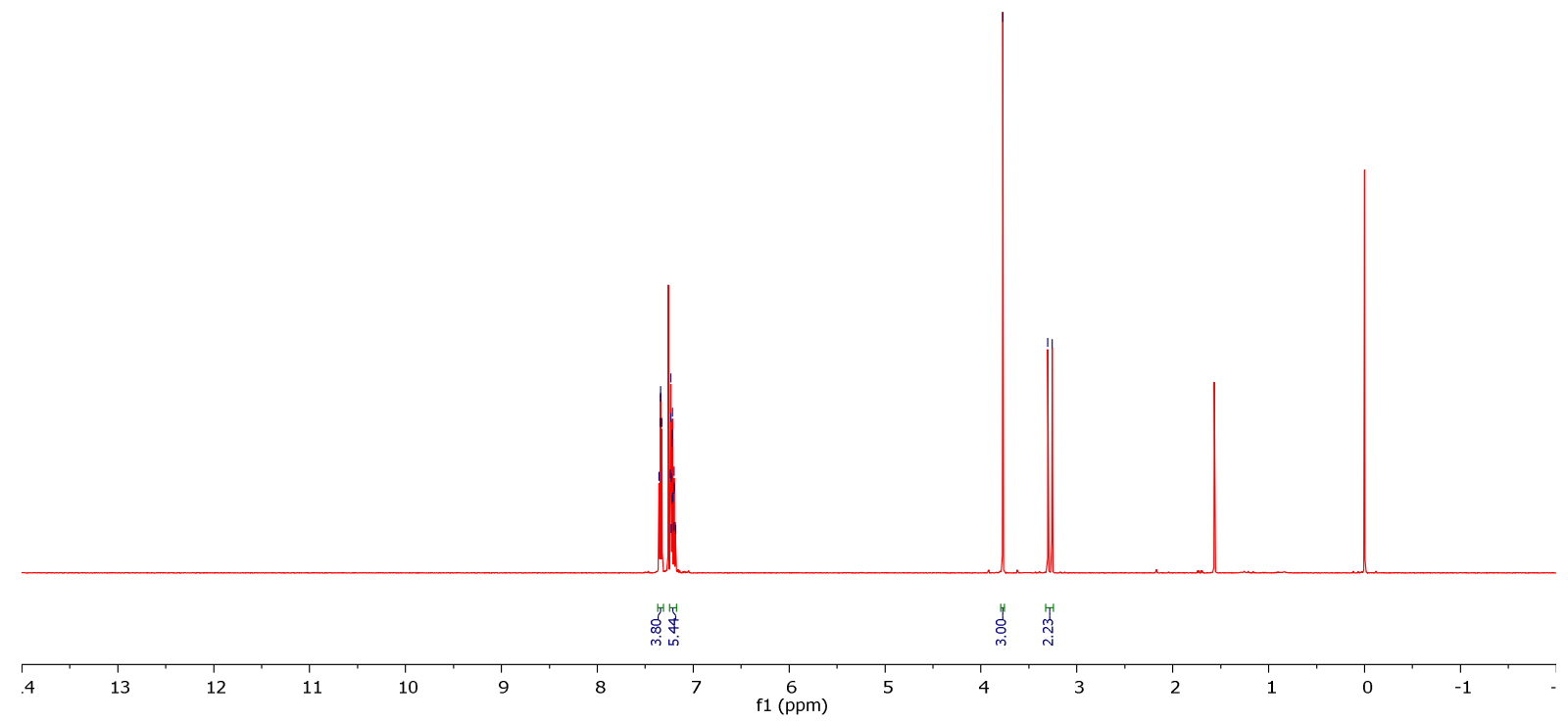

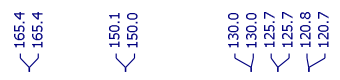

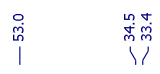

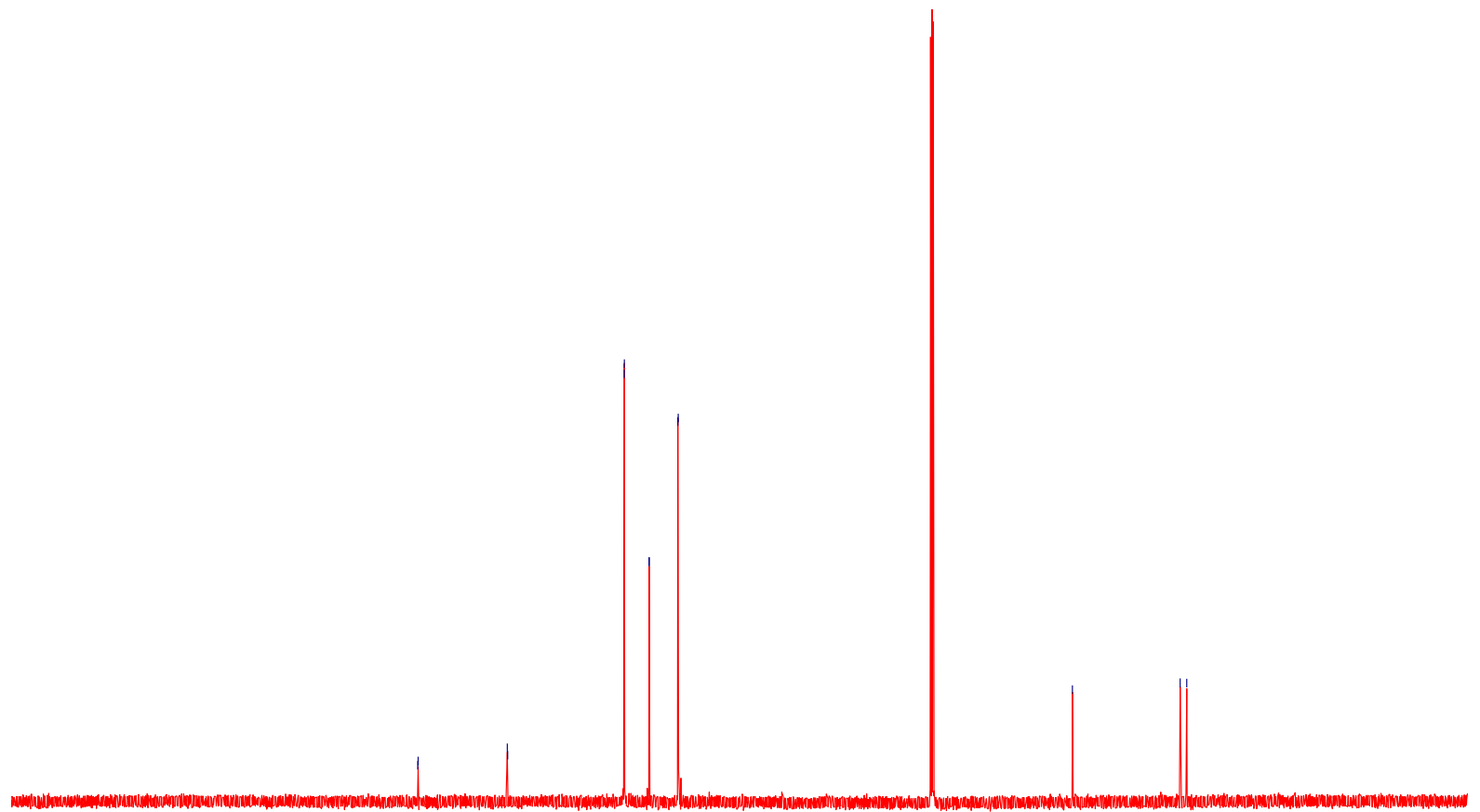

$\begin{array}{llllllllllllllllllllllllllll}230 & 220 & 210 & 200 & 190 & 180 & 170 & 160 & 150 & 140 & 130 & 120 & 110 & 100 & 90 & 80 & 70 & 60 & 50 & 40 & 30 & 20 & 10 & 0 & -10\end{array}$ 


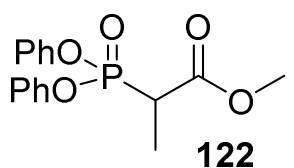

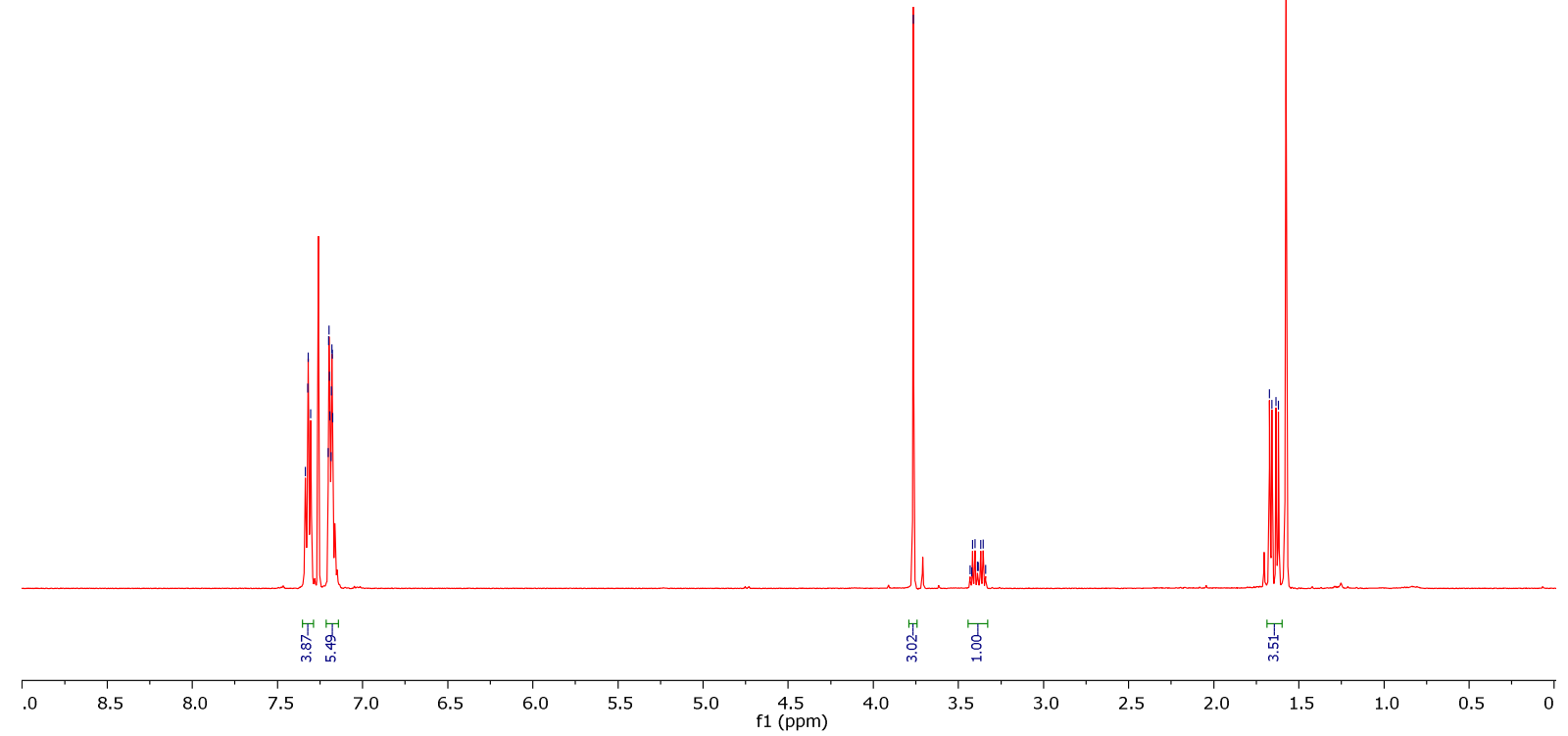

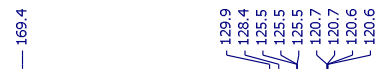

郘 高哭

ํํำ

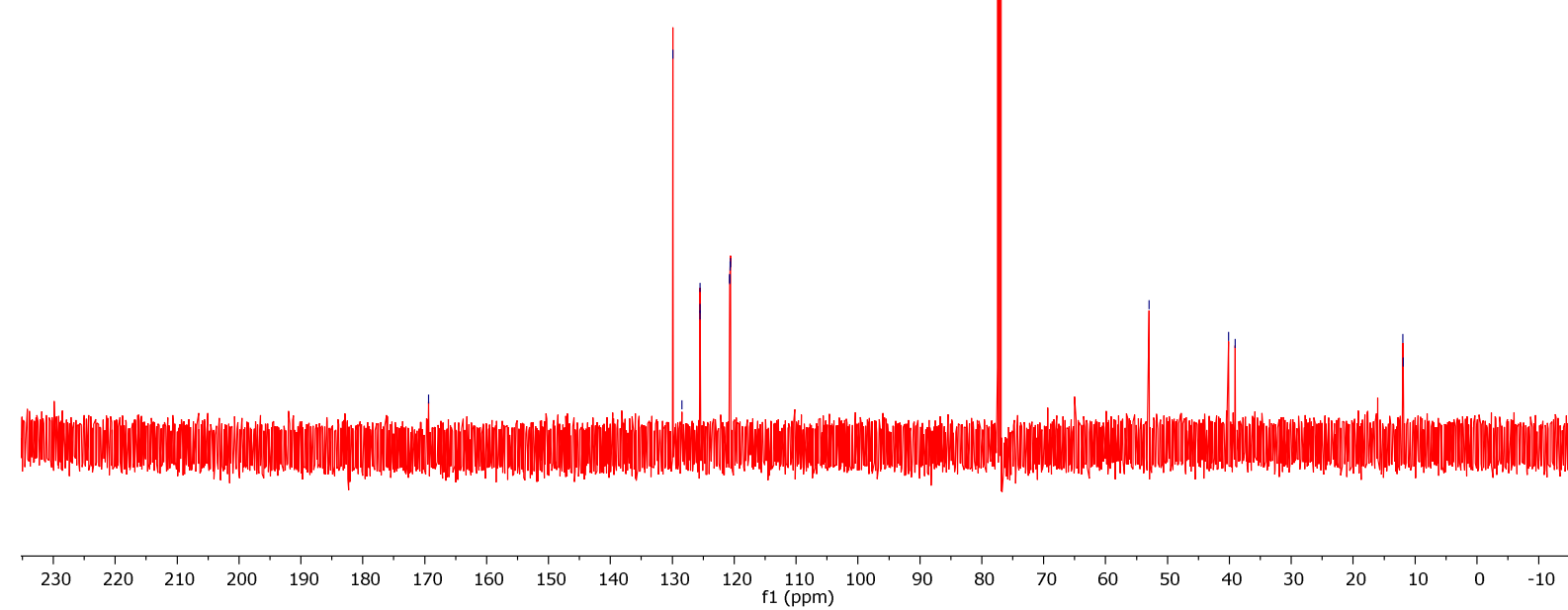



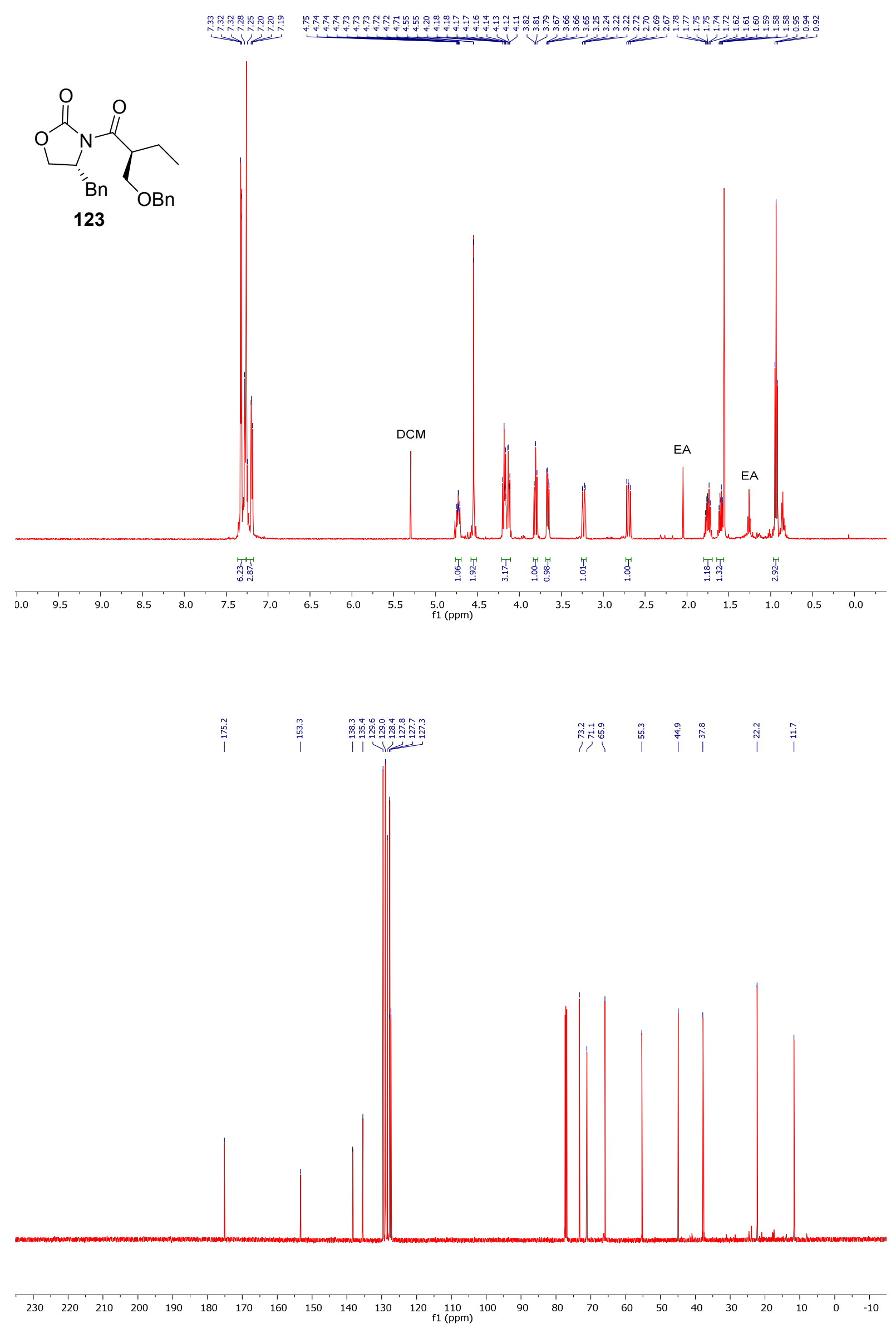


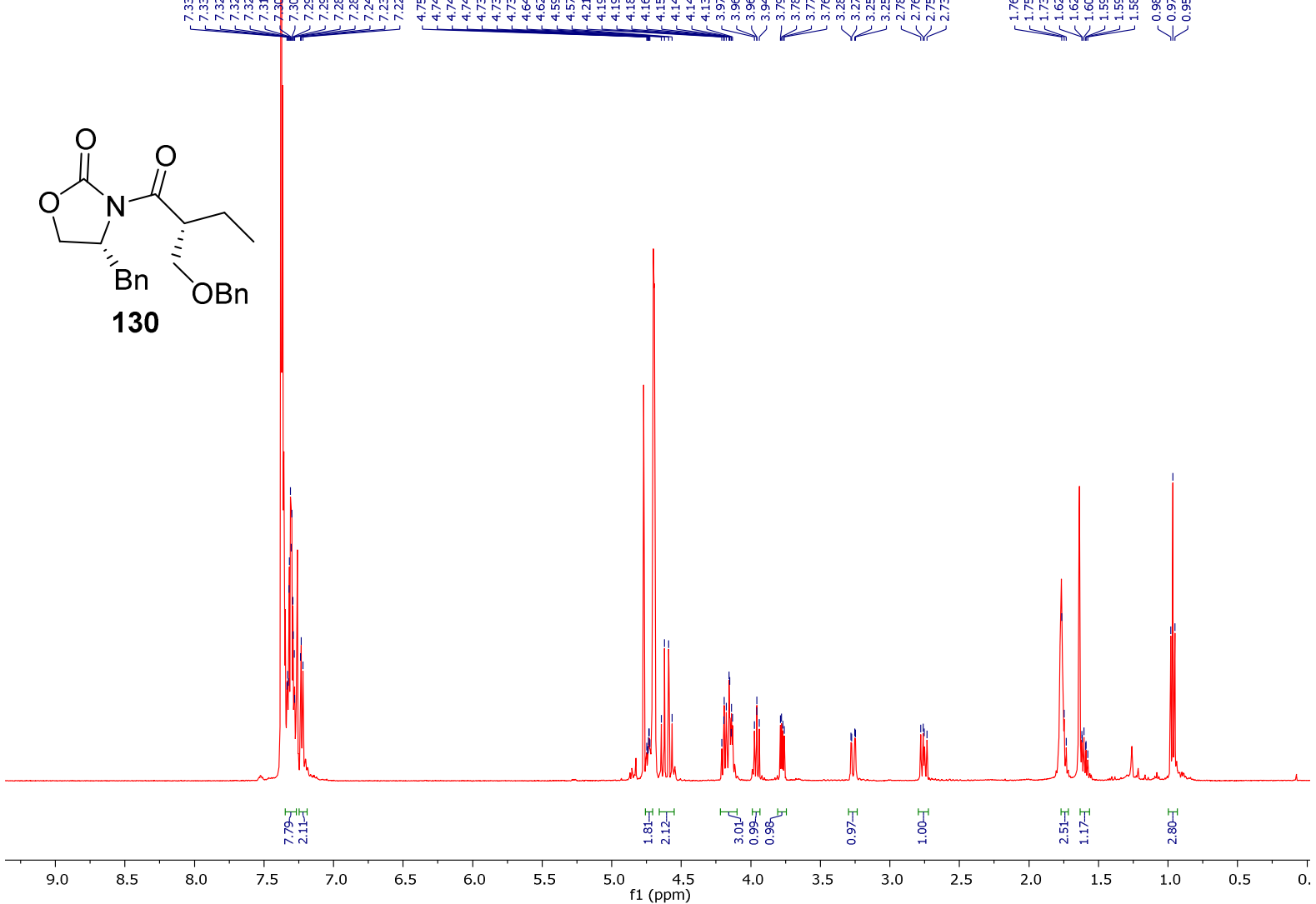

|

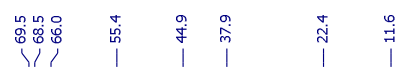

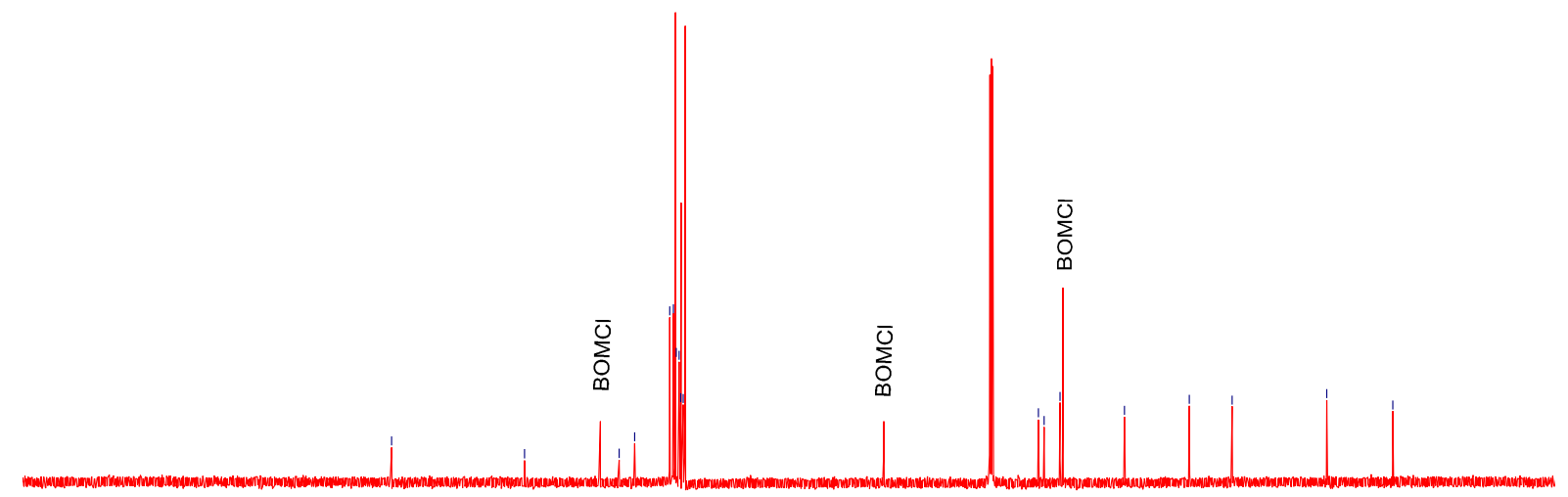

$\begin{array}{llllllllllllllllllllllllllllll}230 & 220 & 210 & 200 & 190 & 180 & 170 & 160 & 150 & 140 & 130 & 120 & 110 & 100 & 90 & 80 & 70 & 60 & 50 & 40 & 30 & 20 & 10 & 0 & -10\end{array}$ 
<smiles>[Z16][14CH2]C[C@H](CC)CO</smiles>

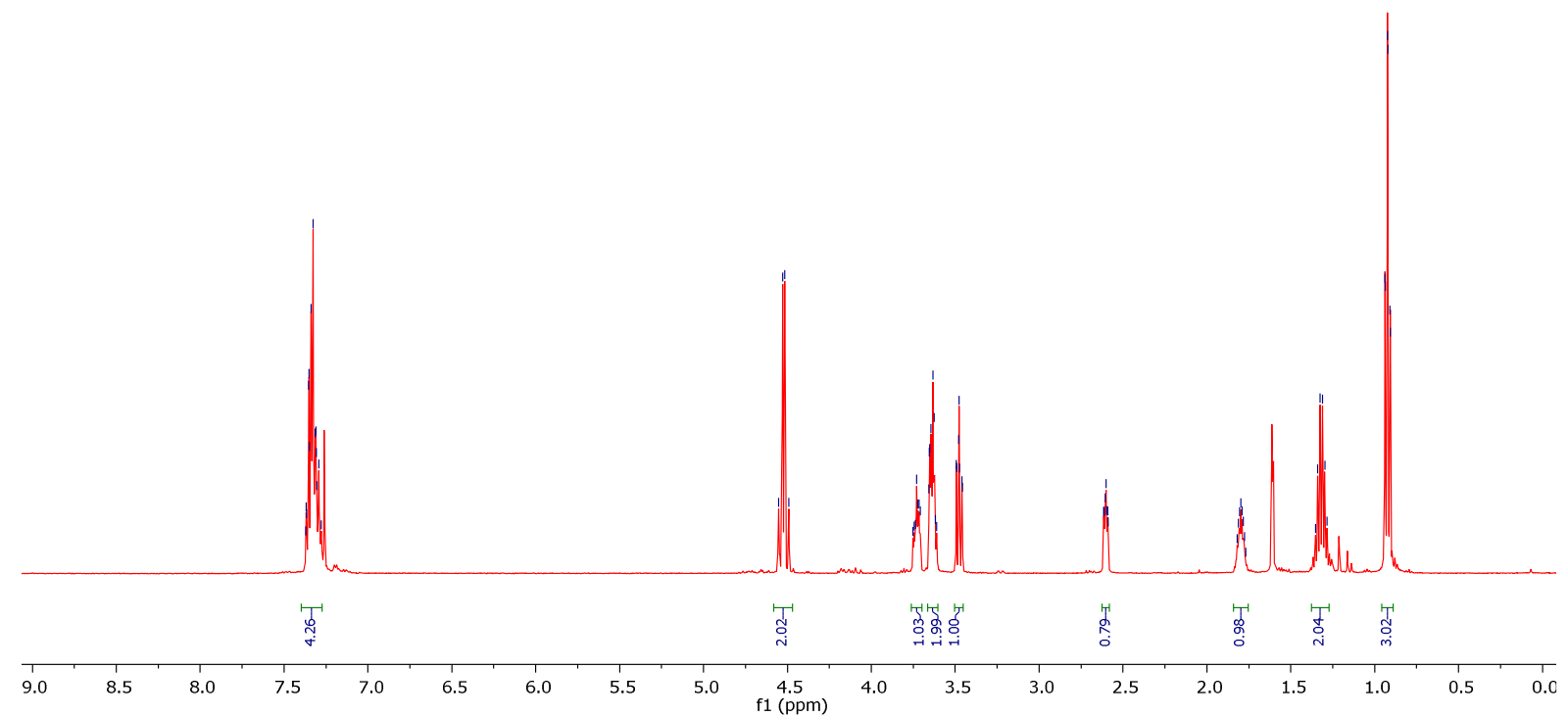

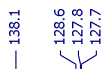

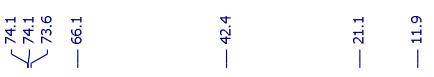

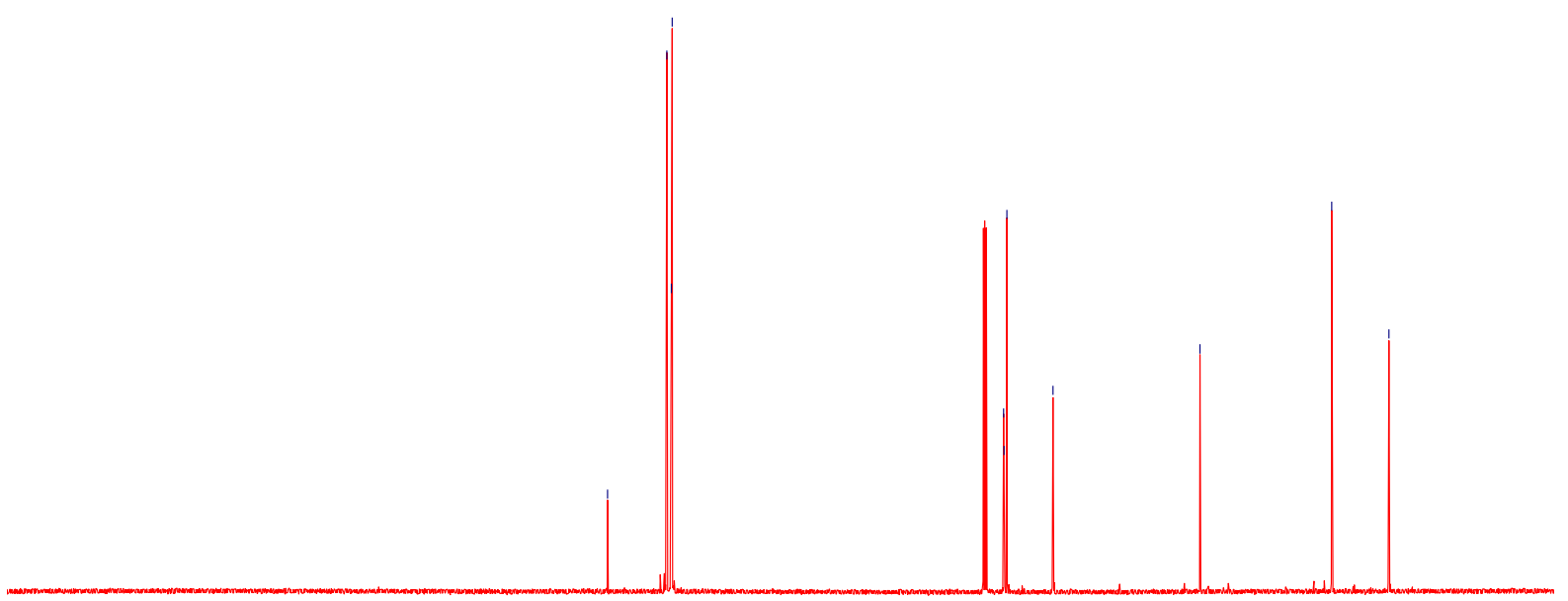

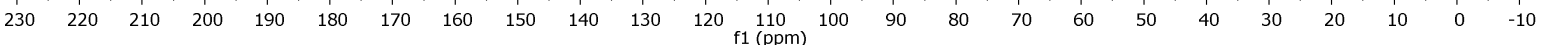



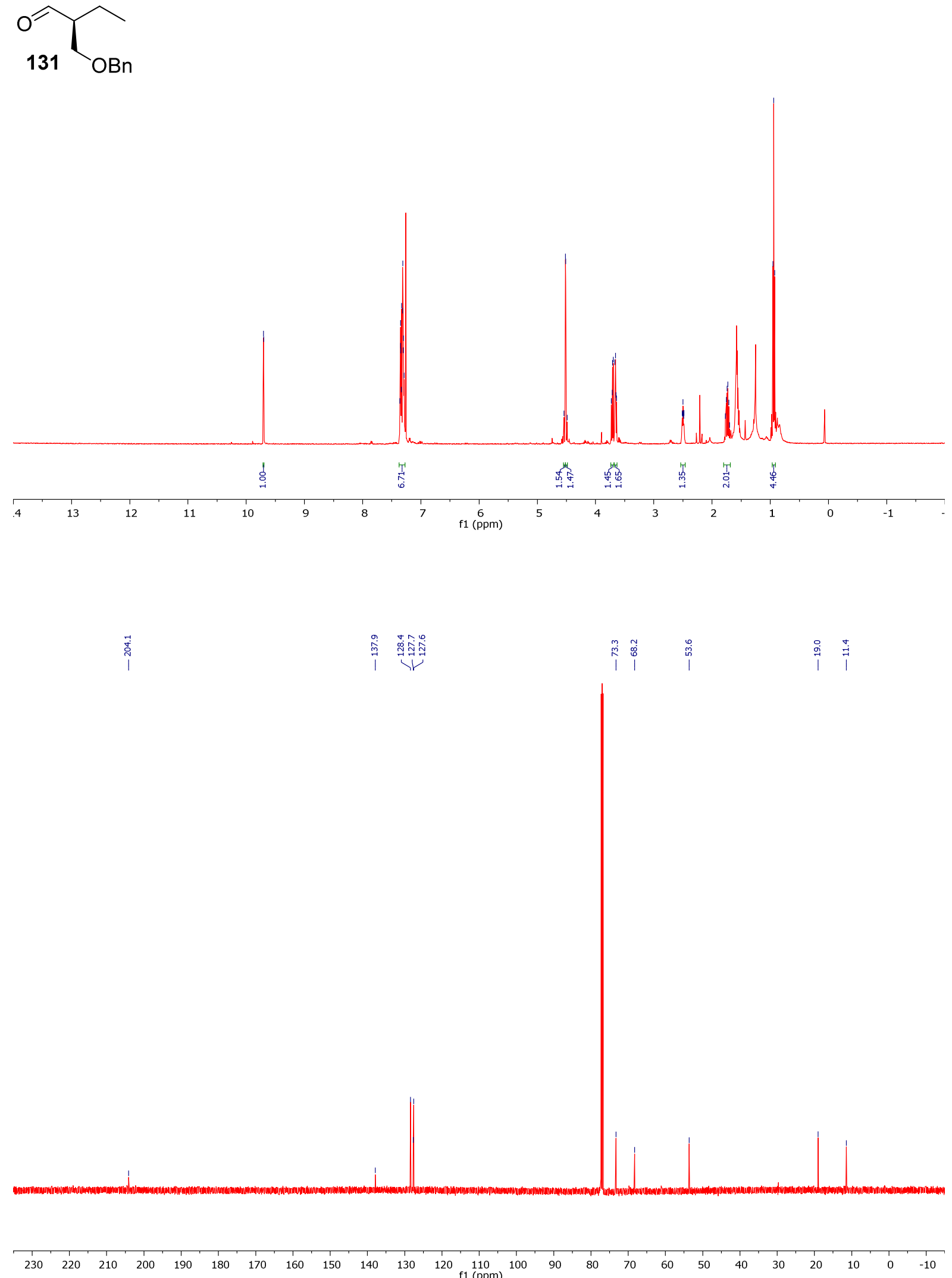
<smiles>CCC(/C=C(/C)C(=O)OC)COCc1ccccc1</smiles>

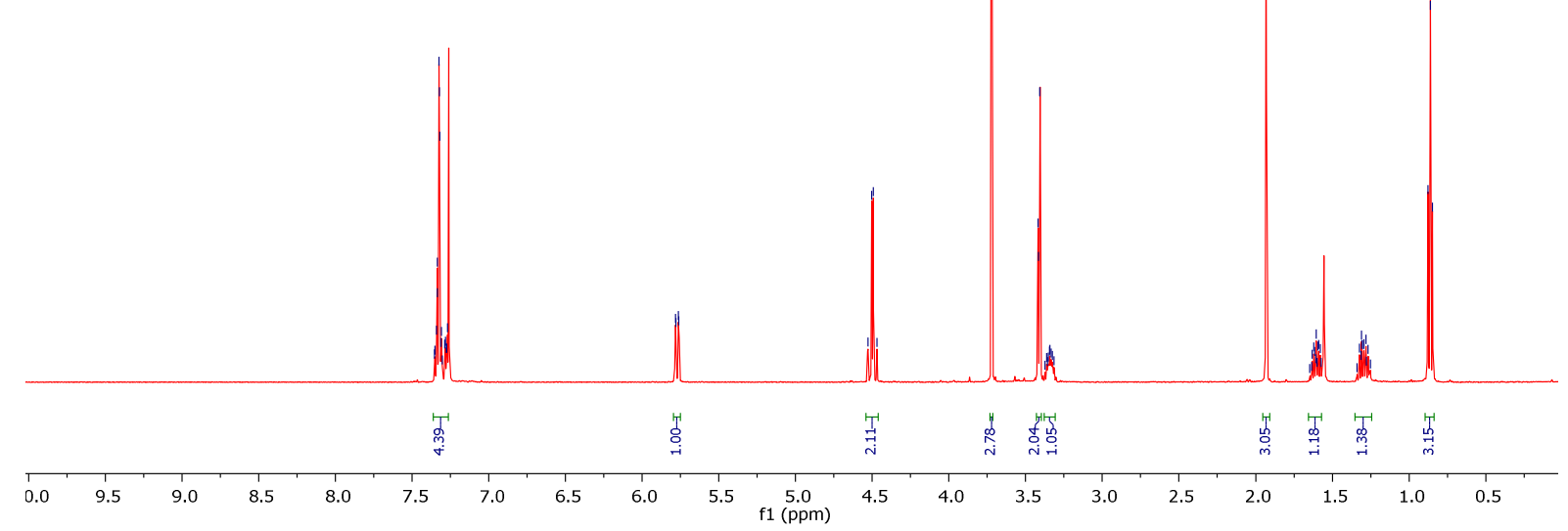

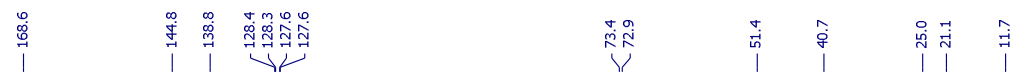

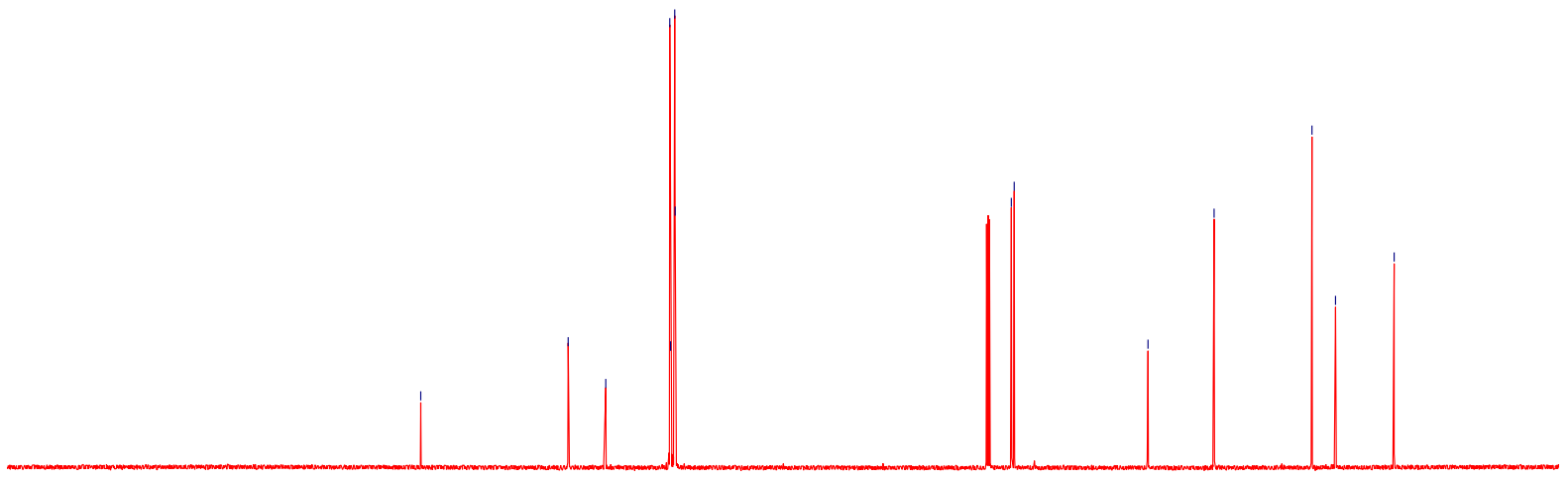

$\begin{array}{lllllllllllllllllllllllllll}230 & 220 & 210 & 200 & 190 & 180 & 170 & 160 & 150 & 140 & 130 & 120 & 110 & 100 & 90 & 80 & 70 & 60 & 50 & 40 & 30 & 20 & 10 & 0 & -10\end{array}$ 
<smiles>CCC(/C=C(/C)CO)COCc1ccccc1</smiles>

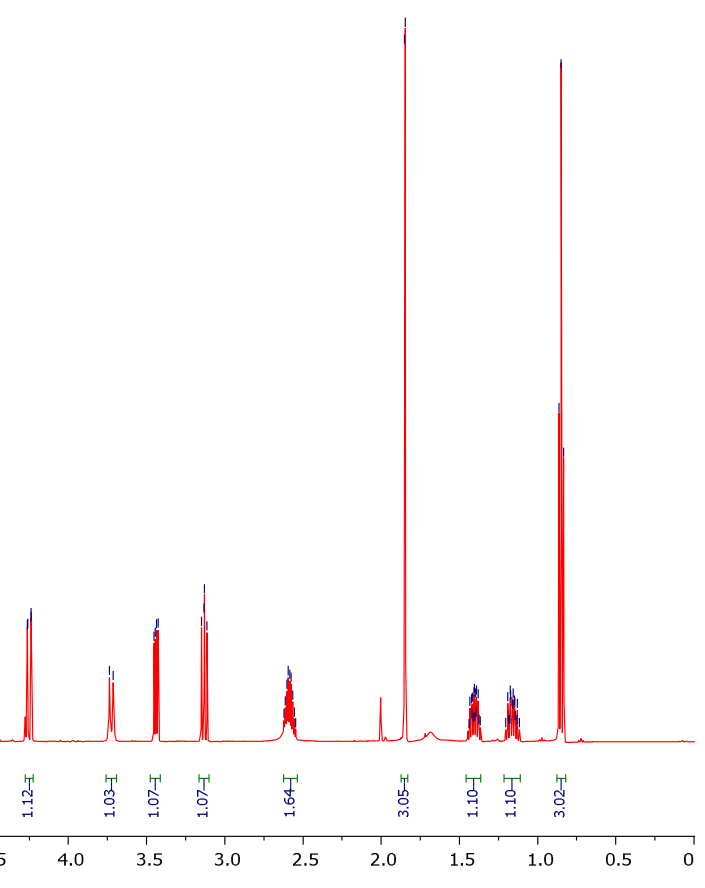

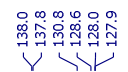

崖

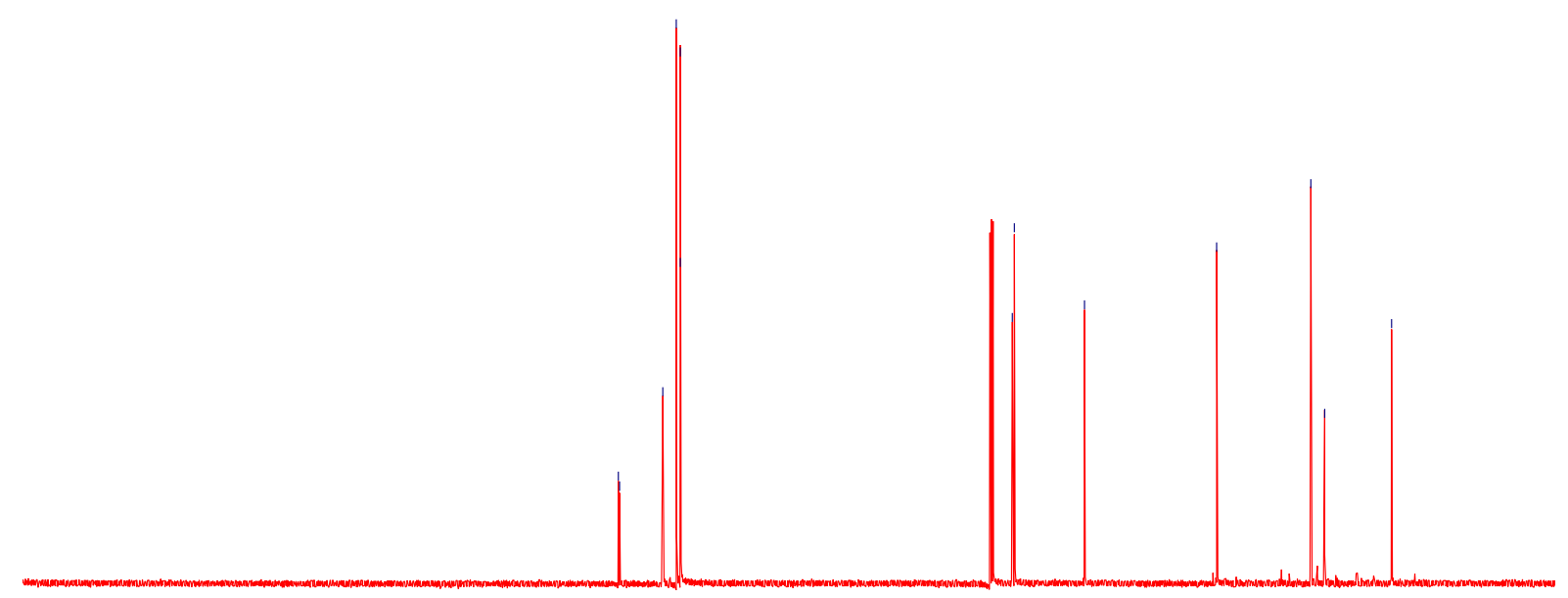

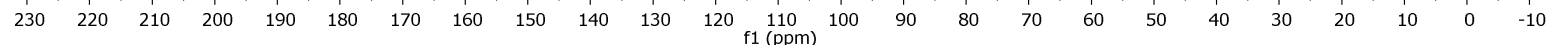


<smiles>CCC(/C=C(/C)C=O)CC(=[Pt])Cc1ccccc1</smiles>
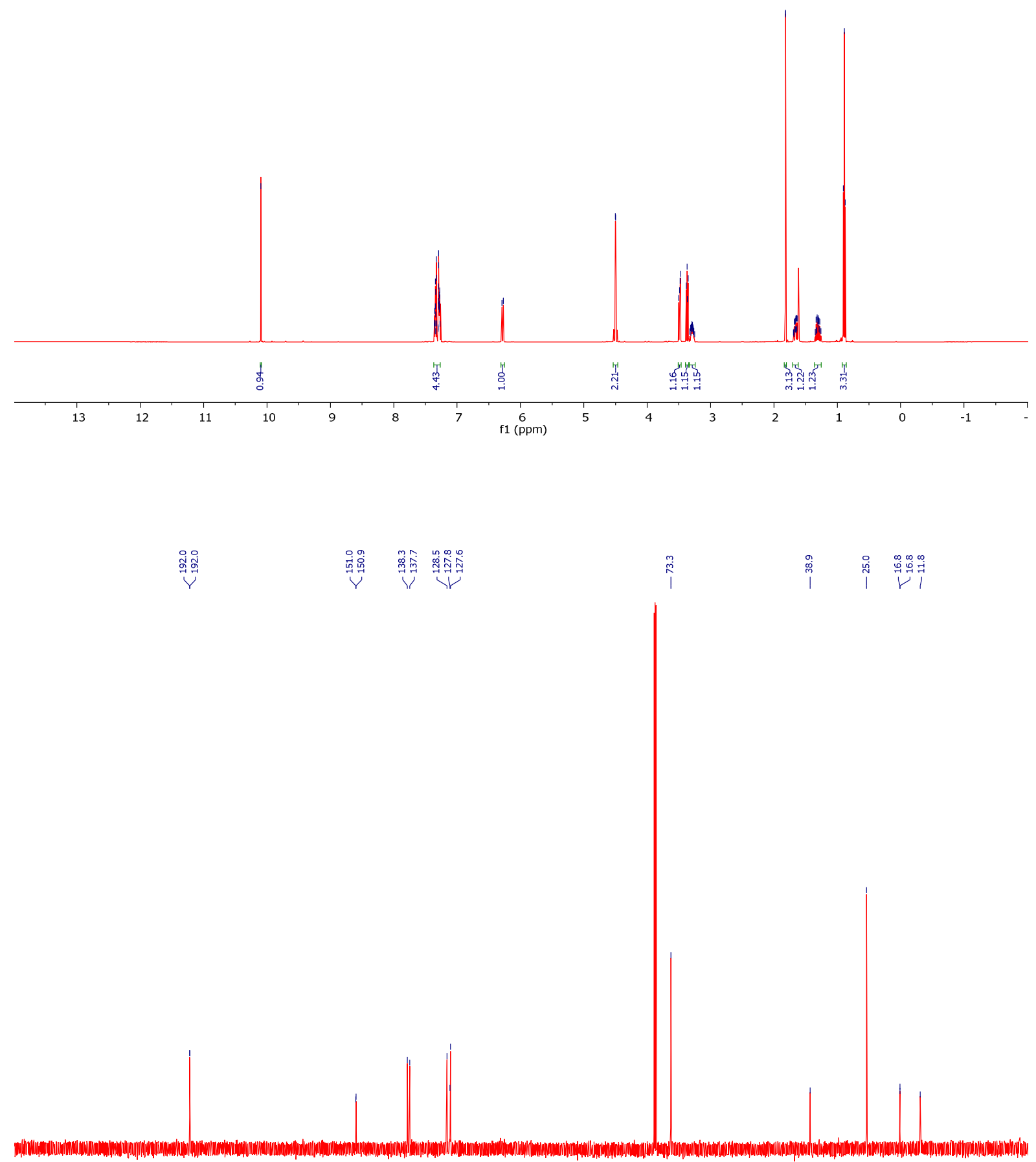

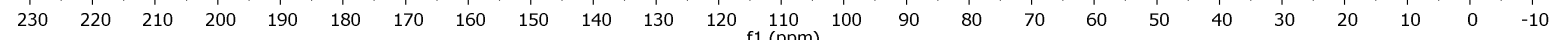


<smiles>CCC(C=C(C)[C@@H](O)CC(C)=O)CC(C)=O</smiles>
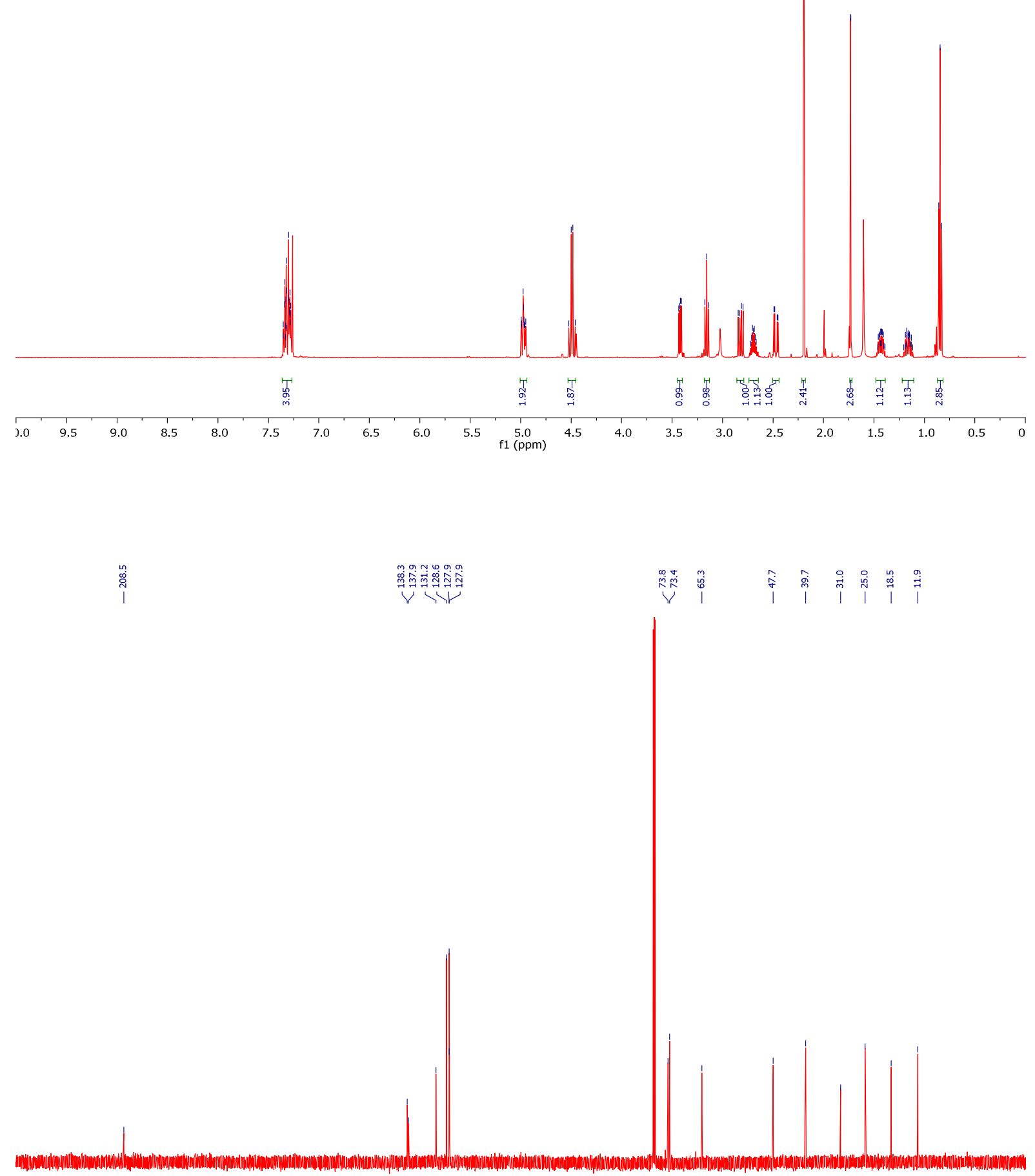

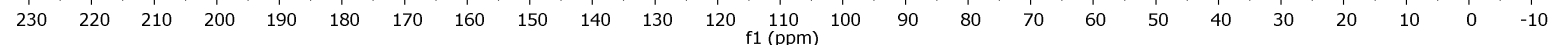


<smiles>CCC(/C=C(/C)[C@H](O)CC(C)=O)COCc1ccccc1</smiles>

135

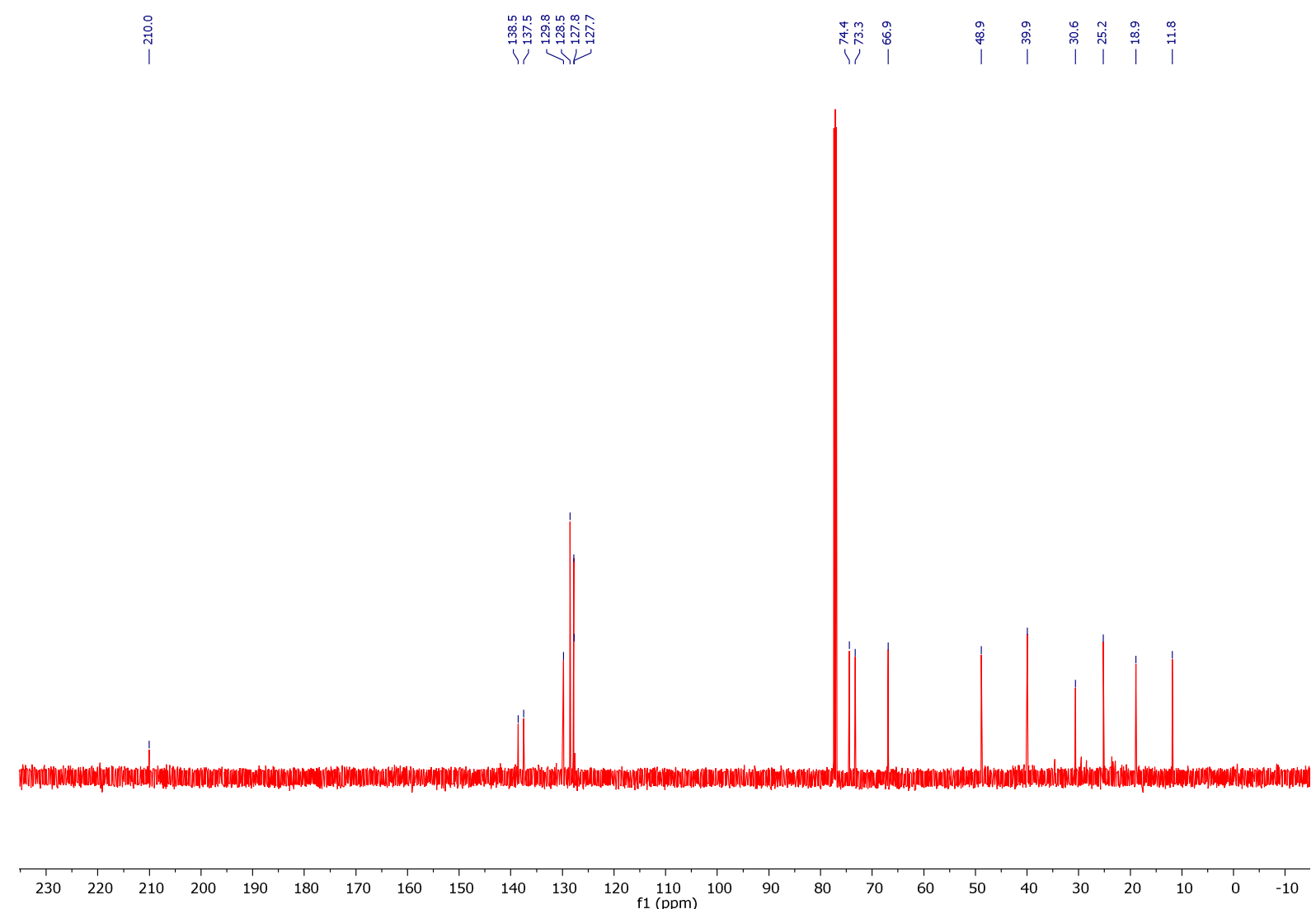




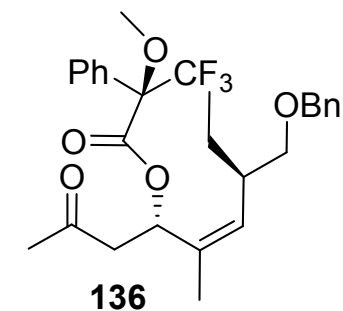

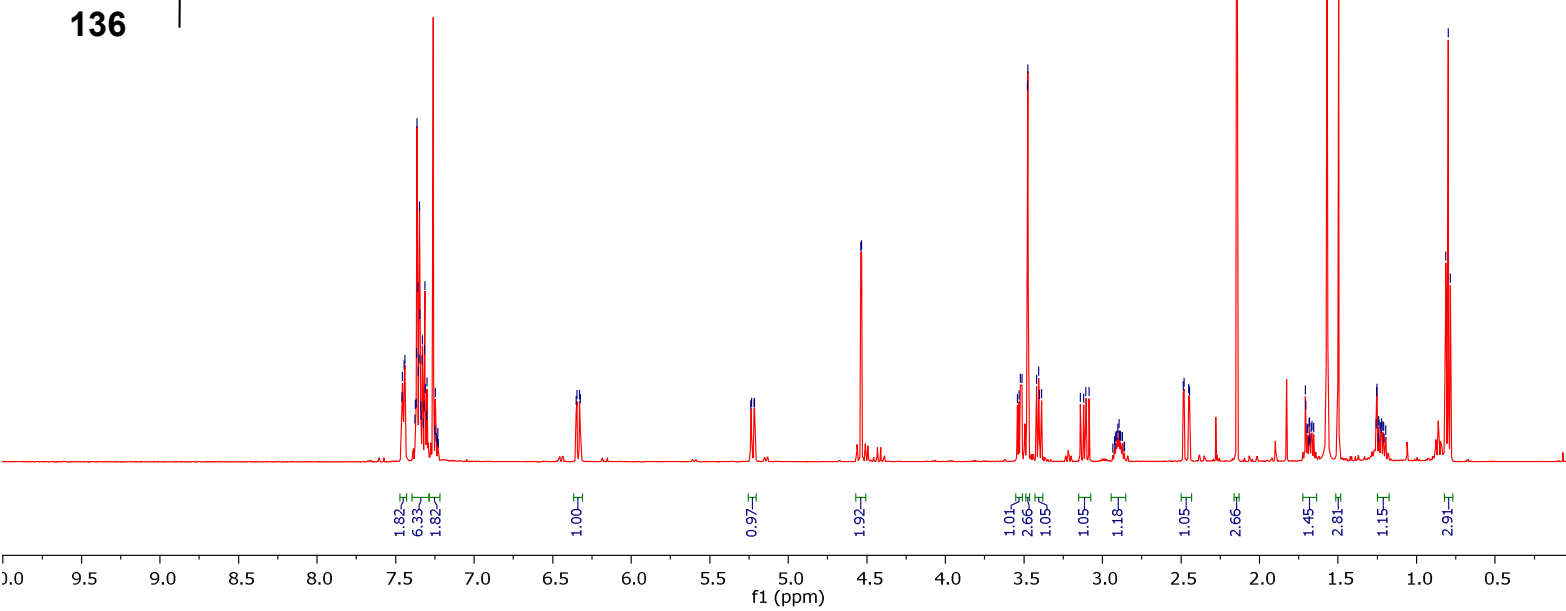

\begin{tabular}{|c|c|c|c|}
\hline & 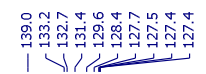 & 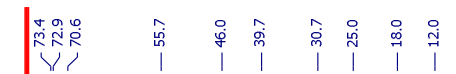 \\
\hline
\end{tabular}

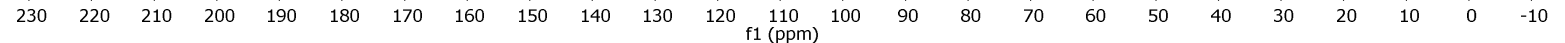


<smiles>COC(=O)C(OC)(c1ccccc1)C(CC(C)=O)CC(CC(C)=O)CC(C)Br</smiles>

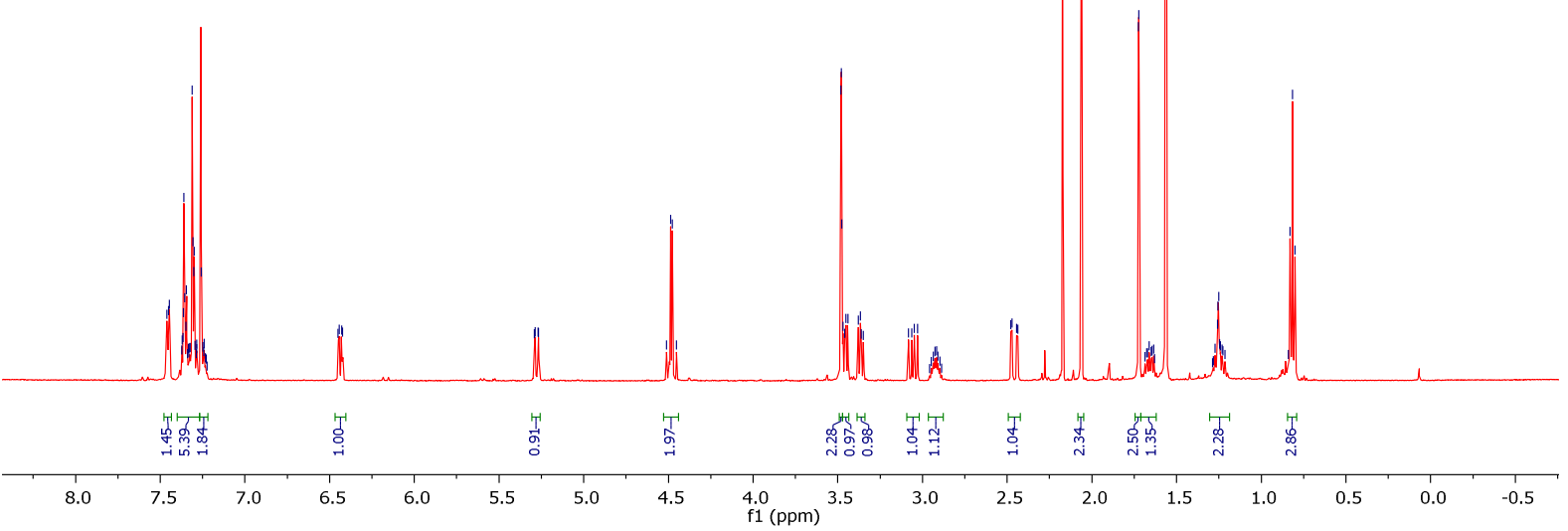




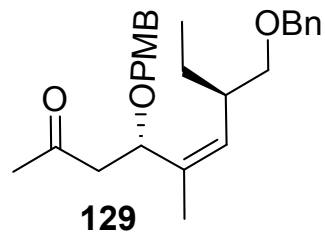

129

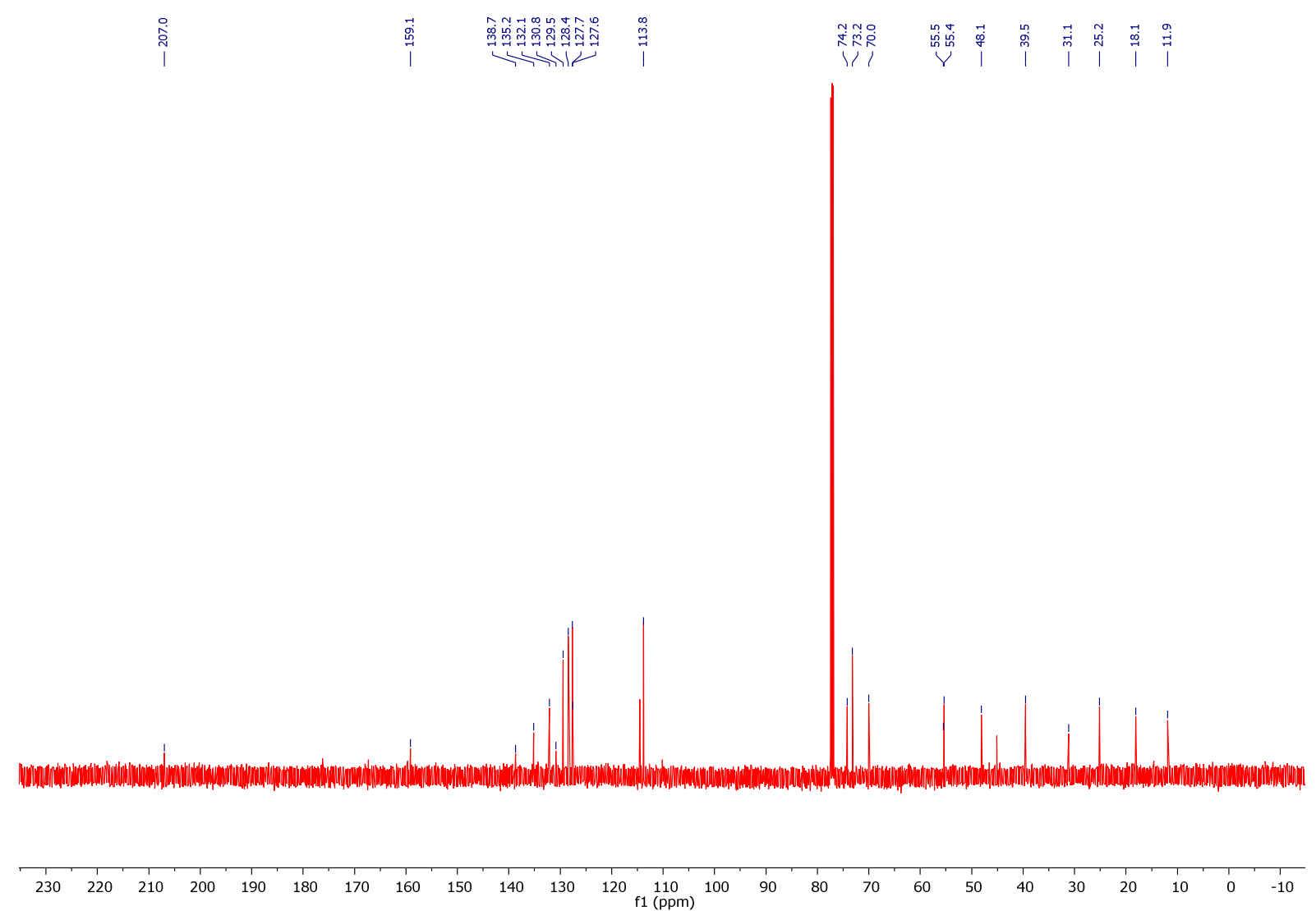



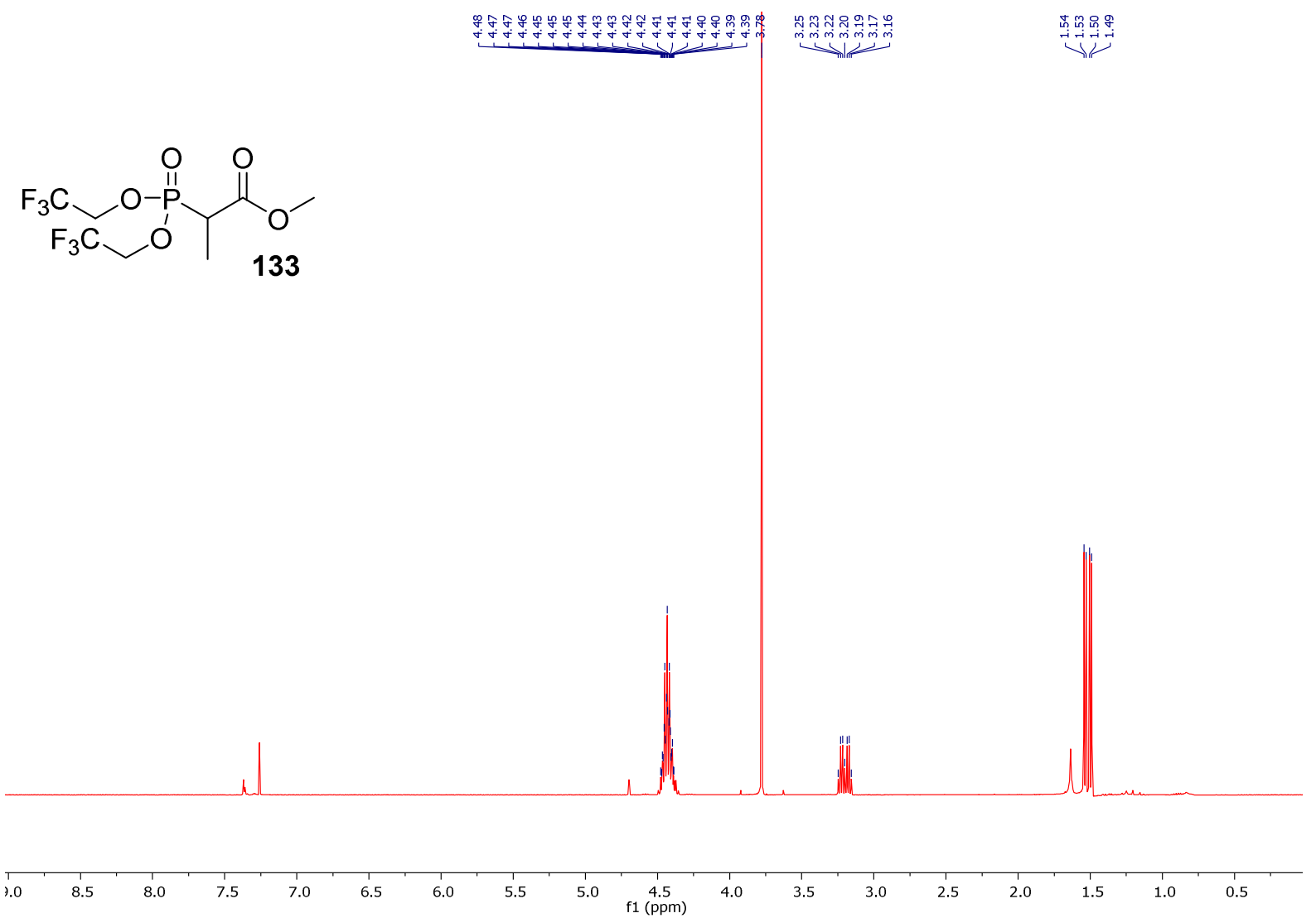

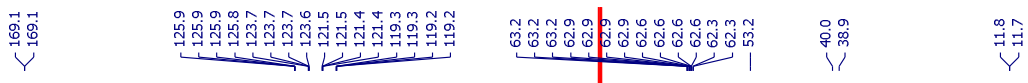

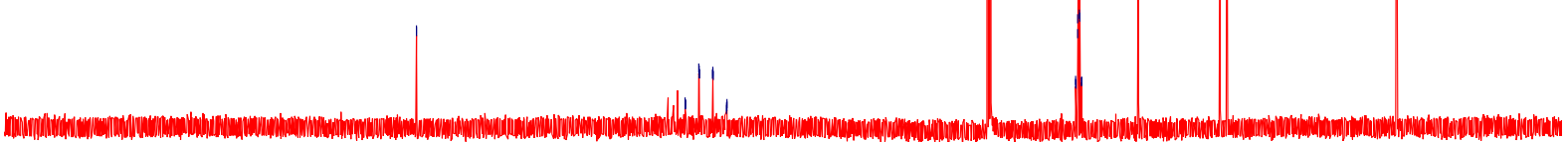

$\begin{array}{lllllllllllllllllllllllllllll}230 & 220 & 210 & 200 & 190 & 180 & 170 & 160 & 150 & 140 & 130 & 120 & 110 & 100 & 90 & 80 & 70 & 60 & 50 & 40 & 30 & 20 & 10 & 0 & -10\end{array}$ 
$\mathrm{BnO}^{\mathrm{OH}}$

66

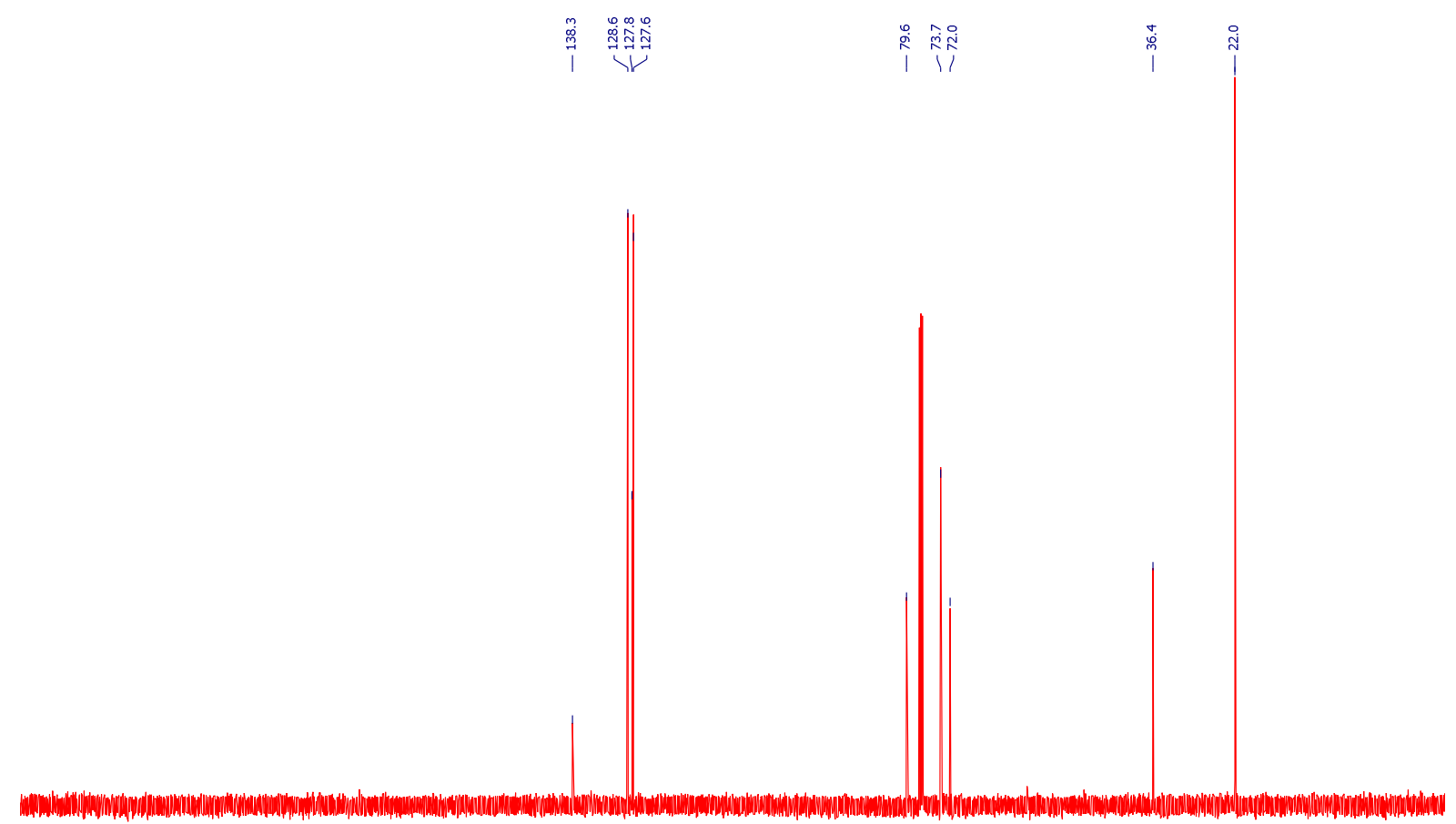

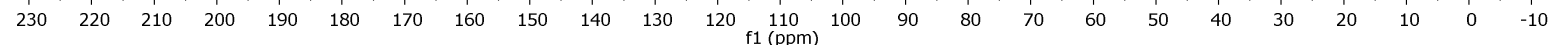




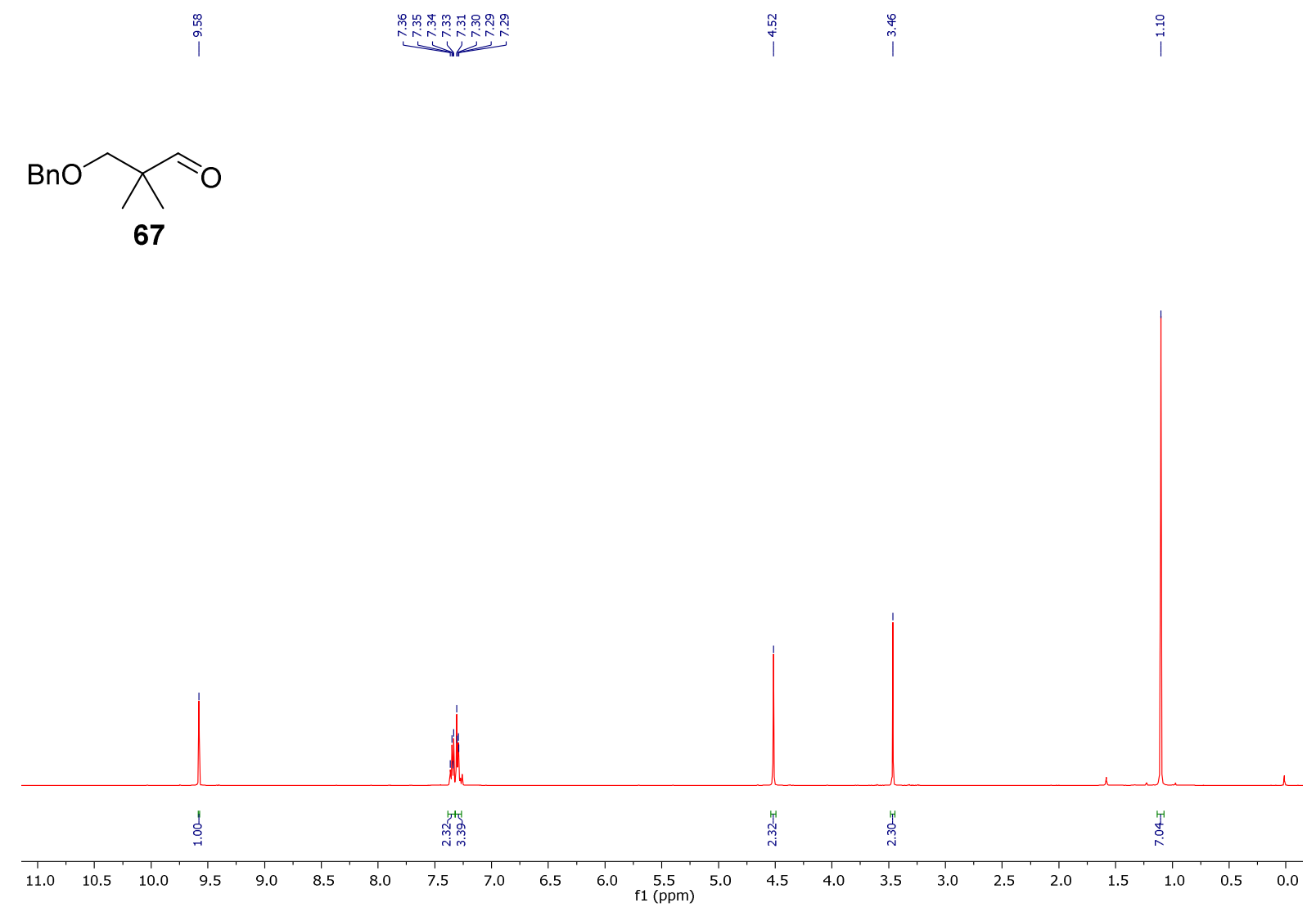

㶾

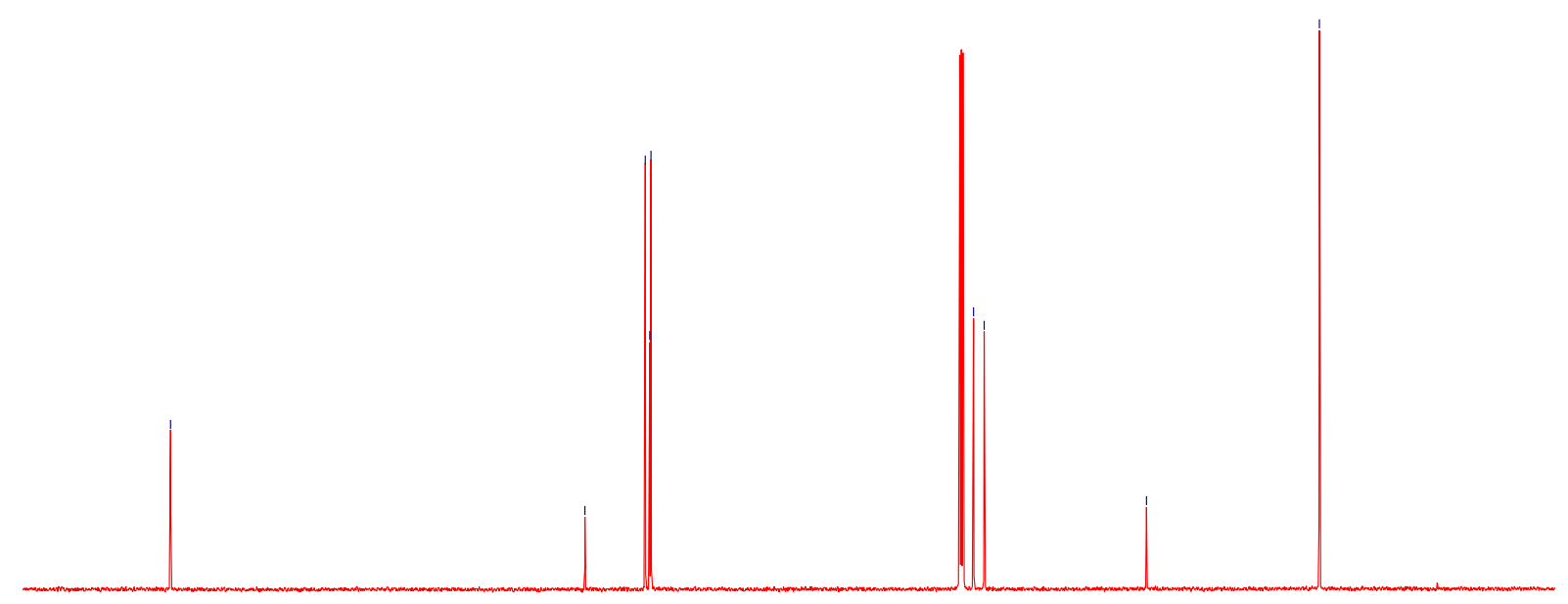

$\begin{array}{lllllllllllllllllllllllll}\mid & 1 \\ 220 & 210 & 200 & 190 & 180 & 170 & 160 & 150 & 140 & 130 & 120 & \underset{\mathrm{f} 1}{110}(\mathrm{ppm}) & 100 & 90 & 80 & 70 & 60 & 50 & 40 & 30 & 20 & 10 & 0 & -10\end{array}$ 


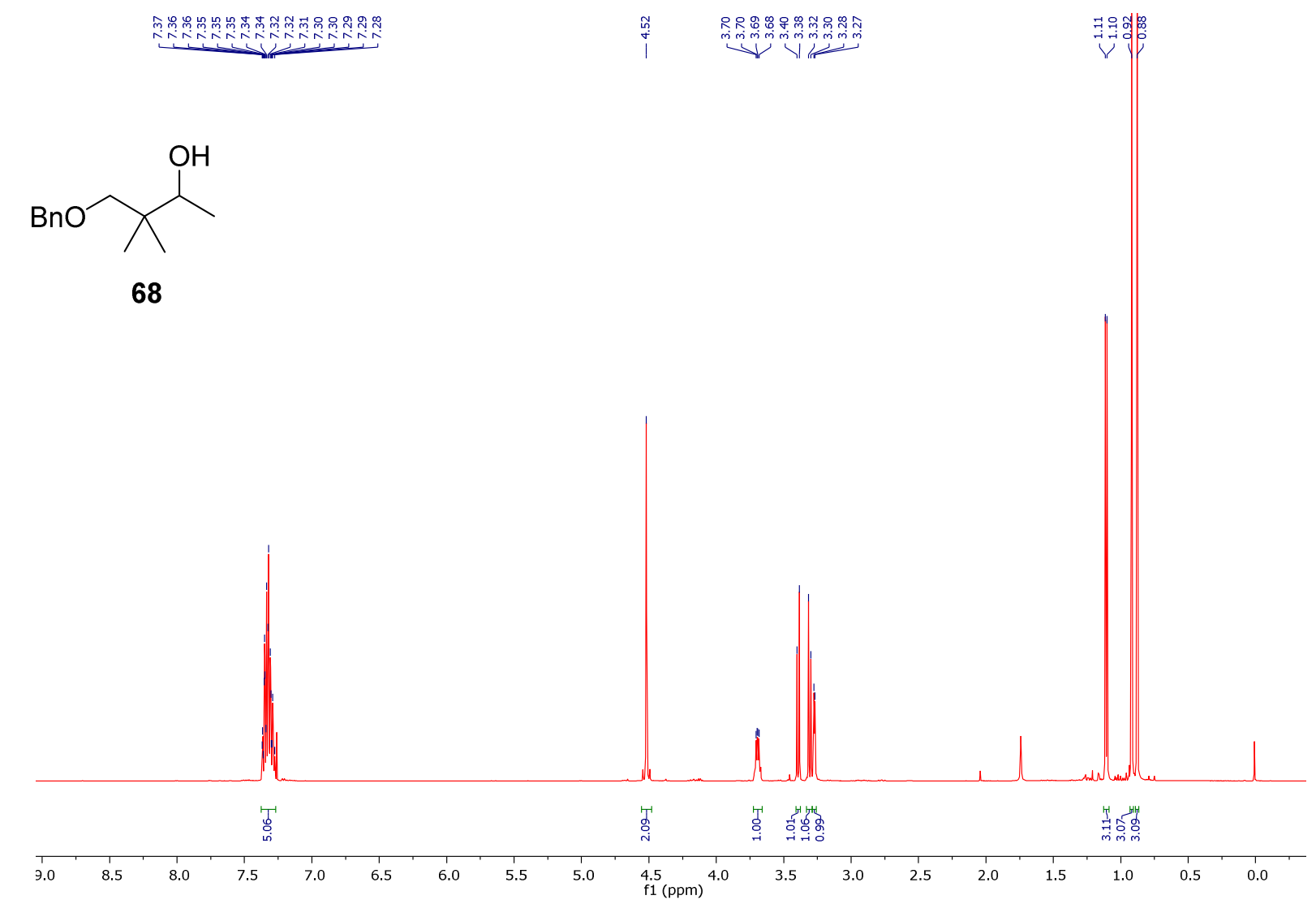

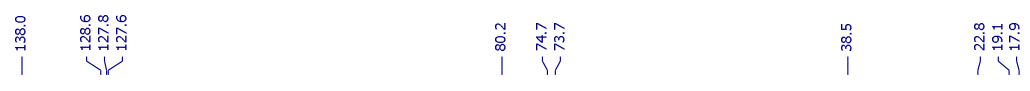

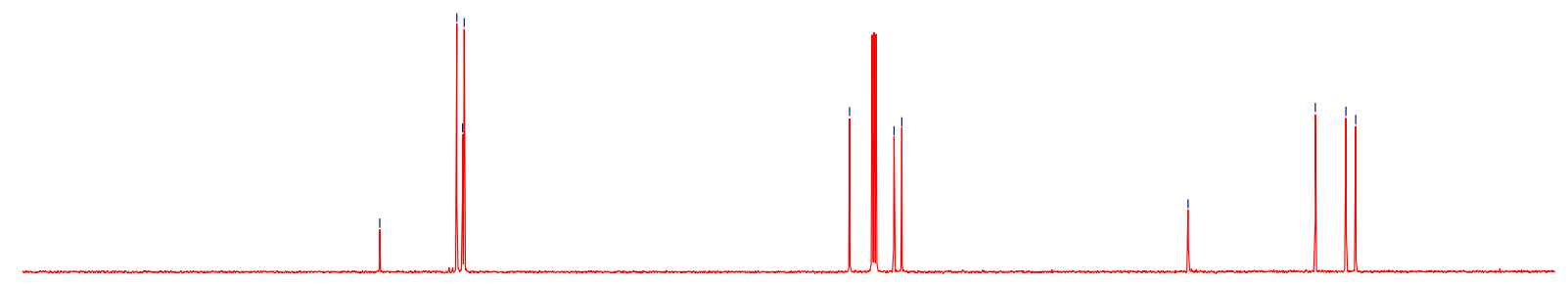

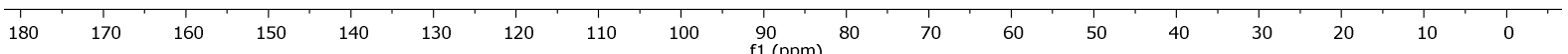


<smiles>[Z4]C(C)(COc1ccccc1)C(C)=O</smiles>
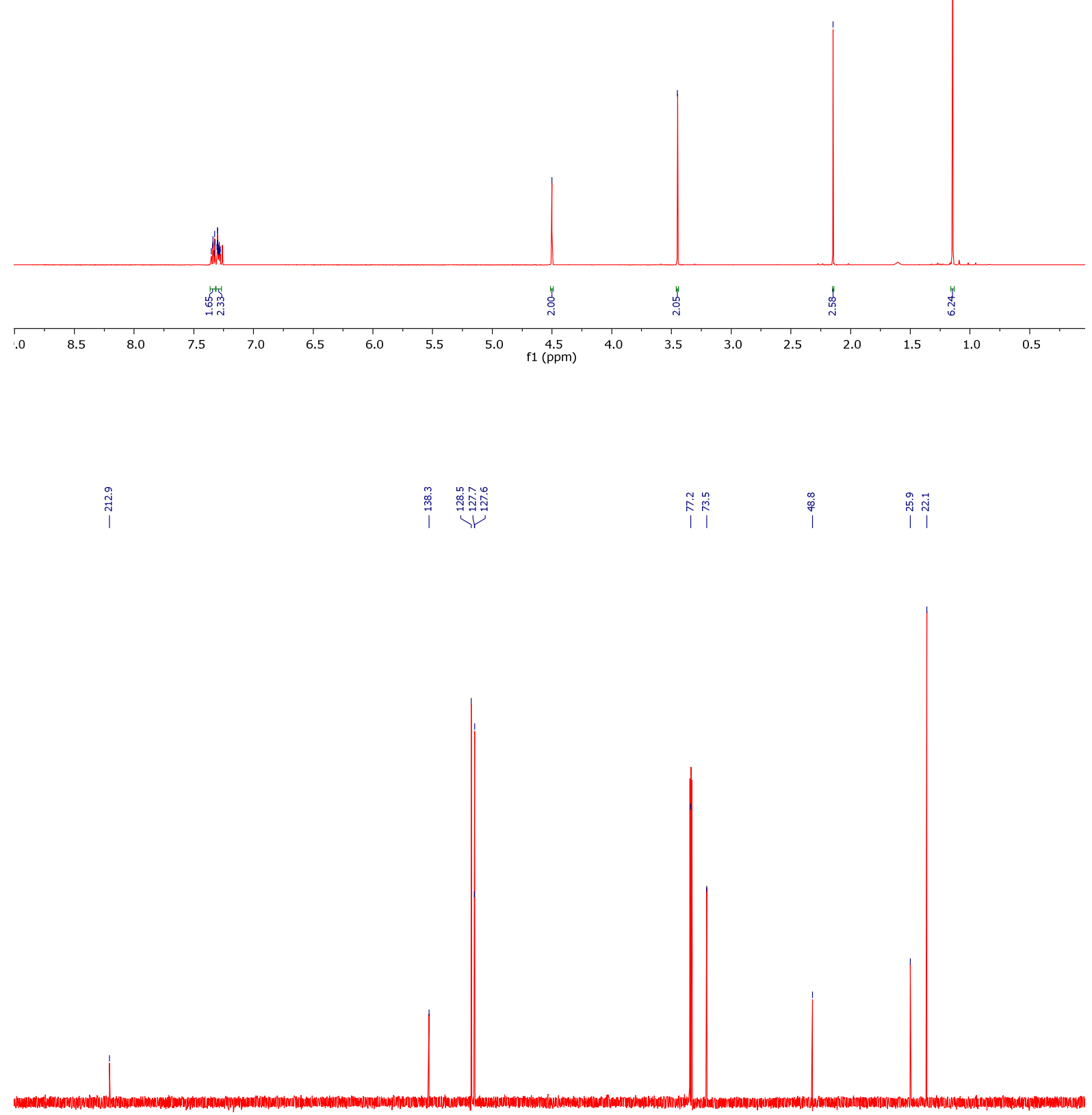

$\begin{array}{llllllllllllllllllllllllllll}230 & 220 & 210 & 200 & 190 & 180 & 170 & 160 & 150 & 140 & 130 & 120 & 110 & 100 & 90 & 80 & 70 & 60 & 50 & 40 & 30 & 20 & 10 & 0 & -10\end{array}$ 
<smiles>CC(=O)C(C)(C)CO</smiles>

71
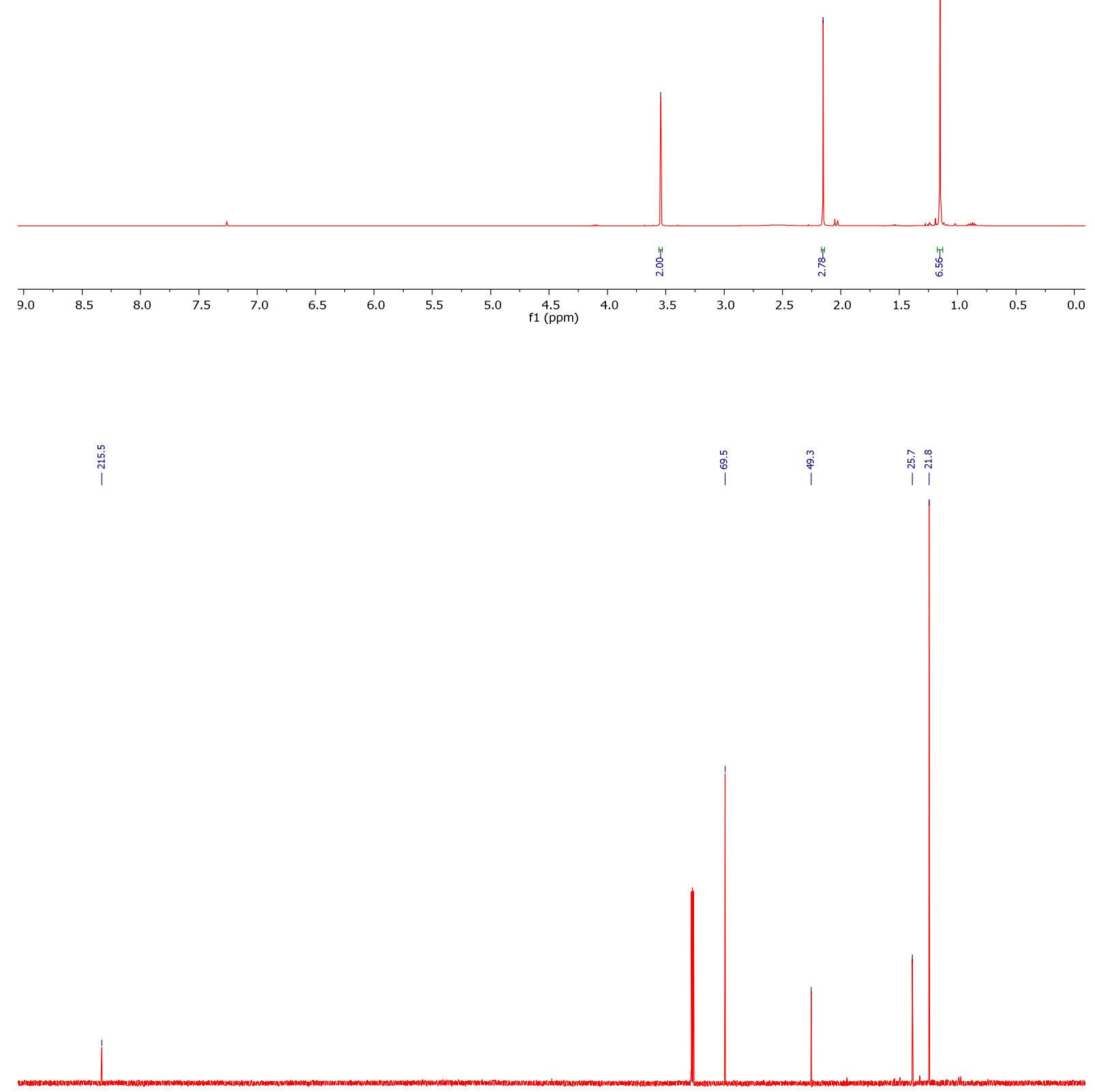

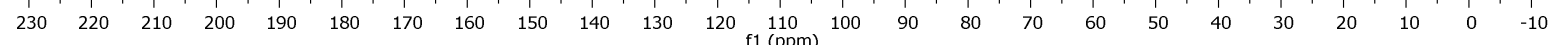




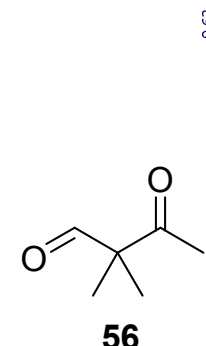

임

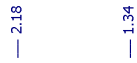

56

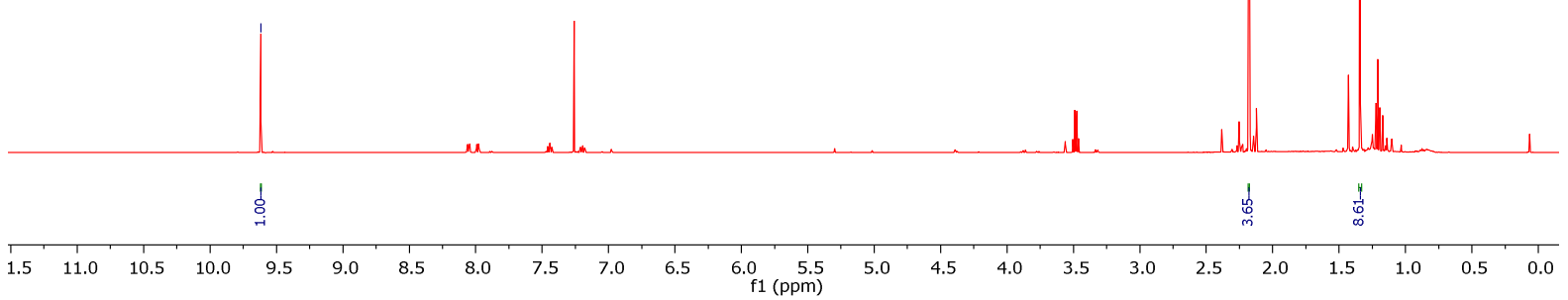




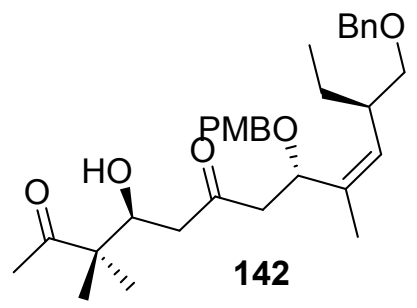

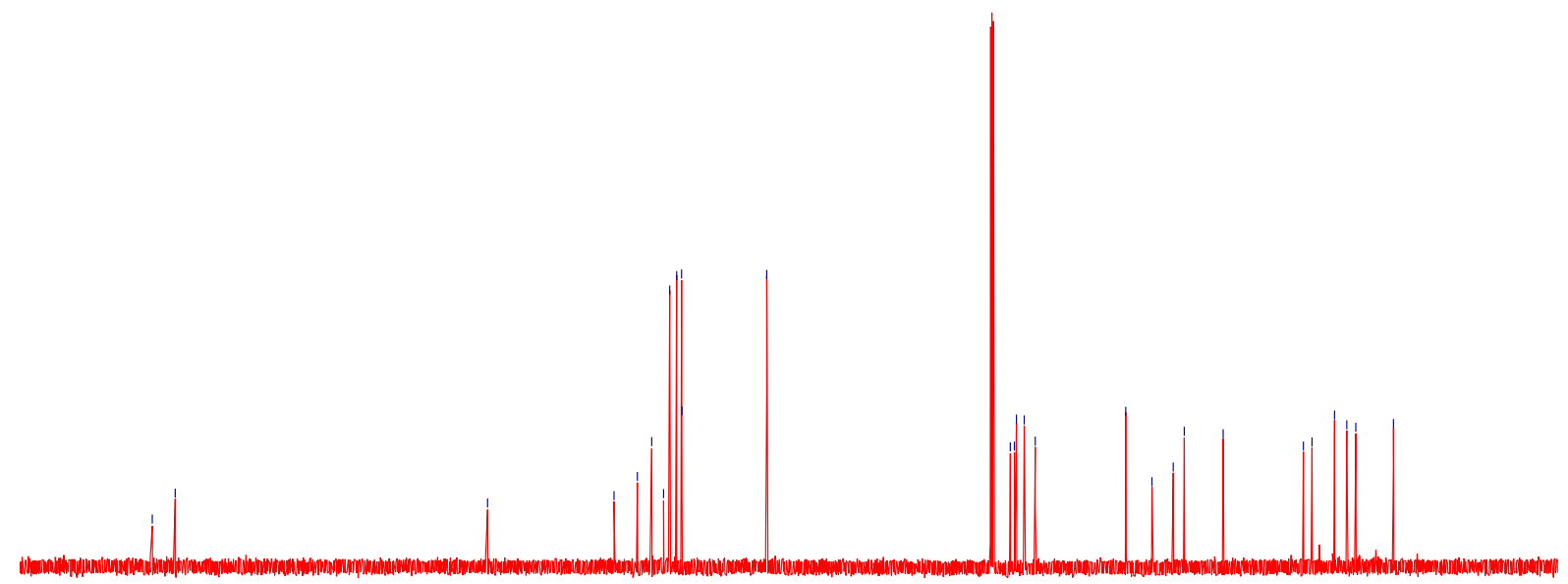

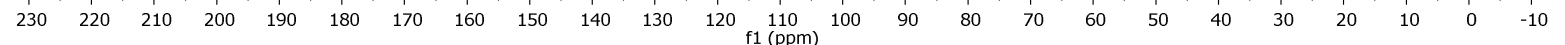



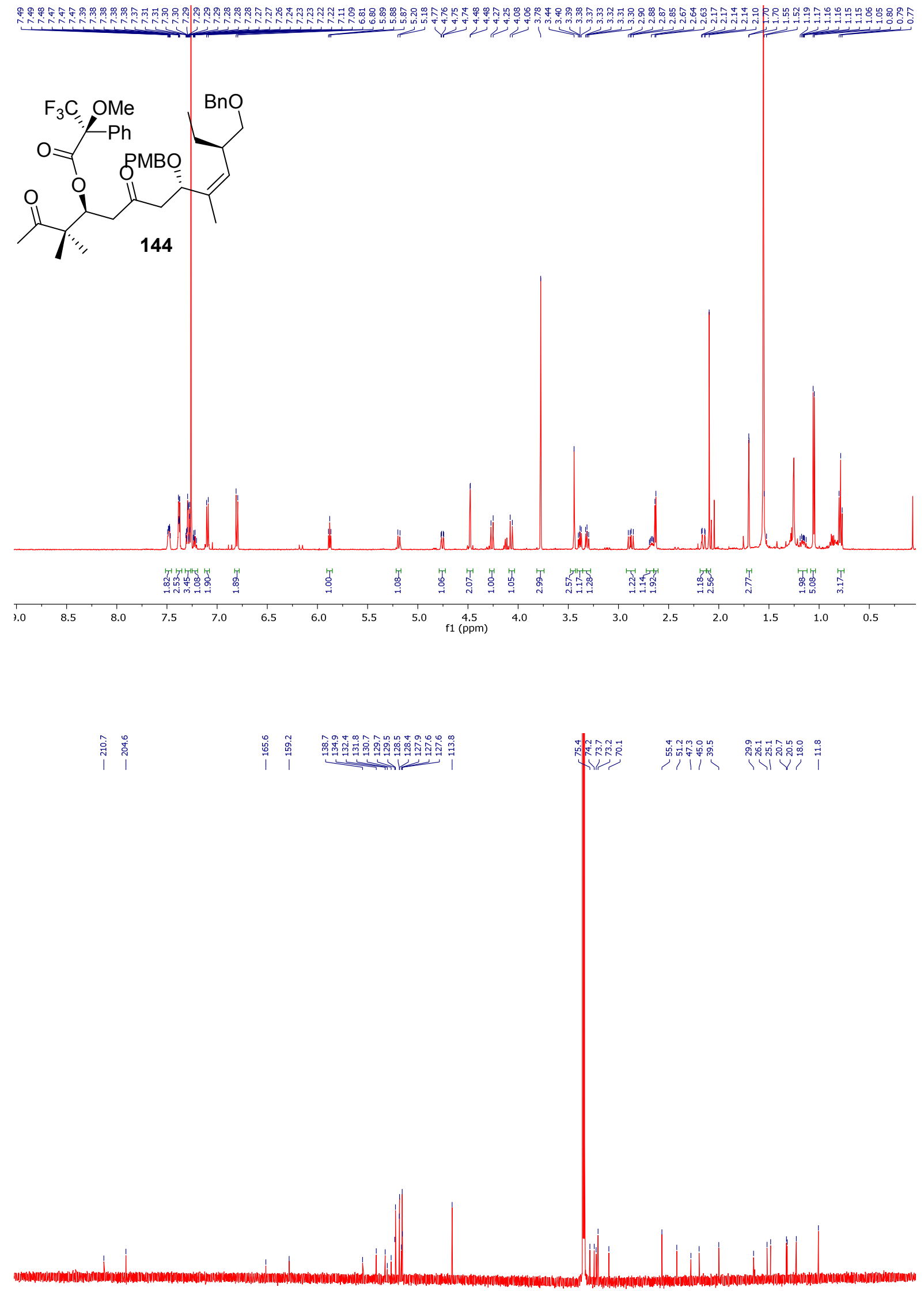

$\begin{array}{lllllllllllllllllllllllllll}230 & 220 & 210 & 200 & 190 & 180 & 170 & 160 & 150 & 140 & 130 & 120 & 110 & 100 & 90 & 80 & 70 & 60 & 50 & 40 & 30 & 20 & 10 & 0 & -10\end{array}$ 


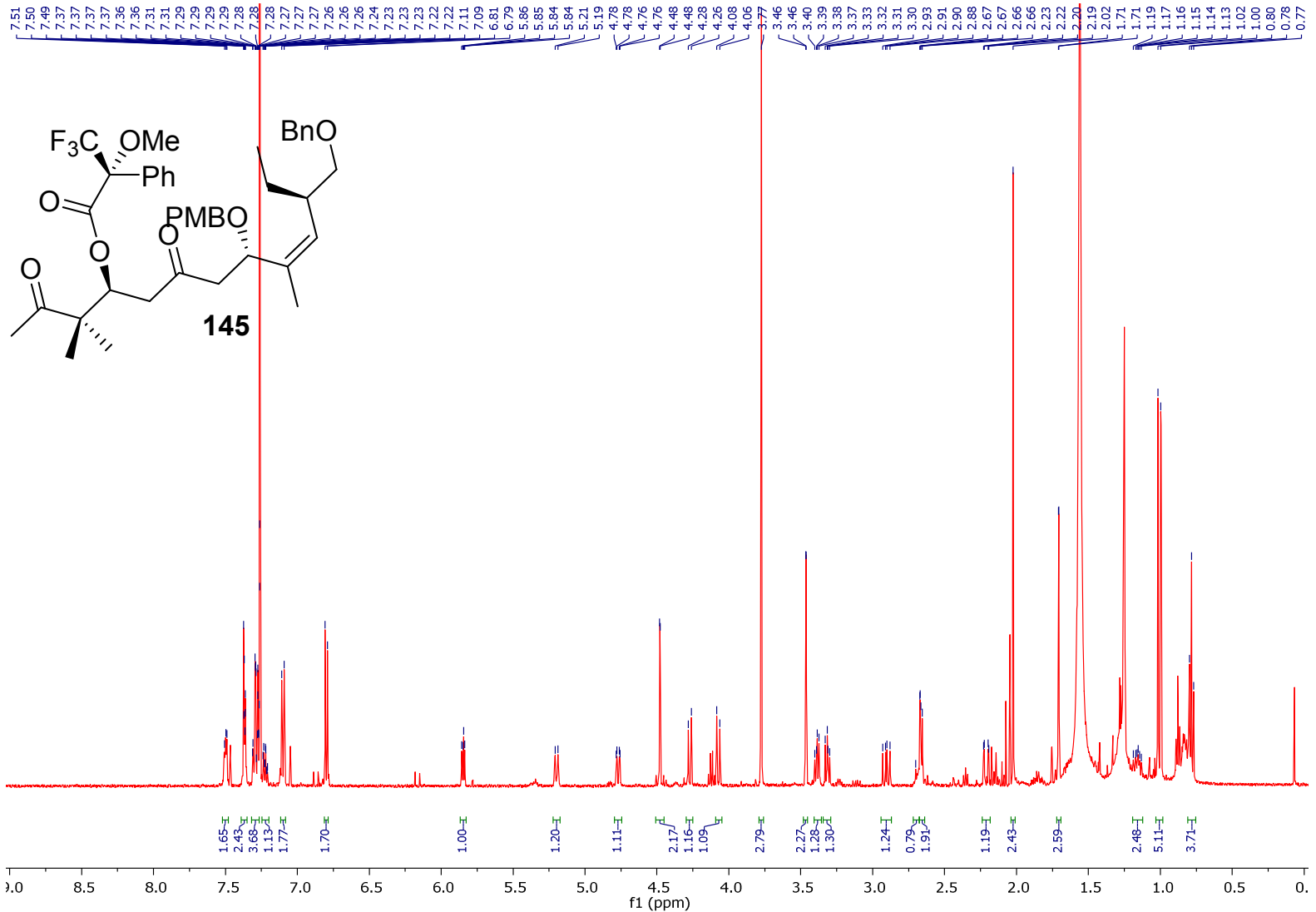


<smiles></smiles>

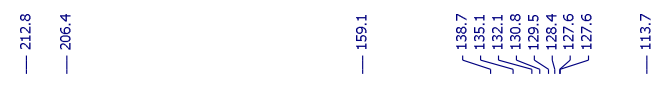

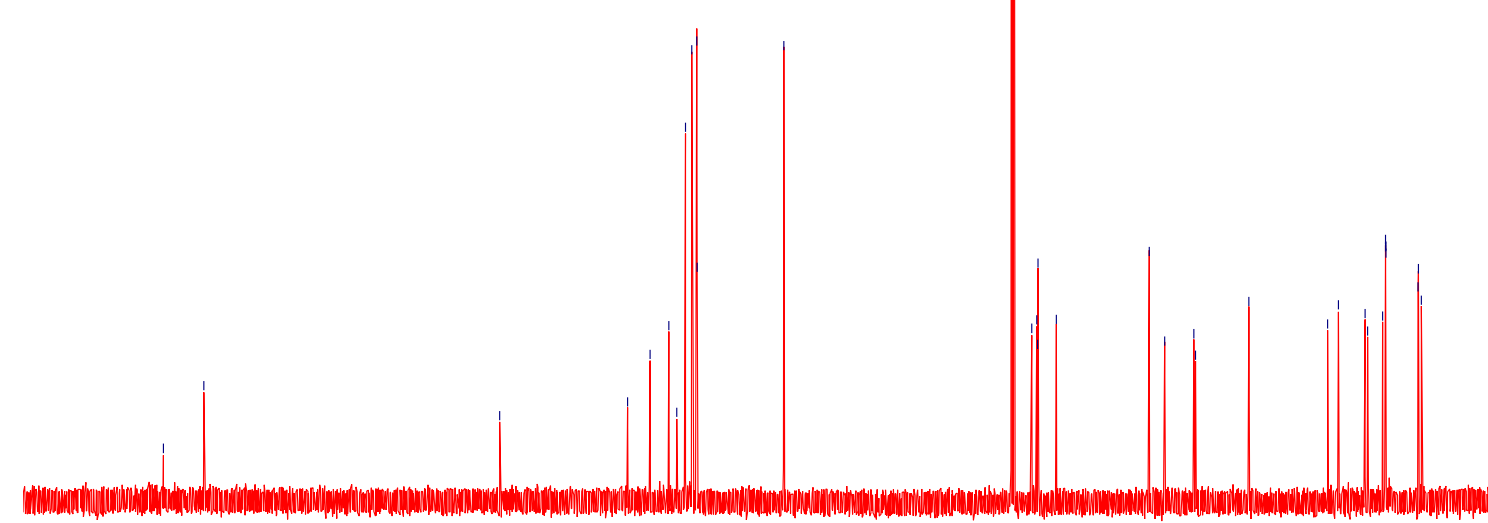

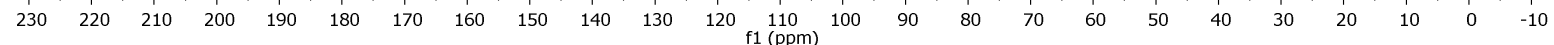




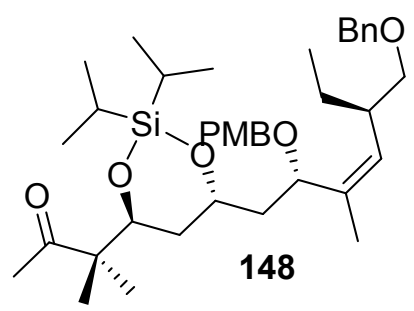
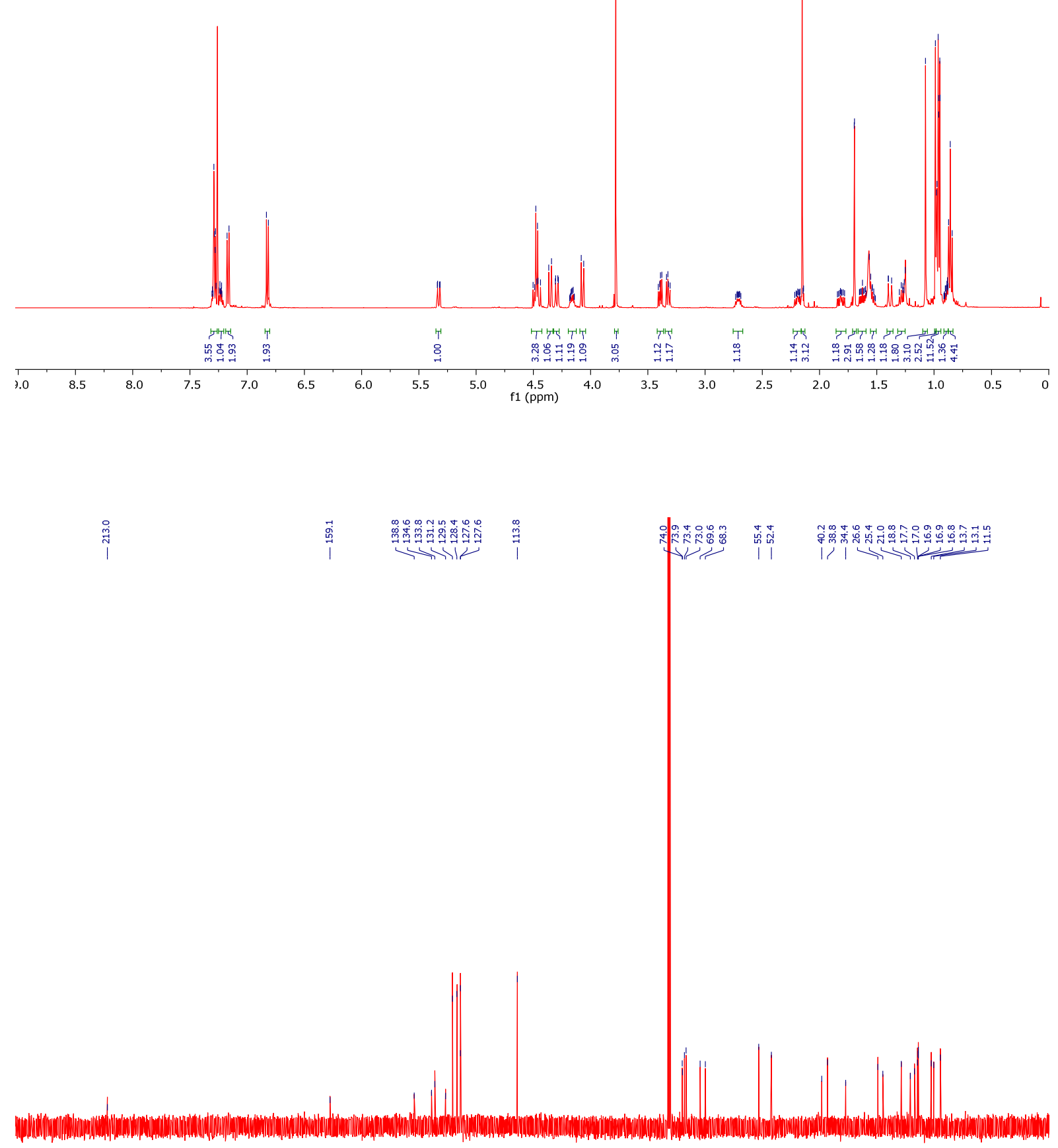

$\begin{array}{lllllllllllllllllllllllll}230 & 220 & 210 & 200 & 190 & 180 & 170 & 160 & 150 & 140 & 130 & 120 & 110 & 100 & 90 & 80 & 70 & 60 & 50 & 40 & 30 & 20 & 10 & 0 & -10\end{array}$ 


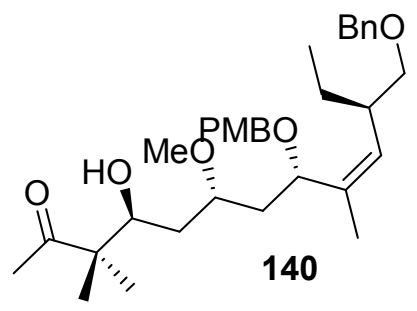
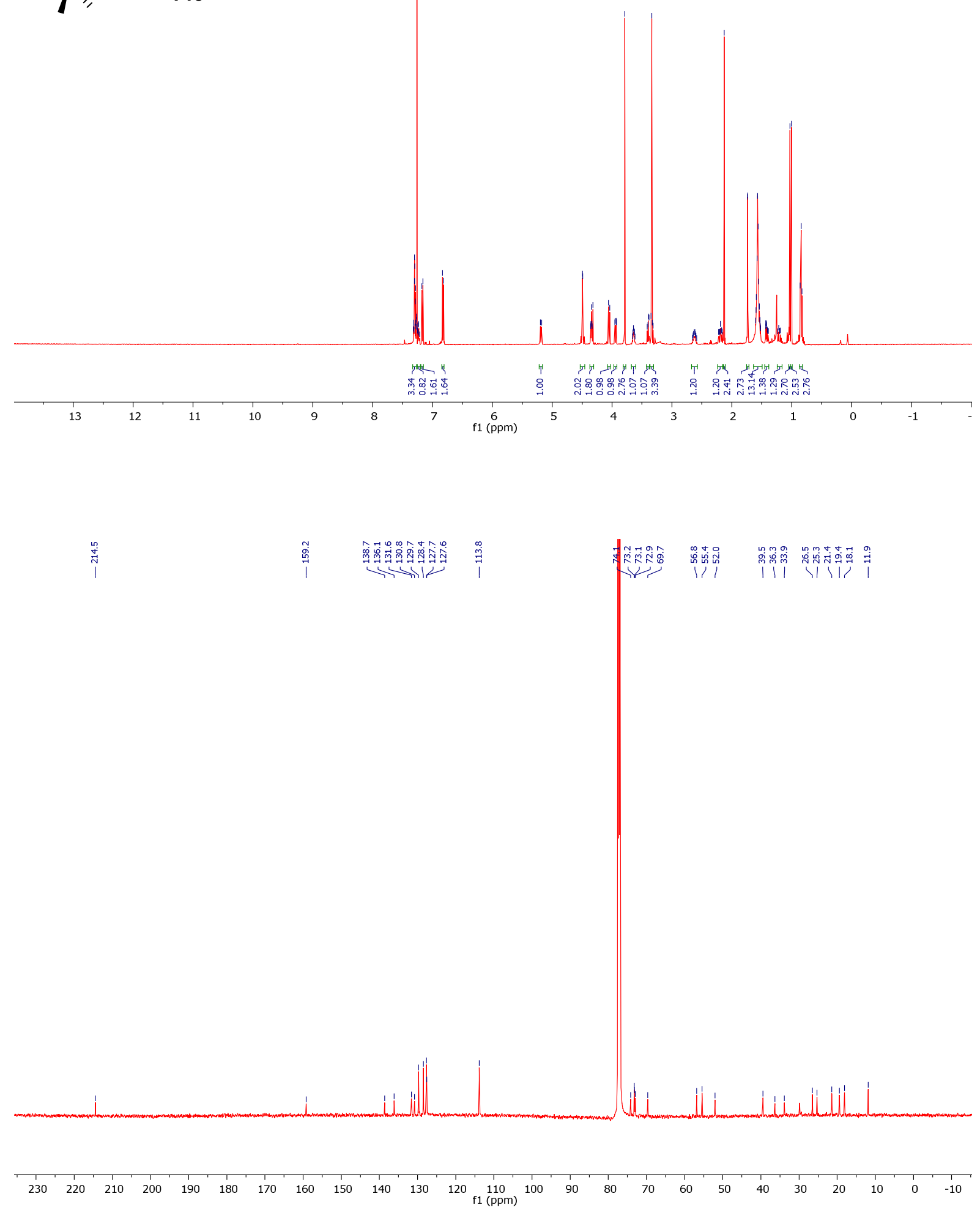
<smiles>C=CC(O)C(C)(C)COc1ccccc1</smiles>

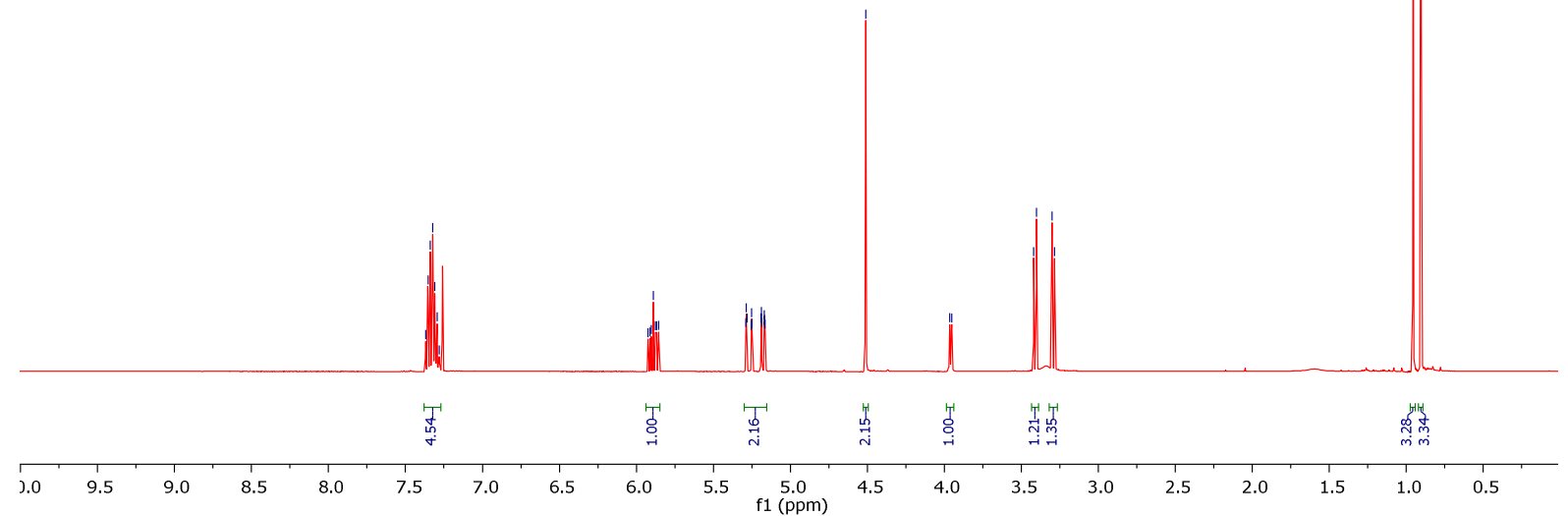

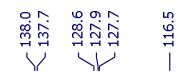

in

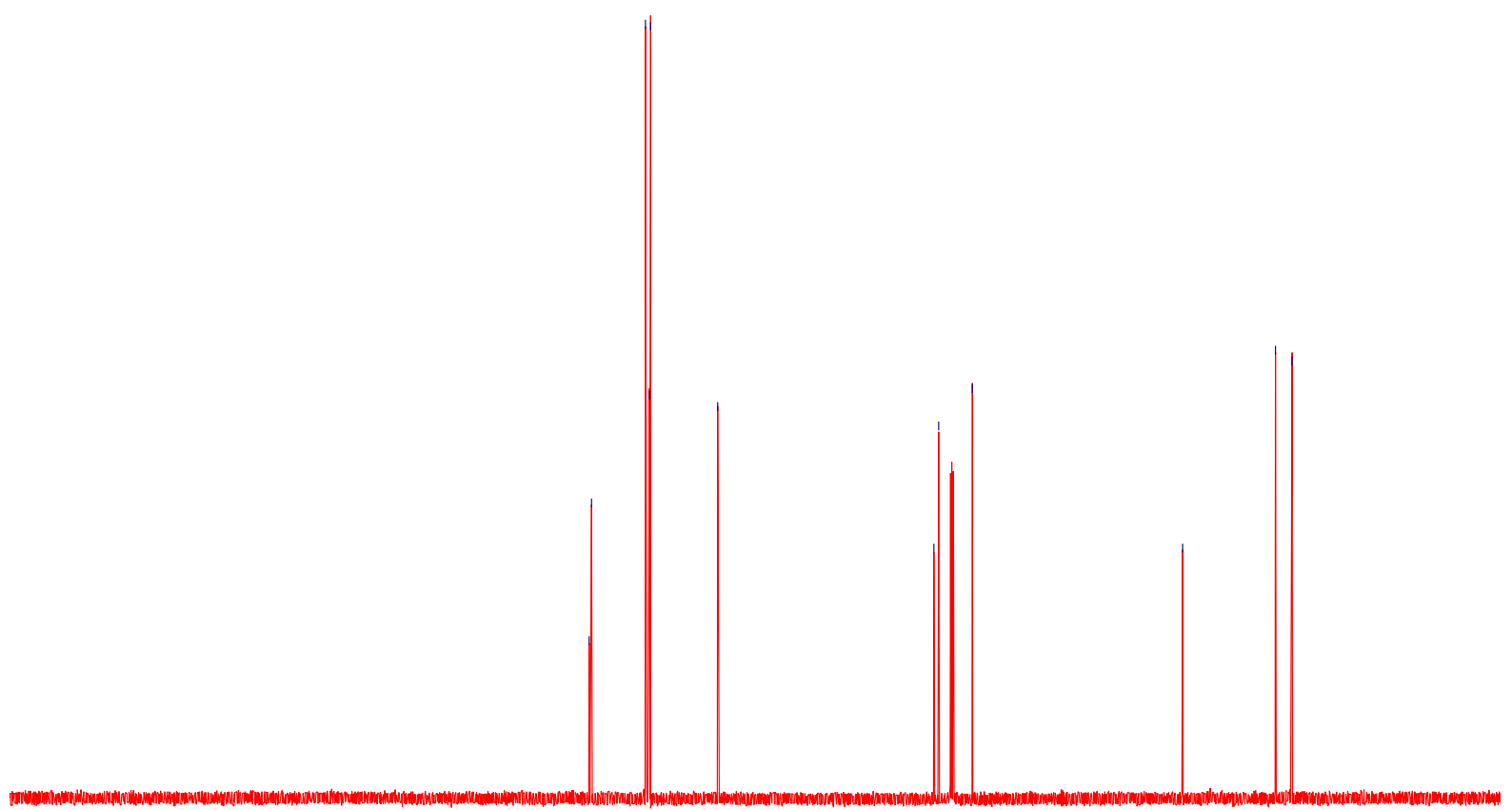

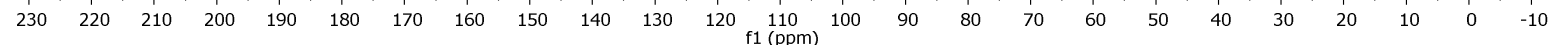




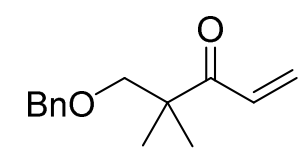

70
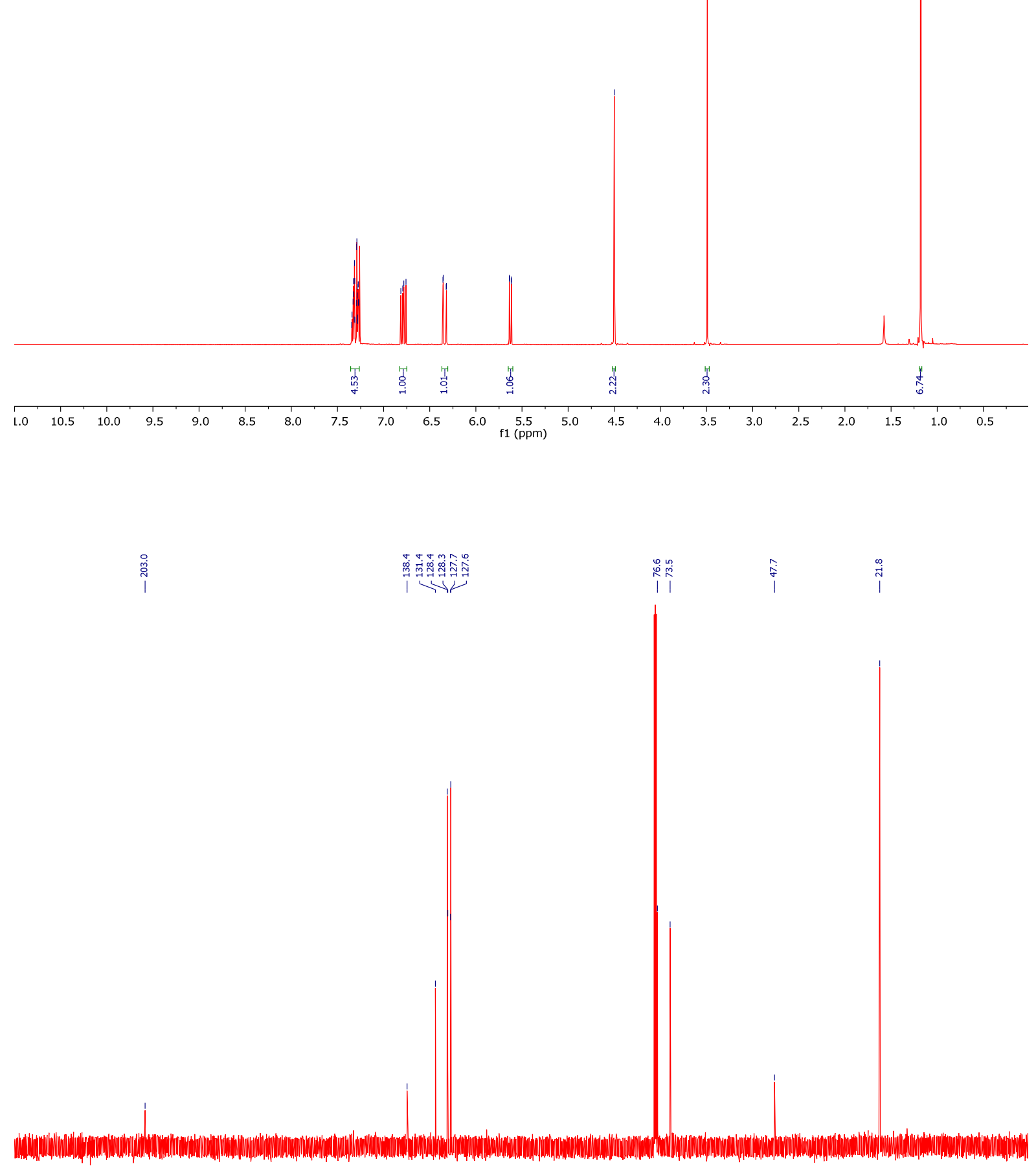

$\begin{array}{llllllllllllllllllllllllllll}230 & 220 & 210 & 200 & 190 & 180 & 170 & 160 & 150 & 140 & 130 & 120 & 110 & 100 & 90 & 80 & 70 & 60 & 50 & 40 & 30 & 20 & 10 & 0 & -10\end{array}$ 


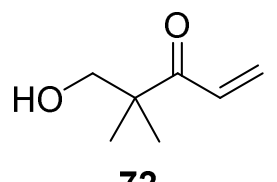

72
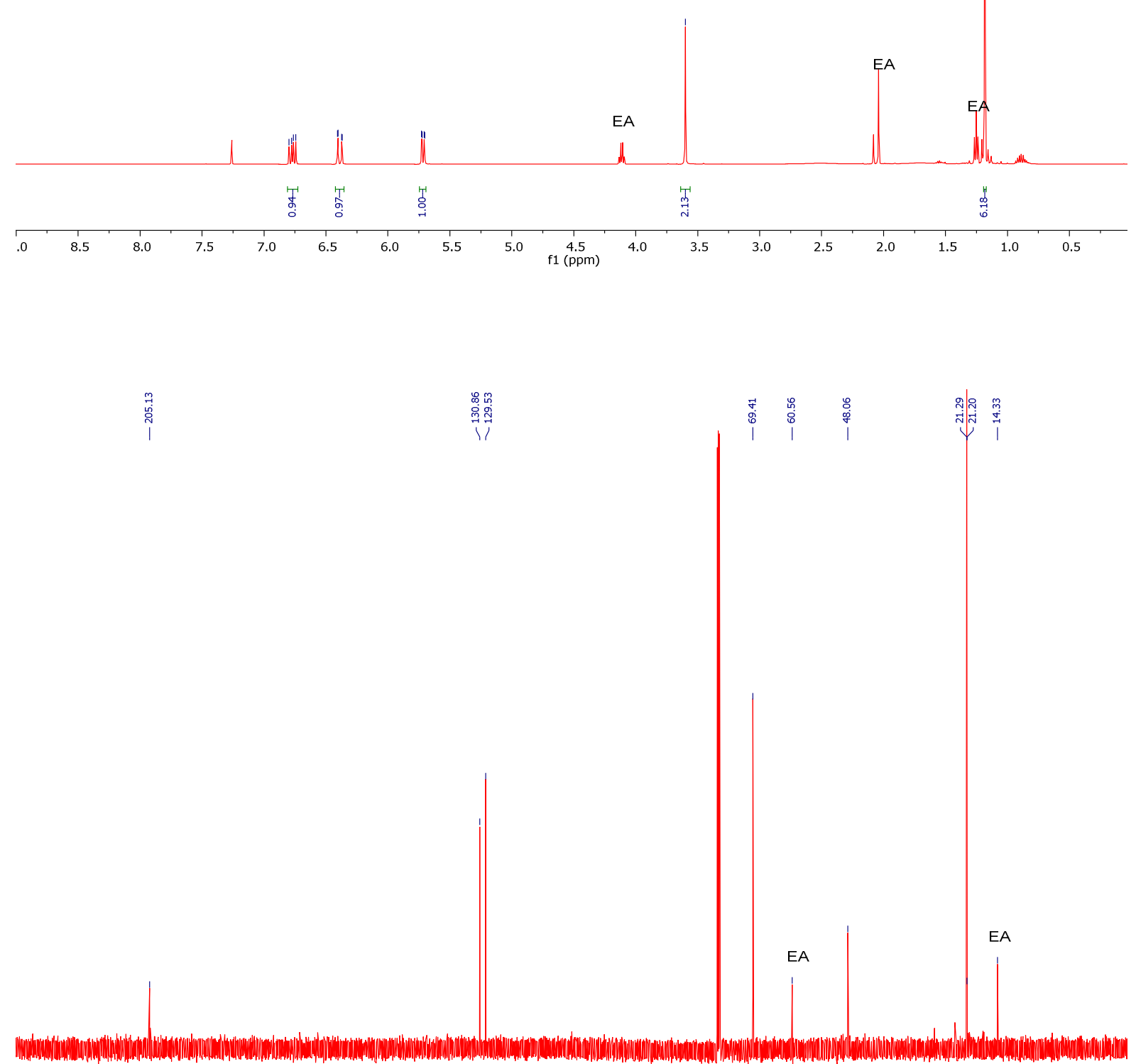

$\begin{array}{llllllllllllllllllllllllllll}230 & 220 & 210 & 200 & 190 & 180 & 170 & 160 & 150 & 140 & 130 & 120 & 110 & 100 & 90 & 80 & 70 & 60 & 50 & 40 & 30 & 20 & 10 & 0 & -10\end{array}$ 
<smiles>[R15]OCC1OC(C)(C)O[C@H]1CO</smiles>

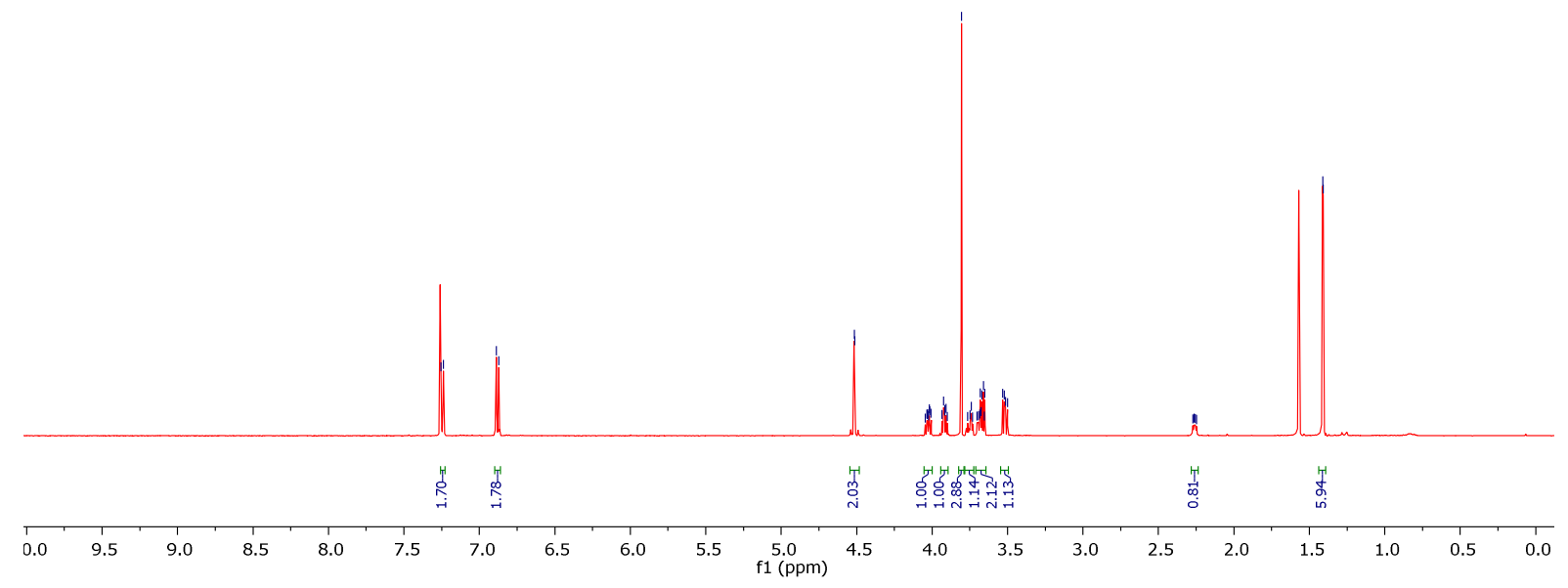

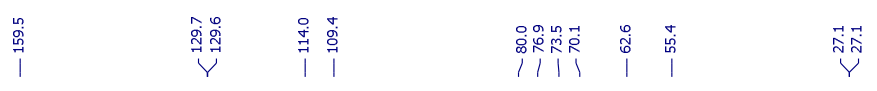

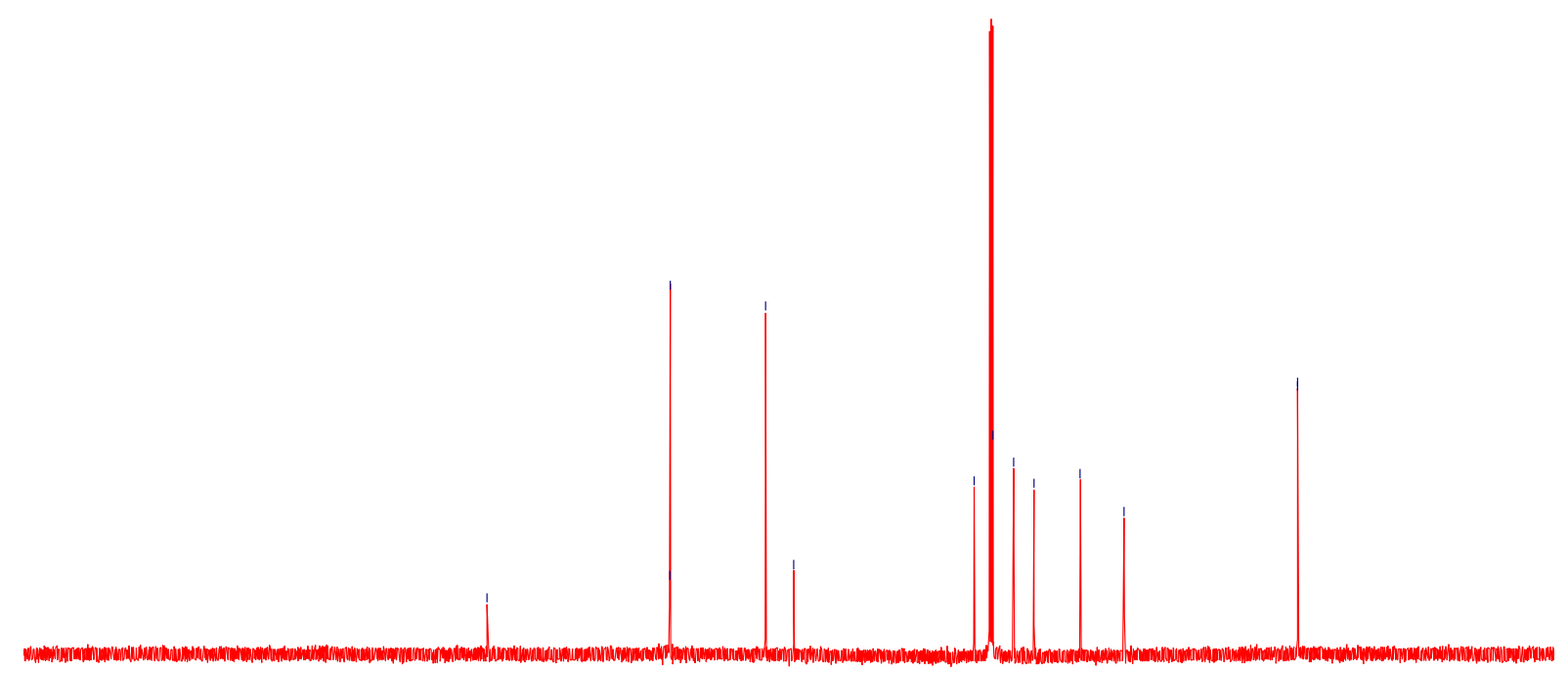

\begin{tabular}{llllllllllllllllllllllllllllllll}
\hline 230 & 220 & 210 & 200 & 190 & 180 & 170 & 160 & 150 & 140 & 130 & 120 & 110 & 100 & 90 & 80 & 70 & 60 & 50 & 40 & 30 & 20 & 10 & 0 & -10
\end{tabular} 

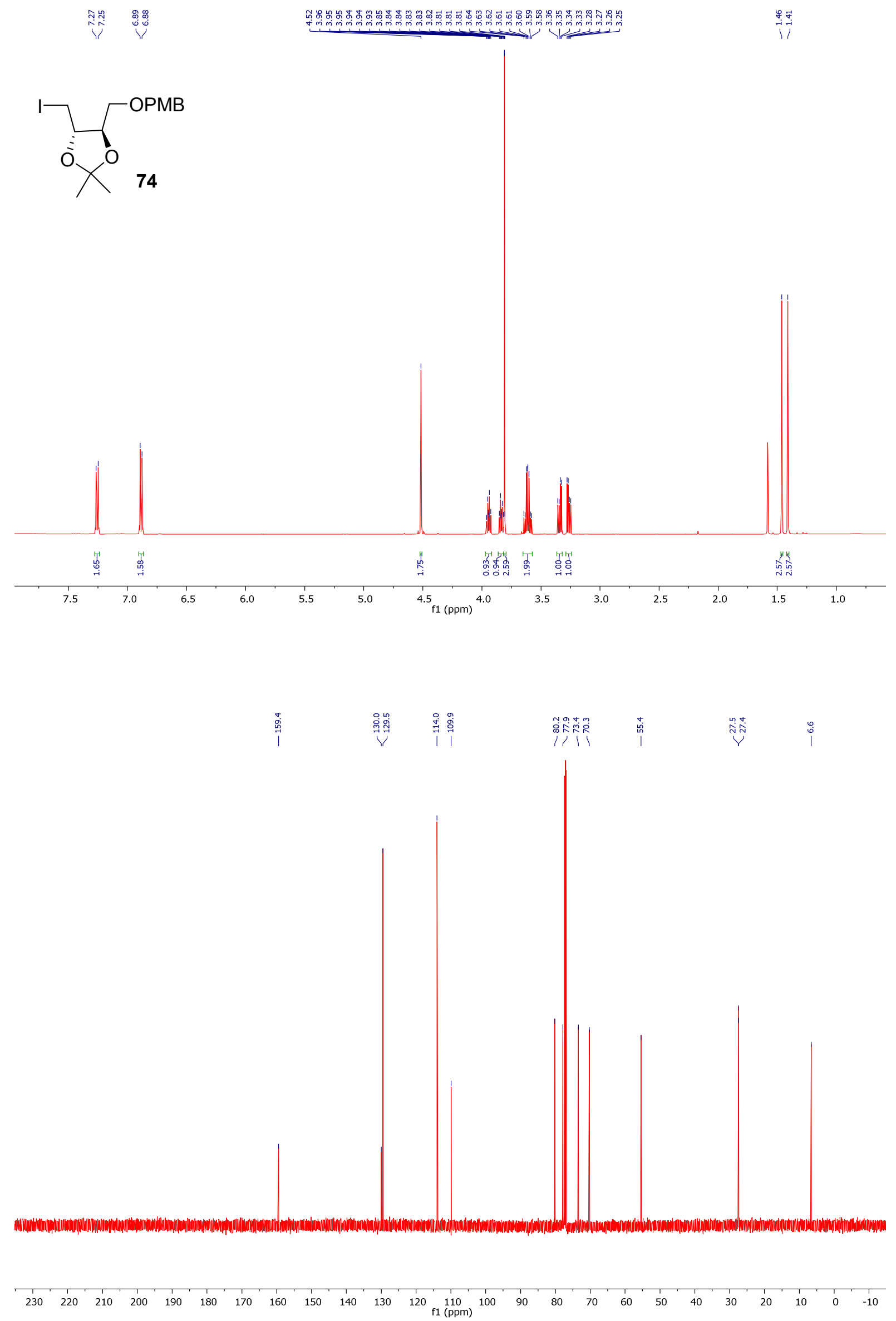


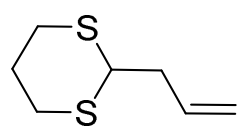

161
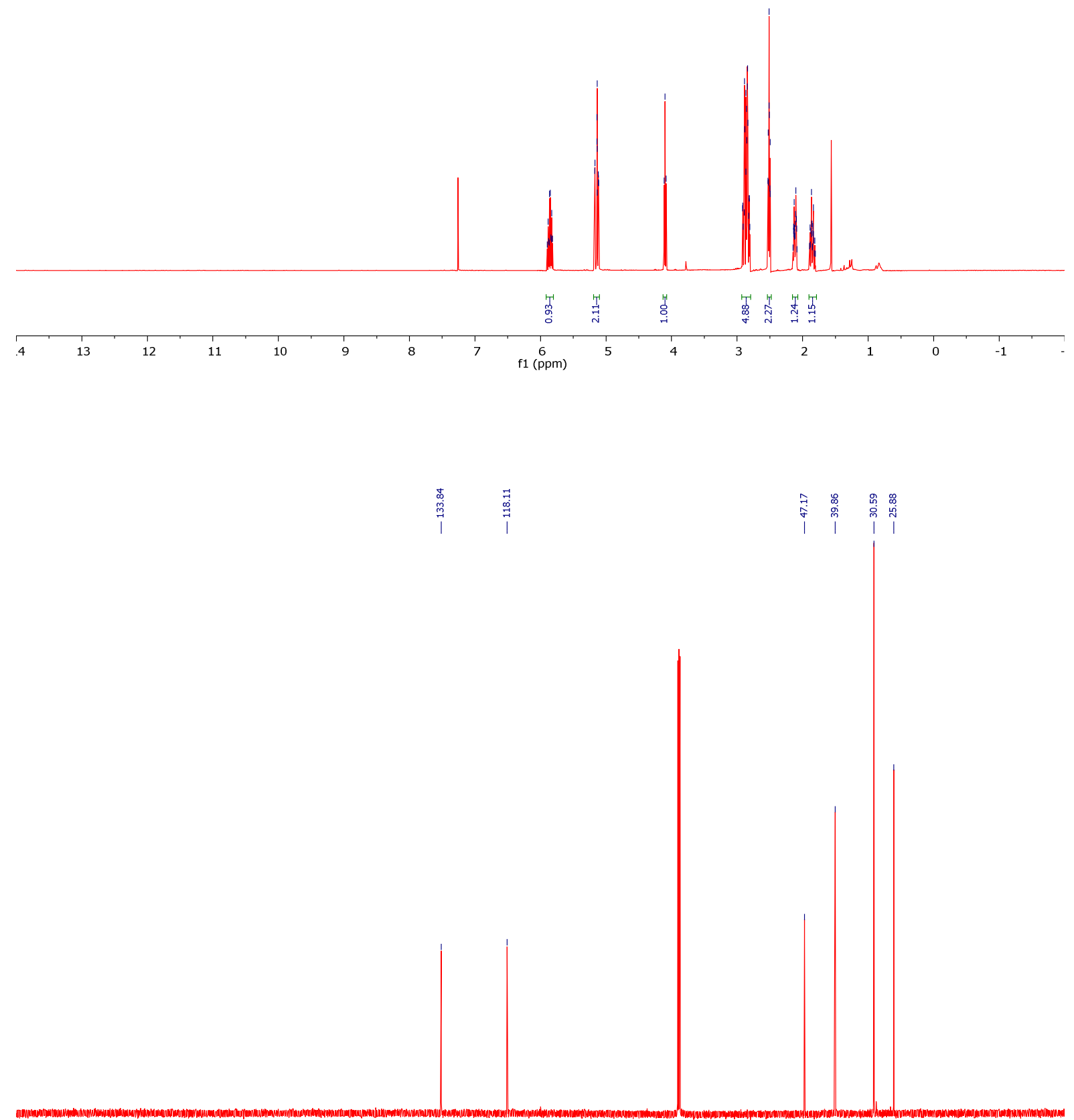

$\begin{array}{llllllllllllllllllllllllllllllllllll}230 & 220 & 210 & 200 & 190 & 180 & 170 & 160 & 150 & 140 & 130 & 120 & 110 & 100 & 90 & 80 & 70 & 60 & 50 & 40 & 30 & 20 & 10 & 0 & -10\end{array}$ 

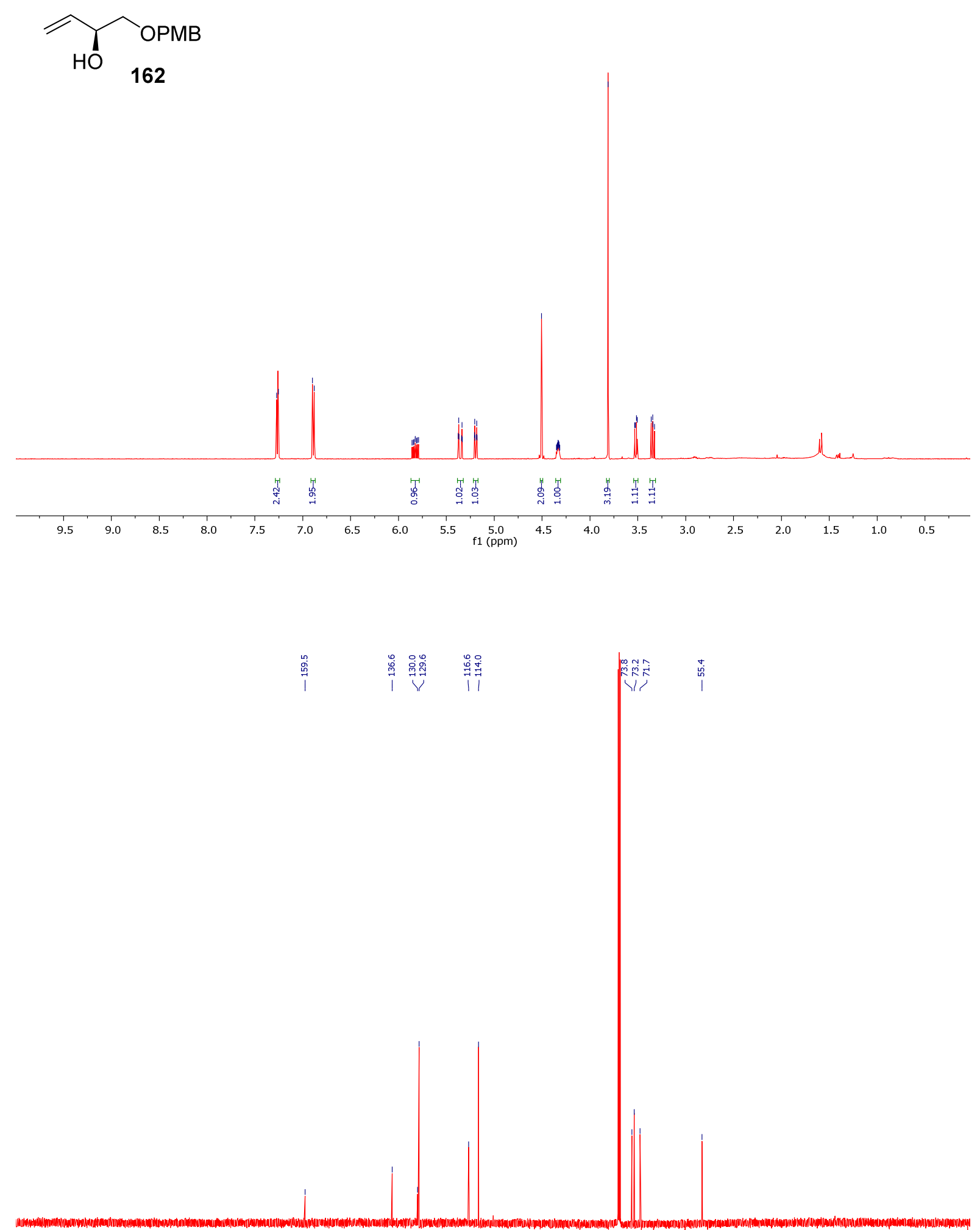

$\begin{array}{lllllllllllllllllllllllllllll}230 & 220 & 210 & 200 & 190 & 180 & 170 & 160 & 150 & 140 & 130 & 120 & 110 & 100 & 90 & 80 & 70 & 60 & 50 & 40 & 30 & 20 & 10 & 0 & -10\end{array}$ 


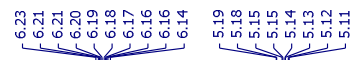

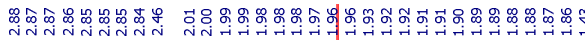

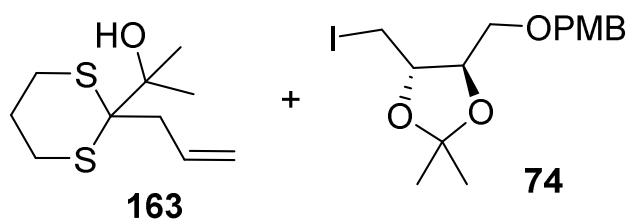

163
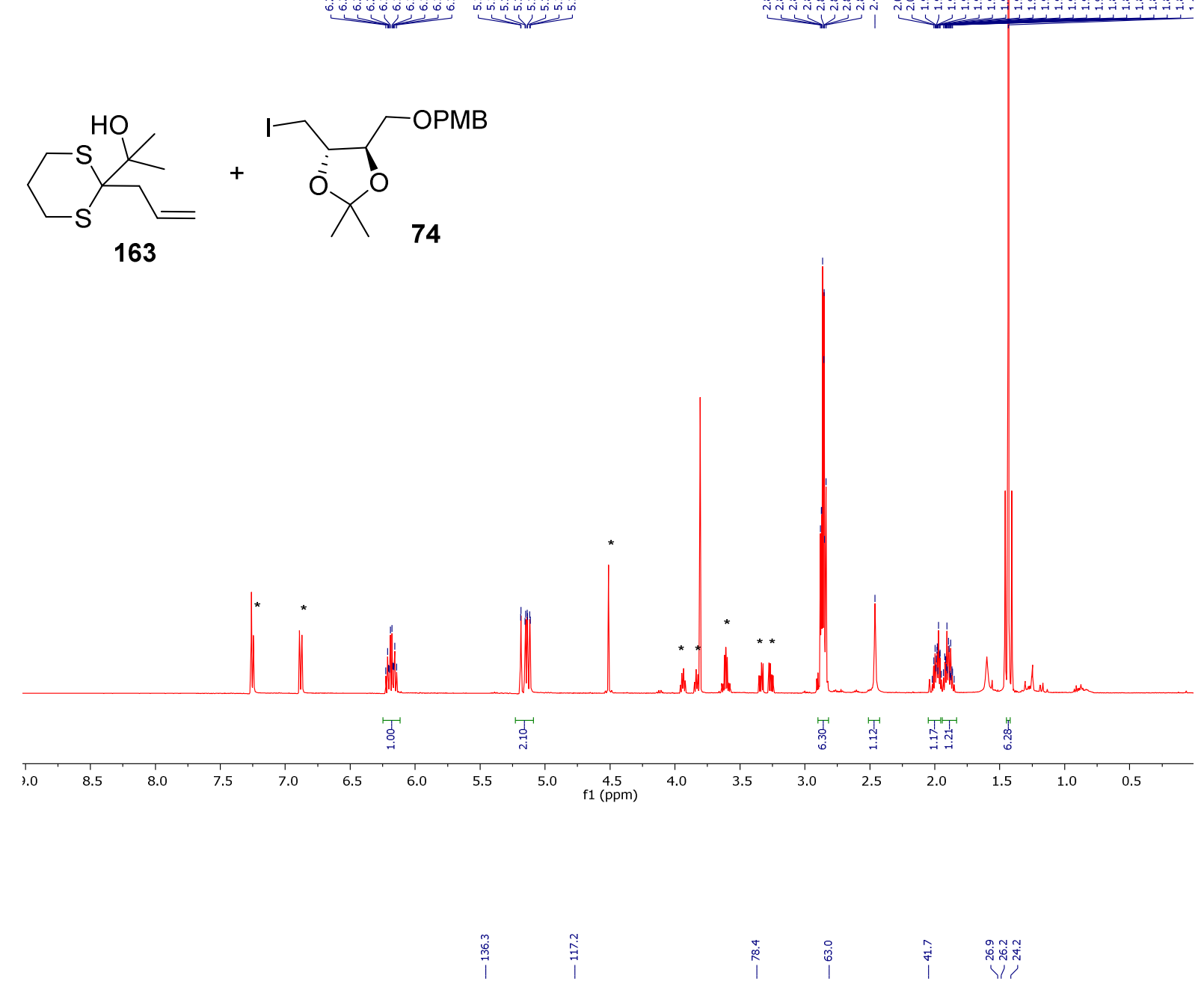

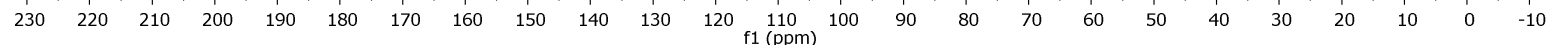




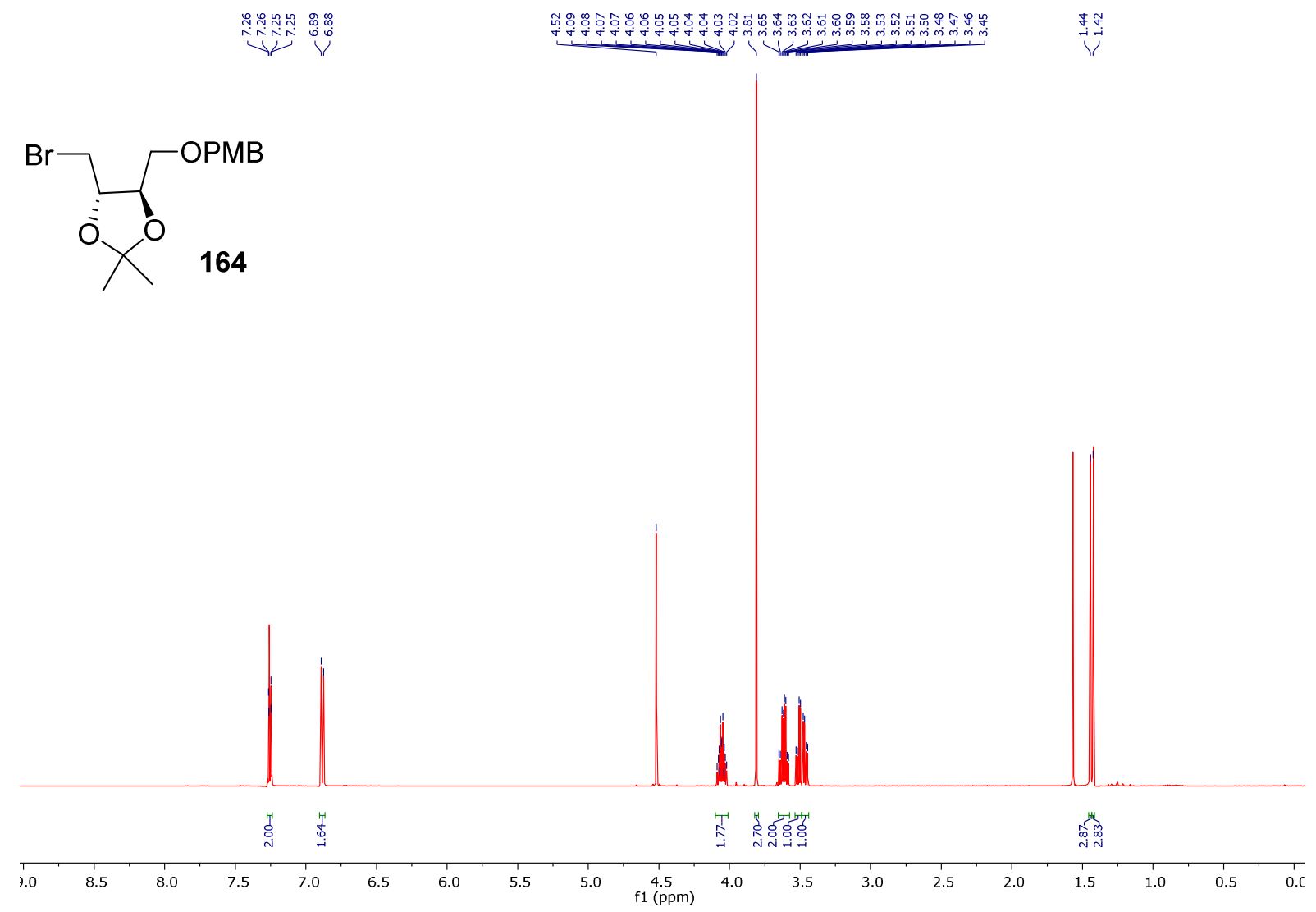

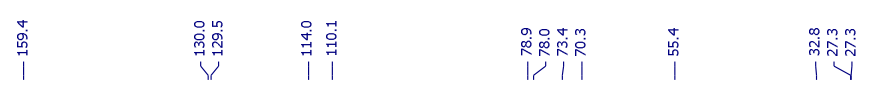

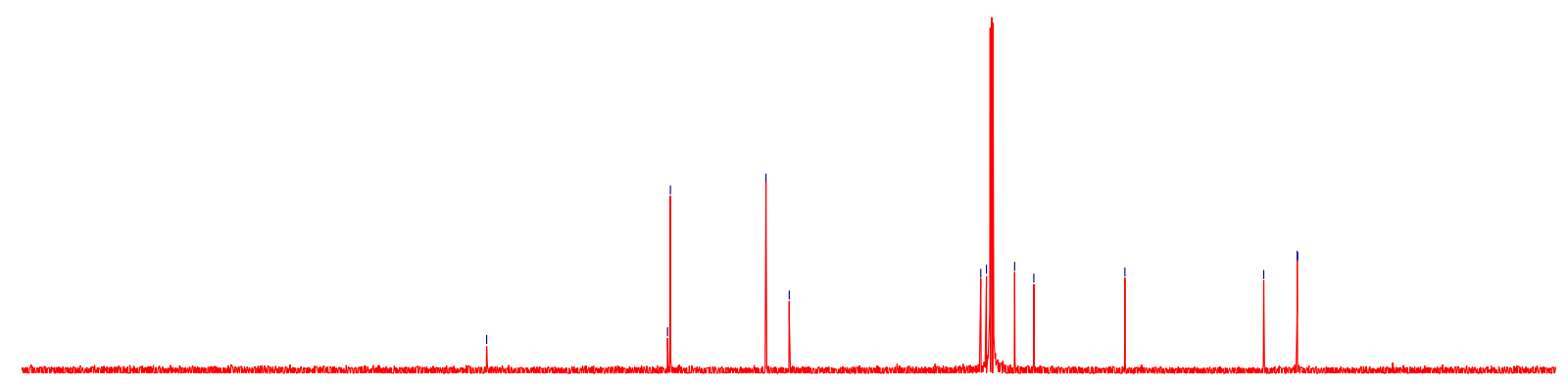

\begin{tabular}{llllllllllllllllllllllllllllllll}
\hline 230 & 220 & 210 & 200 & 190 & 180 & 170 & 160 & 150 & 140 & 130 & 120 & 110 & 100 & 90 & 80 & 70 & 60 & 50 & 40 & 30 & 20 & 10 & 0 & -10
\end{tabular} 

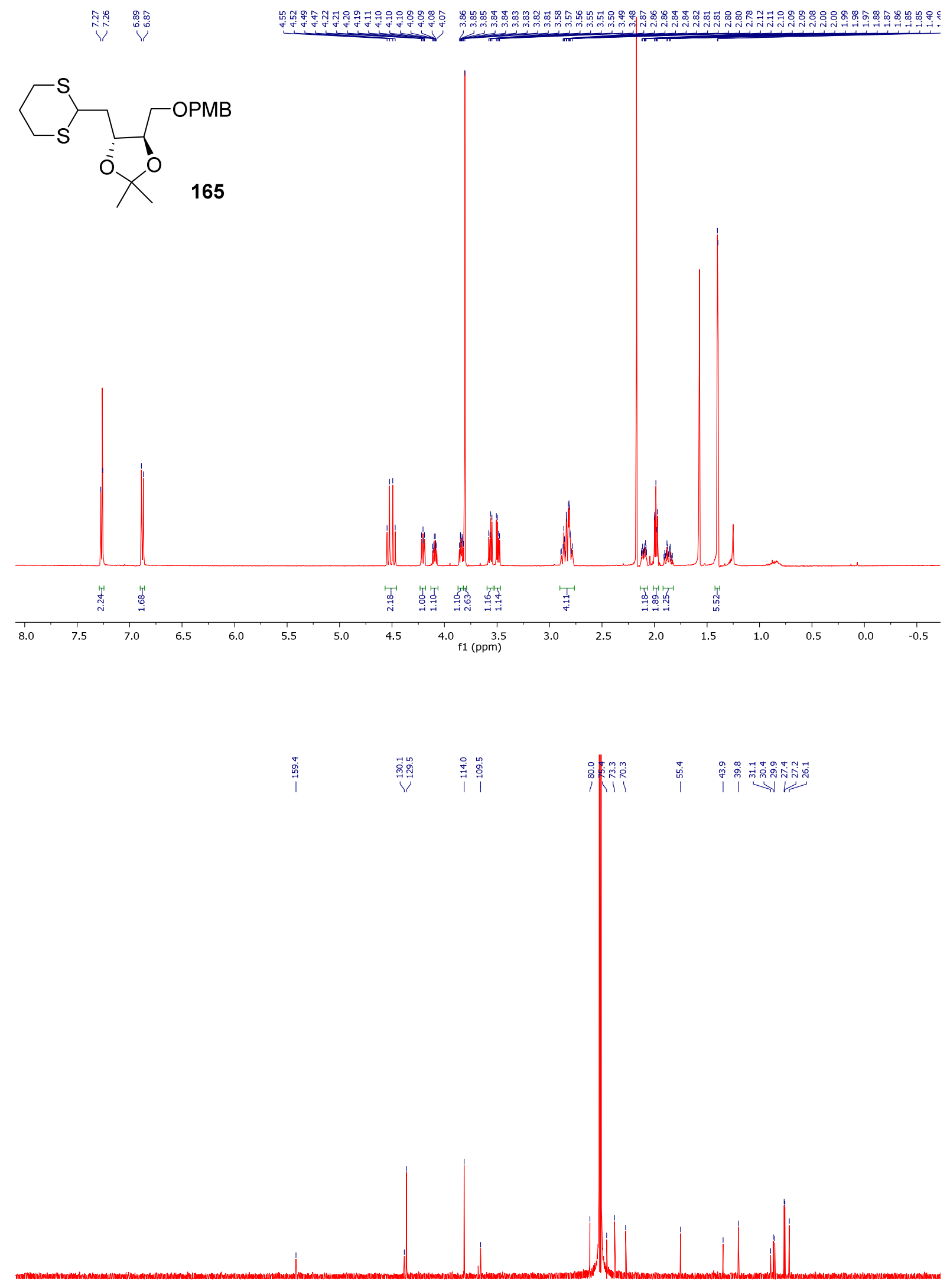

\begin{tabular}{llllllllllllllllllllllllllllllll}
\hline 230 & 220 & 210 & 200 & 190 & 180 & 170 & 160 & 150 & 140 & 130 & 120 & 110 & 100 & 90 & 80 & 70 & 60 & 50 & 40 & 30 & 20 & 10 & 0 & -10
\end{tabular} 


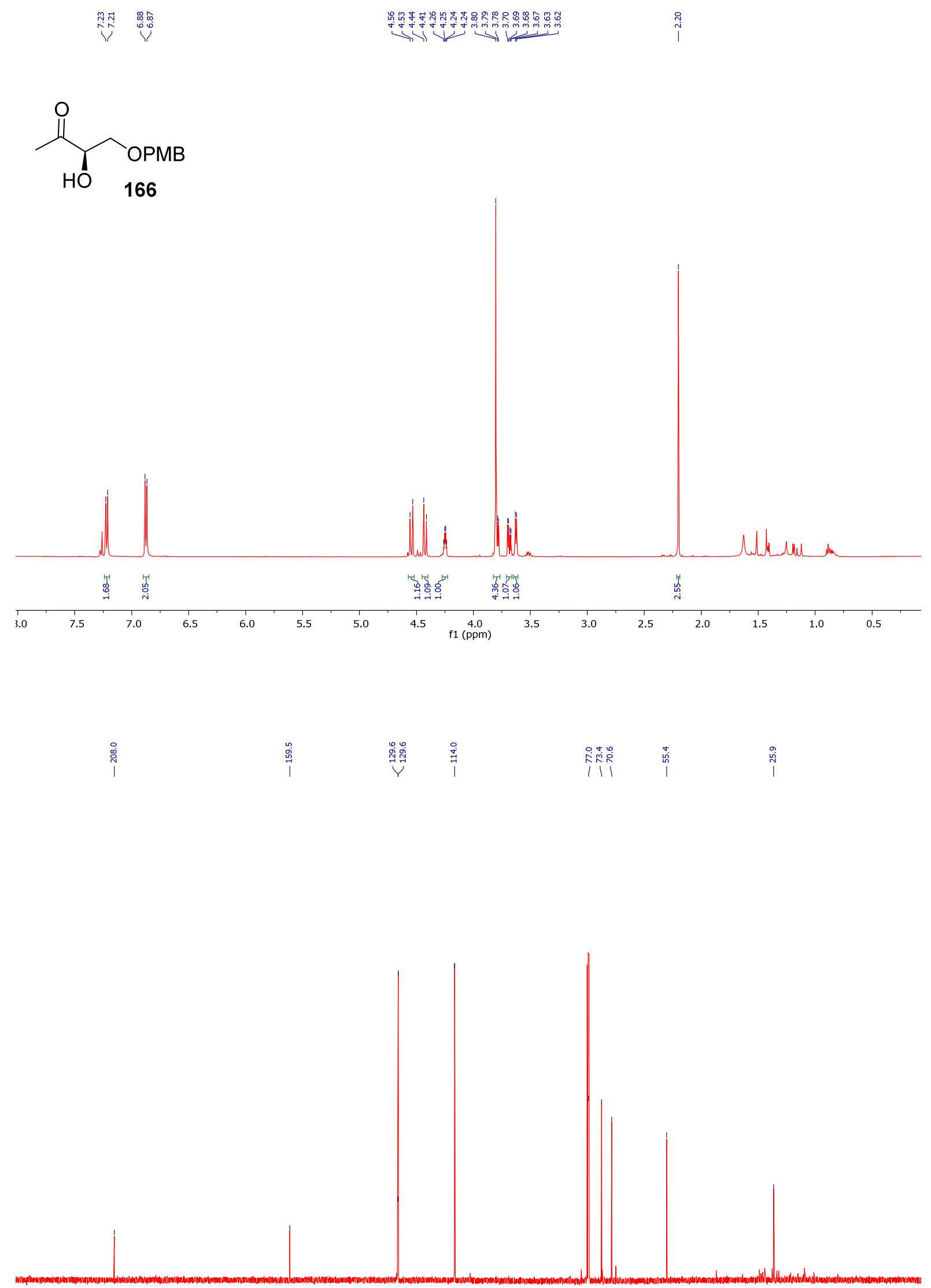

$\begin{array}{lllllllllllllllllllllllllllllllll}230 & 220 & 210 & 200 & 190 & 180 & 170 & 160 & 150 & 140 & 130 & 120 & 110 & 100 & 90 & 80 & 70 & 60 & 50 & 40 & 30 & 20 & 10 & 0 & -10\end{array}$ 


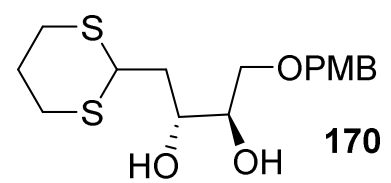

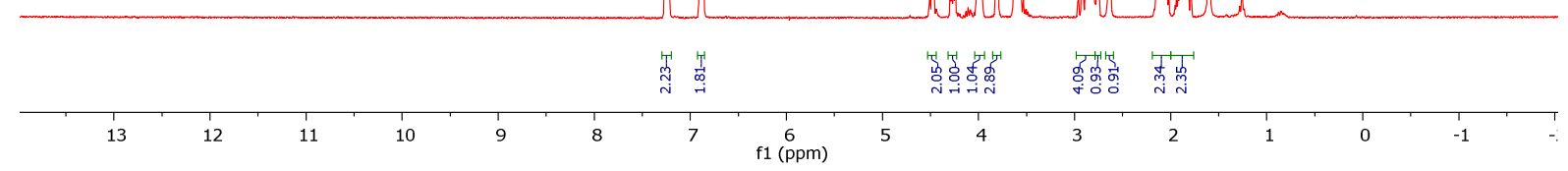



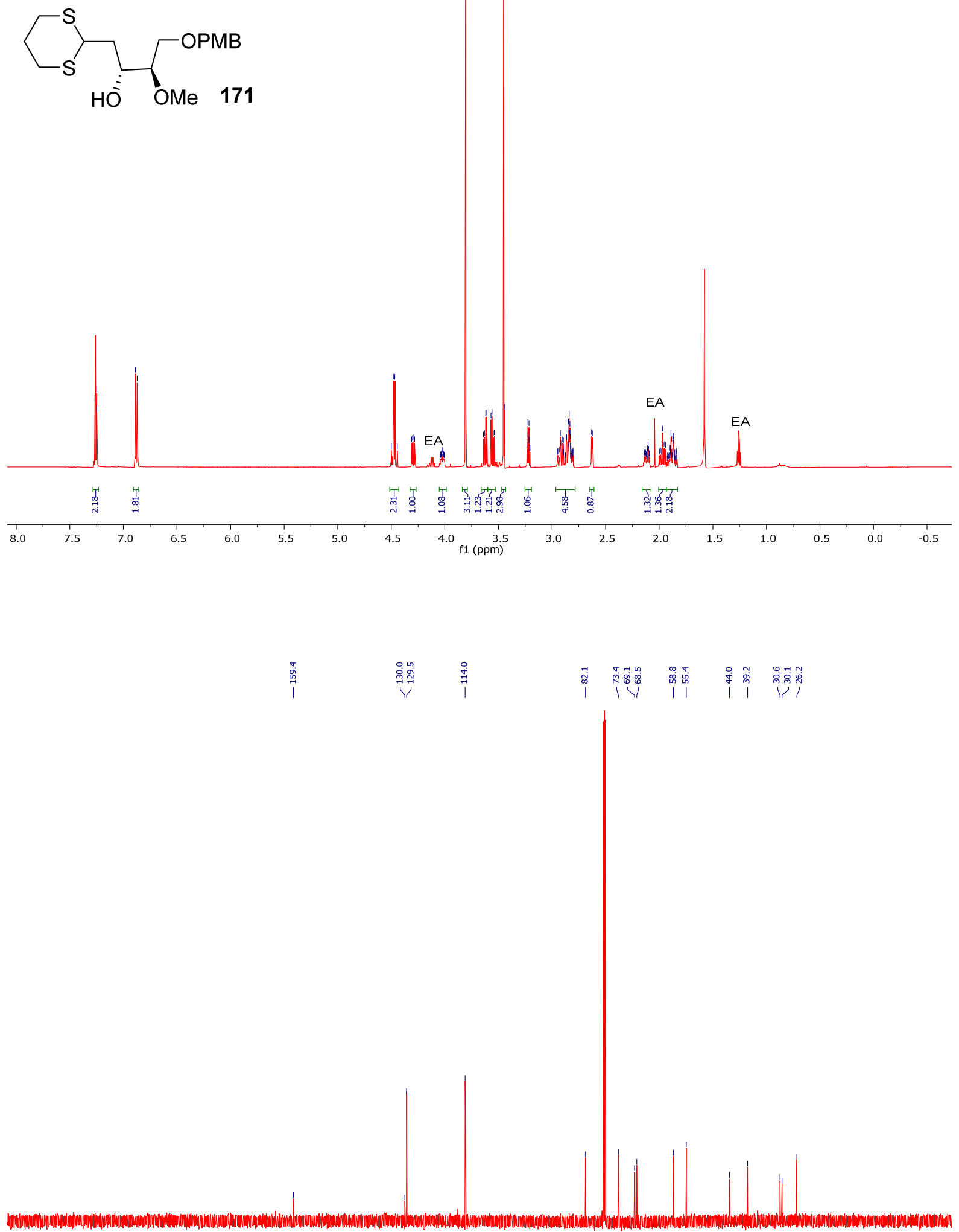

$\begin{array}{lllllllllllllllllllllllllllllllll}230 & 220 & 210 & 200 & 190 & 180 & 170 & 160 & 150 & 140 & 130 & 120 & 110 & 100 & 90 & 80 & 70 & 60 & 50 & 40 & 30 & 20 & 10 & 0 & -10\end{array}$ 

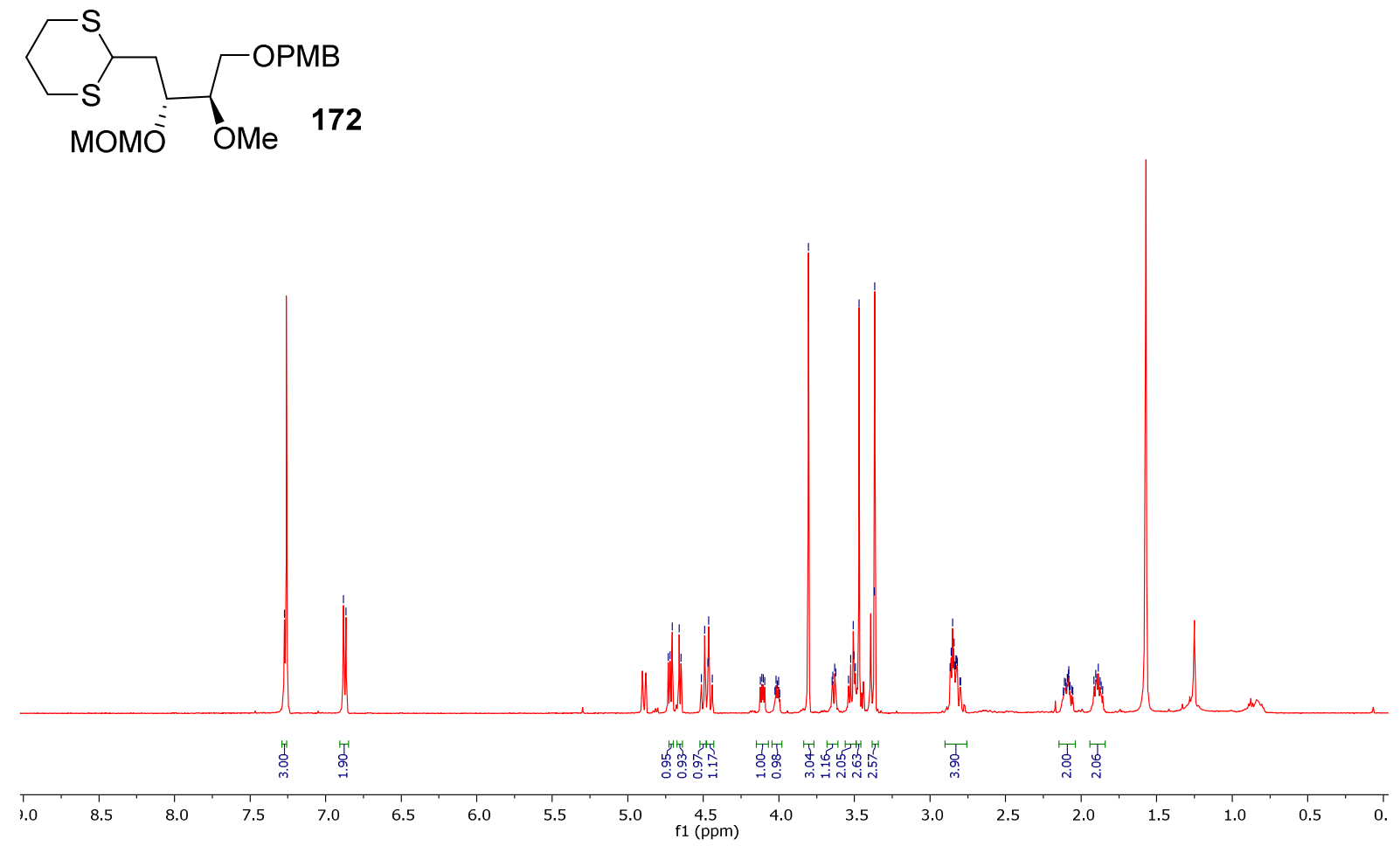

兽

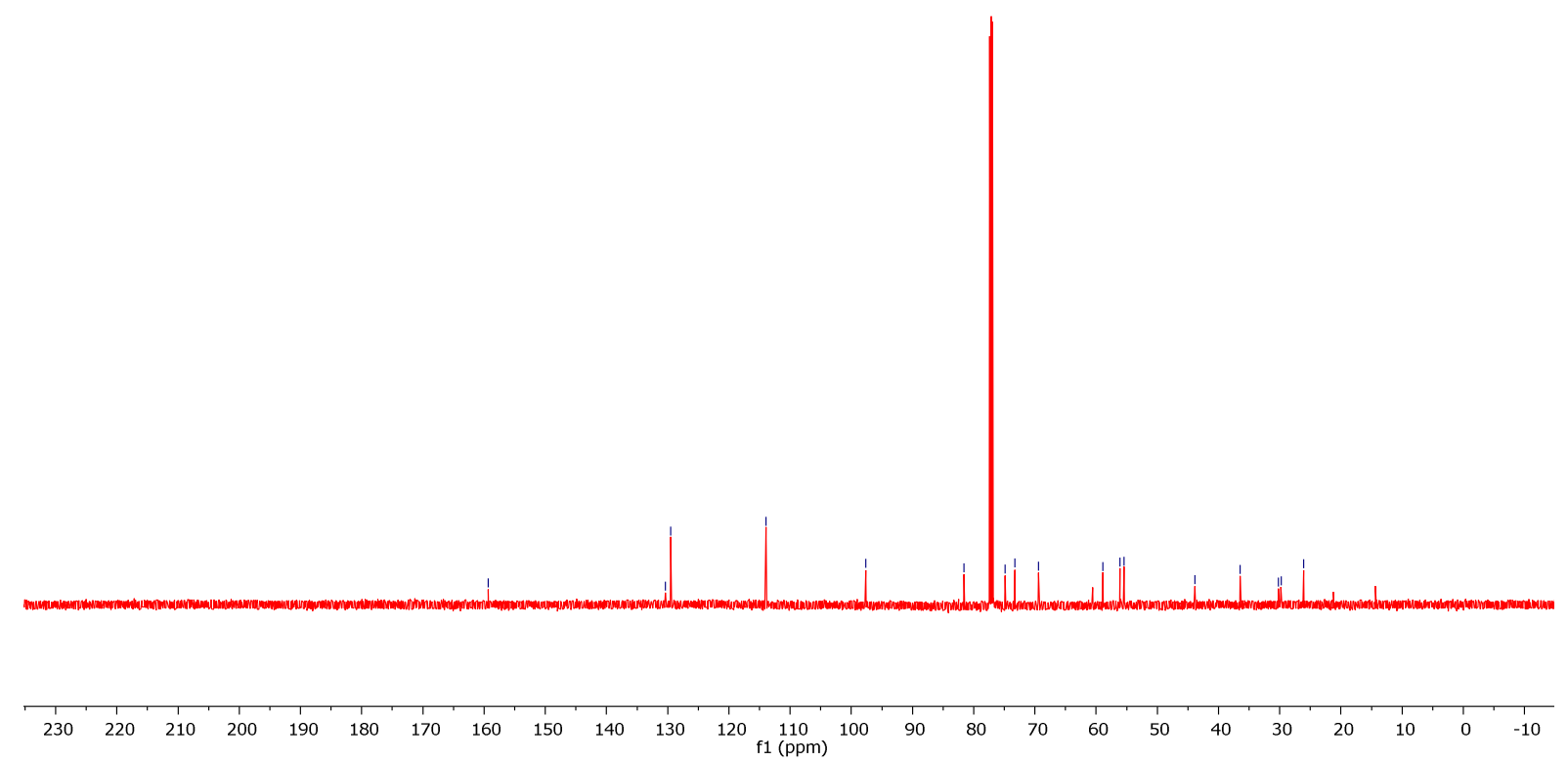




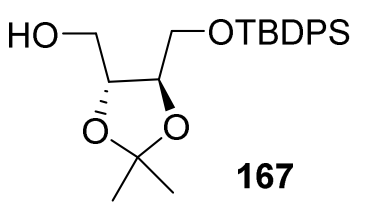

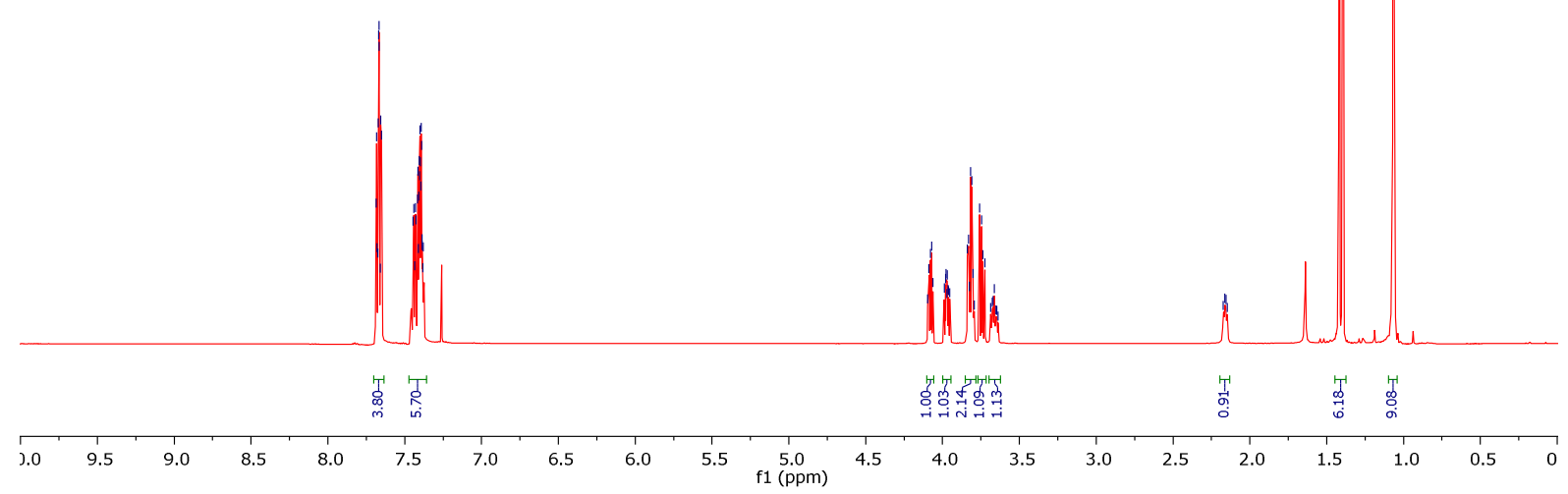

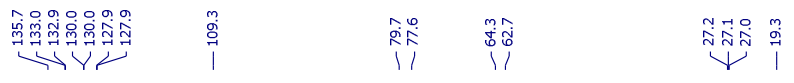

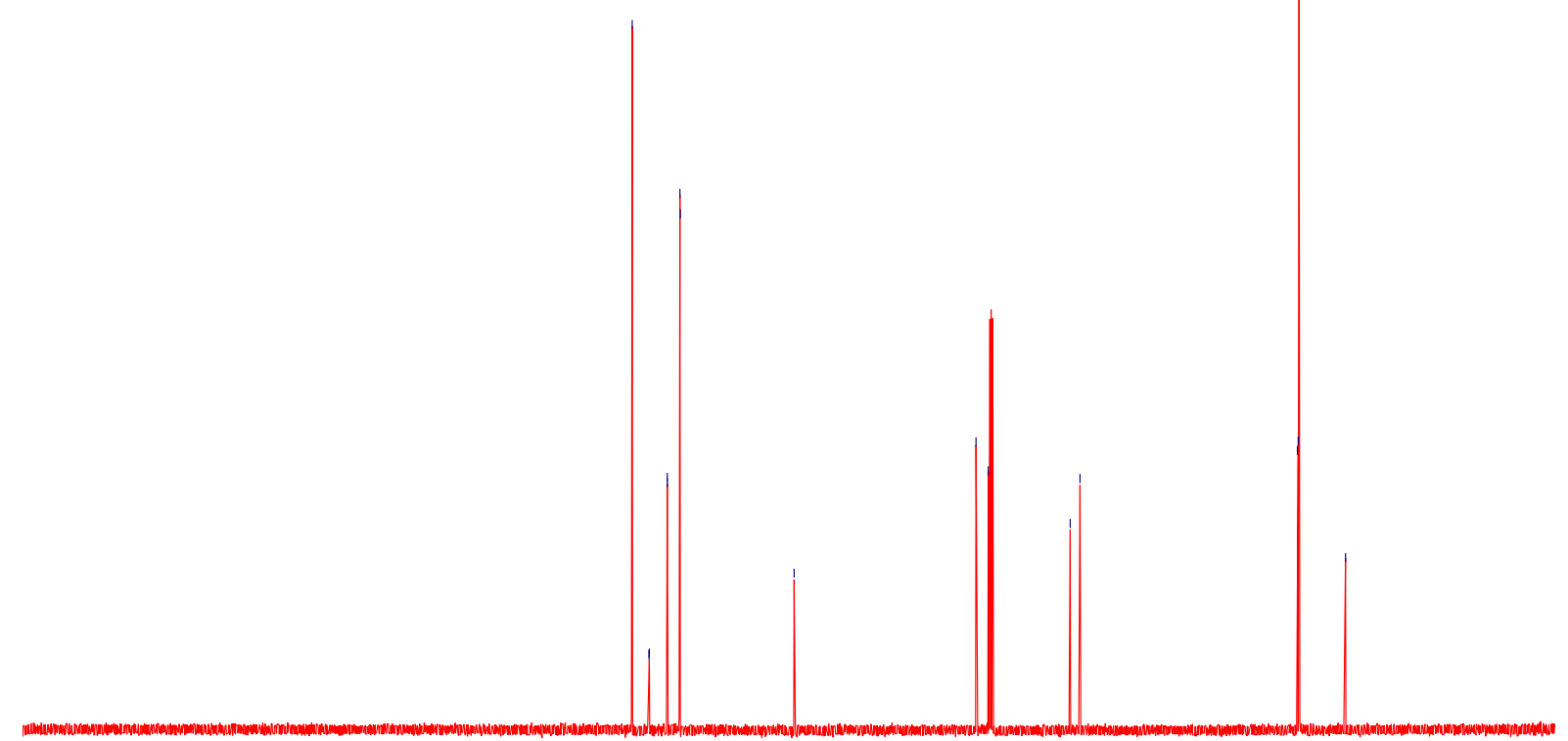

$\begin{array}{llllllllllllllllllllllllllllllll}230 & 220 & 210 & 200 & 190 & 180 & 170 & 160 & 150 & 140 & 130 & 120 & 110 & 100 & 90 & 80 & 70 & 60 & 50 & 40 & 30 & 20 & 10 & 0 & -10\end{array}$ 


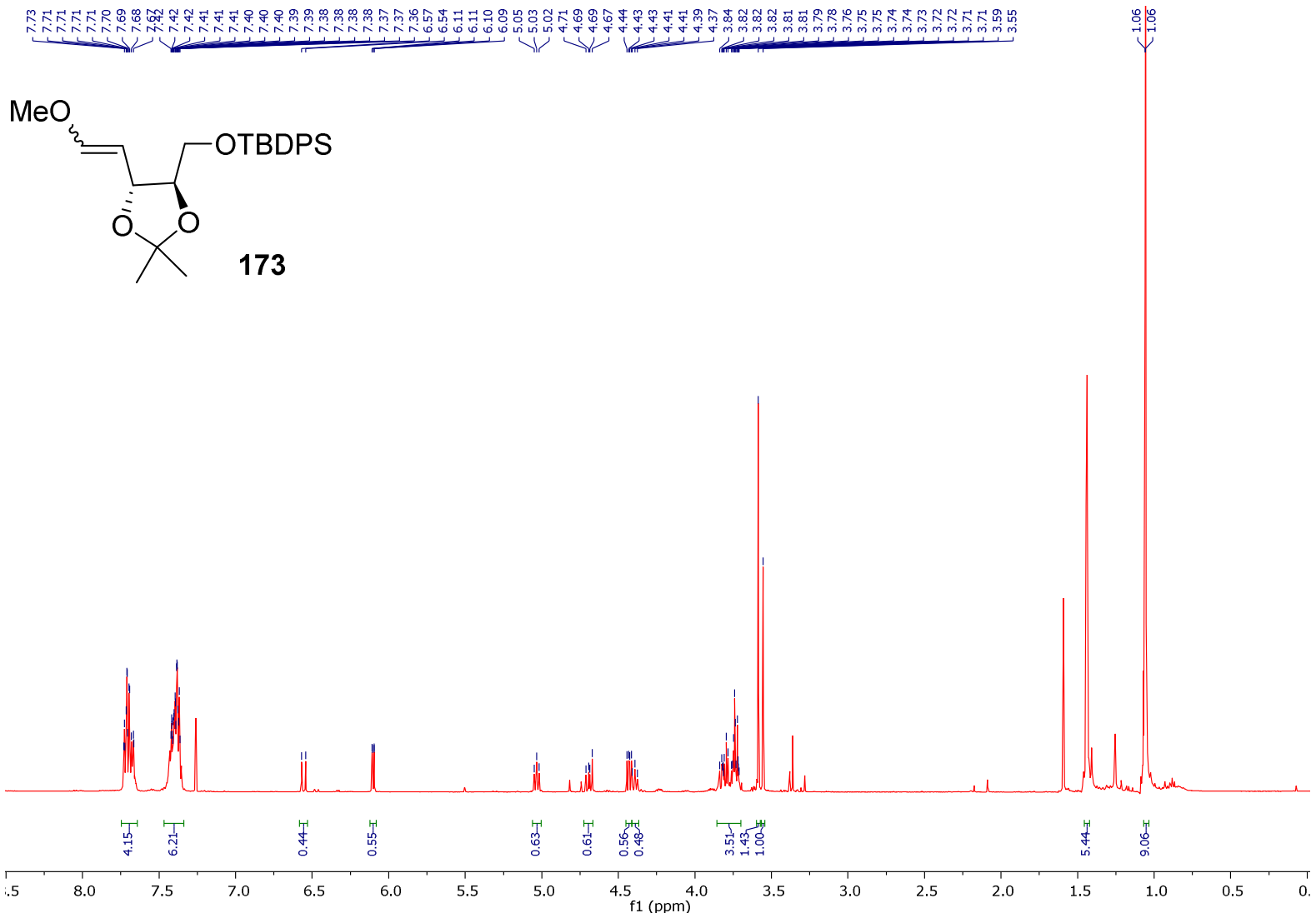

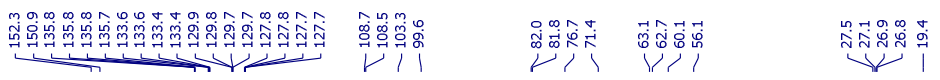

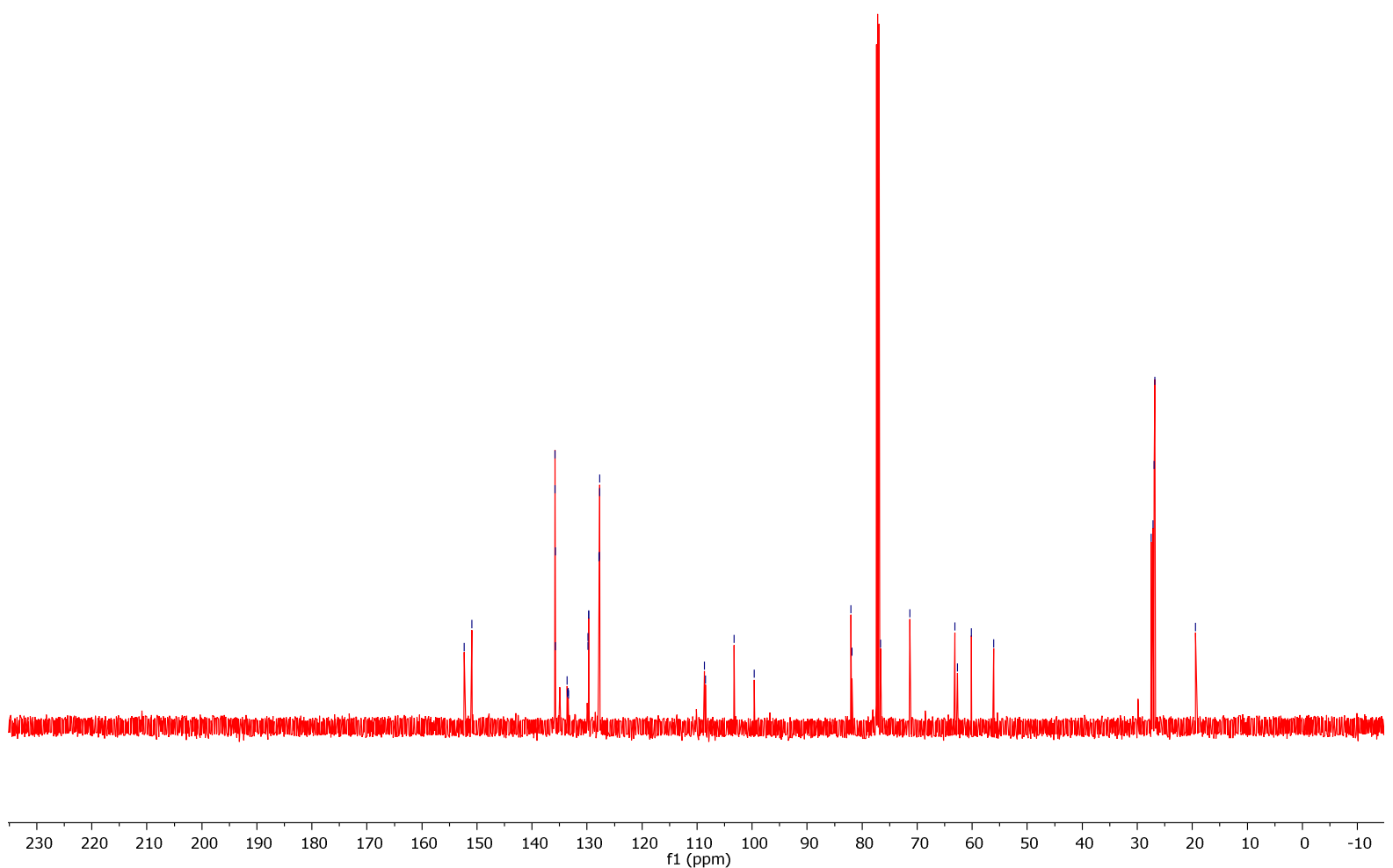



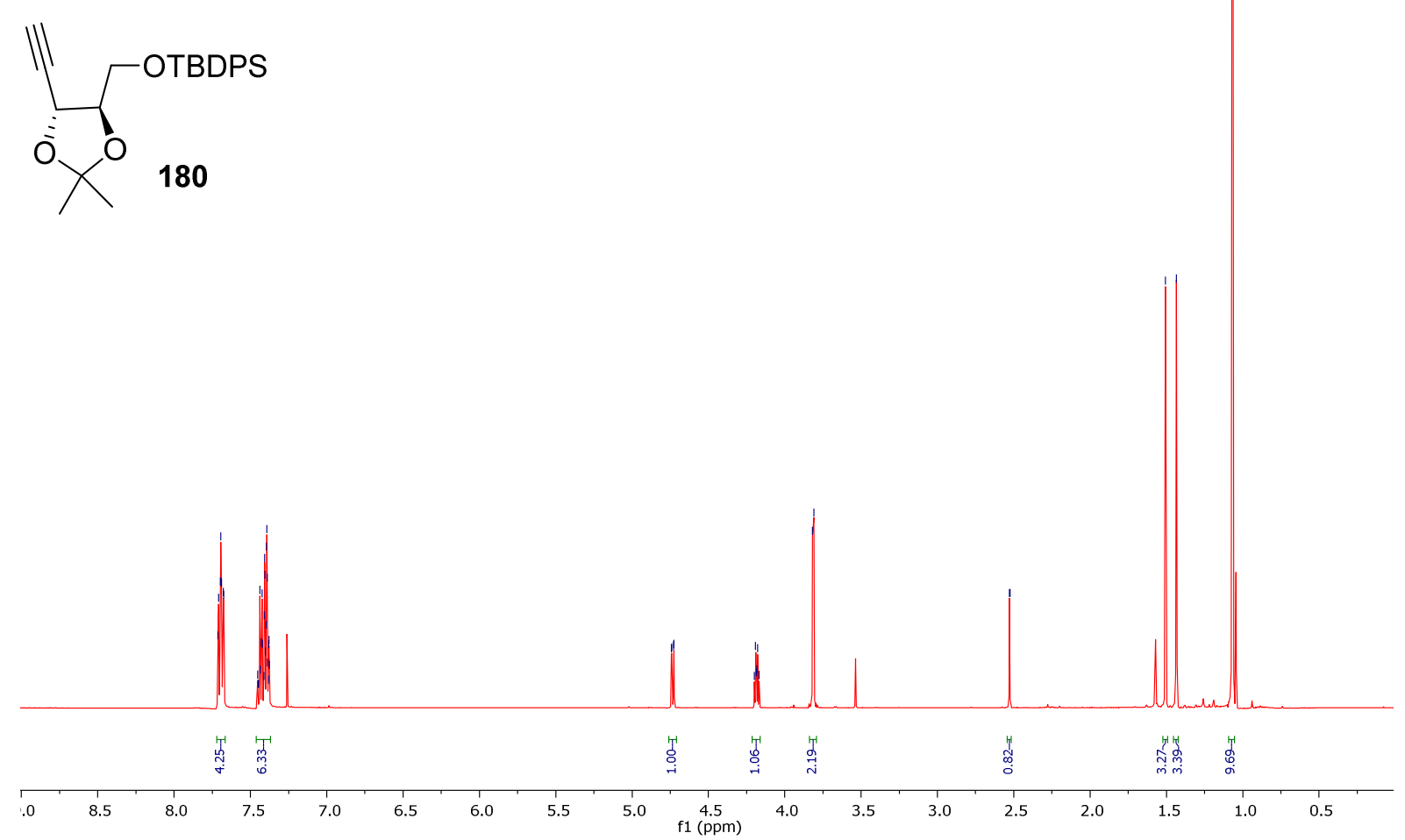

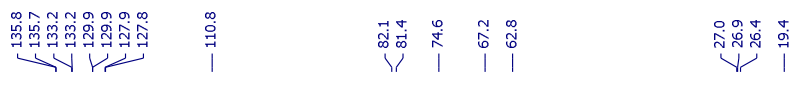

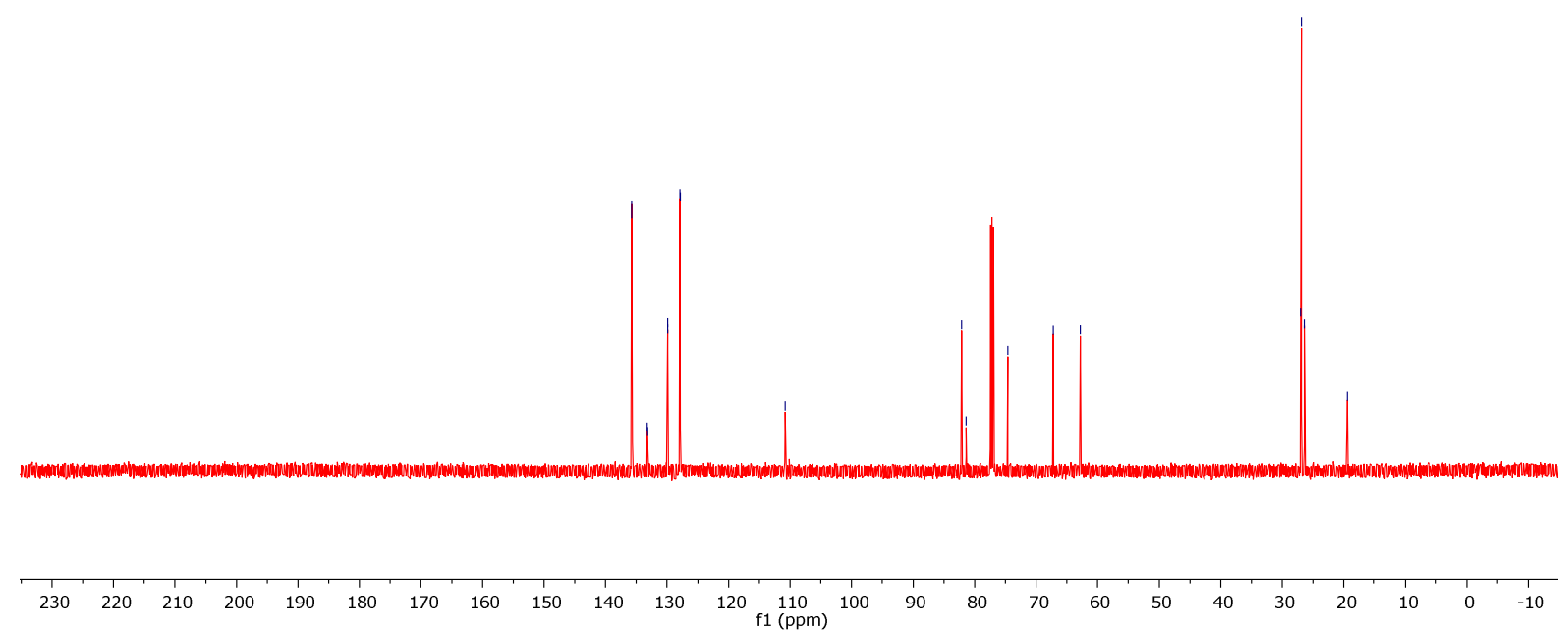




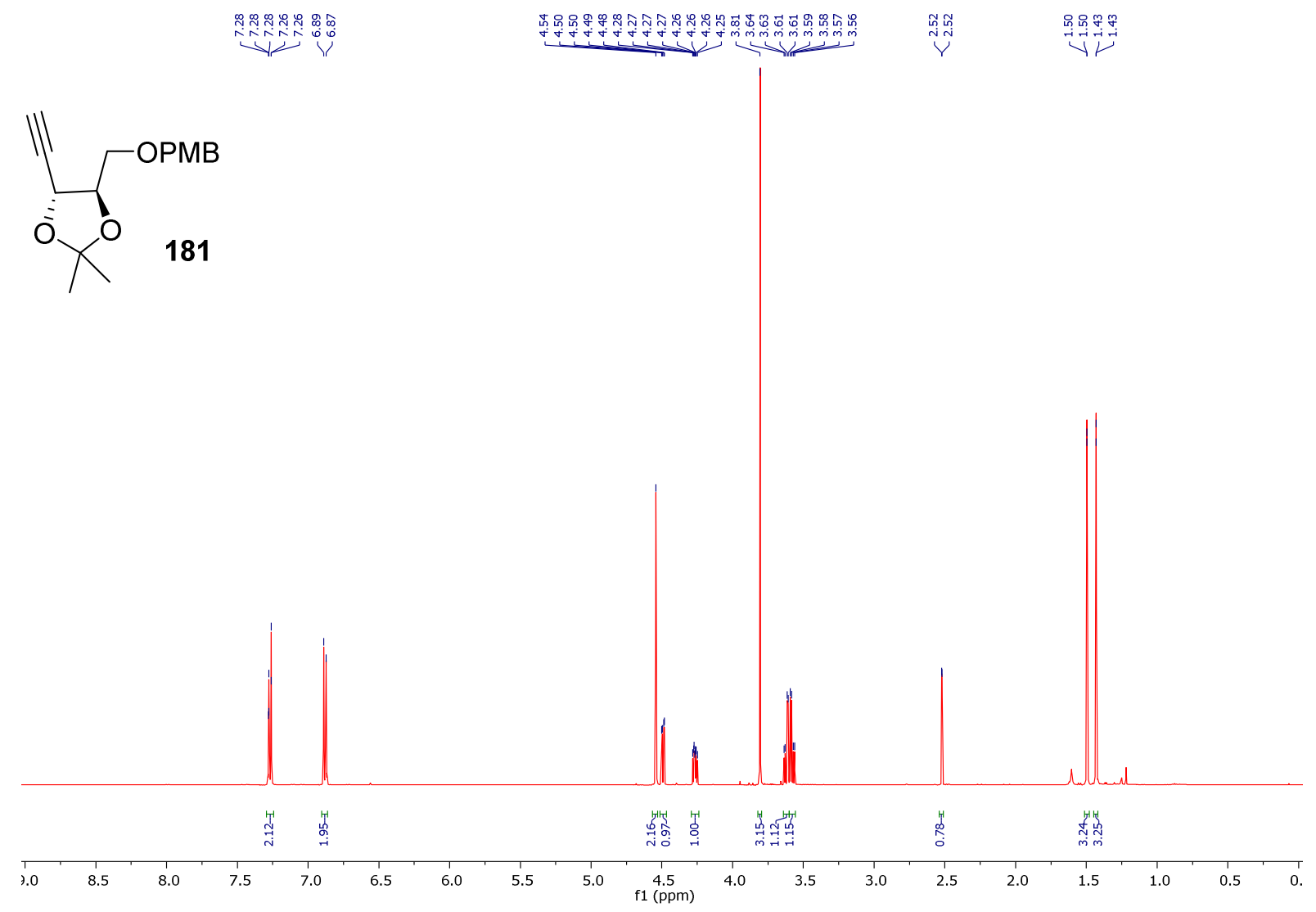

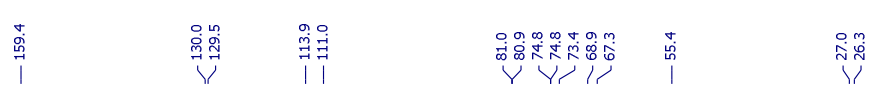

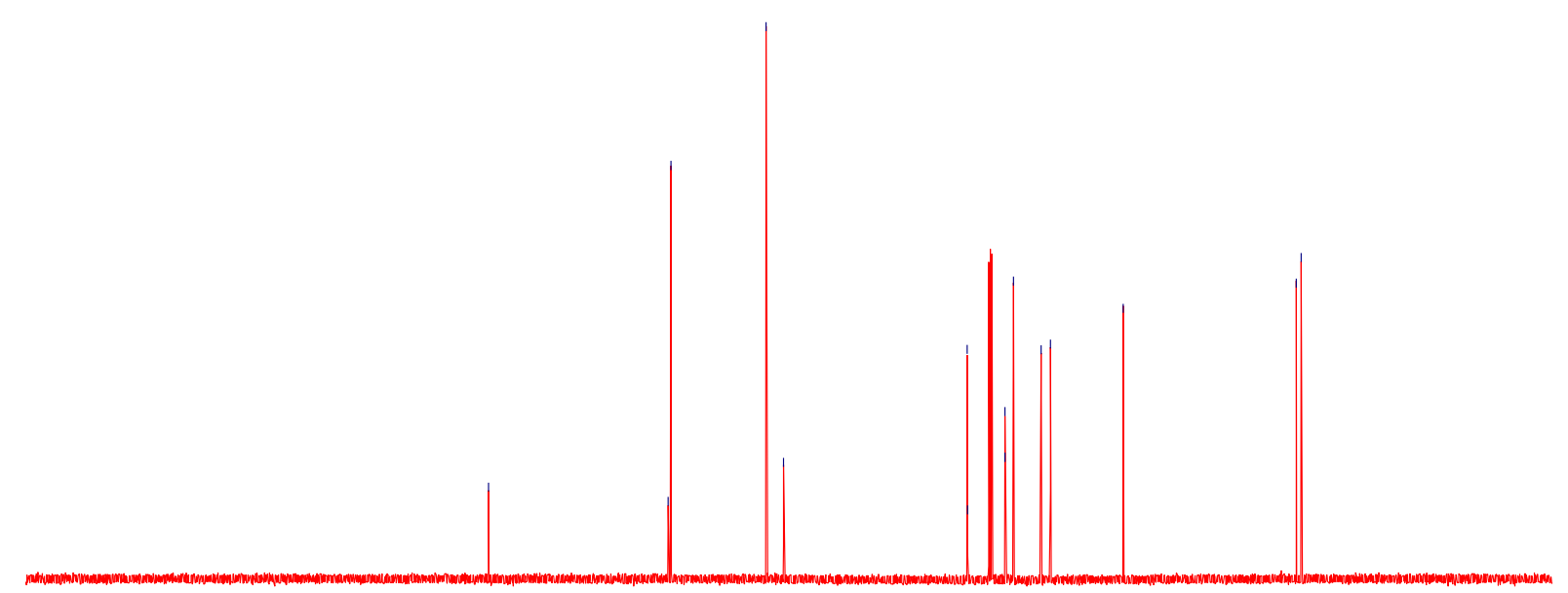

\begin{tabular}{llllllllllllllllllllllllllllll}
\hline 230 & 220 & 210 & 200 & 190 & 180 & 170 & 160 & 150 & 140 & 130 & 120 & 110 & 100 & 90 & 80 & 70 & 60 & 50 & 40 & 30 & 20 & 10 & 0 & -10
\end{tabular} 


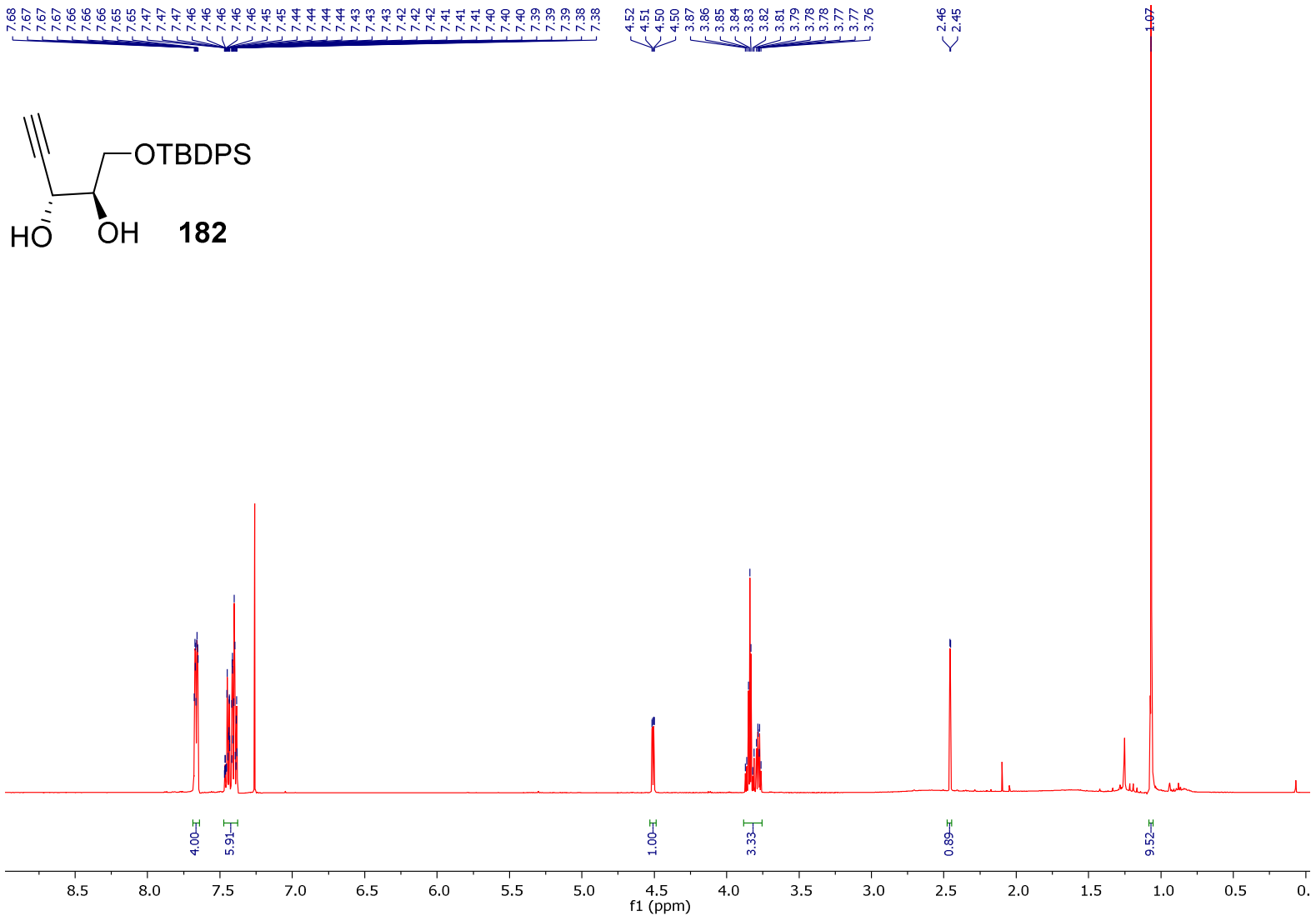

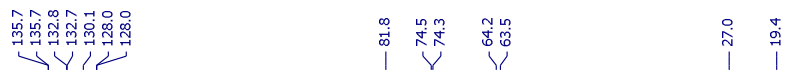

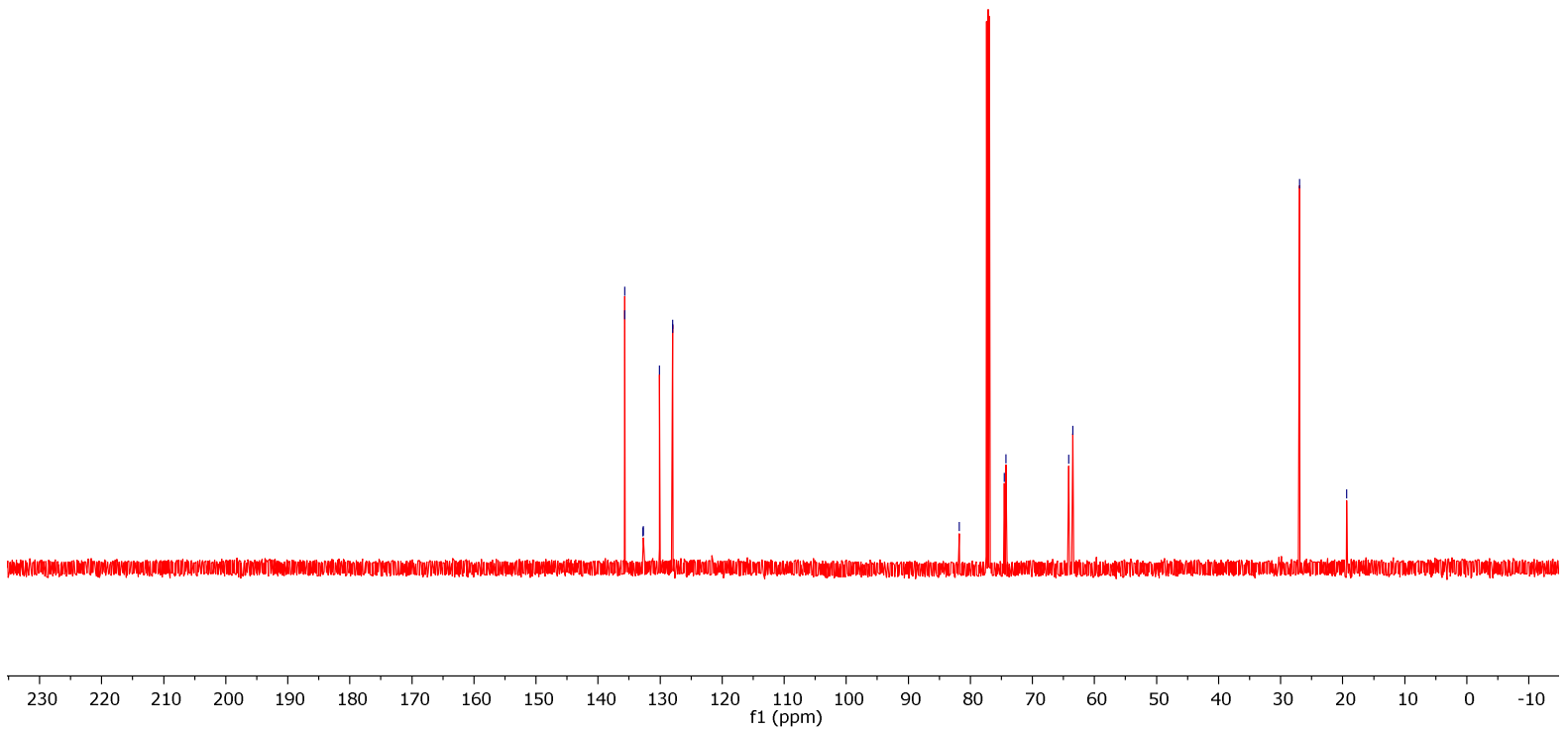



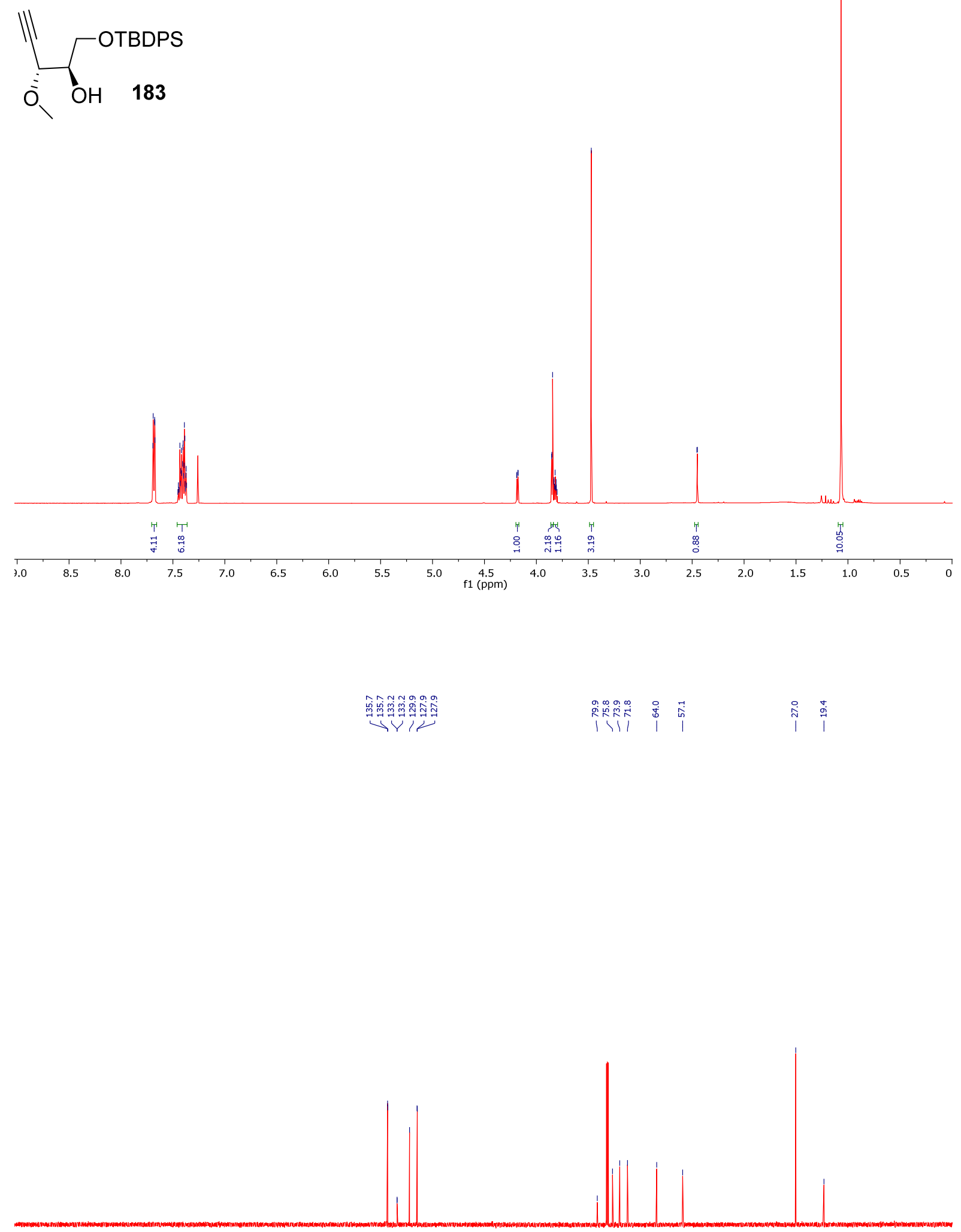

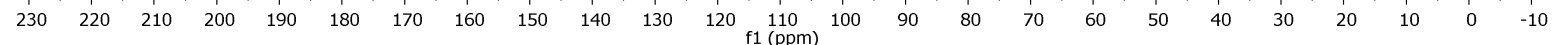




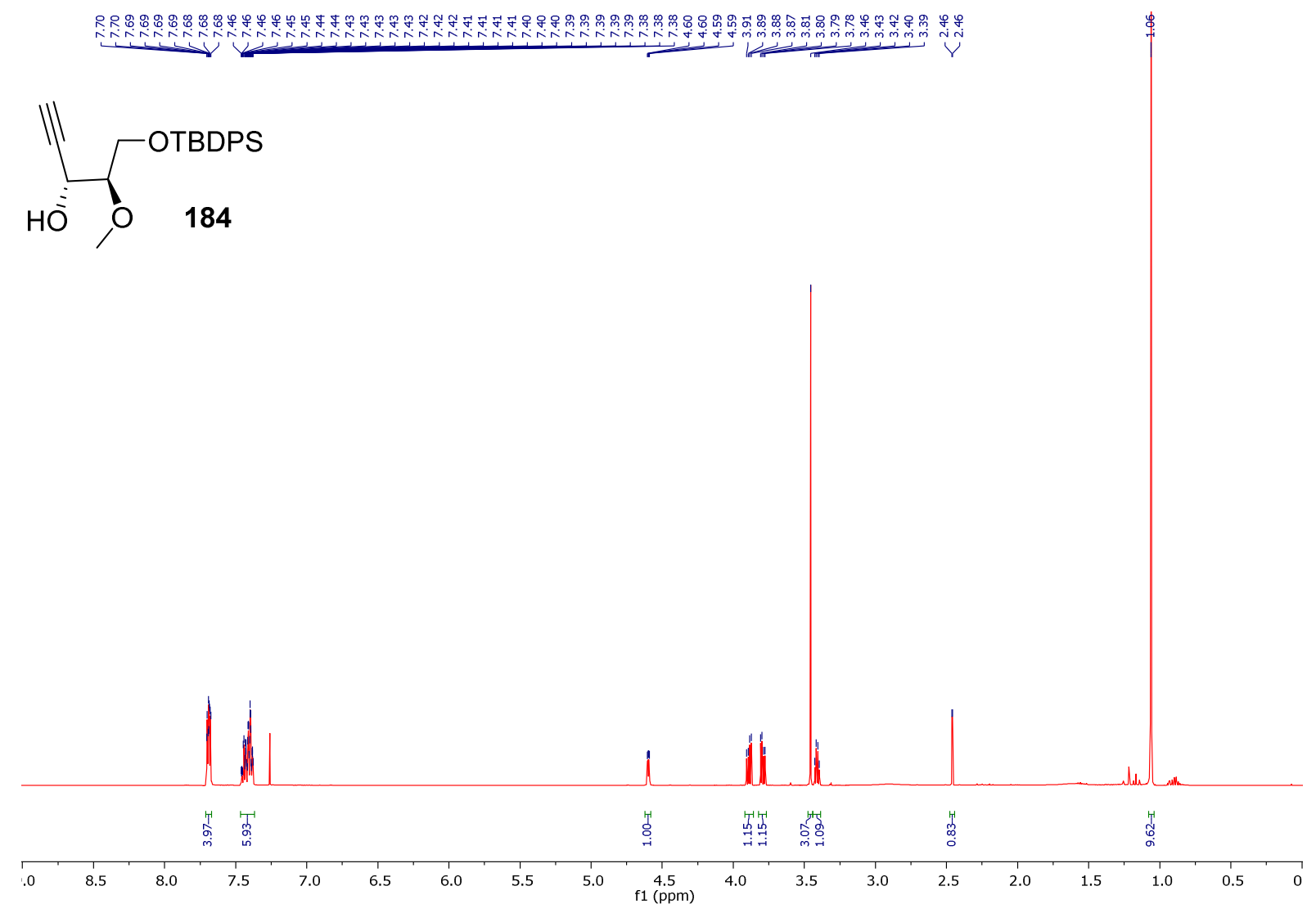

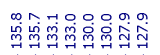

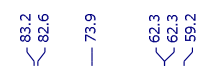

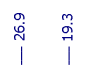

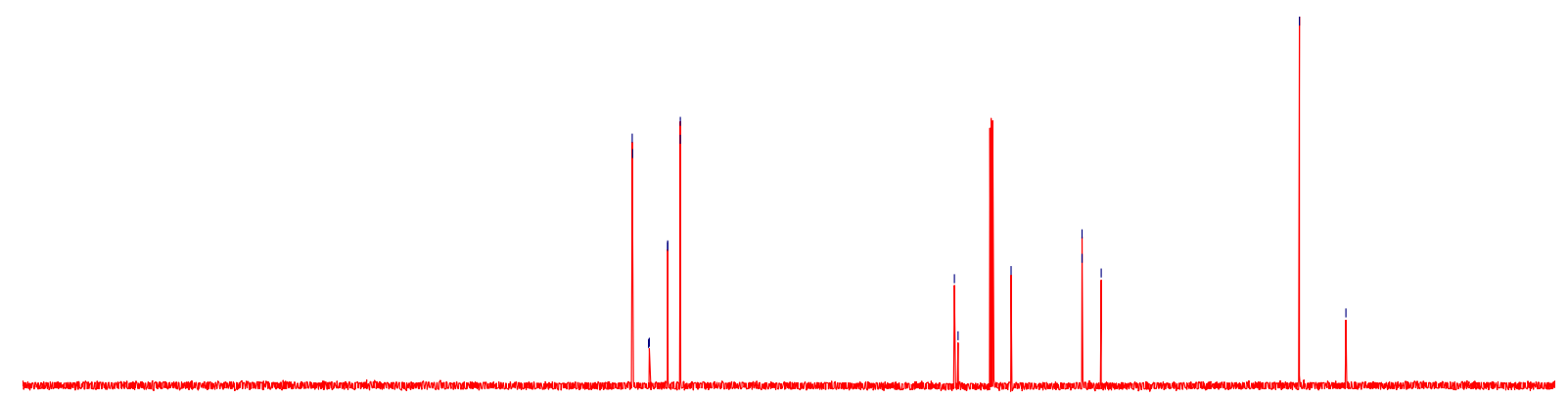

\begin{tabular}{llllllllllllllllllllllllllllllll}
\hline 230 & 220 & 210 & 200 & 190 & 180 & 170 & 160 & 150 & 140 & 130 & 120 & 110 & 100 & 90 & 80 & 70 & 60 & 50 & 40 & 30 & 20 & 10 & 0 & -10
\end{tabular} 
$\underbrace{\| \text { OMOM }}_{\text {MeO }} 185$

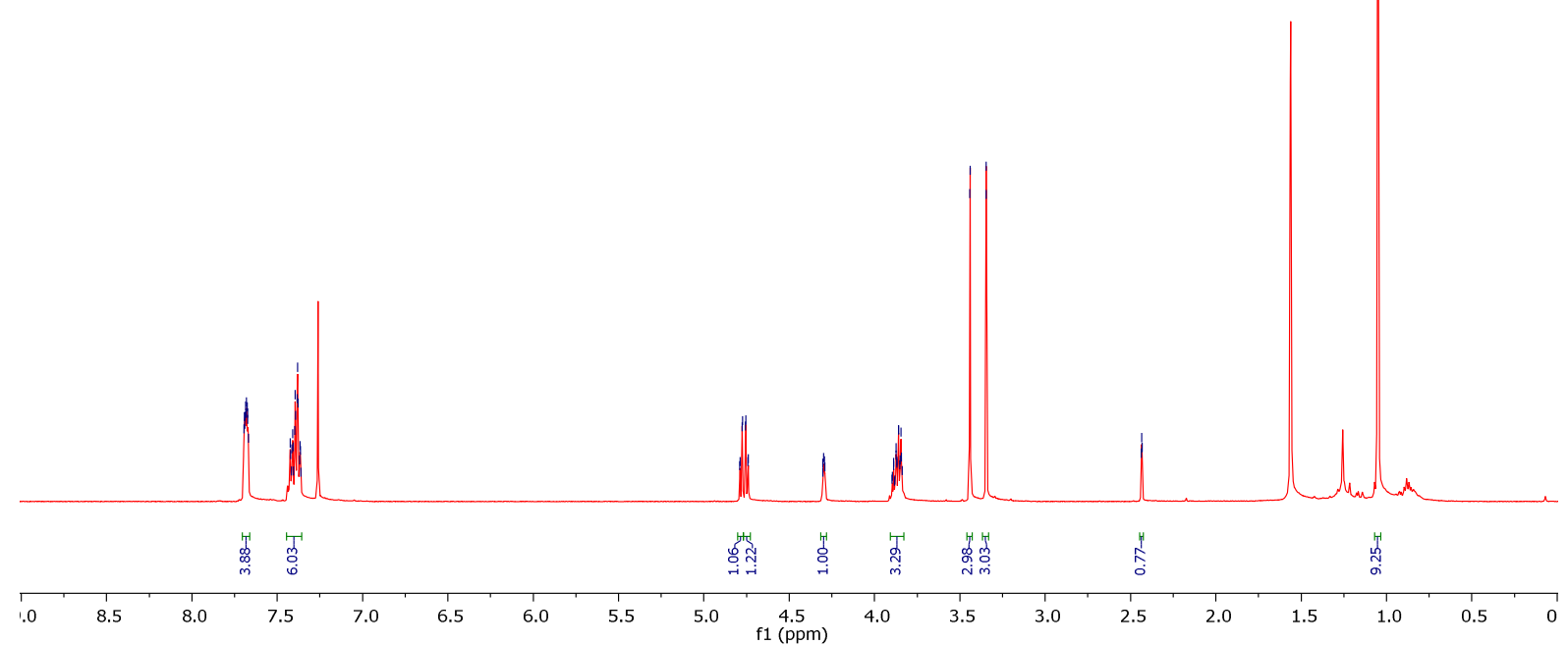

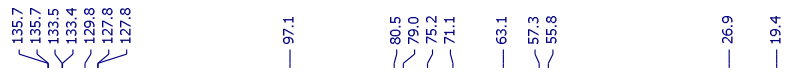

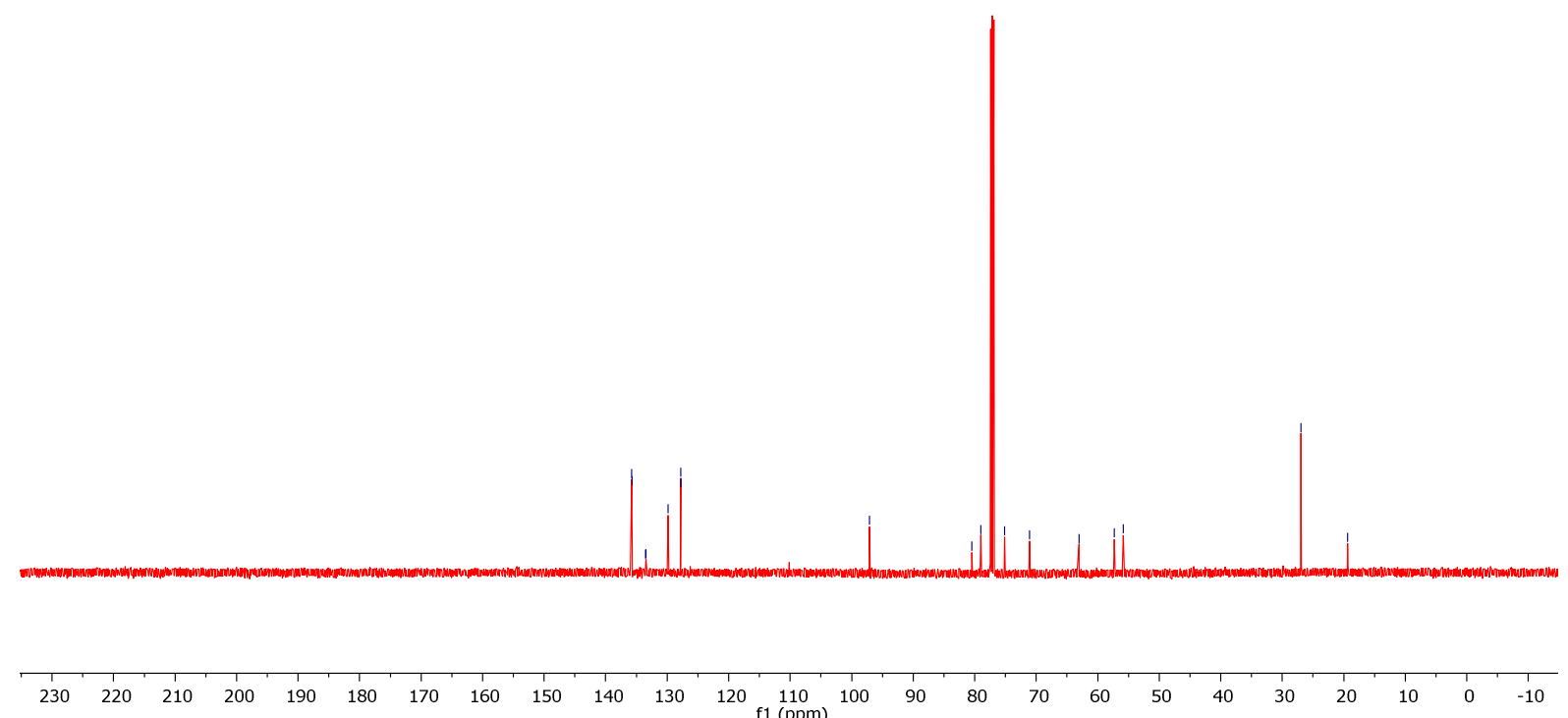




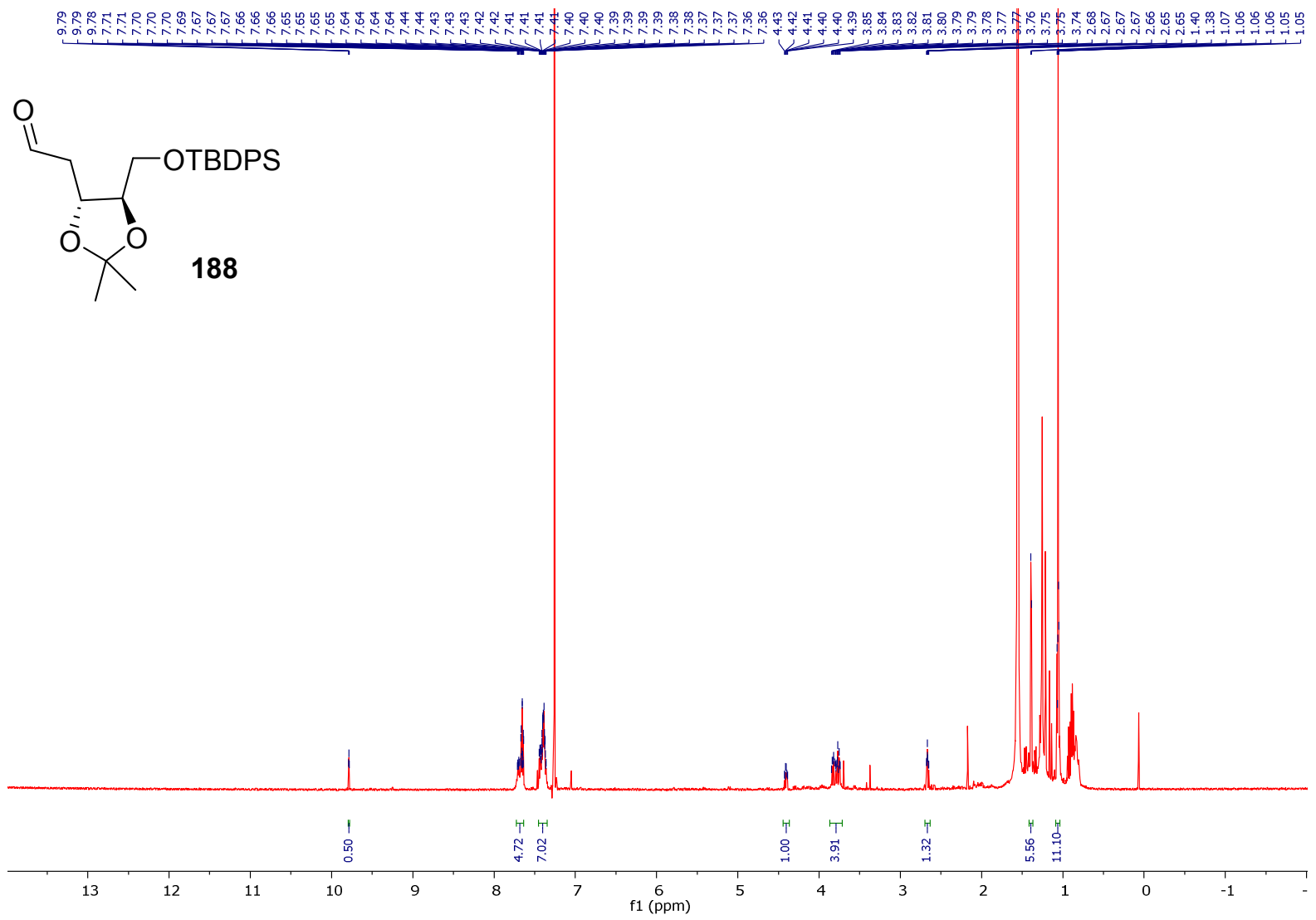



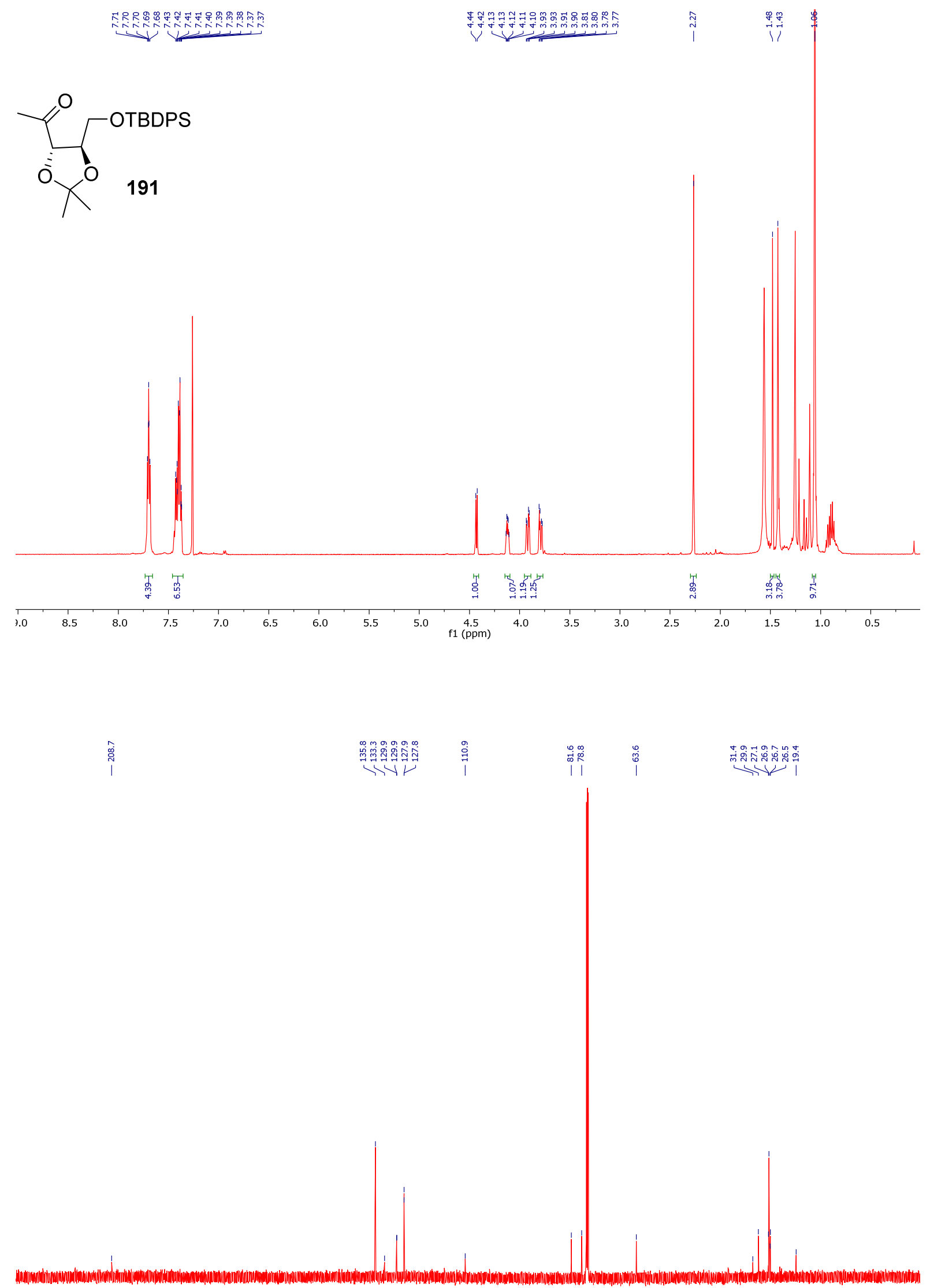

$\begin{array}{llllllllllllllllllllllllllll}230 & 220 & 210 & 200 & 190 & 180 & 170 & 160 & 150 & 140 & 130 & 120 & 110 & 100 & 90 & 80 & 70 & 60 & 50 & 40 & 30 & 20 & 10 & 0 & -10\end{array}$ 

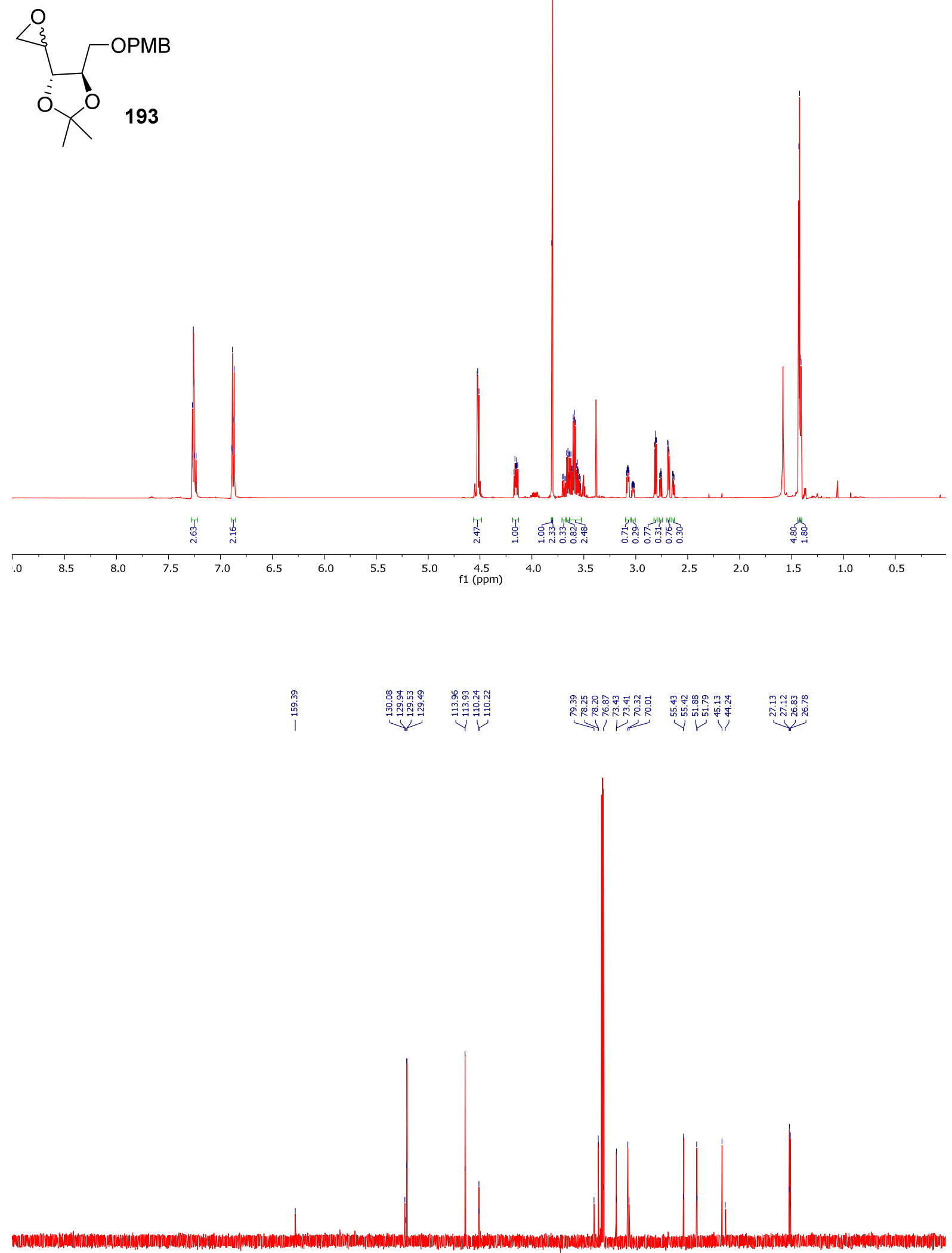

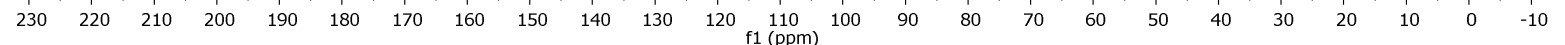




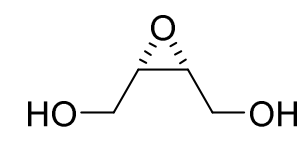

199

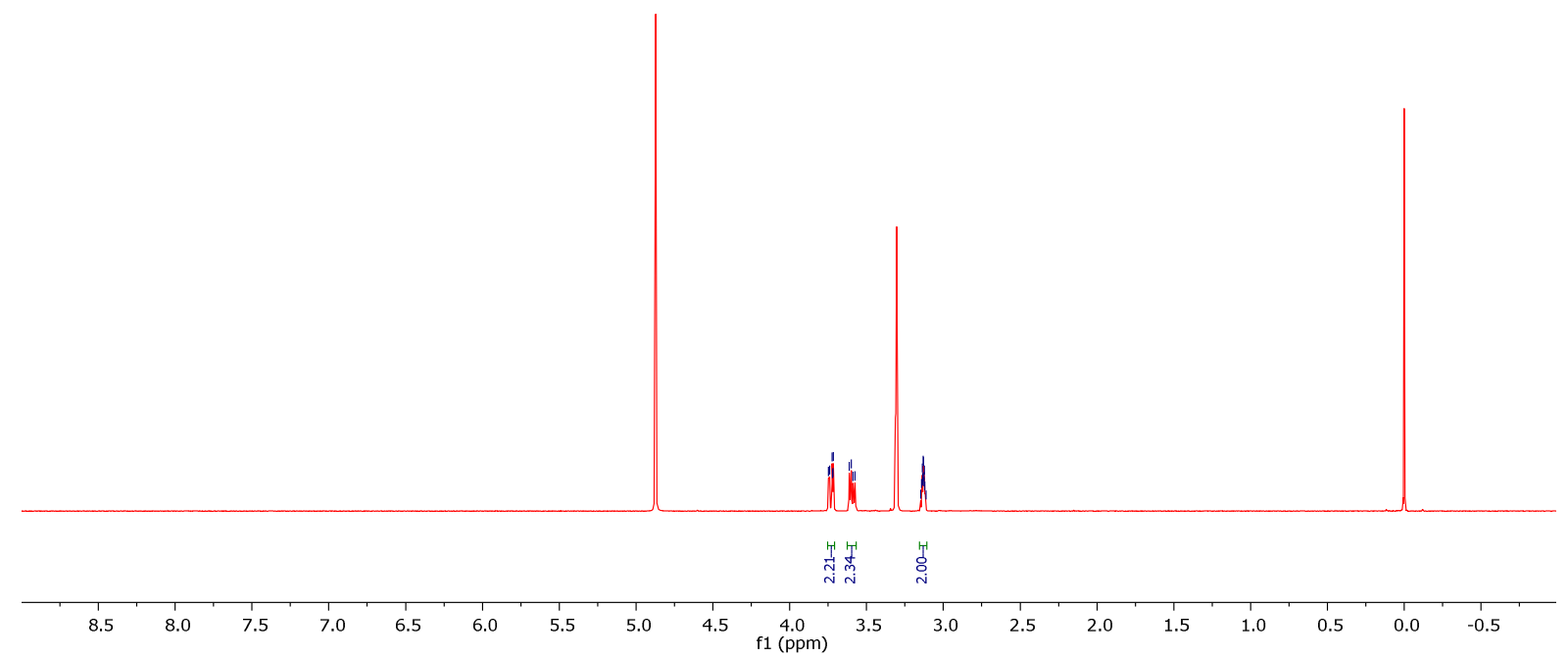

กับ

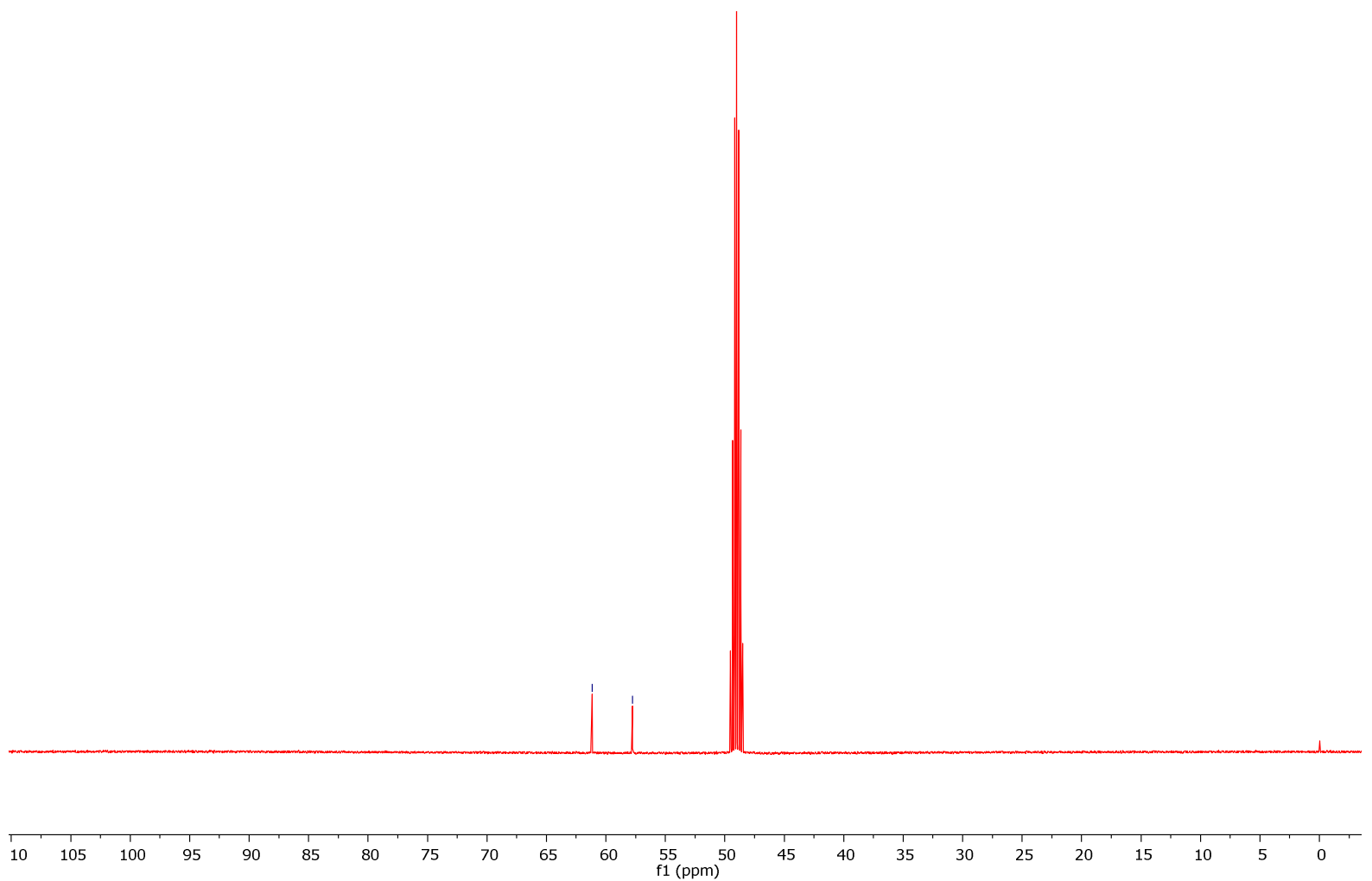



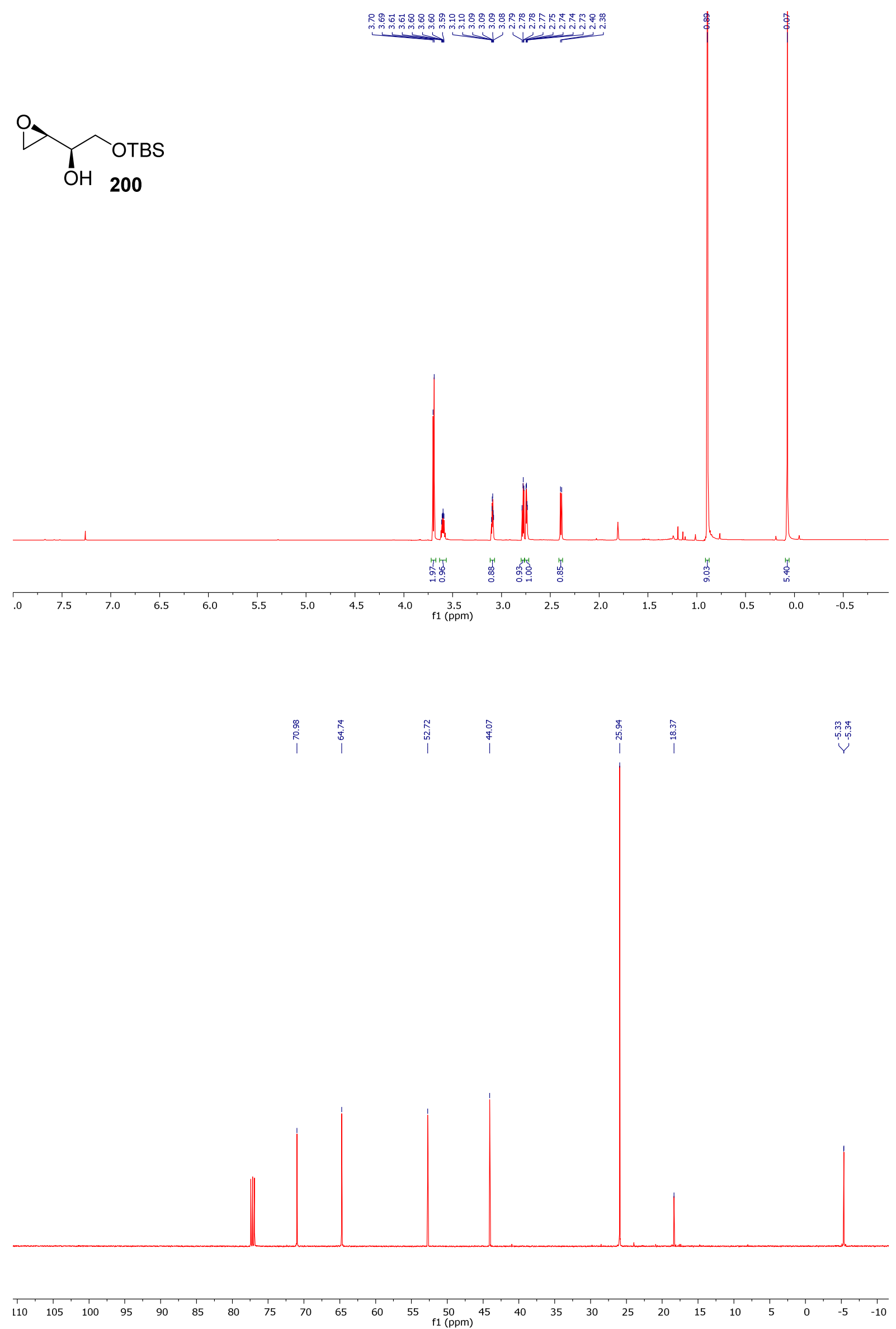

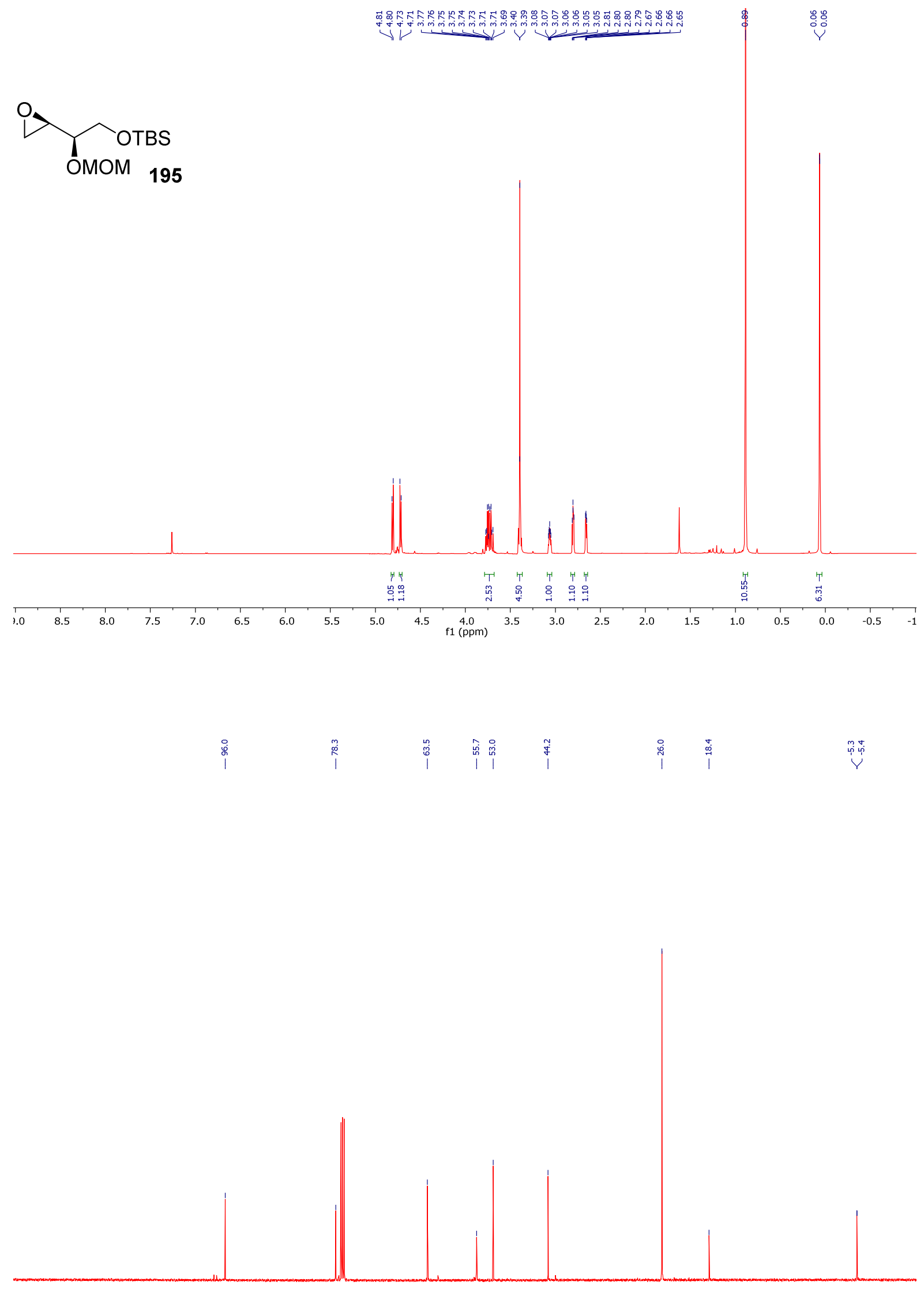

$\begin{array}{llllllllllllllllllllllllllllllllllllll}125 & 120 & 115 & 110 & 105 & 100 & 95 & 90 & 85 & 80 & 75 & 70 & 65 & 60 & 55 & 50 & 45 & 40 & 35 & 30 & 25 & 20 & 15 & 10 & 5 & 0 & -5 & -10\end{array}$ 

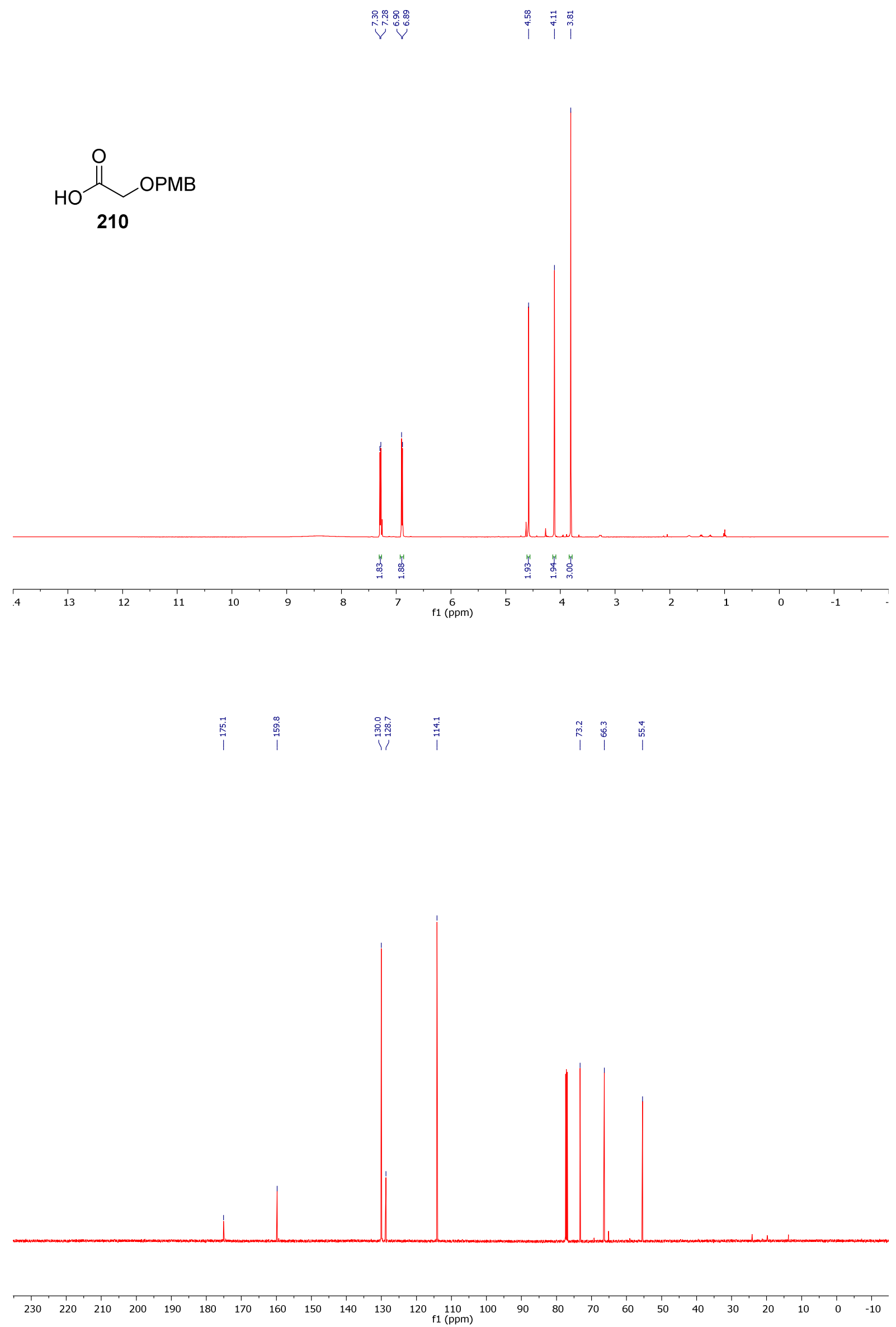
<smiles>CCCCCCC(=O)OCC(=O)N1C(=O)OCC1Br</smiles>

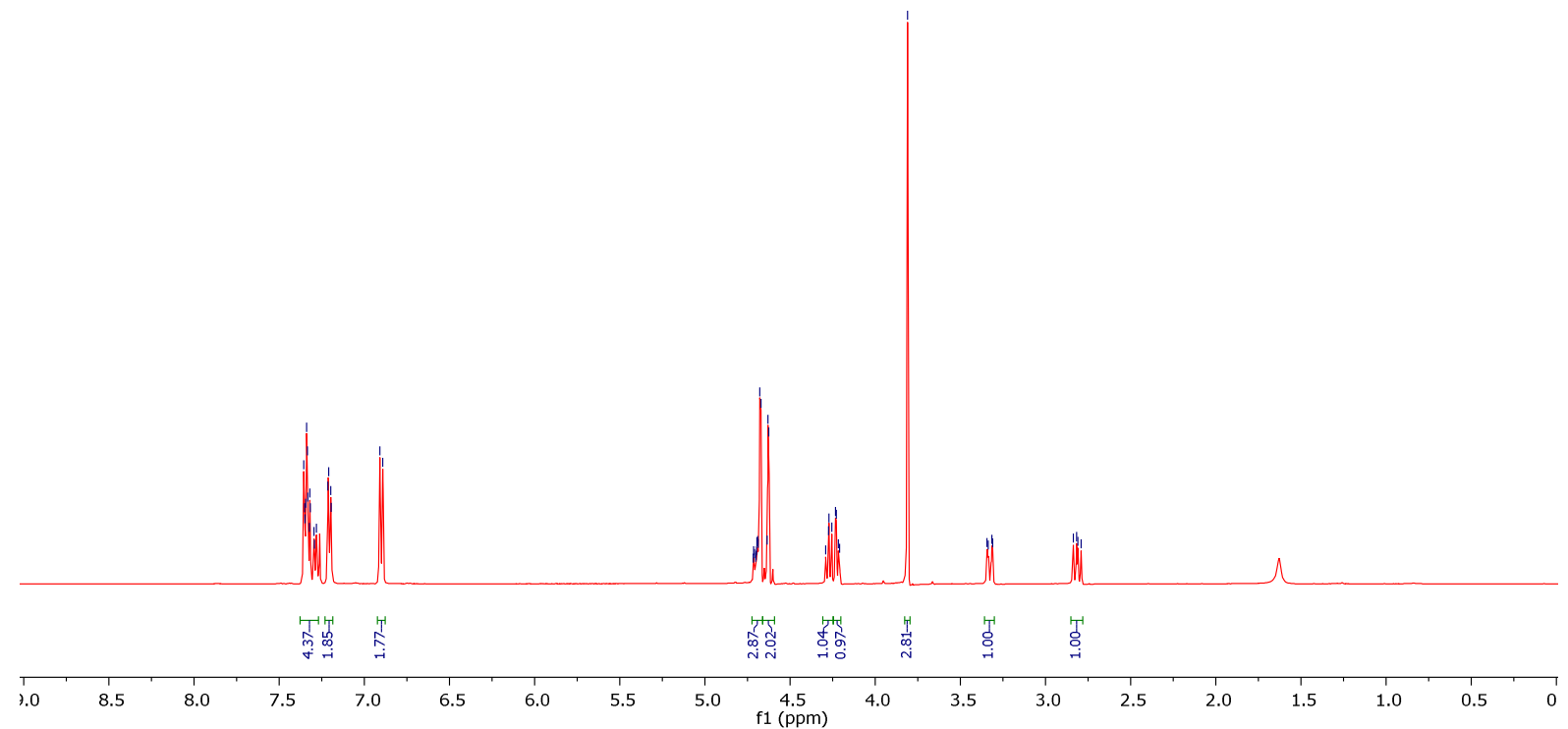

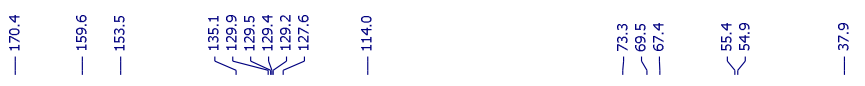

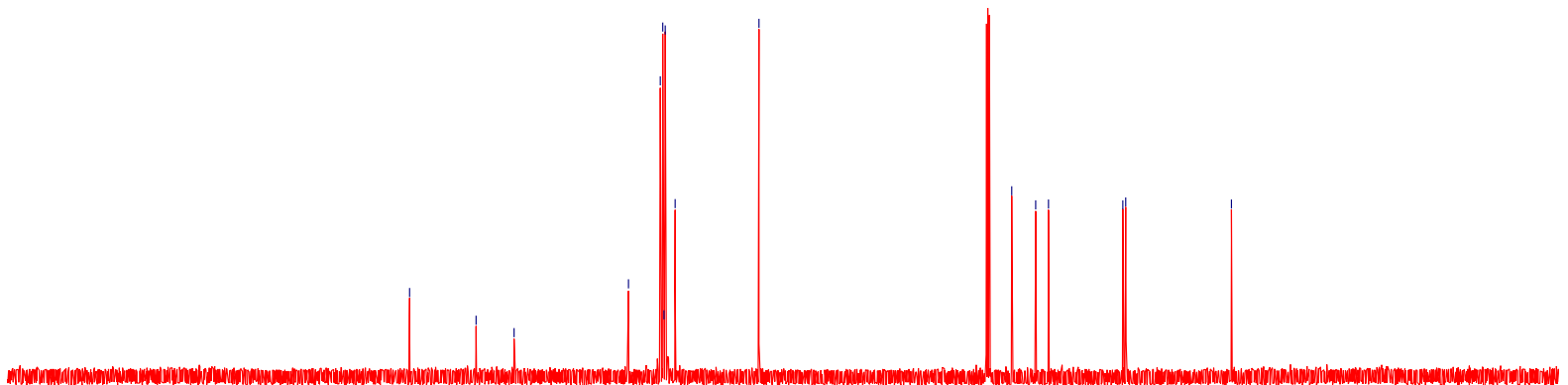

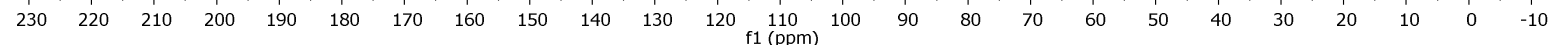


<smiles>C1CSC(CC2CO2)SC1</smiles>

203

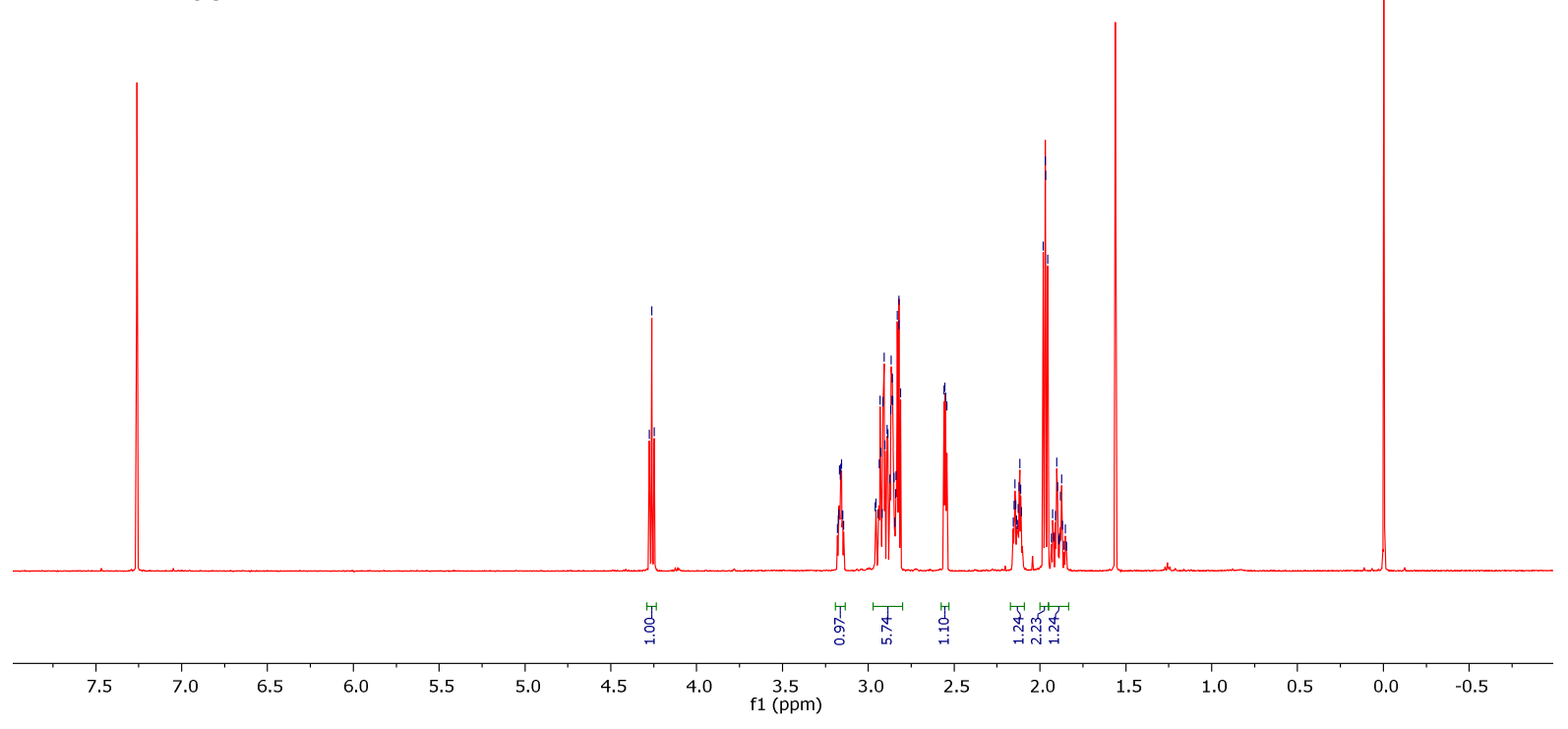

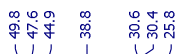

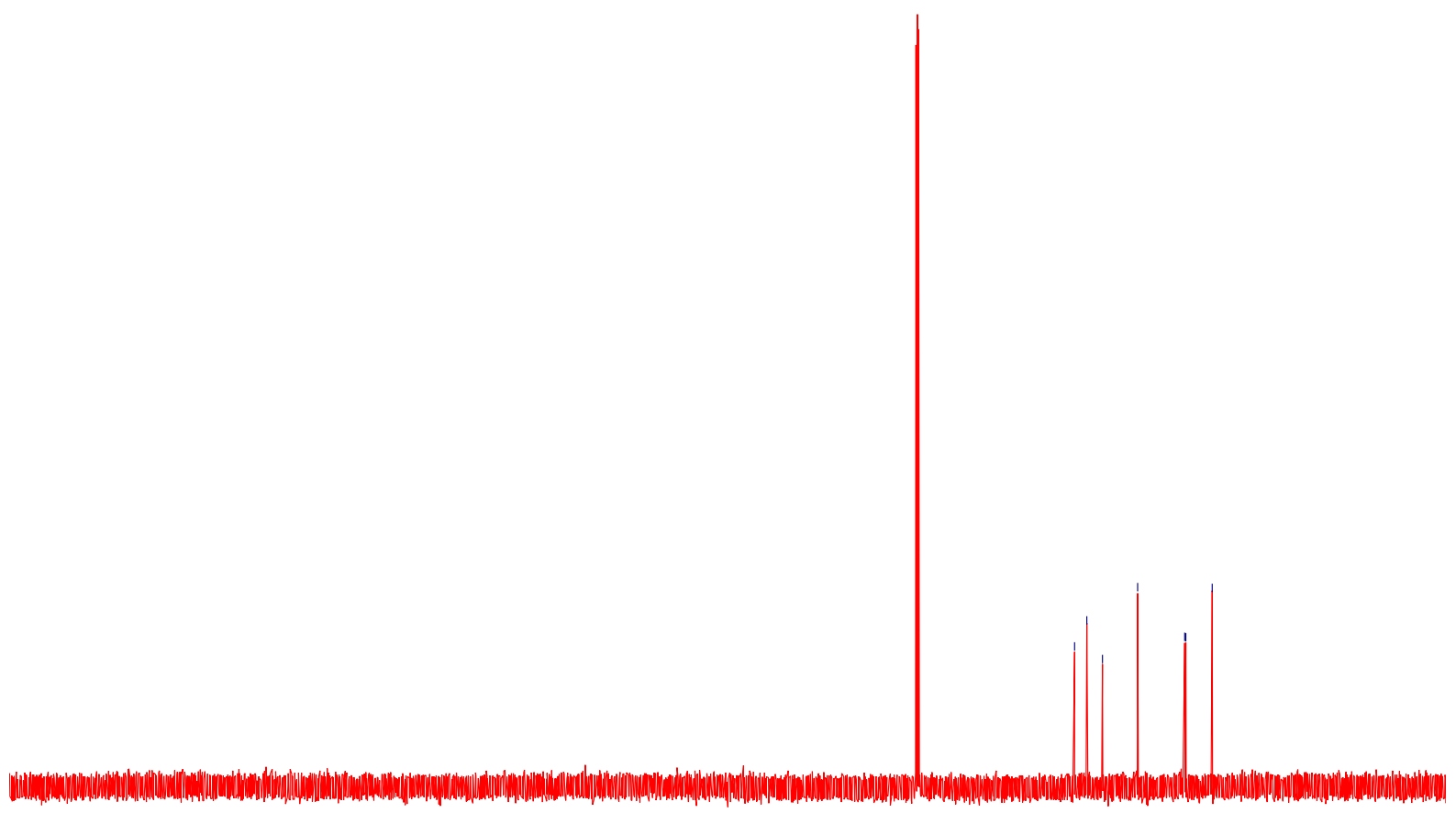

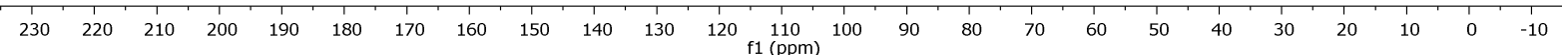


<smiles>C=CCC(O)CC1SCCCS1</smiles>

204

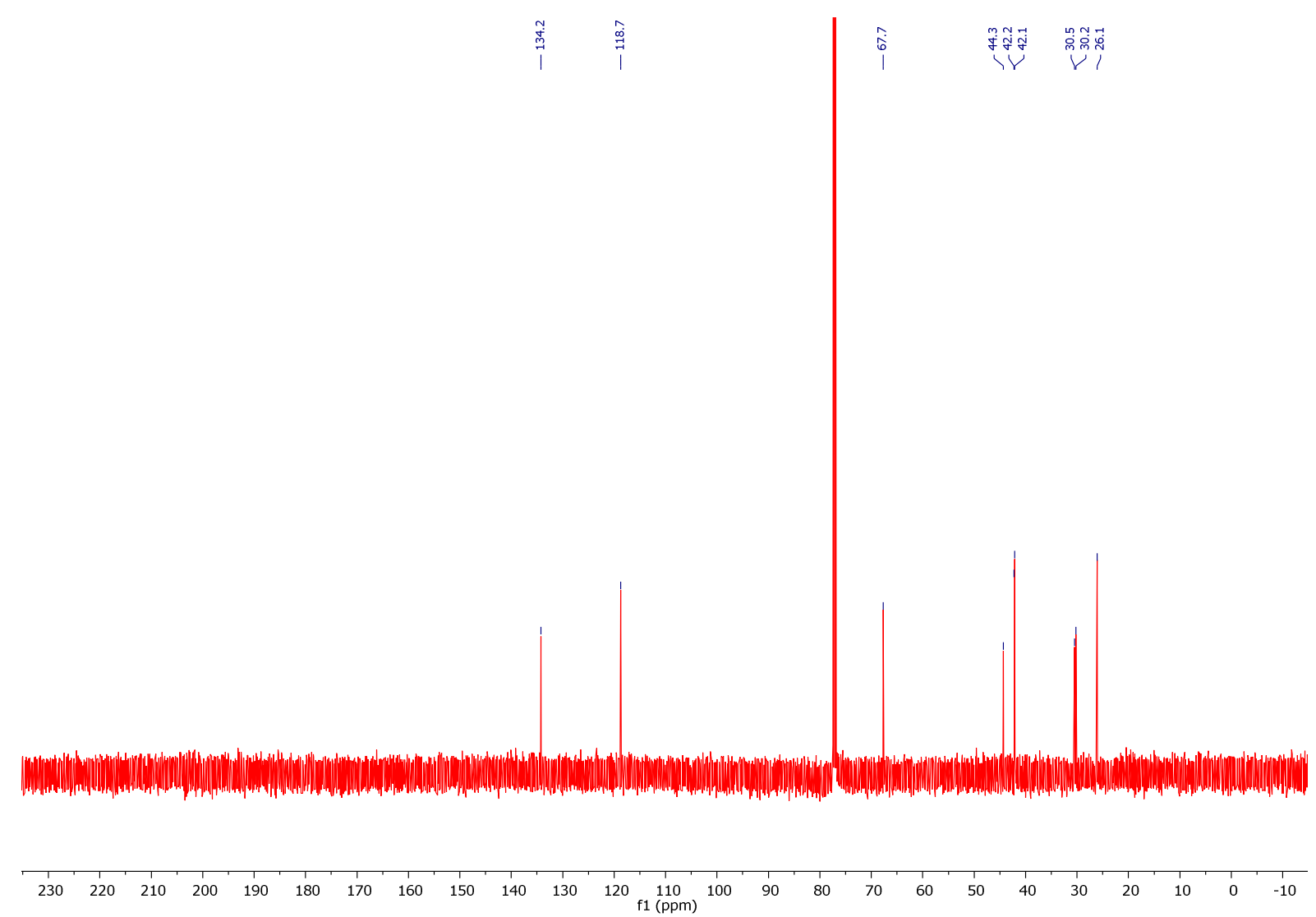



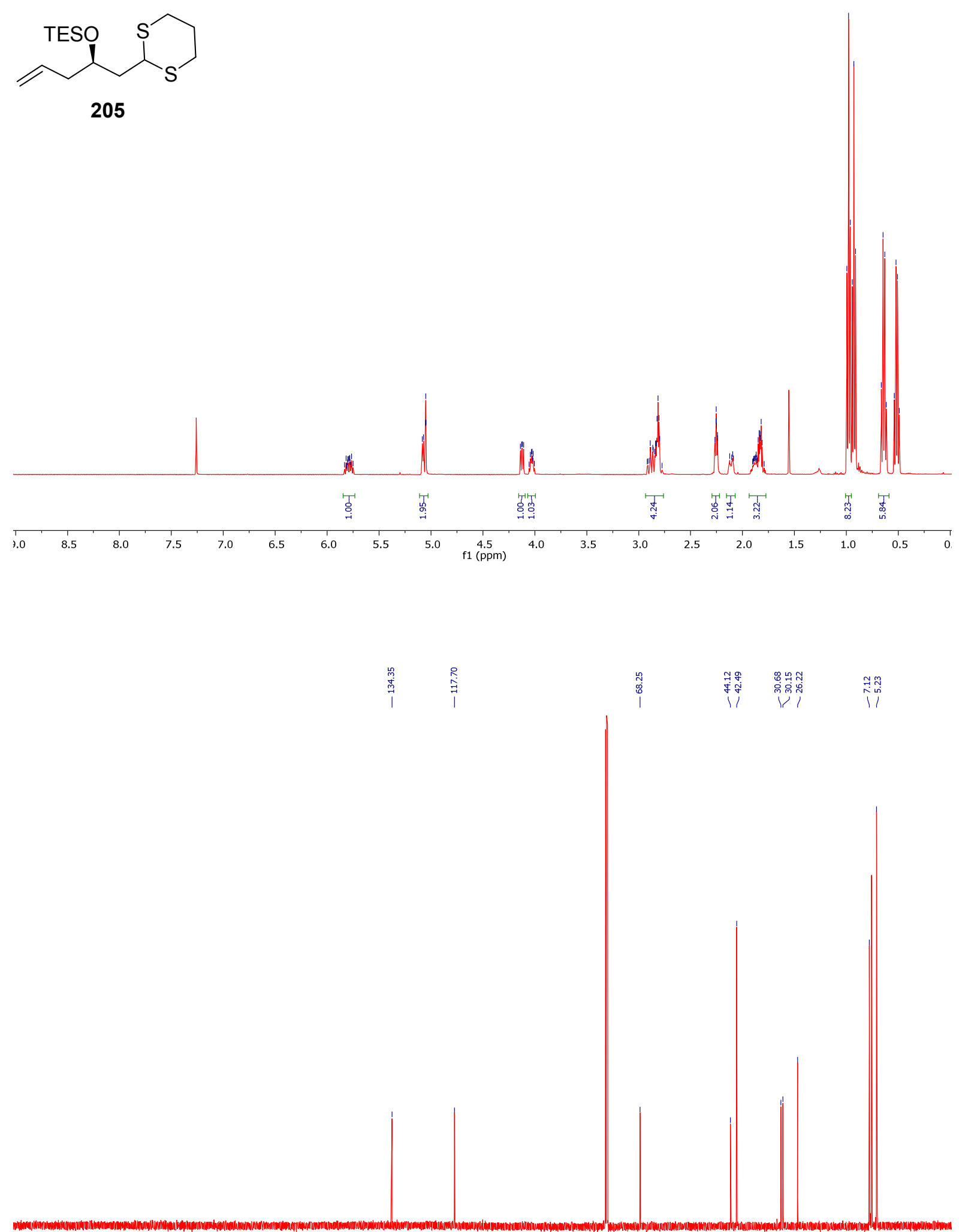

$\begin{array}{lllllllllllllllllllllllllllllll}230 & 220 & 210 & 200 & 190 & 180 & 170 & 160 & 150 & 140 & 130 & 120 & 110 & 100 & 90 & 80 & 70 & 60 & 50 & 40 & 30 & 20 & 10 & 0 & -10\end{array}$ 
<smiles>C=CCC(CC1SCCCS1)OS(C)(=O)=O</smiles>

212

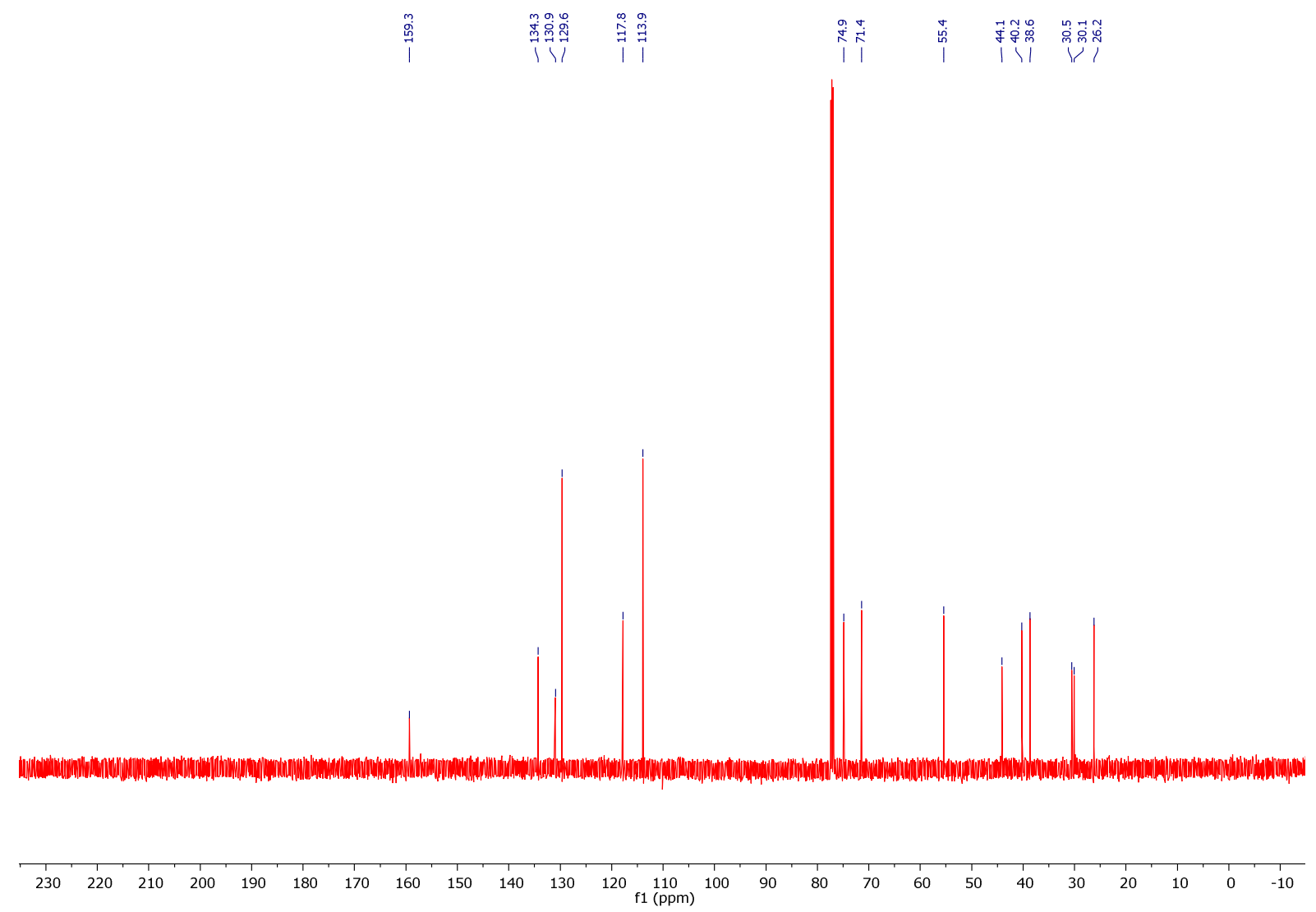


<smiles>[R16]OC(CC=C)CC=O</smiles>

213

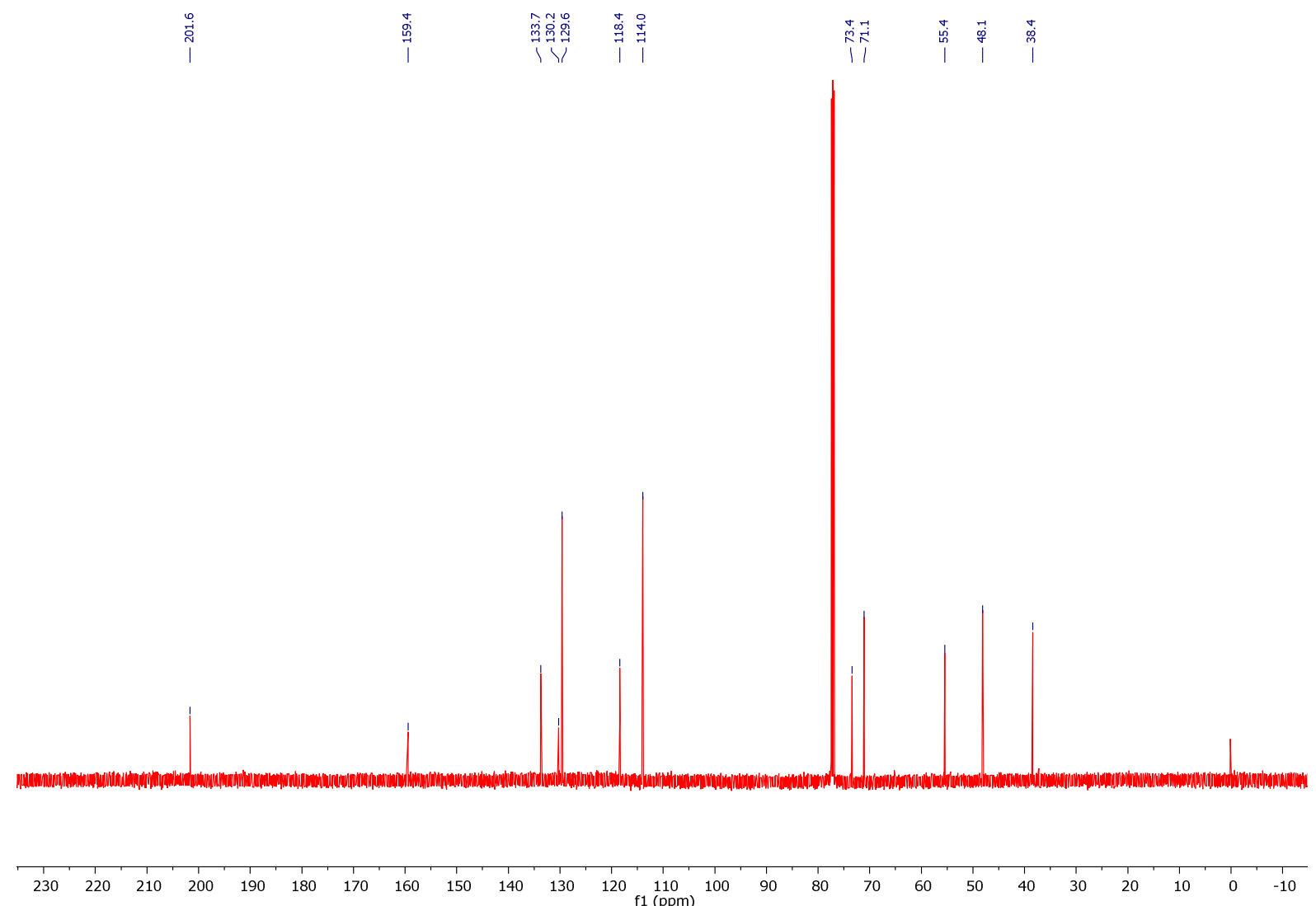




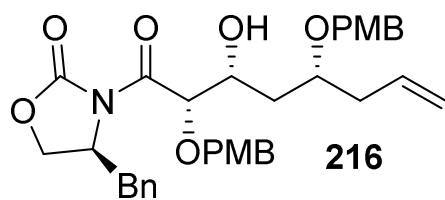

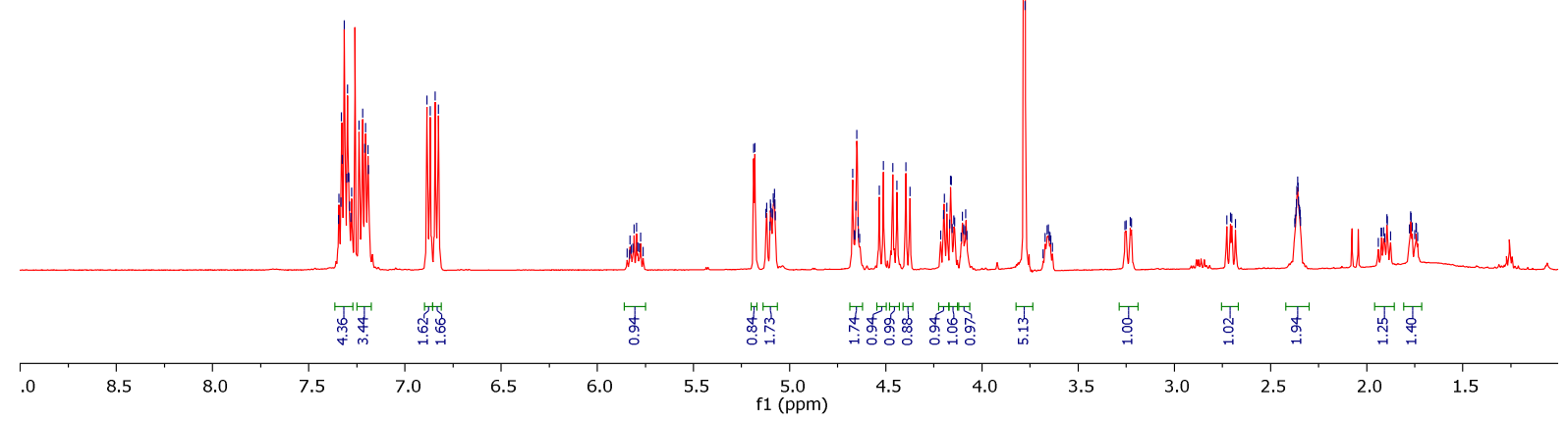

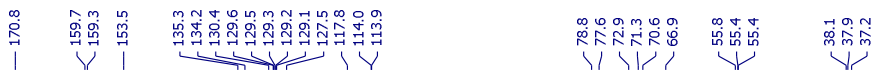

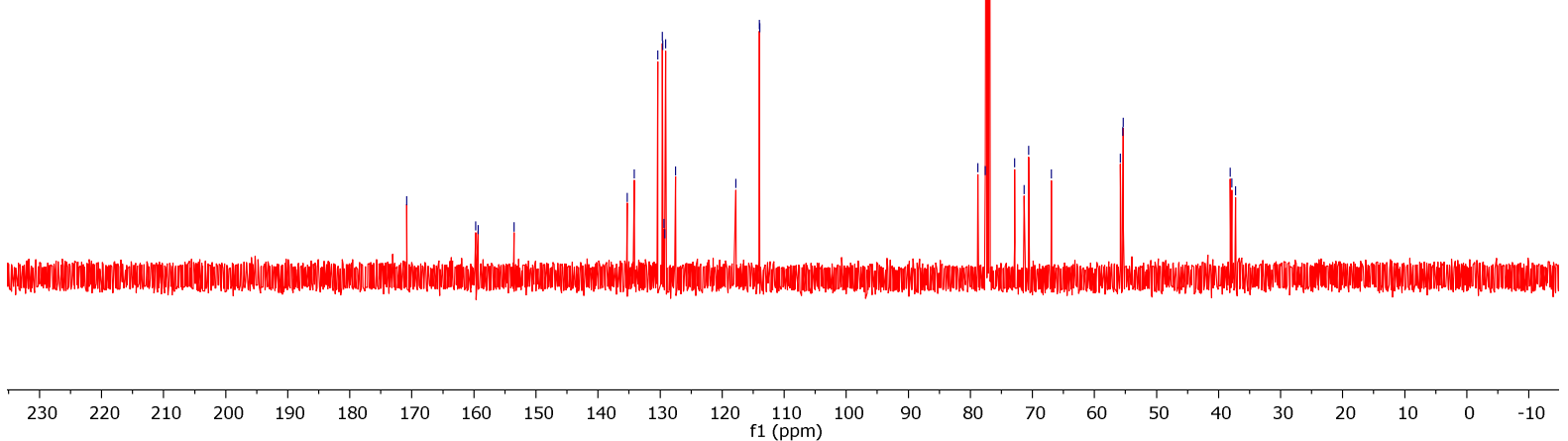



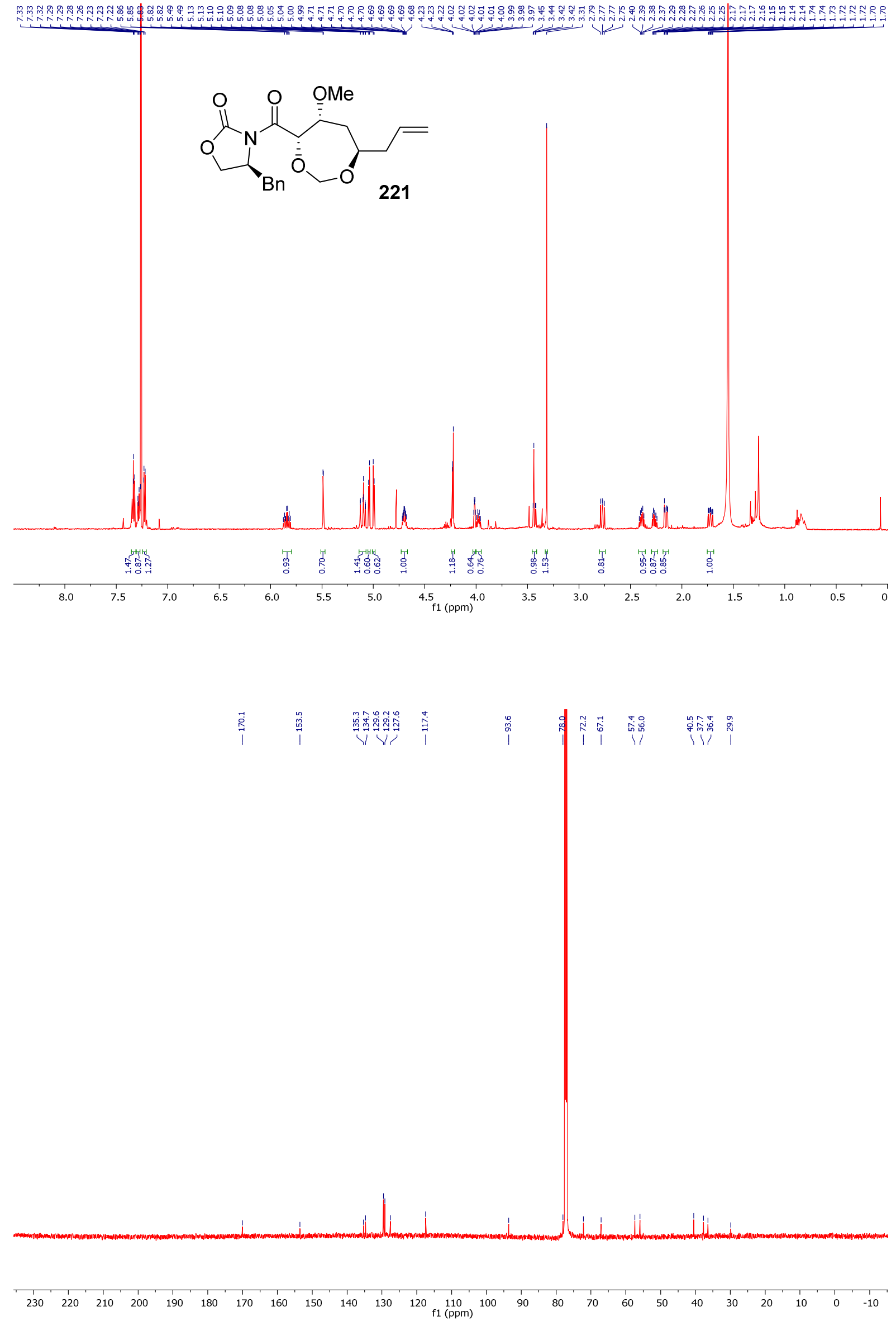

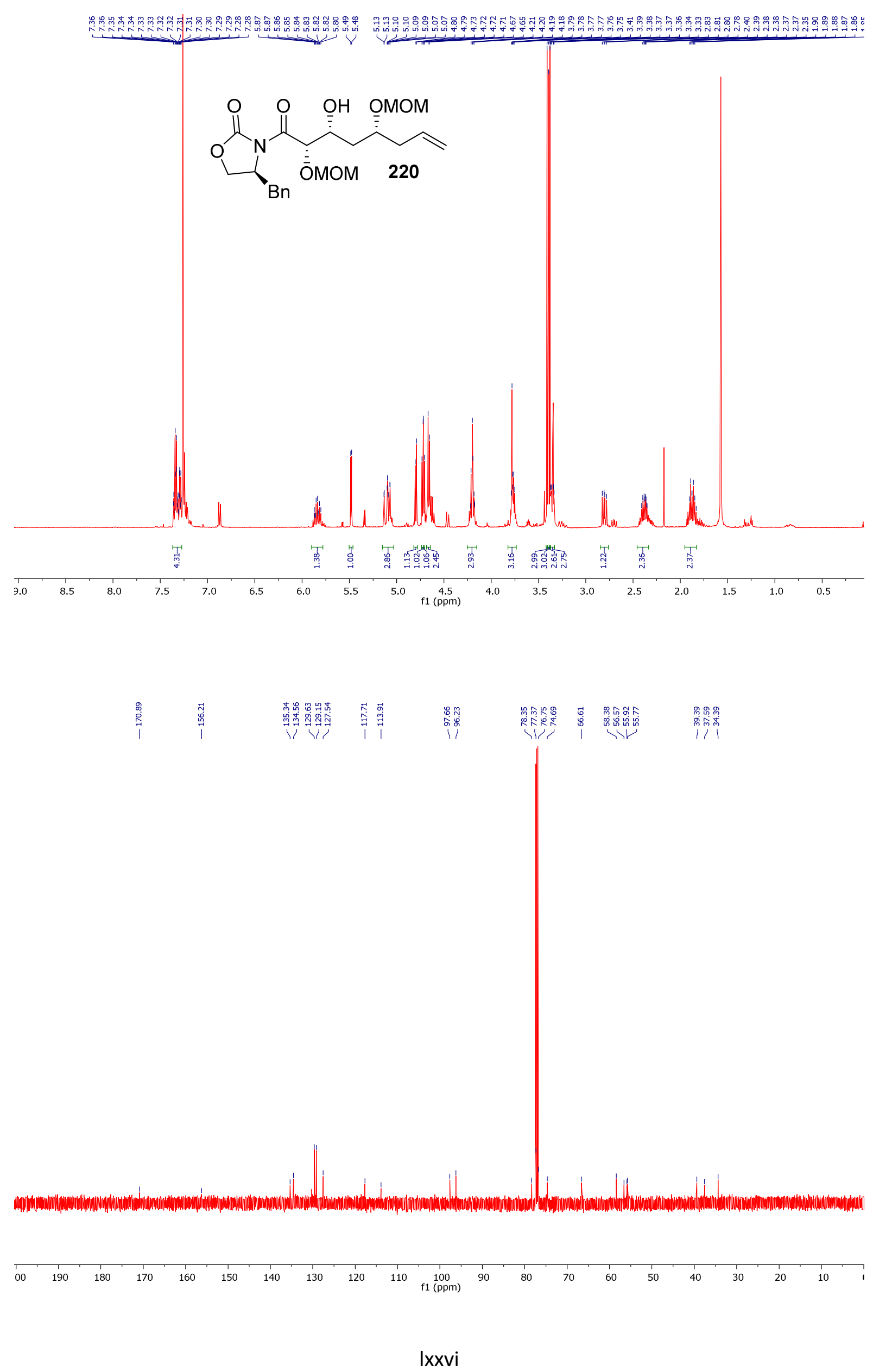
